\title{
Cleanup Verification Package for the 118-F-8:4 Fuel Storage Basin West Side Adjacent and Side Slope Soils
}

Prepared for the U.S. Department of Energy by Washington Closure Hanford

November 2007 


\section{EXECUTIVE SUMMARY}

This cleanup verification package documents completion of remedial action, sampling activities, and compliance with cleanup criteria for the 118-F-8:4 Fuel Storage Basin West Side Adjacent and Side Slope Soils (118-F-8:4 waste site). The 118-F-8:4 waste site, part of the 100-FR-1 Operable Unit, is located in the 100-F Area of the Hanford Site in southeastern Washington State. The fuel storage basin was a rectangular-shaped concrete basin located on the south side of the 105-F Reactor building. It served as an underwater collection, storage, and transfer facility for irradiated fuel elements discharged from the reactor. The reactor was in operation between February 1945 and June 1965.

Remediation of the 118-F-8:4 waste site began on March 14, 2007, and was completed on June 22, 2007. Remedial action activities involved removing the uncontaminated overburden, the buried contaminated material, and the underlying contaminated soil for disposal.

Following excavation, verification sampling was performed to determine if the remedial action was adequate to support interim site closure. Results of the verification sampling, laboratory analyses, data evaluations, and modeling for the 118-F-8:4 waste site indicate that all remedial action objectives for direct exposure, protection of groundwater, and protection of the Columbia River have been met (see Table ES-1). 
Table ES-1. Summary of Remedial Action Goals for the 118-F-8:4 Fuel Storage Basin. (2 Pages)

\begin{tabular}{|c|c|c|c|c|}
\hline $\begin{array}{l}\text { Regulatory } \\
\text { Requirement }\end{array}$ & Remedial Action Goals & Results & $\begin{array}{l}\text { Remedial } \\
\text { Action } \\
\text { Objectives } \\
\text { Attained? } \\
\end{array}$ & Ref. \\
\hline $\begin{array}{l}\text { Direct Exposure - } \\
\text { Radionuclides }\end{array}$ & $\begin{array}{l}\text { Attain } 15 \mathrm{mrem} / \mathrm{yr} \text { dose } \\
\text { rate above background } \\
\text { over } 1,000 \text { years. }\end{array}$ & $\begin{array}{l}\text { The maximum all-pathways dose rate } \\
\text { calculated by RESRAD is } 1.70 \text { and } \\
1.31 \text { mrem/yr at year zero }(2007) \text { from } \\
\text { the overburden stockpile area and } \\
\text { combined excavation shallow and } \\
\text { deep zone, respectively. }\end{array}$ & Yes & $a$ \\
\hline $\begin{array}{l}\text { Direct Exposure - } \\
\text { Nonradionuclides }\end{array}$ & $\begin{array}{l}\text { Attain individual } \\
\text { COC/COPC RAGs. }\end{array}$ & $\begin{array}{l}\text { All individual COC concentrations are } \\
\text { below the direct exposure criteria. }\end{array}$ & Yes & $a, b$ \\
\hline \multirow[t]{4}{*}{$\begin{array}{l}\text { Nonradionuclide } \\
\text { Risk Requirements }\end{array}$} & $\begin{array}{l}\text { Attain hazard quotient of } \\
<1 \text { for all individual } \\
\text { noncarcinogens. }\end{array}$ & All individual hazard quotients are $<1$. & \multirow{4}{*}{ Yes } & C \\
\hline & $\begin{array}{l}\text { Attain a cumulative } \\
\text { hazard quotient of }<1 \text { for } \\
\text { noncarcinogens. }\end{array}$ & $\begin{array}{l}\text { The cumulative hazard quotient }(3.9 \times \\
\left.10^{-2}\right) \text { is }<1 \text {. }\end{array}$ & & c \\
\hline & $\begin{array}{l}\text { Attain an excess cancer } \\
\text { risk of }<1 \times 10^{-6} \text { for } \\
\text { individual carcinogens. }\end{array}$ & $\begin{array}{l}\text { All individual excess carcinogenic risk } \\
\text { values are }<1 \times 10^{-6} \text {. }\end{array}$ & & C \\
\hline & $\begin{array}{l}\text { Attain a total excess } \\
\text { cancer risk of }<1 \times 10^{-5} \\
\text { for carcinogens. }\end{array}$ & $\begin{array}{l}\text { The cumulative excess carcinogenic } \\
\text { risk value }\left(2.6 \times 10^{-7}\right) \text { is }<1 \times 10^{-5} \text {. }\end{array}$ & & C \\
\hline \multirow[t]{2}{*}{$\begin{array}{l}\text { Groundwater/River } \\
\text { Protection - } \\
\text { Radionuclides }\end{array}$} & $\begin{array}{l}\text { Attain single COC/COPC } \\
\text { groundwater and river } \\
\text { protection RAGS. }\end{array}$ & $\begin{array}{l}\text { Cesium-137, cobalt- } 60 \text {, nickel- } 63 \text {, and } \\
\text { strontium-90 are calculated to reach } \\
\text { groundwater in the } 1,000 \text { years of the } \\
\text { RESRAD model run. However, none } \\
\text { of these constituents is predicted to } \\
\text { migrate to groundwater (and thus the } \\
\text { Columbia River) at concentrations } \\
\text { exceeding groundwater or river } \\
\text { criteria within } 1,000 \text { years. Therefore, } \\
\text { residual concentrations achieve the } \\
\text { remedial action objectives for } \\
\text { groundwater and river protection. }\end{array}$ & \multirow[t]{2}{*}{ Yes } & $a$ \\
\hline & $\begin{array}{l}\text { Attain national primary } \\
\text { drinking water } \\
\text { regulations: }^{d} \text { 4-mrem/yr } \\
\text { (beta/gamma) dose rate } \\
\text { to target receptor/organs. }\end{array}$ & $\begin{array}{l}\text { All organ-specific doses are below the } \\
\text { 4-mrem/yr dose standard. }\end{array}$ & & e \\
\hline
\end{tabular}




\section{Table ES-1. Summary of Remedial Action Goals for the 118-F-8:4 Fuel Storage Basin. (2 Pages)}

\begin{tabular}{|c|c|c|c|c|}
\hline $\begin{array}{l}\text { Regulatory } \\
\text { Requirement }\end{array}$ & Remedial Action Goals & Results & \begin{tabular}{|l|} 
Remedial \\
Action \\
Objectives \\
Attained? \\
\end{tabular} & Ref. \\
\hline & $\begin{array}{l}\text { Meet drinking water } \\
\text { standards for alpha } \\
\text { emitters: the more } \\
\text { stringent of } 15 \mathrm{pCi} / \mathrm{L} \mathrm{MCL} \\
\text { or } 1 / 25^{\text {th }} \text { of the derived } \\
\text { concentration guide for } \\
\text { DOE Order } 5400.5 .^{\text {f }}\end{array}$ & $\begin{array}{l}\text { RESRAD modeling indicates that the } \\
\text { alpha-emitting COCs will not impact } \\
\text { groundwater. Therefore, the alpha } \\
\text { activity is } 0 \mathrm{pCi} / \mathrm{L} \text { for all years. }\end{array}$ & Yes & e \\
\hline & $\begin{array}{l}\text { Meet total uranium } \\
\text { standard of } 21.2 \mathrm{pCi} / \mathrm{L} .{ }^{\mathrm{g}}\end{array}$ & $\begin{array}{l}\text { The total uranium COCs } \\
\text { (uranium-233/234, uranium-235, and } \\
\text { uranium-238) are present at } \\
\text { concentrations less than natural } \\
\text { background. }\end{array}$ & Yes & $a$ \\
\hline $\begin{array}{l}\text { Groundwater/River } \\
\text { Protection - } \\
\text { Nonradionuclides }\end{array}$ & $\begin{array}{l}\text { Attain individual } \\
\text { nonradionuclide } \\
\text { groundwater and river } \\
\text { cleanup requirements. }\end{array}$ & $\begin{array}{l}\text { Residual concentrations of lead, } \\
\text { mercury, and Aroclor- } 1260 \text { exceeded } \\
\text { soil RAGs for the protection of } \\
\text { groundwater and/or the Columbia } \\
\text { River. However, it is predicted that } \\
\text { these constituents will not migrate to } \\
\text { groundwater (and thus the Columbia } \\
\text { River) at concentrations exceeding } \\
\text { groundwater or river criteria within } \\
1,000 \text { years. Therefore, residual } \\
\text { concentrations achieve the remedial } \\
\text { action objectives for groundwater and } \\
\text { river protection. }\end{array}$ & Yes & h \\
\hline \multirow{2}{*}{$\begin{array}{l}\text { Other Supporting } \\
\text { Information }\end{array}$} & \multicolumn{3}{|c|}{ Sample design calculation brief. } & $\mathrm{i}$ \\
\hline & \multicolumn{3}{|c|}{ Variance sampling calculation briefs } & $\mathrm{j}, \mathrm{k}$ \\
\hline \multicolumn{5}{|c|}{ 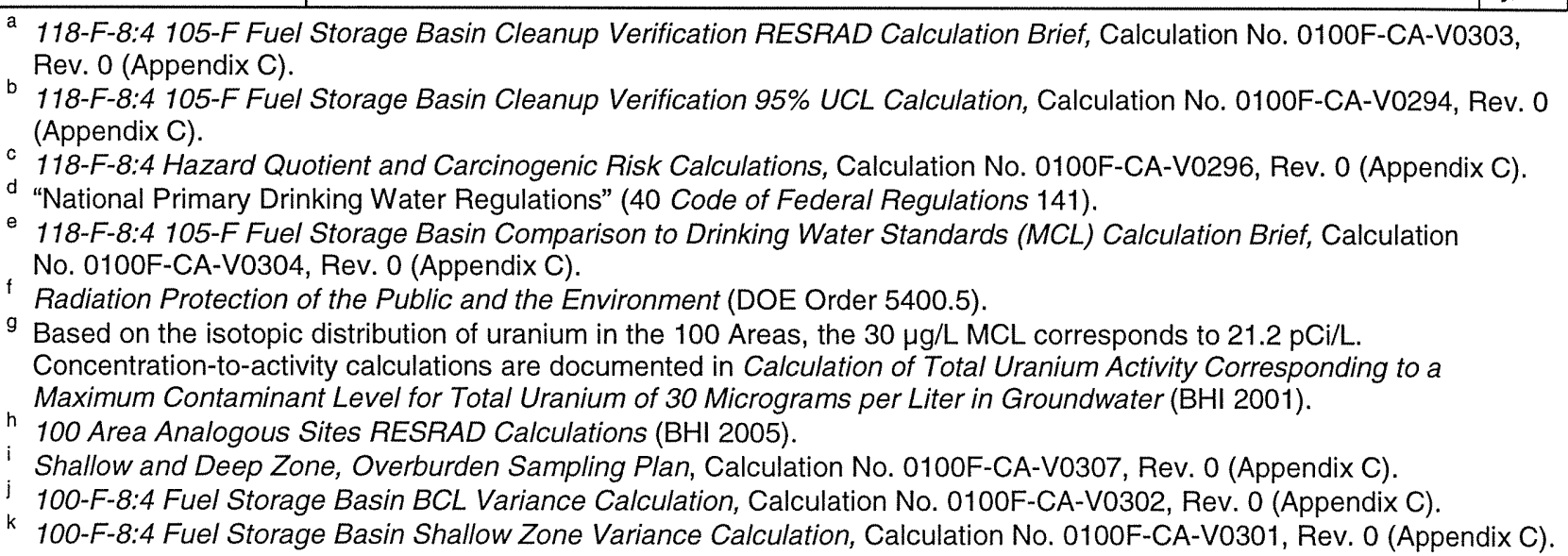 } \\
\hline \multicolumn{5}{|c|}{$\begin{array}{ll}\mathrm{COC}=\text { contaminant of concern } & \text { RAG }=\text { remedial action goal } \\
\mathrm{COPC}=\text { contaminant of potential concern } & \text { RESRAD }=\text { RESidual RADioactivity (dose model) } \\
\mathrm{MCL}=\text { maximum contaminant level (drinking water standard) } & \end{array}$} \\
\hline
\end{tabular}


A comparison against ecological risk screening levels has been made for the site contaminants of concern and other constituents. Screening levels were not exceeded for the site constituents, with the exception of aluminum, antimony, boron, manganese, mercury, selenium, and vanadium. Exceedance of screening values does not necessarily indicate the existence of risk to ecological receptors. It is believed that the presence of these constituents does not pose a risk to ecological receptors because concentrations of aluminum, antimony, manganese, and vanadium are below site background levels; mercury and selenium are within the range of Hanford Site background levels; and boron concentrations are consistent with concentrations at other locations on the Hanford Site (no established background value is available for boron). A more complete quantitative ecological risk assessment will be presented in the baseline risk assessment for the river corridor portion of the Hanford Site and will be used to support the final closeout decision for this site.

This evaluation supports a reclassification of this waste site to Interim Closed Out, in accordance with the Hanford Federal Facility Agreement and Consent Order (Ecology et al. 1989) and the Tri-Party Agreement Handbook Management Procedures, Waste Site Reclassification Guideline TPA-MP-14 (DOE-RL 2007). A copy of the waste site reclassification form is included as Attachment ES-1. 
Attachment ES-1. Waste Site Reclassification Form.

\begin{tabular}{|c|c|c|}
\hline \multirow{2}{*}{ Date Submitted: $11 / 05 / 07$} & WASTE SITE RECLASSIFICATION FORM & \multirow{5}{*}{ Control Number: $2007-027$} \\
\hline & Operable Unit(s): $\quad 100-F R-1$ & \\
\hline Originator: $\quad$ L.M. Dittmer & Waste Site Code: $\quad 118-F-8: 4$ & \\
\hline \multirow[t]{2}{*}{ Phone: $\quad 372-9227$} & Type of Reclassification Action: & \\
\hline & $\begin{array}{l}\text { Closed Out } \square \quad \text { Interim Closed Out } \square \text { No Action } \square \\
\text { RCRA Postclosure } \square \quad \text { Rejected } \square \quad \text { Consolidated } \square\end{array}$ & \\
\hline
\end{tabular}

This form documents agreement among parties listed authorizing classification of the subject unit as Closed Out, Interim Closed Out, No Action, RCRA Postclosure, Rejected, or Consolidated. This form also authorizes backfill of the waste management unit, if appropriate, for Closed Out and Interim Closed Out units. Final removal from the NPL of No Action and Closed Out waste management units will occur at a future date.

Description of current waste site condition:

The 118-F-8:4 Fuel Storage Basin West Side Adjacent and Side Slope Soils were portions of the 118-F-8:3 waste site deferred for remediation in December 2002. The site has been remediated and was backfilled during October 2007. Remediation, verification sampling, and RESidual RADioactivity (RESRAD) modeling have been performed in accordance with remedial action objectives and goals established by the Action Memorandum, USDOE Hanford 100 Area National Priorities List (NPL) 105-F and 105-DR Reactor Buildings and Ancillary Facilities, Hanford Site, Benton County, Washington, Washington State Department of Ecology, U.S. Environmental Protection Agency, and U.S. Department of Energy, Richland Operations Office, Richland, Washington. The selected remedy involved (1) excavating the site to the extent required to meet specified soil cleanup levels, (2) disposing of contaminated excavation materials at the Environmental Restoration Disposal Facility at the 200 Area of the Hanford Site, (3) demonstrating through verification sampling and RESRAD modeling that cleanup goals have been achieved, and (4) proposing the site for reclassification as Interim Closed Out.

Basis for reclassification:

In accordance with this evaluation, the verification sampling and modeling results support a reclassification of this site to Interim Closed Out. The current site conditions achieve the remedial action objectives established in the Action Memorandum. The results show that residual contaminant concentrations do not preclude any future uses (as bounded by the rural-residential

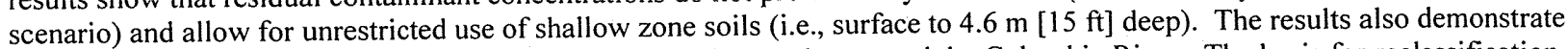
that residual contaminant concentrations are protective of groundwater and the Columbia River. The basis for reclassification is described in detail in the Cleanup Verification Package for the 118-F-8:4 Fuel Storage Basin West Side Adjacent and Side Slope Soils (CVP-2007-00004), Washington Closure Hanford, Richland, Washington. The cleanup verification package does not

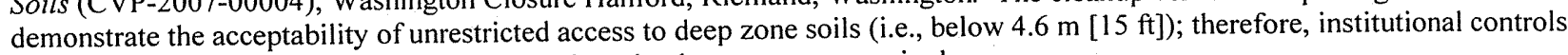
to prevent uncontrolled drilling or excavation into the deep zone are required.

Waste Site Controls:

Engineered Controls: Yes $\square$ No $\square \quad$ Institutional Controls: Yes $\square$ No $\square \quad$ O\&M requirements: Yes $\square$ No $\square$ If any of the Waste Site Controls are checked Yes specify control requirements including reference to the Record of Decision, TSD Closure Letter, or other relevant documents.

Deed restrictions to prevent uncontrolled drilling or excavation into the deep zone (greater than $4.6 \mathrm{~m}$ [ $15 \mathrm{ft}$ ] below the ground surface).

S. L. Charboneau

DOE Federal Project Director (printed)
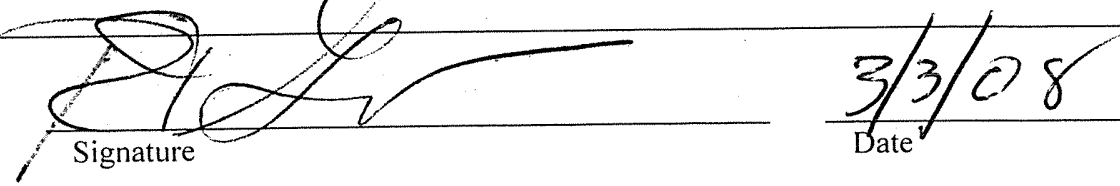

NA

Ecology Project Manager (printed)

R. A. Lobos

EPA Project Manager (printed)
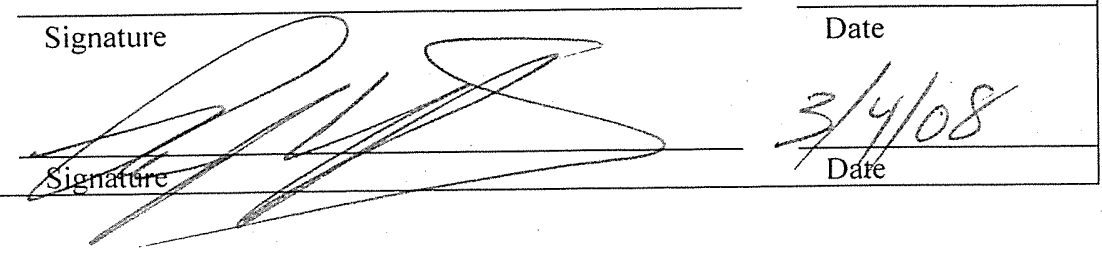



\section{CONTENTS}

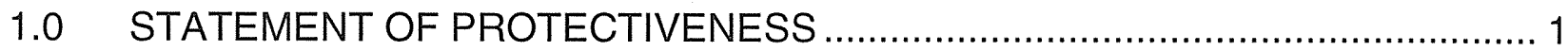

2.0 BACKGROUND AND GENERAL SITE INFORMATION ................................ 1

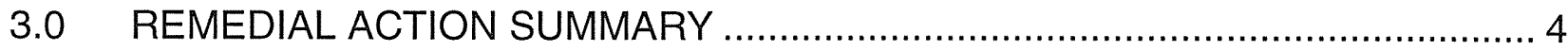

3.1 EXCAVATION AND DISPOSAL INFORMATION ................................. 4

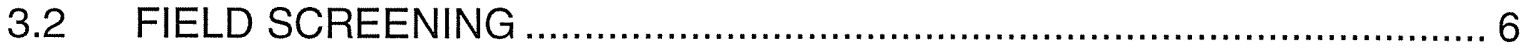

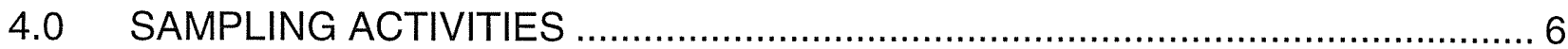

4.1 CONTAMINANTS OF CONCERN FOR VERIFICATION SAMPLING ....... 7

4.2 SAMPLING DESIGN SELECTION AND BASIS ................................... 7

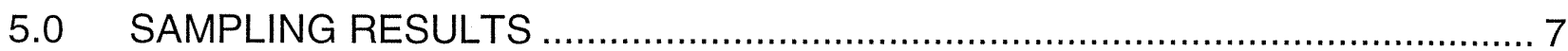

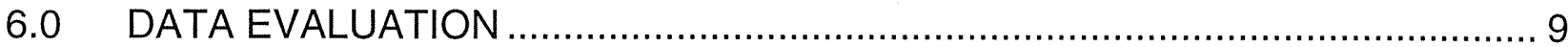

6.1 COMPARISON OF SAMPLE DATA TO RAGS .................................. 16

6.2 COMPARISON OF SAMPLE DATA TO ECOLOGICAL RISK SCREENING LEVELS ................................................................... 16

6.3 HUMAN HEALTH RISK EVALUATION FOR NONRADIONUCLIDES..... 16

6.4 WAC THREE-PART TEST FOR NONRADIONUCLIDES …................. 17

6.5 RESRAD MODELING FOR RADIONUCLIDES................................ 17

6.5.1 Attainment of Radionuclide Direct Exposure RAG ..................... 18

6.5.2 Attainment of Radionuclide Excess Lifetime Cancer Risk Goal .... 18

6.5.3 Attainment of Radionuclide Groundwater and River Protection

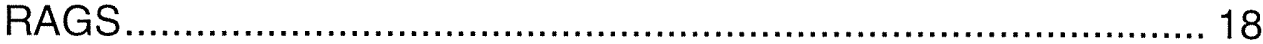

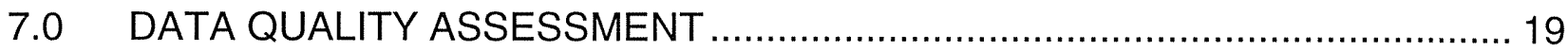

8.0 SUMMARY FOR WASTE SITE RECLASSIFICATION ................................... 20

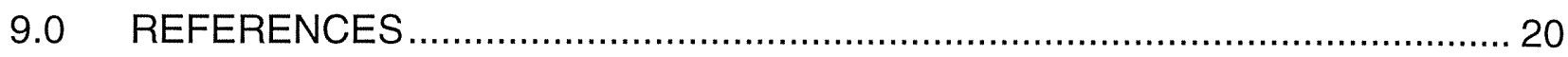

\section{APPENDICES}

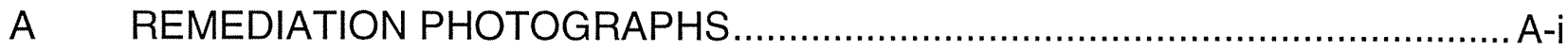

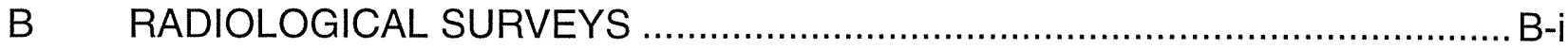

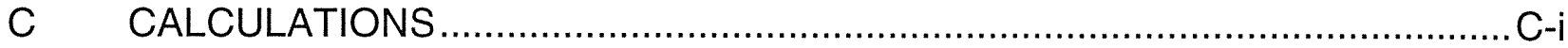

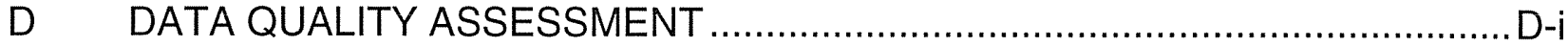




\section{FIGURES}

1. Location of the 118-F-8:4 Fuel Storage Basin West Side Adjacent and Side Slope Soils (Post-Remediation Boundary). 2

2. 105-F Reactor Plan View of Below-Grade Structures (Pre-Remediation Boundaries) ...................................................................................... 3

3. 118-F-8:4 Waste Site Post-Excavation Aerial Photograph (August 2007)............. 4

4. 118-F-8:4 Waste Site Post-Excavation Topography ..................................... 5

5. General Overview of Sample Designs for the 118-F-8:4 Waste Site....................... 8

\section{TABLES}

1. Comparison of Statistical Contaminant Concentrations to Action Levels for the 118-F-8:4 Shallow Zone Excavation Verification Sampling 10

2. Comparison of Statistical Contaminant Concentrations to Action Levels for the 118-F-8:4 Deep Zone Excavation Verification Sampling 12

3. Comparison of Statistical Contaminant Concentrations to Action Levels for the 118-F-8:4 Overburden/BCL Verification Sampling 


\section{ACRONYMS AND ABBREVIATIONS}

$\begin{array}{ll}\text { BCM } & \text { bank cubic meters } \\ \text { BCY } & \text { bank cubic yards } \\ \text { BCL } & \text { below cleanup level } \\ \text { CFR } & \text { Code of Federal Regulations } \\ \text { COC } & \text { contaminant of concern } \\ \text { DQA } & \text { data quality assessment } \\ \text { EPA } & \text { U.S. Environmental Protection Agency } \\ \text { FSB } & \text { fuel storage basin } \\ \text { GPERS } & \text { Global Positioning Environmental Radiological Surveyor } \\ \text { LARADS } & \text { Laser-Assisted Ranging and Data System } \\ \text { MCL } & \text { maximum contaminant level (drinking water standard) } \\ \text { RAG } & \text { remedial action goal } \\ \text { RDL } & \text { required detection limit } \\ \text { RDR/RAWP } & \text { remedial design report/remedial action work plan } \\ \text { RESRAD } & \text { RESidual RADioactivity dose assessment model } \\ \text { SAP } & \text { sampling and analysis plan } \\ \text { UCL } & \text { upper confidence limit } \\ \text { WAC } & \text { Washington Administrative Code }\end{array}$


CVP-2007-00004

Rev. 0 


\subsection{STATEMENT OF PROTECTIVENESS}

This report demonstrates that the 118-F-8:4 Fuel Storage Basin West Side Adjacent and Side Slope Soils (118-F-8:4 waste site) was remediated in accordance with the Removal Action Work Plan for 105-DR and 105-F Building Interim Safe Storage Projects and Ancillary Buildings (DOE-RL 2002) and meets the objectives and goals for interim closure as established in the Action Memorandum (EPA et al. 1998) and, by reference, the 100 Area Remedial Action Sampling and Analysis Plan (100 Area SAP) (DOE-RL 2005a) and the Remedial Design Report/Remedial Action Work Plan for the 100 Area (100 Area RDR/RAWP) (DOE-RL 2005b).

The results of verification sampling show that residual contaminant concentrations do not preclude any future uses (as bounded by the rural-residential scenario) and allow for unrestricted use of shallow zone soils (i.e., surface to $4.6 \mathrm{~m}$ [15 ft] deep). The results also demonstrate that residual contaminant concentrations are protective of groundwater and the Columbia River. The acceptability of unrestricted direct exposure to deep zone soils has not been demonstrated due to the presence of residual activities from multiple radionuclides; therefore, institutional controls to prevent uncontrolled

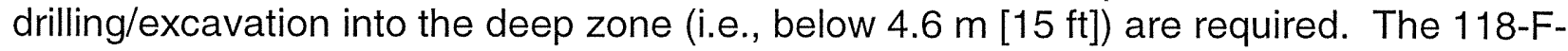
8:4 waste site is verified to meet protectiveness standards in accordance with the Action Memorandum (EPA et al. 1998).

\subsection{BACKGROUND AND GENERAL SITE INFORMATION}

The 118-F-8:4 waste site is located southwest of the 105-F Building (Figure 1) near the former location of the fuel storage basin (FSB). The FSB held dichromate-treated reactor cooling water and served as an underwater collection, storage, and transfer facility for irradiated fuel elements discharged from the reactor. The water was primarily contaminated by activated elements spilled into the FSB during fuel discharge and fission products, uranium, and transuranics introduced by fuel cladding failures. The FSB was approximately 23.8 by 21.6 by $6.4 \mathrm{~m}$ ( 78 by 71 by $21 \mathrm{ft}$ ) deep. Construction of the 105-F Reactor began in December 1943, with operations commencing in February 1945. The 105-F Reactor was retired in June 1965. Deactivation of the FSB occurred in 1970. Deactivation was accomplished by pumping the majority of the water from the basin and filling it with sand.

Decontamination and decommissioning of the FSB was completed in December 2002. The soils directly below the FSB were excavated to support interim closure (BHI 2004a). Concurrent with these activities, the Remedial Action/Waste Disposal Project began excavation and removal of the 105-F pipelines (100-F-19:2). The Remedial Action/Waste Disposal Project pipeline removal activities encroached on the east, south, and a small portion of the southwest side-slope soils of the FSB (Figure 2). Cleanup verification of these soils was documented with the interim closure of the 100F-19:2 waste site (BHI 2003). 
Figure 1. Location of the 118-F-8:4 Fuel Storage Basin West Side Adjacent and Side Slope Soils (Post-Remediation Boundary).

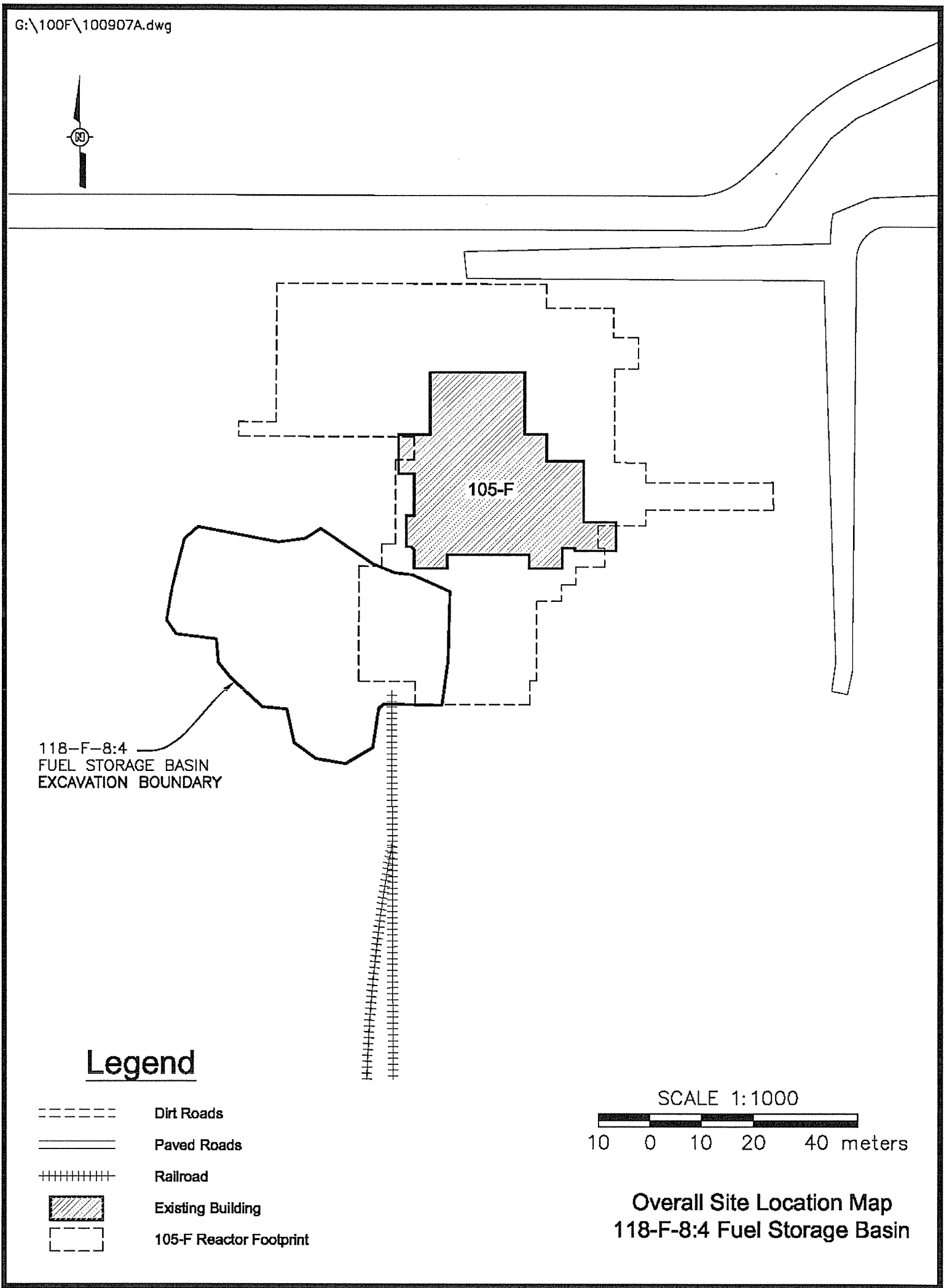


Figure 2. 105-F Reactor Plan View of Below-Grade Structures (Pre-Remediation Boundaries).

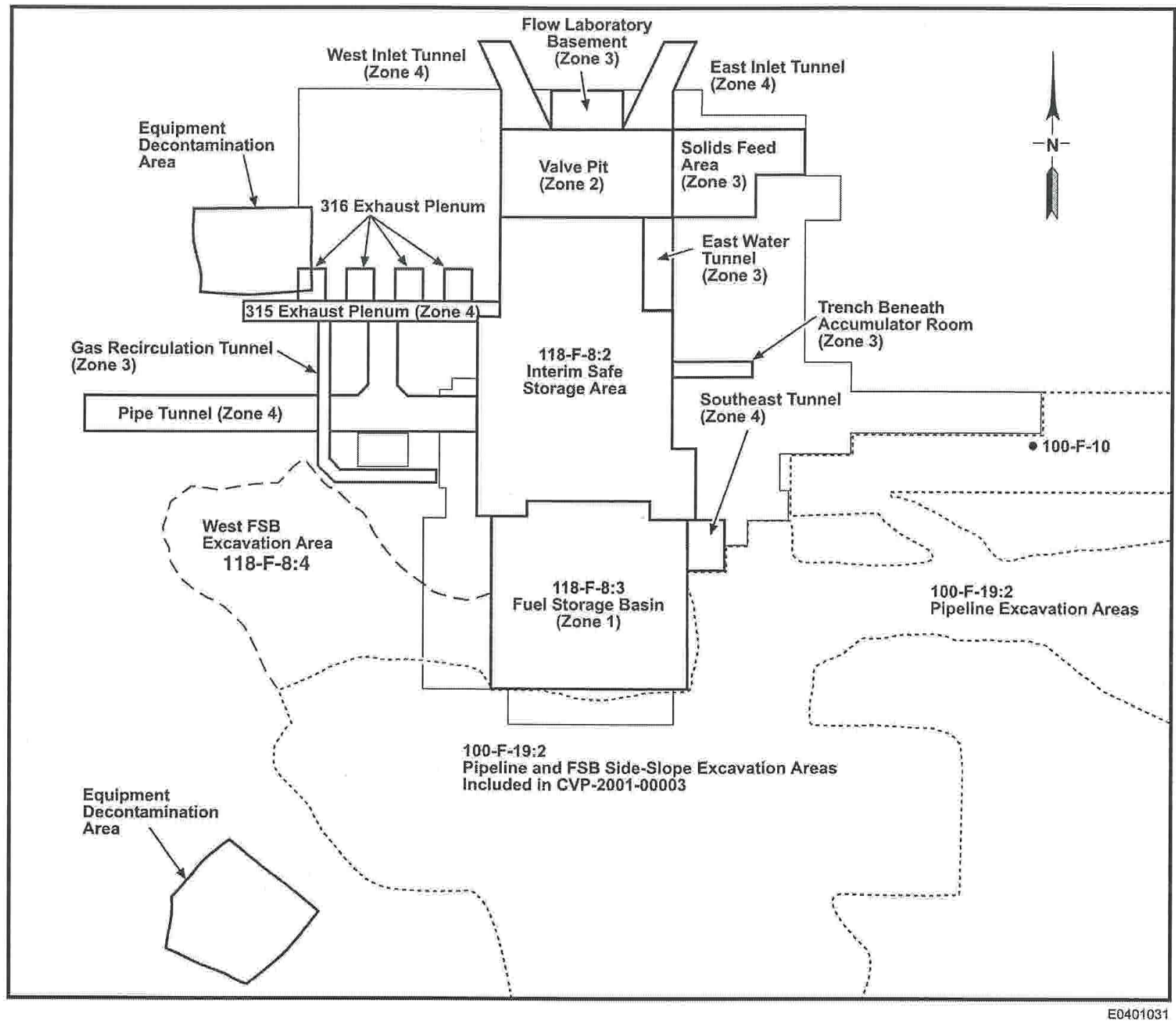




\subsection{REMEDIAL ACTION SUMMARY}

This section contains specific information about the excavation and disposal activities for the 118-F-8:4 waste site. It also contains information about the types of wastes encountered and the field screening that was conducted.

\subsection{EXCAVATION AND DISPOSAL INFORMATION}

Remedial action of the 118-F-8:4 waste site began on March 14, 2007, and was completed on June 22, 2007. Excavation of the site involved removing the uncontaminated overburden and the underlying contaminated soil. Approximately 2,950 bank cubic meters (BCM) (3,859 bank cubic yards [BCY]) of uncontaminated overburden soil was removed and stockpiled near the excavation for subsequent use as backfill. Approximately $1,650 \mathrm{BCM}(2,158 \mathrm{BCY})$ of contaminated soil was disposed at the Environmental Restoration Disposal Facility.

At the conclusion of remediation activities, the elevation at the deepest part of the excavation was approximately $118.5 \mathrm{~m}$ (389 ft) above mean sea level with a maximum depth of approximately $8 \mathrm{~m}$ ( $26 \mathrm{ft}$ ) below ground surface. The remediation excavation was approximately $1,634 \mathrm{~m}^{2}\left(17,580 \mathrm{ft}^{2}\right)$ in area. A post-excavation aerial photograph is shown in Figure 3, and the post-excavation topography is shown in Figure 4. Additional photographs of the remediation activities are included in Appendix A. No anomalies or stained soil were discovered during remediation.

\section{Figure 3. 118-F-8:4 Waste Site Post-Excavation Aerial Photograph (August 2007).}

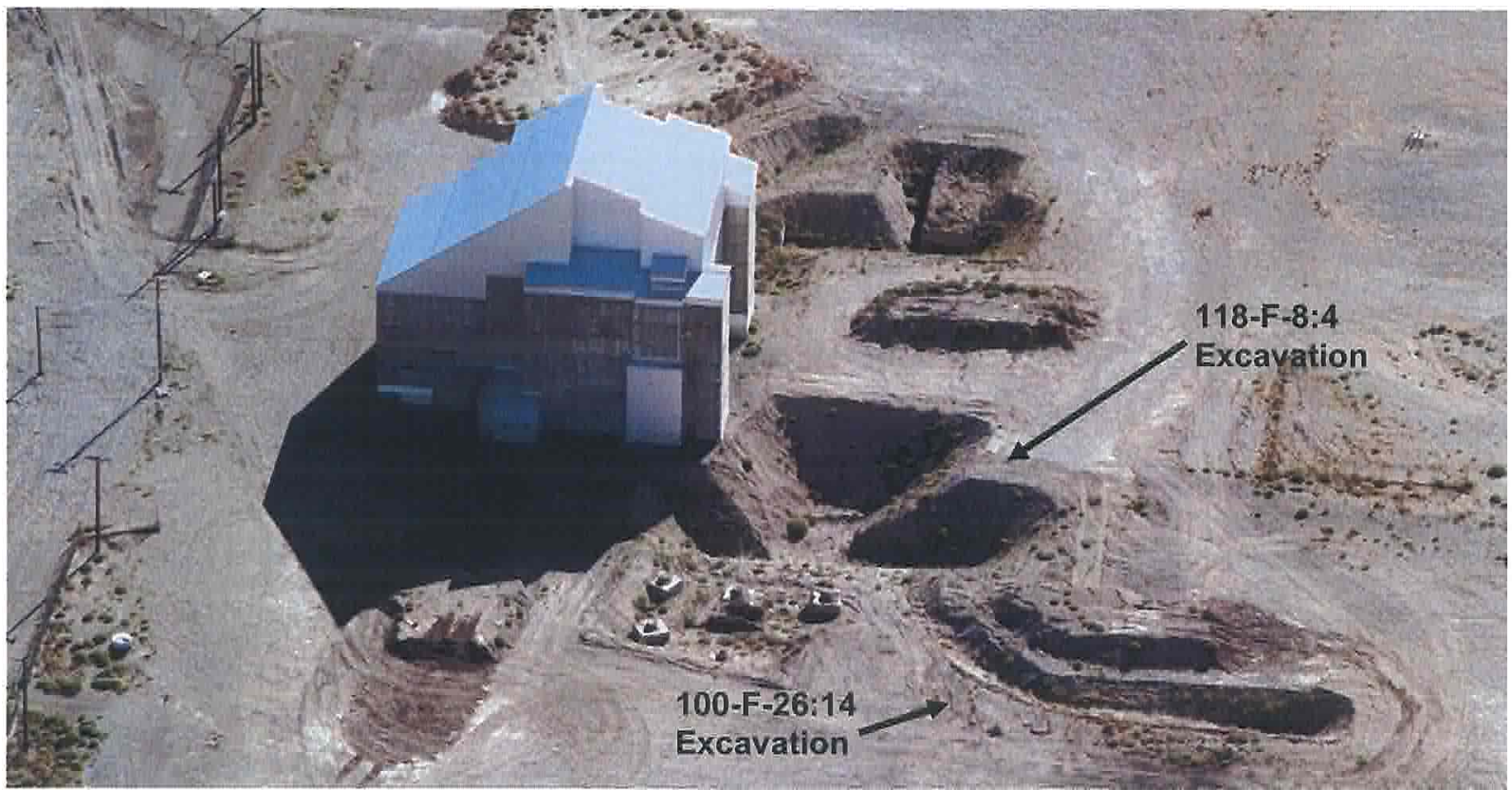


Figure 4. 118-F-8:4 Waste Site Post-Excavation Topography.

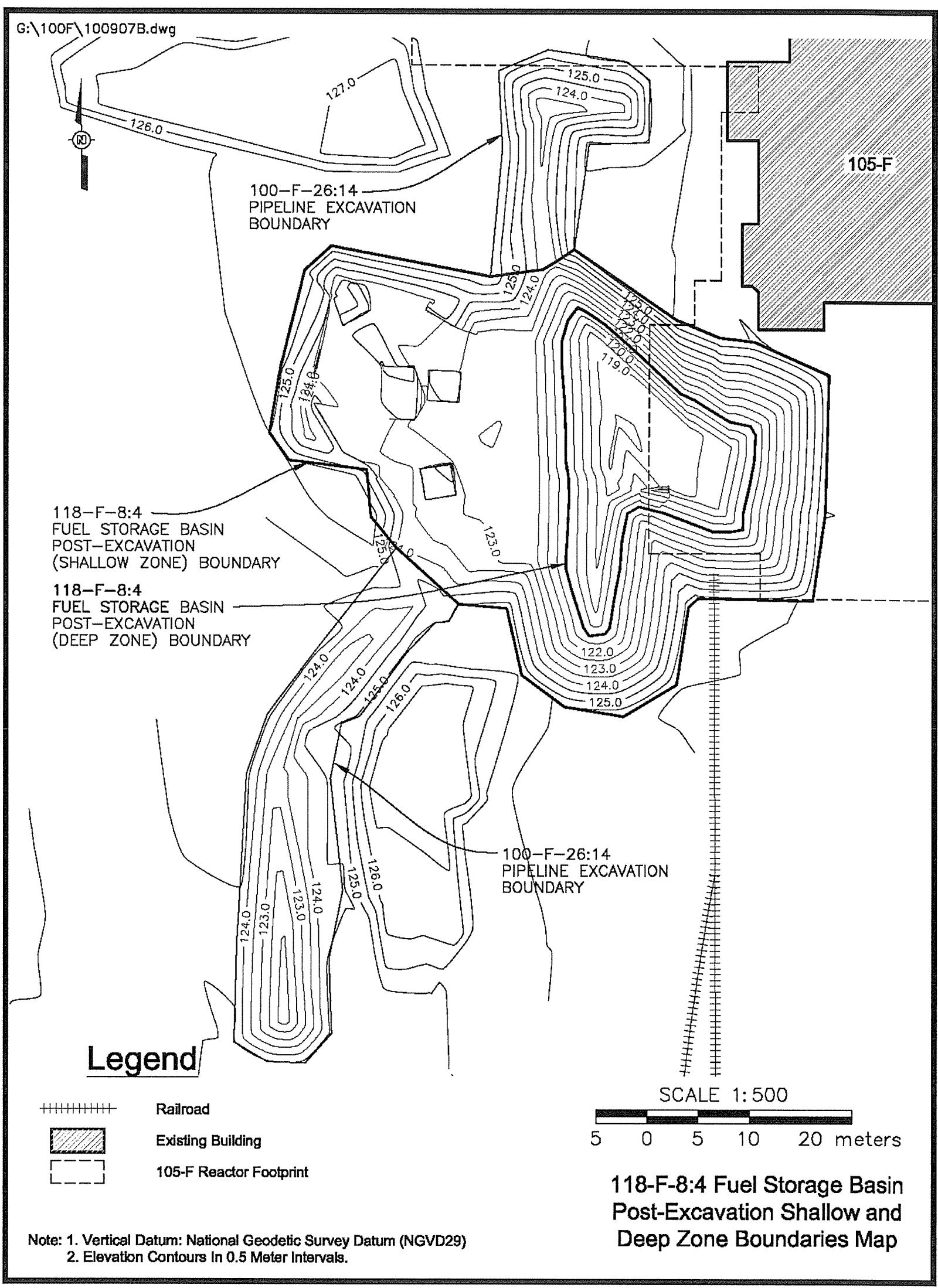




\subsection{FIELD SCREENING}

Radiological field screening was conducted during and after the site remedial actions as specified in the 100 Area SAP (DOE-RL 2005a). Field screening was used to guide the excavation and quickly assess the presence and level of contamination. Field screening at the site included using a Global Positioning Environmental Radiological Surveyor (GPERS) with instrumentation specific to the detection of radiation associated with gamma-emitting radionuclides.

The GPERS survey demonstrated that excavation was effective in remediating the 118-F-8:4 waste site. Consistent with the deep zone institutional controls specified for the other FSB subsites (BHI 2004a), the GPERS survey and verification sample results from deep zone sample area $A 4$ indicate levels of contamination that require deep zone institution controls for the 118-F-8:4 FSB subsite. Verification sampling and RESidual RADioactivity (RESRAD) modeling indicate that the 118-F-8:4 FSB deep zone meets the applicable deep zone cleanup goals for protection of groundwater and the river. Institutional controls for prevention of uncontrolled excavation in the deep zone of the area around the 105-F Reactor were already necessary as indicated by the previously submitted cleanup verification package for the FSB (BHI 2004a). The radiological surveys for the 118-F-8:4 FSB are provided in Appendix B.

\subsection{SAMPLING ACTIVITIES}

Following remediation and field screening of the 118-F-8:4 waste site, verification sampling was conducted between May 31 and June 22, 2007 (WCH 2007a, WCH 2007b). Verification sampling is performed to collect data to determine if the remedial action goals (RAGs) have been met. RAGs are the specific numeric goals against which the verification data are evaluated to demonstrate attainment of the remedial action objectives as established in the Action Memorandum (EPA et al. 1998).

The requirements for verification sampling were established in an interoffice memorandum deferring remediation of the adjacent and side slope areas of the FSB (subsequently assigned to the 118-F-8:4 waste site) (Nielson 2002). As provided in the interoffice memorandum, sampling was performed in accordance with the 100 Area SAP (DOE-RL 2005a), except that the contaminants of concern (COCs) for these areas were those listed in the Sampling and Analysis Plan for the 105-F Phase IV Fuel Storage Basin (FSB SAP) (DOE-RL 2000).

The following subsections provide additional discussion of the information used to develop the COCs for verification sampling, as well as the sampling design selection and basis. 


\subsection{CONTAMINANTS OF CONCERN FOR VERIFICATION SAMPLING}

The FSB SAP (DOE-RL 2000) identified the COCs for the side slope soils as americium-241, carbon-14, cobalt-60, cesium-137, europium-152, europium-154, europium-155, helium-3, nickel-63, plutonium-238, plutonium-239/240, strontium-90, uranium-234, uranium-235, uranium-238, hexavalent chromium, mercury, lead, barium, and PCBs. Barium-133 was included as a $\mathrm{COC}$ to be consistent with the analytical requirements for the adjacent and related 118-F-8:3 waste site previously interim closed under CVP-2003-00017 (BHI 2004a).

\subsection{SAMPLING DESIGN SELECTION AND BASIS}

The sampling design for the 118-F-8:4 waste site was established using the 100 Area SAP. The site was divided into decision units (e.g., shallow zone and deep zone) based on the size of the site and depth of the excavation. This division determines the number of verification samples to collect in each decision unit. The division of the site into decision units is also a function of the applicable RAGs. The direct exposure, groundwater protection, and river protection RAGs are applicable to soils within $4.6 \mathrm{~m}$ $(15 \mathrm{ft})$ of the ground surface. This soil zone is referred to as the shallow zone. The groundwater protection and river protection RAGs are applicable to soils greater than

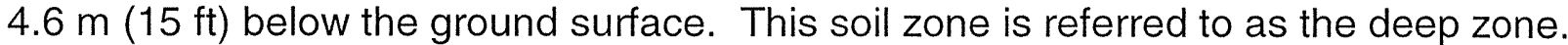

The 118-F-8:4 waste site contains three decision units: (1) shallow zone excavation, (2) deep zone excavation, and (3) overburden stockpiles, which combines two overburden stockpile areas (also referred to as below cleanup level [BCL] stockpiles) into one decision unit. The calculation of the number of verification samples to collect in each of the decision units resulted in 4 composite samples collected per decision unit for the shallow and deep zones and 16 composite samples collected for the overburden stockpile. This calculation, and the sample locations, is located in the sample design calculation in Appendix C. Figure 5 provides an overview of the three sample designs.

\subsection{SAMPLING RESULTS}

The verification samples were submitted to offsite laboratories for analysis using approved EPA analytical methods, as required per the 100 Area SAP (DOE-RL 2005a). The laboratory-reported data results from the verification sampling were used in the statistical calculations and are included with the 95\% upper confidence limit (UCL) calculations in Appendix C. 
Figure 5. General Overview of Sample Designs for the 118-F-8:4 Waste Site.

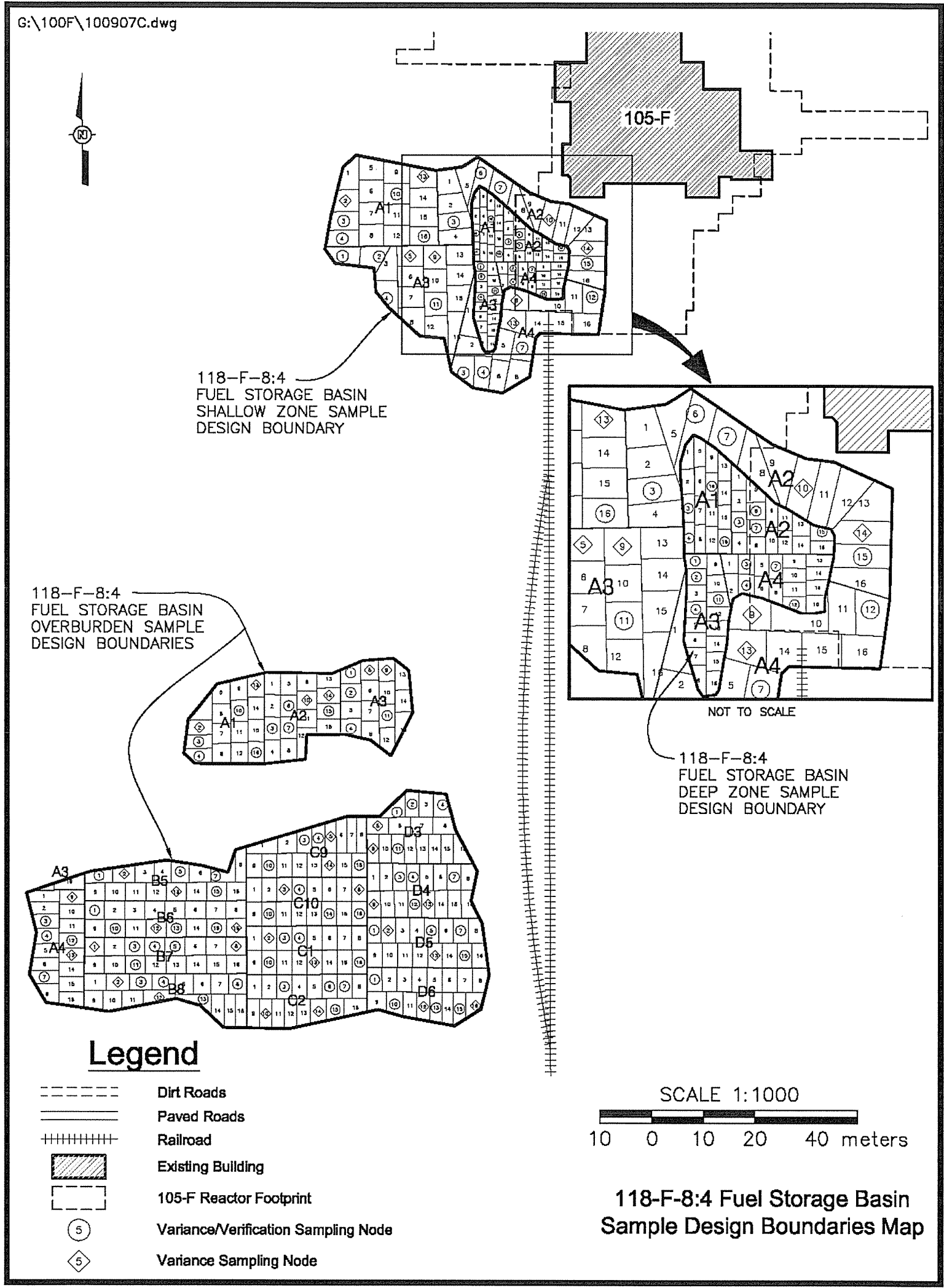


The primary statistical calculation to evaluate compliance with cleanup standards is the $95 \%$ UCL on the arithmetic mean of the data. The $95 \%$ UCL values for each COC are computed for each of the 118-F-8:4 waste site decision units as specified by the 100 Area RDR/RAWP (DOE-RL 2005b). When a nonradionuclide COC was detected in fewer than $50 \%$ of the verification samples collected, the maximum detected value was used for comparison against the RAGs.

Comparisons of the statistical results for site COCs with the RAGs (cleanup criteria) for each of the 118-F-8:4 waste site decision units (shallow zone excavation, deep zone excavation, and overburden stockpiles) are listed in Tables 1, 2, and 3. The standard laboratory analysis performed to quantify the concentrations of the COCs also detected other analytes. These were not included in the statistical calculations unless they exceeded an applicable RAG. The only non-COC analyte to which this applied was selenium. It was detected above the river protection RAG in a duplicate sample (J15511) in the shallow zone.

Initial results for the smaller of the two overburden stockpiles indicated that one area (sample area A1) contained elevated carbon-14 levels. Additional remediation was completed in this area. Following additional remedial excavation, resampling was done on October 22, 2007. The sample (J15690) was analyzed for carbon-14 and tritium. The original cleanup verification sample (J15574) result for carbon-14 was replaced with the resampling data (J15690); the tritium data for the original sample (J15574) was used for statistical calculations (tritium was not detected in either sample).

\subsection{DATA EVALUATION}

This section demonstrates that remedial actions at the 118-F-8:4 FSB have achieved the applicable RAGs, comply with ecological screening levels, and comply with applicable nonradionuclide risk requirements and Washington Administrative Code (WAC) 173-340-740(7)(e) three-part test criteria. This section also describes the RESRAD modeling performed that predicts the dose rate, the excess lifetime cancer risk, and the impact on groundwater and the river from residual radionuclide COC concentrations. 
Table 1. Comparison of Statistical Contaminant Concentrations to Action Levels for the 118-F-8:4 Shallow Zone Excavation Verification Sampling. (2 Pages)

\begin{tabular}{|c|c|c|c|c|c|c|}
\hline \multirow[b]{2}{*}{ COC } & \multirow[b]{2}{*}{$\begin{array}{c}\text { Statistical } \\
\text { Result } \\
\text { (pCi/g) }\end{array}$} & \multicolumn{3}{|c|}{ Generic Site Lookup Values $^{a}$ (pCi/g) } & \multirow{2}{*}{$\begin{array}{l}\text { Does the } \\
\text { Statistical } \\
\text { Result } \\
\text { Exceed } \\
\text { Lookup } \\
\text { Values? }\end{array}$} & \multirow{2}{*}{$\begin{array}{c}\text { Does the } \\
\text { Statistical } \\
\text { Result } \\
\text { Pass } \\
\text { RESRAD } \\
\text { Modeling? }\end{array}$} \\
\hline & & $\begin{array}{l}\text { Shallow } \\
\text { Zone } \\
\text { Lookup } \\
\text { Value }^{\text {b }}\end{array}$ & $\begin{array}{l}\text { Groundwater } \\
\text { Protection } \\
\text { Lookup } \\
\text { Value }\end{array}$ & $\begin{array}{l}\text { River } \\
\text { Protection } \\
\text { Lookup } \\
\text { Value }\end{array}$ & & \\
\hline Americium-241 & $0.110 \mathrm{U}$ & 2.38 & $--^{c}$ & $--^{c}$ & No & -- \\
\hline Barium-133 & $0.020 U$ & 11.8 & $--^{c}$ & $--^{c}$ & No & -- \\
\hline Carbon-14 & $-0.325 \mathrm{U}$ & 5.16 & $--^{c}$ & $--^{c}$ & No & - \\
\hline Cesium-137 & 0.281 & 6.2 & $--^{c}$ & $--^{c}$ & No & -- \\
\hline Cobalt-60 & $0.019 \mathrm{U}$ & 1.4 & $--^{c}$ & $--^{c}$ & No & -- \\
\hline Europium-152 & 0.160 & 3.3 & $--^{c}$ & $--^{c}$ & No & -- \\
\hline Europium-154 & $0.061 \mathrm{U}$ & 3.0 & $--^{c}$ & $--^{c}$ & No & -- \\
\hline Europium-155 & $0.050 \mathrm{U}$ & 125 & $--^{c}$ & $-^{c}$ & No & -- \\
\hline Nickel-63 & $1.26 \mathrm{U}$ & $4,013^{d}$ & $--^{c}$ & $--^{c}$ & No & -- \\
\hline Plutonium-238 & $0.023 \mathrm{U}$ & 37.4 & $--^{c}$ & $--^{c}$ & No & -- \\
\hline Plutonium-239/240 & $0.063 \mathrm{U}$ & 33.9 & $-{ }^{c}$ & $--^{c}$ & No & -- \\
\hline Strontium-90 & $0.151 \mathrm{U}$ & 4.5 & $--^{c}$ & $--^{c}$ & No & -- \\
\hline Tritium & $2.85 \mathrm{U}$ & 15.8 & 15.8 & 15.8 & No & -- \\
\hline Uranium-233/234 & $0(<B G)$ & $1.1^{d}$ & $1.1^{\mathrm{d}}$ & $1.1^{\mathrm{d}}$ & No & -- \\
\hline Uranium-235 & $0(<B G)$ & 0.84 & 0.27 & 0.5 & No & -- \\
\hline Uranium-238 & $0(<B G)$ & $1.1^{d}$ & $1.1^{\mathrm{d}}$ & $1.1^{\mathrm{d}}$ & No & -- \\
\hline \multirow[b]{2}{*}{$\mathrm{coc}$} & \multirow[b]{2}{*}{$\begin{array}{c}\text { Statistical } \\
\text { Result } \\
\text { (mg/kg) }\end{array}$} & \multicolumn{3}{|c|}{ Remedial Action Goals ${ }^{\mathrm{a}}$ (mg/kg) } & \multirow[b]{2}{*}{$\begin{array}{c}\text { Does the } \\
\text { Statistical } \\
\text { Result } \\
\text { Exceed } \\
\text { RAGs? }\end{array}$} & \multirow[b]{2}{*}{$\begin{array}{c}\text { Does the } \\
\text { Statistical } \\
\text { Result } \\
\text { Pass } \\
\text { RESRAD } \\
\text { Modeling? }\end{array}$} \\
\hline & & $\begin{array}{c}\text { Direct } \\
\text { Exposure }\end{array}$ & $\begin{array}{l}\text { Soil Cleanup } \\
\text { Level for } \\
\text { Groundwater } \\
\text { Protection }\end{array}$ & $\begin{array}{c}\text { Soil } \\
\text { Cleanup } \\
\text { Level for } \\
\text { River } \\
\text { Protection }\end{array}$ & & \\
\hline Barium & 70.1 & 5,600 & $132^{d}$ & 224 & No & -- \\
\hline $\begin{array}{l}\text { Hexavalent } \\
\text { chromium }\end{array}$ & 0.26 & $2.1^{f}$ & 4.8 & 2 & No & -- \\
\hline Lead & 9.0 & 353 & $10.2^{d}$ & $10.2^{d}$ & No & -- \\
\hline Mercury & 0.41 & 24 & $0.33^{d}$ & $0.33^{d}$ & No & -- \\
\hline Selenium & 1.5 & 400 & 5 & 1 & Yes & $Y_{e s}^{i}$ \\
\hline Aroclor-1016 & $0.014 \mathrm{U}$ & $5.6^{\mathrm{g}}$ & 0.112 & $0.017^{e}$ & No & -- \\
\hline Aroclor-1221 & $0.014 \mathrm{U}$ & $0.5^{\mathrm{h}}$ & $0.017^{e}$ & $0.017^{e}$ & No & -- \\
\hline
\end{tabular}


Table 1. Comparison of Statistical Contaminant Concentrations to Action Levels for the 118-F-8:4 Shallow Zone Excavation Verification Sampling. (2 Pages)

\begin{tabular}{|c|c|c|c|c|c|c|}
\hline \multirow[b]{2}{*}{$\mathrm{COC}$} & \multirow[b]{2}{*}{$\begin{array}{c}\text { Statistical } \\
\text { Result } \\
\text { (mg/kg) }\end{array}$} & \multicolumn{3}{|c|}{ Remedial Action Goals ${ }^{a}(\mathrm{mg} / \mathrm{kg})$} & \multirow[b]{2}{*}{$\begin{array}{c}\text { Does the } \\
\text { Statistical } \\
\text { Result } \\
\text { Exceed } \\
\text { RAGs? }\end{array}$} & \multirow[b]{2}{*}{$\begin{array}{c}\text { Does the } \\
\text { Statistical } \\
\text { Result } \\
\text { Pass } \\
\text { RESRAD } \\
\text { Modeling? }\end{array}$} \\
\hline & & $\begin{array}{c}\text { Direct } \\
\text { Exposure }\end{array}$ & $\begin{array}{l}\text { Soil Cleanup } \\
\text { Level for } \\
\text { Groundwater } \\
\text { Protection }\end{array}$ & $\begin{array}{c}\text { Soil } \\
\text { Cleanup } \\
\text { Level for } \\
\text { River } \\
\text { Protection }\end{array}$ & & \\
\hline Aroclor-1232 & $0.014 \mathrm{U}$ & $0.5^{\mathrm{h}}$ & $0.017^{\mathrm{e}}$ & $0.017^{e}$ & No & -- \\
\hline Aroclor-1242 & $0.014 \mathrm{U}$ & $0.5^{\mathrm{h}}$ & $0.017^{e}$ & $0.017^{e}$ & No & - \\
\hline Aroclor-1248 & $0.014 \mathrm{U}$ & $0.5^{h}$ & $0.017^{\mathrm{e}}$ & $0.017^{\mathrm{e}}$ & No & -- \\
\hline Aroclor-1254 & $0.014 \mathrm{U}$ & $0.5^{\mathrm{h}}$ & $0.017^{\mathrm{e}}$ & $0.017^{\mathrm{e}}$ & No & -- \\
\hline Aroclor-1260 & $0.014 \mathrm{U}$ & $0.5^{\mathrm{h}}$ & $0.017^{e}$ & $0.017^{e}$ & No & -- \\
\hline
\end{tabular}

a Lookup values, RAGs, and/or background values obtained from the Remedial Design Report/Remedial Action Work Plan for the 100 Area (DOE-RL 2005b) or calculated per WAC 173-340-720, WAC 173-340-730, or WAC 173-340-740, Method B, 1996, unless otherwise noted.

b Activity corresponding to a single-radionuclide $15 \mathrm{mrem} / \mathrm{yr}$ exposure as calculated using the RESRAD model (DOE-RL 2005b).

c The 100 Area RDR/RAWP (DOE-RL 2005b) does not provide soil cleanup levels for this contaminant to be protective of groundwater and the Columbia River. Based on the lowest radionuclide soil partitioning coefficient (for strontium-90 [25 mL/g]), this contaminant is not predicted to migrate more than $3 \mathrm{~m}(10 \mathrm{ft}$ ) vertically in 1,000 years (BHI 2005). The vadose zone underlying this waste site is approximately $7.8 \mathrm{~m}(25 \mathrm{ft})$ thick. Therefore, residual concentrations of this contaminant are predicted to be protective of groundwater and the Columbia River.

d The calculated cleanup level is below the Hanford-Site specific statistical soil background. The value presented is the Hanford Site-specific statistical soil background (DOE-RL 2005b) per WAC 173-340-700[4][d] (1996).

${ }^{e}$ Where cleanup levels are less than required detection limits (RDLs), cleanup levels default to RDLs (WAC 173-340-740[3], 1996, and DOE-RL 2005b).

Carcinogenic cleanup level calculated based on the inhalation exposure pathway; WAC 173-340-750(3), 1996.

g Noncarcinogenic cleanup level calculated from WAC 173-340-740(3), Method B, 1996.

h Carcinogenic cleanup level calculated per WAC 173-340-740(3), Method B, 1996.

' Based on 100 Area Analogous Sites RESRAD Calculations (BHI 2005), selenium with a soil partitioning coefficient of $150 \mathrm{~mL} / \mathrm{g}$ will not migrate more than $1 \mathrm{~m}(3.3 \mathrm{ft})$ in 1,000 years. The vadose zone underlying this waste site is approximately $7.8 \mathrm{~m}(25 \mathrm{ft})$ thick. Therefore, residual concentrations of this contaminant are predicted to be protective of groundwater and the Columbia River.

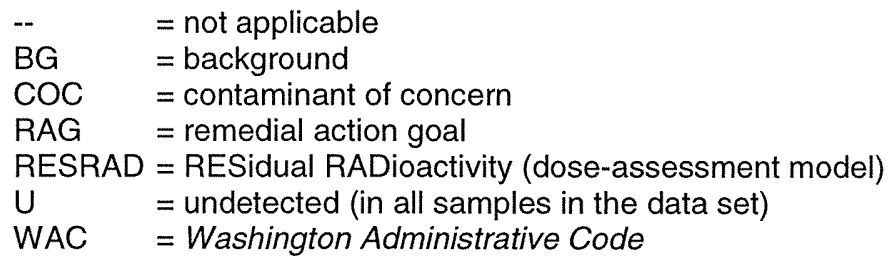


Table 2. Comparison of Statistical Contaminant Concentrations to Action Levels for the 118-F-8:4 Deep Zone Excavation Verification Sampling. (2 Pages)

\begin{tabular}{|c|c|c|c|c|c|}
\hline \multirow[b]{2}{*}{$\mathrm{COC}$} & \multirow[b]{2}{*}{$\begin{array}{l}\text { Statistical } \\
\text { Result } \\
(\mathrm{pCi} / \mathrm{g})^{\mathrm{b}}\end{array}$} & \multicolumn{2}{|c|}{$\begin{array}{c}\text { Generic Site Lookup } \\
\text { Values }{ }^{a}(\mathrm{pCi} / \mathrm{g})\end{array}$} & \multirow{2}{*}{$\begin{array}{c}\text { Does the } \\
\text { Statistical } \\
\text { Result Exceed } \\
\text { Lookup Values? }\end{array}$} & \multirow[b]{2}{*}{$\begin{array}{c}\text { Does the Statistical } \\
\text { Result Pass } \\
\text { RESRAD } \\
\text { Modeling? }\end{array}$} \\
\hline & & $\begin{array}{l}\text { Groundwater } \\
\text { Protection } \\
\text { Lookup Value }\end{array}$ & $\begin{array}{l}\text { River } \\
\text { Protection } \\
\text { Lookup } \\
\text { Value }\end{array}$ & & \\
\hline Americium-241 & 0.081 & $--^{c}$ & $--^{c}$ & No & -- \\
\hline Barium-133 & $0.022 \mathrm{U}$ & $--^{c}$ & $--^{c}$ & No & -- \\
\hline Carbon-14 & $0.070 \mathrm{U}$ & $--^{c}$ & $-^{c}$ & No & -- \\
\hline Cesium-137 & 4.52 & $--^{c}$ & $--^{c}$ & No & -- \\
\hline Cobalt-60 & 0.186 & $--^{c}$ & $--^{c}$ & No & -- \\
\hline Europium-152 & 3.01 & $--^{c}$ & $-^{c}$ & No & -- \\
\hline Europium-154 & $0.181 \mathrm{U}$ & $--^{c}$ &..$-^{c}$ & No & -- \\
\hline Europium-155 & $0.057 \mathrm{U}$ & $--^{c}$ & $--^{c}$ & No & - \\
\hline Nickel-63 & 18.7 & $--^{c}$ & $--^{c}$ & No & -- \\
\hline Plutonium-238 & $0.056 \mathrm{U}$ & $--^{c}$ & $--^{c}$ & No & -- \\
\hline $\begin{array}{l}\text { Plutonium- } \\
239 / 240\end{array}$ & 0.520 & $--^{c}$ & $--^{c}$ & No & -- \\
\hline Strontium-90 & 2.20 & $--^{c}$ & $-^{c}$ & No & -- \\
\hline Tritium & $1.38 \mathrm{U}$ & 15.8 & 15.8 & No & -- \\
\hline $\begin{array}{l}\text { Uranium- } \\
233 / 234\end{array}$ & $0(<B G)$ & $1.1^{\mathrm{d}}$ & $1.1^{\mathrm{d}}$ & No & -- \\
\hline Uranium-235 & $0(<B G)$ & 0.27 & 0.5 & No & -- \\
\hline Uranium-238 & $0(<B G)$ & $1.1^{d}$ & $1.1^{\mathrm{d}}$ & No & -- \\
\hline \multirow[b]{2}{*}{$\mathrm{coc}$} & \multirow{2}{*}{$\begin{array}{l}\text { Statistical } \\
\text { Result } \\
(\mathrm{mg} / \mathrm{kg})\end{array}$} & \multicolumn{2}{|c|}{$\begin{array}{l}\text { Remedial Action Goals }{ }^{a} \\
(\mathrm{mg} / \mathrm{kg})\end{array}$} & \multirow{2}{*}{$\begin{array}{l}\text { Does the } \\
\text { Statistical } \\
\text { Result Exceed } \\
\text { RAGs? }\end{array}$} & \multirow{2}{*}{$\begin{array}{c}\text { Does the Statistical } \\
\text { Result Pass } \\
\text { RESRAD } \\
\text { Modeling? }\end{array}$} \\
\hline & & $\begin{array}{l}\text { Soil Cleanup } \\
\text { Level for } \\
\text { Groundwate } \\
\text { r Protection }\end{array}$ & $\begin{array}{l}\text { Soil Cleanup } \\
\text { Level for } \\
\text { River } \\
\text { Protection }\end{array}$ & & \\
\hline Barium & 52.6 & $132^{d}$ & 224 & No & - \\
\hline $\begin{array}{l}\text { Hexavalent } \\
\text { chromium }\end{array}$ & 0.26 & 4.8 & 2 & No & -- \\
\hline Lead & 5.5 & $10.2^{d}$ & $10.2^{d}$ & No & - \\
\hline Mercury & 0.05 & $0.33^{d}$ & $0.33^{d}$ & No & -- \\
\hline Aroclor-1016 & $0.014 \mathrm{U}$ & 0.112 & $0.017^{e}$ & No & -- \\
\hline Aroclor-1221 & $0.014 \mathrm{U}$ & $0.017^{\mathrm{e}}$ & $0.017^{\mathrm{e}}$ & No & -- \\
\hline
\end{tabular}


Table 2. Comparison of Statistical Contaminant Concentrations to Action Levels for the 118-F-8:4 Deep Zone Excavation Verification Sampling. (2 Pages)

\begin{tabular}{|c|c|c|c|c|c|}
\hline \multirow[b]{2}{*}{$\mathrm{coc}$} & \multirow{2}{*}{$\begin{array}{l}\text { Statistical } \\
\text { Result } \\
\text { (mg/kg) }\end{array}$} & \multicolumn{2}{|c|}{$\begin{array}{c}\text { Remedial Action Goals }{ }^{\mathrm{a}} \\
(\mathrm{mg} / \mathrm{kg})\end{array}$} & \multirow{2}{*}{$\begin{array}{l}\text { Does the } \\
\text { Statistical } \\
\text { Result Exceed } \\
\text { RAGs? }\end{array}$} & \multirow{2}{*}{$\begin{array}{c}\text { Does the Statistical } \\
\text { Result Pass } \\
\text { RESRAD } \\
\text { Modeling? }\end{array}$} \\
\hline & & $\begin{array}{l}\text { Soil Cleanup } \\
\text { Level for } \\
\text { Groundwate } \\
\text { r Protection }\end{array}$ & $\begin{array}{l}\text { Soil Cleanup } \\
\text { Level for } \\
\text { River } \\
\text { Protection }\end{array}$ & & \\
\hline Aroclor-1232 & $0.014 \mathrm{U}$ & $0.017^{\mathrm{e}}$ & $0.017^{\mathrm{e}}$ & No & -- \\
\hline Aroclor-1242 & $0.014 \mathrm{U}$ & $0.017^{\mathrm{e}}$ & $0.017^{\mathrm{e}}$ & No & -- \\
\hline Aroclor-1248 & $0.014 \mathrm{U}$ & $0.017^{e}$ & $0.017^{\mathrm{e}}$ & No & -- \\
\hline Aroclor-1254 & $0.014 \mathrm{U}$ & $0.017^{e}$ & $0.017^{e}$ & No & -- \\
\hline Aroclor-1260 & 0.049 & $0.017^{e}$ & $0.017^{e}$ & No & $Y_{e s}^{\dagger}$ \\
\hline
\end{tabular}

${ }^{2}$ Lookup values, RAGs, and/or background values obtained from the Remedial Design Report/Remedial Action Work Plan for the 100 Area (DOE-RL 2005b) or calculated per WAC 173-340-720, WAC 173-340-730, or WAC 173-340-740, Method B, 1996, unless otherwise noted.

b Activity corresponding to a single-radionuclide $15 \mathrm{mrem} / \mathrm{yr}$ exposure as calculated using the RESRAD model (DOE-RL 2005b).

c The 100 Area RDR/RAWP (DOE-RL 2005b) does not provide soil cleanup levels for this contaminant to be protective of groundwater and the Columbia River. Based on the lowest radionuclide soil partitioning coefficient (for strontium-90 [25 mL/g]), this contaminant is not predicted to migrate more than $3 \mathrm{~m}(10 \mathrm{ft}$ ) vertically in 1,000

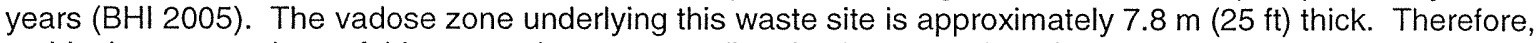
residual concentrations of this contaminant are predicted to be protective of groundwater and the Columbia River.

d The calculated cleanup level is below the Hanford-Site specific statistical soil background. The value presented is the Hanford Site-specific statistical soil background (DOE-RL 2005b) per WAC 173-340-700[4][d]) (1996).

e Where cleanup levels are less than required detection limits (RDLs), cleanup levels default to RDLs (WAC 173-340-740[3], 1996, and DOE-RL 2005b).

f Based on 100 Area Analogous Sites RESRAD Calculations (BHI 2005), Aroclor-1260 with a soil partitioning

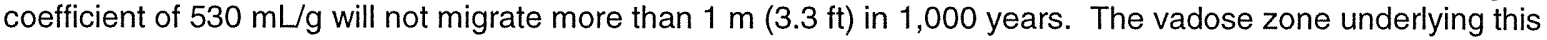

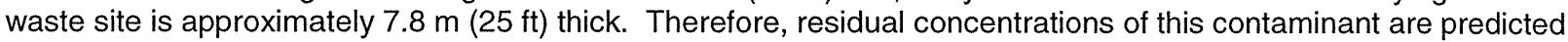
to be protective of groundwater and the Columbia River.

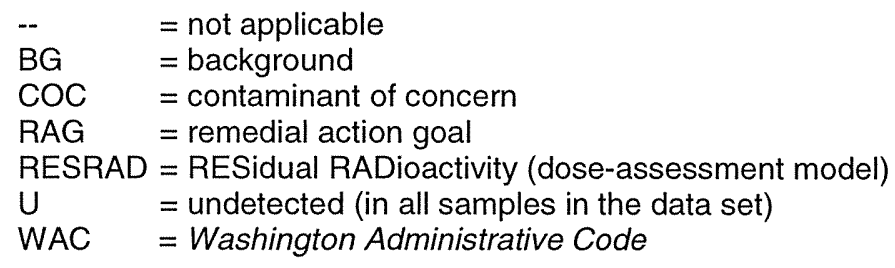


Table 3. Comparison of Statistical Contaminant Concentrations to Action Levels for the 118-F-8:4 Overburden/BCL Verification Sampling. (2 Pages)

\begin{tabular}{|c|c|c|c|c|c|c|}
\hline \multirow[b]{2}{*}{$\mathrm{COC}$} & \multirow[b]{2}{*}{$\begin{array}{c}\text { Statistical } \\
\text { Result } \\
(\mathrm{pCi} / \mathrm{g})\end{array}$} & \multicolumn{3}{|c|}{ Generic Site Lookup Values ${ }^{a}(p C i / g)$} & \multirow{2}{*}{$\begin{array}{l}\text { Does the } \\
\text { Statistical } \\
\text { Result } \\
\text { Exceed } \\
\text { Lookup } \\
\text { Values? }\end{array}$} & \multirow{2}{*}{$\begin{array}{c}\text { Does the } \\
\text { Statistical } \\
\text { Result } \\
\text { Pass } \\
\text { RESRAD } \\
\text { Modeling? }\end{array}$} \\
\hline & & $\begin{array}{l}\text { Shallow } \\
\text { Zone } \\
\text { Lookup } \\
\text { Value }^{b}\end{array}$ & $\begin{array}{l}\text { Groundwater } \\
\text { Protection } \\
\text { Lookup } \\
\text { Value }\end{array}$ & $\begin{array}{l}\text { River } \\
\text { Protection } \\
\text { Lookup } \\
\text { Value }\end{array}$ & & \\
\hline Americium-241 & $0.027 \mathrm{U}$ & 2.38 & $\ldots$ & $--^{c}$ & No & - \\
\hline Barium-133 & $0.018 \mathrm{U}$ & 11.8 & $--^{c}$ & $-n^{c}$ & No & -- \\
\hline Carbon-14 & $-0.7 U$ & 5.16 & $--^{c}$ & $--^{c}$ & No & -- \\
\hline Cesium-137 & 0.184 & 6.2 & $--^{c}$ & $--^{c}$ & No & -- \\
\hline Cobalt-60 & 0.016 & 1.4 & $--^{c}$ & $--^{c}$ & No & -- \\
\hline Europium-152 & 0.240 & 3.3 & $--^{c}$ & $--^{c}$ & No & -- \\
\hline Europium-154 & $0.050 \mathrm{U}$ & 3.0 & $--^{c}$ & $--^{c}$ & No & -- \\
\hline Europium-155 & $0.044 \mathrm{U}$ & 125 & $--^{c}$ & $--^{c}$ & No & -- \\
\hline Nickel-63 & $0.637 \mathrm{U}$ & 4,013 & $--^{c}$ & $--^{c}$ & No & -- \\
\hline Plutonium-238 & $0.008 \mathrm{U}$ & 37.4 & $--^{c}$ & $--^{c}$ & No & -- \\
\hline Plutonium-239/240 & 0.154 & 33.9 & $--^{c}$ & $--^{c}$ & No & -- \\
\hline Strontium-90 & $0.036 \mathrm{U}$ & 4.5 & $--^{c}$ & $--^{c}$ & No & -- \\
\hline Tritium & $0.857 \mathrm{U}$ & 15.8 & 15.8 & 15.8 & No & -- \\
\hline Uranium-233/234 & $0(<B G)$ & $1.1^{d}$ & $1.1^{d}$ & $1.1^{\mathrm{d}}$ & No & -- \\
\hline Uranium-235 & $0(<B G)$ & 0.84 & 0.27 & 0.5 & No & -- \\
\hline Uranium-238 & $0(<B G)$ & $1.1^{\mathrm{d}}$ & $1.1^{\mathrm{d}}$ & $1.1^{\mathrm{d}}$ & No & -- \\
\hline \multirow[b]{2}{*}{$\mathrm{COC}$} & \multirow[b]{2}{*}{$\begin{array}{c}\text { Statistical } \\
\text { Result } \\
(\mathbf{m g} / \mathrm{kg})\end{array}$} & \multicolumn{3}{|c|}{ Remedial Action Goals ${ }^{\mathrm{a}}(\mathrm{mg} / \mathrm{kg})$} & \multirow[b]{2}{*}{$\begin{array}{l}\text { Does the } \\
\text { Statistical } \\
\text { Result } \\
\text { Exceed } \\
\text { RAGs? }\end{array}$} & \multirow[b]{2}{*}{$\begin{array}{c}\text { Does the } \\
\text { Statistical } \\
\text { Result } \\
\text { Pass } \\
\text { RESRAD } \\
\text { Modeling? }\end{array}$} \\
\hline & & $\begin{array}{c}\text { Direct } \\
\text { Exposure }\end{array}$ & $\begin{array}{l}\text { Soil Cleanup } \\
\text { Level for } \\
\text { Groundwater } \\
\text { Protection }\end{array}$ & $\begin{array}{c}\text { Soil } \\
\text { Cleanup } \\
\text { Level for } \\
\text { River } \\
\text { Protection }\end{array}$ & & \\
\hline Barium & 67.8 & 5,600 & $132^{\circ}$ & 224 & No & -- \\
\hline $\begin{array}{l}\text { Hexavalent } \\
\text { chromium }\end{array}$ & 0.27 & $2.1^{\dagger}$ & 4.8 & 2 & No & -- \\
\hline Lead & 5.5 & 353 & $10.2^{e}$ & $10.2^{e}$ & No & -- \\
\hline Mercury & 0.57 & 24 & $0.33^{\mathrm{e}}$ & $0.33^{\mathrm{e}}$ & Yes & $Y_{e s}{ }^{g}$ \\
\hline Aroclor-1016 & $0.016 \mathrm{U}$ & $5.6^{h}$ & 0.112 & $0.017^{e}$ & No & -- \\
\hline Aroclor-1221 & $0.016 \mathrm{U}$ & $0.5^{i}$ & $0.017^{e}$ & $0.017^{e}$ & No & -- \\
\hline Aroclor-1232 & $0.016 \mathrm{U}$ & $0.5^{\mathrm{i}}$ & $0.017^{e}$ & $0.017^{e}$ & No & -- \\
\hline
\end{tabular}


Table 3. Comparison of Statistical Contaminant Concentrations to Action Levels for the 118-F-8:4 Overburden/BCL Verification Sampling. (2 Pages)

\begin{tabular}{|c|c|c|c|c|c|c|}
\hline \multirow[b]{2}{*}{$\mathrm{COC}$} & \multirow[b]{2}{*}{$\begin{array}{c}\text { Statistical } \\
\text { Result } \\
\text { (mg/kg) }\end{array}$} & \multicolumn{3}{|c|}{ Remedial Action Goals ${ }^{a}(\mathrm{mg} / \mathrm{kg})$} & \multirow[b]{2}{*}{$\begin{array}{c}\text { Does the } \\
\text { Statistical } \\
\text { Result } \\
\text { Exceed } \\
\text { RAGs? }\end{array}$} & \multirow[b]{2}{*}{$\begin{array}{c}\text { Does the } \\
\text { Statistical } \\
\text { Result } \\
\text { Pass } \\
\text { RESRAD } \\
\text { Modeling? }\end{array}$} \\
\hline & & $\begin{array}{c}\text { Direct } \\
\text { Exposure }\end{array}$ & $\begin{array}{l}\text { Soil Cleanup } \\
\text { Level for } \\
\text { Groundwater } \\
\text { Protection }\end{array}$ & $\begin{array}{c}\text { Soil } \\
\text { Cleanup } \\
\text { Level for } \\
\text { River } \\
\text { Protection }\end{array}$ & & \\
\hline Aroclor-1242 & $0.016 \mathrm{U}$ & $0.5^{i}$ & $0.017^{\mathrm{e}}$ & $0.017^{\mathrm{e}}$ & No & -- \\
\hline Aroclor-1248 & $0.016 \mathrm{U}$ & $0.5^{i}$ & $0.017^{e}$ & $0.017^{e}$ & No & -- \\
\hline Aroclor-1254 & 0.016 & $0.5^{i}$ & $0.017^{e}$ & $0.017^{e}$ & No & -- \\
\hline Aroclor-1260 & $0.016 \mathrm{U}$ & $0.5^{i}$ & $0.017^{e}$ & $0.017^{\ominus}$ & No & -- \\
\hline
\end{tabular}

${ }^{a}$ Lookup values, RAGs, and/or background values obtained from the Remedial Design Report/Remedial Action Work Plan for the 100 Area (DOE-RL 2005b) or calculated per WAC 173-340-720, WAC 173-340-730, or WAC 173-340-740, Method B, 1996, unless otherwise noted.

b Activity corresponding to a single-radionuclide $15 \mathrm{mrem} / \mathrm{yr}$ exposure as calculated using the RESRAD model (DOE-RL 2005b).

c The 100 Area RDR/RAWP (DOE-RL 2005b) does not provide soil cleanup levels for this contaminant to be protective of groundwater and the Columbia River. Based on the lowest radionuclide soil partitioning coefficient (for strontium-90 [25 mL/g]), this contaminant is not predicted to migrate more than $3 \mathrm{~m}$ (10 ft) vertically in 1,000 years (BHI 2005). The vadose zone underlying this waste site is approximately $7.8 \mathrm{~m}(25 \mathrm{ft}$ ) thick. Therefore, residual concentrations of this contaminant are predicted to be protective of groundwater and the Columbia River.

d The calculated cleanup level is below the Hanford-Site specific statistical soil background. The value presented is the Hanford Site-specific statistical soil background (DOE-RL 2005b) per WAC 173-340-700[4][d]) (1996).

${ }^{\ominus}$ Where cleanup levels are less than required detection limits (RDLs), cleanup levels default to the RDLs (WAC 173-340-740[3], 1996, and DOE-RL 2005b).

${ }^{1}$ Carcinogenic cleanup level calculated based on the inhalation exposure pathway; WAC 173-340-750(3), 1996.

9 Based on 100 Area Analogous Sites RESRAD Calculations (BHI 2005), mercury with a soil partitioning coefficient of $30 \mathrm{~mL} / \mathrm{g}$ will not migrate more than $2 \mathrm{~m}(6.6 \mathrm{ft})$ in 1,000 years. The vadose zone underlying this waste site is approximately $7.8 \mathrm{~m}(25 \mathrm{ft})$ thick. Therefore, residual concentrations of this contaminant are predicted to be protective of groundwater and the Columbia River.

h Noncarcinogenic cleanup level calculated from WAC 173-340-740(3), Method B, 1996.

'Carcinogenic cleanup level calculated per WAC 173-340-740(3), Method B, 1996.

$--\quad=$ not applicable

$\mathrm{BCL} \quad=$ below cleanup level

$\mathrm{BG} \quad=$ background

$\mathrm{COC}=$ contaminant of concern

RAG = remedial action goal

RESRAD = RESidual RADioactivity (dose-assessment model)

$U \quad=$ undetected (in all samples in the data set)

WAC = Washington Administrative Code 


\subsection{COMPARISON OF SAMPLE DATA TO RAGS}

Evaluation of the results listed in Tables 1,2, and 3 from the verification sampling at the 118-F-8:4 waste site indicates that all COCs were undetected and/or quantified below RAGs and lookup values, except for Aroclor-1260, mercury, and selenium. Residual concentrations of Aroclor-1260 in the deep zone decision unit $(0.049 \mathrm{mg} / \mathrm{kg})$ slightly exceed the soil RAGs for groundwater and river protection (both $0.017 \mathrm{mg} / \mathrm{kg}$ ). Residual concentrations of mercury in the shallow zone and overburden decision units $(0.41 \mathrm{mg} / \mathrm{kg}$ and $0.57 \mathrm{mg} / \mathrm{kg}$, respectively) slightly exceed the soil RAGs for groundwater and river protection (both $0.33 \mathrm{mg} / \mathrm{kg}$ ). Residual concentrations of selenium in the shallow zone decision unit $(1.5 \mathrm{mg} / \mathrm{kg})$ slightly exceed the soil RAG for river protection $(1 \mathrm{mg} / \mathrm{kg})$.

Data were not collected on the vertical extent of contamination for this area, but given the soil-partitioning coefficient for these contaminants (Aroclor-1260, $530 \mathrm{~mL} / \mathrm{g}$; mercury, $30 \mathrm{~mL} / \mathrm{g}$; and selenium, $150 \mathrm{~mL} / \mathrm{g}$ ), they would not be expected to migrate more than $2 \mathrm{~m}$ (6.6 ft) vertically in 1,000 years (BHI 2005). The vadose zone underlying

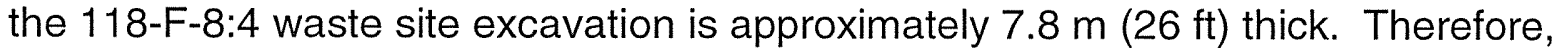
residual concentrations of this contaminant are predicted to be protective of the Columbia River.

\subsection{COMPARISON OF SAMPLE DATA TO ECOLOGICAL RISK SCREENING LEVELS}

A comparison against ecological risk screening levels has been made for the site COCs and other constituents. Screening levels were not exceeded for the site constituents, with the exception of aluminum, antimony, boron, manganese, mercury, selenium, and vanadium. Exceedance of screening values does not necessarily indicate the existence of risk to ecological receptors. It is believed that the presence of these constituents does not pose a risk to ecological receptors because concentrations of aluminum, antimony, manganese, and vanadium are below site background levels; mercury and selenium are within the range of Hanford Site background levels; and boron concentrations are consistent with concentrations at other locations on the Hanford Site (no established background value is available for boron). A more complete quantitative ecological risk assessment will be presented in the baseline risk assessment for the river corridor portion of the Hanford Site and will be used to support the final closeout decision for this site.

\subsection{HUMAN HEALTH RISK EVALUATION FOR NONRADIONUCLIDES}

The human health risk evaluations are calculated for nonradionuclide COCs only per WAC 173-340-740(3)(a)(iii)(A) and (B). The requirements include a hazard quotient of less than 1.0 for all individual noncarcinogens, a cumulative hazard quotient of less than 1.0 , an individual contaminant carcinogenic risk of less than $1 \times 10^{-6}$, and a cumulative 
excess carcinogenic risk of less than $1 \times 10^{-5}$. The details of the hazard quotient calculations are provided in Appendix C.

Hazard quotient calculations were performed for the 118-F-8:4 waste site using the highest of the statistical values from all sampling areas. Risk values were not calculated for constituents that were not detected or were detected at concentrations below Hanford Site or Washington State background values. All individual hazard quotients are below 1.0, and all individual excess carcinogenic risk values are below $1 \times 10^{-6}$. The cumulative hazard quotient for the 118-F-8:4 waste site is $3.9 \times 10^{-2}$ and the cumulative excess carcinogenic risk value is $2.6 \times 10^{-7}$, satisfying the criteria of less than 1.0 and less than $1 \times 10^{-5}$, respectively. Therefore, nonradionuclide risk requirements are met.

\subsection{WAC THREE-PART TEST FOR NONRADIONUCLIDES}

When using a statistical sampling approach, a RAG requirement for nonradionuclides is the WAC 173-340-740(7)(e) three-part test. The WAC 173-340 three-part test consists of the following criteria: (1) the cleanup verification $95 \%$ UCL value must be less than the cleanup level, (2) no single detection can exceed two times the cleanup criteria, and (3) the percentage of samples exceeding the cleanup criteria must be less than $10 \%$ of the data set.

The application of the three-part test for the 118-F-8:4 waste site is included in the $95 \%$ UCL calculation (Appendix C). The results of this evaluation indicate that lead, mercury, selenium, and Aroclor-1260 fail one or more components of the three-part test when compared to the most stringent RAG (soil RAG for the protection of the Columbia River). Lead and mercury are the most mobile of the four analytes having the lowest soil-partitioning coefficient (both $30 \mathrm{~mL} / \mathrm{g}$ ), and neither is expected to migrate more than $2 \mathrm{~m}$ (6.6 ft) vertically in 1,000 years (BHI 2005). Therefore, lead, mercury, and the other two less mobile contaminants are not anticipated to migrate to groundwater or the Columbia River in 1,000 years. All of these contaminants satisfy the three-part test criteria in comparison to the direct exposure RAG. Therefore, the requirements of the three-part test are met.

\subsection{RESRAD MODELING FOR RADIONUCLIDES}

For radionuclide contaminants, RESRAD modeling (ANL 2005) is used to determine contaminant migration and to predict the dose rate, the excess lifetime cancer risk, and the impact on groundwater and the river from residual radionuclide $\mathrm{COC}$ concentrations (DOE-RL 2005b). For the 118-F-8:4 waste site, the individual radionuclide cleanup statistical values were entered into the RESRAD computer code with the results included in the RESRAD calculations in Appendix $C$, as well as summarized in the following sections. 


\subsubsection{Attainment of Radionuclide Direct Exposure RAG}

The direct exposure RAG for radionuclide COCs is expressed in terms of an allowable radiation dose rate above background, which is $15 \mathrm{mrem} / \mathrm{yr}$ over 1,000 years. For the 118-F-8:4 waste site, separate RESRAD runs were performed for each of the decision units (shallow zone excavation, deep zone excavation, and overburden stockpiles). The maximum dose rate calculated by RESRAD occurs at the present year (2007) and is $1.70 \mathrm{mrem} / \mathrm{yr}$ (from the overburden stockpile). The maximum dose rate in 1,000 years decreases to $0.0591 \mathrm{mrem} / \mathrm{yr}$ (from the overburden stockpile); therefore, this requirement is attained.

\subsubsection{Attainment of Radionuclide Excess Lifetime Cancer Risk Goal}

The "National Oil and Hazardous Substances Pollution Contingency Plan" (40 Code of Federal Regulations [CFR] 300) presents a target range for residual radionuclide risk of $10^{-4}$ to $10^{-6}$. The RESRAD model calculates the excess lifetime cancer risk associated with the estimated radiation dose rates. For the 118-F-8:4 waste site, the maximum excess lifetime cancer risk occurs at the present year (2007) and is $1.99 \times 10^{-5}$ (from the overburden stockpile). The maximum excess lifetime cancer risk in 1,000 years decreases to $1.14 \times 10^{-7}$ (from the overburden stockpile); therefore, this requirement is attained.

\subsubsection{Attainment of Radionuclide Groundwater and River Protection RAGS}

The attainment of groundwater and river protection RAGs for radionuclides is determined by four criteria: (1) attain single COC groundwater and river RAGs, (2) attain "National Primary Drinking Water Regulations" (40 CFR 141) $4 \mathrm{mrem} / \mathrm{yr}$ (beta/gamma) dose standard to target receptor/organ; (3) meet drinking water standards for nonuranium alpha emitters (the more stringent of $15 \mathrm{pCi} / \mathrm{L}$ maximum contaminant level [MCL] or 1/25th of the derived concentration guide [DOE Order 5400.5]), and (4) meet total uranium standard of $21.2 \mathrm{pCi} / \mathrm{L}$.

The first criterion is determined by comparing peak concentrations of radionuclide COCs to the RAGs. The peak concentration is the maximum predicted value from the three decision units, as shown in the MCL calculation in Appendix C. Among the radionuclide COCs for the 118-F-8:4 waste site, cobalt-60, cesium-137, nickel-63, and strontium-90 are calculated by the RESRAD model to reach groundwater in 1,000 years; however, all are at concentrations significantly below the RAGs.

The peak concentration of cobalt- 60 reaching the groundwater from the three decision units is predicted to occur in year 7 at $0.00134 \mathrm{pCi} / \mathrm{L}$. Both the groundwater and river protection RAGs for cobalt- 60 are $100 \mathrm{pCi} / \mathrm{L}$; therefore, this criterion is attained.

The peak concentration of cesium-137 reaching the groundwater from the three decision units is predicted to occur in year 43 at $0.185 \mathrm{pCi} / \mathrm{L}$. Both the groundwater and river protection RAGs for cesium-137 are $60 \mathrm{pCi} / \mathrm{L}$; therefore, this criterion is attained. 
The peak concentration of nickel-63 reaching the groundwater from the three decision units is predicted to occur in year 135 at $11.2 \mathrm{pCi} / \mathrm{L}$. Both the groundwater and river protection RAGs for nickel-63 are $50 \mathrm{pCi} / \mathrm{L}$; therefore, this criterion is attained.

The peak concentration of strontium-90 reaching the groundwater from the three decision units is predicted to occur in year 43 at $0.636 \mathrm{pCi} / \mathrm{L}$. Both the groundwater and river protection RAGs for strontium-90 are $8 \mathrm{pCi} / \mathrm{L}$; therefore, this criterion is attained.

The second criterion, attainment of the National Primary Drinking Water Regulations $4 \mathrm{mrem} / \mathrm{yr}$ (beta/gamma) above background dose standard to target receptor/organ, is demonstrated in the MCL calculation provided in Appendix C. The results predict a maximum dose of $1.01 \mathrm{mrem} / \mathrm{yr}$ dose to any organ (total body, bone, liver, and gastrointestinal trace [lower large intestine]) within the 1,000 years of the evaluation period. Therefore, this criterion is attained.

The third criterion, meet drinking water standards for nonuranium alpha emitters (the more stringent of $15 \mathrm{pCi} / \mathrm{L} \mathrm{MCL}$ or $1 / 25$ th of the derived concentration guide [DOE Order 5400.5]) is demonstrated in the MCL calculation in Appendix C. The results indicate that the alpha-emitting COCs (americium-241, plutonium-238, and plutonium-239/240) will not impact groundwater. Therefore, concentrations are less than the maximum allowable gross particle activity.

For the last criterion, meet the total uranium standard of $21.2 \mathrm{pCi} / \mathrm{L}$, no uranium isotopes were detected above background levels in the 118-F-8:4 waste site verification samples. Therefore, this standard is met.

\subsection{DATA QUALITY ASSESSMENT}

A data quality assessment (DQA) is performed to compare the verification sampling approach and resulting analytical data with the sampling and data quality requirements specified by the project objectives and performance specifications.

The DQA for the 118-F-8:4 waste site established that the data are of the right type, quality, and quantity to support site verification decisions within specified error tolerances. All analytical data were found to be acceptable for decision-making purposes. The evaluation verified that the sample design was sufficient for the purpose of clean site verification. The detailed DQA is presented in Appendix D. 


\subsection{SUMMARY FOR WASTE SITE RECLASSIFICATION}

This cleanup verification package demonstrates that remedial action at the 118-F-8:4 waste site has achieved the remedial action objectives and corresponding RAGs established in the Action Memorandum (EPA et al. 1998a) and by reference, the 100 Area SAP (DOE-RL 2005a) and RDR/RAWP (DOE-RL 2005b). The remaining soils at this site have been sampled, analyzed, and modeled. The results indicate that the residual concentrations of COCs at this site do not preclude any future uses (as bounded by a rural-residential scenario) and allow for unrestricted use of the shallowzone. The acceptability of unrestricted direct exposure to deep zone soils has not been demonstrated due to the presence of residual activities from multiple radionuclides; therefore, institutional controls to prevent uncontrolled drilling/excavation into the deep zone (i.e., below $4.6 \mathrm{~m}[15 \mathrm{ft}]$ ) are required. The results also demonstrate that residual contaminant concentrations are protective of groundwater and the Columbia River.

In accordance with this evaluation, the verification sampling and modeling results support a reclassification of the 118-F-8:4 waste site to Interim Closed Out.

\subsection{REFERENCES}

40 CFR 141, "National Primary Drinking Water Regulations," Code of Federal Regulations, as amended.

40 CFR 300, "National Oil and Hazardous Substances Pollution Contingency Plan," Code of Federal Regulations, as amended.

ANL, 2005, RESRAD for Windows, Version 6.30, Argonne National Laboratory, Environmental Assessment Division, Argonne, Illinois.

BHI, 2001, Calculation of Total Uranium Activity Corresponding to a Maximum Contaminant Level for Total Uranium of 30 Micrograms per Liter in Groundwater, 0100X-CA-V0038, Rev. 0, Bechtel Hanford, Inc., Richland, Washington.

BHI, 2003, Cleanup Verification Package for the 100-F-19:2 Reactor Cooling Water Effluent Pipeline, 116-F-11 Cushion Corridor French Drain, UPR-100-F-1 Sewer Line Leak, and 100-F-29 Experimental Animal Farm Process Sewer Pipelines, CVP-2001-00003, Rev. 0, Bechtel Hanford, Inc., Richland, Washington.

BHI, 2004a, Cleanup Verification Package for the 118-F-8:1, 105-F Reactor BelowGrade Structures and Underlying Soils; the 118-F-8:3, 105-F Fuel Storage Basin Underlying Soils; and the 100-F-10 French Drain, CVP-2003-00017, Rev. 0, Bechtel Hanford, Inc., Richland, Washington. 
BHI, 2004b, 100 Area Radionuclide and Nonradionuclide Lookup Values for the 1995 Interim Remedial Action Record of Decision, 0100X-CA-V0046, Rev. 0, Bechtel Hanford Inc., Richland, Washington.

BHI, 2005, 100 Area Analogous Sites RESRAD Calculations, 0100X-CA-V0050, Rev. 0, Bechtel Hanford, Inc., Richland, Washington.

DOE Order 5400.5, Radiation Protection of the Public and the Environment, U.S. Department of Energy, Washington, D.C.

DOE-RL, 2000, Sampling and Analysis Plan for the 105-F Phase IV Fuel Storage Basin, DOE/RL-2000-54, Rev. 0, U.S. Department of Energy, Richland Operations Office, Richland, Washington.

DOE-RL, 2002, Removal Action Work Plan for 105-DR and 105-F Building Interim Safe Storage Projects and Ancillary Buildings, DOE/RL-98-37, Rev. 5, U.S. Department of Energy, Richland Operations Office, Richland, Washington.

DOE-RL, 2005a, 100 Area Remedial Action Sampling and Analysis Plan, DOE/RL-96-22, Rev. 4, U.S. Department of Energy, Richland Operations Office, Richland, Washington.

DOE-RL, 2005b, Remedial Design Report/Remedial Action Work Plan for the 100 Area, DOE/RL-96-17, Rev. 5, U.S. Department of Energy, Richland Operations Office, Richland, Washington.

DOE-RL, 2007, Tri-Party Agreement Handbook Management Procedures, RL-TPA-90-0001, Rev. 1, Guideline Number TPA-MP-14, "Maintenance of the Waste Information Data System (WIDS)," U.S. Department of Energy, Richland Operations Office, Richland, Washington.

Ecology, EPA, and DOE, 1989, Hanford Federal Facility Agreement and Consent Order, 2 vols., as amended, Washington State Department of Ecology, U.S. Environmental Protection Agency, and U.S. Department of Energy, Olympia, Washington.

EPA, Ecology, and RL, 1998, Action Memorandum, USDOE Hanford 100 Area National Priorities List (NPL) 105-F and 105-DR Reactor Buildings and Ancillary Facilities, Hanford Site, Benton County, Washington, U.S. Environmental Protection Agency, Region 10, Seattle, Washington.

Nielsen, R. R., 2002, Proposal to Defer Sections of the 105-F Fuel Storage Basin Adjacent and Side Slope Soils To Remedial Action/Waste Disposal Project, CCN 103659 dated December 4, 2002, to D. A. Faulk, U.S. Environmental Protection Agency, and R. Bond, Washington State Department of Ecology, Bechtel Hanford, Inc., Richland, Washington. 
WAC 173-340, 1996, "Model Toxics Control Act - Cleanup," Washington Administrative Code.

WCH, 2007a, 100F Remedial Sampling, Logbook EFL-1174-2, pp. 93-96, 99-100, Washington Closure Hanford, Richland, Washington.

WCH, 2007b, 100F Remedial Sampling, Logbook EFL-1174-3, pp. 1-10, 16, Washington Closure Hanford, Richland, Washington. 
APPENDIX A

SITE PHOTOGRAPHS

A-i 
Rev. 0 
Excavator loading can with material from the 118-F-8:4 waste site (looking west: March 23, 2007).

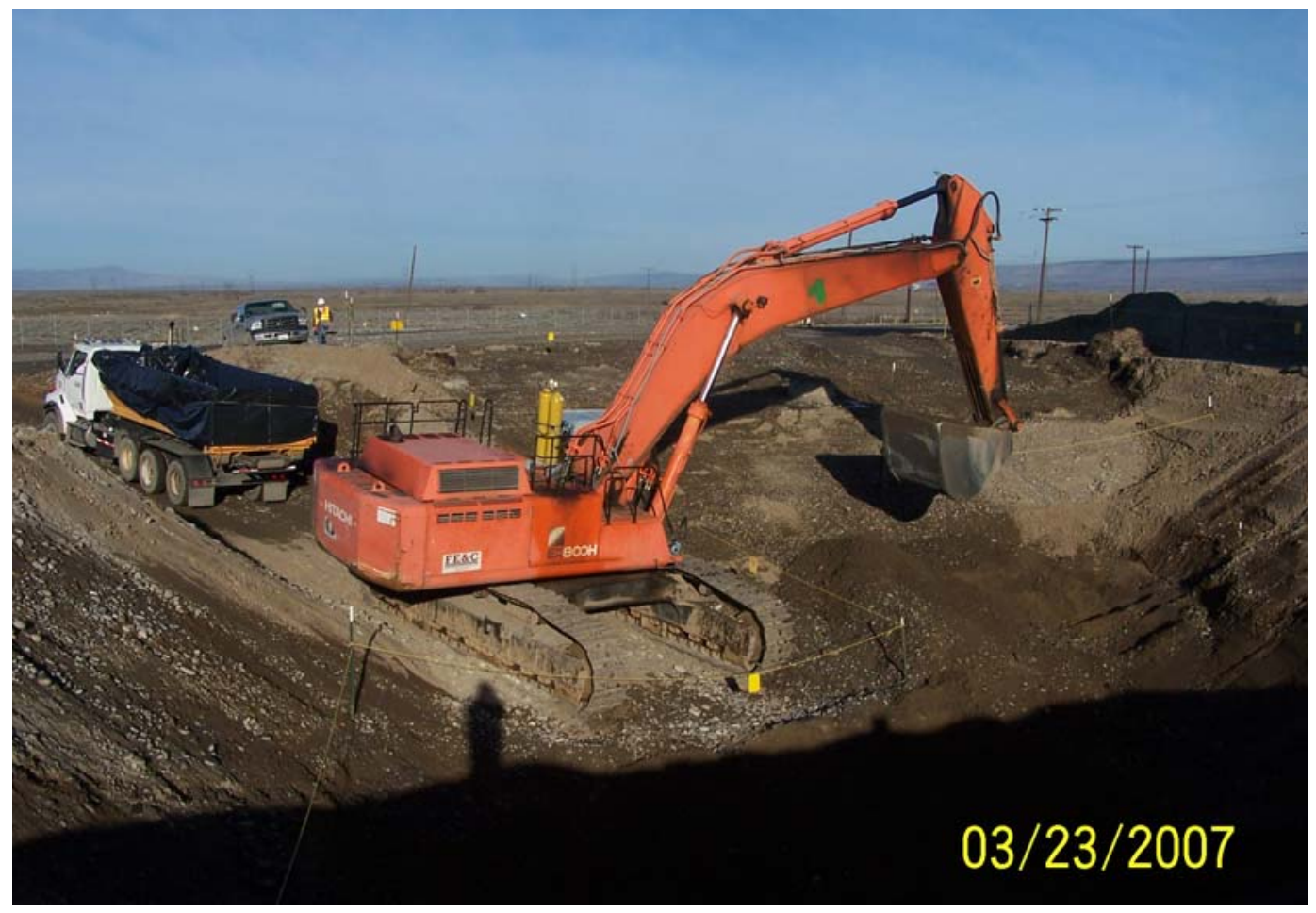

Excavator loading can with material from the 118-F-8:4 waste site (looking north: March 23, 2007).

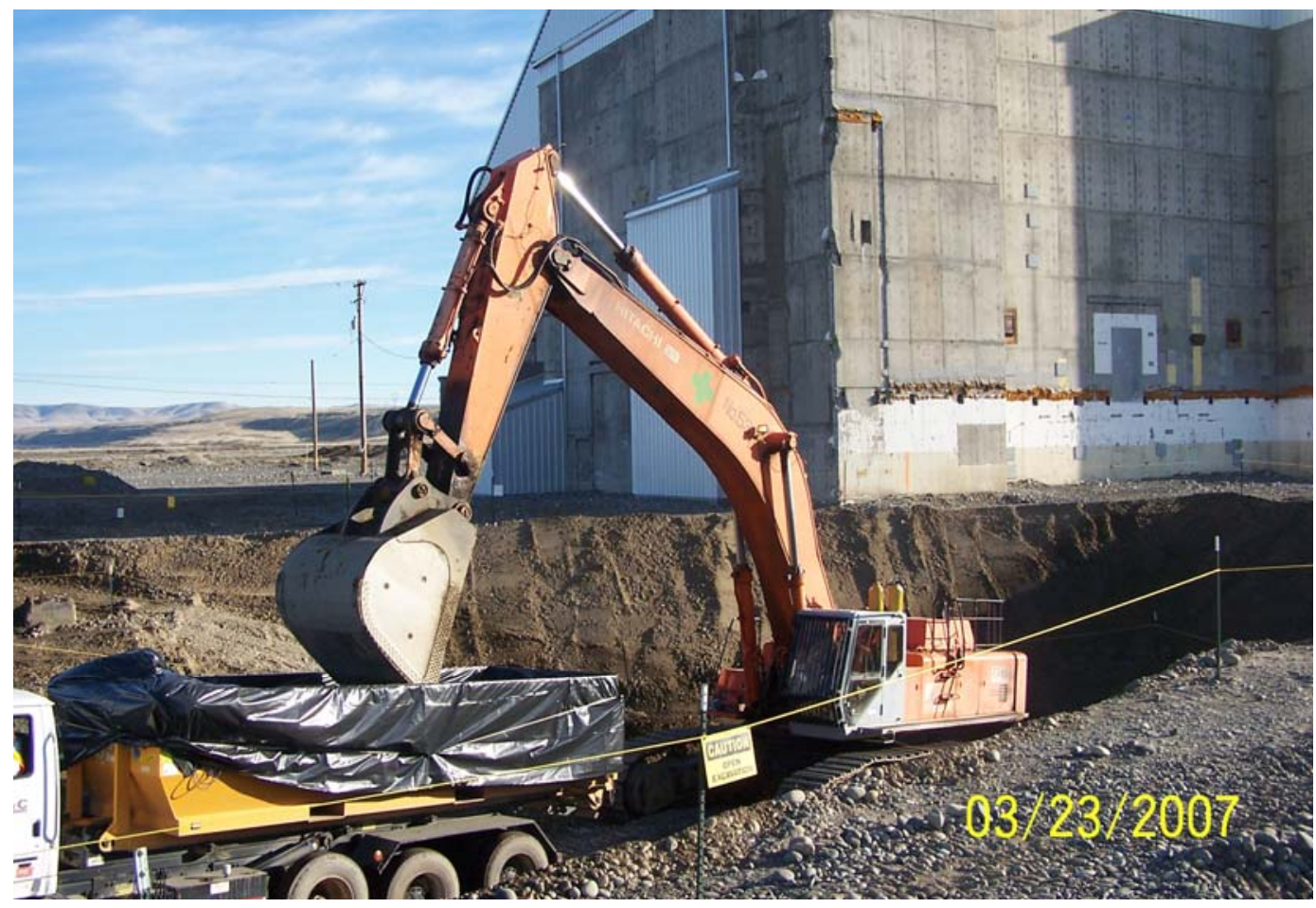


118-F-8:4 Fuel Storage Basin West Side Adjacent and Side Slope Soils excavation (looking southwest: March 21, 2007).

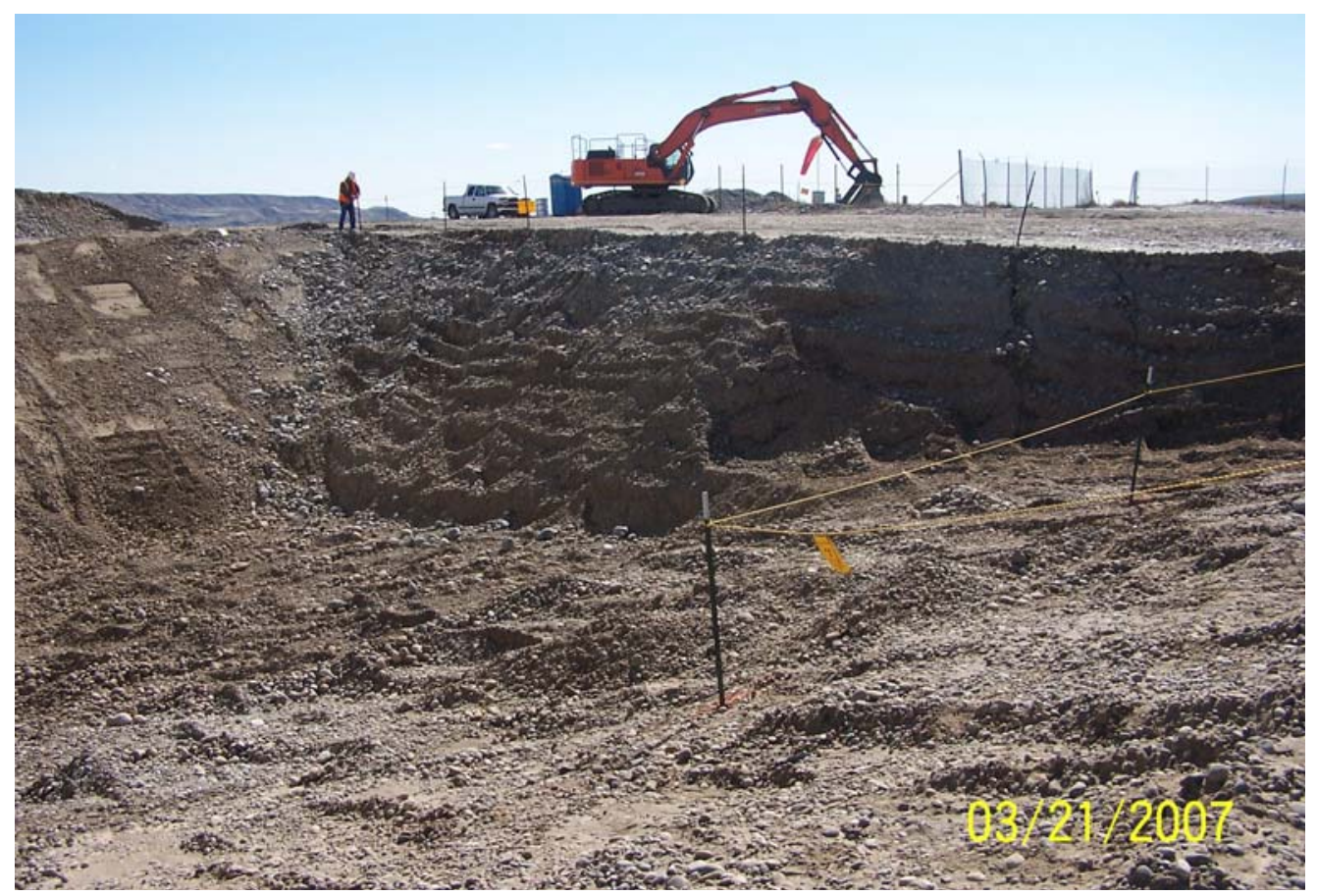

118-F-8:4 waste site being excavated. Elevated water tank foundation is visible in the foreground (looking west: April 18, 2007).

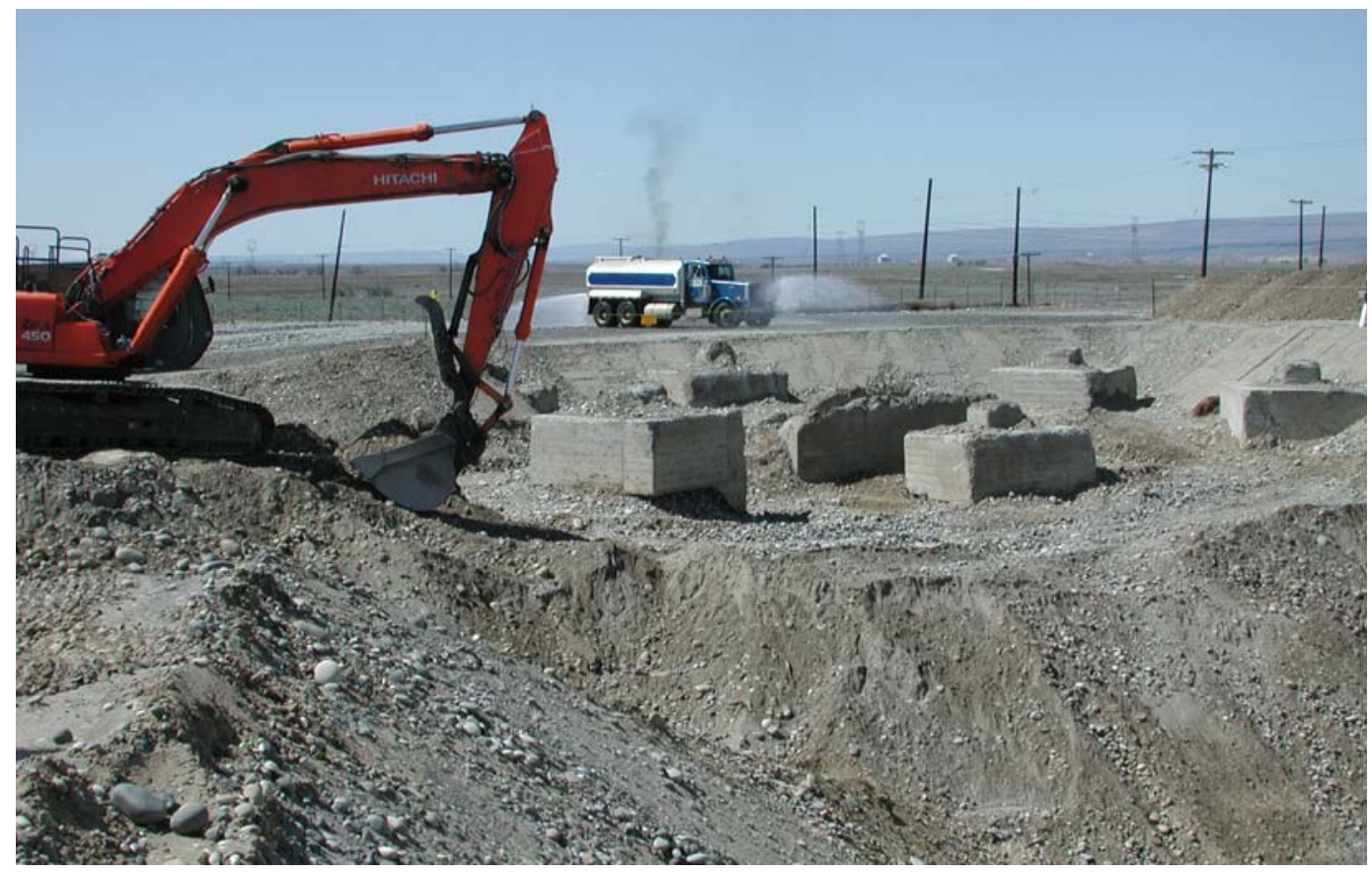


118-F-8:4 waste site excavation (looking northwest: April 18, 2007).

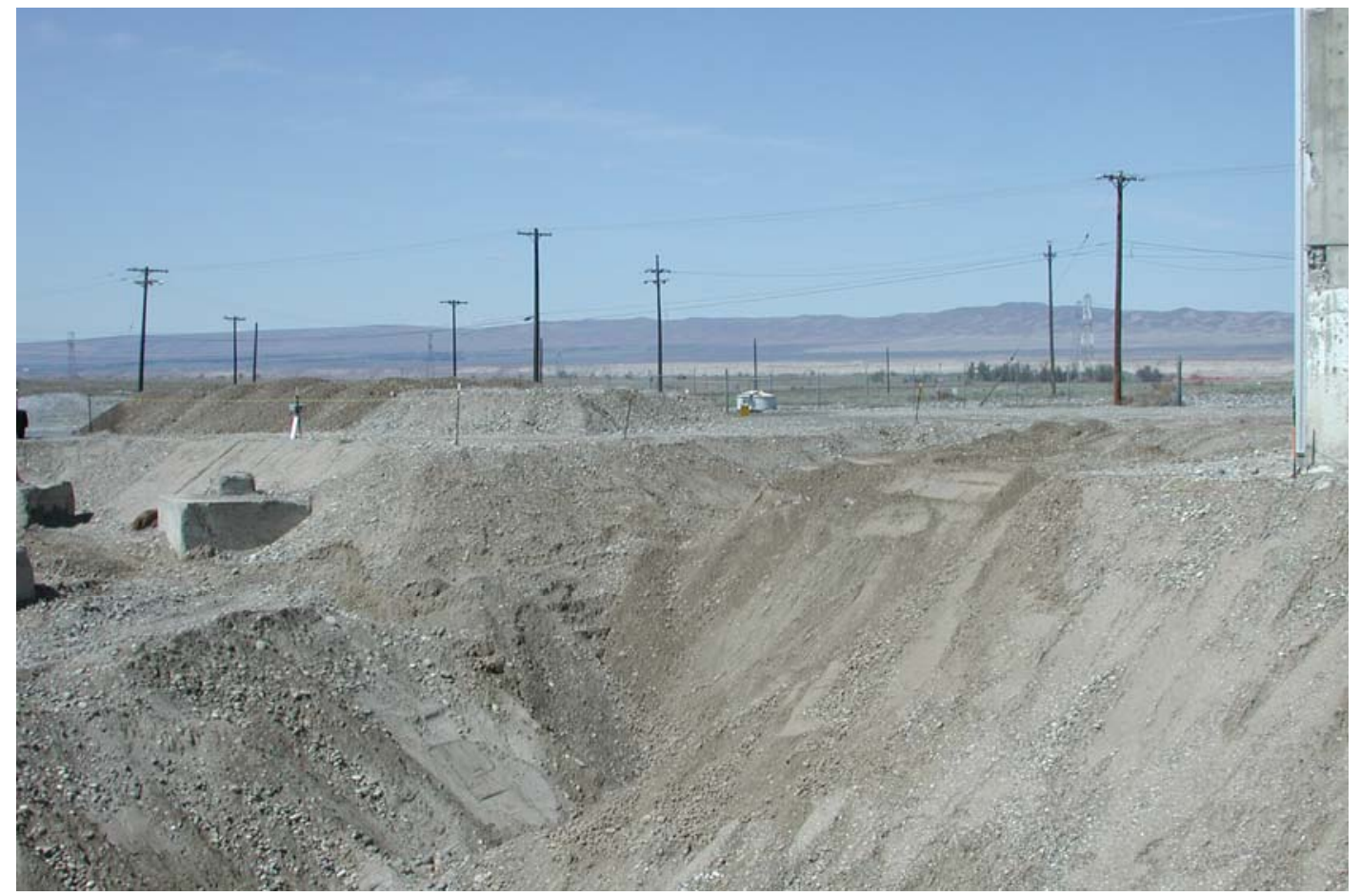

118-F-8:4 waste site excavation (close-up view of bottom of excavation: April 18, 2007).

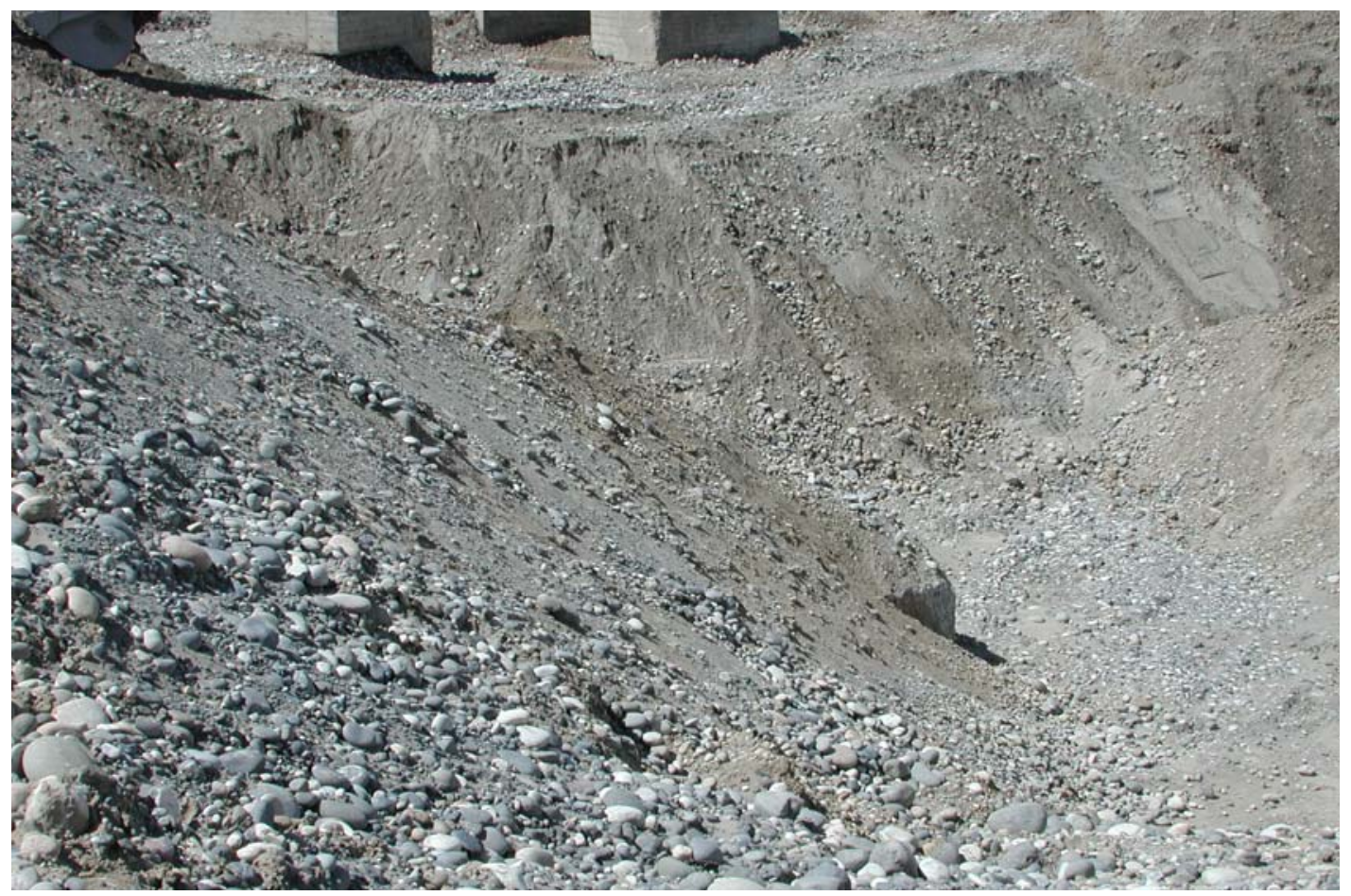


CVP-2007-00004

Rev. 0 
Rev. 0

APPENDIX B

RADIOLOGICAL SURVEYS

$B-\mathrm{i}$ 
CVP-2007-00004

Rev. 0

B-ii 


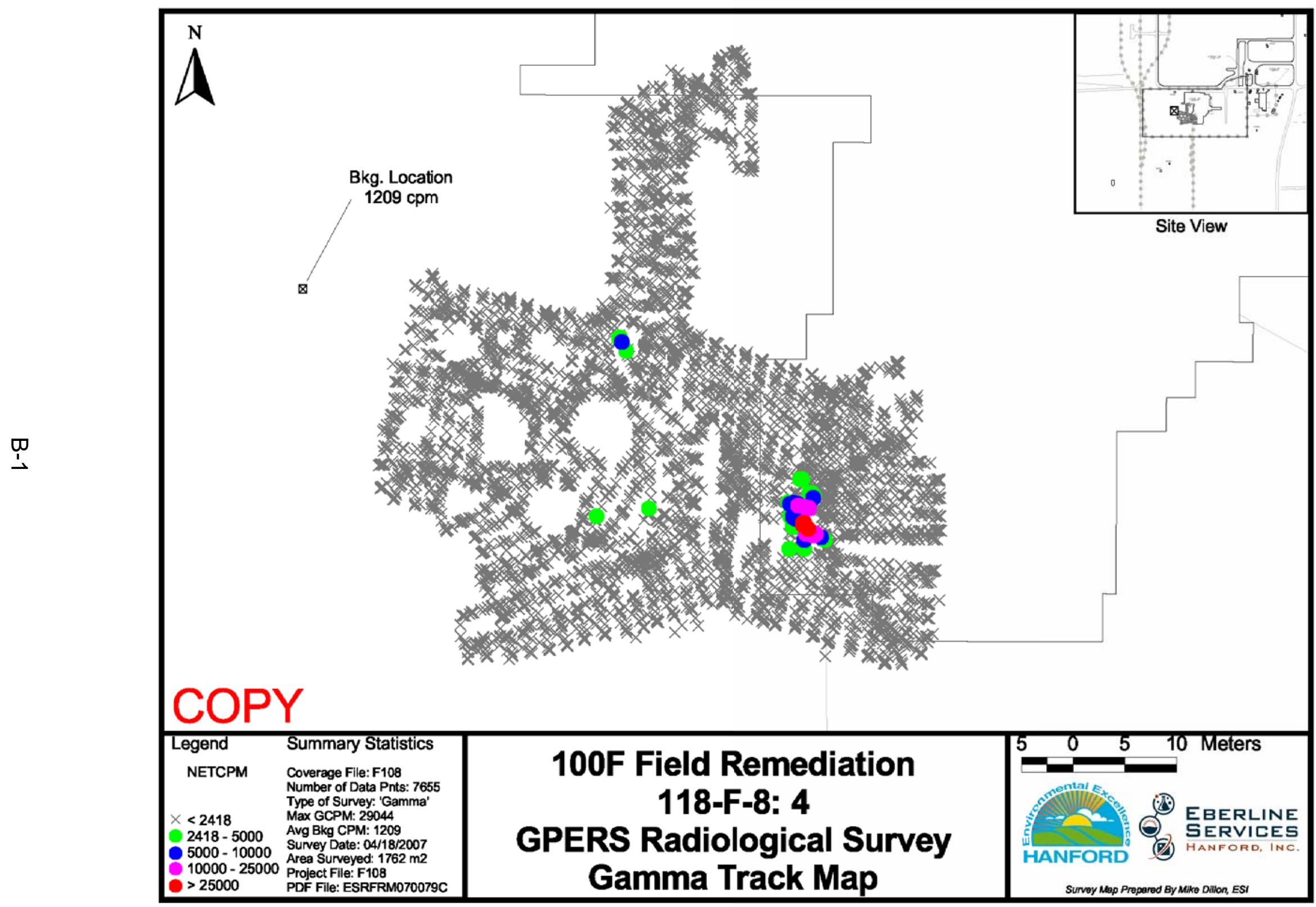



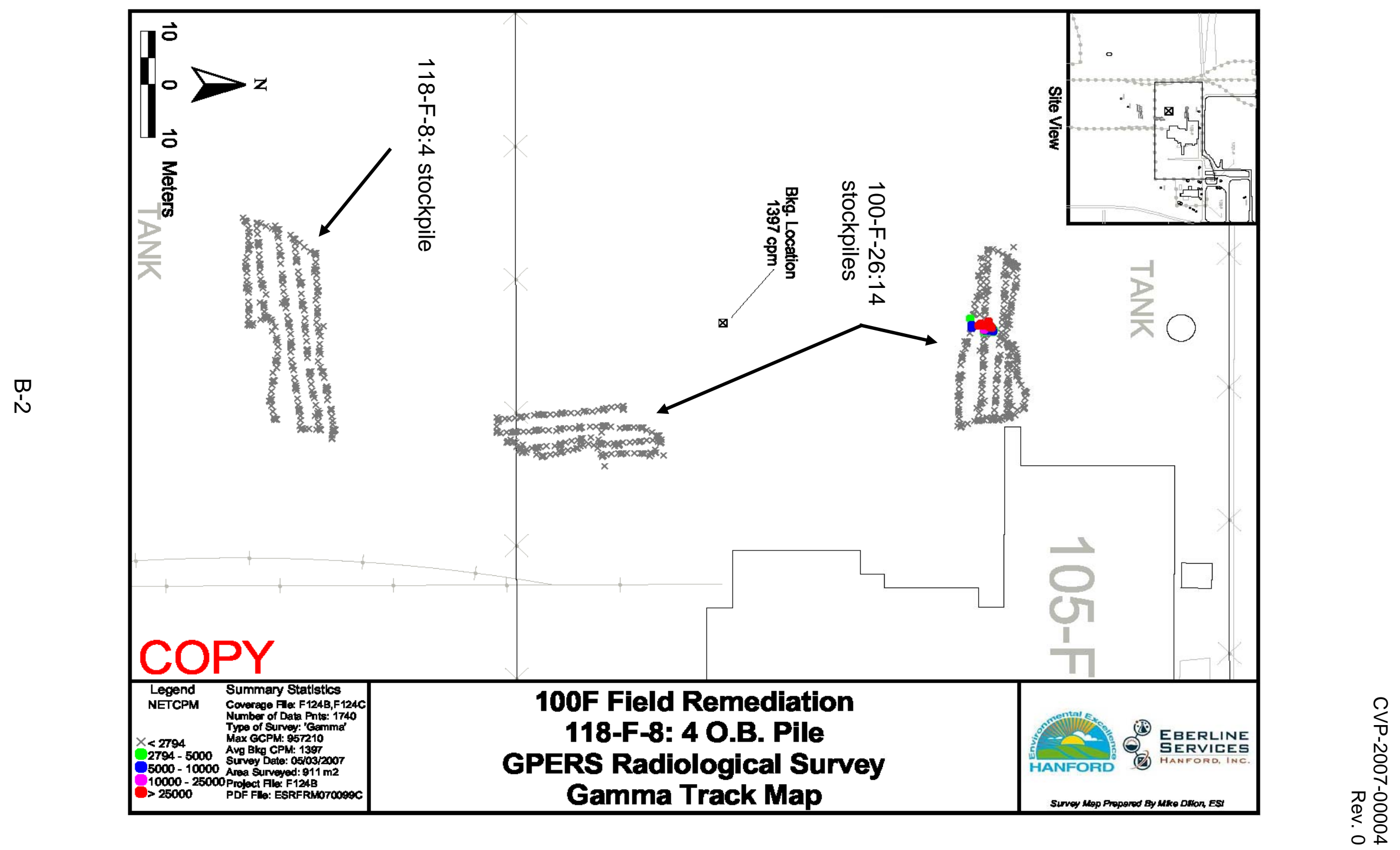


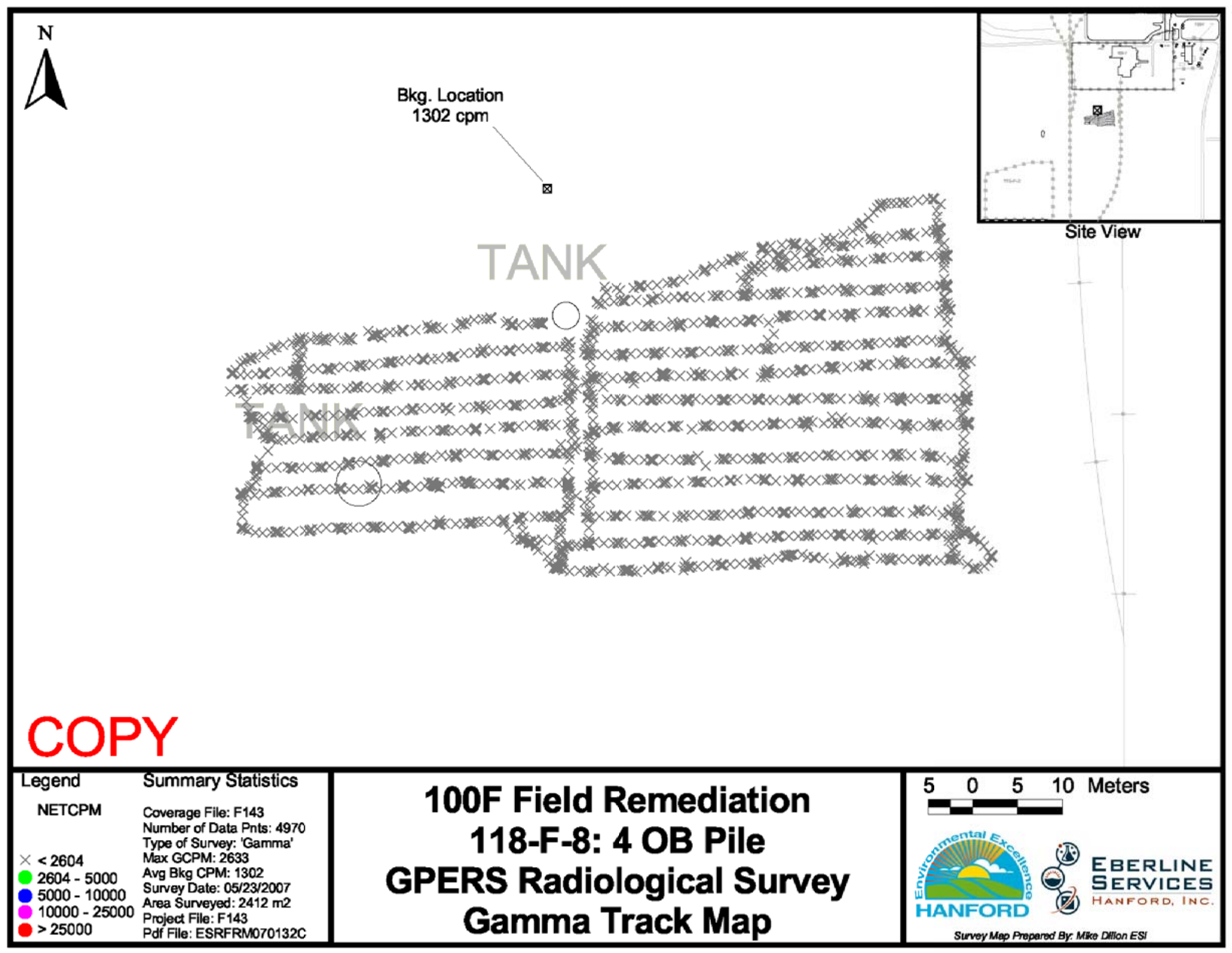

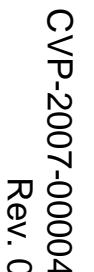


CVP-2007-00004

Rev. 0

B-4 


\section{APPENDIX C}

CALCULATIONS 


\section{DISCLAIMER FOR CALCULATIONS}

The calculations that are provided in the following appendix have been generated to document compliance with established cleanup levels. These calculations should be used in conjunction with other relevant documents in the administrative record. 


\section{CALCULATION BRIEFS}

The following calculation briefs have been prepared in accordance with ENG-1, Engineering Services, ENG-1-4.5, "Project Calculations," Washington Closure Hanford, Richland, Washington.

118-F-8:4 105-F Fuel Storage Basin Cleanup Verification RESRAD Calculation Brief, Calculation No. 0100F-CA-V0303, Rev. 0.

118-F-8:4 105-F Fuel Storage Basin Cleanup Verification 95\% UCL Calculation, Calculation No. 0100F-CA-V0294, Rev. 0.

118-F-8:4 Hazard Quotient and Carcinogenic Risk Calculations, Calculation No. 0100F-CA-V0296, Rev. 0.

118-F-8:4 105-F Fuel Storage Basin Comparison to Drinking Water Standards (MCL) Calculation Brief, Calculation No. 0100F-CA-V0304, Rev. 0.

Shallow and Deep Zone, Overburden Sampling Plan, Calculation No. 0100F-CA-V0307, Rev. 0.

100-F-8:4 Fuel Storage Basin BCL Variance Calculation, Calculation No. 0100F-CA-V0302, Rev. 0.

100-F-8:4 Fuel Storage Basin Shallow Zone Variance Calculation, Calculation No. 0100F-CA-V0301, Rev. 0. 
CVP-2007-00004

Rev. 0

C-iv 


\section{CALCULATION COVER SHEET}

Project Title: Field Remediation

Job No. 14655

Area: $\quad 100-\mathrm{F}$

Discipline: Environmental

Frue $\frac{0}{4 / 19 / \%}$ ${ }^{*}$ Calculation No: 01006-CA-V0303

Subject: $\quad$ 118-F-8:4 105-F Fuel Storage Basin Cleanup Verification RESRAD Calculation Brief Computer Program: RESRAD

Program No: Version 6.3

The attached calculations have been generated to document compliance with established cleanup levels. These calculations should be used in conjunction with other relevant documents in the administrative record.

Committed Calculation $\bigotimes$

Preliminary

Superseded

Voided

\begin{tabular}{|c|c|c|c|c|c|c|}
\hline Rev & Sheet Numbers & Originator & Checker & Reviewer & Approval & Date \\
\hline 0 & \begin{tabular}{|l|} 
Cover $-1 \mathrm{pg}$ \\
Summary $-6 \mathrm{pg}$ \\
Attm. $1-1 \mathrm{pg}$ \\
Attm. $2-19 \mathrm{pg}$ \\
Attm. $3-21 \mathrm{pg}$ \\
Attm. $4-10 \mathrm{pg}$ \\
Attm. $5-27 \mathrm{pg}$ \\
Attm. $6-41 \mathrm{pg}$ \\
Attm. $7-19 \mathrm{pg}$ \\
Attm. $8-24 \mathrm{pg}$ \\
Attm. $9-31 \mathrm{pg}$ \\
Attm. $10-10 \mathrm{pg}$ \\
Total $-210 \mathrm{pages}$ \\
\end{tabular} & $\begin{array}{c}\text { R.u. elana } \\
\text { S. W. Clark }\end{array}$ & $\begin{array}{l}\text { melfiett } \\
\text { M.W.Perrott }\end{array}$ & N/A & S4late & 9.25 .07 \\
\hline & & & & & & \\
\hline & & & & & & \\
\hline & & & & & & \\
\hline
\end{tabular}

SUMMARY OF REVISION

\begin{tabular}{|l|l|}
\hline & \\
\hline & \\
\hline & \\
\hline & \\
\hline
\end{tabular}




Originator: S. W Clark D T Te CALCULATION SHEET

Date: $\quad 107$ Calc. No.: 0100F-CA-V0303

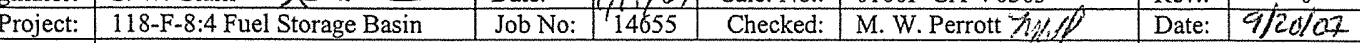

\section{1}

2

\section{PURPOSE:}

Calculate the soil and groundwater concentrations, dose, and risk contributions from remaining radionuclide contaminants in the remediated 118-F-8:4 105-F Fuel Storage Basin areas (the excavation shallow zone, excavation deep zone, and overburden/below cleanup level soil areas) over a period of 1,000 years.

\section{GIVEN/REFERENCES:}

1) Cleanup verification data from 118-F-8:4 105-F Fuel Storage Basin 95\% UCL Calculation, Calculation No. 0100F-CA-V0294, Rev. 0, Washington Closure Hanford, Richland, Washington.

2) Remedial Design Report/Remedial Action Work Plan for the 100 Area (RDR/RAWP), DOE/RL-96-17, Rev. 5, U.S. Department of Energy, Richland Operations Office, Richland, Washington.

3) 118-F-8:4 is a portion of the 118-F-8:3 Fuel Storage Basin that was deferred for remediation (see the Cleanup Verification Package for the 118-F-8:1, 105-F Reactor Below-Grade Structures and Underlying Soils; the 118-F-8:3, 105-F Fuel Storage Basin Underlying Soils; and the 100-F-10 French Drain, CVP-2003-00017, Rev. 0, 2004, Bechtel Hanford, Inc., Richland, Washington). Radioactive and nonradioactive contaminants of concern for the fuel storage basin are from the Sampling and Analysis Plan for the 105-Phase IV Fuel Storage Basin, DOE/RL-2000-54, Rev. 0, U.S. Department of Energy, Richland Operations Office, Richland, Washington. For the purpose of these RESRAD calculations, the radioactive contaminants of concern (COCs) are americium-241, barium-133, carbon-14, cesium-137, cobalt-60, europium-152, europium-154, europium-155, nickel-63, plutonium-238, plutonium-239/240, strontium-90, tritium $(\mathrm{H}-3)$, uranium-234, uranium-235, and uranium-238. The nonradionuclide contaminants of concern are barium, hexavalent chromium, lead, mercury, and PCBs. Attainment of remedial action goals (RAGs) by residual concentrations of nonradionuclides is discussed in the 118-F-8:4 105-F Fuel Storage Basin 95\% UCL Calculation. All nonradionuclide direct exposure RAGs are met and protection of groundwater and the river can be demonstrated by the results of vertical migration modeling in the 100 Area Analogous Sites RESRAD Calculations, 0100X-CA-V0050, Rev. 0, Bechtel Hanford, Inc., Richland, Washington, which predicts that none of the nonradionuclides will migrate to groundwater (and thus the Columbia River) within 1,000 years.

4) RESidual RADioactivity (RESRAD) computer code, version 6.3, to calculate compliance with residual radioactivity guidelines, developed for the U.S. Department of Energy by the Environmental Assessment Division of Argonne National Laboratory, Argonne, Illinois.

5) Sample design data from the 118-F-8:4 Fuel Storage Basin Sampling Plan, Calculation No. 0100F-CA-V0307, Rev. 0, Washington Closure Hanford, Richland, Washington.

6) Groundwater elevation from Hanford Groundwater Monitoring for Fiscal Year 2006, PNNL-16346, Pacific Northwest National Laboratory, Richland, Washington. 
Washington Closure Hanford

CALCULATION SHEET

\begin{tabular}{|c|c|c|c|c|c|c|}
\hline Originator: & S. W. Clark & $\$ 1 / 969$ & Calc. No.: & $0100 \mathrm{~F}-\mathrm{CA}-\mathrm{V} 0303$ & Rev: & 0 \\
\hline Project: & 118-F-8:4 Fuel Storage Basin & Job No: 14655 & Checked: & M. W. Perrott /h/lof & Date: & $9 / 20 / 07$ \\
\hline
\end{tabular}

Subject: $118-\mathrm{F}-8: 4$ 105-F Fuel Storage Basin Cleanup Verification RESRAD Calculation Brief

\section{SOLUTION:}

1) Separate RESRAD runs were performed for the 118-F-8:4 Fuel Storage Basin Excavation Shallow Zone, Excavation Deep Zone, and Overburden/Below Cleanup Level Stockpile (OB/BCL) soils. Table 1 shows the elevations (NAVD88) and thickness of each soil horizon. Attachment 1 shows representative dimensions of soil horizons and contaminant pathways considered for dose, risk, and groundwater protection. Input factors for each run are shown in the "Summary" section of the RESRAD "Mixture Sums and Single Radionuclide Guidelines" printouts in the Attachments to this Calculation Summary.

\begin{tabular}{|l|c|c|c|c|}
\hline \multicolumn{5}{|c|}{ Table 1. Waste Site Dimensions for RESRAD Modeling } \\
\hline \multicolumn{1}{|c|}{ Parameter } & Units & $\begin{array}{c}\text { Excavation } \\
\text { Shallow Zone }\end{array}$ & $\begin{array}{c}\text { Excavation } \\
\text { Deep Zone }\end{array}$ & OB/BCL \\
\hline \multicolumn{5}{|c|}{ Contaminated Zone Dimensions } \\
\hline Cover Depth & $\mathrm{m}$ & 0 & 4.6 & 0 \\
\hline Area of Contaminated Zone (CZ) & $\mathrm{m}^{2}$ & 1,634 & 1,634 & 3,558 \\
\hline Length Parallel to Aquifer Flow & $\mathrm{m}$ & 42 & 42 & 69 \\
\hline \multicolumn{2}{|c|}{ Elevations of Vadose Zone Horizons } \\
\hline Elevation: Surface & $\mathrm{m}$ & 126.4 & 126.4 & 126.4 \\
\hline Elevation: Groundwater & $\mathrm{m}$ & 114.0 & 114.0 & 114.0 \\
\hline Thickness: Contaminated Zone & $\mathrm{m}$ & 4.6 & 7.8 & 4.6 \\
\hline Thickness: Unsaturated Zone & $\mathrm{m}$ & 7.8 & 0 & 7.8 \\
\hline OB/BCL = Overburden/Below Cleanup Levels Stockpile & & \\
\hline
\end{tabular}

2) The year where the peak dose (or concentration) occurs from each individual radionuclide $\mathrm{COC}$ and layer is determined by a preliminary run. This year is then added for all horizons for the final RESRAD runs. For the direct exposure pathway (i.e. soil ingestion and inhalation and external radiation), the peak year occurred at year zero (year 2007) for all COCs. For the water pathways (i.e. drinking water and food ingestion) the peak year was year 7 for cobalt-60, year 43 for cesium-137 and strontium-90, and year 135 for nickel-63. The 7-, 43-, and 135-year time periods were added to all RESRAD runs.

\section{METHODOLOGY:}

1) Runs of RESRAD version 6.3 were completed for the 118-F-8:4 Fuel Storage Basin Excavation Shallow Zone, Excavation Deep Zone, and Overburden/Below Cleanup Level Stockpile (OB/BCL) soils using the radionuclide concentrations shown in Table 2. RESRAD numerical output reports for dose, risk, and concentration are presented in the Attachments to this calculation summary. 


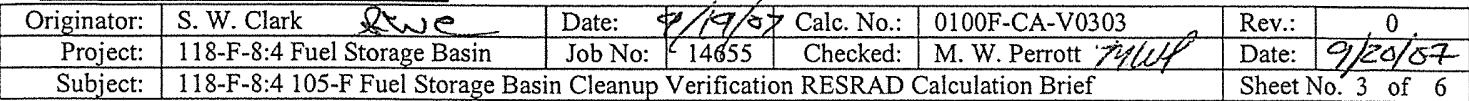

\begin{tabular}{|c|c|c|c|}
\hline \multicolumn{4}{|c|}{ Table 2. Cleanup Verification Data Set ${ }^{\text {a }}$} \\
\hline $\mathrm{COCs}$ & $\begin{array}{c}\text { Excavation } \\
\text { Shallow Zone }\end{array}$ & $\begin{array}{l}\text { Excavation } \\
\text { Deep Zone }\end{array}$ & $\mathrm{OB} / \mathrm{BCL}$ \\
\hline \multicolumn{4}{|c|}{ Radionuclide Activity ( $p C i / g$ ) } \\
\hline Americium-241 & $0.110 \mathrm{U}$ & 0.081 & $0.027 \mathrm{U}$ \\
\hline Barium-133 & $0.020 \mathrm{U}$ & $0.022 \mathrm{U}$ & $0.018 \mathrm{U}$ \\
\hline Carbon-14 & $-0.325 \mathrm{U}$ & $0.070 \mathrm{U}$ & $-0.7 \mathrm{U}$ \\
\hline Cesium-137 & 0.281 & 4.52 & 0.184 \\
\hline Cobalt- 60 & $0.019 \mathrm{U}$ & 0.186 & 0.016 \\
\hline Europium-152 & 0.160 & 3.01 & 0.240 \\
\hline Europium-154 & $0.061 \mathrm{U}$ & $0.181 \mathrm{U}$ & $0.050 \mathrm{U}$ \\
\hline Europium-155 & $0.050 \mathrm{U}$ & $0.057 \mathrm{U}$ & $0.044 \mathrm{U}$ \\
\hline Nickel-63 & $1.26 \mathrm{U}$ & 18.7 & $0.637 \mathrm{U}$ \\
\hline Plutonium-238 & $0.023 \mathrm{U}$ & $0.056 \mathrm{U}$ & $0.008 \mathrm{U}$ \\
\hline Plutonium-239 & $0.051 \mathrm{U}$ & 0.42 & 0.124 \\
\hline Plutonium-240 & $0.012 \mathrm{U}$ & 0.10 & 0.03 \\
\hline Strontium-90 & $0.151 \mathrm{U}$ & 2.20 & $0.036 \mathrm{U}$ \\
\hline Tritium $(\mathrm{H}-3)$ & $2.85 \mathrm{U}$ & $1.38 \mathrm{U}$ & $0.857 \mathrm{U}$ \\
\hline Uranium-233/234 & $0(<\mathrm{BG})$ & $O(<\mathrm{BG})$ & $0(<\mathrm{BG})$ \\
\hline Uranium-235 & $0(<\mathrm{BG})$ & $0(<\mathrm{BG})$ & $0(<\mathrm{BG})$ \\
\hline Uranium-238 & $0(<\mathrm{BG})$ & $0(<\mathrm{BG})$ & $0(<\mathrm{BG})$ \\
\hline \multicolumn{4}{|c|}{$\begin{array}{l}\mathrm{U}=\text { Undetected. Not evaluated in RESRAD modeling. } \\
\mathrm{OB} / \mathrm{BCL}=\text { Overburden/Below Cleanup Levels Stockpile } \\
\text { a } \\
\text { Soil concentration values are from the } 118-F-8: 4105-F \text { Fuel Storage Basin } 95 \% \text { UCL Calculation, } \\
\text { Calculation No. O100F-CA-V0294, Rev. 0, Washington Closure Hanford, Richland, Washington. Refer to } \\
\text { the } 95 \% \text { UCL for nonradionuclide concentrations. Nonradionuclides are not evaluated in this RESRAD } \\
\text { calculation. }\end{array}$} \\
\hline
\end{tabular}

\section{RESULTS:}

\section{1) Radionuclide "All Pathways" Dose Rate}

The "all pathways" (maximum) dose rates are shown in Table 3. The maximum all pathways dose rate from the 118-F-8:4 Fuel Storage Basin is $1.70 \mathrm{mrem} / \mathrm{yr}$ at year zero (2007) from the Overburden/Below Cleanup Level Stockpile (OB/BCL) area. The maximum all-pathways dose rates from the combined Excavation Shallow and Deep Zone is $1.31 \mathrm{mrem} / \mathrm{yr}$ at year zero (2007).

\begin{tabular}{|c|c|c|c|c|c|c|c|c|c|c|}
\hline \multicolumn{8}{|c|}{ Table 3. All Pathways Dose Rate (mrem/yr) } \\
\hline $\begin{array}{c}\text { RESRAD } \\
\text { Run }\end{array}$ & $\begin{array}{c}\text { Vadose Zone } \\
\text { Horizons }\end{array}$ & \multicolumn{8}{c|}{ "All Pathways" Dose Contributions in mrem/yr at Each Time Slice (yr) } \\
\cline { 3 - 11 } & 0 & 1 & 3 & 7 & 11 & 43 & 135 & 300 & 1000 \\
\hline Excavation & Shallow Zone & $1.31 \mathrm{E}+00$ & $1.26 \mathrm{E}+00$ & $1.17 \mathrm{E}+00$ & $1.01 \mathrm{E}+00$ & $8.70 \mathrm{E}-01$ & $3.02 \mathrm{E}-01$ & $2.73 \mathrm{E}-02$ & $5.69 \mathrm{E}-04$ & $4.63 \mathrm{E}-11$ \\
\hline Excavation & Deep Zone & $8.89 \mathrm{E}-24$ & $5.93 \mathrm{E}-03$ & $1.72 \mathrm{E}-02$ & $3.66 \mathrm{E}-02$ & $5.25 \mathrm{E}-02$ & $9.73 \mathrm{E}-02$ & $3.89 \mathrm{E}-02$ & $5.05 \mathrm{E}-03$ & $3.41 \mathrm{E}-05$ \\
\hline Excavation & Total & $1.31 \mathrm{E}+00$ & $1.27 \mathrm{E}+00$ & $1.19 \mathrm{E}+00$ & $1.04 \mathrm{E}+00$ & $9.23 \mathrm{E}-01$ & $3.99 \mathrm{E}-01$ & $6.62 \mathrm{E}-02$ & $5.62 \mathrm{E}-03$ & $3.41 \mathrm{E}-05$ \\
\hline $\mathrm{OB} / \mathrm{BCL}$ & Shallow Zone & $1.70 \mathrm{E}+00$ & $1.62 \mathrm{E}+00$ & $1.47 \mathrm{E}+00$ & $1.22 \mathrm{E}+00$ & $1.02 \mathrm{E}+00$ & $3.32 \mathrm{E}-01$ & $8.33 \mathrm{E}-02$ & $6.37 \mathrm{E}-02$ & $5.91 \mathrm{E}-02$ \\
\hline $\mathrm{OB} / \mathrm{BCL}=$ Overburden/Below Cleanup Levels Stockpile Material \\
\hline
\end{tabular}


Washington Closure Hanford

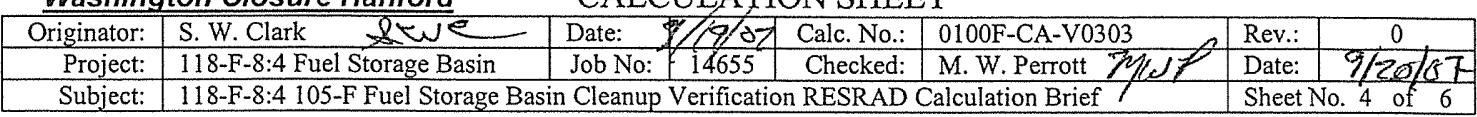

1 2) Radionuclide Excess Lifetime Cancer Risk

2 The radionuclide excess lifetime cancer risk (ELCR) results are shown in Table 4. The

3 maximum ELCR for the 118-F-8:4 Fuel Storage Basin is $1.99 \times 10^{-5}$ at year zero (2007) for the

4 Overburden/Below Cleanup Level Stockpile (OB/BCL) area. The maximum ELCR result for

5 the combined Excavation Shallow and Deep Zone is $1.89 \times 10^{-5}$.

6

\begin{tabular}{|c|c|c|c|c|c|c|c|c|c|c|}
\hline \multicolumn{10}{|c|}{ Table 4. Radionuclide Excess Lifetime Cancer Risk } \\
\hline $\begin{array}{c}\text { RESRAD } \\
\text { Run }\end{array}$ & $\begin{array}{c}\text { Vadose Zone } \\
\text { Horizons }\end{array}$ & 0 & 1 & 3 & 7 & 11 & 43 & 135 & 300 & 1000 \\
\hline & & 0 & \multicolumn{7}{c|}{ Excess Cancer Risk at Each Time Slice (yr) } \\
\hline Excavation & Shallow Zone & $1.79 \mathrm{E}-05$ & $1.73 \mathrm{E}-05$ & $1.61 \mathrm{E}-05$ & $1.40 \mathrm{E}-05$ & $1.22 \mathrm{E}-05$ & $4.52 \mathrm{E}-06$ & $4.37 \mathrm{E}-07$ & $9.17 \mathrm{E}-09$ & $7.46 \mathrm{E}-16$ \\
\hline Excavation & Deep Zone & $9.55 \mathrm{E}-07$ & $1.00 \mathrm{E}-06$ & $1.09 \mathrm{E}-06$ & $1.24 \mathrm{E}-06$ & $1.36 \mathrm{E}-06$ & $1.54 \mathrm{E}-06$ & $6.32 \mathrm{E}-07$ & $1.46 \mathrm{E}-07$ & $8.63 \mathrm{E}-10$ \\
\hline Excavation & Total & $1.89 \mathrm{E}-05$ & $1.83 \mathrm{E}-05$ & $1.72 \mathrm{E}-05$ & $1.53 \mathrm{E}-05$ & $1.36 \mathrm{E}-05$ & $6.06 \mathrm{E}-06$ & $1.07 \mathrm{E}-06$ & $1.56 \mathrm{E}-07$ & $8.63 \mathrm{E}-10$ \\
\hline OB/BCL & Shallow Zone & $1.99 \mathrm{E}-05$ & $1.90 \mathrm{E}-05$ & $1.74 \mathrm{E}-05$ & $1.46 \mathrm{E}-05$ & $1.24 \mathrm{E}-05$ & $3.90 \mathrm{E}-06$ & $4.25 \mathrm{E}-07$ & $1.29 \mathrm{E}-07$ & $1.14 \mathrm{E}-07$ \\
\hline OB/BCL = Overburden/Below Cleanup Levels Stockpile & \multicolumn{10}{c|}{} \\
\hline
\end{tabular}

7

8 3) Radionuclide Groundwater Protection

9 The radionuclide concentrations in groundwater calculated by the RESRAD model are

10 summarized in Table 5. Among the radionuclide contaminants of concern cobalt-60,

11 cesium-137, nickel-63, and strontium-90 are calculated to reach groundwater in the 1,000 years

12 of the RESRAD model evaluation. The organ specific dose via the groundwater (and river)

13 pathway is presented in a separate calculation brief [118-F-8:4 Fuel Storage Basin Comparison

14 To Drinking Water Standards (MCL) Calculation, Calculation No. 0100F-CA-V0304, Rev. 0,

15 Washington Closure Hanford, Richland, Washington]. Only concentrations are presented here.

16

\begin{tabular}{|c|c|c|c|c|c|c|c|c|c|c|c|}
\hline \multirow{2}{*}{$\begin{array}{c}\text { Radio- } \\
\text { nuclides }\end{array}$} & \multirow{2}{*}{ RESRAD Run } & \multicolumn{9}{|c|}{ Groundwater Concentrations in $\mathrm{pCi} / \mathrm{L}$ at Each Time Slice (yr) } & \multirow{2}{*}{$\begin{array}{l}\text { RAGs, } \\
\mathrm{pCi} / \mathrm{L}\end{array}$} \\
\hline & & 0 & 1 & 3 & 7 & 11 & 43 & 135 & 300 & 1000 & \\
\hline \multirow[t]{4}{*}{$\mathrm{Co}-60$} & Excavation $\mathrm{SZ}$ & 0 & 0 & 0 & 0 & 0 & 0 & 0 & 0 & 0 & \multirow[t]{4}{*}{100} \\
\hline & Excavation DZ & 0 & $4.20 \mathrm{E}-04$ & $9.68 \mathrm{E}-04$ & $1.34 \mathrm{E}-03$ & $1.24 \mathrm{E}-03$ & $7.20 \mathrm{E}-05$ & 1.26E-09 & $1.05 \mathrm{E}-18$ & 0 & \\
\hline & Excav. Total & 0 & 4.20E-04 & $9.68 \mathrm{E}-04$ & \begin{tabular}{|l|}
$1.34 \mathrm{E}-03$ \\
\end{tabular} & $1.24 \mathrm{E}-03$ & $7.20 \mathrm{E}-05$ & $1.26 \mathrm{E}-09$ & $1.05 \mathrm{E}-18$ & 0 & \\
\hline & $\mathrm{OB} / \mathrm{BCL}$ & 0 & 0 & 0 & 0 & 0 & 0 & 0 & 0 & 0 & \\
\hline \multirow[t]{4}{*}{ Cs- 137} & Excavation $\mathrm{SZ}$ & 0 & 0 & 0 & 0 & 0 & 0 & 0 & 0 & 0 & \multirow[t]{4}{*}{60} \\
\hline & Excavation DZ & 0 & $1.14 \mathrm{E}-02$ & $3.26 \mathrm{E}-02$ & $6.93 \mathrm{E}-02$ & $9.93 \mathrm{E}-02$ & $1.85 \mathrm{E}-01$ & $6.92 \mathrm{E}-02$ & $3.39 \mathrm{E}-03$ & $8.55 \mathrm{E}-10$ & \\
\hline & Excav. Total & 0 & $1.14 \mathrm{E}-02$ & $3.26 \mathrm{E}-02$ & $6.93 \mathrm{E}-02$ & $9.93 \mathrm{E}-02$ & $1.85 \mathrm{E}-01$ & 6.92E-02 & $3.39 \mathrm{E}-03$ & $8.55 \mathrm{E}-10$ & \\
\hline & $\mathrm{OB} / \mathrm{BCL}$ & 0 & 0 & 0 & 0 & 0 & 0 & 0 & 0 & 0 & \\
\hline \multirow[t]{4}{*}{$\mathrm{Ni}-63$} & Excavation $\mathrm{SZ}$ & 0 & 0 & 0 & 0 & 0 & 0 & 0 & 0 & 0 & \multirow[t]{4}{*}{50} \\
\hline & Excavation DZ & 0 & $2.21 \mathrm{E}-01$ & $6.53 \mathrm{E}-01$ & $1.48 \mathrm{E}+00$ & $2.26 \mathrm{E}+00$ & $6.99 \mathrm{E}+00$ & $1.12 \mathrm{E}+01$ & $7.36 \mathrm{E}+00$ & 4.28E-02 & \\
\hline & Excav. Total & 0 & $2.21 \mathrm{E}-01$ & $6.53 \mathrm{E}-01$ & $1.48 \mathrm{E}+00$ & $2.26 \mathrm{E}+00$ & $6.99 \mathrm{E}+00$ & $1.12 \mathrm{E}+0 \mathrm{I}$ & $7.36 \mathrm{E}+00$ & $4.28 \mathrm{E}-02$ & \\
\hline & $\mathrm{OB} / \mathrm{BCL}$ & 0 & 0 & 0 & 0 & 0 & 0 & 0 & 0 & 0 & \\
\hline
\end{tabular}


Washington Closure Hanford CALCULATION SHEET

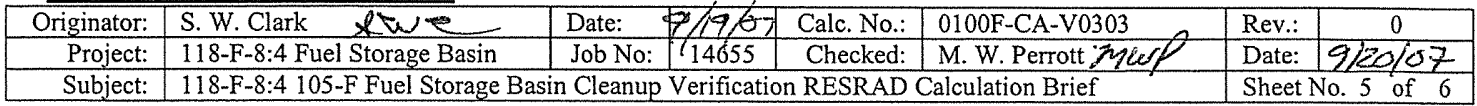

Table 5. Predicted Groundwater (Well Water/Drinking Water) Concentrations (2 Pages)

\begin{tabular}{|c|c|c|c|c|c|c|c|c|c|c|c|}
\hline \multirow{2}{*}{$\begin{array}{c}\text { Radio- } \\
\text { nuclides }\end{array}$} & \multirow{2}{*}{ RESRAD Run } & \multicolumn{9}{|c|}{ Groundwater Concentrations in $\mathrm{pCi} / \mathrm{L}$ at Each Time Slice (yr) } & \multirow{2}{*}{$\begin{array}{c}\text { RAGs, } \\
\mathrm{pCi} / \mathrm{L}\end{array}$} \\
\hline & & 0 & 1 & 3 & 7 & 11 & 43 & 135 & 300 & 1000 & \\
\hline \multirow[t]{4}{*}{ Sr-90 } & Excavation $\mathrm{SZ}$ & 0 & 0 & 0 & 0 & 0 & 0 & 0 & 0 & 0 & \multirow[t]{4}{*}{8} \\
\hline & Excavation DZ & 0 & $4.04 \mathrm{E}-02$ & $1.15 \mathrm{E}-01$ & $2.45 \mathrm{E}-01$ & $3.50 \mathrm{E}-01$ & $6.36 \mathrm{E}-01$ & $2.22 \mathrm{E}-01$ & $7.09 \mathrm{E}-03$ & $3.64 \mathrm{E}-10$ & \\
\hline & Excav. Total & 0 & $4.04 \mathrm{E}-02$ & 1.15E-01 & $2.45 \mathrm{E}-01$ & 3.50E-01 & $6.36 \mathrm{E}-01$ & $2.22 \mathrm{E}-01$ & $7.09 \mathrm{E}-03$ & $3.64 \mathrm{E}-10$ & \\
\hline & $\mathrm{OB} / \mathrm{BCL}$ & 0 & 0 & 0 & 0 & 0 & 0 & 0 & 0 & 0 & \\
\hline
\end{tabular}

\section{CONCLUSIONS:}

- The "all pathways" (maximum) dose rates are shown in Table 3. The maximum all pathways dose rate from the 118-F-8:4 Fuel Storage Basin is $1.70 \mathrm{mrem} / \mathrm{yr}$ at year zero (2007) from the Overburden/Below Cleanup Level Stockpile (OB/BCL) area. The maximum all-pathways dose rates from the combined Excavation Shallow and Deep Zone is $1.31 \mathrm{mrem} / \mathrm{yr}$ at year zero (2007).

- The radionuclide excess lifetime cancer risk (ELCR) results are shown in Table 4. The maximum ELCR for the 118-F-8:4 Fuel Storage Basin is $1.99 \times 10^{-5}$ at year zero (2007) for the Overburden/Below Cleanup Level Stockpile (OB/BCL) area. The maximum ELCR result for the combined Excavation Shallow and Deep Zone is $1.89 \times 10^{-5}$.

- The dominant pathway for the RESRAD evaluations dose rate for the combined Excavation Shallow and Deep Zone and the Overburden/Below Cleanup Level Stockpile (OB/BCL) area is direct external exposure due to cesium-137 and europium-152.

- None of the site COCs are projected to exceed remedial action goals (RAGs).

- Among the radionuclide contaminants of concern cobalt-60, cesium-137, nickel-63, and strontium-90 are calculated to reach groundwater in the 1,000 years of the RESRAD model evaluation. All of these contaminants are predicted to reach groundwater at concentrations significantly below the RAGs.

\section{ATTACHMENTS:}

1. Graphic showing 118-F-8:4 Cleanup Verification Model (1 page)

2. RESRAD Output: 118-F-8:4 Excavation Shallow Zone Radionuclides - Mixture Sums and Single Radionuclide Guidelines (19 pages)

3. RESRAD Output: 118-F-8:4 Excavation Shallow Zone Radionuclides - Intake Quantities and Health Risk Factors (21 pages) 
Washington Closure Hanford CALCULATION SHEET

\begin{tabular}{|c|c|c|c|c|c|c|c|}
\hline Originator: & S. W. Clark & Date: & $9 / 1967$ & Calc. No.: & $0100 \mathrm{~F}-\mathrm{CA}-\mathrm{V} 0303$ & Rev.: & 0 \\
\hline Project: & 118-F-8:4 Fuel Storage Basin & Job No: & 14655 & Checked: & M. W. Perrott $7 / 20 /$ & Date: & $9 / 20 / 67$ \\
\hline
\end{tabular}

1 4. RESRAD Output: 118-F-8:4 Excavation Shallow Zone Radionuclides - Concentration of Radionuclides, (10 pages)

5. RESRAD Output: 118-F-8:4 Excavation Deep Zone Radionuclides - Mixture Sums and Single Radionuclide Guidelines (27 pages)

6. RESRAD Output: 118-F-8:4 Excavation Deep Zone Radionuclides Radionuclides - Intake Quantities and Health Risk Factors (41 pages)

7. RESRAD Output: 118-F-8:4 Excavation Deep Zone Radionuclides Radionuclides Concentration of Radionuclides (19 pages)

8. RESRAD Output: 118-F-8:4 Overburden/Below Cleanup Level Radionuclides - Mixture Sums and Single Radionuclide Guidelines (24 pages)

9. RESRAD Output: 118-F-8:4 Overburden/Below Cleanup Level Radionuclides Radionuclides - Intake Quantities and Health Risk Factors (31 pages)

10. RESRAD Output: 118-F-8:4 Overburden/Below Cleanup Level Radionuclides Radionuclides - Concentration of Radionuclides (10 pages) 
118-F-8:4 105-F Fuel Storage Basin Cleanup Verification Model

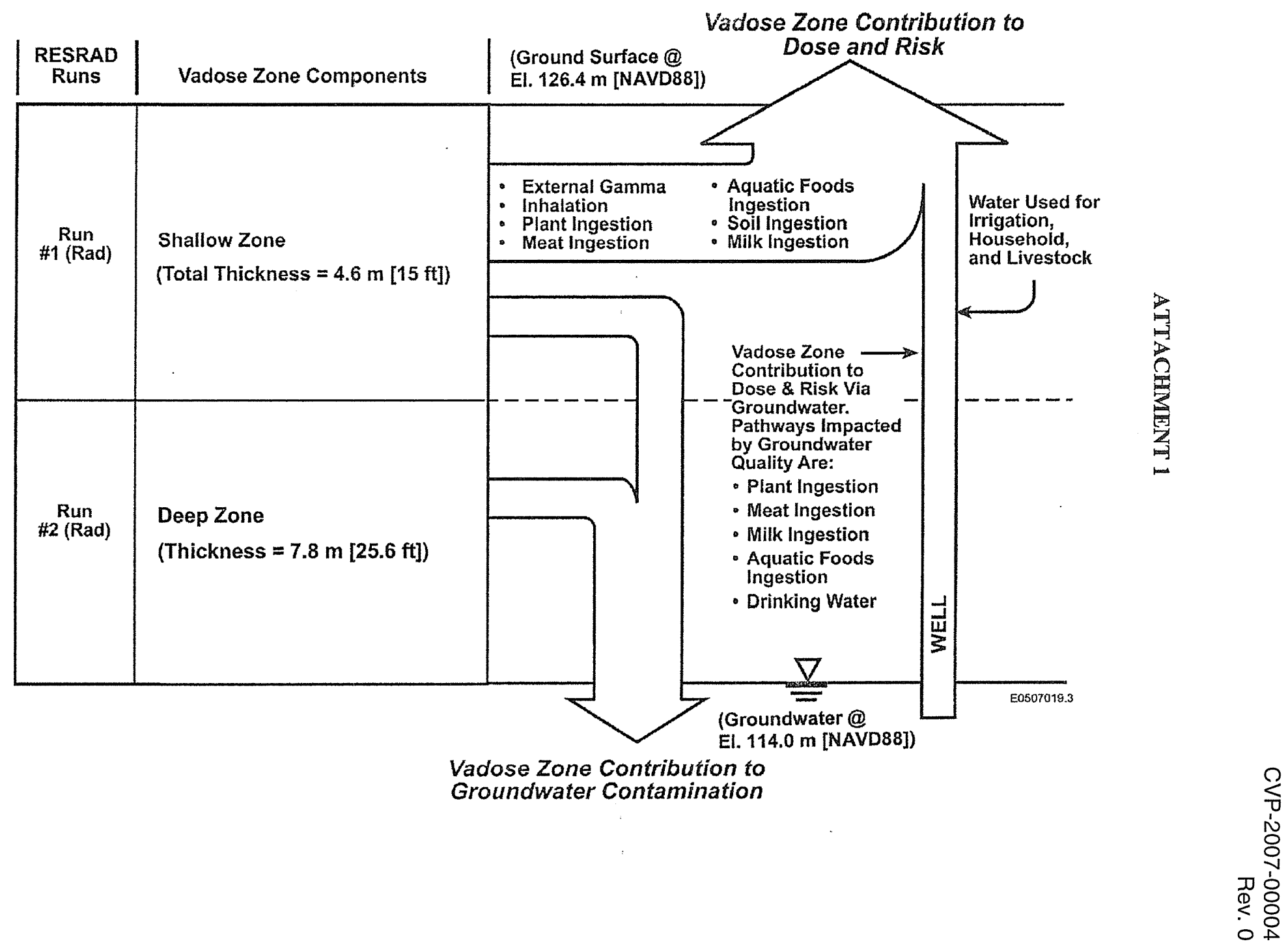




\section{ATTACHMENT 2}

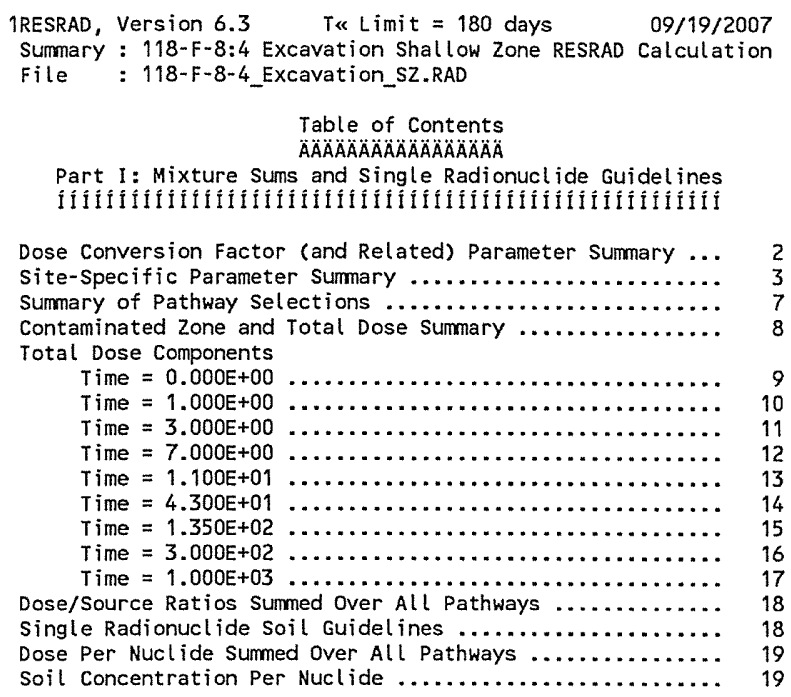

Attachment 2 Sheet No. 1 of 19 Originator: S.W. Clark \&5Je Date $9 \sqrt{14 / 07}$ Chk'd By M.W. Perrott Zfue Date \$polot Calc. No. $0100 \mathrm{~F}-\mathrm{CA}-\mathrm{V} 0303$ Rev. No. 0 


\section{ATTACHMENT 2}

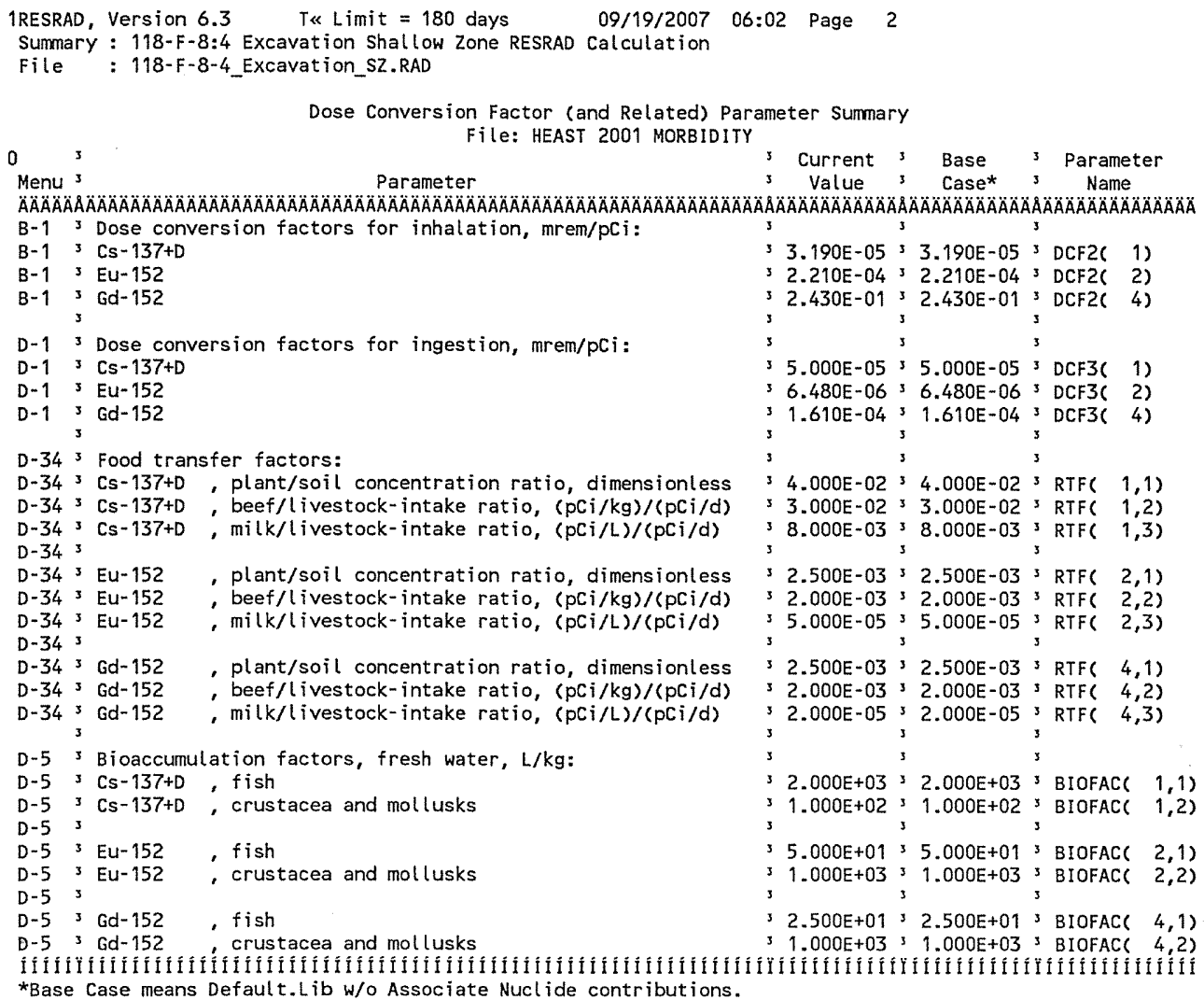

$\begin{array}{ll}\text { Attachment } \frac{2}{\text { S. Clark }} & \text { Sheet No. } 2 \text { of } 19 \\ \text { Originator: } \frac{\text { S.W. }}{\text { M. W. Perrott }} & \text { Date } \\ \text { Chk'd By } & \text { Rev. No. } 0 \\ \text { Calc. No. } 0100 \text { F-CA-V0303 } & \text { Rev. }\end{array}$




\section{ATTACHMENT 2}

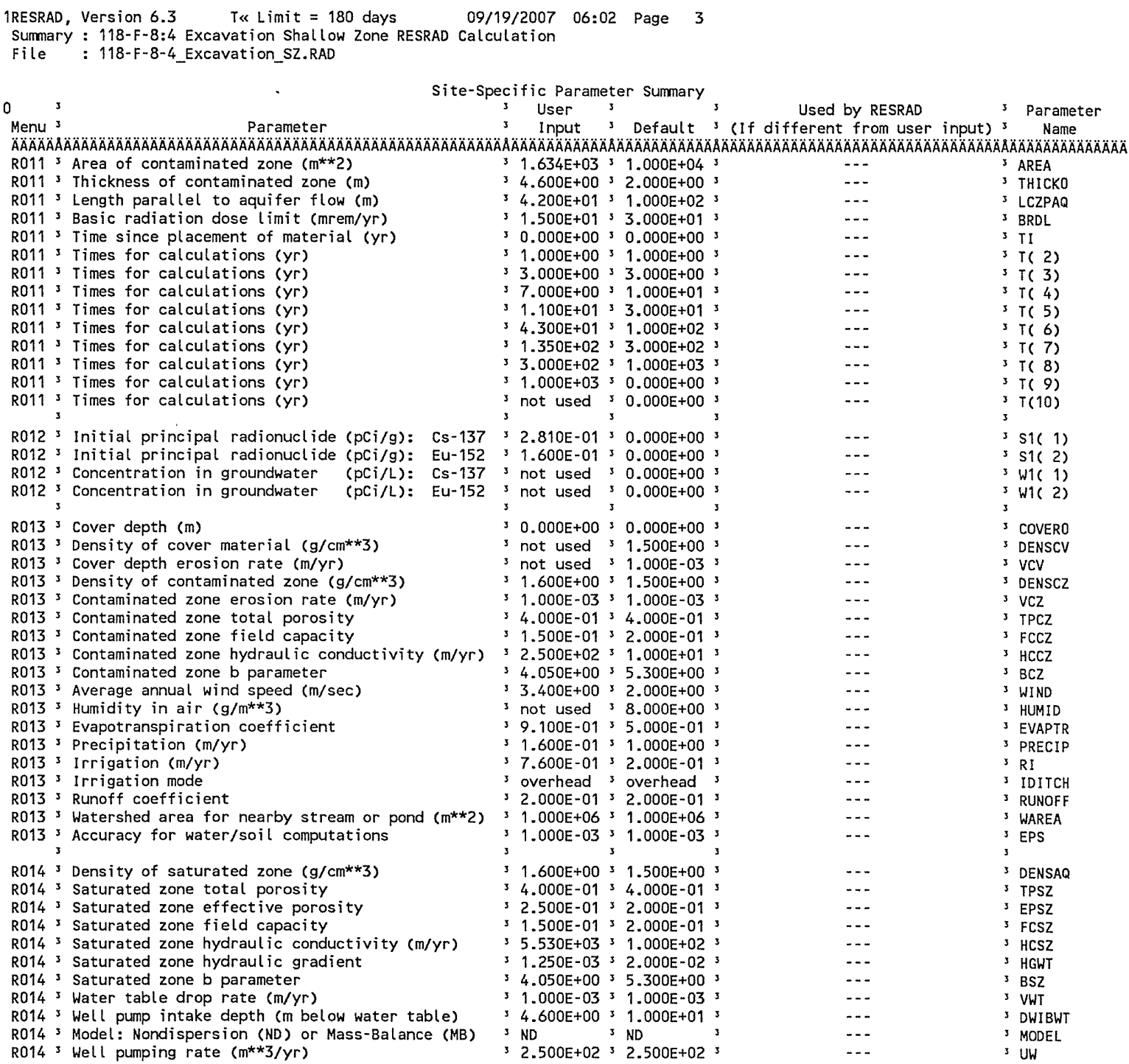

Attachment Originator: S.W. Clark 2 Calc. No. 0100F-CA-V0303
Sheet No. $\underline{3}$ of 19

Rev. No. 0 


\section{ATTACHMENT 2}

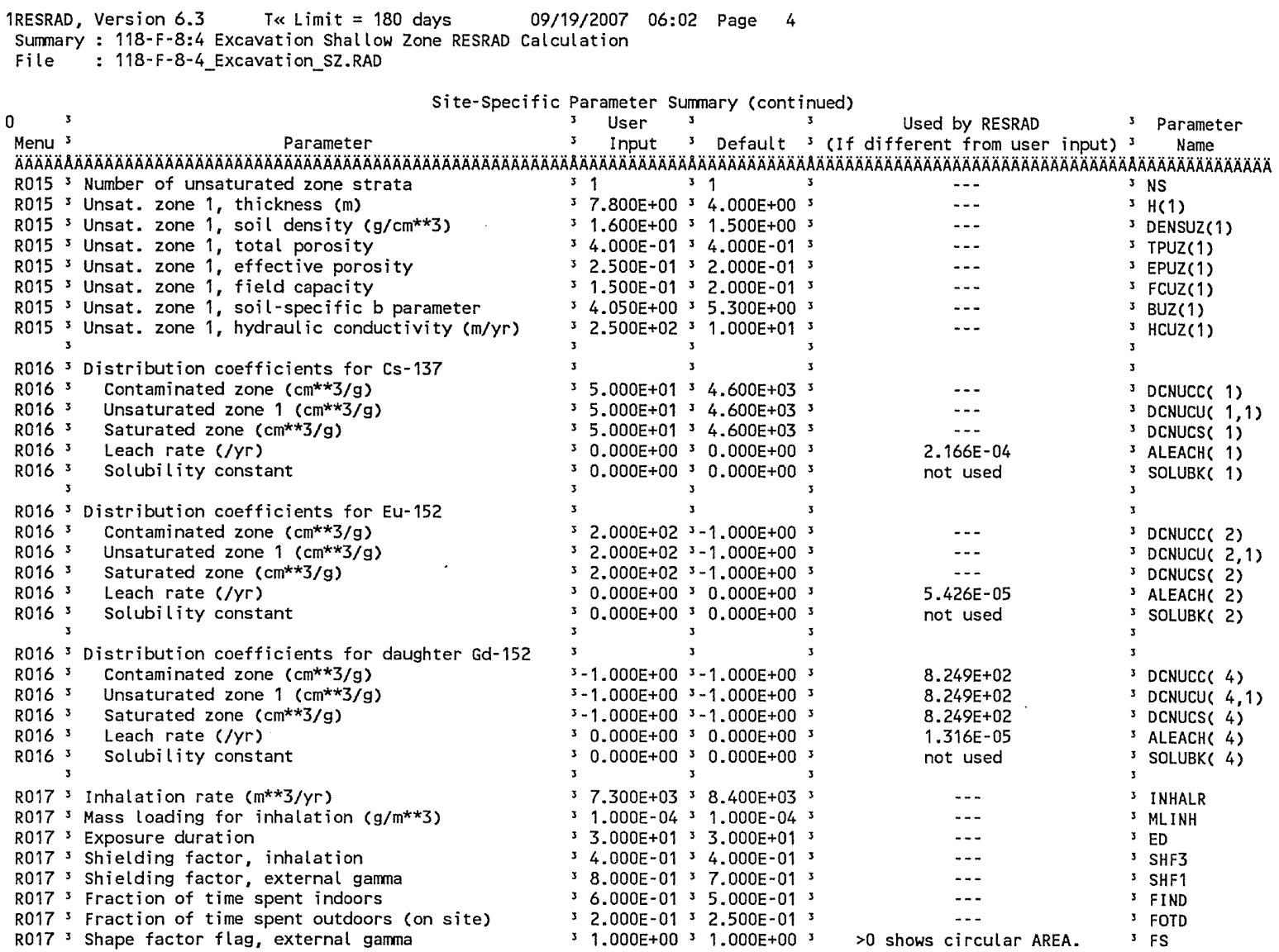

$\begin{array}{ll}\text { Attachment } \frac{2}{\text { O. W. Clark }} & \text { Sheet No. } 4 \text { of } 19 \\ \text { Originator: } & \text { Date } \\ \text { Chk'd By } & \text { Date } \\ \text { Calc. No. N. Perrott } & \text { Rev. No. } \quad 0\end{array}$




\section{ATTACHMENT 2}

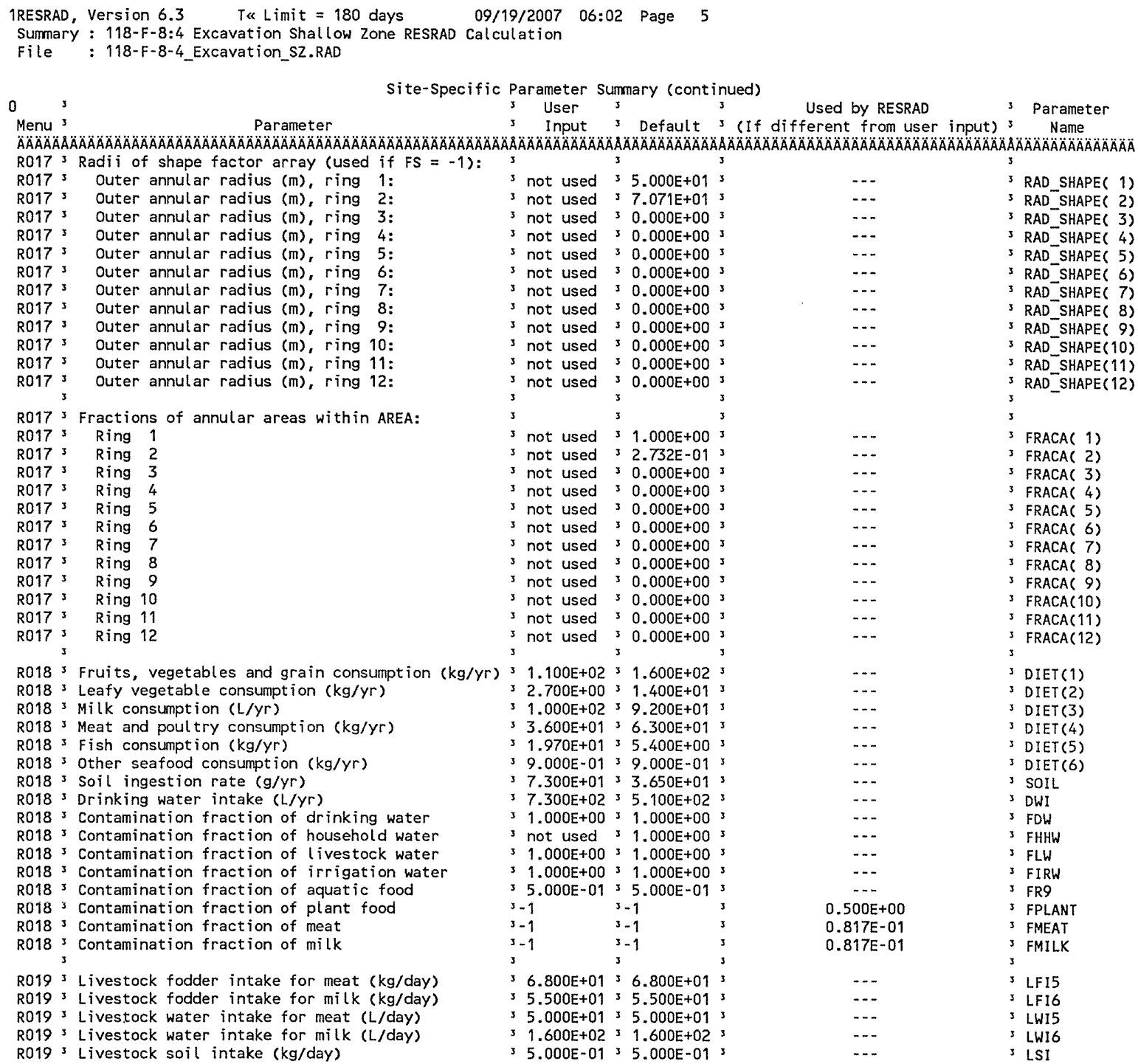

Attachment 


\section{ATTACHMENT 2}

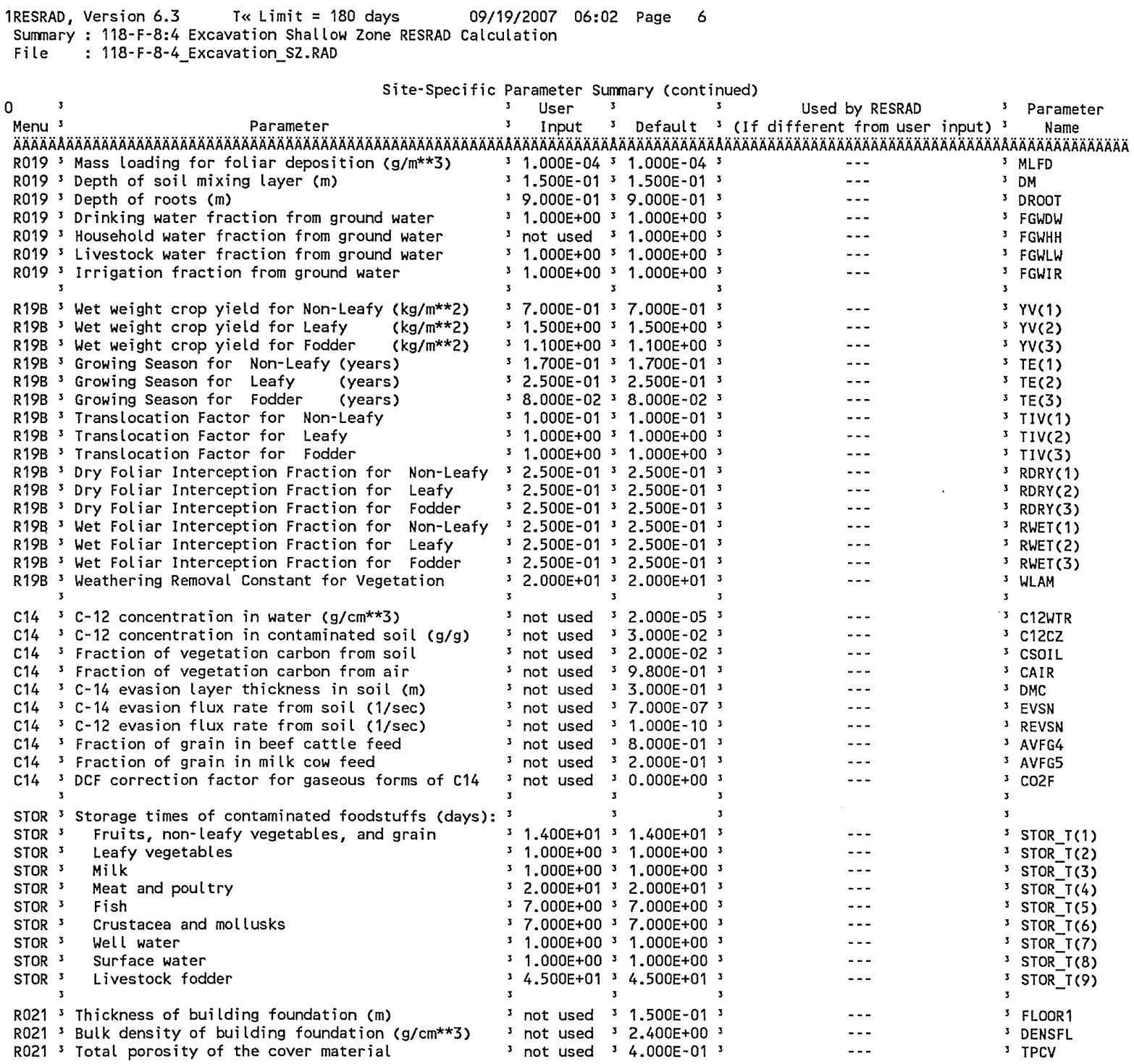

Attachment Originator: S.W. Clark 2 Chk'd By M.W. Perrott Calc. No. 0100F-CA-V0303
Sheet No. $\underline{6}$ of 19 Date Rev. No. 0 


\section{ATTACHMENT 2}

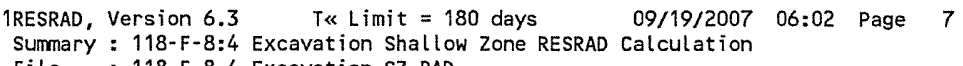

Summary of Pathway Selections

Pathway 3 User Selection

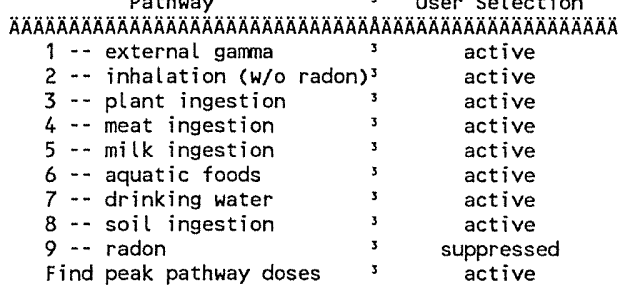

$\begin{array}{ll}\text { Attachment } \frac{2}{\text { S. Clark }} & \text { Sheet No. } I \text { of } 19 \\ \text { Originator: } & \text { Sate } \\ \text { Chk'd By } & \text { Date } \\ \text { Calc. No. Perrott } & \text { Rev. No. } 0\end{array}$




\section{ATTACHMENT 2}

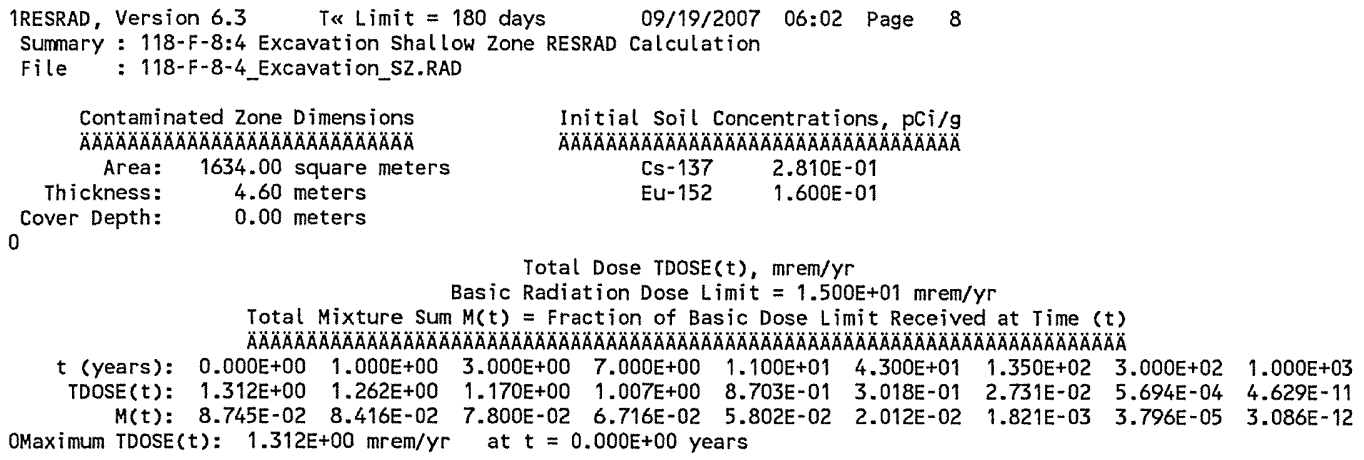

\begin{tabular}{|c|c|}
\hline \multirow{2}{*}{\multicolumn{2}{|c|}{$\begin{array}{c}\text { Attachment } \frac{2}{2} \\
\text { Originator: S.W. Clark }\end{array}$}} \\
\hline & \\
\hline M. W. Perrott & Date \\
\hline $0100 F-C A-V 0303$ & Rev. No. \\
\hline
\end{tabular}




\section{ATTACHMENT 2}

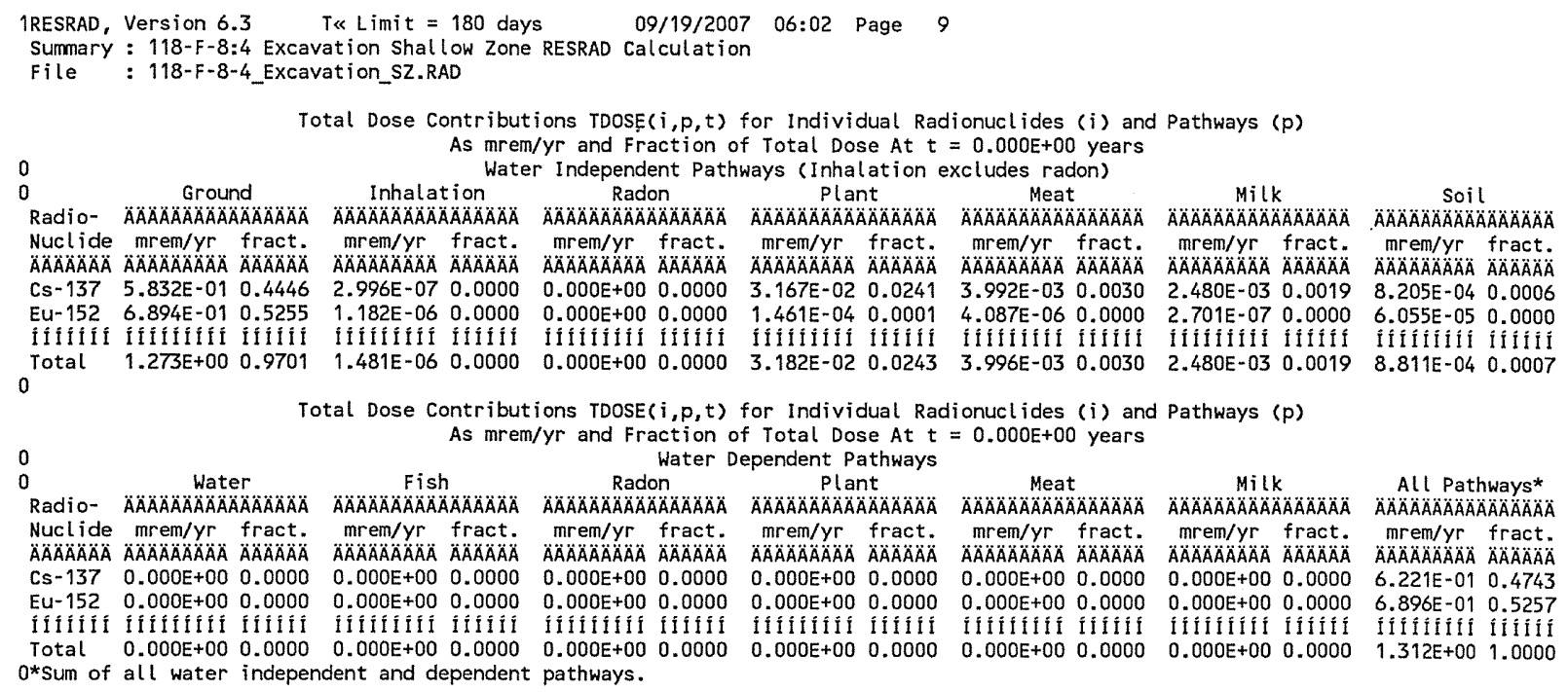

$\begin{array}{ll}\text { Attachment } \frac{2}{\text { W. Clark }} & \text { Sheet No. } \underline{9} \text { of } \underline{19} \\ \text { Originator: } \frac{\text { S.W. }}{\text { M.W. Perrott }} & \text { Date } \\ \text { Chk'd By } & \\ \text { Calc. No. } & \text { Rev. No. } \underline{0100 \text { F-CA-V0303 }}\end{array}$




\section{ATTACHMENT 2}

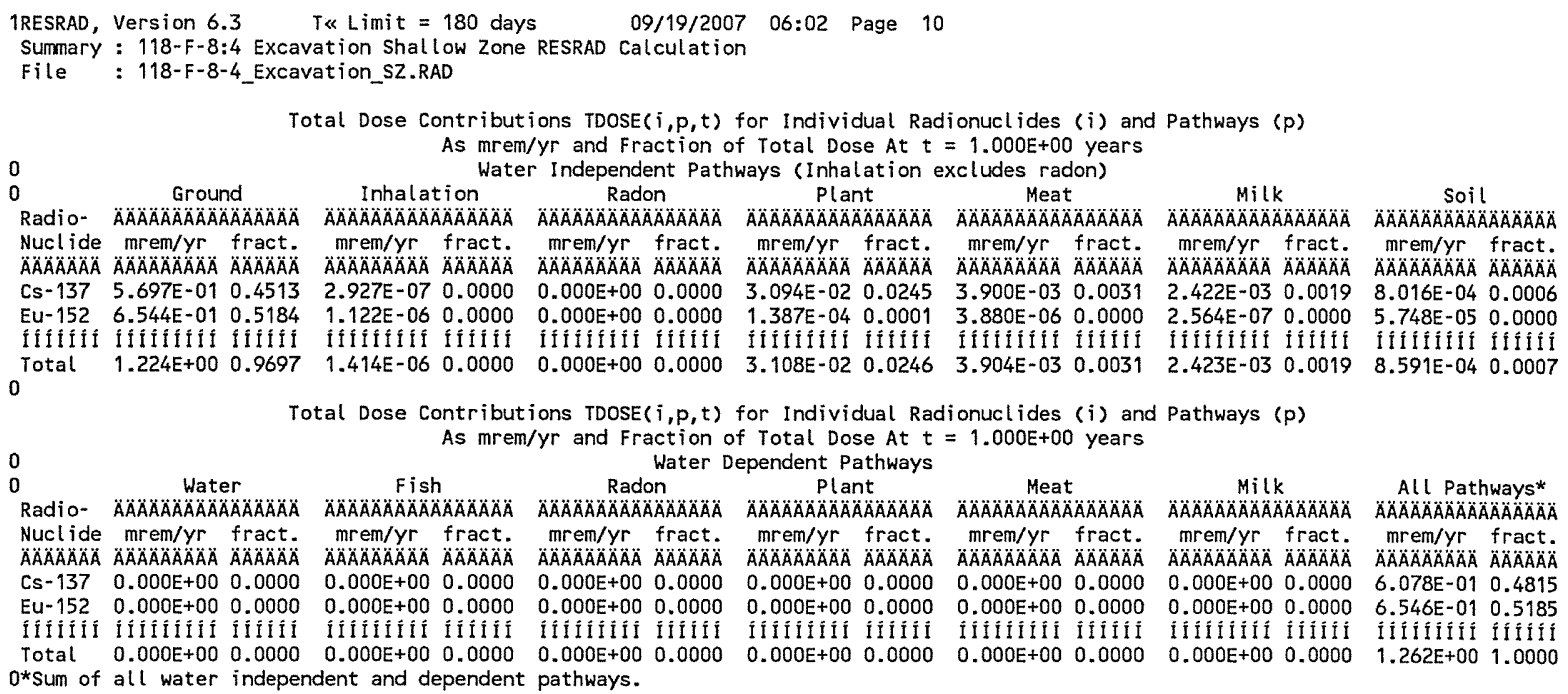

Attachment 


\section{AI"IACHMENI 2}

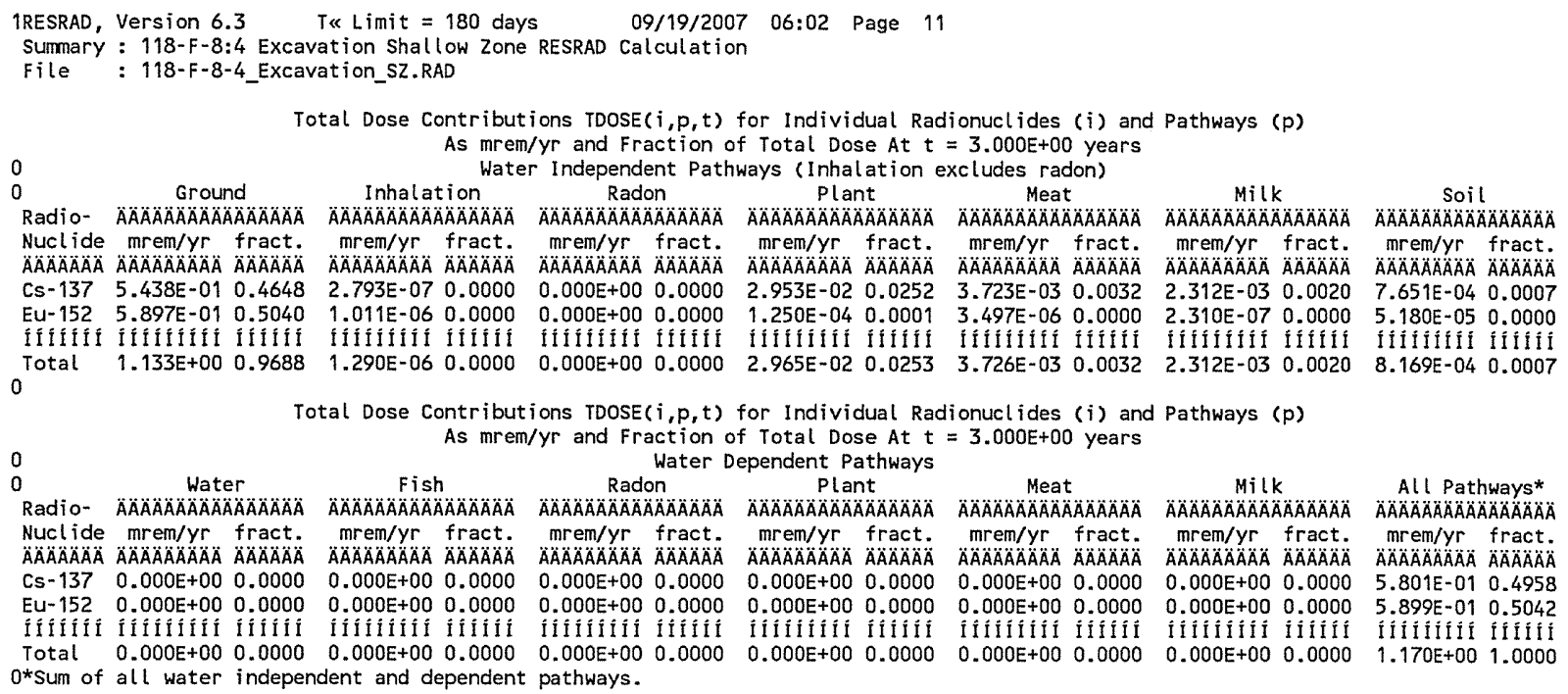

Total Dose Contributions $\operatorname{TDOSE}(i, p, t)$ for Individual Radionuclides (i) and Pathways ( $p$ ) As $\mathrm{mrem} / \mathrm{yr}$ and Fraction of Total Dose At $t=3.000 \mathrm{E}+00$ years

0 Water Wish Water Dependent Pathways

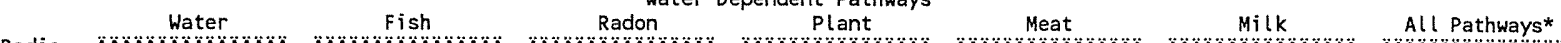

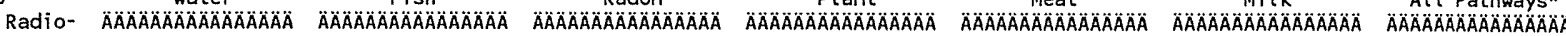
Nuclide mrem/yr fract. mrem/yr fract. mrem/yr fract. mrem/yr fract. mrem/yr fract. mrem/yr fract. mrem/yr fract.

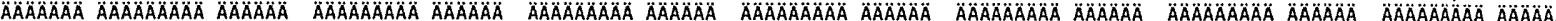
CS-137 0.000E+00 0.0000 0.000E+00 0.0000 0.000E+00 0.0000 $0.000 E+000.0000$ 0.000E+00 0.0000 $0.000 E+000.0000-5.801 E-010.4958$ Cs-137 $0.000 E+00.00000 .000 E+00.00000 .000 E+00 \quad 0.0000 \quad 0.000 E+00 \quad 0.0000 \quad 0.000 E+00 \quad 0.0000 \quad 0.000 E+00 \quad 0.0000 \quad 5.801 E-01 \quad 0.4958$

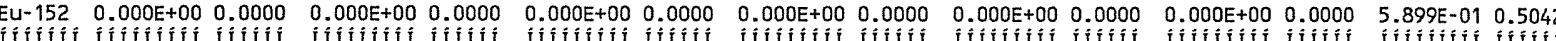

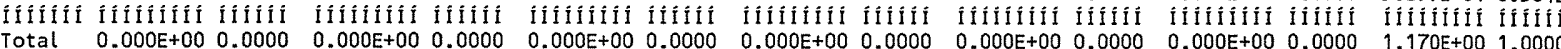
$\begin{array}{llllllllllllllllllllllllll}\text { Total } & 0.000 E+00 & 0.0000 & 0.000 E+00 & 0.0000 & 0.000 E+00 & 0.0000 & 0.000 E+00 & 0.0000 & 0.000 E+00 & 0.0000 & 0.000 E+00 & 0.0000 & 1.170 E+00 & 1.0000\end{array}$ 0 * Sum of all water independent and dependent pathways.

\begin{tabular}{|c|c|}
\hline Attachment & Sheet No. 11 of 19 \\
\hline Originator: S.W. Clark & Date \\
\hline Chk'd By M.W.Perrott & Date \\
\hline Calc. No. $\quad 0100 \mathrm{~F}-\mathrm{CA}-\mathrm{V} 0303$ & Rev. No. \\
\hline
\end{tabular}




\section{ATTACHMENT 2}

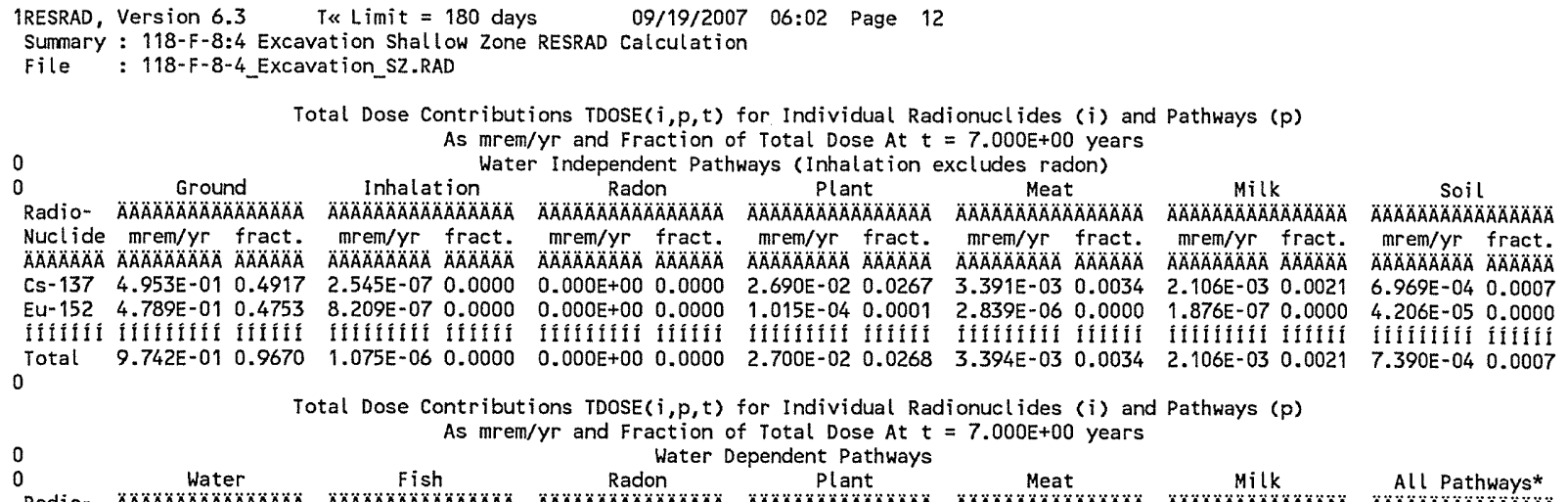

Total Dose Contributions $\operatorname{TDOSE}(i, p, t)$ for Individual Radionuclides (i) and Pathways ( $p$ ) As mrem/yr and Fraction of Total Dose At $t=7.000 E+00$ years

0 Water Dependent Pathways 


\section{ATTACHMENT 2}

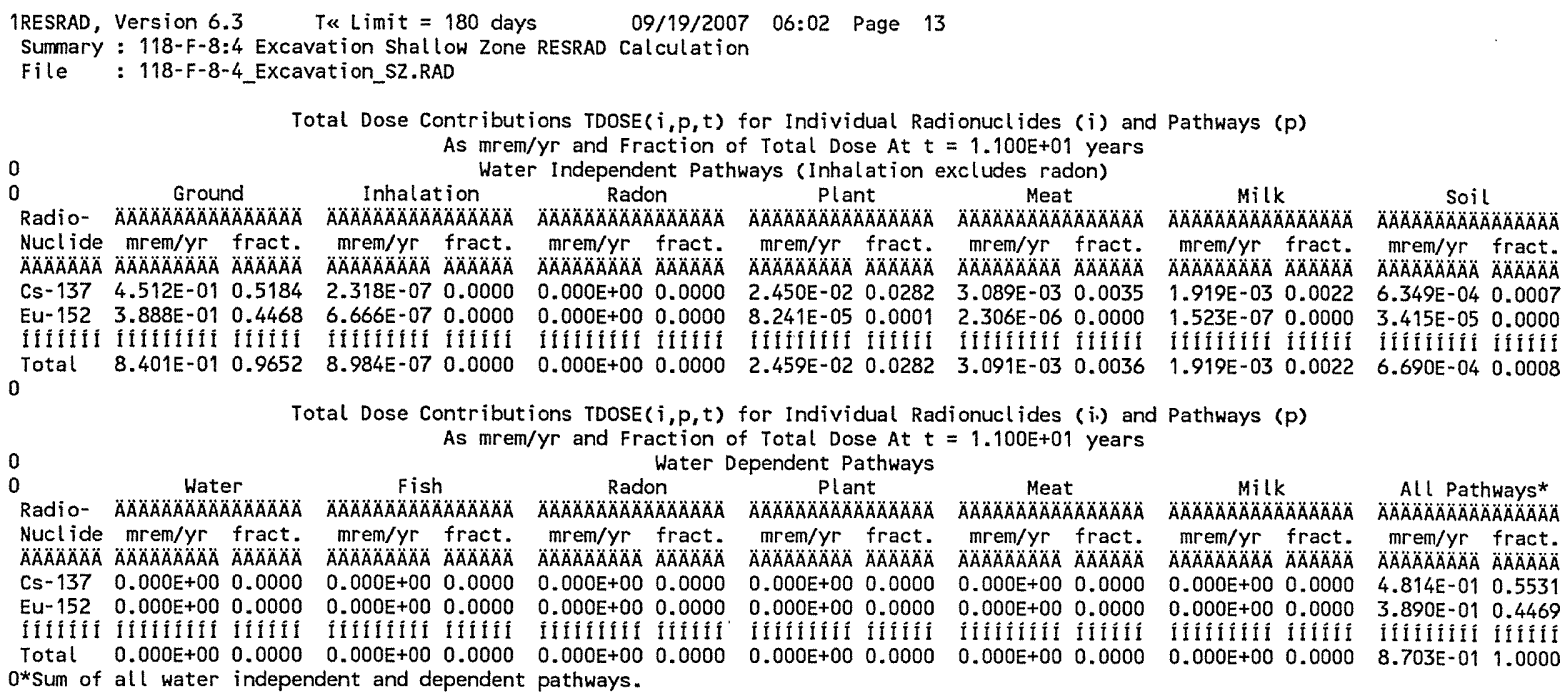




\section{ATTACHMENT 2}

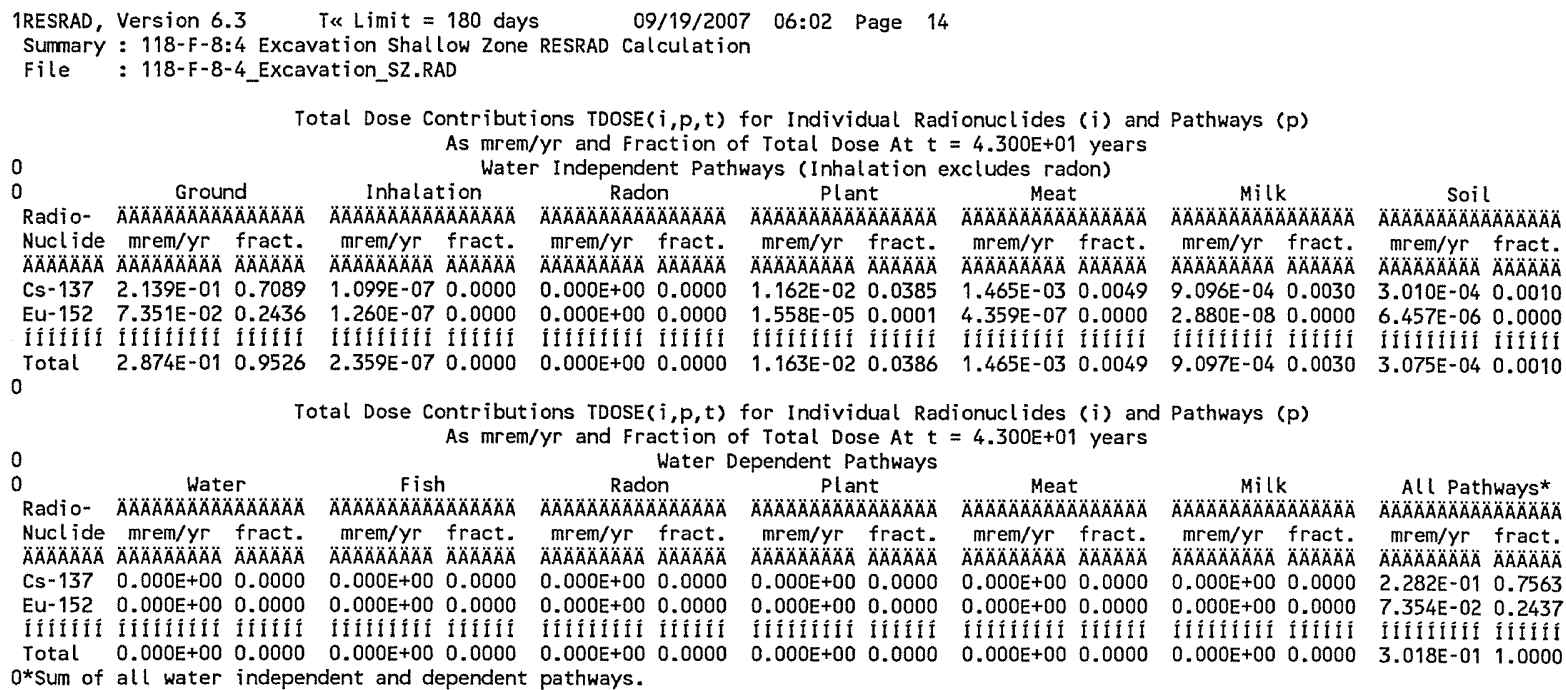

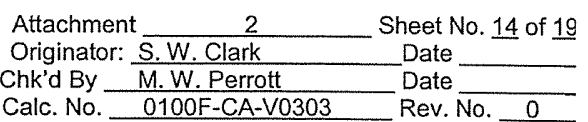




\section{ATTACHMENT 2}

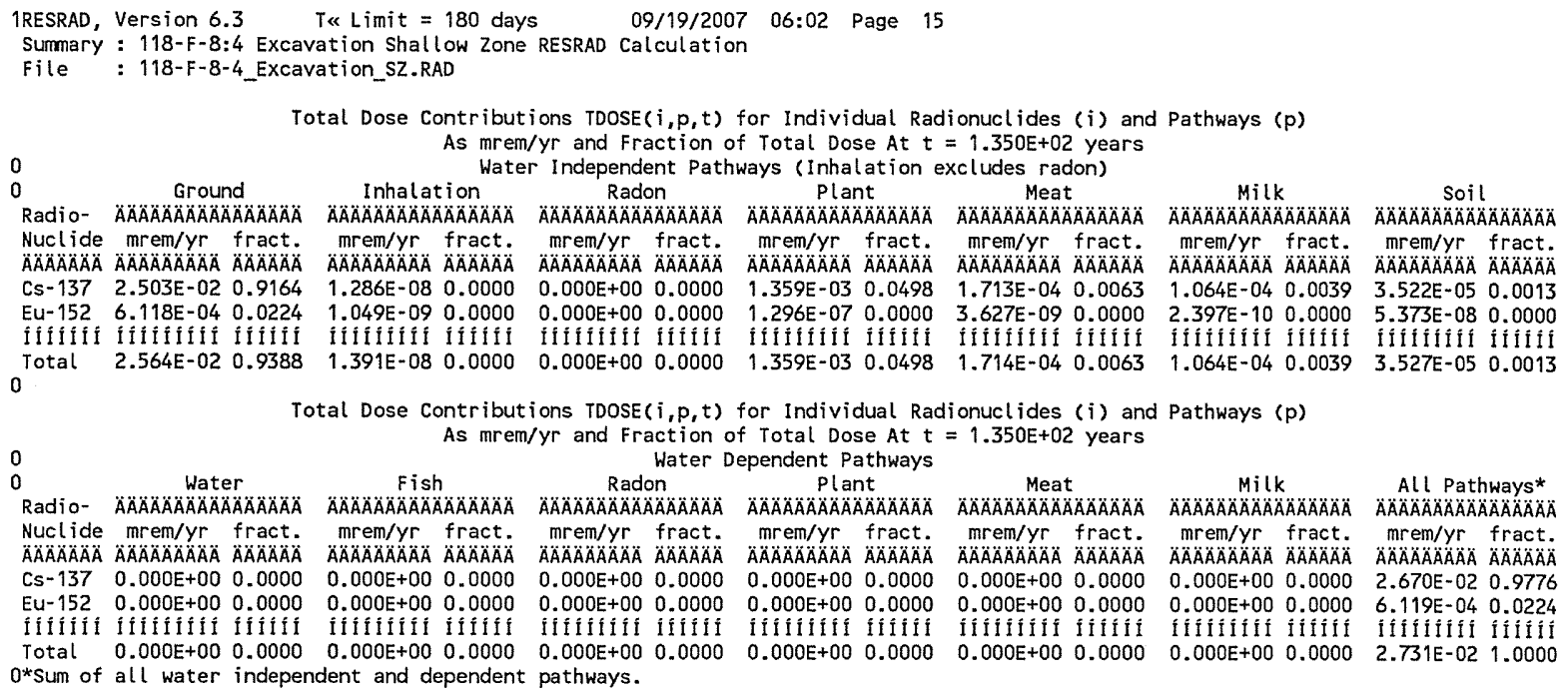




\section{ATTACHMENT 2}

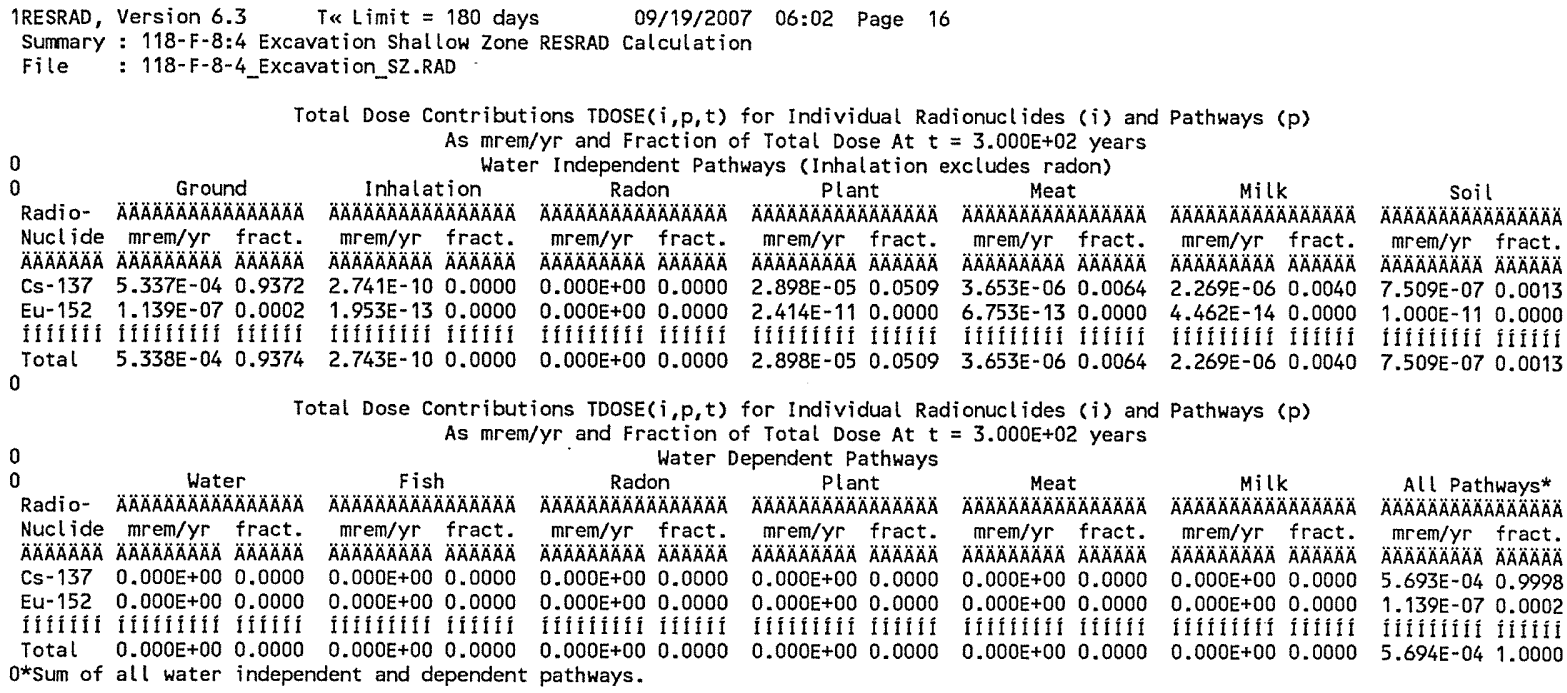

\begin{tabular}{|c|c|}
\hline Attachment & Sheet No. 16 of 19 \\
\hline Originator: S.W. Clark & Date \\
\hline M.W. Perrott & Date \\
\hline $0100 \mathrm{~F}-\mathrm{CA}-\mathrm{V} 0303$ & Rev. No. 0 \\
\hline
\end{tabular}




\section{ATTACHMENT 2}

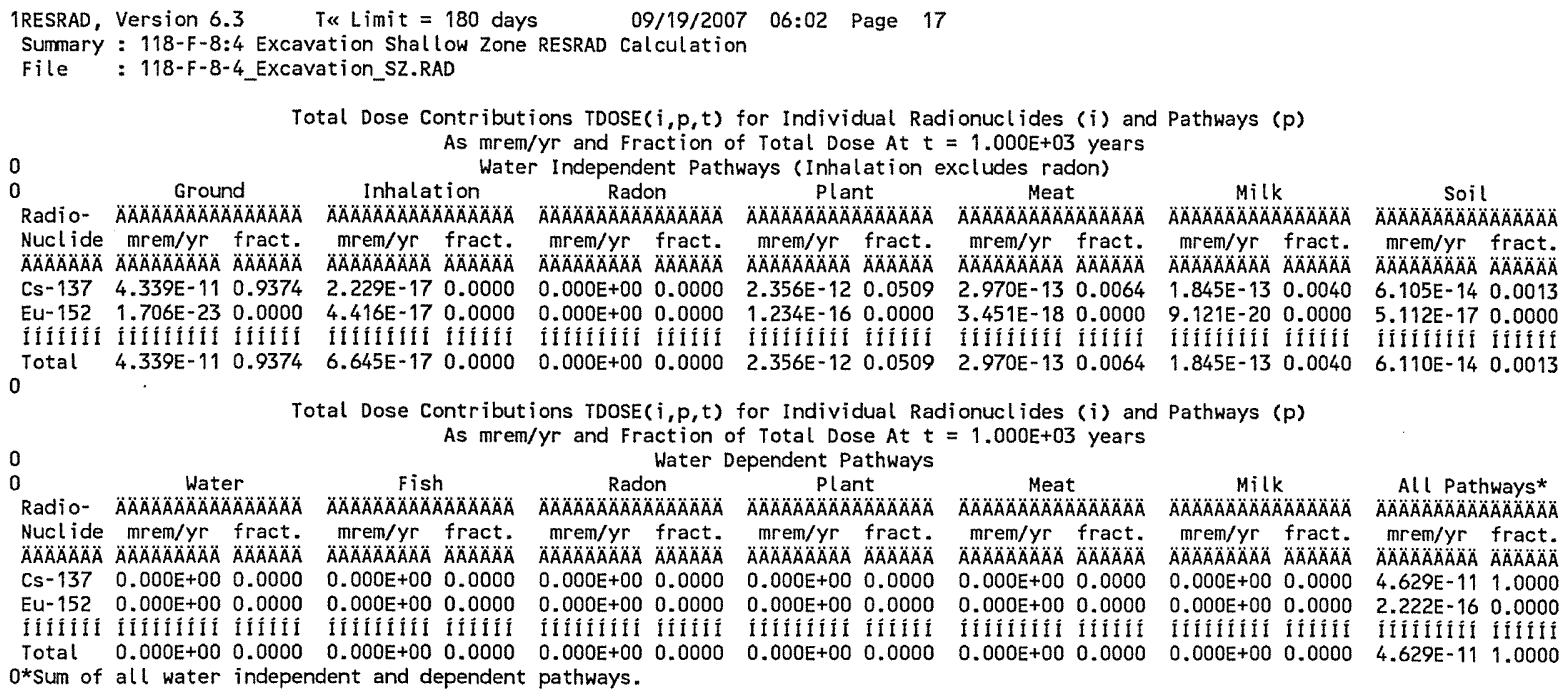

\begin{tabular}{|c|c|}
\hline Attachment & Sheet No. 17 of 19 \\
\hline Originator: S.W. Clark & Dat \\
\hline M. W. Perrott & Date \\
\hline Calc. No. $0100 F-C A-V 0303$ & Rev. No. 0 \\
\hline
\end{tabular}




\section{ATTACHMENT 2}

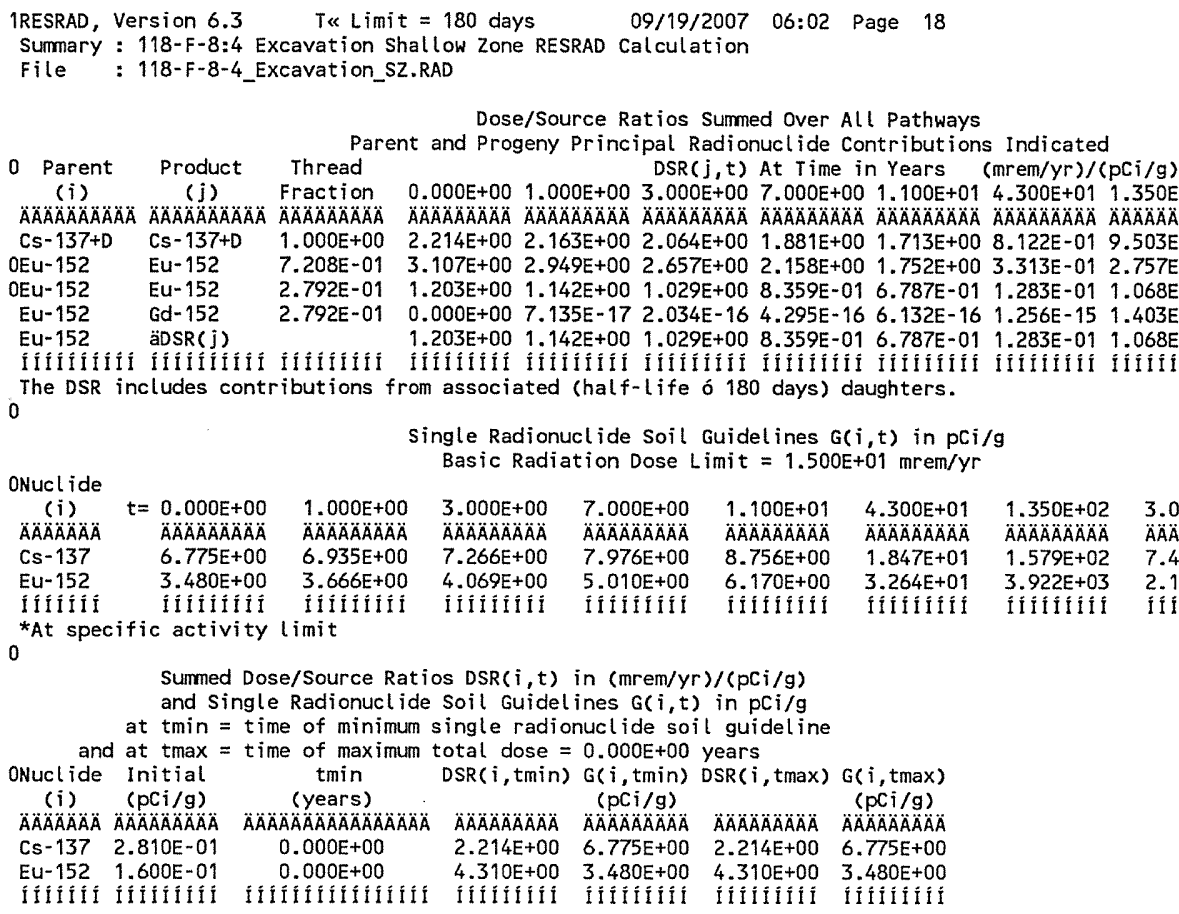

$\begin{array}{llr}1.350 E+02 & 3.000 E+02 & 1.000 E+03 \\ \text { AAAAAAAAAA } & \text { ÄÄÄAAAÄ } & \text { ÄAAAAAAAA } \\ 1.579 E+02 & 7.403 E+03 & 9.106 E+10 \\ 3.922 E+03 & 2.107 E+07 & * 1.765 E+14 \\ \text { IIIIIIII } & \text { IIIIIIIII } & \text { IIIIIIIII }\end{array}$




\section{ATTACHMENT 2}

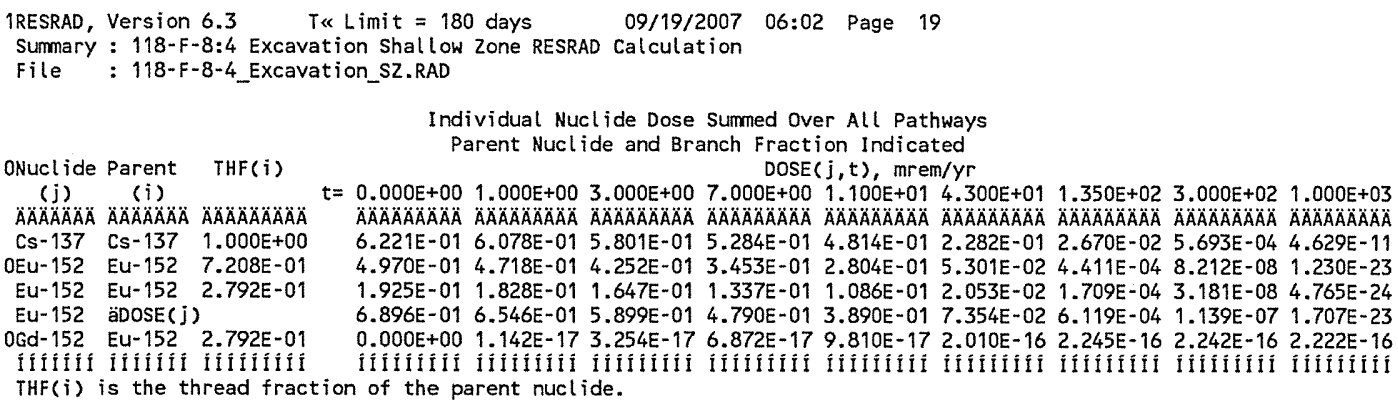

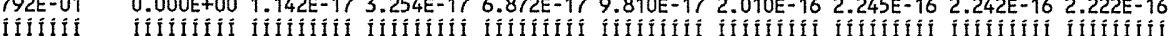

$t=0.000 E+001.000 E+00 \quad 3.000 E+007.000 E+001, t), m r e m / y r$

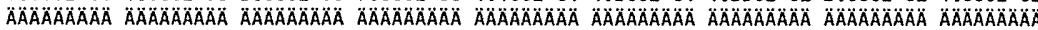

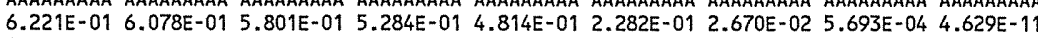
4.970E-01 4.718E-01 4.252E-01 3.453E-01 2.804E-01 5.301E-02 4.411E-04 8.212E-08 1.230E-23 1.925E-01 1.828E-01 1.647E-01 1.337E-01 1.086E-01 2.053E-02 $1.709 \mathrm{E}-04 \quad 3.181 \mathrm{E}-08$ 4.765E-24 6.896E-01 6.546E-01 5.899E-01 4.790E-01 3.890E-01 7.354E-02 6.119E-04 1.139E-07 1.707E-23

Parent Nuclide and Branch Fraction Indicated

ONuclide Parent THF(i)

(j) (i) $S(j, t), p C i / g$ AAAAAAA AAAAAAAA AAAAAAAAA

Cs-137 CS-137 1.000E+00

OEU-152 EU-152 7.208E-0

Eu-152 Eu-152 2.792E-01

Eu-152 äS(j):

OGd-152 EU-152 2.792E-01

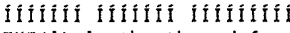

THF(i) is the thread fraction

$=0.000 E+001.000 E+003.000 E+007.000 E+001.100 E+01 \quad 4.300 E+011.350 E+023.000 E+021.000 E+03$

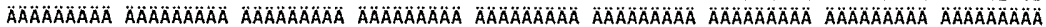

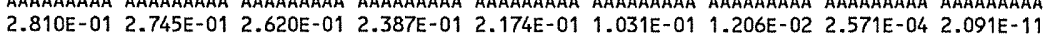

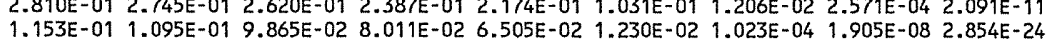
4.467E-02 4.241E-02 3.821E-02 3.103E-02 2.520E-02 4.764E-03 $3.964 E-05 \quad 7.381 E-09 \quad 1.106 E-24$

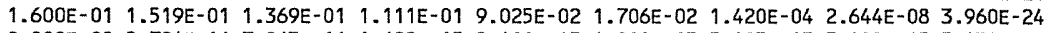
$0.000 E+00 \quad 2.794 \mathrm{E}-16 \quad 7.963 \mathrm{E}-16 \quad 1.682 \mathrm{E}-15 \quad 2.401 \mathrm{E}-15 \quad 4.919 \mathrm{E}-15 \quad 5.495 \mathrm{E}-15 \quad 5.488 \mathrm{E}-15 \quad 5.437 \mathrm{E}-15$

ORESCALC.EXE execution time $=8.56$ seconds

\begin{tabular}{|c|c|}
\hline Attachment & Sheet No. 19 of 19 \\
\hline Originator: S.W. Clark & Date \\
\hline M. W. Perrott & Date \\
\hline $0100 F-C A-V 0303$ & Rev. No. \\
\hline
\end{tabular}




\section{ATTACHMENT 3}

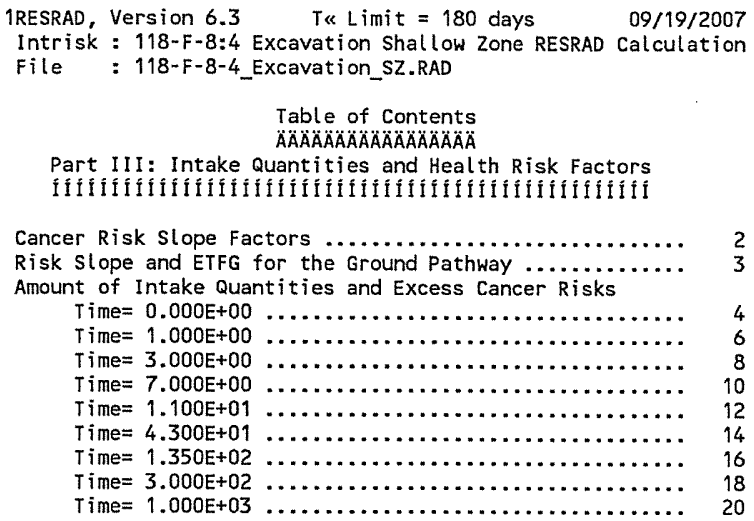

Attachment 


\section{ATTACHMENT 3}

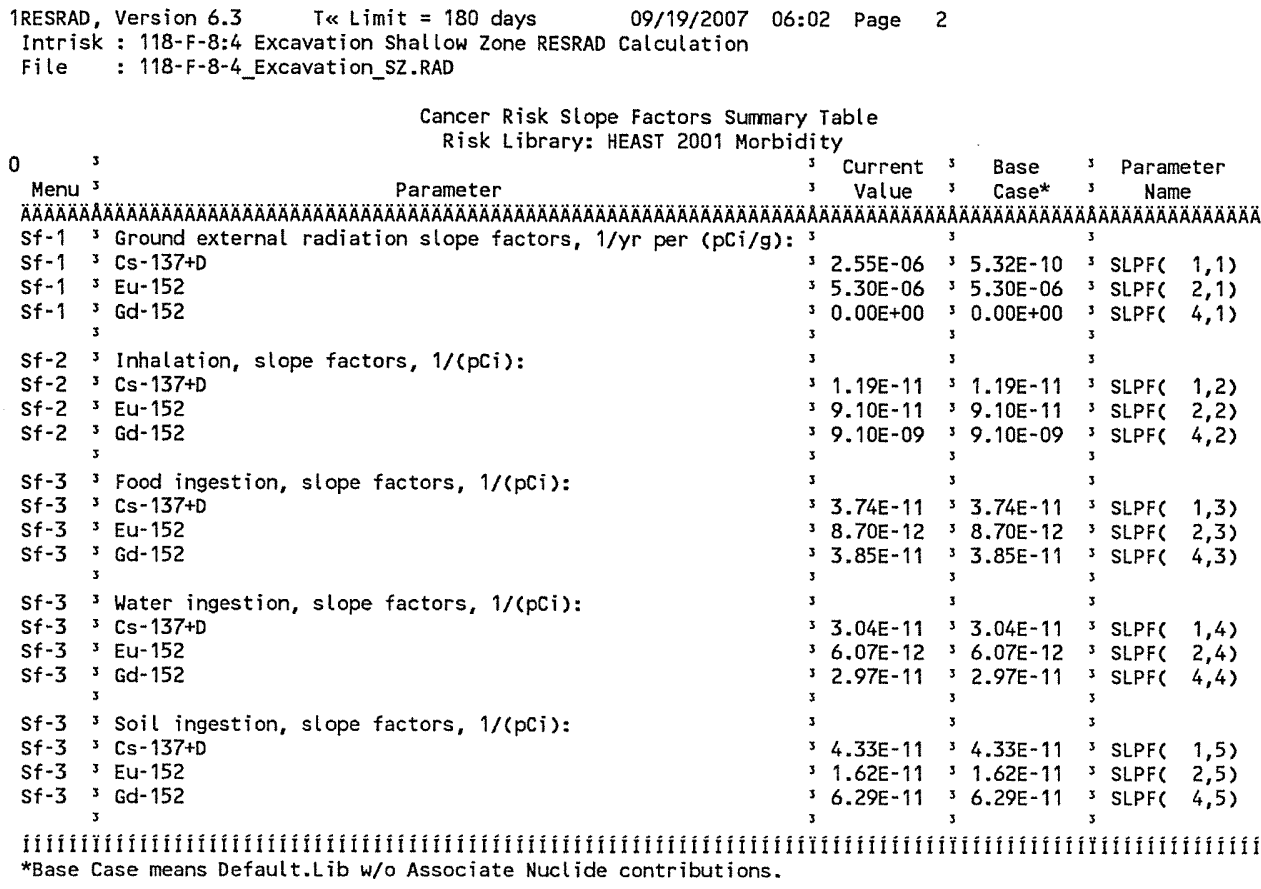

\begin{tabular}{ll} 
Attachment $\frac{3}{\text { W. Clark }}$ & Sheet No. 2 of 21 \\
Originator: $\frac{\text { S.W. }}{\text { M.W. Perrott }}$ & Date \\
Chk'd By & Rate \\
Calc. No. & 0100 F.CA-V0303 \\
\hline
\end{tabular}




\section{ATTACHMENT 3}

\begin{tabular}{|c|c|c|c|c|c|c|c|c|c|c|}
\hline $\begin{array}{l}\text { IRESRAD, } \\
\text { Intrisk } \\
\text { File }\end{array}$ & $\begin{array}{l}\text { Version } 6.3 \\
: 118-F-8: 4 \\
: 118-F-8-4\end{array}$ & $\begin{array}{l}\text { T« Lin } \\
\text { Excavation } \\
\text { Excavation }\end{array}$ & $\begin{array}{l}\text { it }=180 \text { day } \\
\text { Shall low Zone } \\
\text { SZ.RAD }\end{array}$ & Ys & $\begin{array}{r}09 / 19 / 2007 \\
\text { Calculation }\end{array}$ & 06:02 Page & 3 & & & \\
\hline ONucl ide & Slope $(i)^{*}$ & & & $\begin{array}{l}\text { ment } \\
\text { ETFC }\end{array}$ & $\begin{array}{l}\text { tal Transpo } \\
=G(i, t) \text { At }\end{array}$ & $\begin{array}{l}\text { Factors for } \\
\text { me in Years }\end{array}$ & $\begin{array}{l}\text { the Ground } \\
\text { (dimension }\end{array}$ & $\begin{array}{l}\text { Pathway } \\
\text { (ess) }\end{array}$ & & \\
\hline (i) & & $=0.000 E+00$ & $.000 E+00$ & $3.000 E+O C$ & $307.000 E+00$ & $1.100 \mathrm{E}+01$ & $4.300 E+01$ & $1.350 E+02$ & $3.000 E+02$ & $1.000 E+03$ \\
\hline$\triangle A A ̈ A ̈ A ̈ A ̈ A ̈$ & $A A A ̈ A$ & $\triangle A A A A B A A A ̈ A$ & ÄÄAिÄÄÄÄ & 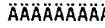 & 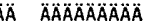 & $\triangle A A A A A A A A O A$ & $\triangle A A A O A O A ̈ A ̈ A ̈ A ̈$ & ÄÄÄÄÄÄÄÄ & $\triangle A O A A A O A O A ̈ A ̈$ & $\triangle A A O A O A O A O A O A ̈$ \\
\hline $\mathrm{Ba}-137 \mathrm{~m}$ & $2.690 \mathrm{E}-06$ & $6.084 E-01$ & $6.084 E-01$ & $6.084 E-0$ & $11 \quad 6.084 \mathrm{E}-01$ & $6.084 E-01$ & $6.084 E-01$ & $6.084 E-01$ & $6.084 E-01$ & $6.084 E-01$ \\
\hline Cs -137 & $5.320 E-10$ & $6.239 E-01$ & $6.239 E-01$ & $6.239 E-0$ & $01 \quad 6.239 \mathrm{E}-01$ & $6.239 \mathrm{E}-01$ & $6.239 E-01$ & $6.239 \mathrm{E}-01$ & $6.239 E-01$ & $6.239 E-01$ \\
\hline Eu-152 & $5.300 E-06$ & 6.151E-01 & $6.151 \mathrm{E}-01$ & $6.151 \mathrm{E}-0$ & $01 \quad 6.151 \mathrm{E}-01$ & $6.151 E-01$ & $6.151 \mathrm{E}-01$ & $6.151 \mathrm{E}-01$ & $6.151 E-01$ & $6.151 \mathrm{E}-01$ \\
\hline$G d-152$ & $0.000 E+00$ & $0.000 E+00$ & $0.000 E+00$ & $0.000 E+00$ & $30 \quad 0.000 E+00$ & $0.000 E+00$ & $0.000 E+00$ & $0.000 E+00$ & $0.000 E+00$ & $0.000 E+00$ \\
\hline fififit & fifitifit & Ififfifí & fiffifif & ifififit & Iifitifiti & fifififit & IIIIIIifi & Iifílíî́n & Ififílíín & Iífítílí \\
\hline
\end{tabular}

\begin{tabular}{|c|c|}
\hline Attachment & Sheet No \\
\hline Originator: S.W. Clark & Date \\
\hline M.W. Perrott & Date \\
\hline $0100 F-C A-V 0303$ & Rev. No. 0 \\
\hline
\end{tabular}




\section{ATTACHMENT 3}

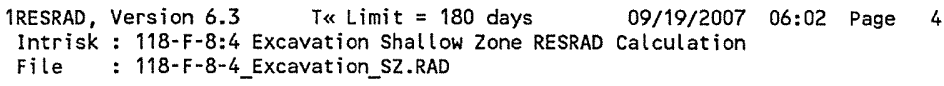

Water Dependent Pathways

Water
Radio-

and water dependent water, fish, plant, meat, milk pathways

$\begin{array}{ll}\text { Attachment } \frac{3}{\text { W. Clark }} & \text { Sheet No. } 4 \text { of } 21 \\ \text { Originator: } \frac{\text { S.W. }}{\text { M.W.Perrott }} & \text { Date } \\ \text { Chk'd By } & \text { Date } \\ \text { Calc. No. } & \text { Rev. No. } 0100 \text { F-CA-V0303 }\end{array}$




\section{ATTACHMENT 3}

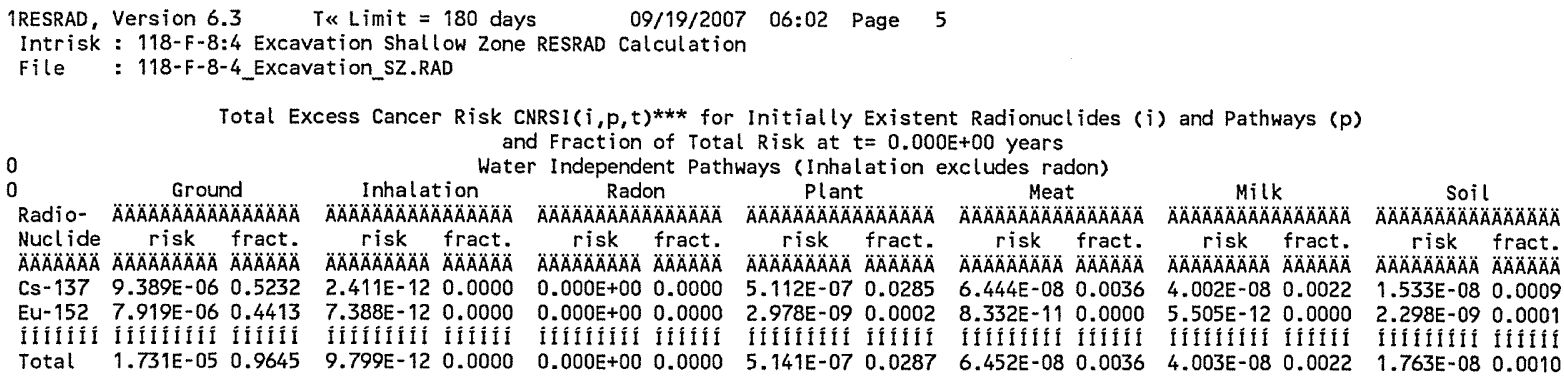

Water Dependent Pathways

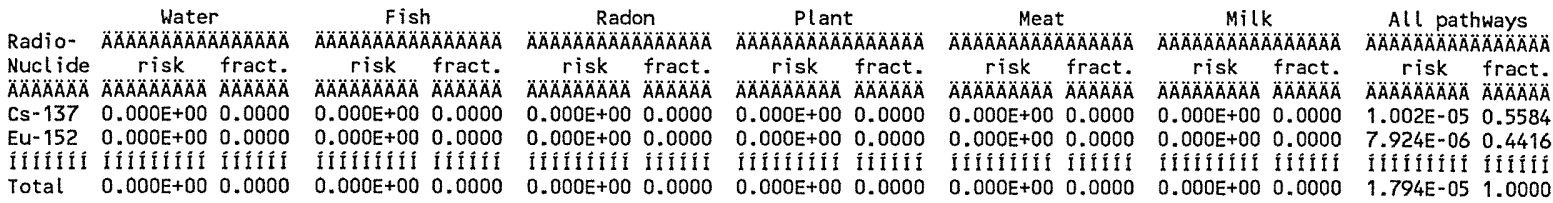

$* * * \operatorname{CNRSI}(i, p, t)$ includes contribution from decay daughter radionucl ides

$\begin{array}{ll}\text { Attachment } \frac{3}{\text { W. Clark }} & \text { Sheet No. } \underline{5} \text { of } 21 \\ \text { Originator: } \frac{\text { S.W. }}{\text { M.W. Perrott }} & \text { Date } \\ \text { Chk'd By } & \text { Rev. No. _ } \frac{0}{\text { O100F-CA-V0303 }}\end{array}$




\section{ATTACHMENT 3}

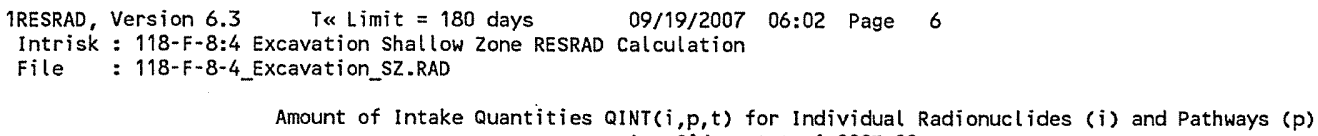

Excess Cancer Risks CNRS( $i, p, t)$ for Individual Radionuclides (i) and Pathways ( $p$ ) and Fraction of Total Risk at $t=1.000 E+00$ years

\section{Water Dependent Pathways}

\begin{tabular}{|c|c|c|c|c|c|c|}
\hline & $\begin{array}{c}\text { Water } \\
\text { AAAAOAOAOAAAOAOAOA }\end{array}$ & $\begin{array}{c}\text { Fish } \\
\text { AAAAAÄÄÄÄÄÄÄÄ }\end{array}$ & $\begin{array}{c}\text { Plant } \\
\triangle A O A A A A A A A A A O A O A B\end{array}$ & $\begin{array}{c}\text { Meat } \\
\text { ÄÄÄÄÄÄÄÄÄÄA }\end{array}$ & 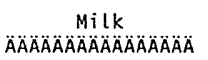 & 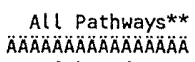 \\
\hline $\begin{array}{l}\triangle C l \text { ide } \\
A \triangle A A O A O A B\end{array}$ & 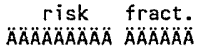 & $\begin{array}{r}\text { risk fract. } \\
\text { AAAAAAAAB } \triangle A A A B A B A\end{array}$ & $\begin{array}{c}\text { risk fract. } \\
A O A B A B A O A O A B A O A\end{array}$ & $\begin{array}{l}\text { risk fract. } \\
A \ddot{A} A O A B A B A O A O A A B A\end{array}$ & 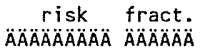 & $\begin{array}{cc}\text { risk } & f \\
A \triangle A ̈ A ̈ A ̈ A ̈ A ̈ & A ̈\end{array}$ \\
\hline & 000. & & & & 1000 & \\
\hline & 00. & 000. & 00 & & & \\
\hline & & $\begin{array}{ll}00 & 0.00 \\
\text { II IIIII }\end{array}$ & $\begin{array}{ll}00 & 0.0 \\
11 & \end{array}$ & 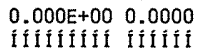 & $\begin{array}{ll}00 & 0 . \\
\text { If } & \text { If }\end{array}$ & 20 \\
\hline & $+00 \quad 0.0$ & +000. & +000.0000 & $+\infty 00.0000$ & $E+00 \quad 0.0000$ & 05 \\
\hline
\end{tabular}

** Sum of water independent ground, inhalation, plant, meat, milk, soil and water dependent water, fish, plant, meat, milk pathways

\begin{tabular}{|c|c|c|}
\hline \multicolumn{2}{|c|}{ Attachment $\quad 3$} & \multirow{2}{*}{$\begin{array}{l}\text { Sheet No. } \underline{6} \text { of } 21 \\
\text { Date }\end{array}$} \\
\hline Originator: & S. W. Clark & \\
\hline Chk'd B & M. W. Perrott & Date \\
\hline Calc. No. & $0100 F-C A-V 0303$ & Rev. No. \\
\hline
\end{tabular}




\section{ATTACHMENT 3}

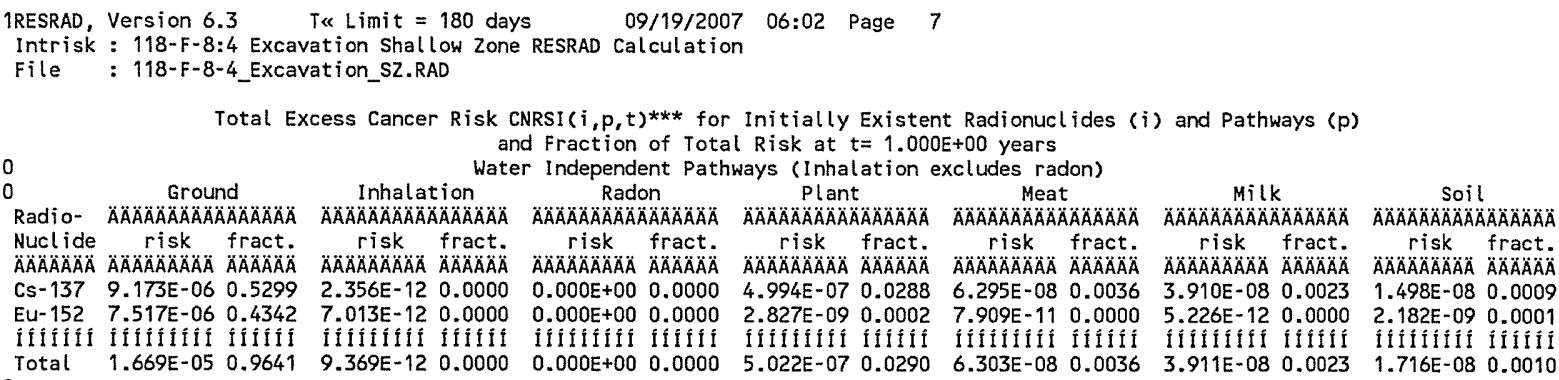

Total Excess Cancer Risk CNRSI $(i, p, t) * * *$ for Initially Existent Radionuclides (i) and Pathways (p) and Fraction of Total Risk at $t=1.000 E+00$ years

Water Dependent Pathways

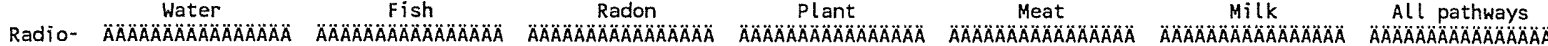

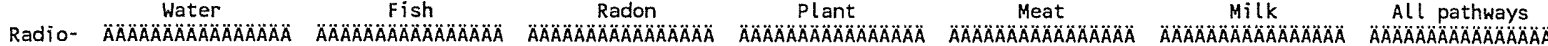

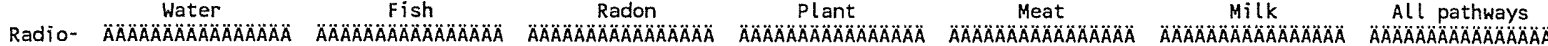

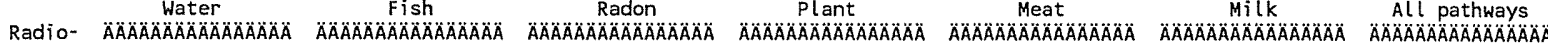

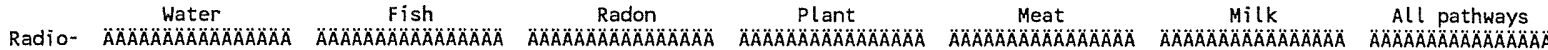

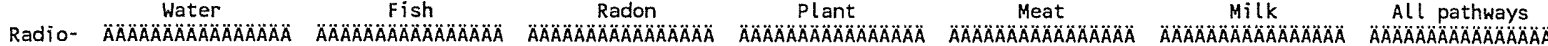
Nuclide risk fract. risk fract. risk fract. risk fract. risk fract. risk fract. risk fract.

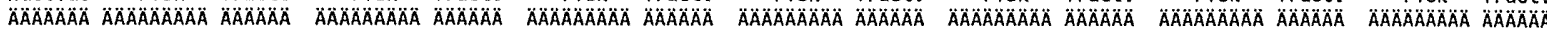

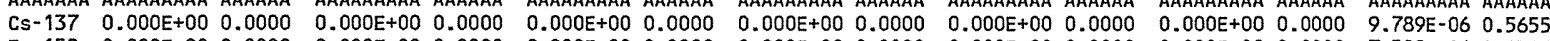
$\begin{array}{lllllllllllllll}\mathrm{Eu}-152 & 0.000 E+00 & 0.0000 & 0.000 E+00 & 0.0000 & 0.000 E+00 & 0.0000 & 0.000 E+00 & 0.0000 & 0.000 E+00 & 0.0000 & 0.000 E+00 & 0.0000 & 7.522 E-06 & 0.4345\end{array}$

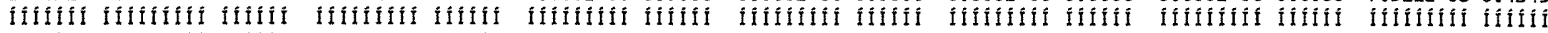

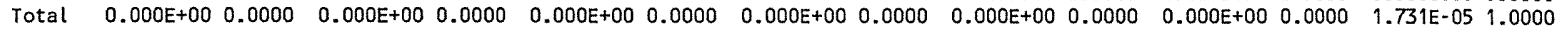

${ }^{* * *}$ CNRSI $(i, p, t)$ includes contribution from decay daughter radionuclides

\begin{tabular}{|c|c|c|}
\hline \multicolumn{2}{|c|}{ Attachment $\quad 3$} & \multirow{2}{*}{$\begin{array}{l}\text { Sheet No. } I \text { of } 21 \\
\text { Date }\end{array}$} \\
\hline Originator: $₫$ & S. W. Clark & \\
\hline Chk'd By & M. W. Perrott & Date \\
\hline Calc. No. & $0100 F-C A-V 0303$ & Rev. No. \\
\hline
\end{tabular}




\section{ATTACHMENT 3}

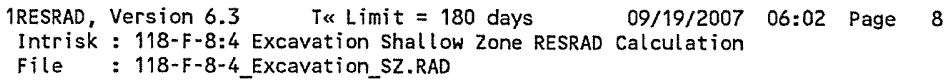

$\begin{array}{ll}\text { Attachment } \frac{3}{\text { S. Clark }} & \text { Sheet No. } \underline{8} \text { of } 21 \\ \text { Originator: } \frac{\text { S. W. }}{\text { M. W. Perrott }} & \text { Date } \\ \text { Chk'd By } & \text { Rev. No. } \underline{0} \\ \text { Calc. No. } & \text { O100F-CA-V0303 }\end{array}$




\section{ATTACHMENT 3}

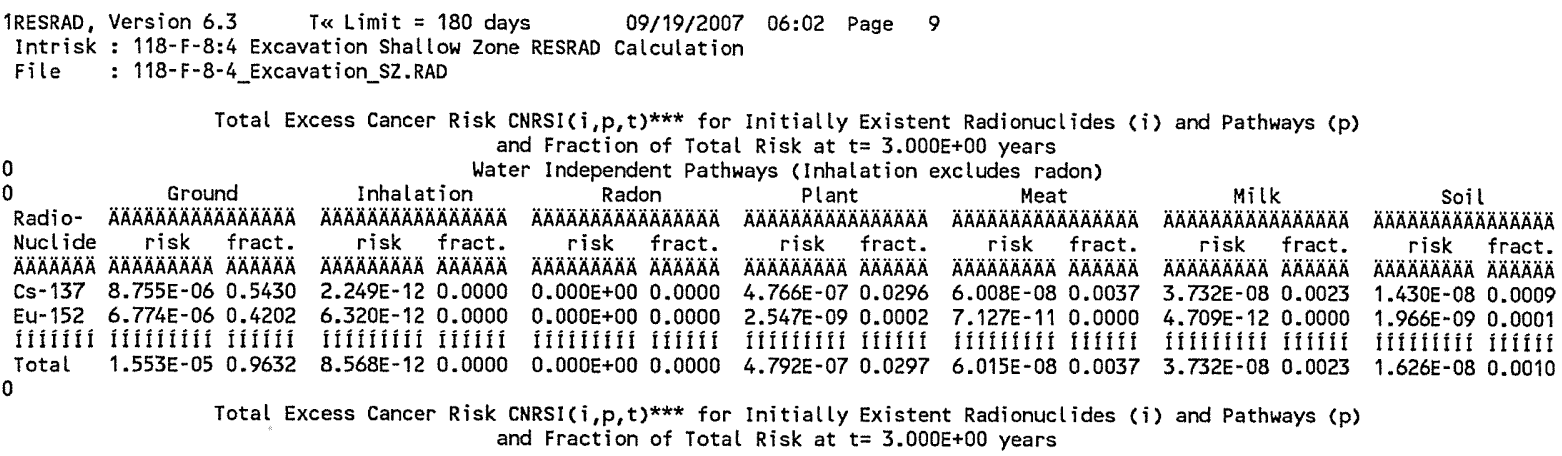

Water Dependent Pathways

\begin{tabular}{|c|c|c|c|c|c|c|c|}
\hline Radio- & 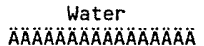 & 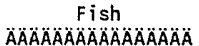 & 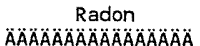 & $\begin{array}{c}P l \text { ant } \\
\triangle A A B A B A B A B A B A B A B A O A B\end{array}$ & 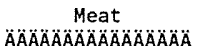 & $\begin{array}{c}\text { Milk } \\
\text { AAOAAAAOABAAAOAOA }\end{array}$ & $\begin{array}{l}\text { All pathways } \\
\text { ALAAAAABAAAAAAAA }\end{array}$ \\
\hline $\begin{array}{l}\text { Nuclide } \\
\text { ÄAAAAAAA }\end{array}$ & $\begin{array}{r}\text { risk fract. } \\
\text { AAAAÄAAOA AOAAOÄ }\end{array}$ & $\begin{array}{r}\text { risk fract. } \\
\text { ÄAÄAAAOA ÄÄÄÄ }\end{array}$ & $\begin{array}{l}\text { risk fract. } \\
\text { AAAAAÄÄÄ ÄÄÄÄA }\end{array}$ & $\begin{array}{r}\text { risk fract. } \\
\text { ÄÄÄ̈̈ÄÄÄA ÄÄÄÄÄA }\end{array}$ & $\begin{array}{r}\text { risk fract. } \\
\text { ÄÄÄÄÄÄÄÄA ÄÄÄÄÄ̈ }\end{array}$ & $\begin{array}{r}\text { risk fract. } \\
\text { ÄÄ̈̈̈̈ÄÄÄA ÄÄÄÄÄA }\end{array}$ & $\begin{array}{r}\text { TiSk fract. } \\
\text { ÄÄÄAOAOA AOABÄA }\end{array}$ \\
\hline Cs -137 & $0.000 E+00 \quad 0.0000$ & $0.000 E+00 \quad 0.0000$ & $0.000 E+00 \quad 0.0000$ & $0.000 E+00 \quad 0.0000$ & $0.000 E+00 \quad 0.0000$ & $0.000 E+00 \quad 0.0000$ & $9.343 \mathrm{E}-06 \quad 0.5795$ \\
\hline Eu-152 & $0.000 E+00 \quad 0.0000$ & $0.000 E+00 \quad 0.0000$ & $0.000 \mathrm{E}+00 \quad 0.0000$ & $0.000 E+00 \quad 0.0000$ & $0.000 E+00 \quad 0.0000$ & $0.000 E+00 \quad 0.0000$ & $6.779 \mathrm{E}-06 \quad 0.4205$ \\
\hline İî́î́î́n & 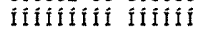 & 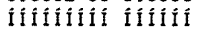 & IIIIIIIAI IIIIIII & IIIIIIIII IIIIII & IIIIIIIII finín & ilifitif itifit & Ifififith ifi \\
\hline Total & $0.000 E+00 \quad 0.0000$ & $0.000 E+00 \quad 0.0000$ & $0.000 \mathrm{E}+00 \quad 0.0000$ & $0.000 E+00 \quad 0.0000$ & $0.000 E+00 \quad 0.0000$ & $0.000 E+00 \quad 0.0000$ & $1.612 \mathrm{E}-05 \quad 1.0000$ \\
\hline
\end{tabular}




\section{ATTACHMENT 3}

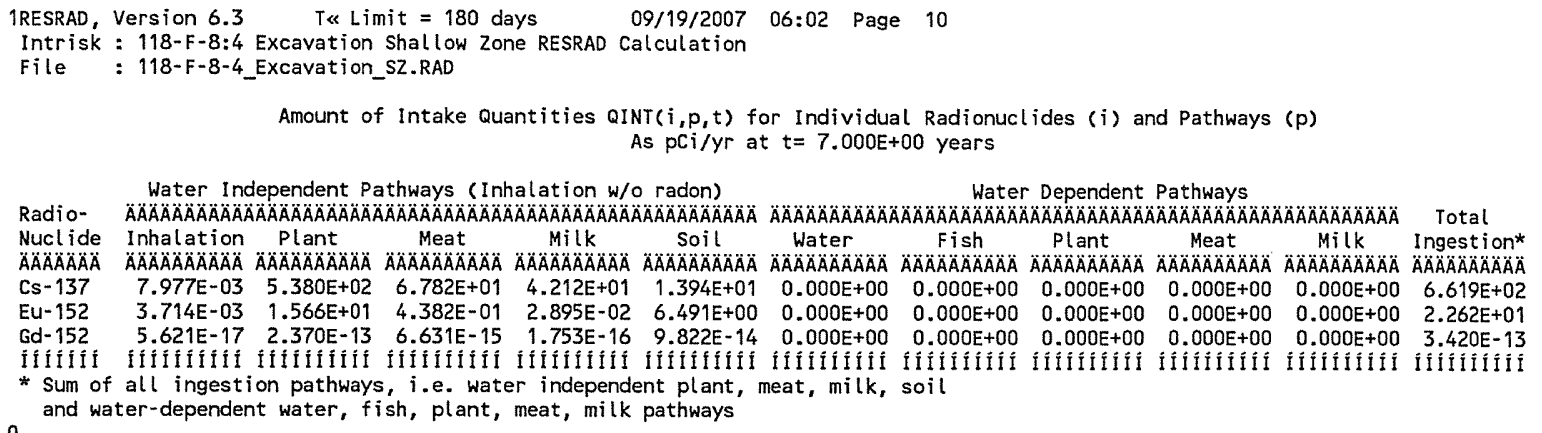

Excess Cancer Risks CNRS $(i, p, t)$ for Individual Radionuclides $(i)$ and Pathways $(p)$ and Fraction of Total Risk at $t=7.000 E+00$ years

Water Dependent Pathways

\begin{tabular}{|c|c|c|c|c|c|c|}
\hline & 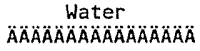 & $\begin{array}{c}F i s h \\
\text { ANAAAAAAAAAAAAAOAOA }\end{array}$ & 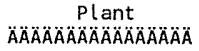 & 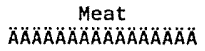 & $\begin{array}{c}\text { Milk } \\
\text { AAAAAAAOAOAOAOAOAOAOA }\end{array}$ & $\begin{array}{r}\text { All Pathways } \\
\text { A** } \\
\text { AAAAAAAAAAAAAAAA }\end{array}$ \\
\hline $\begin{array}{l}\text { clide } \\
\text { ÄÄÄÄA }\end{array}$ & $\begin{array}{l}\text { risk fract. } \\
\text { ÄAAAAAA AAAAAAA }\end{array}$ & 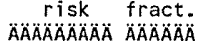 & 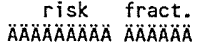 & 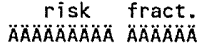 & 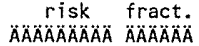 & $\begin{array}{l}\text { risk fract. } \\
A B A A A B A O A B A O A O A\end{array}$ \\
\hline & $0.000 E+00 \quad 0.0000$ & $=+000$ & 000.0 & 000 & & \\
\hline & & & & 000 & +000 & \\
\hline & & 00 & & & & \\
\hline & & & & & & \\
\hline & $.000 E+00 \quad 0.0000$ & $.000 E+00 \quad 0.0000$ & $0.000 E+00 \quad 0.0000$ & $0.000 E+00 \quad 0.0000$ & $0.000 \mathrm{E}+00 \quad 0.0000$ & $1.402 E-05 \quad 1.0000$ \\
\hline
\end{tabular}

** Sum of water independent ground, inhalation, plant, meat, milk, soit

and water dependent water, fish, plant, meat, milk pathways

\begin{tabular}{|c|c|}
\hline Attachment & Sheet No. 10 \\
\hline Originator: S.W. Clark & Date \\
\hline M.W. Perrott & Date \\
\hline $0100 \mathrm{~F}-\mathrm{CA}-\mathrm{V} 0303$ & Rev. No. \\
\hline
\end{tabular}




\section{ATTACHMENT 3}

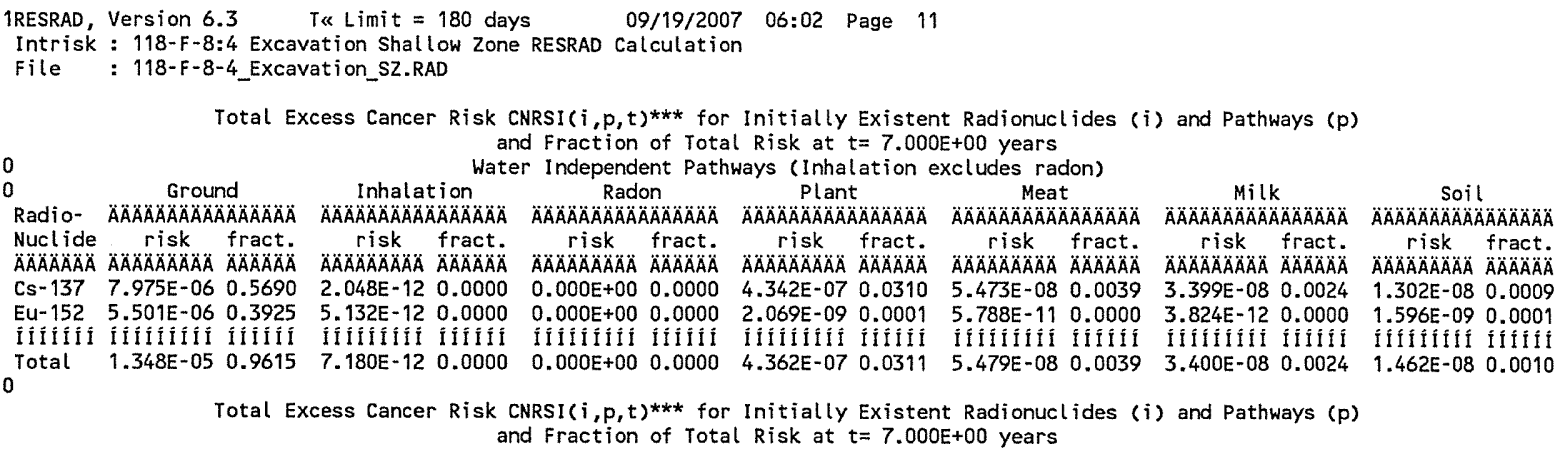

Water Dependent Pathways

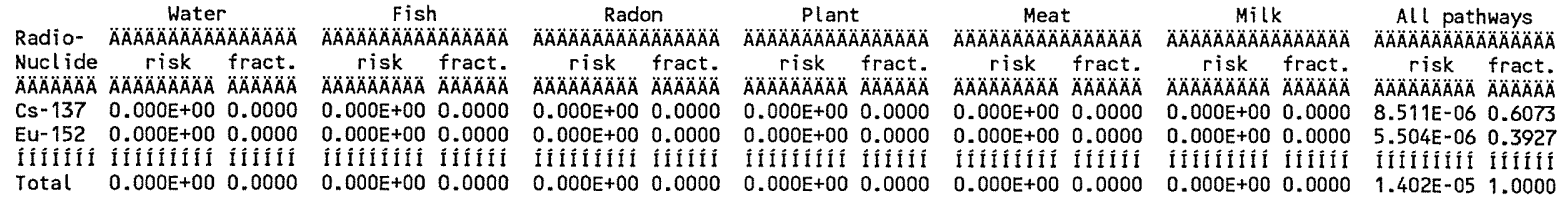

***CNRSI $(i, p, t)$ includes contribution from decay daughter radionuclides

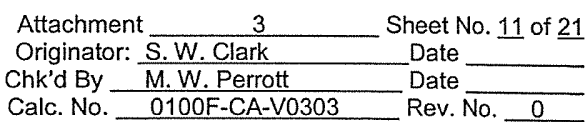




\section{ATTACHMENT 3}

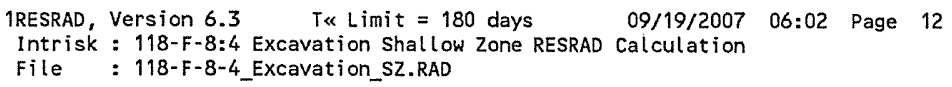

Water Dependent Pathways

\begin{tabular}{|c|c|c|c|c|c|c|c|c|c|c|c|c|}
\hline & $\begin{array}{r}\text { Wat } \\
\hdashline A A A B A A B\end{array}$ & $\ddot{A} \ddot{A} A \ddot{A} \ddot{A}$ & $\begin{array}{r}F i s \\
A A B A B A B A B A\end{array}$ & 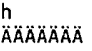 & 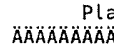 & $\begin{array}{l}n t \\
\because A A M A O A O A O A\end{array}$ & $\begin{array}{r}M e a \\
A \cap A ̈ A ̈ A ̈ A ̈ A ̈ A ̈ A ̈\end{array}$ & ÄÄÄÄÄÄ & $\begin{array}{r}M i l l \\
A A ̈ A B A O A B A ̈\end{array}$ & ÄÄÄÄÄÄ & $\begin{array}{l}A l l \text { Pat } \\
A O A A A B A B A\end{array}$ & 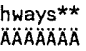 \\
\hline & risk & $\begin{array}{l}\text { fract. } \\
\ddot{A} A ̈ A ̈ A ̈ A ̈ A\end{array}$ & & & & fract: & risk & & risk & fra & risk & \\
\hline & & & & & & & & & & & & \\
\hline & & & & & & & & & & & & \\
\hline & & & & & & & & & & & & \\
\hline 36 & $+\infty 0$ & 00 & $.000 E+00$ & 0.0000 & 0.00 & 0.0000 & +00 & 0.0000 & $E+00$ & 0.0000 & $=-05$ & 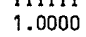 \\
\hline
\end{tabular}

** Sum of water independent ground, inhalation, plant, meat, milk, soil

and water dependent water, fish, plant, meat, milk pathways

\begin{tabular}{|c|c|}
\hline Attachment & Sheet No. 12 of 21 \\
\hline Originator: S.W. Clark & Date \\
\hline Chk'd By M.W. Perrott & Date \\
\hline $0100 \mathrm{~F}-\mathrm{CA}-\mathrm{V} 0303$ & Rev. No. 0 \\
\hline
\end{tabular}




\section{ATTACHMENT 3}

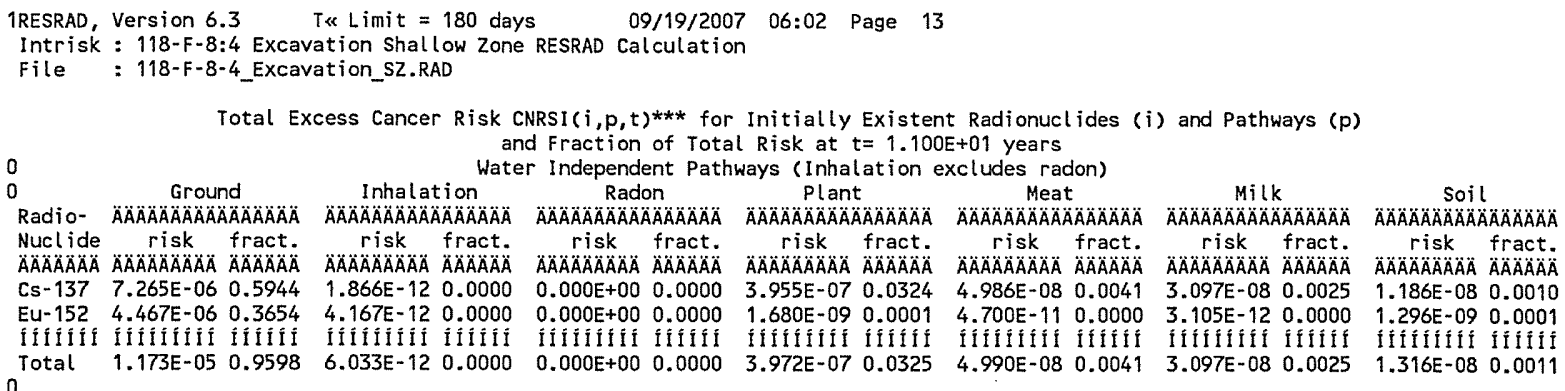

Water Dependent Pathways

\begin{tabular}{|c|c|c|c|c|c|c|c|}
\hline Radio- & $\begin{array}{c}\text { Water } \\
\text { ÄÄAAAAAAAAAAAAAÄ }\end{array}$ & $\begin{array}{c}F i s h \\
\text { ÄÂAAAAAAAAAAOÄÄÄ }\end{array}$ & $\begin{array}{c}\text { Radon } \\
\text { AAAAAAAAAAAAAAAAÄÄ }\end{array}$ & $\begin{array}{c}\text { Plant } \\
\text { AAAAAAAAAAAAAAAAAA }\end{array}$ & $\begin{array}{c}\text { Meat } \\
\text { AAAAAAAAAAAAAOAAAA }\end{array}$ & $\begin{array}{c}M i l k \\
\text { AAAAAAAAAAAAAAAAAÄ }\end{array}$ & $\begin{array}{c}\text { All pathways } \\
\text { AAAAAAAAAAAAAAOAOA }\end{array}$ \\
\hline $\begin{array}{l}\text { Nucl ide } \\
\text { ÄÄÄÄÄÄ }\end{array}$ & 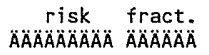 & 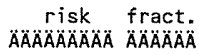 & $\begin{array}{cc}\text { risk fract. } \\
\text { ÄAAÄÄÄÄA ÄÄÄÄÄ }\end{array}$ & 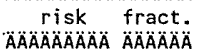 & $\begin{array}{r}\text { risk fract. } \\
\text { AAAAAAAAA AAAAAAOA }\end{array}$ & $\begin{array}{c}\text { risk fract. } \\
\text { AAAAAAAAAAA AAÄÄÄ }\end{array}$ & 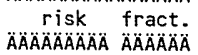 \\
\hline Cs -137 & $0.000 E+00 \quad 0.0000$ & $0.000 E+00 \quad 0.0000$ & $0.000 E+00 \quad 0.0000$ & $0.000 E+00 \quad 0.0000$ & $0.000 E+00 \quad 0.0000$ & $0.000 E+00 \quad 0.0000$ & $\begin{array}{ll}7.753 E-06 & 0.6343\end{array}$ \\
\hline Eu- 152 & $0.000 E+00 \quad 0.0000$ & $0.000 \mathrm{E}+00 \quad 0.0000$ & $0.000 E+00 \quad 0.0000$ & $0.000 E+00 \quad 0.0000$ & $0.000 E+00 \quad 0.0000$ & $0.000 E+00 \quad 0.0000$ & $\begin{array}{lll}4.470 E-06 & 0.3657\end{array}$ \\
\hline IIIIIIII & 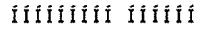 & 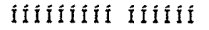 & IIIIIIIIIII İIIIII & IIIIIYIII IIIIII & 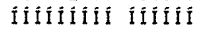 & 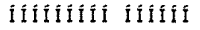 & Difififi ifiti \\
\hline Total & $0.000 E+00 \quad 0.0000$ & $0.000 E+00 \quad 0.0000$ & $0.000 E+00 \quad 0.0000$ & $0.000 E+00 \quad 0.0000$ & $0.000 E+00 \quad 0.0000$ & $0.000 E+00 \quad 0.0000$ & $1.222 \mathrm{E}-05 \quad 1.0000$ \\
\hline
\end{tabular}

$* * * \operatorname{CNRSI}(i, p, t)$ includes contribution from decay daughter radionuclides

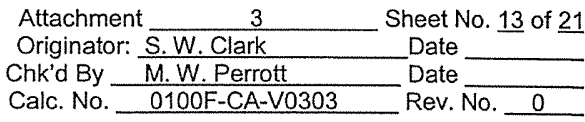




\section{ATTACHMENT 3}

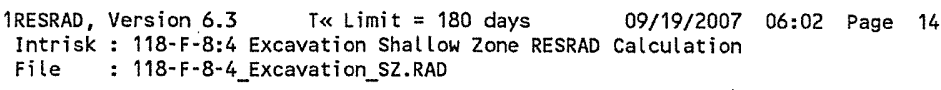

Excess Cancer Risks CNRS $(i, p, t)$ for Individual Radionuclides (i) and Pathways $(p)$ and Fraction of Total Risk at $t=4.300 E+01$ years

Water Dependent Pathways

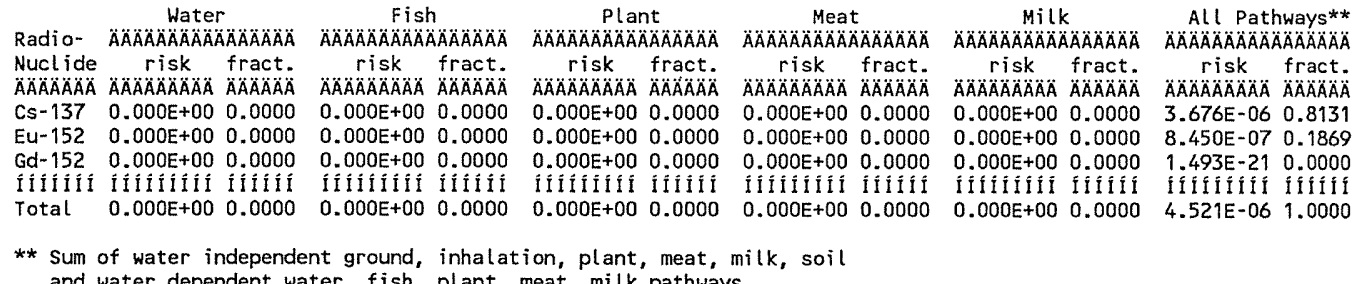

and water dependent water, fish, plant, meat, milk pathways

$\begin{array}{ll}\text { Attachment } \frac{3}{\text { S.W. Clark }} & \text { Sheet No. } 14 \text { of } 21 \\ \text { Originator: } & \text { Date } \\ \text { Chk'd By } \frac{\text { M.W.Perrott }}{0100 \text { F-CA-V0303 }} & \text { Date } \\ \text { Calc. No. No. No. } 0\end{array}$




\section{ATTACHMENT 3}

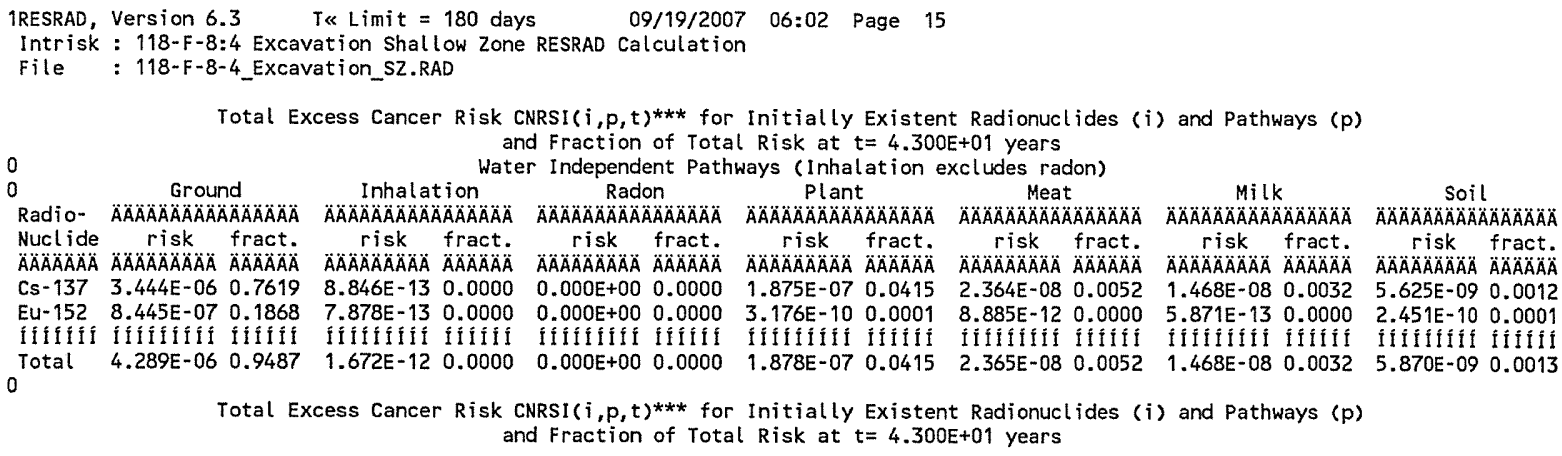

Water Dependent Pathways

\begin{tabular}{|c|c|c|c|c|c|c|c|c|c|c|c|c|c|}
\hline & 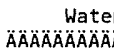 & $r$ & $\begin{array}{r}\text { Fist } \\
A \ddot{A B A B A O}\end{array}$ & $\begin{array}{l}h \\
\text { ÄĂĀÄÄÄ }\end{array}$ & $\begin{array}{r}R a \\
A ̈ A ̈ A ̈ A ̈ A\end{array}$ & $\ddot{A} A ̈ A ̈ A ̈ A ̈$ & $\begin{array}{r}P l a \\
A B A A A A A\end{array}$ & $\triangle \triangle A \cap A \cap A ̈ A ̈$ & $\begin{array}{r}M e \\
A \cap A A A B A O\end{array}$ & $\triangle A \cap A O A B$ & $\begin{array}{r}M i l l \\
A \cap A A B A A B\end{array}$ & ÄÄÄÄÄ & hways \\
\hline & isk & $\begin{array}{l}\mathrm{fra} \\
\ddot{A} \ddot{A} \ddot{A}\end{array}$ & $\begin{array}{l}S k \\
\dot{A} A ̈ A ̈ A\end{array}$ & $\begin{array}{l}\text { fra } \\
\ddot{A} \ddot{A} \ddot{A}\end{array}$ & risk & & risk & & $\begin{array}{l}\text { risk } \\
A A A B A B A B\end{array}$ & & risk & & \\
\hline & & & & & & & & & & & & & \\
\hline & & & & & & & & & & & & & \\
\hline & & & & & & & & & & & & & \\
\hline & & & & & & & +00 & & +00 & & $E+00$ & & \\
\hline
\end{tabular}

$* * *$ CNRSI $(i, p, t)$ includes contribution from decay daughter radionuclides

\begin{tabular}{|c|c|}
\hline Attachment & Sheet $\mathrm{N}$ \\
\hline Originator: S.W. Clark & Date \\
\hline M. W. Perrott & Date \\
\hline $0100 F-C A-V 0303$ & Rev. $\overline{\text { No }}$ \\
\hline
\end{tabular}




\section{ATTACHMENT 3}

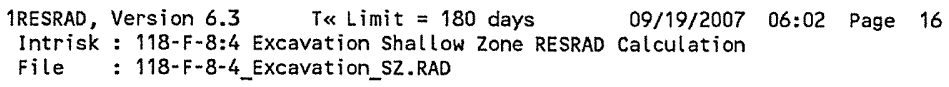

Excess Cancer Risks CNRS( $i, p, t)$ for Individual Radionuclides (i) and Pathways ( $p$ ) and Fraction of Total Risk at $t=1.350 E+02$ years

Water Dependent Pathways

\begin{tabular}{|c|c|c|c|c|c|c|}
\hline & 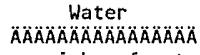 & 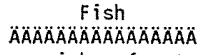 & 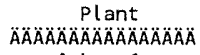 & 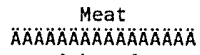 & 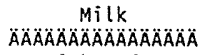 & 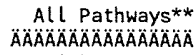 \\
\hline$\stackrel{e}{\ddot{A}}$ & $\begin{array}{ll}\text { risk } & \text { frac } \\
A A A B A O A B & A B A O A B\end{array}$ & $\begin{aligned} \text { risk fract. } \\
A B A O A O A O A\end{aligned}$ & $\begin{array}{l}\text { risk fract. } \\
\triangle A B A O A B A O A A A A B A\end{array}$ & $\begin{array}{c}\text { risk fra } \\
\triangle A ̈ A O A B A O A O A B\end{array}$ & risk fr & risk fract. \\
\hline & 100 & 000 & & & & 37 \\
\hline & & & & & & 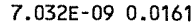 \\
\hline & 10 & & 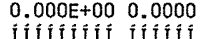 & & & \\
\hline & $+00 \quad 0.0000$ & 000.0000 & $\begin{array}{lll}\text { I I f } & \text { i } \\
+ & 0 & 0\end{array}$ & $+00 \quad 0.0000$ & $\begin{array}{l}\text { if if ifilifi } \\
+000.0000\end{array}$ & $\begin{array}{l}\text { fif fiffifi } \\
-07 \\
-07.0000\end{array}$ \\
\hline
\end{tabular}

** Sum of water independent ground, inhalation, plant, meat, milk, soil

and water dependent water, fish, plant, meat, milk pathways

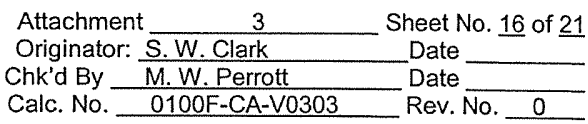




\section{ATTACHMENT 3}

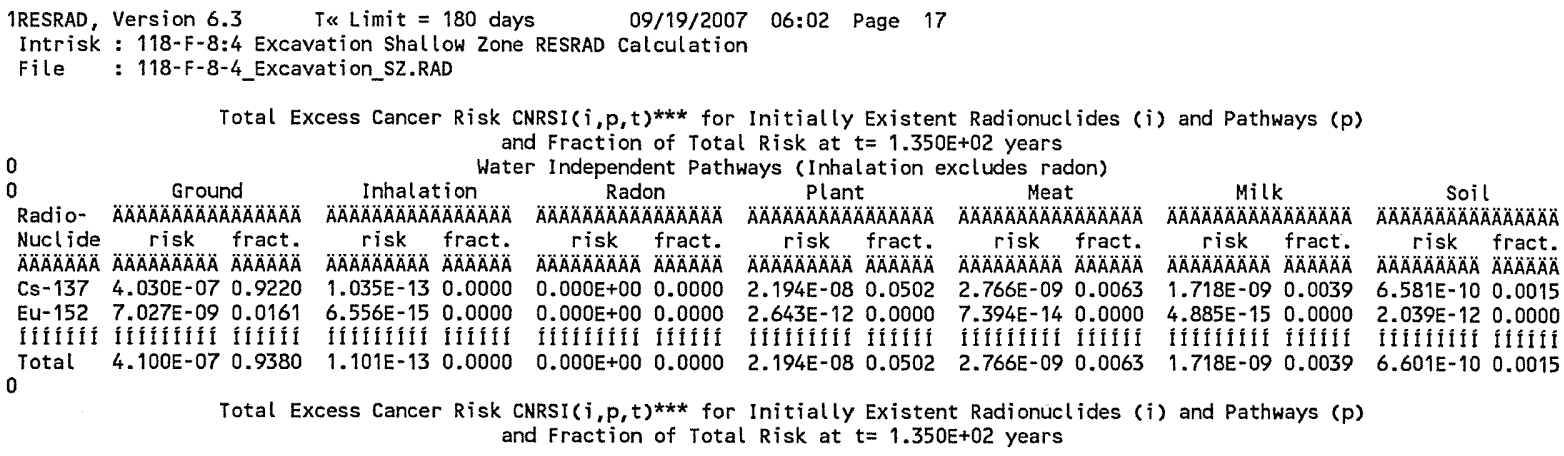

Water Dependent Pathways

\begin{tabular}{|c|c|c|c|c|c|c|c|c|}
\hline & 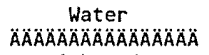 & $\begin{array}{c}F i s h \\
\text { AÄÄÄÄÄÄÄÄÄÄÄÄÄ }\end{array}$ & 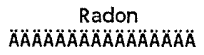 & $\begin{array}{c}\text { Plant } \\
\text { ALAAAAAAAOAAAOAOAO }\end{array}$ & $\begin{array}{c}\text { Meat } \\
\text { AAAAAAAÄÄÄÄÄÄÄÄ }\end{array}$ & $\begin{array}{r}M i \\
M A A ̈ A ̈ A ̈ A ̈\end{array}$ & 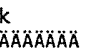 & 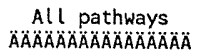 \\
\hline & $\begin{array}{l}\text { fract. } \\
\ddot{A} \ddot{A} \ddot{A} A \ddot{A} \ddot{A}\end{array}$ & 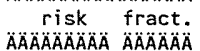 & $\begin{array}{l}\text { risk fract. } \\
\text { ÄÄÄÄA ÄABAAÄ }\end{array}$ & $\begin{array}{l}\text { risk fract. } \\
\text { AAAAAAA AAAAAAA }\end{array}$ & $\begin{array}{l}\text { risk fract. } \\
\text { AAAAAAA AAAAAÄ }\end{array}$ & $\begin{array}{l}\text { risk } \\
\text { AAAOABAA }\end{array}$ & & $\begin{array}{l}\text { risk fract. } \\
\text { AAAAAAA ABAAAOA }\end{array}$ \\
\hline & $100 E+00 \quad 0.0000$ & & & & & & & \\
\hline & 00.000 & $+00 \quad 0.00$ & $\begin{array}{l}0.00 \\
\text { fif }\end{array}$ & Ifo. & 000 & & & \\
\hline Total & $10 E+00 \quad 0.0000$ & $00 \quad 0.0000$ & 00 & 00 & 000 & 500 & 0000 & \\
\hline
\end{tabular}

$* * *$ CNRSI $(i, p, t)$ includes contribution from decay daughter radionuclides

\begin{tabular}{|c|c|}
\hline Attachment & Sheet No \\
\hline Originator: S.W. Clark & Date \\
\hline M. W. Perrott & Date \\
\hline $0100 F-C A-V 0303$ & Rev. No. \\
\hline
\end{tabular}




\section{ATTACHMENT 3}

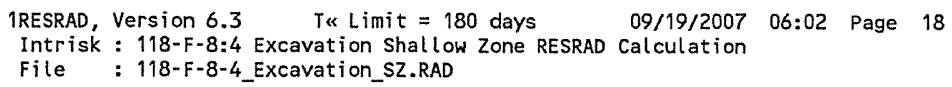

Excess Cancer Risks CNRS $(i, p, t)$ for Individual Radionuclides (i) and Pathways $(p)$ and Fraction of Total Risk at $t=3.000 E+02$ years

Water Dependent Pathways

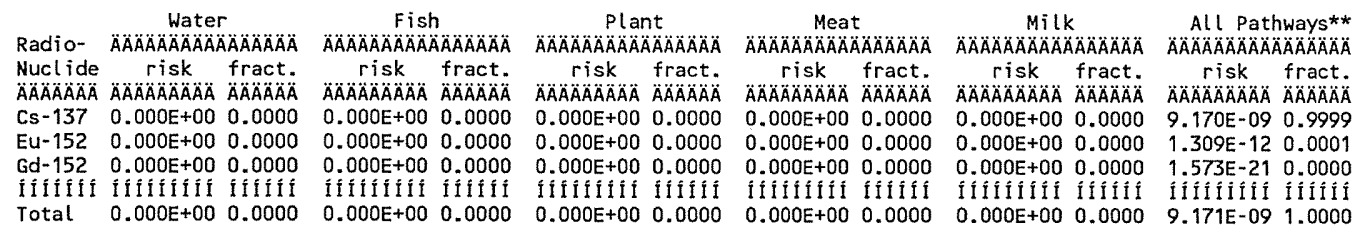

** Sum of water independent ground, inhalation, plant, meat, milk, soil

and water dependent water, fish, plant, meat, milk pathways

\begin{tabular}{|c|c|}
\hline Attachment & Sheet No. 18 of 21 \\
\hline Originator: S.W. Clark & Date \\
\hline Chk'd By M.W. Perrott & Date \\
\hline Calc. No. $0100 \mathrm{~F}-\mathrm{CA}-\mathrm{V} 0303$ & Rev. No. \\
\hline
\end{tabular}




\section{ATTACHMENT 3}

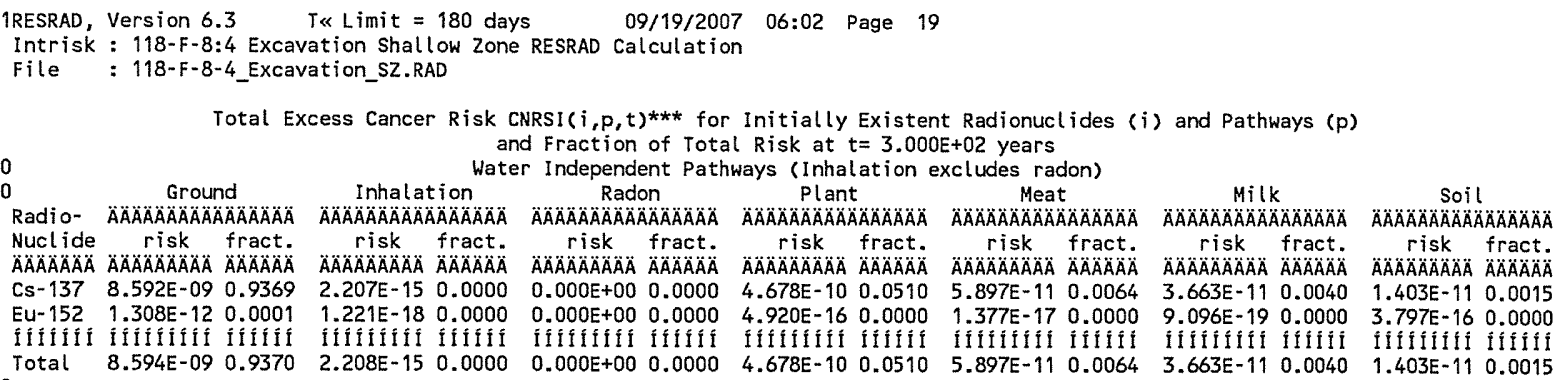

Total Excess Cancer Risk CNRSI $(i, p, t) * * *$ for Initially Existent Radionuclides $(i)$ and Pathways $(p)$ and Fraction of Total Risk at $t=3.000 E+02$ years

Water Dependent Pathways

\begin{tabular}{|c|c|c|c|c|c|c|c|}
\hline Radio- & $\begin{array}{c}\text { Water } \\
\text { ÄÄÄÄÄAAAAAÄÄÄ }\end{array}$ & $\begin{array}{c}\text { Fish } \\
\text { ÄAAAAAAAAAAAAAAA }\end{array}$ & $\begin{array}{c}\text { Radon } \\
\text { AAAAAAAAAAAAAOAOÄA }\end{array}$ & 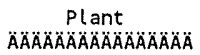 & $\begin{array}{c}\text { Meat } \\
\text { ÄÄÄÄÄÄÄÄÄÄÄÄÄÄ }\end{array}$ & $\begin{array}{c}M i l k \\
\text { ÄÄÄÄÄAOAOAAAOAOÄ }\end{array}$ & $\begin{array}{c}\text { All pathways } \\
\text { AOAAAAAAAAAAAAAAAA }\end{array}$ \\
\hline $\begin{array}{l}\text { Nuclide } \\
\text { ÄAAAAAÄ }\end{array}$ & $\begin{array}{l}\text { risk fract. } \\
\text { AAAAAAAAA AAAAAA }\end{array}$ & 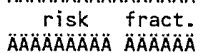 & $\begin{array}{cc}\text { risk } & \text { fract. } \\
\triangle A A A B A B A A A & A B A A A B A\end{array}$ & $\begin{array}{l}\text { risk fract. } \\
\text { AAAAAAAAAA ABAAAA }\end{array}$ & $\begin{array}{l}\text { risk fract. } \\
\text { ÄAAAAAAAA AAAAAÄ }\end{array}$ & $\begin{array}{l}\text { risk fract. } \\
\text { AAAAAAAAOAAAAAA }\end{array}$ & $\begin{array}{l}\text { risk fract. } \\
\triangle A \cap A B A B A B A \text { ÄAABÄA }\end{array}$ \\
\hline Cs- 137 & $0.000 \mathrm{E}+000.0000$ & $0.000 \mathrm{E}+000.0000$ & $0.000 E+000.0000$ & $0.000 E+00 \quad 0.0000$ & $\begin{array}{l}\text { AAAAAAAAA AAAAAA } \\
0.000 E+000.0000\end{array}$ & $\begin{array}{l}\text { AAAAAAAAA AAAAAA } \\
0.000 E+000.0000\end{array}$ & $\begin{array}{l}\text { AAAAAAAAA AAAAAA } \\
9.170 E-09 \quad 0.9999\end{array}$ \\
\hline Eu-152 & $0.000 E+00 \quad 0.0000$ & $0.000 E+00 \quad 0.0000$ & $0.000 E+000.0000$ & $0.000 E+00 \quad 0.0000$ & $0.000 \mathrm{E}+00 \quad 0.0000$ & $0.000 E+00 \quad 0.0000$ & $1.309 \mathrm{E}-12 \quad 0.0001$ \\
\hline fifilif & IIIHIIIII IIIIII & 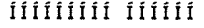 & Ififitifit lififí & 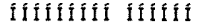 & 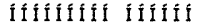 & 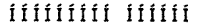 & filiniti lifil \\
\hline Total & $0.000 E+00 \quad 0.0000$ & $0.000 E+00 \quad 0.0000$ & $0.000 E+00 \quad 0.0000$ & $0.000 E+00 \quad 0.0000$ & $0.000 E+00 \quad 0.0000$ & $0.000 E+00 \quad 0.0000$ & $9.171 \mathrm{E}-09 \quad 1.0000$ \\
\hline
\end{tabular}

$* * * \operatorname{CNRSI}(i, p, t)$ includes contribution from decay daughter radionuclides

\begin{tabular}{|c|c|}
\hline Attachment & Sheet No \\
\hline Originator: S.W. Clark & Date \\
\hline Chk'd By M. W. Perrott & Date \\
\hline Calc. No. $0100 \mathrm{~F}-\mathrm{CA}-\mathrm{V} 0303$ & Rev. No. \\
\hline
\end{tabular}




\section{ATTACHMENT 3}

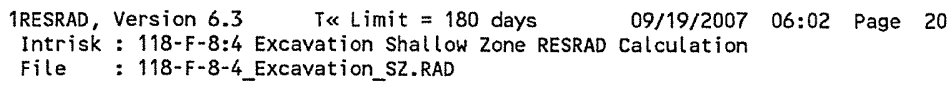

Excess Cancer Risks CNRS $(i, p, t)$ for Individual Radionuclides (i) and Pathways $(p)$ and Fraction of Total Risk at $t=1.000 E+03$ years

Water Dependent Pathways

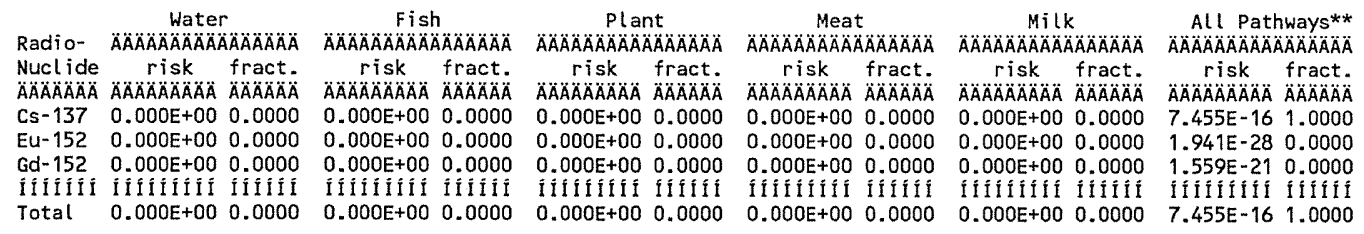

** Sum of water independent ground, inhalation, plant, meat, milk, soil

and water dependent water, fish, plant, meat, milk pathways

\begin{tabular}{|c|c|}
\hline Attachment & Sheet No \\
\hline lator: S.W. Clark & Date \\
\hline M. W. Perrott & Date \\
\hline $0100 F-C A-V 0303$ & Rev. No. \\
\hline
\end{tabular}




\section{ATTACHMENT 3}

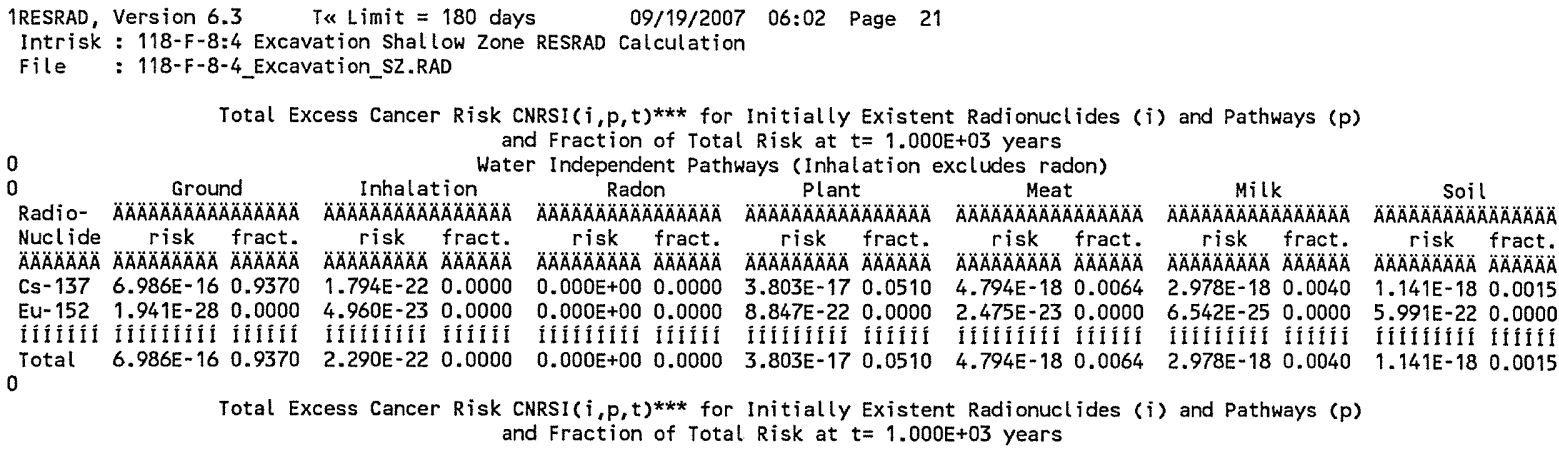

Total Excess Cancer Risk CNRSI $(i, p, t) * * *$ for Initially Existent Radionuclides ( $i$ ) and Pathways $(p)$ and Fraction of Total Risk at $t=1.000 E+03$ years

Water Dependent Pathways

\begin{tabular}{|c|c|c|c|c|c|c|c|c|}
\hline & $\begin{array}{l}\text { Water } \\
\text { ÄAAAAAAAAAAAÄA }\end{array}$ & $\begin{array}{c}\text { Fish } \\
\text { AAAAAAAAAAAOÄA }\end{array}$ & $\begin{array}{c}\text { Radon } \\
\text { ĀÄÄÄÄÄÄÄÄÄÄA }\end{array}$ & $\begin{array}{c}P l a n t \\
\text { ÄAAAAAAAAAAAOAOAOÄ }\end{array}$ & 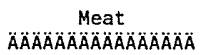 & $\begin{array}{r}M i \\
A A B A A A ̈ A ̈\end{array}$ & $\triangle A O A \cap A \cap A ̈ A ̈$ & $\begin{array}{l}\text { All pathways } \\
A B A A A A B A A A O A B A \\
\end{array}$ \\
\hline & $\begin{array}{l}\text { frac } \\
\triangle A \cap A ̈ A ̊\end{array}$ & $\begin{array}{l}\text { risk frac } \\
\text { ÁÄÄÄ ÄÄÄ }\end{array}$ & $\begin{array}{l}\text { fract. } \\
\triangle A O A O A O A\end{array}$ & $\begin{array}{l}f r a \\
A ̈ A ̈ A ̈\end{array}$ & $\begin{array}{l}\text { risk fra } \\
A B A A B A O A \\
A A B A B\end{array}$ & $\begin{array}{l}\text { risk } \\
\text { ÄÄÄAAA }\end{array}$ & & risk fo \\
\hline & 00.0 & 0.0 & & & & & & \\
\hline & & & & & & & & \\
\hline & & & & & & & & \\
\hline & & & & & 000 & $\mathrm{E}+00$ & & \\
\hline
\end{tabular}

$* * *$ CNRSI $(i, p, t)$ includes contribution from decay daughter radionuclides

\begin{tabular}{|c|c|}
\hline Attachment & Sheet No. 21 of 21 \\
\hline Originator: S.W. Clark & Date \\
\hline M.W. Perrott & Date \\
\hline 0100F-CA-V0303 & Rev. No. \\
\hline
\end{tabular}




\section{ATTACHMENT 4}

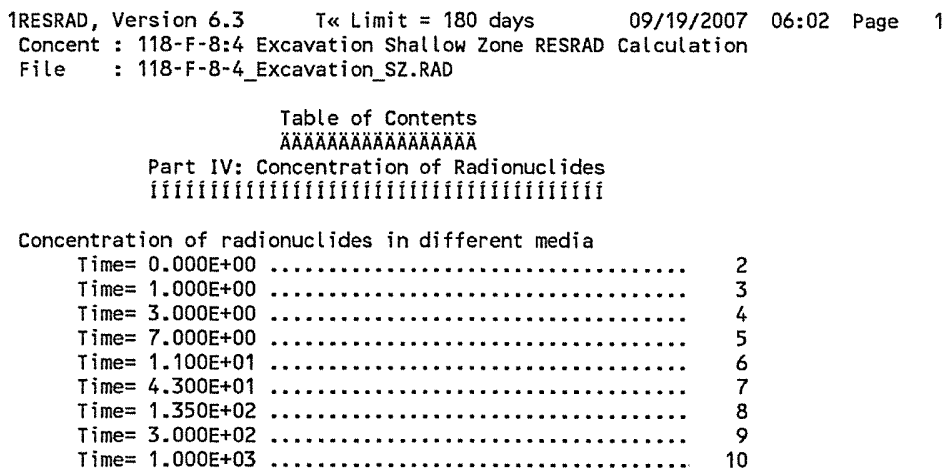




\section{ATTACHMENT 4}

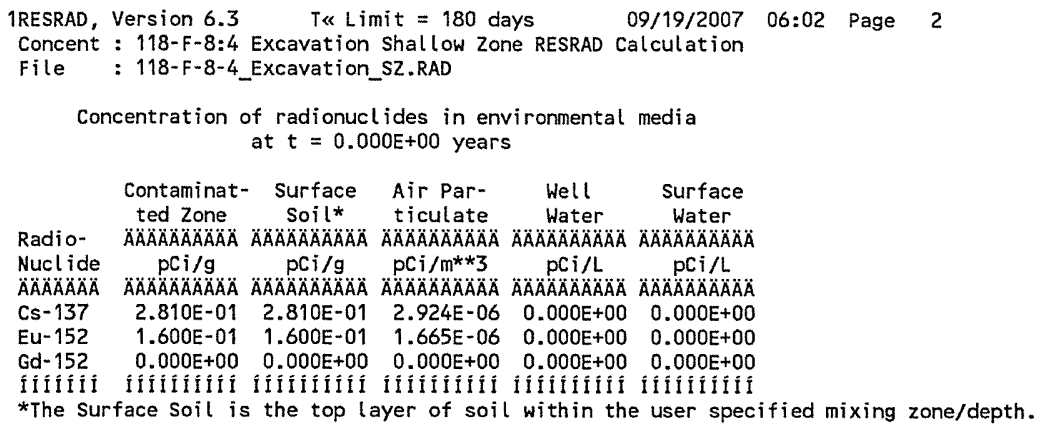

Concentrations in the media occurring in pathways that are suppressed are calculated using the current input parameters, i.e. using parameters appearing in the input screen when the pathways are active.

\begin{tabular}{|c|c|c|c|c|c|c|c|c|c|}
\hline \multicolumn{10}{|c|}{$\begin{array}{c}\text { Concentration of radionuclides in foodstuff media } \\
\text { at } t=0.000 E+00 \text { years* }\end{array}$} \\
\hline & $\begin{array}{l}\text { Drinking } \\
\text { Water }\end{array}$ & $\begin{array}{l}\text { Nonleafy } \\
\text { Vegetable }\end{array}$ & $\begin{array}{l}\text { Leafy } \\
\text { Vegetable }\end{array}$ & $\begin{array}{l}\text { Fodder } \\
\text { Meat }\end{array}$ & $\begin{array}{l}\text { Fodder } \\
\text { Milk }\end{array}$ & Meat & Milk & Fish & Crustacea \\
\hline adio- & $\triangle A \cap A ̈ A ̈ A \cap A ̈ A \cap A ̈ A ̈$ & 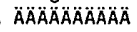 & 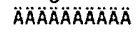 & $\triangle A \cap A \cap A A A A A B A$ & ÄÄÄÄAAAOÄ & 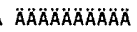 & 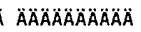 & 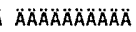 & 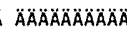 \\
\hline clide & $\mathrm{pCi} / \mathrm{L}$ & $\mathrm{pCi} / \mathrm{kg}$ & $\mathrm{pCi} / \mathrm{kg}$ & $\mathrm{pCi} / \mathrm{kg}$ & $\mathrm{pCi} / \mathrm{kg}$ & $\mathrm{pCi} / \mathrm{kg}$ & $\mathrm{pCi} / \mathrm{L}$ & $\mathrm{pCi} / \mathrm{kg}$ & $\mathrm{pCi} / \mathrm{kg}$ \\
\hline$A ̈ A ̈ A ̈ A ̈ A$ & $A A A A A A A A A A ̈$ & AAAAAAAAAAAÄ & AAAAAAAAAAAA & $\triangle A A A A A A A A A A O A$ & $\triangle A A A A A A A O A O A O A$ & $\triangle A O A A A A A A B A A B$ & $\triangle A O A O A O A O A O A O A ̈$ & 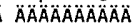 & $\triangle A A A A A A A A A O A$ \\
\hline Cs -137 & $0.000 E+00$ & $1.124 E+01$ & $1.124 \mathrm{E}+01$ & $1.124 \mathrm{E}+01$ & $1.124 \mathrm{E}+01$ & $2.715 \mathrm{E}+01$ & $6.070 E+00$ & $0.000 E+00$ & $0.000 E+00$ \\
\hline$u-152$ & $0.000 E+00$ & $4.001 \mathrm{E}-01$ & $4.004 \mathrm{E}-01$ & 4.005E-01 & $4.005 E-01$ & $2.145 \mathrm{E}-01$ & $5.101 \mathrm{E}-03$ & $0.000 E+00$ & $0.000 E+00$ \\
\hline Gd- 152 & $0.000 E+00$ & $0.000 E+00$ & $0.000 E+00$ & $0.000 E+00$ & $0.000 E+00$ & $0.000 E+00$ & $0.000 E+00$ & $0.000 E+00$ & $0.000 E+00$ \\
\hline & fili & & & 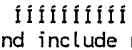 & ffififififí & 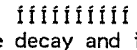 & Iflififilit & iff & fitifititit \\
\hline
\end{tabular}

Concentrations in the media occurring in pathways that are suppressed are calculated using the current input parameters, i.e. using parameters appearing in the input screen when the pathways are active.

$\begin{array}{ll}\text { Attachment } \frac{4}{\text { W. Clark }} & \text { Sheet No. } 2 \text { of } 10 \\ \text { Originator: } & \text { Date } \\ \text { Chk'd By } & \text { Date } \\ \text { Calc. No. Perrott } & \text { Rev. No. } \frac{0}{0100 \text { F-CA-V0303 }}\end{array}$




\section{ATTACHMENT 4}

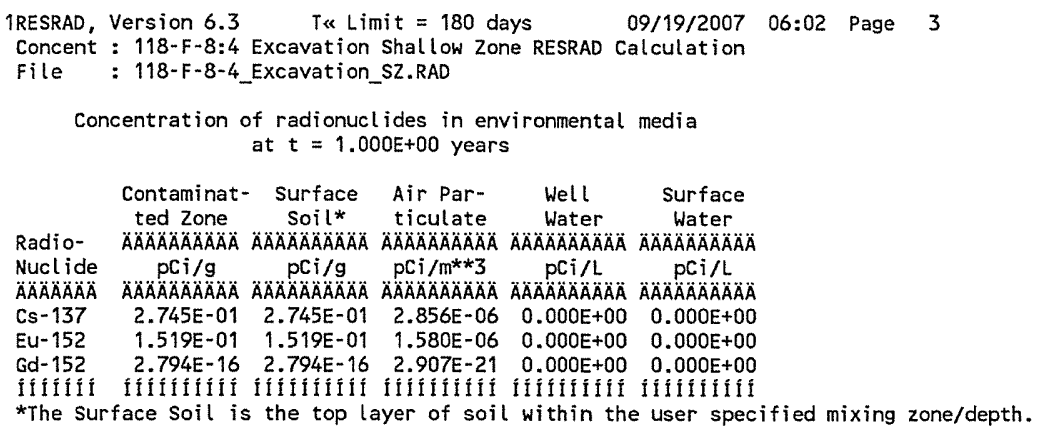

Concentrations in the media occurring in pathways that are suppressed are calculated using the current input parameters, i.e. using parameters appearing in the input screen when the pathways are active.

$\begin{array}{ll}\text { Attachment } \frac{4}{\text { O. W. Clark }} & \text { Sheet No. } \underline{3} \text { of } 10 \\ \text { Originator: } & \text { Date } \\ \text { Chk'd By } & \text { Date } \\ \text { Calc. No. Nerrott } & \text { Rev. No. } 0\end{array}$




\section{ATTACHMENT 4}
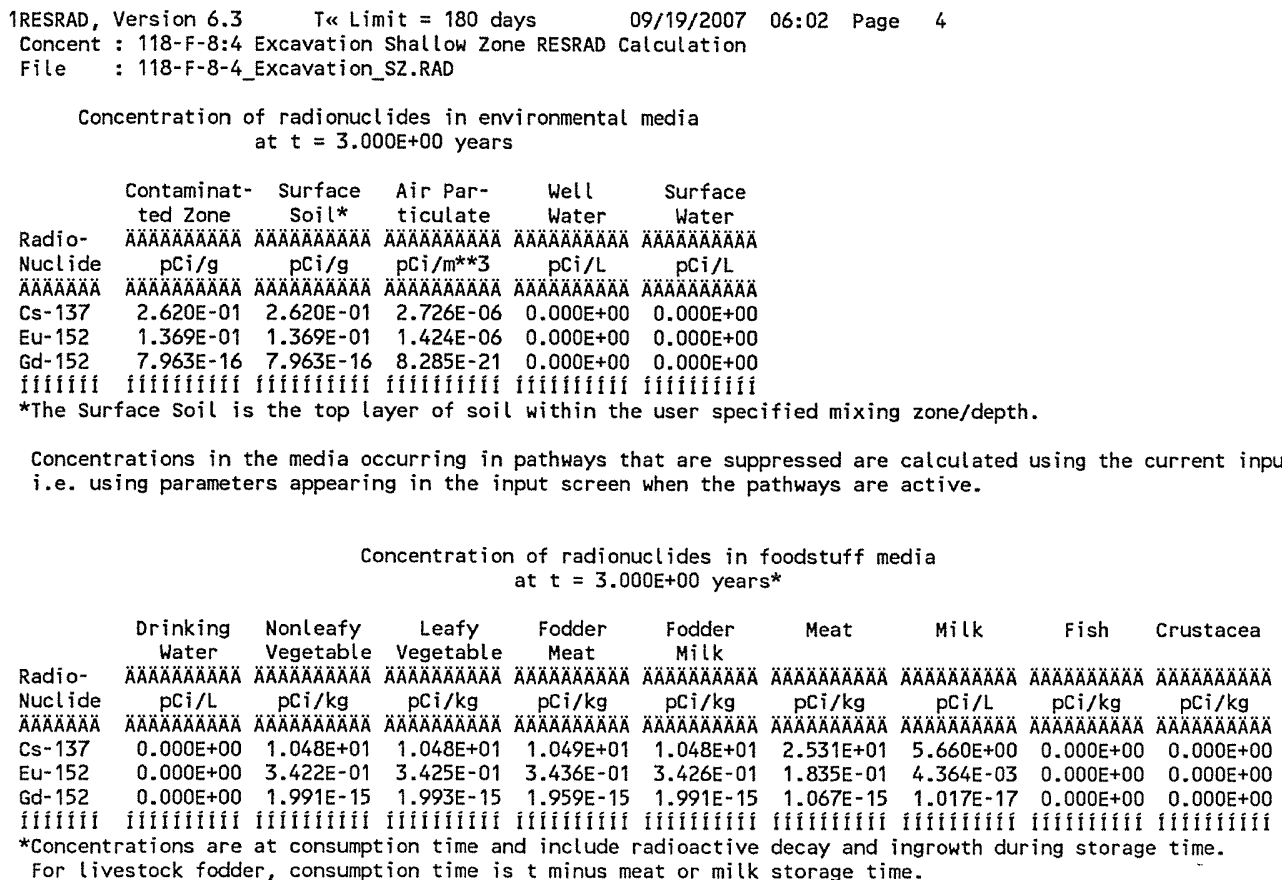

Concentrations in the media occurring in pathways that are suppressed are calculated using the current input parameters, i.e. using parameters appearing in the input screen when the pathways are active.

\begin{tabular}{|c|c|}
\hline Attachment & Sheet No. 4 of 10 \\
\hline Originator: S.W. Clark & Date \\
\hline M. W. Perrott & Date \\
\hline $0100 F-C A-V 0303$ & Rev. No. \\
\hline
\end{tabular}




\section{ATTACHMENT 4}

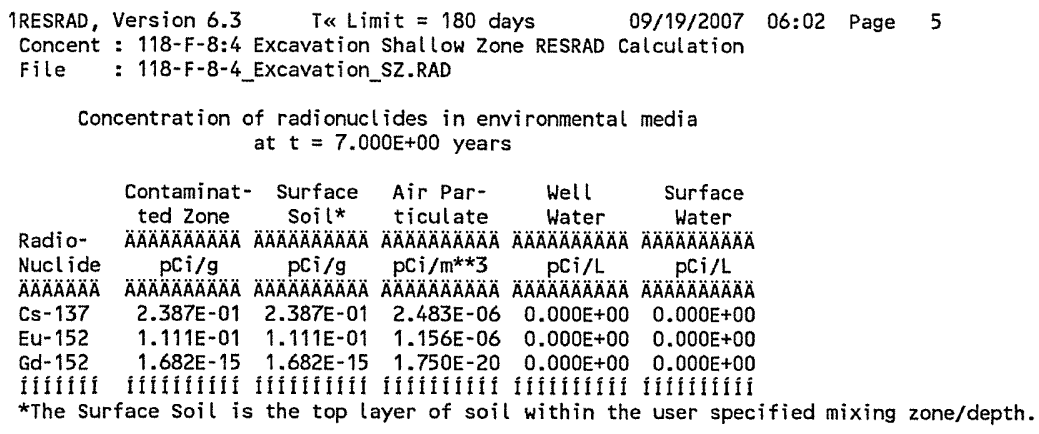

Concentrations in the media occurring in pathways that are suppressed are calculated using the current input parameters, i.e. using parameters appearing in the input screen when the pathways are active.

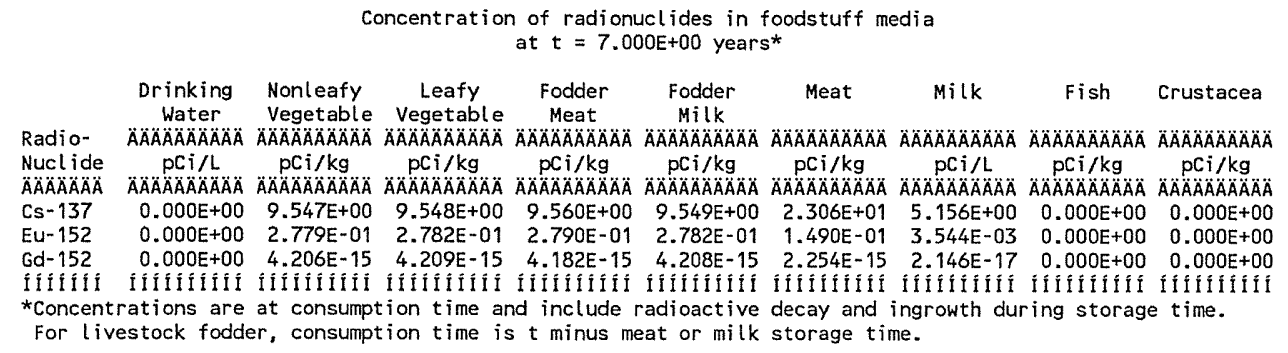

Concentrations in the media occurring in pathways that are suppressed are calculated using the current input parameters, i.e. using parameters appearing in the input screen when the pathways are active.

\begin{tabular}{|c|c|}
\hline Attachment & Sheet N \\
\hline Originator: S.W. Clark & Date \\
\hline M. W. Perrott & Date \\
\hline 0100F-CA-V0303 & Rev. No. \\
\hline
\end{tabular}




\section{ATTACHMENT 4}

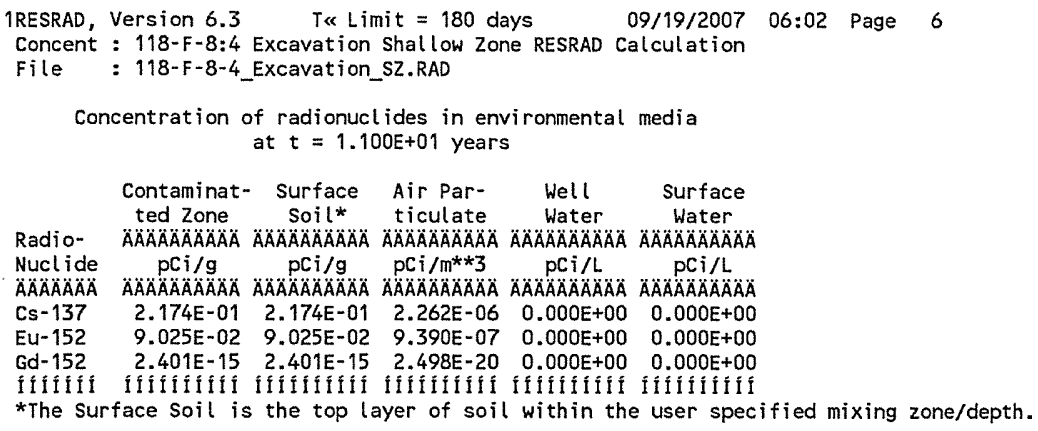

Concentrations in the media occurring in pathways that are suppressed are calculated using the current input parameters, i.e. using parameters appearing in the input screen when the pathways are active.

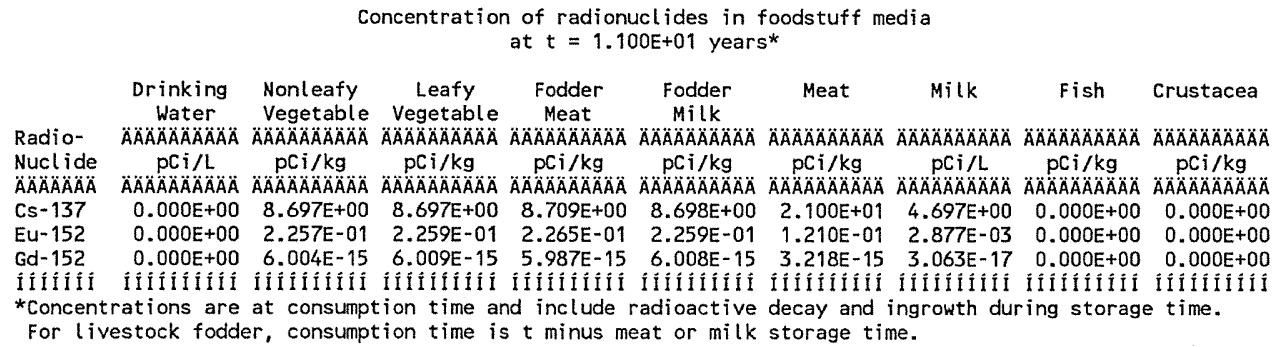

Concentrations in the media occurring in pathways that are suppressed are calculated using the current input parameters, i.e. using parameters appearing in the input screen when the pathways are active.

$\begin{array}{ll}\text { Attachment } \frac{4}{S . W . C l a r k} & \text { Sheet No. } \underline{6} \text { of } 10 \\ \text { Originator: } \frac{\text { S.W. }}{\text { M.W. Perrott }} & \text { Date } \\ \text { Chk'd By } & \text { Date } \\ \text { Calc. No. } & \text { Rev. No. } \frac{0}{0 \text { Nof-CA-V0303 }}\end{array}$




\section{ATTACHMENT 4}

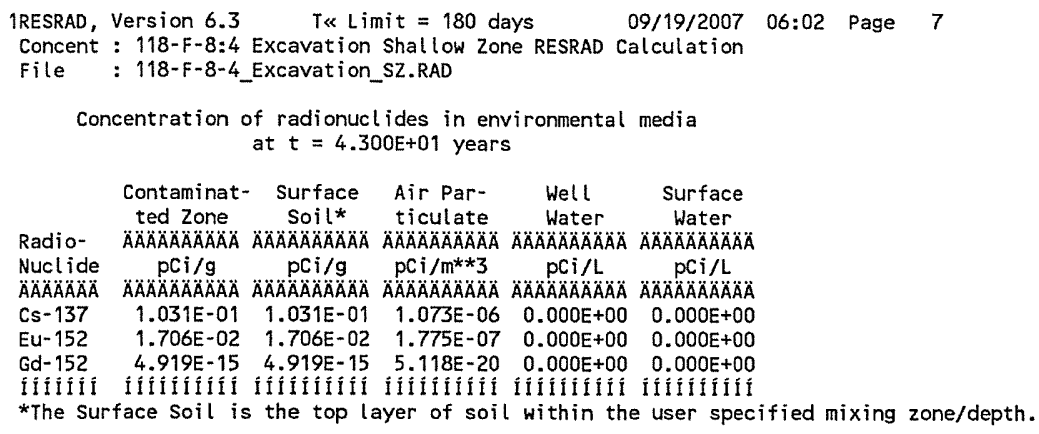

Concentrations in the media occurring in pathways that are suppressed are calculated using the current input parameters, i.e. using parameters appearing in the input screen when the pathways are active.

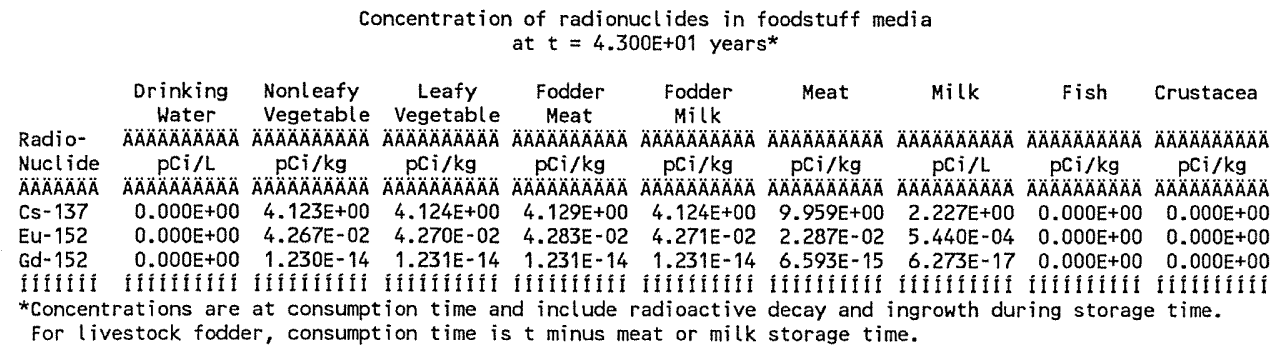

Concentrations in the media occurring in pathways that are suppressed are calculated using the current input parameters, i.e. using parameters appearing in the input screen when the pathways are active.

\begin{tabular}{|c|c|}
\hline Attachment & Sheet No. 7 of 10 \\
\hline Originator: $S . \mathrm{W}$. Clark & Date \\
\hline M. W. Perrott & Date \\
\hline $0100 F-C A-V 0303$ & Rev. No. 0 \\
\hline
\end{tabular}




\section{ATTACHMENT 4}

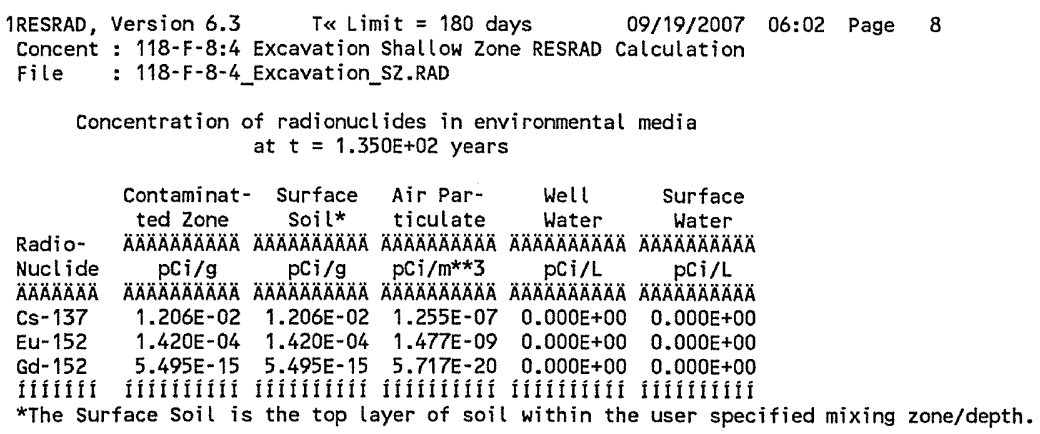

Concentrations in the media occurring in pathways that are suppressed are calculated using the current input parameters, i.e. using parameters appearing in the input screen when the pathways are active.

Concentration of radionuclides in foodstuff media at $t=1.350 E+02$ years*

\begin{tabular}{|c|c|c|c|c|c|c|c|c|c|}
\hline & $\begin{array}{l}\text { Drinking } \\
\text { Water }\end{array}$ & $\begin{array}{l}\text { Nonleafy } \\
\text { Vegetable }\end{array}$ & $\begin{array}{c}\text { Leafy } \\
\text { Vegetable }\end{array}$ & $\begin{array}{l}\text { Fodder } \\
\text { Meat }\end{array}$ & $\begin{array}{l}\text { Fodder } \\
\text { Milk }\end{array}$ & Meat & Milk & Fish & Crustacea \\
\hline & $\triangle A ̈ A \cap A O A O A O A O A$ & $\triangle A A A A A A A A B A B$ & $\triangle A O A O A O A A A O A B A$ & $\triangle A O A O A O A O A O A O A$ & 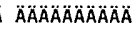 & $\triangle A B A A A A A A B A$ & $\triangle \triangle A A O A O A ̈ A ̈ A ̈ A ̈ A ̈$ & $\triangle \triangle A A A A A A A B A A A$ & $\triangle A A A A B$ \\
\hline & $\mathrm{pCi} /$ & $\mathrm{pC} i$ & $\mathrm{pCi} / \mathrm{kg}$ & $p C i$ & $\mathrm{pCi}$ & & & & \\
\hline$A A A$ & AAÄAAOAAAAAAOA & $\triangle A A A A A A A A A A B A$ & $\triangle A A A B A A A A A B A$ & $\triangle A A A A A A O A A A O A$ & $1 \triangle A B A A B A A O A A O A$ & ÄÄÄ & $A A A A A$ & AAAAAAAAAA & 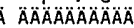 \\
\hline 137 & $0.000 E+00$ & $4.824 E-01$ & $14.825 E-01$ & $E-01$ & $4.825 \mathrm{E}-01$ & $E+00$ & $2.605 E-01$ & $0.000 E+00$ & $0.000 E+00$ \\
\hline 152 & $0.000 E+00$ & 3.5 & $4 \quad 3.55$ & 3. & 3.5 & 04 & & 0.0 & $E+00$ \\
\hline$\alpha^{-}$ & $0.000 E+00$ & $1.374 \mathrm{E}-14$ & $4 \quad 1.375 E-14$ & $1.375 E-14$ & $E-14$ & $E-15$ & -17 & $+\infty$ & $O E+00$ \\
\hline & & & & & & & & & 7,0 \\
\hline 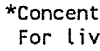 & & & & & & & & & \\
\hline
\end{tabular}

Concentrations in the media occurring in pathways that are suppressed are calculated using the current input parameters, i.e. using parameters appearing in the input screen when the pathways are active.

$\begin{array}{ll}\text { Attachment } \frac{4}{\text { Originator: }} \frac{4 . \text { W. Clark }}{\text { M.W. Perrott }} & \text { Sheet No. } \underline{8} \text { of } 10 \\ \text { Chk'd By } & \text { Date } \\ \text { Calc. No. } & \text { Rev. No. } \frac{0}{0100 \text { F-CA-V0303 }}\end{array}$




\section{ATTACHMENT 4}

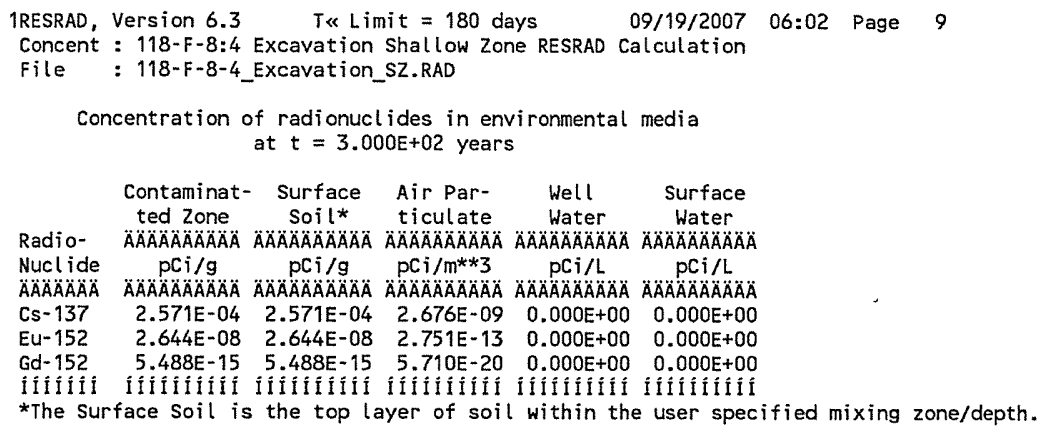

Concentrations in the media occurring in pathways that are suppressed are calculated using the current input parameters, i.e. using parameters appearing in the input screen when the pathways are active.

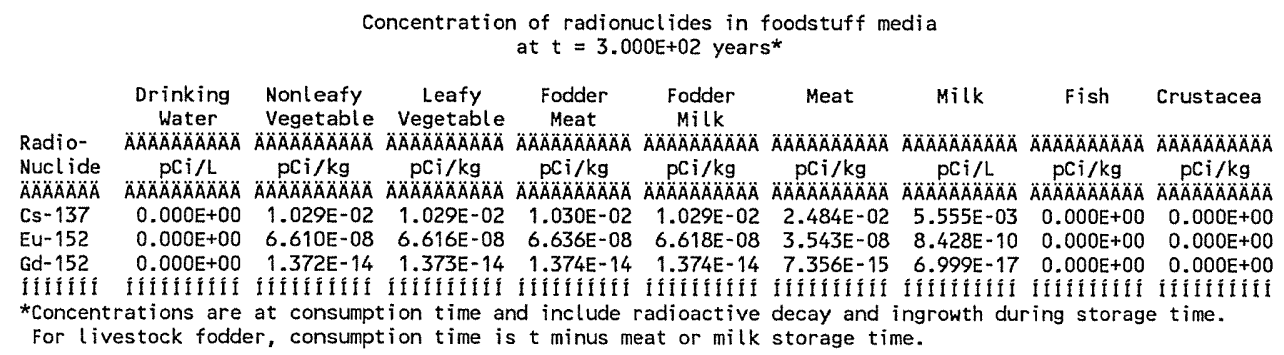

Concentrations in the media occurring in pathways that are suppressed are calculated using the current input parameters, i.e. using parameters appearing in the input screen when the pathways are active.

\begin{tabular}{|c|c|c|}
\hline \multicolumn{2}{|c|}{ Attachment $\quad 4$} & Sheet $\mathrm{No}$ \\
\hline \multicolumn{2}{|c|}{$\begin{array}{l}\text { Attachment } \\
\text { Originator: S. W. Clark }\end{array}$} & Date \\
\hline Chk' & M. W. Perrott & Date \\
\hline Calc. No. & $0100 F-C A-V 0303$ & Rev. No. \\
\hline
\end{tabular}




\section{ATTACHMENT 4}

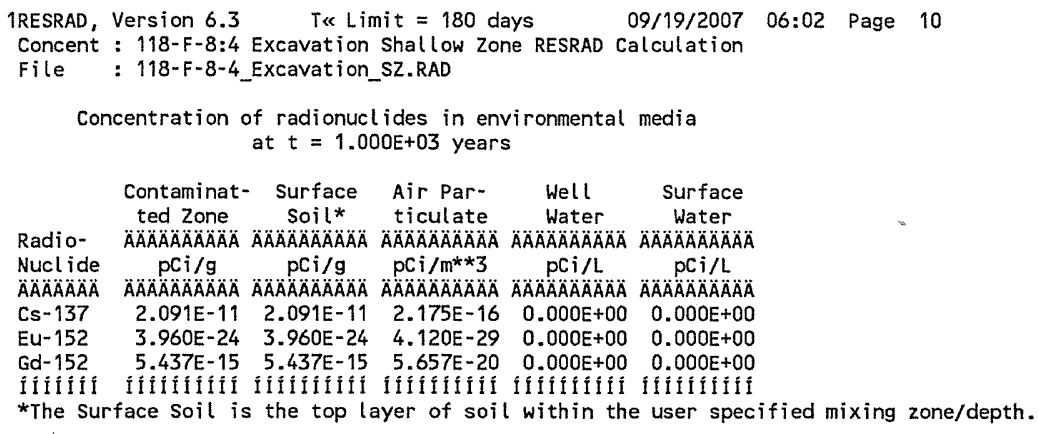

\begin{tabular}{|c|c|}
\hline Attachment & Sheet No. 10 of 10 \\
\hline Originator: S.W. Clark & Date \\
\hline M. W. Perrott & Date \\
\hline Calc. No. $0100 \mathrm{~F}-\mathrm{CA}-\mathrm{V} 0303$ & Rev. No. \\
\hline
\end{tabular}




\section{ATTACHMENT 5}

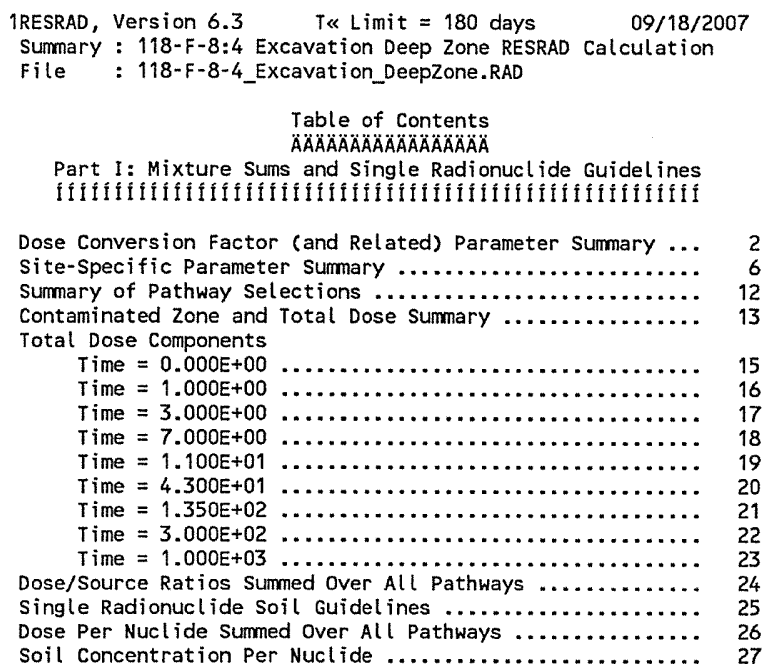




\section{ATTACHMENT 5}

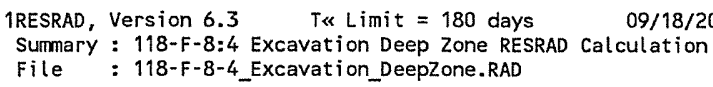

\begin{tabular}{|c|c|}
\hline \multicolumn{2}{|l|}{ Attachment $\quad 5$} \\
\hline Originator: S. W. Clark & Date \\
\hline M. W. Perrott & Date \\
\hline $0100 \mathrm{~F}-\mathrm{CA}-\mathrm{V} 0303$ & Rev. No \\
\hline
\end{tabular}




\section{ATTACHMENT 5}

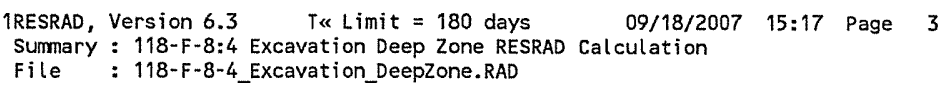

\begin{tabular}{|c|c|}
\hline Attachment & Sheet No. 3 of 27 \\
\hline Originator: S.W. Clark & Date \\
\hline M. W. Perrott & Date \\
\hline $0100 F-C A-V 0303$ & Rev. No. \\
\hline
\end{tabular}




\section{ATTACHMENT 5}

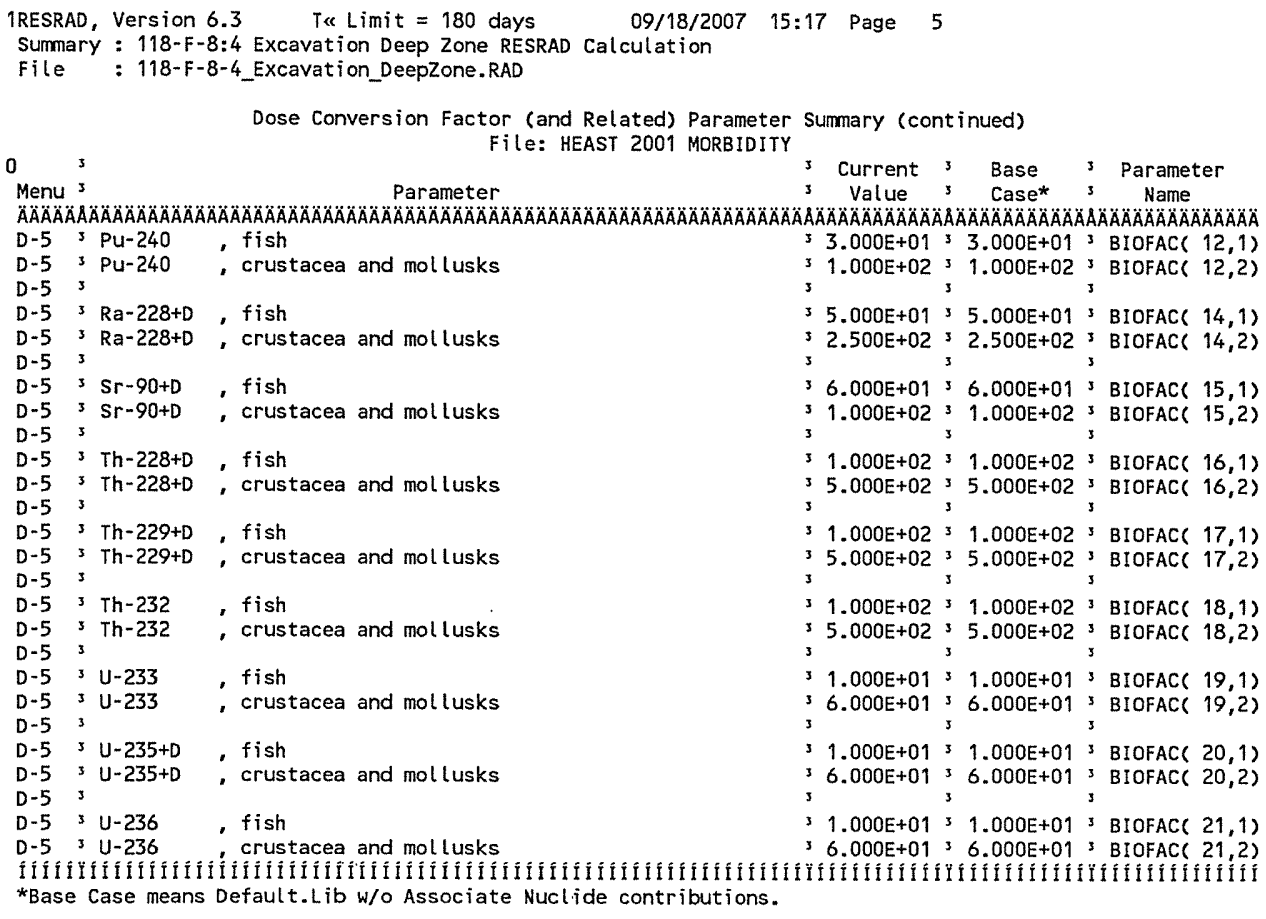

\begin{tabular}{|c|c|}
\hline Attachment & eet No \\
\hline Originator: S.W. Clark & Date \\
\hline M.W. Perrott & Date \\
\hline 0100F-CA-V0303 & Rev. No. $\quad 0$ \\
\hline
\end{tabular}




\section{ATTACHMENT 5}

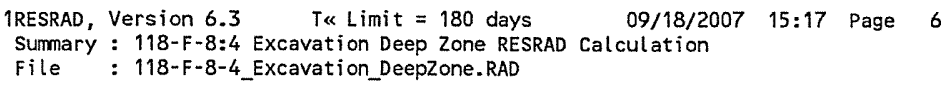

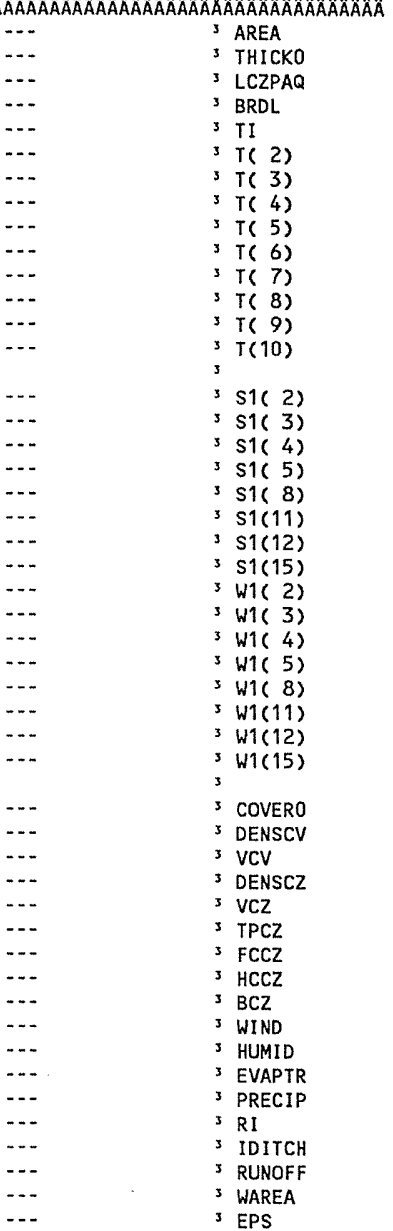

\begin{tabular}{|c|c|}
\hline \multicolumn{2}{|l|}{ Attachment $\frac{5}{\mathrm{~W}}$} \\
\hline Originator: s. W. Clark & Date \\
\hline M. W. Perrott & Date \\
\hline $0100 \mathrm{~F}-\mathrm{CA}-\mathrm{V} 0303$ & Rev. No. 0 \\
\hline
\end{tabular}




\section{ATTACHMENT 5}

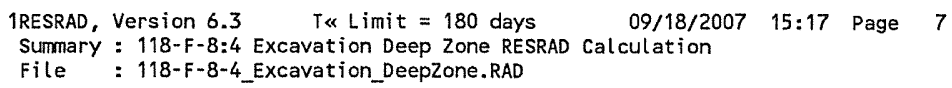

\begin{tabular}{|c|c|c|}
\hline$\ldots$ & & DENSAQ \\
\hline$\ldots$ & 3 & TPSZ \\
\hline$\cdots$ & 3 & EPSZ \\
\hline$\cdots$ & 3 & FCsZ \\
\hline$\cdots$ & 3 & HCSZ \\
\hline$\cdots$ & 3 & HGWT \\
\hline$\cdots$ & 3 & BSZ \\
\hline$\cdots$ & 3 & VWT \\
\hline$\cdots$ & 3 & DWIBWT \\
\hline$\cdots$ & 3 & MODEL \\
\hline.- & 3 & UW \\
\hline$\cdots$ & $\begin{array}{l}3 \\
3 \\
3\end{array}$ & NS \\
\hline$\ldots$ & 3 & DCNUCC ( 2) \\
\hline$\cdots$ & 3 & DCNUCS( 2) \\
\hline $200 \mathrm{E}-05$ & 3 & ALEACH ( 2) \\
\hline ot used & $\begin{array}{l}3 \\
3\end{array}$ & SOLUBK( 2 ) \\
\hline & 3 & \\
\hline$\ldots$ & 3 & DCNUCC ( 3) \\
\hline$\cdots$ & 3 & DCNUCS ( 3) \\
\hline $.278 E-04$ & 3 & ALEACH (3) \\
\hline tt used & $\begin{array}{l}3 \\
3\end{array}$ & SOLUBK $(3)$ \\
\hline & 3 & \\
\hline$\cdots$ & 3 & $\operatorname{DCNUCC}(4)$ \\
\hline - & 3 & DCNUCS (4) \\
\hline $278 E-04$ & 3 & ALEACH ( 4) \\
\hline ot used & 3 & SOLUBK ( 4 ) \\
\hline & 3 & \\
\hline- & 3 & DCNUCC ( 5) \\
\hline & 3 & DCNUCS ( 5 ) \\
\hline $200 E-05$ & 3 & ALEACH ( 5) \\
\hline ot used & $\begin{array}{l}3 \\
3\end{array}$ & SOLUBK( 5) \\
\hline & 3 & \\
\hline - & 3 & DCNUCC ( 8) \\
\hline & 3 & DCNUCS( 8) \\
\hline $.126 E-04$ & 3 & ALEACH $(8)$ \\
\hline used & 3 & SOLLBX ( 8) \\
\hline & 3 & \\
\hline & 3 & DCNUCC (11) \\
\hline & 3 & DCNUCS $(11)$ \\
\hline $.200 E-05$ & 3 & ALEACH $(11)$ \\
\hline used & 3 & SOLUBK (11) \\
\hline
\end{tabular}

$\begin{array}{ll}\text { Attachment } \frac{5}{\text { Originator: }} \frac{5 . \text { W. Clark }}{\text { M.W. Perrott }} & \text { Sheet No. } \underline{7} \text { of } \underline{27} \\ \text { Date } & \text { Date } \\ \text { Chk'd By } & \text { Rev. No. } \frac{0}{0100 \text { F-CA-V0303 }}\end{array}$




\section{ATTACHMENT 5}

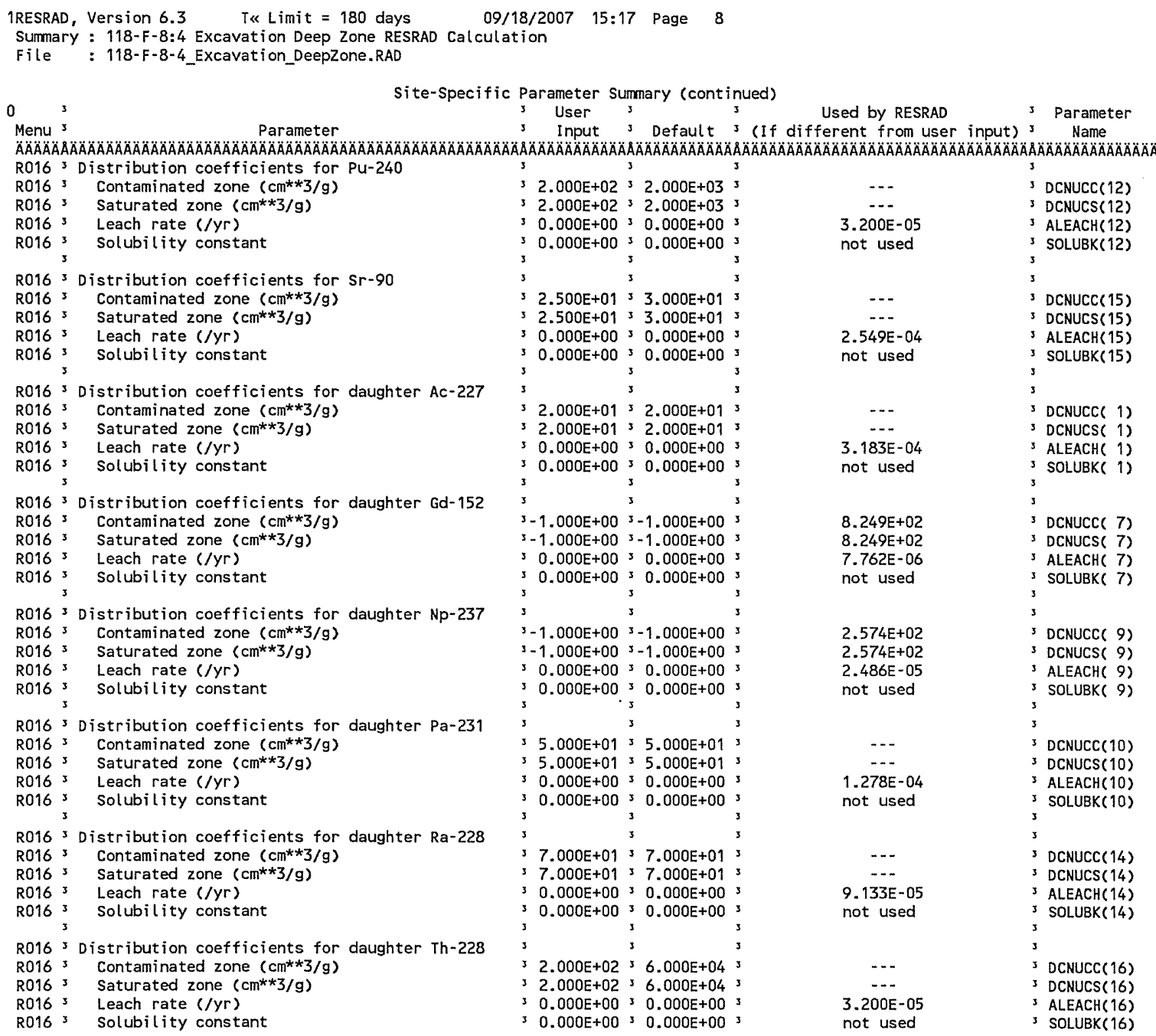

\begin{tabular}{|c|c|}
\hline chment & Sheet No. 8 of 27 \\
\hline Attacnment $\frac{5}{\mathrm{~W} \text {. Clark }}$ & Date \\
\hline M. W. Perrott & Date \\
\hline 0100F-CA-V0303 & Rev. No. 0 \\
\hline
\end{tabular}




\section{ATTACHMENT 5}

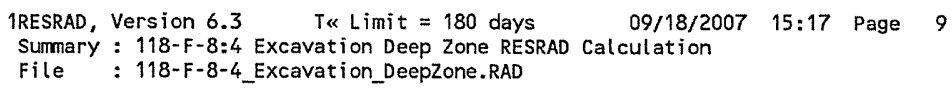

\begin{tabular}{|c|c|c|c|c|c|c|c|c|}
\hline \multirow{4}{*}{$\begin{array}{l}0 \\
\text { Menu } \\
A B A ̈ A ̈\end{array}$} & \multicolumn{8}{|c|}{ Parameter Summary (continued) } \\
\hline & 3 & User & 3 & & s & Used by RESRAD & 3 & Parameter \\
\hline & Parameter & Input & 3 & Default & I $(I f$ & different from user input) & 3 & Name \\
\hline & 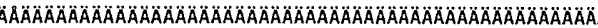 & $\triangle A ̈ A ั A ̈ A ̈ A ̈ A ̈ A ̈ A ̈ A ̈ A ̈ A ̈$ & & $\triangle A \cap A ̈ A O A O A O A ̈ A ̈$ & & 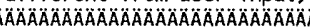 & & 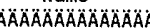 \\
\hline R016 & 3 Distribution coefficients for daughter Th-229 & 3 & s & & 3 & & 3 & \\
\hline R016 & Contaminated zone $(\mathrm{cm} * * 3 / \mathrm{g})$ & $32.000 E+02$ & $2^{3}$ & $6.000 E+04$ & 3 & ... & s & DCNUCC (17) \\
\hline R016 & Saturated zone $\left(\mathrm{cm}^{* * 3 / \mathrm{g})}\right.$ & $32.000 E+02$ & ? & $6.000 \mathrm{E}+04$ & 3 & ... & 3 & DCNUCS (17) \\
\hline R016 & Leach rate $(/ y r)$ & $30.000 \mathrm{E}+00$ & 3 & $0.000 E+00$ & 3 & $3.200 E-05$ & 3 & ALEACH $(17)$ \\
\hline R016 & Solubility constant & $30.000 E+00$ & 3 & $0.000 \mathrm{E}+00$ & 3 & not used & s & SOLUBK(17) \\
\hline & 3 & 3 & 3 & & 3 & & 3 & \\
\hline R016 & Distribution coefficients for daughter Th-232 & $s$ & 3 & & $s$ & & 3 & \\
\hline R016 & 3 Contaminated zone $(\mathrm{cm} * * 3 / \mathrm{g})$ & $32.000 E+02$ & $2^{3}$ & $6.000 E+04$ & s & ... & 3 & DCNUCC (18) \\
\hline R016 & Saturated zone $\left(\mathrm{cm}^{* * 3 / \mathrm{g})}\right.$ & $32.000 E+02$ & $2^{3}$ & $6.000 E+04$ & 3 & ... & 3 & DCNUCS(18) \\
\hline R016 & Leach rate (/yr) & $30.000 E+00$ & 3 & $0.000 E+00$ & 3 & $3.200 E-05$ & 3 & ALEACH (18) \\
\hline R016 & Solubility constant & $30.000 E+00$ & 3 & $0.000 E+00$ & 3 & not used & 3 & SOLUBK(18) \\
\hline & 3 & 3 & 3 & & 3 & & s & \\
\hline R016 & Distribution coefficients for daughter U-233 & 3 & s & & $\mathbf{3}$ & & 3 & \\
\hline R016 & 3 Contaminated zone $\left(\mathrm{cm}^{* * 3 / \mathrm{g}}\right)$ & $32.000 E+00$ & 3 & $5.000 E+01$ & 3 & $\ldots$ & 3 & DCNUCC (19) \\
\hline R016 & Saturated zone $\left(\mathrm{cm}^{* * 3 / \mathrm{g})}\right.$ & $=2.000 E+00$ & 3 & $5.000 E+01$ & 3 & ... & 3 & DCNUCS (19) \\
\hline R016 & Leach rate (/yr) & $=0.000 E+00$ & 3 & $0.000 E+00$ & 3 & $3.019 \mathrm{E}-03$ & 3 & ALEACH (19) \\
\hline R016 & Solubility constant & $=0.000 E+00$ & 3 & $0.000 E+00$ & 3 & not used & 3 & SOLUBK(19) \\
\hline & 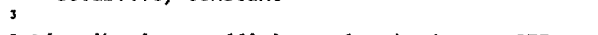 & 3 & 3 & & 3 & & 3 & \\
\hline R016 & ${ }^{3}$ Distribution coefficients for daughter U-235 & 3 & 3 & & 3 & & $\mathbf{3}$ & \\
\hline R016 & 3 Contaminated zone $\left(\mathrm{cm}^{* * 3 / \mathrm{g})}\right.$ & $32.000 E+00$ & 3 & $5.000 E+01$ & 3 & $\cdots$ & 3 & DCNUCC (20) \\
\hline R016 & Saturated zone $\left(\mathrm{cm}^{* * 3 / g}\right)$ & $32.000 E+00$ & 3 & $5.000 E+01$ & s & ... & 3 & DCNUCS(20) \\
\hline R016 & Leach rate $(/ y r)$ & $30.000 E+00$ & ב & $0.000 E+00$ & 3 & $3.019 \mathrm{E}-03$ & 3 & ALEACH(20) \\
\hline R016 & Solubility constant & $30.000 E+00$ & 3 & $0.000 E+00$ & 3 & not used & 3 & SOLUBK(20) \\
\hline & 3 & 3 & 3 & & s & & 3 & \\
\hline R016 & ${ }^{3}$ Distribution coefficients for daughter U-236 & s & 3 & & s & & 3 & \\
\hline R016 & Contaminated zone $\left(\mathrm{cm}^{* * 3 / \mathrm{g})}\right.$ & $32.000 E+00$ & 3 & $5.000 E+01$ & s & ... & $\mathbf{3}$ & DCNUCC(2१) \\
\hline R016 & Saturated zone $\left(\mathrm{cm}^{* * 3 / \mathrm{g})}\right.$ & $3.2 .000 E+00$ & 3 & $5.000 E+01$ & 3 & ... & $\mathrm{s}$ & DCNUCS $(21)$ \\
\hline R016 & Leach rate $(/ y r)$ & $30.000 E+00$ & 3 & $0.000 E+00$ & 3 & $3.019 \mathrm{E}-03$ & 3 & ALEACH(21) \\
\hline R016 & Solubility constant & $50.000 E+00$ & $b^{3}$ & $0.000 E+00$ & s & not used & 3 & SOLUBK(21) \\
\hline & & s & 3 & & 3 & & 3 & \\
\hline R017 & 3 Inhalation rate $\left(m^{* * 3 / y r}\right)$ & $37.300 E+03$ & $3^{3}$ & $8.400 E+03$ & 3 & $\cdots$ & s & INHALR \\
\hline $\mathrm{R} 017$ & 3 Mass loading for inhalation $\left(\mathrm{g} / \mathrm{m}^{\star *}\right)$ & $31.000 E-04$ & 43 & $1.000 \mathrm{E}-04$ & 3 & $\ldots$ & 3 & MLINH \\
\hline R017 & 3 Exposure duration & $33.000 E+01$ & $1^{3}$ & $3.000 E+01$ & 3 & -.. & s & ED \\
\hline R017 & 'shielding factor, inhalation & $34.000 E-01$ & 3 & $4.000 E-01$ & 3 & ... & 3 & SHF3 \\
\hline R017 & 3 Shielding factor, external gamma & $38.000 E-01$ & $1^{3}$ & $7.000 \mathrm{E}-01$ & 3 & ... & 3 & SHF1 \\
\hline R017 & "Fraction of time spent indoors & $36.000 E-01$ & 13 & $5.000 E-01$ & 3 & -.. & 3 & FIND \\
\hline R017 & $s$ Fraction of time spent outdoors (on site) & $32.000 E-01$ & $1^{3}$ & $2.500 E-01$ & 3 & ... & 3 & FOTD \\
\hline R017 & 3 Shape factor flag, external gamma & $=1.000 E+00$ & 3 & $1.000 E+00$ & s & $>0$ shows circular AREA. & s & FS \\
\hline
\end{tabular}

Attachment 5 Sheet No. 9 of $\underline{27}$ 


\section{ATTACHMENT 5}

1RESRAD, Version $6.3 \quad$ T\& Limit $=180$ days
Summary $: 118-F-8: 4$ Excavation Deep Zone RESRAD Calculation

File : 118-F-8-4_Excavation DeepZone.RAD

0

Site-Specific Parameter Summary (cont inued)

3 User 3 Used by RESRAD

3 Parameter

MenU

Parameter Name

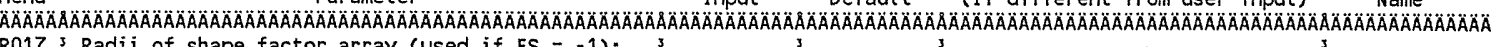
R017' Radí $i$ of shape factor array (used if $F S=-1$ ): 3

R017: Outer annular radius ( $m$ ), ring 1

$2017^{3}$ Outer annular radius (m), ring 2

$R 017^{3}$ Outer annular radius (m), ring 3:

$2017^{3}$ Outer annular radius (m), ring 4:

R017' Outer annular radius (m), ring 5 :

$2017^{3}$ Outer annular radius (m), ring 6:

R017 3 Outer annular radius (m), ring 7

$2017^{3}$ Outer annular radius (m), ring 8

$\mathrm{R}_{017^{3}}{ }^{3}$ Outer annular radius (m), ring 9

$\mathrm{R}_{017^{3}}{ }^{3}$ Outer annular radius (m), ring 10:

$R_{017^{3}}{ }^{3}$ Outer annular radius (m), ring 11

$\mathrm{R}^{017^{3}}$ Outer annular radius (m), ring 12:

R017 3 Fractions of annular areas within AREA:

$\mathrm{R}_{017^{3} \text { Ring }}^{3}$

R017 3 Ring 2

R017 ${ }^{3}$ Ring 3

R017 3 Ring 4

R017 3 Ring 5

R017 3 Ring 6

R017 3 Ring 7

R017's Ring 8

R017's Ring 9

R017 ' Ring 10

not used $35.000 E+01$

not used $37.071 \mathrm{E}+01$,

3 not used $30.000 E+00$ 3

not used $30.000 E+00$ s

3 not used $30.000 E+003$

3 not used $30.000 E+003$

3 not used $30.000 E+00$ s

3 not used $30.000 E+00$ s

not used $30.000 E+00$ 's

3 not used $30.000 E+00$ s

3 not used $30.000 \mathrm{E}+00^{3}$

not used $30.000 E+00$ '

3 not used $31.000 \mathrm{E}+00^{3}$

3 not used $32.732 E-013$

3 not used $30.000 E+00$ s

3 not used $30.000 E+00$ ?

not used $30.000 E+00$ ?

3 not used $30.000 E+00$ '

3 not used $30.000 E+00$ s

3 not used $30.000 E+00$ s

3 not used $s 0.000 E+00^{3}$

3 not used $s 0.000 E+003$

3 not used $30.000 \mathrm{E}+00$,

3 not used $30.000 \mathrm{E}+00$ s

R017: Ring 12

Fruits, vegetables and grain consumpt

R018 3 Milk consunpt

$\mathrm{R} 0183$ Meat and poultry consumption ( $\mathrm{kg} / \mathrm{yr}$ )

R018 3 Fish consumption ( $\mathrm{kg} / \mathrm{yr}$ )

R018 ' Other seafood consumption ( $\mathrm{kg} / \mathrm{yr}$ )

R018 3 Soil ingestion rate $(\mathrm{g} / \mathrm{yr})$

$R 018{ }^{3}$ Drinking water intake ( $\mathrm{L} / \mathrm{y} \mathrm{r}$ )

R018 ' Contamination fraction of drinking water

R018 3 Contamination fraction of household water

R018 3 Contamination fraction of livestock water

R018 3 Contamination fraction of irrigation water

R018 ' Contamination fraction of aquatic food

R018 3 Contamination fraction of plant food

R018 3 Contamination fraction of meat

R018 3 Contamination fraction of milk

3

R019 ' Livestock fodder intake for meat ( $\mathrm{kg} /$ day)

R019 3 Livestock fodder intake for milk ( $\mathrm{kg} /$ day)

R019 3 Livestock water intake for meat (L/day)

R019 3 Livestock water intake for milk (L/day)

R019 3 Livestock soil intake $(\mathrm{kg} /$ day)

$31.100 E+02 ; 1.600 E+02 ;$

$32.700 E+00 \div 1.400 E+013$

$31.000 \mathrm{E}+0239.200 \mathrm{E}+01^{3}$

$3.600 \mathrm{E}+01,6.300 \mathrm{E}+01,3$

3.60E+01 $6.300 \mathrm{E}+01^{3}$

$\mathrm{OE}+00^{3}$

$39.000 \mathrm{E}-0139.000 \mathrm{E}-01^{3}$

$37.300 \mathrm{E}+0133.650 \mathrm{E}+013$

$37.300 \mathrm{E}+0235.100 \mathrm{E}+023$

$31.000 E+00 \div 1.000 E+003$

not used $31.000 E+00$ 's

$31.000 \mathrm{E}+0031.000 \mathrm{E}+003$

$31.000 E+00 \div 1.000 E+003$

$35.000 E-0135.000 E-01$ s

$3-1$

$3-1$

3

$6.800 E+0136.800 E+01$

$5.500 E+0135.500 E+01^{3}$

$35.000 \mathrm{E}+0135.000 \mathrm{E}+01$ '

$31.600 \mathrm{E}+0231.600 \mathrm{E}+02$

5. 5.000E-013 5.000E-013

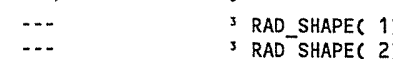

3 RAD_SHAPE( 2)

-..

-.- 3 RAD SHAPE( 5)

(..- 3 RAD SHAPE( 6)

$\begin{array}{ll}-. . & 3 \\ \ldots & \text { RAD_SHAPE( } 7) \\ -.- & \text { RAD } \operatorname{SHAPE}(8)\end{array}$

-.. 3 RAD SHAPE( 9)

-.. 3 RAD SHAPE(10)

... 3 RAD_SHAPE(11)

-.. 3 RAD SHAPE(12)

$\begin{array}{ll}-. & 3 \text { FRACAC (1) } \\ \ldots-. & 3\end{array}$

$\begin{array}{ll}\ldots & 3 \\ \ldots & 3 \text { FRACA( 2) }\end{array}$

3 FRACA( 4)

-.- 1 FRACAC 5)

-.- 3 FRACA( 6)

-.. 3 FRACA( 7)

3 FRACA( 8)

....

-

..-

Chen

-.. 3 DIET(1)

… 3 DIET(2)

‥- $3 \operatorname{DIET}(3)$

-.- 3 DIET(4)

-.. 3 DIET(5)

-.. 3 DIET(6)

... 3 SOIL

-..

-..

FHHW

-..

$0.500 E+00 \quad 3$ FPLANT

$0.817 E-01$ FMEAT

$0.817 E-01 \quad 3$ FMILK

-.. 3 LFI5

..- 3 LFI6

-.- 3 LWI5

$\begin{array}{ll}\ldots & 3 \text { LWI6 } \\ \ldots & \text { s LSI }\end{array}$
Attachment 5

Originator: S.W. Clark

Chk'd By M. W. Perrott

Calc. No. 0100F-CA-V0303
Sheet No. 10 of $\underline{27}$

Date

Date

Rev. No. 0 


\section{ATTACHMENT 5}

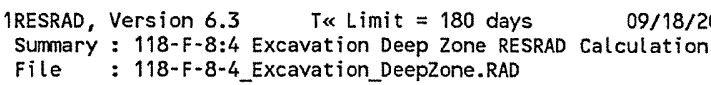

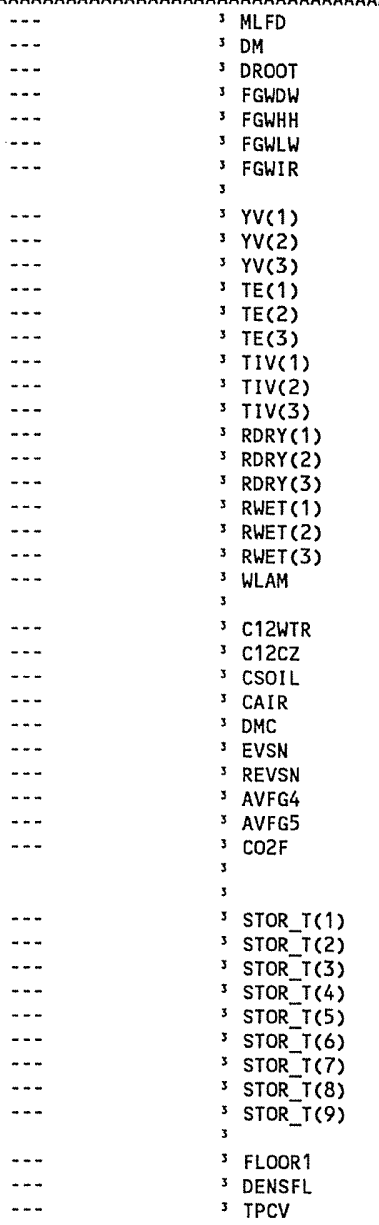




\section{ATTACHMENT 5}

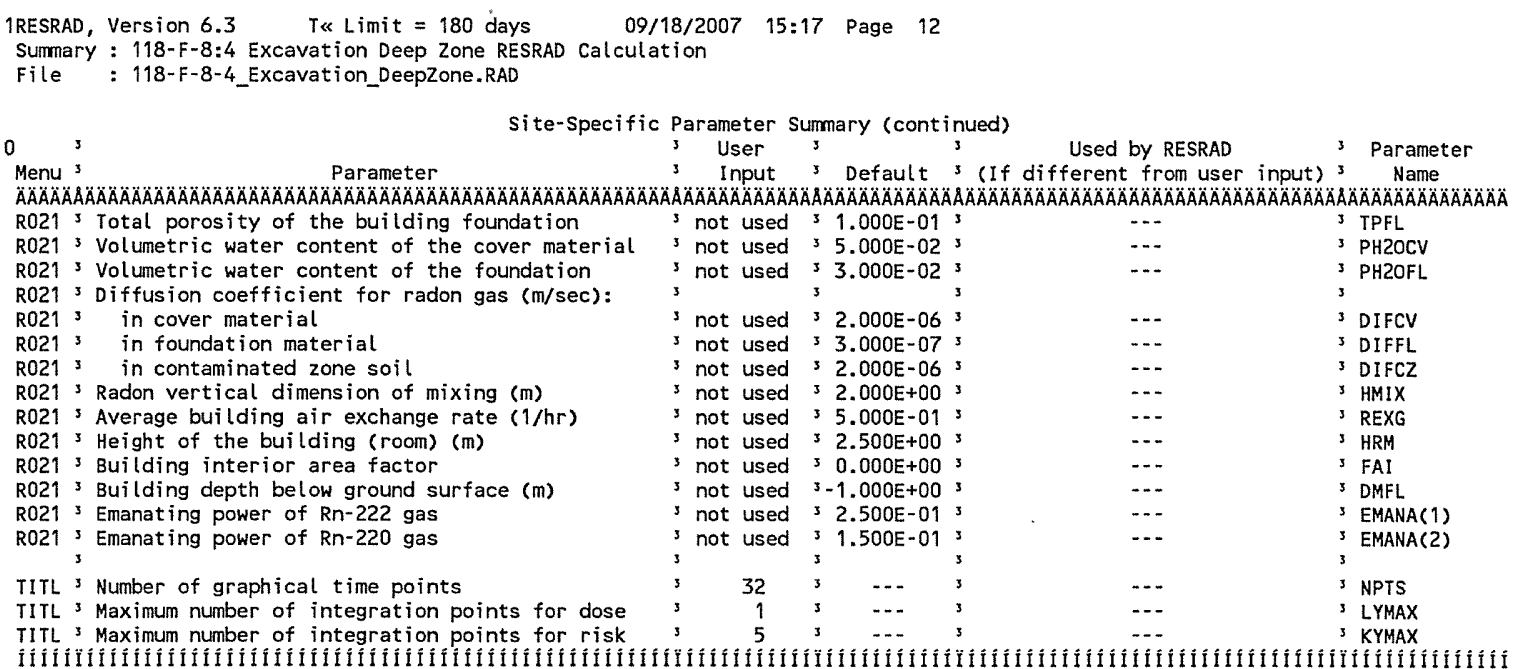

Summary of Pathway Selections

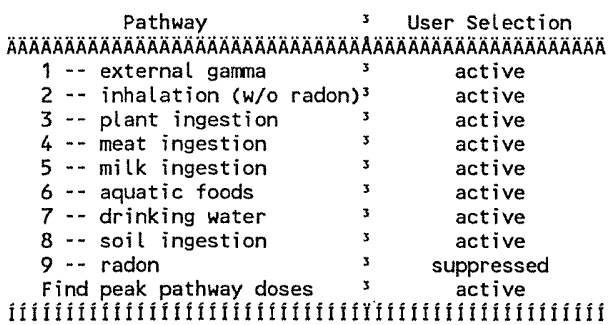

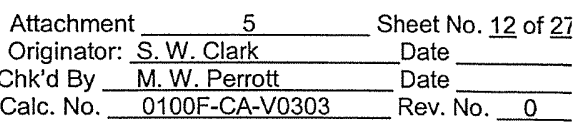




\section{ATTACHMENT 5}

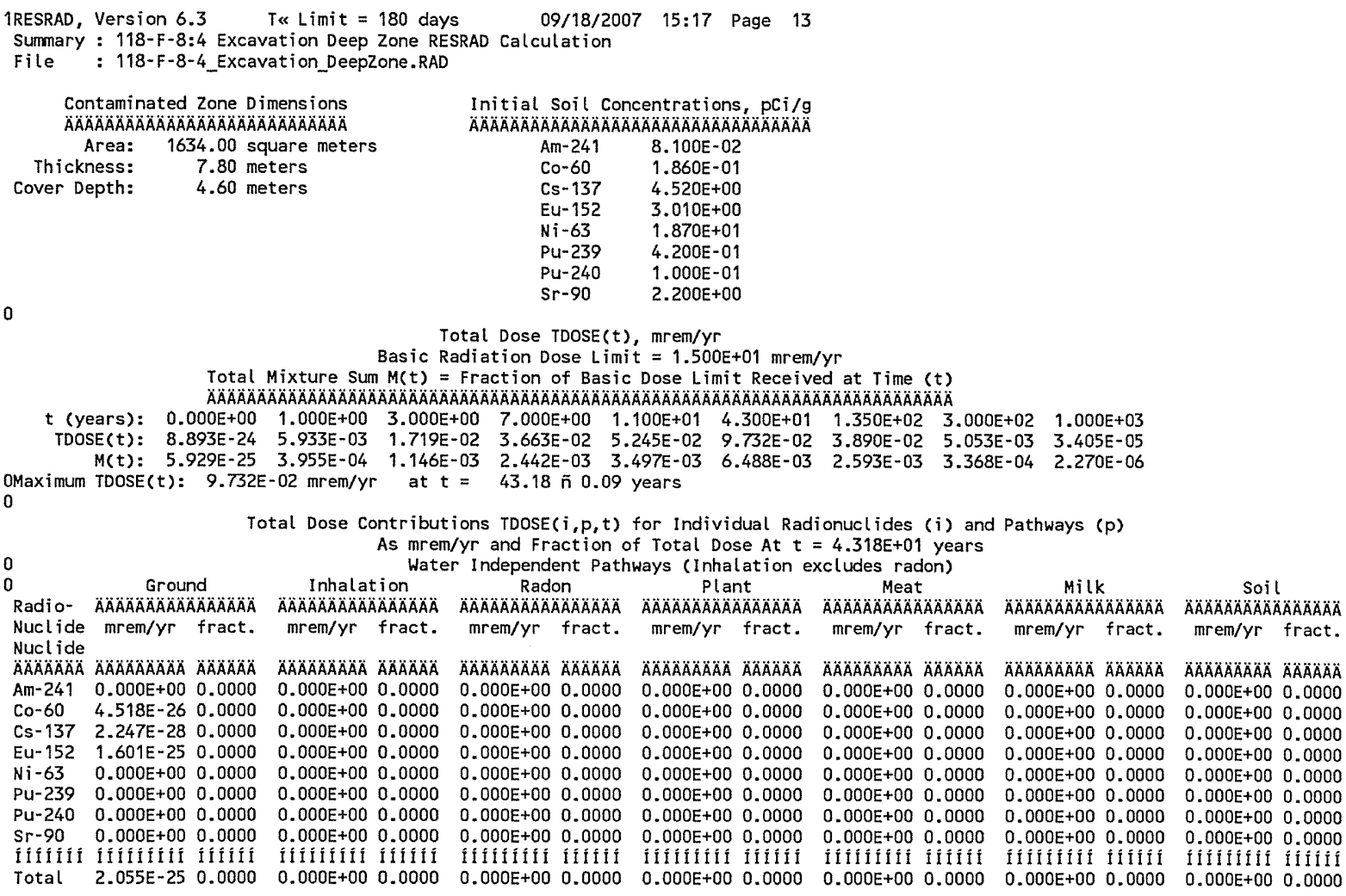

Total Dose TDOSE $(t)$, mrem/yr

Basic Radiation Dose Limit $=1.500 E+01 \mathrm{mrem} / \mathrm{yr}$

Total Mixture Sum $M(t)=$ Fraction of Basic Dose Limit Received at $T$ ime $(t)$

$t$ (years): $\begin{array}{llllllllll}0.000 E+00 & 1.000 E+00 & 3.000 E+00 & 7.000 E+00 & 1.100 E+01 & 4.300 E+01 & 1.350 E+02 & 3.000 E+02 & 1.000 E+03\end{array}$

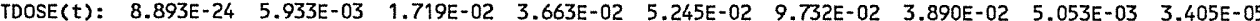

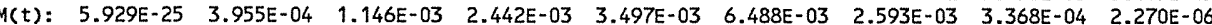
OMaximum TDOSE $(t): 9.732 \mathrm{E}-02 \mathrm{mrem} / \mathrm{yr}$ at $t=43.18 \tilde{\mathrm{n}} 0.09$ years

Total Dose Contributions $\operatorname{TDOSE}(i, p, t)$ for Individual Radionuclides (i) and Pathways $(p)$

As $\mathrm{mrem} / \mathrm{yr}$ and Fraction of Total Dose At $t=4.318 \mathrm{E}+01$ years

Water Independent Pathways (Inhalation excludes radion)

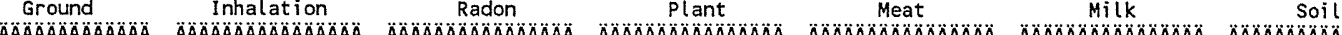

Nuctide mrem/yr fract. mrem/yr fract. mrem/yr fract, mrem/yr fract.

Nuclide

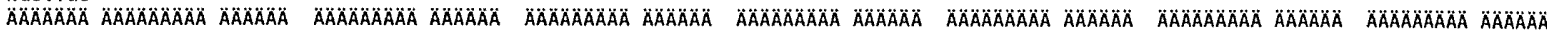

$\begin{array}{llllllllllllllll}A m-241 & 0.000 E+00 & 0.0000 & 0.000 E+00 & 0.0000 & 0.000 E+00 & 0.0000 & 0.000 E+00 & 0.0000 & 0.000 E+00 & 0.0000 & 0.000 E+00 & 0.0000 & 0.000 E+00 & 0.0000\end{array}$

$\begin{array}{llllllllllllllllll}C 0-60 & 4.518 E-26 & 0.0000 & 0.000 E+00 & 0.0000 & 0.000 E+00 & 0.0000 & 0.000 E+00 & 0.0000 & 0.000 E+00 & 0.0000 & 0.000 E+00 & 0.0000 & 0.000 E+00 & 0.0000\end{array}$

$\begin{array}{llllllllllllllll}C s-137 & 2.247 E-28 & 0.0000 & 0.000 E+00 & 0.0000 & 0.000 E+00 & 0.0000 & 0.000 E+00 & 0.0000 & 0.000 E+00 & 0.0000 & 0.000 E+00 & 0.0000 & 0.000 E+00 & 0.0000\end{array}$ $\begin{array}{llllllllllllllllll}\mathrm{Eu}-152 & 1.601 \mathrm{E}-25 & 0.0000 & 0.000 \mathrm{E}+00 & 0.0000 & 0.000 \mathrm{E}+00 & 0.0000 & 0.000 \mathrm{E}+00 & 0.0000 & 0.000 \mathrm{E}+00 & 0.0000 & 0.000 \mathrm{E}+00 & 0.0000 & 0.000 \mathrm{E}+00 & 0.0000\end{array}$

$\begin{array}{lllllllllllllllllll}\mathrm{Ni}-63 & 0.000 \mathrm{E}+00 & 0.0000 & 0.000 \mathrm{E}+00 & 0.0000 & 0.000 \mathrm{E}+00 & 0.0000 & 0.000 \mathrm{E}+00 & 0.0000 & 0.000 \mathrm{E}+00 & 0.0000 & 0.000 \mathrm{E}+00 & 0.0000 & 0.000 \mathrm{E}+00 & 0.0000\end{array}$

PU-239 $0.000 E+00 \quad 0.0000 \quad 0.000 E+00 \quad 0.0000 \quad 0.000 E+00 \quad 0.0000 \quad 0.000 E+00 \quad 0.0000 \quad 0.000 E+00 \quad 0.0000 \quad 0.000 E+000.0000 \quad 0.000 E+00 \quad 0.0000$

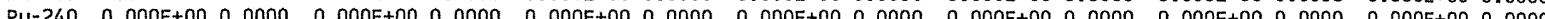

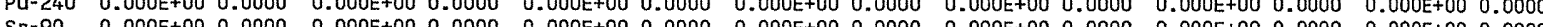

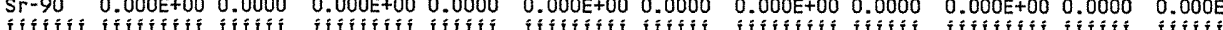

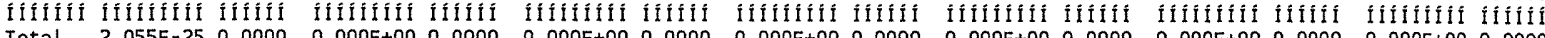

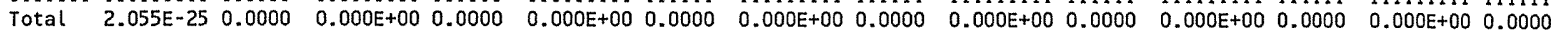

$\begin{array}{ll}\text { Attachment } \frac{5}{\text { S.W. Clark }} & \text { Sheet No. } 13 \text { of } 27 \\ \text { Originator: } & \text { Date } \\ \text { Chk'd By } & \text { Date } \\ \text { M.W. Perrott } & \text { Rev. No. } \quad 0 \\ \text { Calc. No. } & \text { O100F-CA-V0303 }\end{array}$




\section{ATTACHMENT 5}

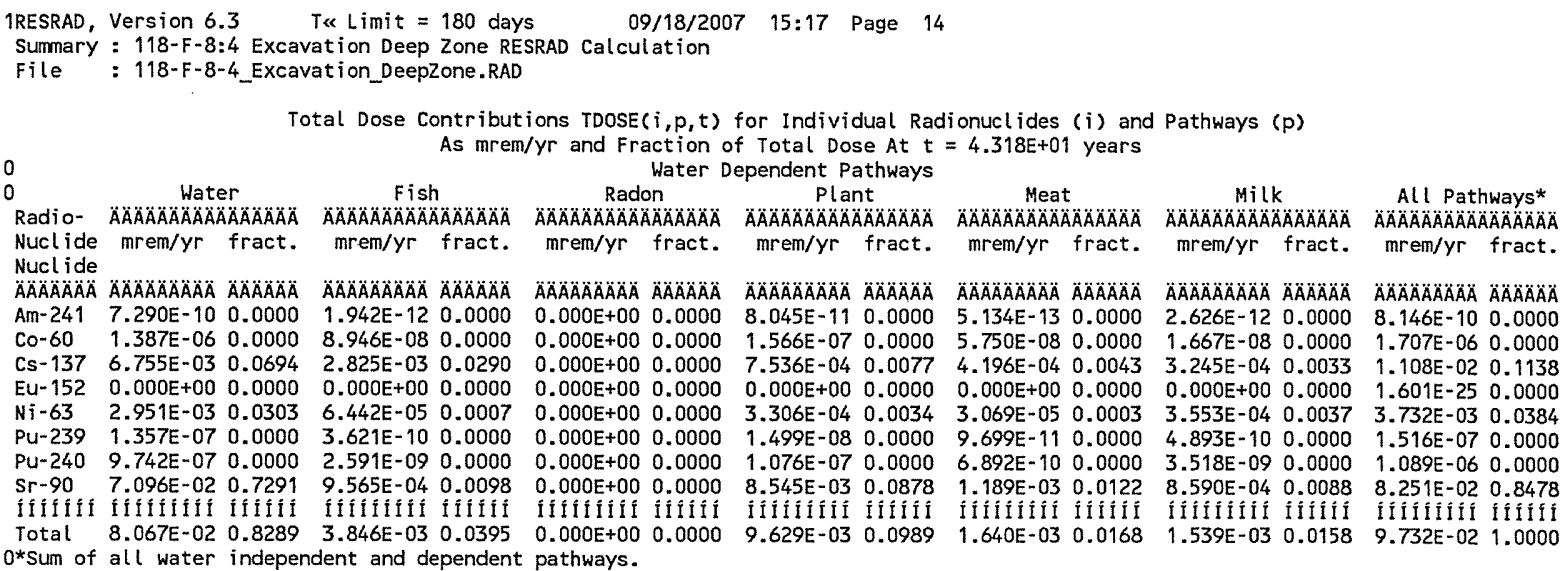

\begin{tabular}{|c|c|}
\hline Attachment & Sheet No. 14 of 27 \\
\hline Originator: S.W. Clark & Date \\
\hline M. W. Perrott & Date \\
\hline $0100 \mathrm{~F}-\mathrm{CA}-\mathrm{V} 0303$ & Rev. No. $\quad 0$ \\
\hline
\end{tabular}




\section{ATTACHMENT 5}

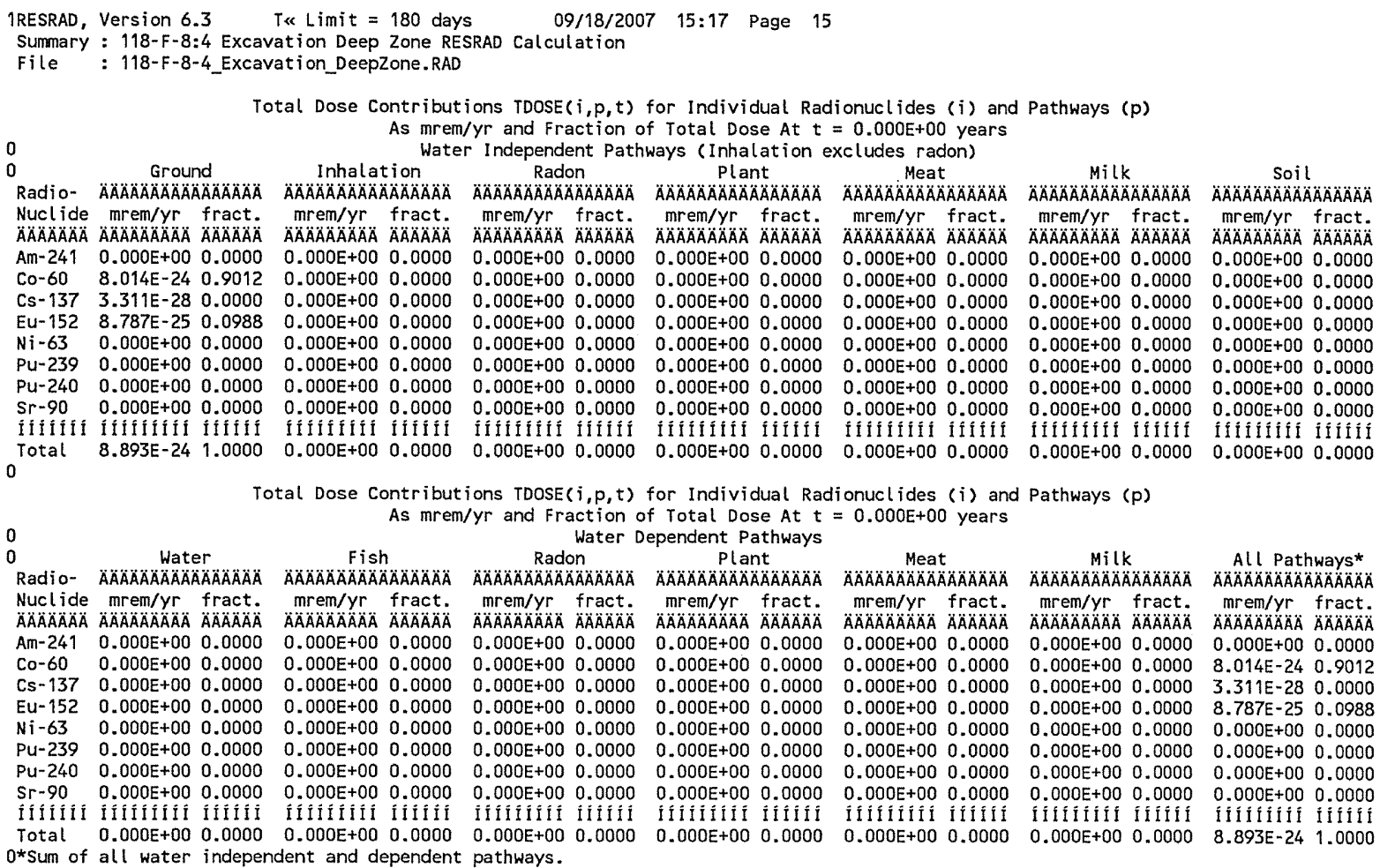

\begin{tabular}{|c|c|}
\hline Attachment & Sheet No. 15 of 27 \\
\hline Originator: S.W. Clark & Date \\
\hline M. W. Perrott & Date \\
\hline 0100F-CA-V0303 & Rev. No. \\
\hline
\end{tabular}




\section{ATTACHMENT 5}

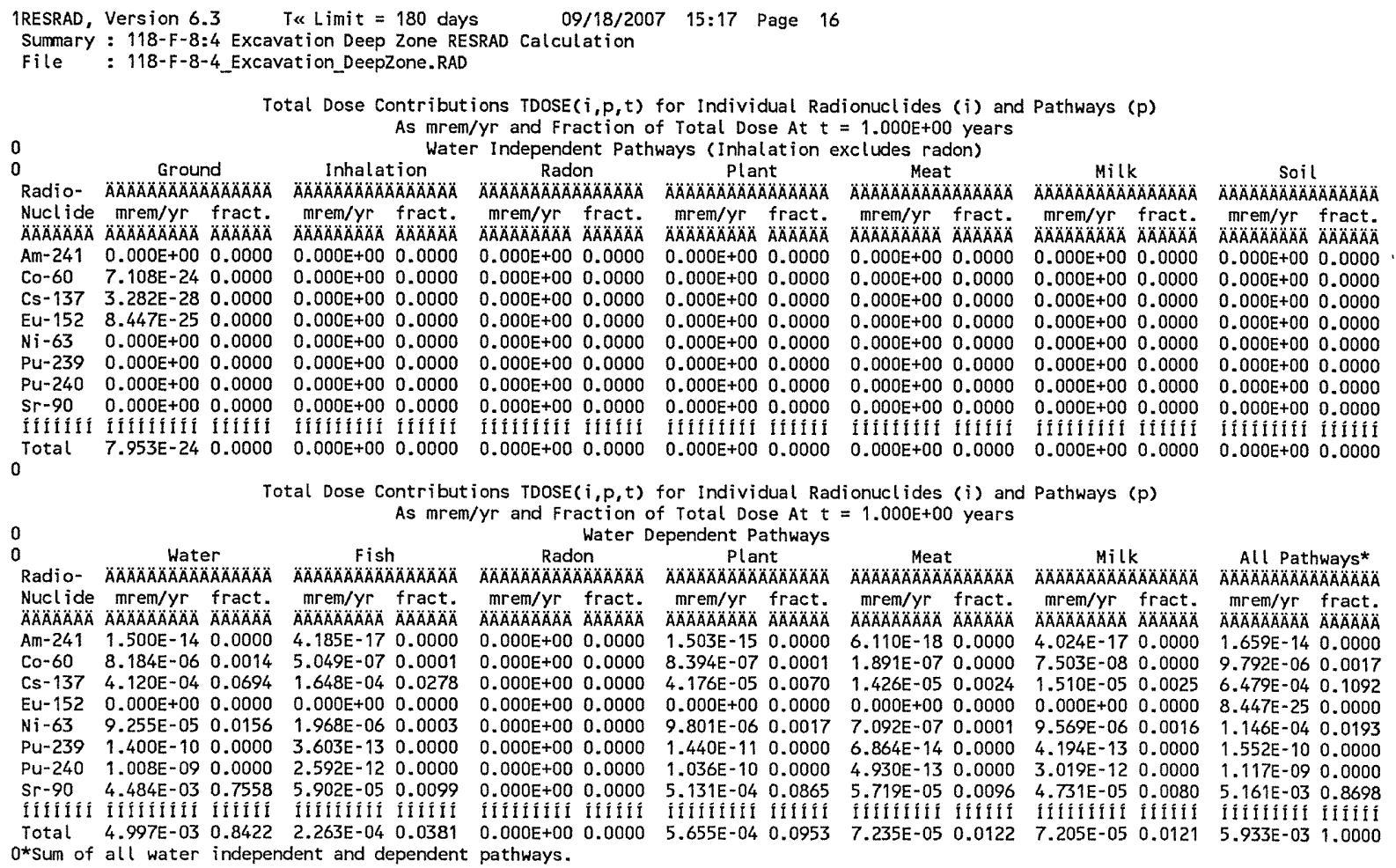

\begin{tabular}{|c|c|}
\hline Attachment & Sheet No. 16 of 27 \\
\hline Originator: S.W. Clark & Date \\
\hline M. W. Perrott & Date \\
\hline $0100 \mathrm{~F}-\mathrm{CA}-\mathrm{V} 0303$ & Rev. No. \\
\hline
\end{tabular}




\section{ATTACHMENT 5}

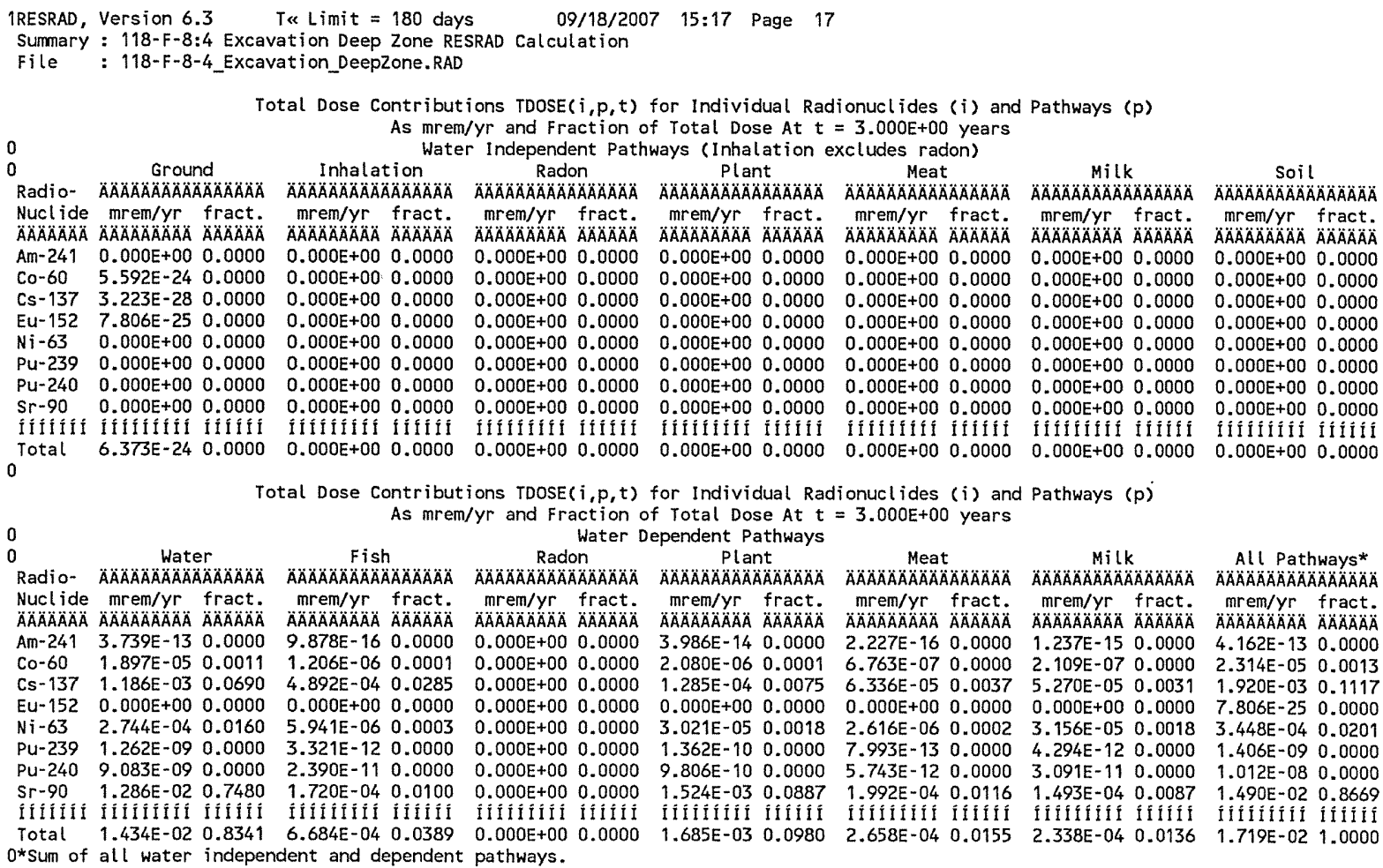

\begin{tabular}{|c|c|}
\hline Attachment & Sheet No. 17 of 27 \\
\hline Originator: S.W. Clark & Date \\
\hline M. W. Perrott & Date \\
\hline 0100F-CA-V0303 & Rev No \\
\hline
\end{tabular}




\section{ATTACHMENT 5}

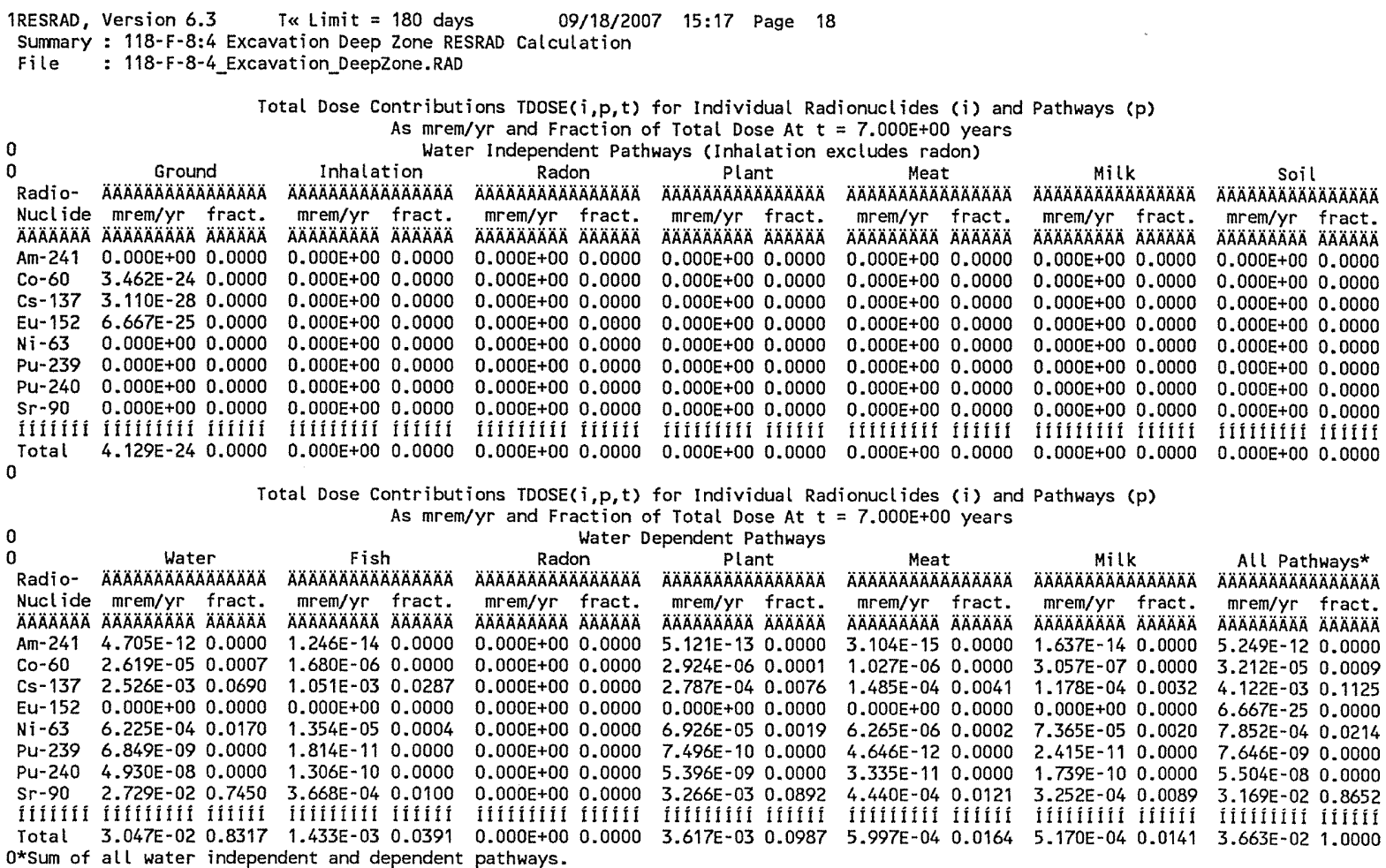

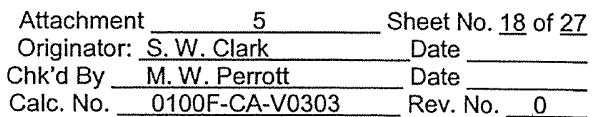




\section{ATTACHMENT 5}

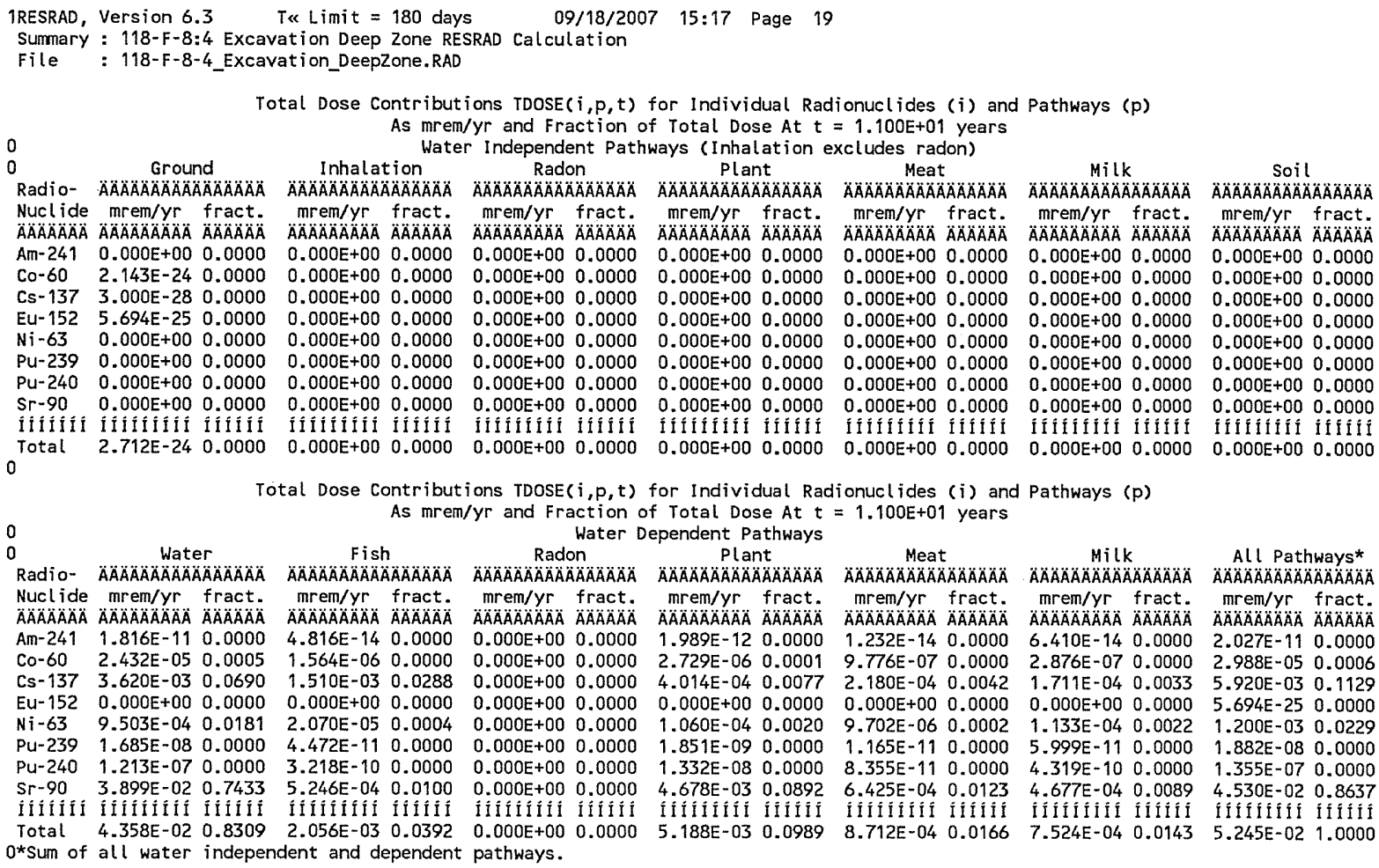

Total Dose Contributions TDOSE $(i, p, t)$ for Individual Radionuclides (i) and Pathways (p) As $\mathrm{mrem} / \mathrm{yr}$ and Fraction of Total Dose At $t=1.100 \mathrm{E}+01$ years

\begin{tabular}{|c|c|c|}
\hline \multirow{2}{*}{\multicolumn{2}{|c|}{ Attachment $\quad 5$}} & Sheet No. 19 of 27 \\
\hline & & Date \\
\hline Chk'd B & M. W. Perrott & Date \\
\hline Calc. No. & $0100 F-C A-V 0303$ & Rev. No. \\
\hline
\end{tabular}




\section{ATTACHMENT 5}

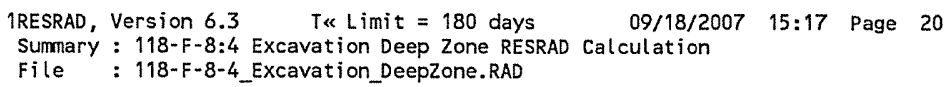

Total Dose Contributions TDOSE $(i, p, t)$ for Individual Radionuclides (i) and Pathways ( $p$ )

As $\mathrm{mrem} / \mathrm{yr}$ and Fraction of Total Dose At $t=4.300 \mathrm{E}+01$ years

0 Water Independent Pathways (Inhalation excludes radon)

0 Ground Inhalation water Independent Pathways (Inhalation excludes radon) Nuclide Ä̈ÄÄA

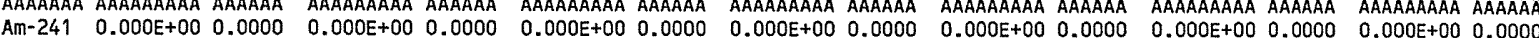
$\begin{array}{lllllllllllllllll}C 0-60 & 4.615 E-26 & 0.0000 & 0.000 E+00 & 0.0000 & 0.000 E+00 & 0.0000 & 0.000 E+00 & 0.0000 & 0.000 E+00 & 0.0000 & 0.000 E+00 & 0.0000 & 0.000 E+00 & 0.0000\end{array}$ Cs-137 2.250E-28 $0.0000 \quad 0.000 E+000.0000 \quad 0.000 E+000.00000 .000 E+000.00000 .000 E+000.0000 \quad 0.000 E+000.0000 \quad 0.0005+000.0000$

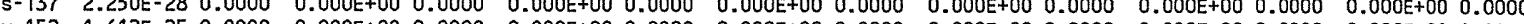

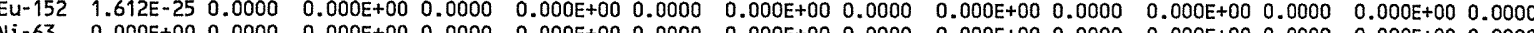
$\begin{array}{llllllllllllllll}N i-63 & 0.000 E+00 & 0.0000 & 0.000 E+00 & 0.0000 & 0.000 E+00 & 0.0000 & 0.000 E+00 & 0.0000 & 0.000 E+00 & 0.0000 & 0.000 E+00 & 0.0000 & 0.000 E+00 & 0.0000\end{array}$ $\begin{array}{llllllllllllllllllll}\text { Pu-239 } & 0.000 E+00 & 0.0000 & 0.000 E+00 & 0.0000 & 0.000 E+00 & 0.0000 & 0.000 E+00 & 0.0000 & 0.000 E+00 & 0.0000 & 0.000 E+00 & 0.0000 & 0.000 E+00 & 0.0000\end{array}$ $\begin{array}{llllllllllllllllll}\text { Pu-240 } & 0.000 E+00 & 0.0000 & 0.000 E+00 & 0.0000 & 0.000 E+00 & 0.0000 & 0.000 E+00 & 0.0000 & 0.000 E+00 & 0.0000 & 0.000 E+00 & 0.0000 & 0.000 E+00 & 0.0000\end{array}$ $\begin{array}{lllllllllllllllll}S r-90 & 0.000 E+00 & 0.0000 & 0.000 E+00 & 0.0000 & 0.000 E+00 & 0.0000 & 0.000 E+00 & 0.0000 & 0.000 E+00 & 0.0000 & 0.000 E+00 & 0.0000 & 0.000 E+00 & 0.0000\end{array}$

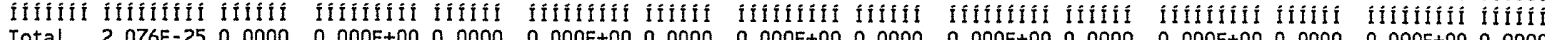

Total Dose Contributions $\operatorname{TDOSE}(i, p, t)$ for Individual Radionuclides (i) and Pathways ( $p$ ) As $\mathrm{mrem} / \mathrm{yr}$ and Fraction of Total Dose At $t=4.300 \mathrm{E}+01$ years

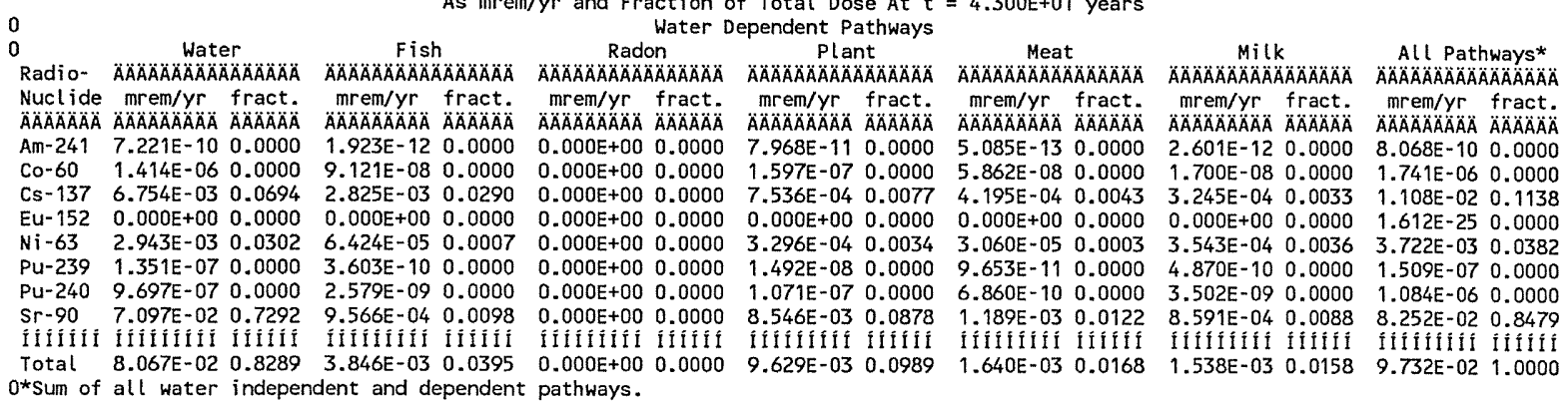

0 *Sum of all water independent and dependent pathways.

\begin{tabular}{|c|c|}
\hline Attachment & Sheet No. 20 of 27 \\
\hline Originator: S.W. Clark & Date \\
\hline Chk'd By M. W. Perrott & Date \\
\hline Calc. No. $\quad 0100 \mathrm{~F}-\mathrm{CA}-\mathrm{V} 0303$ & Rev. No. 0 \\
\hline
\end{tabular}




\section{ATTACHMENT 5}

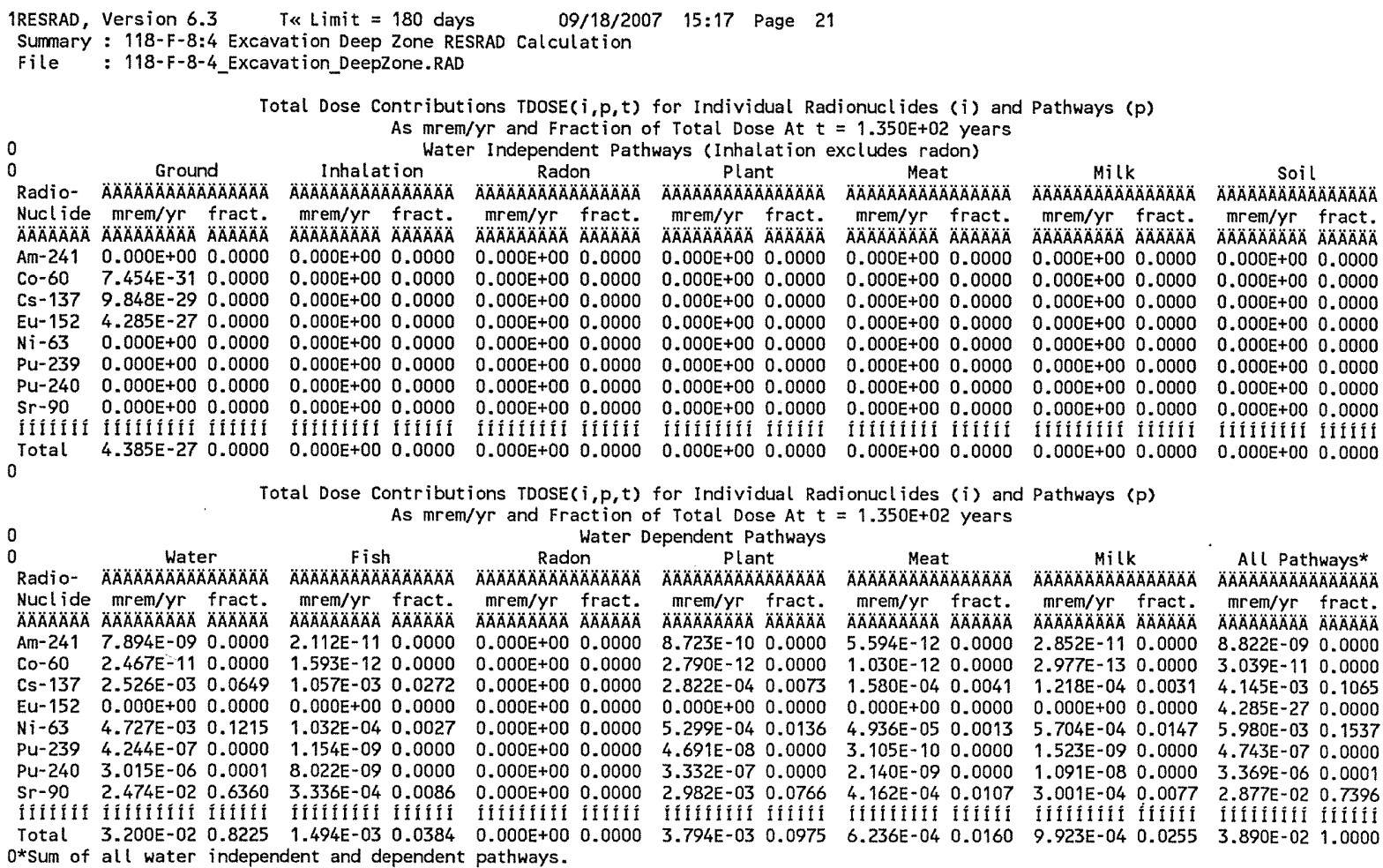

Total Dose Contributions $\operatorname{TDOSE}(i, p, t)$ for Individual Radionuclides $(i)$ and Pathways ( $p$ ) As $\mathrm{mrem} / \mathrm{yr}$ and Fraction of Total Dose At $t=1.350 \mathrm{E}+02$ years

0

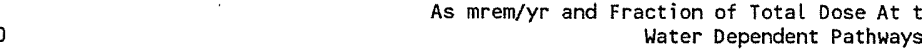

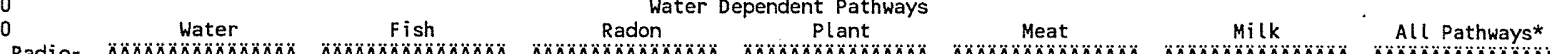

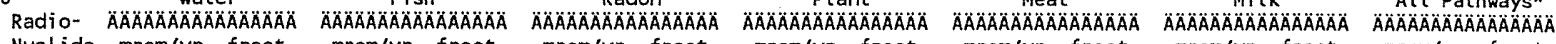
Nuclide mrem/yr fract. mrem/yr fract. mrem/yr fract. mrem/yr fract. mrem/yr fract. mrem/yr fract. mrem/yr fract.

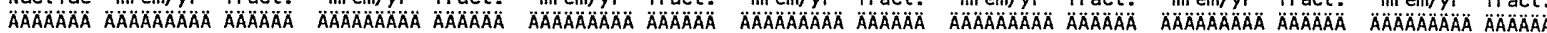
$\begin{array}{lllllllllllllllll}A m-241 & 7.894 E-09 & 0.0000 & 2.112 E-11 & 0.0000 & 0.000 E+00 & 0.0000 & 8.723 E-10 & 0.0000 & 5.594 E-12 & 0.0000 & 2.852 E-11 & 0.0000 & 8.822 E-09 & 0.0000\end{array}$ $\begin{array}{lllllllllllllll}\text { Co-60 } & 2.467 E-11 & 0.0000 & 1.593 E-12 & 0.0000 & 0.000 E+00 & 0.0000 & 2.790 E-12 & 0.0000 & 1.030 E-12 & 0.0000 & 2.977 E-13 & 0.0000 & 3.039 E-11 & 0.0000\end{array}$

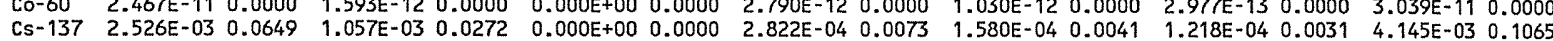

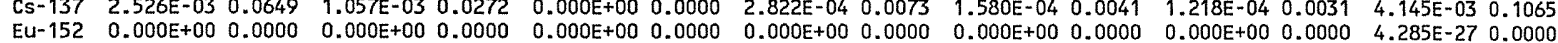

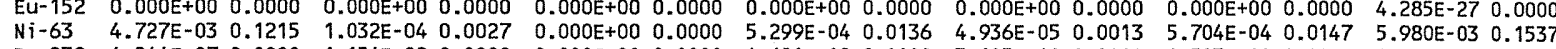
Pu-239 4.244E-07 $0.0000 \quad 1.154 E-09 \quad 0.0000 \quad 0.000 E+00 \quad 0.0000 \quad 4.691 E-08 \quad 0.0000 \quad 3.105 E-10 \quad 0.0000 \quad 1.523 E-09 \quad 0.0000 \quad 4.743 E-07 \quad 0.0000$ $\begin{array}{lllllllllllllllll}\text { Pu-240 } & 3.015 E-06 & 0.0001 & 8.022 E-09 & 0.0000 & 0.000 E+00 & 0.0000 & 3.332 E-07 & 0.0000 & 2.140 E-09 & 0.0000 & 1.091 E-08 & 0.0000 & 3.369 E-06 & 0.0001\end{array}$ $\begin{array}{lllllllllllllll}\mathrm{Sr}-90 & 2.474 \mathrm{E}-02 & 0.6360 & 3.336 \mathrm{E}-04 & 0.0086 & 0.000 \mathrm{E}+00 & 0.0000 & 2.982 \mathrm{E}-03 & 0.0766 & 4.162 \mathrm{E}-04 & 0.0107 & 3.001 E-04 & 0.0077 & 2.877 \mathrm{E}-02 & 0.7396\end{array}$

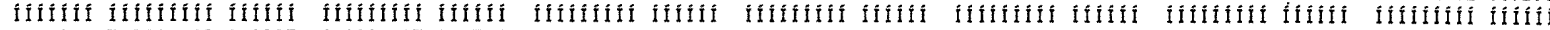
$\begin{array}{llllllllllllllllll}\text { Total } & 3.200 E-02 & 0.8225 & 1.494 E-03 & 0.0384 & 0.000 E+00 & 0.0000 & 3.794 E-03 & 0.0975 & 6.236 E-04 & 0.0160 & 9.923 E-04 & 0.0255 & 3.890 E-02 & 1.0000\end{array}$ 0 * Sum of all water independent and dependent pathways.

\begin{tabular}{|c|c|}
\hline Attachment & Sheet No. 21 of 27 \\
\hline Originator: S.W. Clark & Date \\
\hline Chk'd By M.W. Perrott & Date \\
\hline Calc. No. $\quad 0100 F-C A-V 0303$ & Rev. No. \\
\hline
\end{tabular}




\section{ATTACHMENT 5}

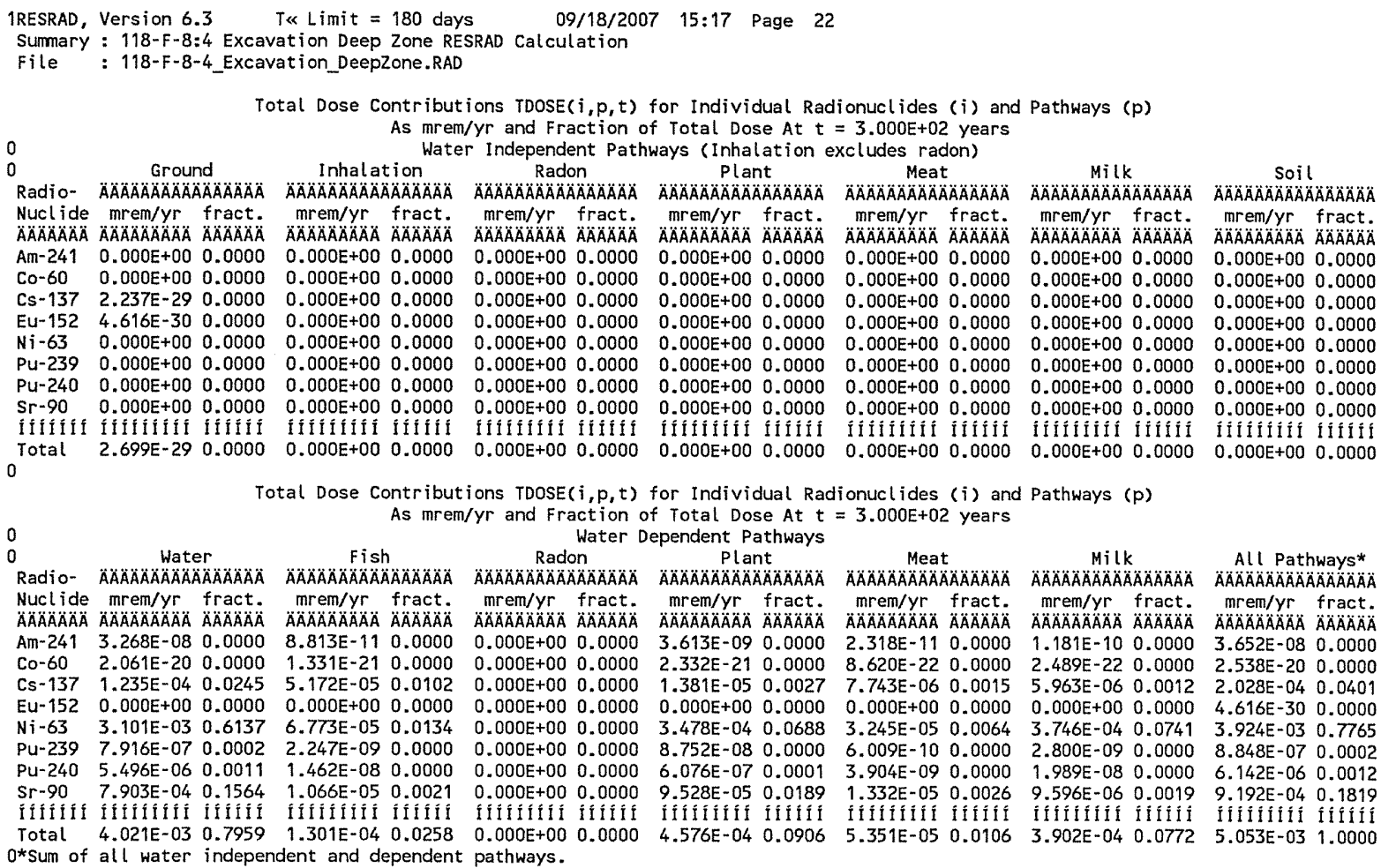

Attachment $\frac{5}{\text { Originator: } \frac{5}{\text { S.W. Clark }} \text { Sheet No. } 22 \text { of } 27}$
Chk'd By $\frac{\text { Date }}{\text { M. W. Perrott }}$ Date
Calc. No. $\frac{}{0100 \text { F-CA-V0303 }}$ Rev. No. $\underline{0}$




\section{ATTACHMENT 5}

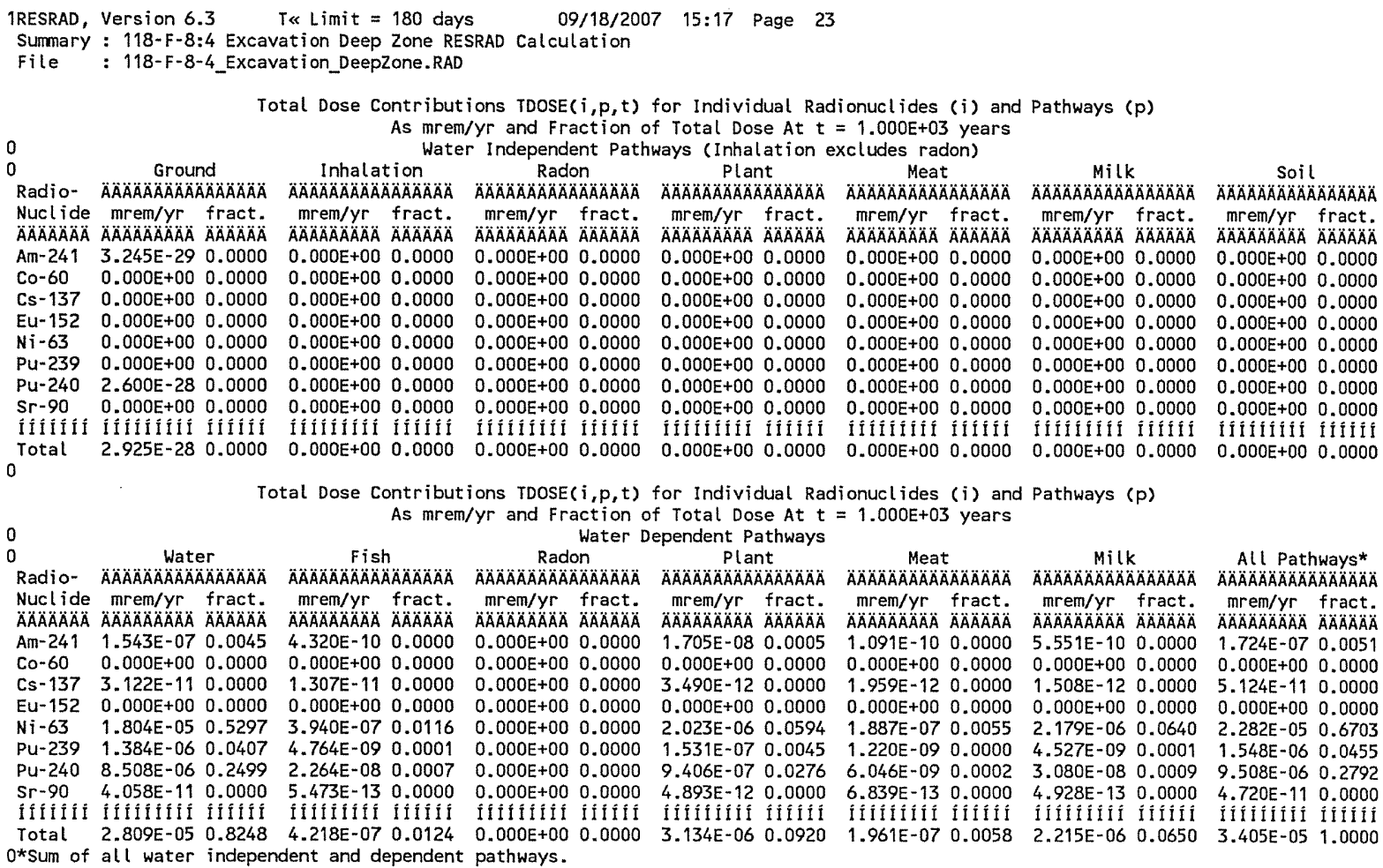

MilK

Soil

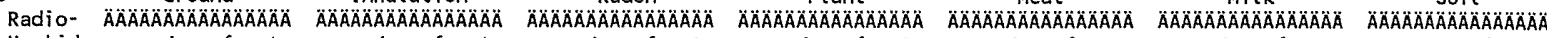
Nuclide $m r e m / y r$ fract. mrem/yr fract. mrem/yr fract. mrem/yr fract. mrem/yr fract. mrem/yr fract. mrem/yr fract.

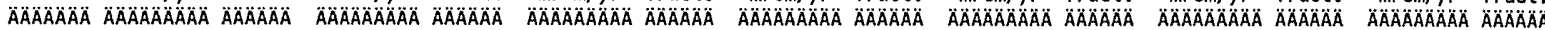

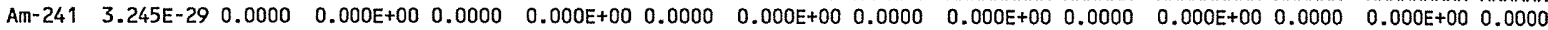
$\begin{array}{lllllllllllllllllllllll}\text { Co-60 } & 0.000 E+00 & 0.0000 & 0.000 E+00 & 0.0000 & 0.000 E+00 & 0.0000 & 0.000 E+00 & 0.0000 & 0.000 E+00 & 0.0000 & 0.000 E+00 & 0.0000 & 0.000 E+00 & 0.0000\end{array}$ $\begin{array}{llllllllllllllllllllllll}\text { Cs }-137 & 0.000 E+00 & 0.0000 & 0.000 E+00 & 0.0000 & 0.000 E+00 & 0.0000 & 0.000 E+00 & 0.0000 & 0.000 E+00 & 0.0000 & 0.000 E+00 & 0.0000 & 0.000 E+00 & 0.0000\end{array}$

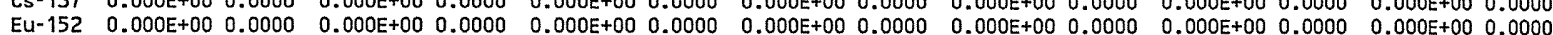

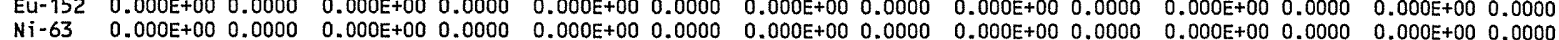
$\begin{array}{llllllllllllllll}N i-63 & 0.000 E+00 & 0.0000 & 0.000 E+00 & 0.0000 & 0.000 E+00 & 0.0000 & 0.000 E+00 & 0.0000 & 0.000 E+00 & 0.0000 & 0.000 E+00 & 0.0000 & 0.000 E+00 & 0.0000\end{array}$ $\begin{array}{lllllllllllllllll}\mathrm{Pu}-239 & 0.000 \mathrm{E}+00 & 0.0000 & 0.000 E+00 & 0.0000 & 0.000 E+00 & 0.0000 & 0.000 E+00 & 0.0000 & 0.000 E+00 & 0.0000 & 0.000 E+00 & 0.0000 & 0.000 E+00 & 0.0000\end{array}$ $\begin{array}{lllllllllllllllll}\text { Pu }-240 & 2.600 E-28 & 0.0000 & 0.000 E+00 & 0.0000 & 0.000 E+00 & 0.0000 & 0.000 E+00 & 0.0000 & 0.000 E+00 & 0.0000 & 0.000 E+00 & 0.0000 & 0.000 E+00 & 0.0000\end{array}$ $\begin{array}{lllllllllllllllll}\mathrm{S} r-90 & 0.000 \mathrm{E}+00 & 0.0000 & 0.000 E+00 & 0.0000 & 0.000 E+00 & 0.0000 & 0.000 E+00 & 0.0000 & 0.000 E+00 & 0.0000 & 0.000 E+00 & 0.0000 & 0.000 E+00 & 0.0000\end{array}$

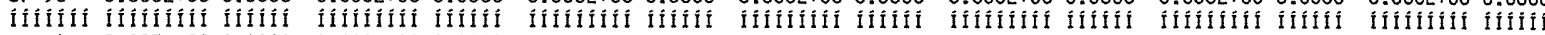

Total Dose Contributions TDOSE $(i, p, t)$ for Individual Radionuclides (i) and Pathways ( $p$ ) As $\mathrm{mrem} / \mathrm{yr}$ and Fraction of Total Dose At $t=1.000 \mathrm{E}+03$ years

0 Radon Plant Meat Milk $0.0005+00$ 0.0000

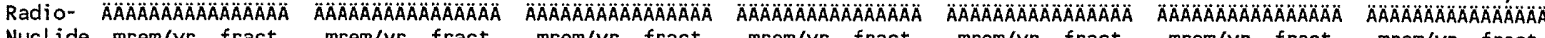
Nuclide $m r e m / y r$ fract. mrem/yr fract. mrem/yr fract. mrem/yr fract. mrem/yr fract. mrem/yr fract. mrem/yr fract.

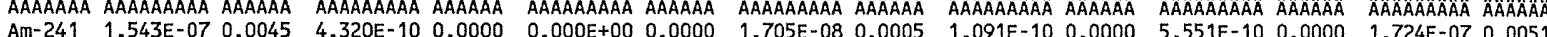

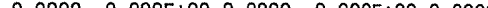
CS-137 $3.122 E-11 \quad 0.0000 \quad 1.307 E-11 \quad 0.0000 \quad 0.000 E+00 \quad 0.0000 \quad 3.490 E-12 \quad 0.0000 \quad 1.959 E-12 \quad 0.0000 \quad 1.508 E-12 \quad 0.0000 \quad 5.124 E-11 \quad 0.0000$ $\begin{array}{lllllllllllllll}\text { EU-152 } & 0.000 E+00 & 0.0000 & 0.000 E+00 & 0.0000 & 0.000 E+00 & 0.0000 & 0.000 E+00 & 0.0000 & 0.000 E+00 & 0.0000 & 0.000 E+00 & 0.0000 & 0.000 E+00 & 0.0000\end{array}$

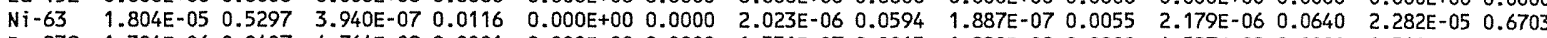

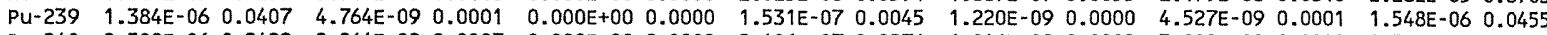
$\begin{array}{llllllllllllllll}\text { PU-240 } & 8.508 E-06 & 0.2499 & 2.264 E-08 & 0.0007 & 0.000 E+00 & 0.0000 & 9.406 E-07 & 0.0276 & 6.046 E-09 & 0.0002 & 3.080 E-08 & 0.0009 & 9.508 E-06 & 0.2792\end{array}$ $\begin{array}{llllllllllllllll}\mathrm{Sr}-90 & 4.058 \mathrm{E}-11 & 0.0000 & 5.473 \mathrm{E}-13 & 0.0000 & 0.000 \mathrm{E}+00 & 0.0000 & 4.893 \mathrm{E}-12 & 0.0000 & 6.839 \mathrm{E}-13 & 0.0000 & 4.928 \mathrm{E}-13 & 0.0000 & 4.720 \mathrm{E}-11 & 0.0000\end{array}$

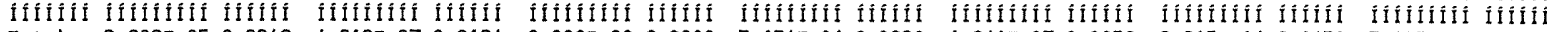
$\begin{array}{llllllllllllllllllllll}\text { Total } & 2.809 E-05 & 0.8248 & 4.218 E-07 & 0.0124 & 0.000 E+00 & 0.0000 & 3.134 E-06 & 0.0920 & 1.961 E-07 & 0.0058 & 2.215 E-06 & 0.0650 & 3.405 E-05 & 1.0000\end{array}$ 0 *Sum of all water independent and dependent pathways.

\begin{tabular}{|c|c|}
\hline Attachment & Sheet No. 23 of 27 \\
\hline Originator: S.W. Clark & Date \\
\hline M. W. Perrott & Date \\
\hline $0100 \mathrm{~F}-\mathrm{CA}-\mathrm{V} 0303$ & Rev. No. 0 \\
\hline
\end{tabular}




\section{ATTACHMENT 5}

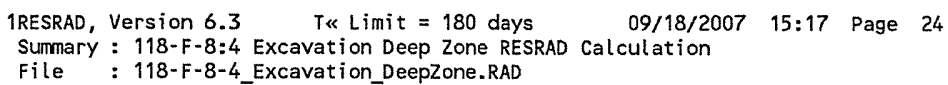

\begin{tabular}{|c|c|}
\hline Attachment & Sheet No. 24 of 27 \\
\hline Originator: S.W. Clark & Date \\
\hline M. W. Perrott & Date \\
\hline Calc. No. $\quad 0100 F-C A-V 0303$ & Rev. No. \\
\hline
\end{tabular}




\section{ATTACHMENT 5}

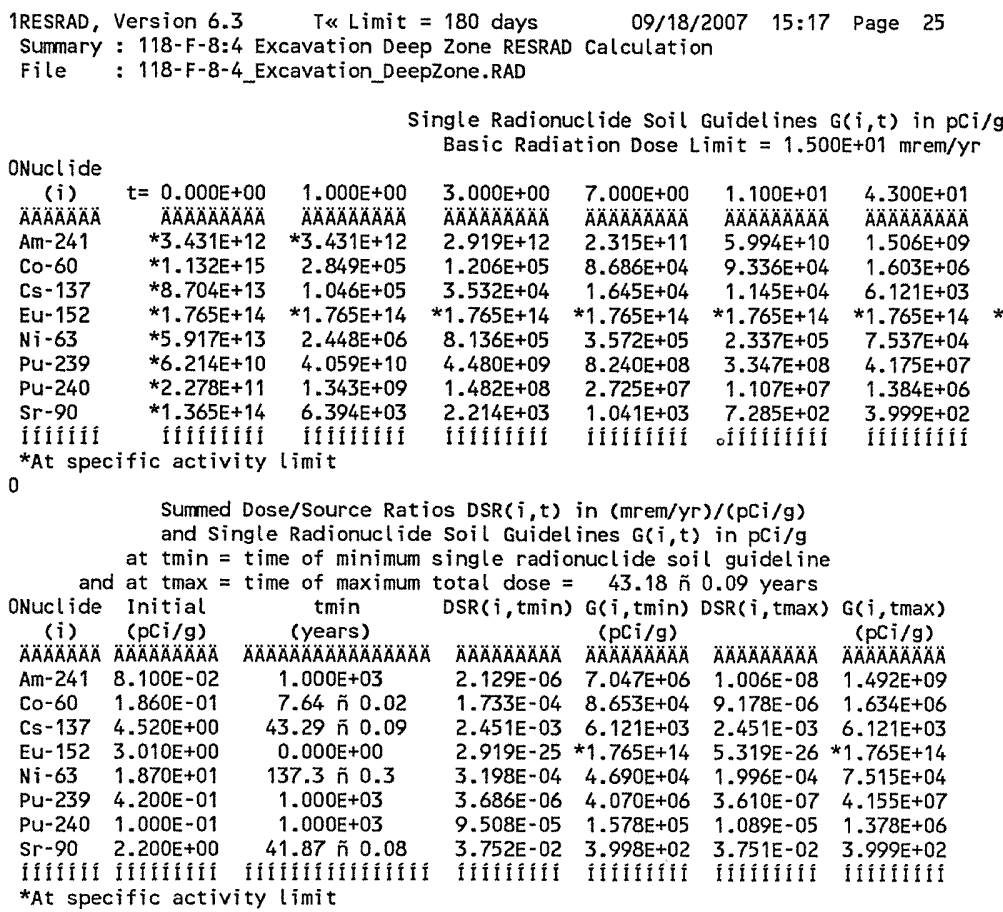

\begin{tabular}{|c|c|c|}
\hline \multicolumn{2}{|c|}{ Attachment $\quad 5$} & \multirow{2}{*}{$\begin{array}{l}\text { Sheet No. } 25 \text { of } 27 \\
\text { Date }\end{array}$} \\
\hline Orig & S.W. Clark & \\
\hline & M. W. Perrott & Date \\
\hline & $0100 F-C A-V 0303$ & Rev. No. \\
\hline
\end{tabular}




\section{ATTACHMENT 5}

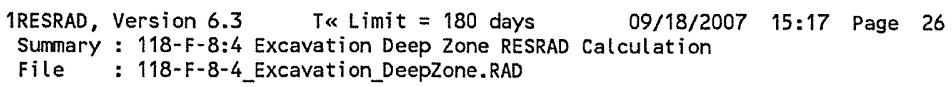

ONucl ide Parent THF(i) (j) (i) Am-241 Am-241 1.000E+OD ONp-237 Am-241 1.000E +00 OU-233 Am-241 1.000E+00 OTh-229 Am-241 1.000E+00 0 CO-60 CO-60 1.000E+00 OCs-137 Cs-137 $1.000 \mathrm{E}+00$ $7.208 \mathrm{C}$ Eu-152 Eu-152 2.792E-01 Eu-152 äDOSE (j) OGd-152 EU-152 2.792E-01 ONi-63 Ni-63 1.000E+00 OPU-239 PU-239 1.000E+00 OU-235 PU-239 1.000E +00 $\mathrm{OPa}-231 \quad \mathrm{PU}-239 \quad 1.000 \mathrm{E}+00$ OAC-227 PU-239 1.000E+00 OPU-240 PU-240 4 . 950E-08 Pu-240 Pu-240 1.000E+00 Pu-240 äDOSE(j) OU-236 PU-240 $1.000 E+00$ OTh-232 PU-240 1.000E+00 ORa-228 Pu-240 1.000E+00 OTh-228 PU-240 1.000E +00 $0 \mathrm{Sr}-90 \mathrm{Sr}-90 \quad 1.000 \mathrm{E}+00$

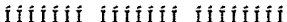
DOSE $(j, t)$, mren

$t=0.000 E+001.000 E+003.000 E+007.000 E+001,1,0014300 E+011350 E+023.000 E+021.000 E+03$

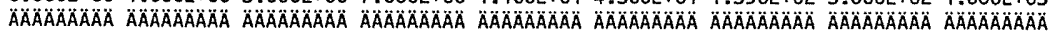
$\begin{array}{lllllllllllllll}0.000 E+00 & 0.000 E+00 & 0.000 E+00 & 0.000 E+00 & 0.000 E+00 & 0.000 E+00 & 0.000 E+00 & 0.000 E+00 & 0.000 E+00\end{array}$ $\begin{array}{llllllllllllllllll}0.000 E+00 & 0.000 E+00 & 0.000 E+00 & 0.000 E+00 & 0.000 E+00 & 0.000 E+00 & 0.000 E+00 & 0.000 E+00 & 0.000 E+00\end{array}$

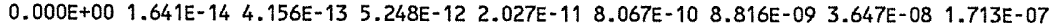
$0.000 E+00 \quad 1.803 E-16 \quad 5.364 E-16 \quad 1.623 E-15 \quad 3.017 E-15 \quad 1.623 E-13 \quad 5.810 E-12 \quad 5.780 E-11 \quad 1.063 E-09$ 8.014E-24 9.792E-06 2.314E-05 3.212E-05 2.988E-05 1.741E-06 $3.039 E-11 \quad 2.538 E-20 \quad 0.000 E+00$

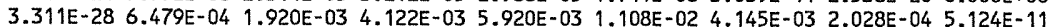

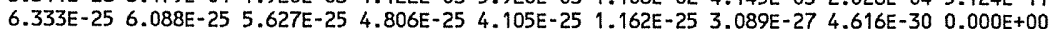

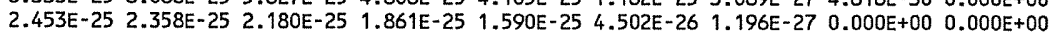

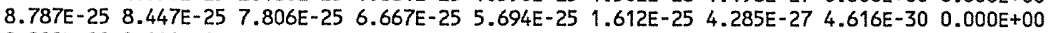
$\begin{array}{llllllllllllll}0.000 E+00 & 0.000 E+00 & 0.000 E+00 & 0.000 E+00 & 0.000 E+00 & 0.000 E+00 & 0.000 E+00 & 0.000 E+00 & 0.000 E+00\end{array}$ $0.000 \mathrm{E}+001.146 \mathrm{E}-04 \quad 3.448 \mathrm{E}-04 \quad 7.852 \mathrm{E}-04 \quad 1.200 \mathrm{E}-03 \quad 3.722 \mathrm{E}-03 \quad 5.980 \mathrm{E}-03 \quad 3.924 \mathrm{E}-03$ 2.282E-05 $\begin{array}{lllllllllll}0.000 E+00 & 0.000 E+00 & 0.000 E+00 & 0.000 E+00 & 0.000 E+00 & 0.000 E+00 & 0.000 E+00 & 0.000 E+00 & 0.000 E+00\end{array}$ $0.000 \mathrm{E}+00 \quad 1.552 \mathrm{E}-10 \quad 1.406 \mathrm{E}-09 \quad 7.645 \mathrm{E}-09 \quad 1.882 \mathrm{E}-08 \quad 1.507 \mathrm{E}-07 \quad 4.703 \mathrm{E}-07 \quad 8.638 \mathrm{E}-07 \quad 1.393 \mathrm{E}-06$ $0.000 \mathrm{E}+001.118 \mathrm{E}-14 \quad 8.511 \mathrm{E}-14$ 8.086E-13 $2.861 \mathrm{E}-121.086 \mathrm{E}-101.205 \mathrm{E}-09$ 5.422E-09 $3.528 \mathrm{E}-08$ 0.00012 .030 $0.000 E+00$ 2.933E-14 $8.588 E-14$. 4 . $\begin{array}{lllllllllll}0.000 E+00 & 0.000 E+00 & 0.000 E+00 & 0.000 E+00 & 0.000 E+00 & 0.000 E+00 & 0.000 E+00 & 0.000 E+00 & 0.000 E+00\end{array}$

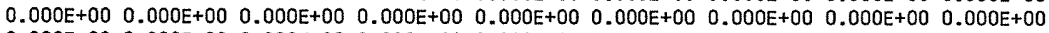
$\begin{array}{llllllllllll}0.000 E+00 & 0.000 E+00 & 0.000 E+00 & 0.000 E+00 & 0.000 E+00 & 0.000 E+00 & 0.000 E+00 & 0.000 E+00 & 0.000 E+00\end{array}$ $0.000 \mathrm{E}+00 \quad 1.117 \mathrm{E}-09 \quad 1.012 \mathrm{E}-08 \quad 5.504 \mathrm{E}-08 \quad 1.355 \mathrm{E}-07 \quad 1.084 \mathrm{E}-06 \quad 3.369 \mathrm{E}-06 \quad 6.142 \mathrm{E}-06 \quad 9.508 \mathrm{E}-06$

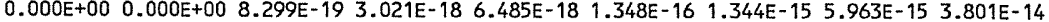
$0.000 \mathrm{E}+00 \quad 7.570 \mathrm{E}-24 \quad 1.141 \mathrm{E}-18 \quad 3.237 \mathrm{E}-18 \quad 6.156 \mathrm{E}-18 \quad 1.390 \mathrm{E}-16 \quad 1.842 \mathrm{E}-15 \quad 8.541 \mathrm{E}-15 \quad 5.701 \mathrm{E}-14$

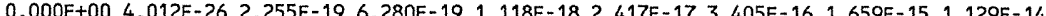
$0.000 E+005.161 E-03 \quad 1.490 E-023.169 E-024.530 E-02$ 8.252E-02 $2.877 E-02$ 9.192E-04 4.720E-11

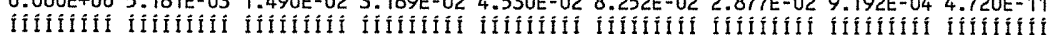

\begin{tabular}{|c|c|}
\hline Attachment & Sheet No. 26 of 27 \\
\hline Originator: S. W. Clark & Date \\
\hline M. W. Perrott & Date \\
\hline $0100 F-C A-V 0$ & Rev. No. \\
\hline
\end{tabular}




\section{ATTACHMENT 5}

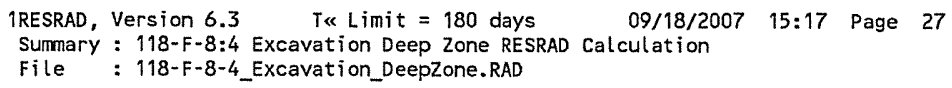

ONuclide Parent THF(i) (j) (i) (i)

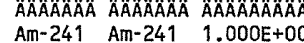
$\begin{array}{rrr}A m-241 & A m-241 & 1.000 E+00 \\ 0 N p-237 & A m-241 & 1.000 E+00\end{array}$ $\begin{array}{lll}\text { ONp-237 } & \mathrm{Am}-241 & 1.000 \mathrm{E}+00 \\ 0 \mathrm{U}-233 & \mathrm{Am}-241 & 1.000 \mathrm{E}+00\end{array}$ 0 Th-229 Am-241 1.000E +00 0 Co-60 Co-60 $1.000 \mathrm{E}+00$ $0 \mathrm{Cs}-137$ Cs-137 1.000E+00 OCs -137 CS-137 1.000E+00 OEU-152 Eu-152 7.208E-01 EU-152 EU-152 2.792E-01 $\mathrm{Eu}-152$ äs( $\mathrm{j})$

OGd-152 Eu-152 2.792E-01 ONi-63 Ni-63 1.000E+00 OPU-239 Pu-239 1.000E +00 OU-235 PU-239 1.000E +00 OPa-231 PU-239 $1.000 E+00$ OAC-227 PU-230 $1.000 E+00$ OPU-240 PU-240 $4.950 E-08$ Pu-240 PU-240 $1.000 E+00$ $\mathrm{Pu}-240$ äS $(\mathrm{j})$ :

OU-236 PU-240 $1.000 E+00$ OTh-232 PU-240 1.000E+00 ORa-228 PU-240 $1.000 E+00$ $0 T h-228 \quad \mathrm{PU}-240 \quad 1.000 \mathrm{E}+00$ OS $-90 \quad \mathrm{Sr}-90 \quad 1.000 \mathrm{E}+00$

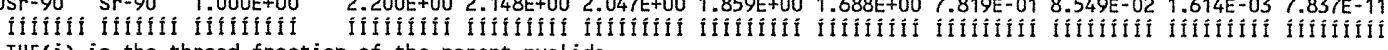
ORESCALC. EXE execution time $=15.19$ seconds

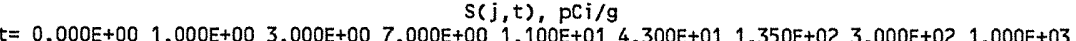

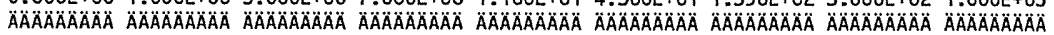
8.100E-02 8.087E-02 8.060E-02 8.008E-02 7.956E-02 7.550E-02 $6.495 \mathrm{E}-02 \quad 4.959 \mathrm{E}-02 \quad 1.578 \mathrm{E}-02$ $0.000 E+00 \quad 2.621 E-08 \quad 7.851 E-08 \quad 1.826 E-07 \quad 2.860 E-07 \quad 1.089 E-06 \quad 3.172 E-06 \quad 6.195 E-06 \quad 1.271 E-05$

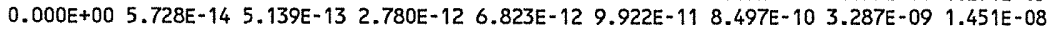

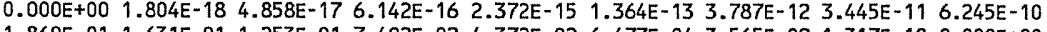

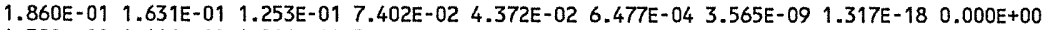

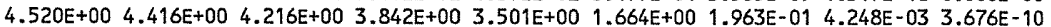

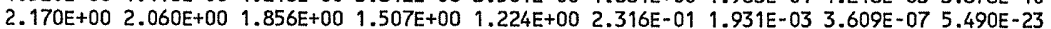
8.404E-01 7.978E-01 7.189E-01 5.839E-01 4.742E-01 8.971E-02 7.480E-04 1.398E-07 2.127E-23

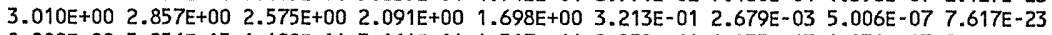

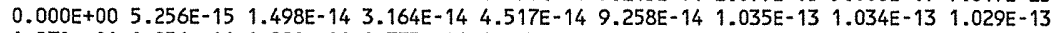

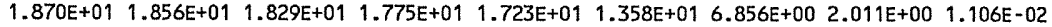
4.200E-01 4.200E-01 4.199E-01 4.198E-01 4.197E-01 4.189E-01 4.166E-01 4.124E-01 3.952E-01 $0.000 \mathrm{E}+00 \quad 4.130 \mathrm{E}-10 \quad 1.235 \mathrm{E}-09 \quad 2.865 \mathrm{E}-09 \quad 4.474 \mathrm{E}-09 \quad 1.666 \mathrm{E}-08 \quad 4.566 \mathrm{E}-08 \quad 8.077 \mathrm{E}-08 \quad 1.247 \mathrm{E}-07$ $0.000 E+00 \quad 4.371 E-15 \quad 3.926 E-14 \quad 2.128 E-13 \quad 5.233 E-13 \quad 7.729 E-12 \quad 6.926 E-11 \quad 2.910 E-10 \quad 1.827 E-09$ $0.000 \mathrm{E}+00 \quad 4.603 \mathrm{E}-17 \quad 1.221 \mathrm{E}-15 \quad 1.498 \mathrm{E}-14 \quad 5.620 \mathrm{E}-14 \quad 2.604 \mathrm{E}-12 \quad 4.502 \mathrm{E}-11 \quad 2.403 \mathrm{E}-10 \quad 1.736 \mathrm{E}-09$ 4.950E-09 4.949E-09 4.948E-09 4.945E-09 4.942E-09 4.921E-09 4.859E-09 4.749E-09 4.312E-09 1.000E-01 9.999E-02 9.996E-02 9.990E-02 9.985E-02 9.941E-02 9.815E-02 9.594E-02 8.711E-02 1.000E-01 9.999E-02 9.996E-02 9.990E-02 9.985E-02 9.941E-02 9.815E-02 9.594E-02 8.711E-02

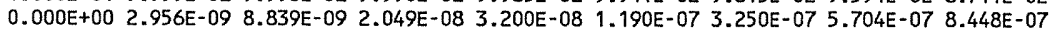
$0.000 E+00 \quad 7.294 E-20 \quad 6.551 E-19 \quad 3.552 E-18 \quad 8.733 E-18 \quad 1.290 E-16 \quad 1.158 E-15 \quad 4.877 E-15 \quad 3.097 E-14$ $0.000 \mathrm{E}+00 \quad 2.845 \mathrm{E}-21 \quad 7.237 \mathrm{E}-20 \quad 8.206 \mathrm{E}-19 \quad 2.863 \mathrm{E}-18 \quad 8.926 \mathrm{E}-17 \quad 1.031 \mathrm{E}-15 \quad 4.645 \mathrm{E}-15 \quad 3.061 \mathrm{E}-14$ $0.000 E+002.415 E-221.631 E-203.473 E-191.580 E-187.704 E-179.899 E-16 \quad 4.570 E-153.050 E-14$

\begin{tabular}{|c|c|}
\hline \multirow{2}{*}{\multicolumn{2}{|c|}{$\begin{array}{l}\text { Attachment } \frac{5}{\text { Originator: S.W. Clark }} \quad \begin{array}{c}\text { Sheet No. } 27 \text { of } 27 \\
\text { Date }\end{array}\end{array}$}} \\
\hline & \\
\hline M. W. Perrott & Date \\
\hline $0100 \mathrm{~F}-\mathrm{CA}$ & Rev. No. \\
\hline
\end{tabular}




\title{
ATTACHMENT 6
}

\author{
1RESRAD, Version $6.3 \quad$ T« Limit $=180$ days $\quad 09 / 18 / 2007 \quad 15: 17$ Page 1
Intrisk : $118-F-8: 4$ Excavation Deep Zone RESRAD Calculation \\ File : 118-F-8-4_Excavation_DeepZone.RAD \\ Table of Contents \\ Part III: Intake Quantities and Health Risk Factors

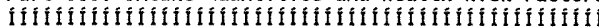

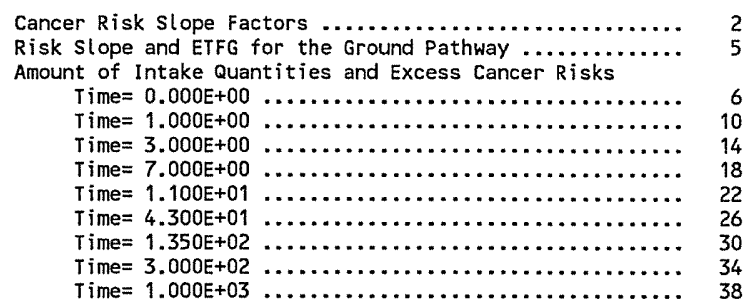

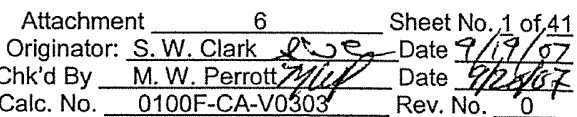




\section{ATTACHMENT 6}

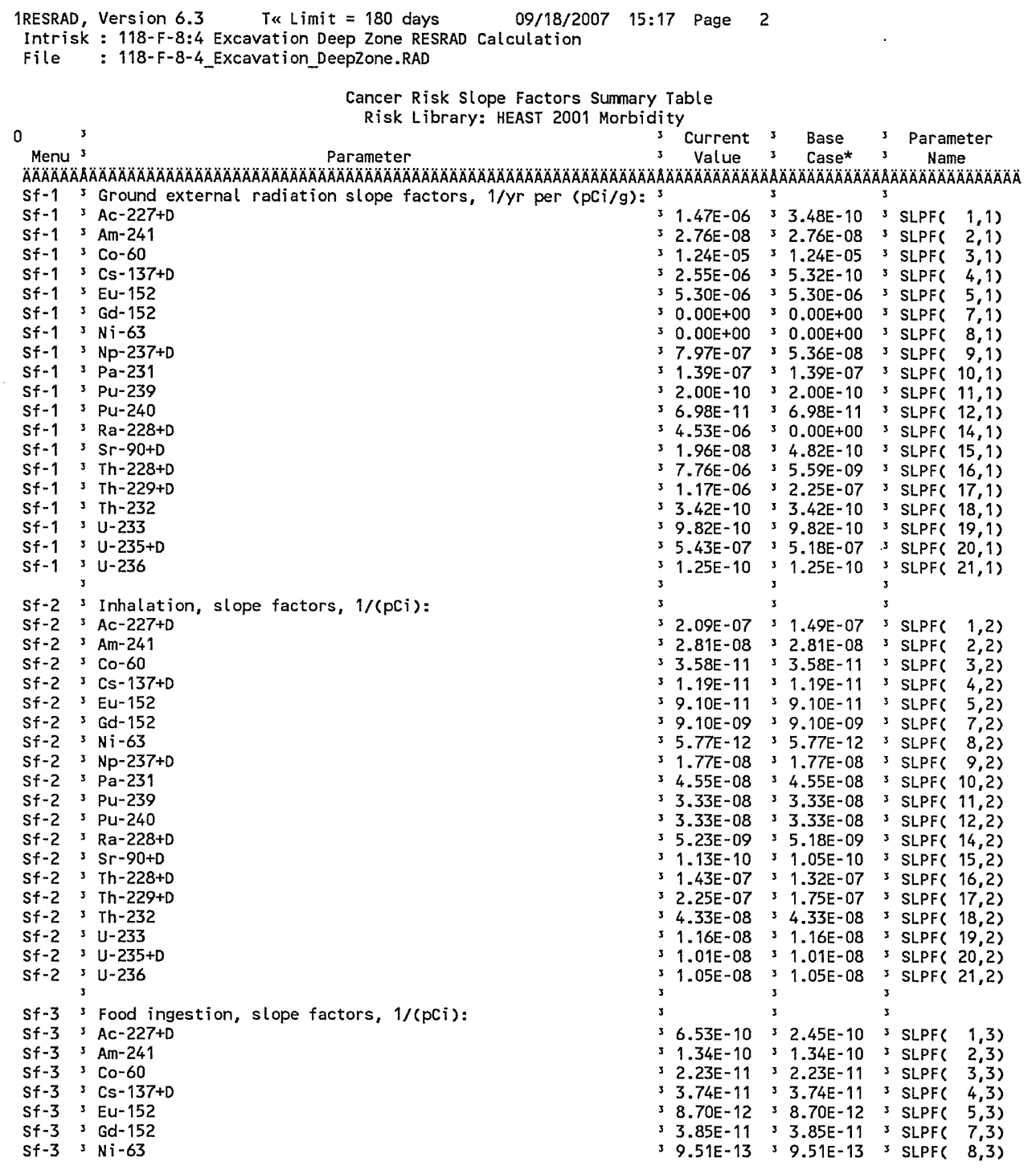

\begin{tabular}{|c|c|}
\hline Attachment & Sheet No. 2 of 41 \\
\hline Originator: S.W. Clark & Date \\
\hline M. W. Perrott & Date \\
\hline $0100 \mathrm{~F}-\mathrm{CA}-\mathrm{V} 0303$ & Rev. No. \\
\hline
\end{tabular}




\section{ATTACHMENT 6}

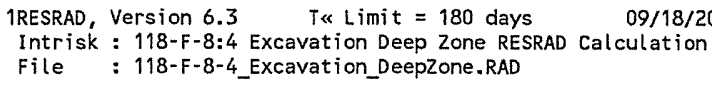

$31.29 \mathrm{E}-09=4.96 \mathrm{E}-10 ; \operatorname{SLPF}(17,5)$

\begin{tabular}{|c|c|}
\hline Attachment & Sheet No. $\underline{3}$ of 41 \\
\hline Originator: S.W. Clark & Date \\
\hline M. W. Perrott & Date \\
\hline $0100 F-C A-V 0303$ & Rev. No. \\
\hline
\end{tabular}




\section{ATTACHMENT 6}

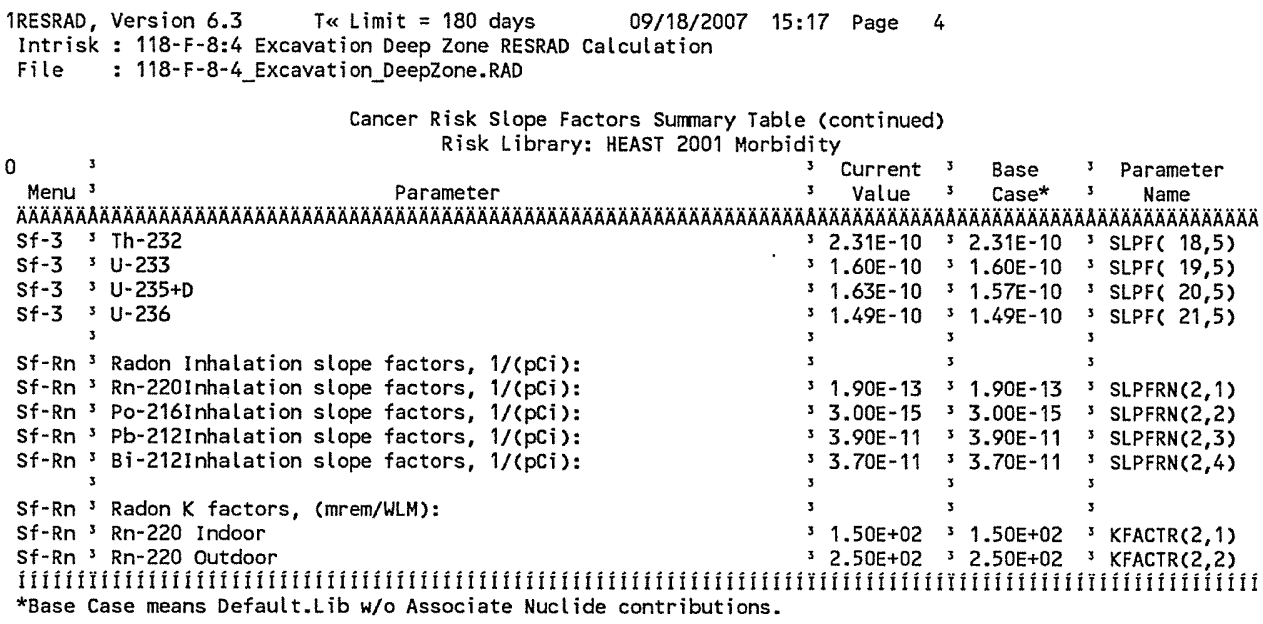

\begin{tabular}{|c|c|}
\hline Attachment & Sheet No. 4 of 41 \\
\hline Originator: $S . W$. Clark & Date \\
\hline M.W. Perrott & Date \\
\hline $0100 F-C A-V 0303$ & Rev. No. 0 \\
\hline
\end{tabular}




\section{ATTACHMENT 6}

\begin{tabular}{|c|c|c|c|c|c|c|c|c|c|c|}
\hline $\begin{array}{l}\text { 1RESRAD } \\
\text { Intris } \\
\text { File }\end{array}$ & $\begin{array}{l}118-F-8: 4 \\
118-F-8-4\end{array}$ & tic & מרח & & tion & & 5 & & & \\
\hline & sioperts & & & & & & & & & \\
\hline & & & & $00 E+00$ & $100 E+00$ & & & $1.350 \mathrm{E}+02$ & $3.000 \mathrm{E}+02$ & $1.000 \mathrm{E}+$ \\
\hline w & $\ddot{A}$ & 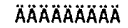 & & $\triangle A A A A A A A A O A$ & $\triangle A A A A O A A A O A O A$ & 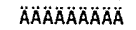 & $\triangle A O A O A A O A$ & $\triangle A A A A A A A A A A A$ & $\triangle A A A A A A A A A$ & $\triangle A A A A A A O A O A B$ \\
\hline & -08 & $0.000 E+00$ & $0.000 E+00$ & $0.000 E+00$ & $0.000 E+00$ & $1.401 \mathrm{E}-45$ & $01 E-45$ & $7.006 \mathrm{E}-45$ & $.111 \mathrm{E}-43$ & $.389 E-36$ \\
\hline & & $0.000 E+00$ & $0.000 E+00$ & $0.000 E+00$ & $0.000 E+00$ & 0.000 & & & & \\
\hline & & $8.308 E-26$ & $2 E-26$ & $8.624 E-26$ & $9.065 E-26$ & $9.528 E-26$ & -25 & $61 E-25$ & & \\
\hline 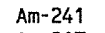 & $2.760 \mathrm{E}-08$ & $0.000 \mathrm{E}+00$ & $0.000 \mathrm{E}+00$ & $0.000 E+00$ & $0.000 E+00$ & $0.000 E+00$ & $0.000 E+00$ & $0.000 E+00$ & $0.000 E+00$ & $0.000 E+00$ \\
\hline & & $775=-31$ & -31 & $7.087 E-31$ & $E-31$ & & & $3 E-30$ & & $=21$ \\
\hline & 16 & & & & -29 & & & & & \\
\hline & & & & & & & & & & \\
\hline & & & & $E-26$ & $E-26$ & & & & & \\
\hline & & & & $=-30$ & -30 & & & & & \\
\hline & & & & & & & & & & -19 \\
\hline & 10 & & & +00 & $E+00$ & & & & & $E-38$ \\
\hline & & & & & & & & & & \\
\hline & & & & 10 & & & & & & \\
\hline & 1 & & & & -41 & & & & & -32 \\
\hline & & & & & +00 & & & & & \\
\hline & & & & 00 & & & & & & +00 \\
\hline & & & & & +00 & & & & & -44 \\
\hline & & & & & & & & & & -28 \\
\hline & & & & & & & & & & \\
\hline & & & & & +00 & & & 00 & $+\infty 0$ & $=-39$ \\
\hline & & & & 29 & & & & & & -2 \\
\hline & & & & & & & & & & -3 \\
\hline & & & & 7 & -28 & & & & & -22 \\
\hline & & & & 30 & +00 & & & & & +00 \\
\hline & & & & & & & & & & $a_{0}+2$ \\
\hline & & & & & & & & & & \\
\hline & & & & 27 & 2 & & & & & -2 \\
\hline & & & & & -41 & & & & & -3 \\
\hline & & & & & & & & & & \\
\hline & & & & & & & & & & \\
\hline & & & & & & & & & & -30 \\
\hline & & & & & & & & & & +00 \\
\hline & & & & & & & & & & \\
\hline & & & & & & & & & & a 5 \\
\hline & & & & & & & & & & -63 \\
\hline & & & & & & & & & & -41 \\
\hline & & & & & & & & & & 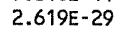 \\
\hline & & & & & & & & & & \\
\hline & & & & & & & & & & \\
\hline & & & & & & & & & & \\
\hline & & $D E+00$ & & $\mathrm{EE}+00$ & $E+00$ & & & & & $E+\infty$ \\
\hline & & & & & & & & & & -23 \\
\hline & & & & & & & & & & \\
\hline & & $=64$ & 1. & 1.3 & $E-24$ & & & & -23 & -19 \\
\hline & & & & & $E-42$ & & & & & \\
\hline & & & & & $E-42$ & & & & & \\
\hline w & & $=0$ & & & & & & & 00 & +00 \\
\hline & & & & & & & & & & $39 F-38$ \\
\hline & & & & 2114 & III & Iififili & 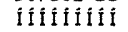 & ПMПП & 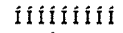 & 1111111 \\
\hline
\end{tabular}

* - Units are $1 / y r$ per $(\mathrm{pCi} / \mathrm{g})$ at infinite depth and area. Multiplication by $\mathrm{ETFG}(\mathrm{i}, \mathrm{t})$ converts to site conditions.

$\begin{array}{ll}\text { Attachment } \frac{6}{6} & \text { Sheet No. } 5 \text { of } \underline{41} \\ \text { Originator: } \frac{\text { S.W. Clark }}{\text { M. W. Perrott }} & \text { Date } \\ \text { Chk'd By } & \text { Date } \\ \text { Calc. No. } \frac{0100 \text { F-CA-V0303 }}{0 \text { Nov. No. } \quad 0}\end{array}$




\section{ATTACHMENT 6}

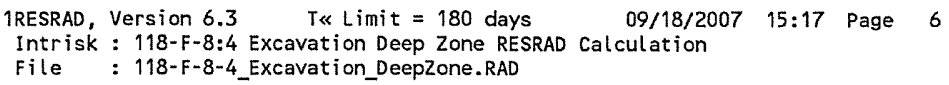

$\begin{array}{ll}\text { Attachment } \frac{6}{S} & \text { Sheet No. } \underline{6} \text { of } \underline{41} \\ \text { Originator: } \frac{\text { S.W.Clark }}{\text { W.W.Perrott }} & \text { Date } \\ \text { Chk'd By } & \text { Date } \\ \text { Calc. No. } & \text { Rev. No. } \quad 0\end{array}$




\section{ATTACHMENT 6}

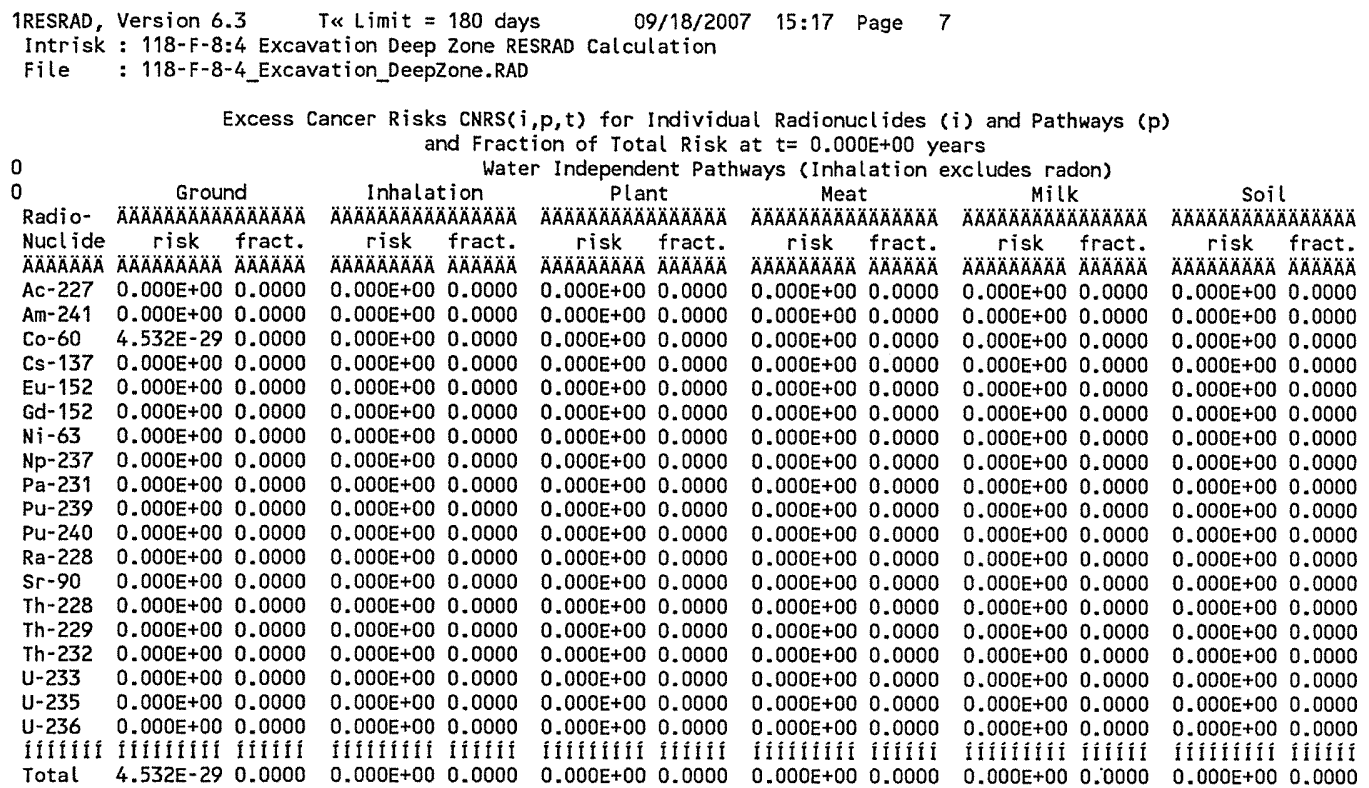

\begin{tabular}{|c|c|}
\hline Attachment & Sheet No. 7 of 41 \\
\hline Originator: S. W. Clark & Date \\
\hline M. W. Perrott & Date \\
\hline $0100 F-C A-V 0303$ & Rev. No. $\quad 0$ \\
\hline
\end{tabular}




\section{ATTACHMENT 6}

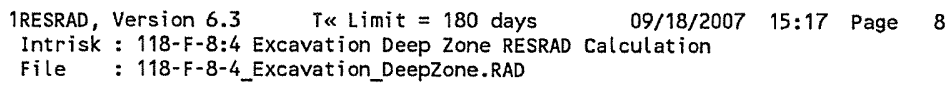

Water Dependent Pathways

\begin{tabular}{|c|c|c|c|c|c|c|c|c|c|c|c|}
\hline \multirow{2}{*}{$\begin{array}{l}\text { Radio- } \\
\text { Nuclide } \\
\ddot{A} \ddot{A} \ddot{A} A \ddot{A} \ddot{A} \ddot{A} \ddot{A}\end{array}$} & $\begin{array}{r}\text { Wate } \\
\text { ÄAिÄÄÄÄ }\end{array}$ & $\because r$ & \multicolumn{2}{|c|}{$\begin{array}{c}F i s h \\
\text { ÄÄÄAิÄÄÄÄÄÄÄÄÄ }\end{array}$} & \multicolumn{2}{|c|}{ 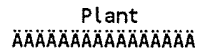 } & \multicolumn{2}{|c|}{$\begin{array}{c}\text { Meat } \\
\text { ÄAAAAAAAAAAAAAOAOÄ }\end{array}$} & \multicolumn{2}{|c|}{$\begin{array}{c}M i l k \\
\triangle A \cap A O A A A O A O A O A A O A O A\end{array}$} & 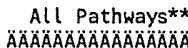 \\
\hline & $\begin{array}{c}\text { risk } \\
\triangle A A B A A A A A A\end{array}$ & $\begin{array}{l}\text { fract. } \\
A A A A A A A\end{array}$ & $\begin{array}{c}\text { risk } \\
\text { ÁÄÄÄÄÄÄÄ }\end{array}$ & $\begin{array}{l}\text { fract. } \\
\not ̈ A ̈ A ̈ A ̈ A ̈\end{array}$ & 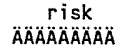 & $\begin{array}{l}\text { fract. } \\
\triangle A ̈ A ̈ A ̈ A ̈ A ̈ A\end{array}$ & 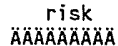 & 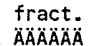 & $\begin{array}{c}\text { risk } \\
\triangle A \cap A ̈ A ̈ A ̈ A ̈ A ̈ A ̈ A\end{array}$ & $\begin{array}{l}\text { fract. } \\
\qquad \ddot{A} \ddot{A} \ddot{A} \ddot{A} \ddot{A}\end{array}$ & 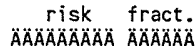 \\
\hline$=-227$ & $7.113 E-18$ & 0.0000 & $1.206 \mathrm{E}-19$ & 0.0000 & $0 E-18$ & 0000 & $8.677 \mathrm{E}-22$ & 0.0000 & $34 E-21$ & .0000 & $8.286 E-18$ \\
\hline-241 & $30 E+00$ & 000 & $0 \mathrm{E}+00$ & 000 & $=+00$ & 100 & $000 E+00$ & 0.0000 & $0.000 E+00$ & 0.0000 & $.000 E+00$ \\
\hline & & & & 0000 & & & & 0.0000 & & 30 & 10 \\
\hline & & 0. & -08 & 0399 & & 106 & & 1058 & & & \\
\hline-152 & $0.000 E+00$ & 0. & $1 O E+00$ & 1000 & $0.000 E+00$ & 0.0000 & $0.000 E+00$ & 0.0000 & $0.000 \varepsilon+00$ & 0.0000 & $+00 \mathrm{c}$ \\
\hline & +00 & 0.0000 & $=+00$ & 0 . & 0. & 00 & $=0$ & 00 & $+\infty$ & 100 & .00 \\
\hline & & & & & & & & & & & \\
\hline & 0.0 & & & & & & +00 & & 00 & 100 & 00 \\
\hline 231 & 5.4 & 0. & -20 & 0 & 8 & & -20 & & 22 & & 18 \\
\hline & & & $=00$ & 0. & & 0 & $E+00$ & 00 & 00 & & \\
\hline & & & 0. & & 0 & 0. & $E+00$ & 0 & $E+00$ & 00 & $+00 \mathrm{c}$ \\
\hline & $E-22$ & & -24 & & & & -25 & & & & \\
\hline & & & -08 & & & & & & & & \\
\hline & & & & & & 0.0 & $1.452 E-26$ & 0.0 & $2.079 E-27$ & & -230 \\
\hline & $=-30$ & & $-21+31+3$ & & & & & & & & -20 \\
\hline & & & & & 3 & & & & & & \\
\hline & $6.217 E-16$ & 0.0000 & $2.228 E-18$ & 0.0000 & 7 & & 5 & & & & -16 \\
\hline & & & $.020 E-15$ & & & & & & $1.379 E-15$ & 0.0000 & $2 E-13 \quad 0.0000$ \\
\hline & & & & & & & & & & & \\
\hline & ilifititi & & IIIItîtîn & 11111 & IIIIIIIII & $M$ & IIfIIIIf & & MIM & & IIIIIIIIÁ IIIIII \\
\hline & $.490 E-07$ & 0.7842 & $.038 \mathrm{E}-08$ & 0.0527 & $147 E-07$ & 0.1201 & $69 E-08$ & 0.0206 & $2.133 \mathrm{E}-08$ & 0.0223 & $1 E-071.0000$ \\
\hline
\end{tabular}

** Sum of water independent ground, inhalation, plant, meat, milk, soil

0 and water dependent water, fish, plant, meat, milk pathways

Excess Cancer Risks CNRS9(irn, $i, t)$ and CNRSPW( $i r n, i, t)$ for Inhalation of Radon and its Decay Products at $t=0.000 E+00$ years $\begin{array}{llllll} & \end{array}$

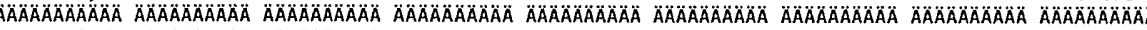
$\begin{array}{lllllllll}\text { Water-ind. } & 0.000 E+00 & 0.000 E+00 & 0.000 E+00 & 0.000 E+00 & 0.000 E+00 & 0.000 E+00 & 0.000 E+00 & 0.000 E+00\end{array}$ Water-dep. $\quad \begin{array}{llllllll}0.000 E+00 & 0.000 E+00 & 0.000 E+00 & 0.000 E+00 & 0.000 E+00 & 0.000 E+00 & 0.000 E+00 & 0.000 E+00\end{array}$

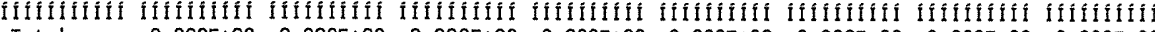

Total $\quad 0.000 \mathrm{E}+00 \quad 0.000 \mathrm{E}+00 \quad 0.000 \mathrm{E}+00 \quad 0.000 \mathrm{E}+00 \quad 0.000 \mathrm{E}+00 \quad 0.000 \mathrm{E}+00 \quad 0.000 \mathrm{E}+00 \quad 0.000 \mathrm{E}+00$

Water-ind. = = Water-independent Water-dep. = Water-dependent

\begin{tabular}{|c|c|c|}
\hline \multirow{2}{*}{\multicolumn{3}{|c|}{$\begin{array}{ll}\text { Attachment } & 6 \\
\text { Originator: S.W. Clark } & \text { Sheet No. } 8 \text { of } 41 \\
\text { Date }\end{array}$}} \\
\hline & & \\
\hline & U. W. Perrott & \\
\hline & 0100 & No. \\
\hline
\end{tabular}




\section{ATTACHMENT 6}

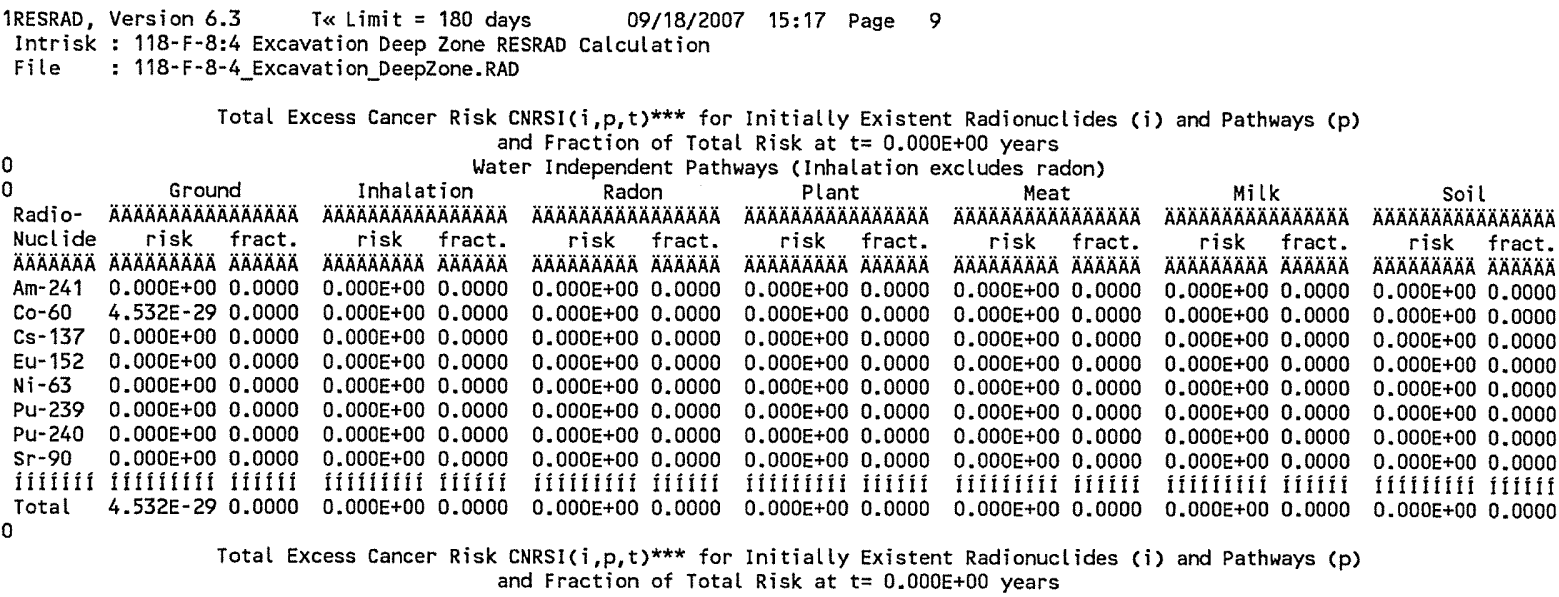

Total Excess Cancer Risk CNRSI $(i, p, t)^{* * *}$ for Initially Existent Radionuclides (i) and Pathways (p) and Fraction of Total Risk at $\mathrm{t}=0.000 \mathrm{E}+00$ years

Water Dependent Pathways

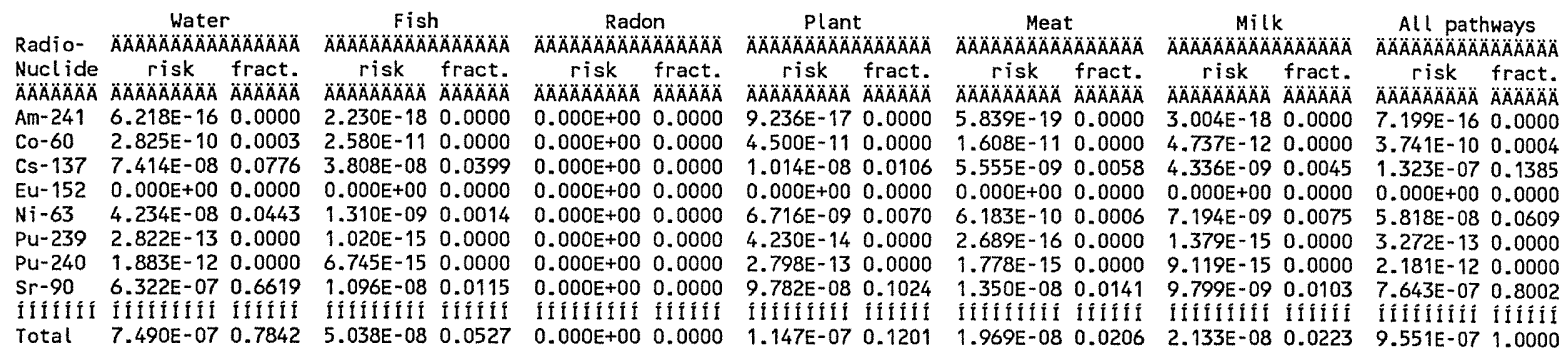

***CNRSI $(i, p, t)$ includes contribution from decay daughter radionuclides

$\begin{array}{ll}\text { Attachment } \frac{6}{6} & \text { Sheet No. } 9 \text { of } 41 \\ \text { Originator: S.W. Clark } & \text { Date } \\ \text { Chk'd By } & \text { Date } \\ \text { Calc. No. Nerrott } & \text { Rev. No. } \frac{0}{0100 \text { F-CA-V0303 }}\end{array}$


CVP-2007-00004

Rev. 0

\section{ATTACHMENT 6}

\begin{tabular}{|c|c|c|c|c|c|c|c|c|c|c|c|c|}
\hline & $\begin{array}{l}\text { Version } \\
: 118-\mathrm{F}- \\
: 118-\mathrm{F}-\end{array}$ & $\begin{array}{l}.3 \\
: 4 \text { Exc } \\
-4 \_E x c\end{array}$ & $\begin{array}{l}\text { ion Dee } \\
\text { ion_Dee }\end{array}$ & $\begin{array}{l}\text { one } \\
\text { ne. } R\end{array}$ & D Calc & tion & 7 & ge & & & & \\
\hline & \multicolumn{6}{|c|}{ Exces } & \\
\hline & Grour & & Inha & tion & $p$ & & Mea & & $\mathrm{Mil}$ & & Soil & \\
\hline & 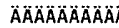 & $\ddot{A A ̈ A ̈}$ & $\triangle A ̈ A \cap A ̈$ & $\triangle A A O A ̈$ & $\triangle A A A A A O A B$ & $\triangle A A A A A A ̈ A ̈$ & $\triangle A A A A A A A A A$ & $\triangle A A O A O A ̈ A ̈ A$ & $\triangle A A A A A A A A B A O A$ & $\triangle A O A A A A B A$ & $\triangle A A A A A B A A A A$ & $\triangle A A A A A B A$ \\
\hline $\begin{array}{l}\text { Nuclide } \\
\ddot{A} \ddot{A} \ddot{A} A \ddot{A} \ddot{A} A\end{array}$ & risk & $\begin{array}{l}\text { fract. } \\
\triangle A A A A A B A\end{array}$ & $\begin{array}{c}\text { risk } \\
A B A B A B A A A\end{array}$ & $\begin{array}{l}\text { fract. } \\
\triangle A A A M A O A\end{array}$ & $\begin{array}{c}\text { risk } \\
\triangle A A A O A O A B A A A\end{array}$ & $\begin{array}{l}\text { fract. } \\
\text { ÄÄÄÄÄA }\end{array}$ & $\begin{array}{c}\text { risk } \\
A ̈ A ̈ A ̈ A B A\end{array}$ & $\begin{array}{l}\text { fract. } \\
\ddot{A} \ddot{A} \ddot{A} A \ddot{A} \ddot{A}\end{array}$ & $\begin{array}{c}\text { risk } \\
\triangle A ̈ A ̈ A ̈ A ̈ A O A\end{array}$ & $\begin{array}{l}\text { fract. } \\
\triangle A A A A A A B A\end{array}$ & $\begin{array}{c}\text { risk } \\
A \triangle A ̈ A ̈ A ̈ A ̈ A ̈ A\end{array}$ & $\begin{array}{l}\text { fract. } \\
\triangle A O A O A O A O A\end{array}$ \\
\hline Ac- 227 & $E+00$ & 0.0000 & $E+00$ & 0.0000 & $0.000 E+00$ & 0.0000 & $E+\infty$ & 0.0000 & $0.000 \mathrm{E}+00$ & 0.0 & $E+00$ & 0.0000 \\
\hline Am= & & 0.0000 & & & $0.000 E+00$ & & +00 & 0.0000 & & 0.0 & +00 & 0.0000 \\
\hline Co- & & & 0. & 0.0000 & $0.000 E+00$ & & 0. & & & & & 00 \\
\hline & & 1000 & & 0.0000 & 0.0 & & 0.0 & 00 & & & & \\
\hline & & 0.0000 & & 0.0000 & $0.000 \mathrm{E}+00$ & 0.0 & 0.0 & 0.0000 & 0. & 0.0 & 00 & 000 \\
\hline & & & & 0.0000 & 0.0 & & & & & & & 00 \\
\hline & & 0 & & 00 & 0.00 & bo & 0.0 & 300 & & & .00 & 000 \\
\hline $\mathrm{Np}$ & & 0 & & 0.0000 & 0.0 & 0.0 & 0. & 0. & & 0.0 & .00 & 000 \\
\hline & & & & & & & & & & & & \\
\hline & & & & 00 & 0.0 & & 0.0 & 0. & & & & 100 \\
\hline $\mathrm{Pu}$ & & 0 & & 0.0000 & 0.00 & & $+\infty$ & 0.0000 & & 0.0 & +00 & 0.0000 \\
\hline & & & & & 0.0 & & & & & & 0 & \\
\hline $\mathrm{Sr}$ & & 0 & 0 & & 0.0 & & 0.0 & & & & & \\
\hline & & 0.0 & & 0.0 & 0.00 & 0.0 & 0.0 & & & 0. & -00 & 000 \\
\hline & & & & 0.0 & $0.000 E+00$ & & 0.0 & & & & & 000 \\
\hline & $0.000 E+00$ & 0.0000 & 0.0 & & $0.000 E+00$ & & 0.0 & & & & 0. & \\
\hline & & & +00 & 0 & $0.000 E+00$ & 0.0 & 0.0 & & & 0.0 & +00 & 0 \\
\hline$U-23$ & $E+00$ & & $0.000 E+00$ & .0000 & $0.000 \mathrm{E}+00$ & 0.0 & 0.00 & 0.0 & 0.0 & & $E+00$ & 0.0000 \\
\hline & & & & & & & & & & & & \\
\hline & & & & & & & & & & & & \\
\hline otal & $3.570 E-29$ & 0.0000 & $.000 E+00$ & 0.0000 & $0.000 E+00$ & 0.0000 & $0.000 \mathrm{E}+00$ & 0.0000 & $0.000 E+00$ & 0.0000 & $0.000 \mathrm{E}+00$ & .00 \\
\hline
\end{tabular}

Attachment 6 


\section{ATTACHMENT 6}

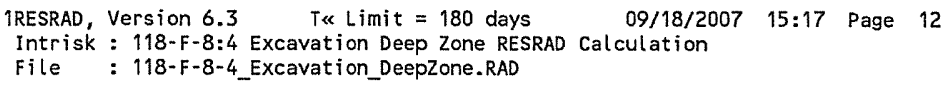

Water Dependent Pathways

\begin{tabular}{|c|c|c|c|c|c|c|c|c|c|c|c|c|}
\hline \multirow[b]{2}{*}{$\begin{array}{l}\text { Nuclide } \\
\ddot{A} A ̈ A ̈ A ̈ A ̈ A ̈ A\end{array}$} & \multicolumn{2}{|c|}{ 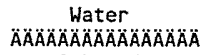 } & \multicolumn{2}{|c|}{ 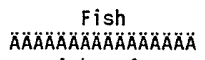 } & \multicolumn{2}{|c|}{ 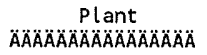 } & \multicolumn{2}{|c|}{ 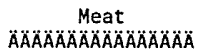 } & \multicolumn{2}{|c|}{$\begin{array}{c}\text { Milk } \\
\text { AOAAAAAAAOAOAOÄA }\end{array}$} & \multicolumn{2}{|c|}{$\begin{array}{r}\text { All Pathways** } \\
\text { AAAAAAAAAAAAAAOAAAA }\end{array}$} \\
\hline & 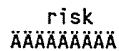 & $\begin{array}{l}\text { fract. } \\
\because A ̈ A ̈ A ̈ A ̈ A\end{array}$ & $\begin{array}{c}r i s k \\
\triangle A \cap A ̈ A ̈ A ̈ A A A A B A\end{array}$ & $\begin{array}{l}\text { fract. } \\
\ddot{A} \ddot{A} \ddot{A} \ddot{A} \ddot{A} \dot{A}\end{array}$ & $\begin{array}{c}r i s k \\
A \cap A B A B A B A B A B\end{array}$ & $\begin{array}{l}\text { fract. } \\
A B A B A B A B A\end{array}$ & $\begin{array}{c}r i s k \\
A B A B A B A A B A\end{array}$ & fract. & $\begin{array}{c}\text { risk } \\
\triangle A B A A B A B A B A ̈\end{array}$ & fract. & risk & $\begin{array}{l}\text { fract. } \\
\triangle A \cap A B A B A A B\end{array}$ \\
\hline$A C-227$ & $8.203 E-18$ & 0.0000 & $1.387 \mathrm{E}-19$ & 0.0000 & & 0.0000 & $9.820 \mathrm{E}-22$ & 0.0000 & & 0.0000 & & 0.0000 \\
\hline & $30 E+00$ & 300 & $E+\infty 0$ & & & 000 & $200 E+00$ & 0.0000 & $30 E+00$ & .0000 & $30 E+00$ & .0000 \\
\hline & & & & & & & & & & & & \\
\hline & & & & & & & & & & & & \\
\hline & $0.000 E+00$ & & & & $D E+\infty O$ & 000 & $30 E+00$ & & $=00$ & & $+\infty$ & \\
\hline & 0.0 & & bo & & 00 & & 00 & & .00 & & & \\
\hline & & & & & & & & & & & & \\
\hline & 0.00 & & & & & & & & & & & \\
\hline & 6.0 & & 20 & & & & & & & & & \\
\hline & & & .00 & & & & & & & & & \\
\hline & 0.00 & & & & 0 & 0 & & & -00 & & & \\
\hline & ? & & & & & & & & & & & \\
\hline & & & & & & & & & & & & \\
\hline & 3.16 & & & & & 0. & & & & & & \\
\hline & ( & & & & & & & & & & & \\
\hline & & & & & & & & & & & & \\
\hline & $=-16$ & & $2.512 E-18$ & & 1. & & 6.5 & & $3.387 E-18$ & & 8. & \\
\hline & & & & & & & & & & & & \\
\hline & & & 10 & & & & & & & & & \\
\hline & if iffifif & & IIfifitio & & IIIIIIIIIII & & & & III & & II I & \\
\hline Total & $7.871 \mathrm{E}-07$ & 2 & $5.293 E-08$ & 0.0 & $1.205 \mathrm{E}-07$ & $\ldots$ & 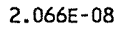 & 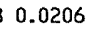 & 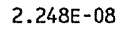 & 24 & E-00 & 000 \\
\hline
\end{tabular}

** Sum of water independent ground, inhalation, plant, meat, milk, soil and water dependent water, fish, plant, meat, milk pathways

0

Excess Cancer Risks CNRSP(irn, $i, t)$ and CNRSPW( $i r n, i, t)$ for Inhalation of

Radon and its Decay Products at $t=1.000 E+00$ years

Radon Radionuclides

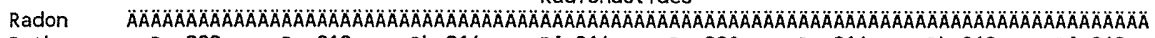

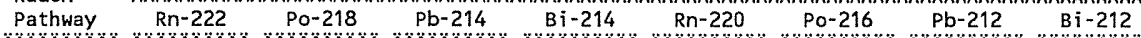

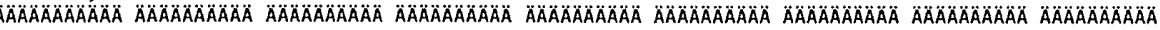
$\begin{array}{lllllllll}\text { Water }- \text { ind. } & 0.000 \mathrm{E}+00 & 0.000 \mathrm{E}+00 & 0.000 \mathrm{E}+00 & 0.000 \mathrm{E}+00 & 0.000 \mathrm{E}+00 & 0.000 \mathrm{E}+00 & 0.000 \mathrm{E}+00 & 0.000 \mathrm{E}+00\end{array}$ $\begin{array}{lllllllll}\text { Water-dep. } & 0.000 \mathrm{E}+00 & 0.000 \mathrm{E}+00 & 0.000 \mathrm{E}+00 & 0.000 \mathrm{E}+00 & 0.000 \mathrm{E}+00 & 0.000 \mathrm{E}+00 & 0.000 \mathrm{E}+00 & 0.000 \mathrm{E}+00\end{array}$

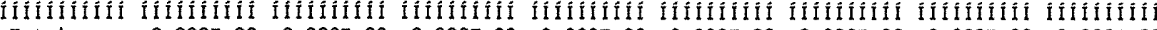

$\begin{array}{lllllllll}\text { Total } & 0.000 E+00 & 0.000 E+00 & 0.000 E+00 & 0.000 E+00 & 0.000 E+00 & 0.000 E+00 & 0.000 E+00 & 0.000 E+00\end{array}$

Water-ind. == Water-independent Water-dep. == Water-dependent

\begin{tabular}{|c|c|}
\hline Attachment & Sheet No. 12 of 41 \\
\hline Originator: S.W. Clark & Date \\
\hline Chk'd By M.W. Perrott & Date \\
\hline Calc. No. $\quad 0100 F-C A-V 0303$ & Rev. No. \\
\hline
\end{tabular}




\section{ATTACHMENT 6}

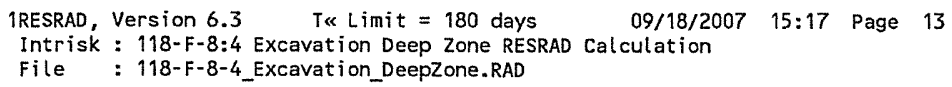

Total Excess Cancer Risk CNRSI $(i, p, t)^{* * *}$ for Initially Existent Radionuclides $(i)$ and Pathways $(p)$ and Fraction of Total Risk at $t=1.000 E+00$ years

Water Dependent Pathways

\begin{tabular}{|c|c|c|c|c|c|c|c|c|c|c|c|c|c|c|}
\hline \multirow[b]{2}{*}{$\begin{array}{l}\text { Nuclide } \\
\text { ÄÄÄÄÄÄA }\end{array}$} & \multicolumn{2}{|c|}{ Water } & \multicolumn{2}{|c|}{ Fish } & \multicolumn{2}{|c|}{ Radon } & \multicolumn{2}{|c|}{$\begin{array}{c}\text { Plant } \\
\text { ÄÄÄÄÄÄÄÄAOAOÄA }\end{array}$} & \multicolumn{2}{|c|}{$\begin{array}{c}\text { Meat } \\
\text { ÄAAAAAAAAAAOAOAOÄ }\end{array}$} & \multicolumn{2}{|c|}{$\begin{array}{c}\text { Milk } \\
\text { ÄÄÄAAAAAAOAAOÄÄA }\end{array}$} & \multicolumn{2}{|c|}{ 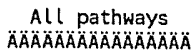 } \\
\hline & $A A$ & $\begin{array}{l}\text { fract. } \\
A A A O A O A O A B\end{array}$ & & & ÄÄ̈ & & 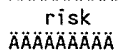 & $\begin{array}{l}\text { fract. } \\
\dddot{A} A \ddot{A} \ddot{A} A \ddot{A}\end{array}$ & (2) & & risk & & & \\
\hline 29 & & & & & & & & & & & & & & \\
\hline & & & & & & & & & & & & & & \\
\hline & & & & & & & & & & & & & & \\
\hline & & & & & & & & & & & & & & \\
\hline & & & & & & & & & & & & & & \\
\hline & & & & & & & & & & & & & & \\
\hline & & & & & & & & & & & & & & \\
\hline & & & & & & & & & & & & & & \\
\hline & & & & & & & & & & & & & & \\
\hline otal & & 0.7842 & & 0.0527 & $0.0005+00$ & 0.0000 & TE- & 0.1201 & $2.066 E-08$ & 0.0206 & $2.248 E-08$ & 0.0224 & $.004 \mathrm{E}-06$ & 1.0000 \\
\hline
\end{tabular}

***CNRSI $(i, p, t)$ includes contribution from decay daughter radionuclides

\begin{tabular}{|c|c|c|}
\hline \multirow{2}{*}{\multicolumn{3}{|c|}{$\begin{array}{ll}\text { Attachment } & 6 \\
\text { Originator: S.W. Clark } & \text { Sheet No. } 13 \text { of } 41 \\
\end{array}$}} \\
\hline & & \\
\hline & M.W. Perrott & 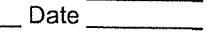 \\
\hline al & $0100 \mathrm{~F}-\mathrm{C}$ & 10. \\
\hline
\end{tabular}




\section{ATTACHMENT 6}

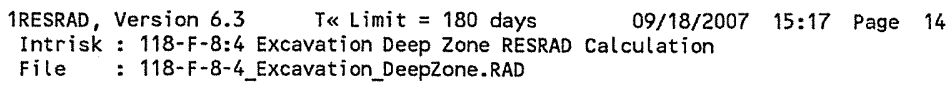

\begin{tabular}{|c|c|}
\hline Attachment & Sheet No. 14 of 41 \\
\hline Originator: S.W. Clark & Date \\
\hline Chk'd By M.W. Perrott & Date \\
\hline Calc. No. $\quad 0100 F-C A-V 0303$ & Rev. No. \\
\hline
\end{tabular}




\section{ATTACHMENT 6}

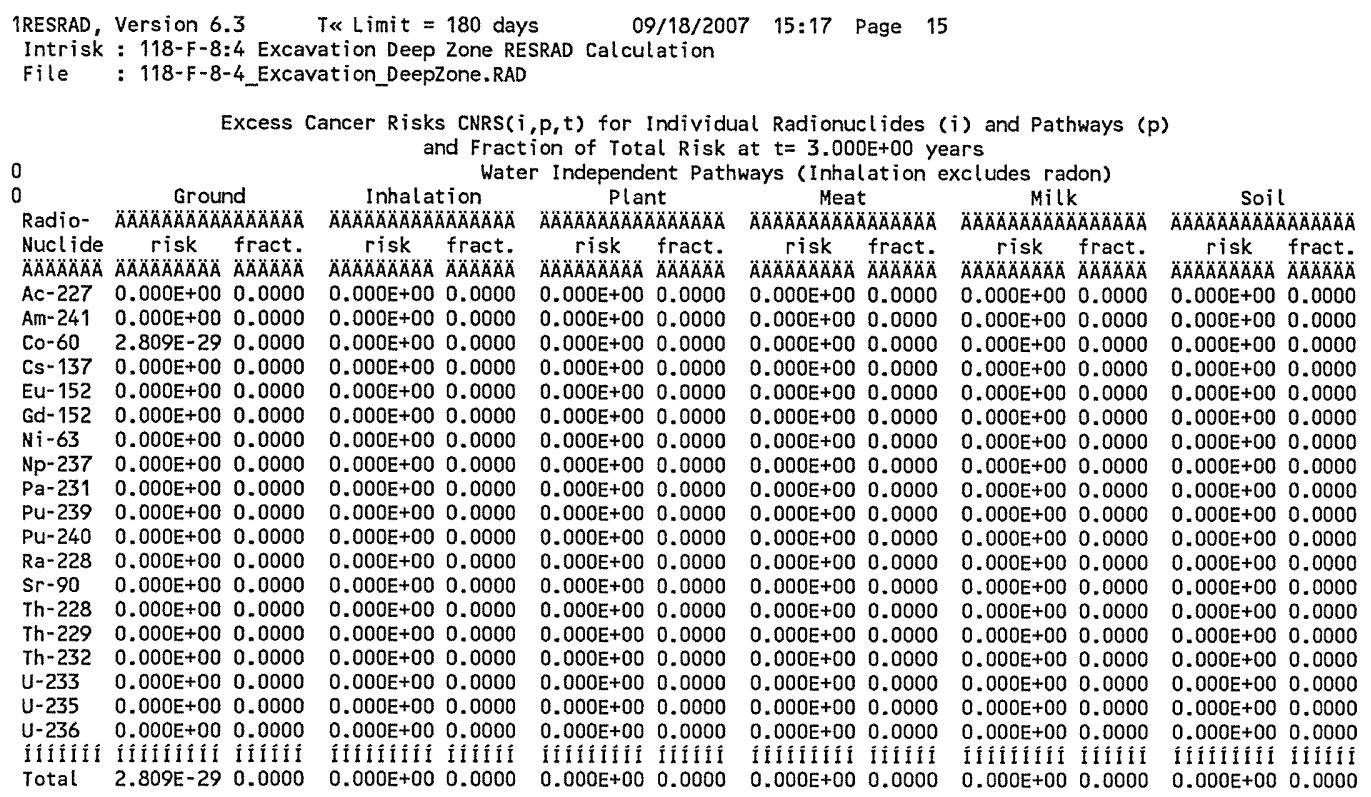

\begin{tabular}{|c|c|}
\hline Attachment & Sheet No. 15 of 41 \\
\hline Originator: S.W. Clark & Date \\
\hline Chk'd By M.W. Perrott & Date \\
\hline Calc. No. $\quad 0100 \mathrm{~F}$-CA-V0303 & Rev. No. \\
\hline
\end{tabular}




\section{ATTACHMENT 6}

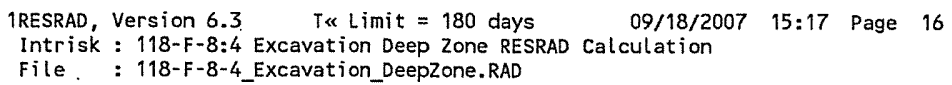

Water Dependent Pathways

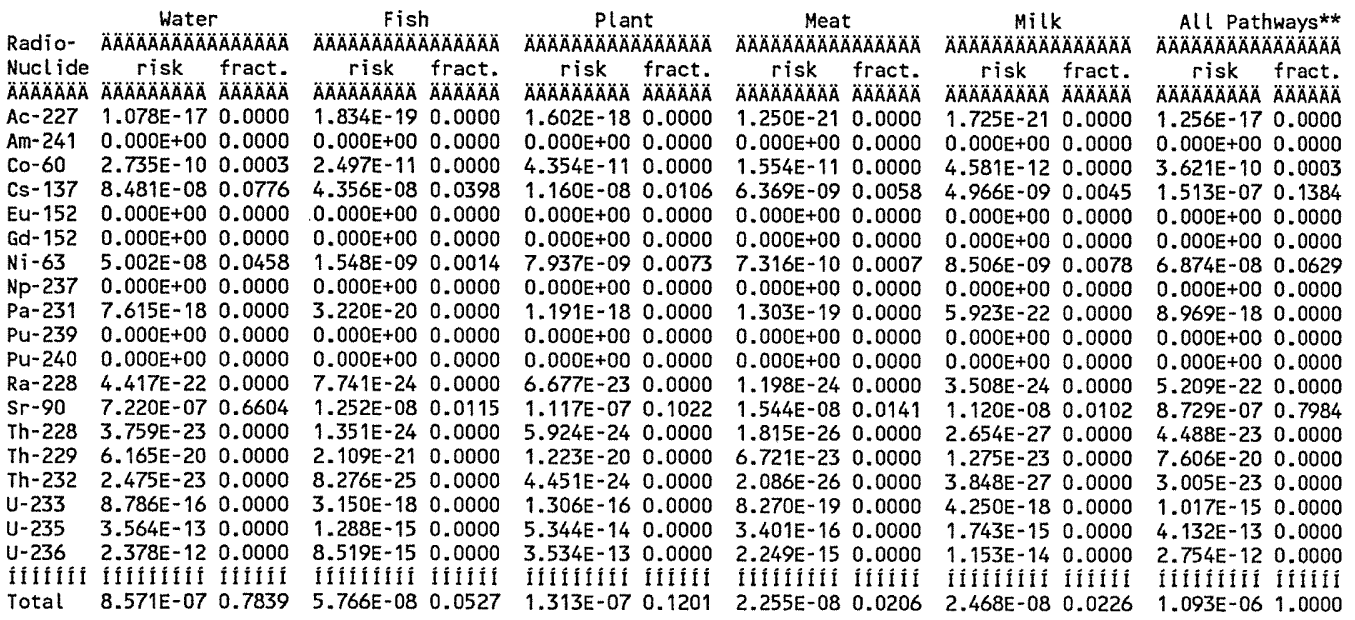

** Sum of water independent ground, inhalation, plant, meat, milk, soil

and water dependent water, fish, plant, meat, milk pathways

Excess Cancer Risks CNRS9( $i r n, i, t$ ) and CNRSOW( $i r n, i, t$ ) for Inhalation of Radon and its Decay Products at $t=3.000 \mathrm{E}+00$ years

Radon Radionucl ides

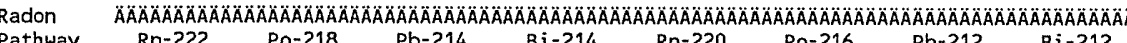
$\begin{array}{lllllll}\text { Pathway Rn-222 Po-218 Pb-214 Bi-214 Rn-220 Po-216 Pb-212 } & \text { B } & \text { Bi-212 }\end{array}$

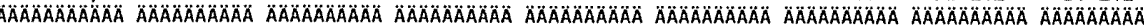
$\begin{array}{lllllllll}\text { Water-ind. } 0.000 \mathrm{E}+00 & 0.000 \mathrm{E}+00 & 0.000 \mathrm{E}+00 & 0.000 \mathrm{E}+00 & 0.000 \mathrm{E}+00 & 0.000 \mathrm{E}+00 & 0.000 \mathrm{E}+00 & 0.000 \mathrm{E}+00\end{array}$ Water-dep. $\quad \begin{array}{llllllll}0.000 E+00 & 0.000 E+00 & 0.000 E+00 & 0.000 E+00 & 0.000 E+00 & 0.000 E+00 & 0.000 E+00 & 0.000 E+00\end{array}$

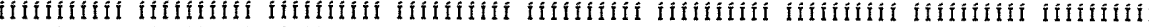

$\begin{array}{lllllllll}\text { Total } & 0.000 E+00 & 0.000 E+00 & 0.000 E+00 & 0.000 E+00 & 0.000 E+00 & 0.000 E+00 & 0.000 E+00 & 0.000 E+00\end{array}$

Water-ind. $=$ = Water-independent Water-dep. $==$ Water-dependent

\begin{tabular}{|c|c|}
\hline Attachment & Sheet No. 1 \\
\hline Originator: S.W. Clark & Date \\
\hline M. W. Perrott & Date \\
\hline $0100 F-C A-V 0303$ & Rev. No. \\
\hline
\end{tabular}




\section{ATTACHMENT 6}

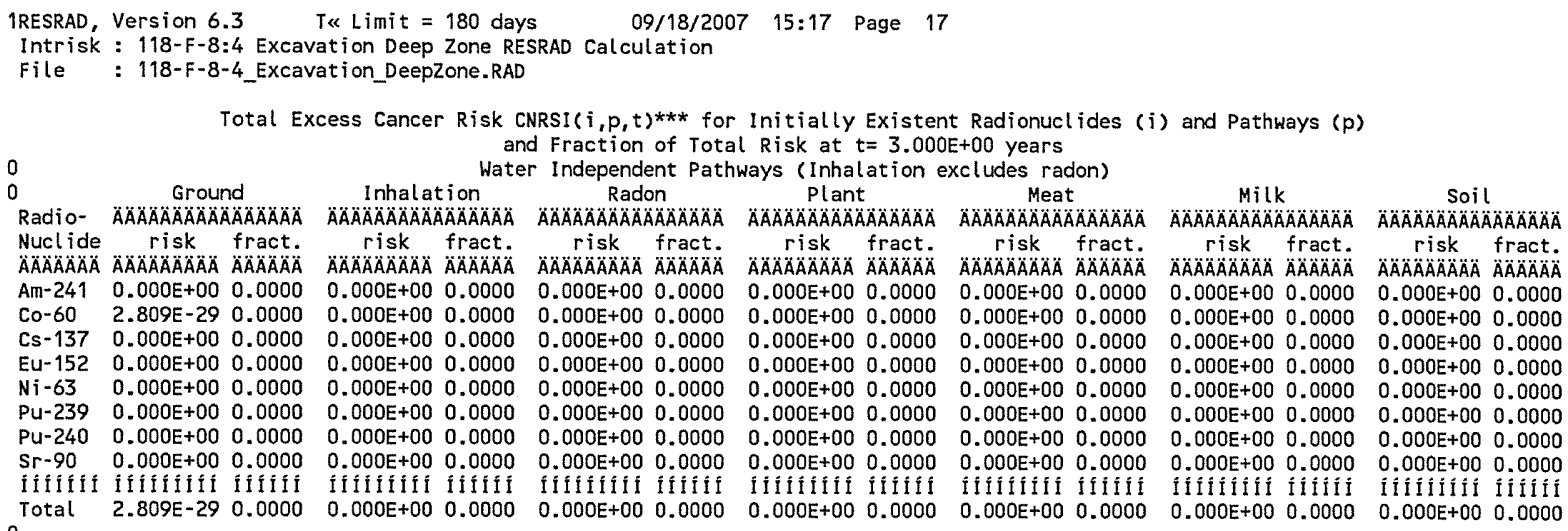

Total Excess Cancer Risk CNRSI $(i, p, t) * * *$ for Initially Existent Radionuclides (i) and Pathways $(p)$

and Fraction of Total Risk at $t=3.000 E+00$ years

\begin{tabular}{|c|c|c|c|c|c|c|c|c|c|c|c|c|c|}
\hline & \multicolumn{13}{|c|}{ Water Independent Pathways (Inhalation excludes radon) } \\
\hline & Grou & & and & tion & Rado & & Plan & & Meat & & Mill & & Soil \\
\hline dio- & $\triangle \triangle A ̈ A ̈ A ̈ A$ & ÄÄÄÃÄÃA & ÄÄÄÄĂÄÄ & $\triangle A O A O A ̈ A ̈ A ̈$ & AÄÄAAOÄÄ & ÄÄÄÄÄÀ & $\triangle A A A A A A A A A ̈$ & ÄÄÄÄÄ & AAÄAAAAAAAA & ÄÄÄÄÄÄ & $\triangle A A O A A O A O A O A$ & $\triangle A O A O A O A ̈ A ̈$ & 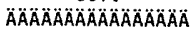 \\
\hline $\begin{array}{l}\text { Nuclide } \\
A \triangle A A A A A B A\end{array}$ & $\begin{array}{c}\text { risk } \\
A A A A A A A A A B A\end{array}$ & $\begin{array}{l}\text { fract. } \\
A A A B A A B\end{array}$ & $\begin{array}{c}\text { risk } \\
\text { AAAAAAAAAAA }\end{array}$ & $\begin{array}{l}\text { fract. } \\
\triangle A A A A A B\end{array}$ & $\begin{array}{c}\text { risk } \\
\text { AAAAAAAAAOA }\end{array}$ & $\begin{array}{l}\text { fract. } \\
\text { ÄÄÄÄÄA }\end{array}$ & $\begin{array}{c}\text { risk } \\
\text { ÄAAAÄÄÄÄ }\end{array}$ & $\begin{array}{l}\text { fract. } \\
A ̈ A ̈ A ̈ A ̈ A ̈\end{array}$ & $\begin{array}{c}\text { risk } \\
\text { ÄAAAAAAÄ }\end{array}$ & $\begin{array}{l}\text { fract. } \\
\text { ÄÄÄÄA }\end{array}$ & $\begin{array}{c}\text { risk } \\
\text { ÄÄÄÄÄÄÄÄ }\end{array}$ & $\begin{array}{l}\text { fract. } \\
A B A B A B A B\end{array}$ & $\begin{array}{r}\text { risk fract. } \\
\text { ÄÄÄÄÄÄÄA ÄÄÄÄÄ }\end{array}$ \\
\hline$A m-241$ & $0.000 E+00$ & 0.0000 & $0.000 \mathrm{E}+00$ & 0.0000 & $0.000 \mathrm{E}+00$ & 0.0000 & $0.000 E+00$ & 0.0000 & $0.000 E+00$ & 0.0000 & $0.000 \mathrm{E}+00$ & 0.0000 & $0.000 E+00 \quad 0.0000$ \\
\hline Co- 60 & 2.80 & 0.0000 & $0.000 E+00$ & 0.0000 & $0.000 \mathrm{E}+00$ & & $0.000 E+00$ & 0 & $E+00$ & & $+\infty$ & & +000.0000 \\
\hline & $E+00$ & 0.0000 & $0.000 E+00$ & 0.0000 & $0.000 E+00$ & 0 & $0.000 E+00$ & 100 & $30 E+00$ & 100 & $+\infty 0$ & 10 & +000.000 \\
\hline & $0.000 E+00$ & 0.0000 & $0.000 \mathrm{E}+00$ & 0.0000 & $0.000 E+00$ & 0.0000 & $0.000 E+00$ & 0 & $E+00$ & 000 & +00 & 0. & +000. \\
\hline & O & 0.0000 & 0.000 & 0.0000 & & & 0 . & & & & $+\infty$ & 0. & $+00 \quad 0.0000$ \\
\hline & 0 & 0.0000 & $.000 E+00$ & 0.0000 & $0.000 E+00$ & 0. & $0.000 \mathrm{E}+00$ & 00 & $00 E+00$ & 0 & 0.0 & 0 & 000 \\
\hline Pu-240 & $0.000 E+00$ & 0.0000 & $.000 E+00$ & 0.0000 & $D E+00$ & 0.0 & $0.000 E+00$ & 0.0 & $0.000 E+00$ & 000 & +00 & 0.0 & $+00 \quad 0.0000$ \\
\hline & & & & & & & & & & & 30 & 0.8 & $+00 \quad 0.0000$ \\
\hline & & & & & & & & & & & & & \\
\hline & -29 & 0.0000 & $10 E+00$ & 0.0000 & 00 & & $0 E+00$ & 0.0000 & $+\infty$ & 0.0000 & $=+00$ & 0.0 & $=000.0$ \\
\hline
\end{tabular}

Total Excess Cancer Risk CNRSI $(i, p, t) * * *$ for Initially Existent Radionuclides $(i)$ and Pathways $(p)$ and Fraction of Total Risk at $t=3.000 E+00$ years

Water Dependent Pathways

\begin{tabular}{|c|c|c|c|c|c|c|c|c|c|c|c|c|c|c|}
\hline & $\begin{array}{r}\text { Wa } \\
\triangle A A ̈ A ̈ A ̈ A ̈\end{array}$ & $\triangle A A ̈ A$ & AOA & $\triangle \triangle A A A A ̈$ & $\begin{array}{r}\text { Rado } \\
\triangle A ̈ A ̈ A ̈ A ̈ A ̈ A ̈ A\end{array}$ & On & $\begin{array}{r}P l a n \\
\triangle A B A O A A O A A\end{array}$ & ÄÄÄÄÄÄA & $\begin{array}{r}M e \\
A \cap A B A ̈ A ̈ A\end{array}$ & $\ddot{A} \ddot{A} A ̈ A ̈ A ̈$ & $\begin{array}{r}M i l \\
\triangle A A A A A A A O\end{array}$ & $\triangle A O A O A B A ̈$ & $\begin{array}{r}\text { All pat } \\
A A A B A B A O A O A\end{array}$ & 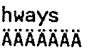 \\
\hline & sk & $\begin{array}{l}\text { fract. } \\
\text { frABABA }\end{array}$ & risk & fract. & risk & fract. & risk & & risk & & risk & & & \\
\hline & 8.7 & & & & m, & & & & & & $E-18$ & & & \\
\hline & 2.7 & & & & 10 & & & & & & & & & \\
\hline & & & & & +00 & & & & & & & & & \\
\hline & & & & & $E+\infty 0$ & & & & 30 & & .00 & & & \\
\hline & 5.0 & & & & 30 & & & & & & & & & \\
\hline & 3.5 & & & & 00 & & & & & & & & & \\
\hline & & & & & & & & & & & & & & \\
\hline & & & & & & & & & & & & & & \\
\hline & & & & & & & & & & & & & & \\
\hline & & & & & .00 & & 7 & & & & 08 & & & \\
\hline
\end{tabular}

***CNRSI $(i, p, t)$ includes contribution from decay daughter radionuclides

$\begin{array}{ll}\text { Attachment } \frac{6}{2} & \text { Sheet No. } 17 \text { of } 41 \\ \text { Originator: } \frac{11}{\text { S.W. Clark }} & \text { Date } \\ \text { Chk'd By } & \text { Date } \\ \text { Calc. No. Nerrott } & \text { Rev. No. } \quad 0\end{array}$




\section{ATTACHMENT 6}

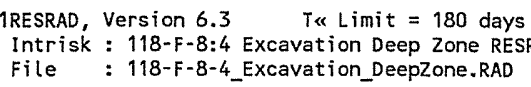

Attachment 6 


\section{ATTACHMENT 6}

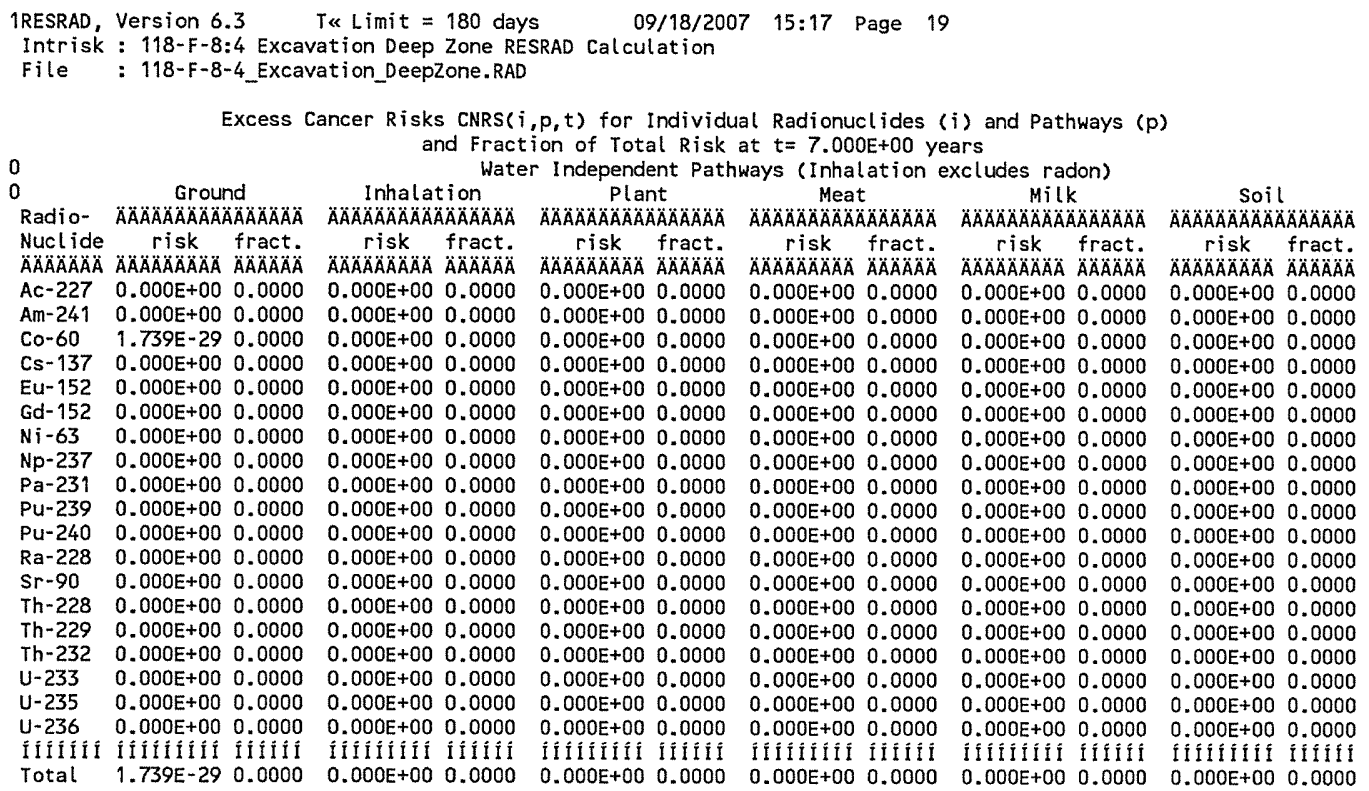

\footnotetext{
Attachment
} 6 Sheet No. 19 of 41 Date Date 


\section{ATTACHMENT 6}

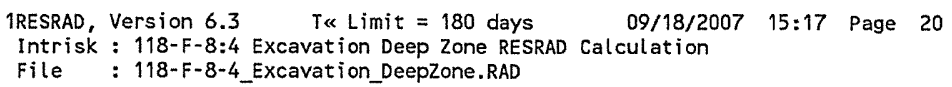

Water Dependent Pathways

\begin{tabular}{|c|c|c|c|c|c|c|c|c|c|c|c|c|}
\hline \multirow{2}{*}{$\begin{array}{l}\text { Radio- } \\
\text { Nuclide } \\
\text { ÄÄÄÄÄÄÄÄ }\end{array}$} & \multicolumn{2}{|c|}{$\begin{array}{c}\text { Water } \\
\text { ÄÄAAÄÄÄÄÄÄÄÄÄÄ }\end{array}$} & \multicolumn{2}{|c|}{$\begin{array}{c}\text { Fish } \\
\text { ĀAAAAAAAAAAAAAAOA }\end{array}$} & \multicolumn{2}{|c|}{$\begin{array}{c}\text { Plant } \\
\text { ALAAAOAOAOAAAAOÄÄ }\end{array}$} & \multicolumn{2}{|c|}{$\frac{\text { Meat }}{\text { ÄÄÄÄÄÄÄÄÄÄÄÄÄ }}$} & \multicolumn{2}{|c|}{ 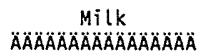 } & \multicolumn{2}{|c|}{$\begin{array}{r}\text { All Pathways** } \\
\text { AAAOAAOAAAAAAAAAOAOAAA }\end{array}$} \\
\hline & $\begin{array}{c}\text { risk } \\
\text { ÄAÄÄÄÄÄÄ }\end{array}$ & $\begin{array}{l}\text { fract. } \\
\ddot{A A B A ̈ A ̈ A ̈ A ̈}\end{array}$ & $\begin{array}{c}\text { risk } \\
\text { ÄAAÄÄÄÄÄ }\end{array}$ & 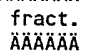 & $\begin{array}{c}\text { risk } \\
\triangle A \cap A ̈ A ̈ A ̈ A \cap A\end{array}$ & $\begin{array}{l}\text { fract. } \\
\ddot{A} \ddot{A} \ddot{A} \ddot{A} \ddot{A} \ddot{A}\end{array}$ & $\begin{array}{c}\text { risk } \\
\text { AAAAAAAOAOÄ }\end{array}$ & $\begin{array}{l}\text { fract. } \\
\text { ÄÄÄÄÄÄ }\end{array}$ & risk & $\begin{array}{l}\text { fract. } \\
\triangle A A A B A O A\end{array}$ & risk & fract. \\
\hline$A C-227$ & & & & & & & & & & & & \\
\hline & $E+00$ & 000 & $1 O E+00$ & 000 & $100 E+00$ & 0000 & $0.000 E+00$ & 0.0000 & $000 E+00$ & 0.0000 & $+\infty$ & \\
\hline & & & & & & & & 0 & & & & \\
\hline & & & & & & & & & & & & \\
\hline & $0.000 E+00$ & & $=+\infty$ & 100 & & & & & & & & \\
\hline & 0. & & & & & & & & & & & \\
\hline & & & & & & & & & & & & \\
\hline & & & & & & & & & & & & \\
\hline & & & & & & & & & & & & \\
\hline & & & 00 & & & & & & & & & 0 \\
\hline & & & 00 & & & 0 & 0. & & & & & 0 \\
\hline & & & & & & & & & & & & 000 \\
\hline & & & & & & & & & & & & \\
\hline & & & & & -24 & & & & & & & \\
\hline & & & & & & & & & -23 & & & 000 \\
\hline & & & & & & & & & & & & \\
\hline & $E-15$ & & -18 & & & & & & & & & 000 \\
\hline & & & & & DE-14 & & & 0. & $E-15$ & & 5. & 0.0000 \\
\hline & & & & & & & & & & & & \\
\hline & & & & & & & & & & & & \\
\hline Total & $43 E-07$ & 7835 & $.556 \mathrm{E}-08$ & 27 & $E-07$ & & $E-08$ & 0.0207 & $2.854 E-08$ & 0.0230 & $:-06$ & 1.0000 \\
\hline
\end{tabular}

** Sum of water independent ground, inhalation, plant, meat, milk, soil

and water dependent water, fish, plant, meat, milk pathways

Excess Cancer Risks CNRS9( $i r n, i, t)$ and CNRSOW(irn, $i, t$ ) for Inhalation of

Radon and its Decay Products at $t=7.000 \mathrm{E}+00$ years

$0 \quad$ Radionuclides

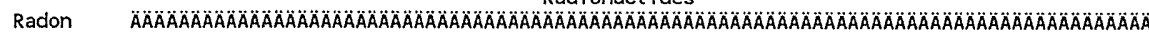

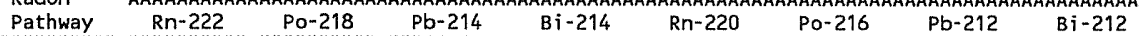

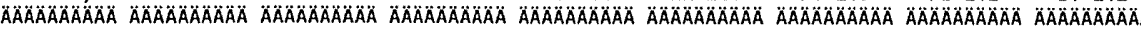

$\begin{array}{lllllllll}\text { Water }- \text { ind. } 0.000 E+00 & 0.000 E+00 & 0.000 E+00 & 0.000 E+00 & 0.000 E+00 & 0.000 E+00 & 0.000 E+00 & 0.000 E+00\end{array}$ $\begin{array}{lllllllll}\text { Water-dep. } & 0.000 E+00 & 0.000 E+00 & 0.000 E+00 & 0.000 E+00 & 0.000 E+00 & 0.000 E+00 & 0.000 E+00 & 0.000 E+00\end{array}$

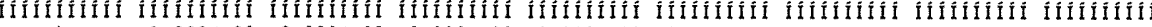

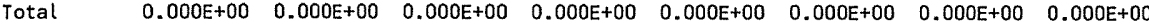

Water-ind. $==$ Water-independent $\quad$ Water-dep. $==$ Water-dependent

\begin{tabular}{|c|c|}
\hline \multirow{2}{*}{\multicolumn{2}{|c|}{$\begin{array}{l}\text { Attachment } \frac{6}{\text { S.W. Clark }} \text { Sheet No. } 20 \text { of } 41 \\
\text { Originator: }\end{array}$}} \\
\hline & \\
\hline M. W. Perrott & \\
\hline $0100 \mathrm{~F}-\mathrm{CA}-\mathrm{V} 0303$ & Rev \\
\hline
\end{tabular}




\section{ATTACHMENT 6}

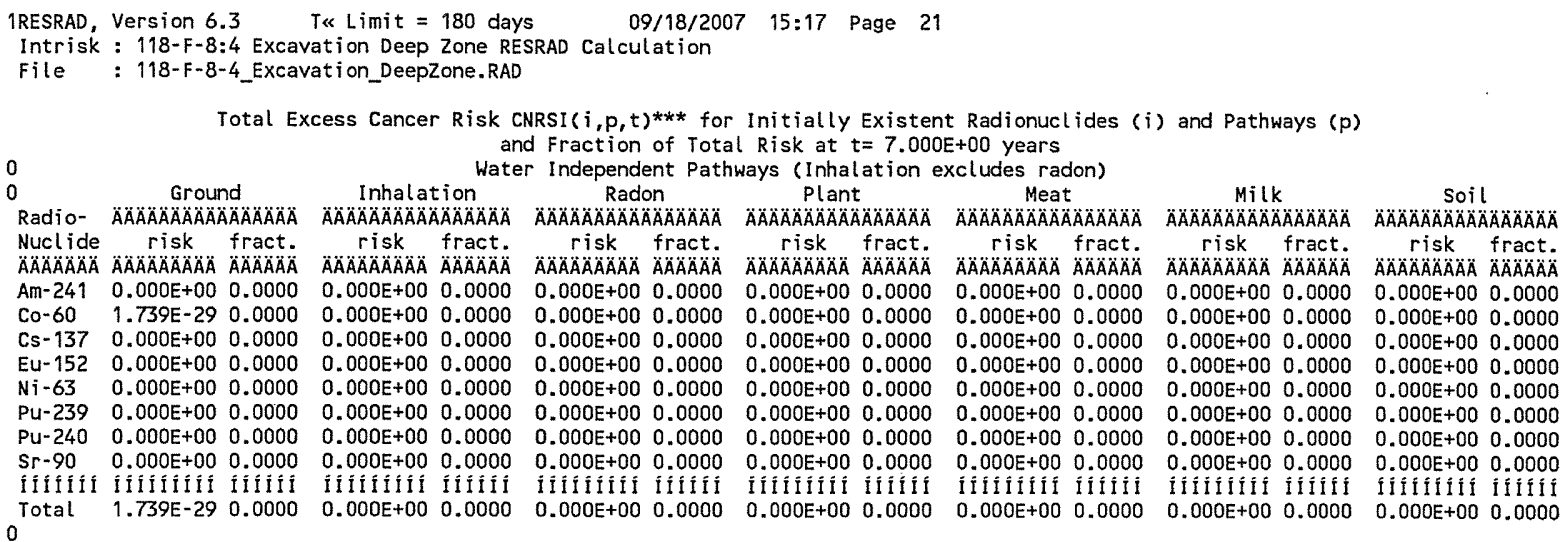

Total Excess Cancer Risk CNRSI $(i, p, t)^{* * *}$ for Initially Existent Radionuclides (i) and Pathways $(p)$ and Fraction of Total Risk at $t=7.000 E+00$ years

Water Dependent Pathways

\begin{tabular}{|c|c|c|c|c|c|c|c|c|c|c|c|c|c|c|}
\hline \multirow{3}{*}{$\begin{array}{l}\text { Radio- } \\
\text { Nuclide } \\
\text { ÄÄÄÄÄÄÄ }\end{array}$} & \multicolumn{2}{|c|}{ Water } & 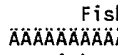 & $\begin{array}{l}\text { h } \\
\ddot{A} A \ddot{A} \ddot{A} \ddot{A} A \ddot{A}\end{array}$ & $\begin{array}{r}\text { Rad } \\
A A A A B A A A O A\end{array}$ & Radon & \multicolumn{2}{|c|}{ 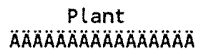 } & \multicolumn{2}{|c|}{$\begin{array}{c}\text { Meat } \\
\text { ÄÄÄÄÄÄÄÄÄÄÄÄÄÄ }\end{array}$} & \multicolumn{2}{|c|}{ 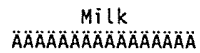 } & \multicolumn{2}{|c|}{$\begin{array}{c}\text { All pathways } \\
\text { ALAAAAAAAAAAAAAAAÄ }\end{array}$} \\
\hline & & & & & & & & fract. & & f & $=0$ & & nit & the \\
\hline & & & & & & & & & & & & & & \\
\hline & & & & & & & & & & & & & & \\
\hline & & & & & & & & & & & & & & \\
\hline & & & & & & & & & & & & & & \\
\hline & & & & & & & & & & & & & & \\
\hline & & & & 0. & & & & & & & & & & \\
\hline & & & & & & & & & & & & & & \\
\hline & & & & & & & & & & & & & & \\
\hline & & & & & & & & & & & & & & \\
\hline & & & & & & & & & & & & & & \\
\hline & & & & & & & & & 00 & & $=00$ & & $1.244 E-06$ & 1.0000 \\
\hline
\end{tabular}

$* * *$ CNRSI $(i, p, t)$ includes contribution from decay daughter radionuclides

\begin{tabular}{|c|c|}
\hline Attachment & Sheet No \\
\hline Originator: S.W. Clark & ate \\
\hline M. W. Perrott & Date \\
\hline Calc. No. $\quad 0100 F-C A-V 0303$ & Rev. No. \\
\hline
\end{tabular}




\section{ATTACHMENT 6}

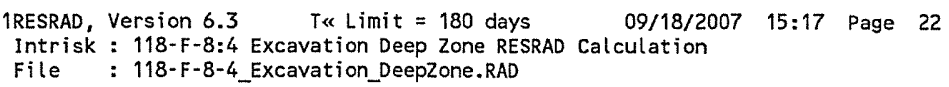

Water-ind. $=$ Water-independent $\quad$ Water-dep. $=$ Water-dependent

\begin{tabular}{|c|c|}
\hline Attachment & Sheet No. 22 of 41 \\
\hline Originator: S.W. Clark & Date \\
\hline M. W. Perrott & Date \\
\hline $0100 F-C A-V 0303$ & Rev. No. 0 \\
\hline
\end{tabular}




\section{ATTACHMENT 6}

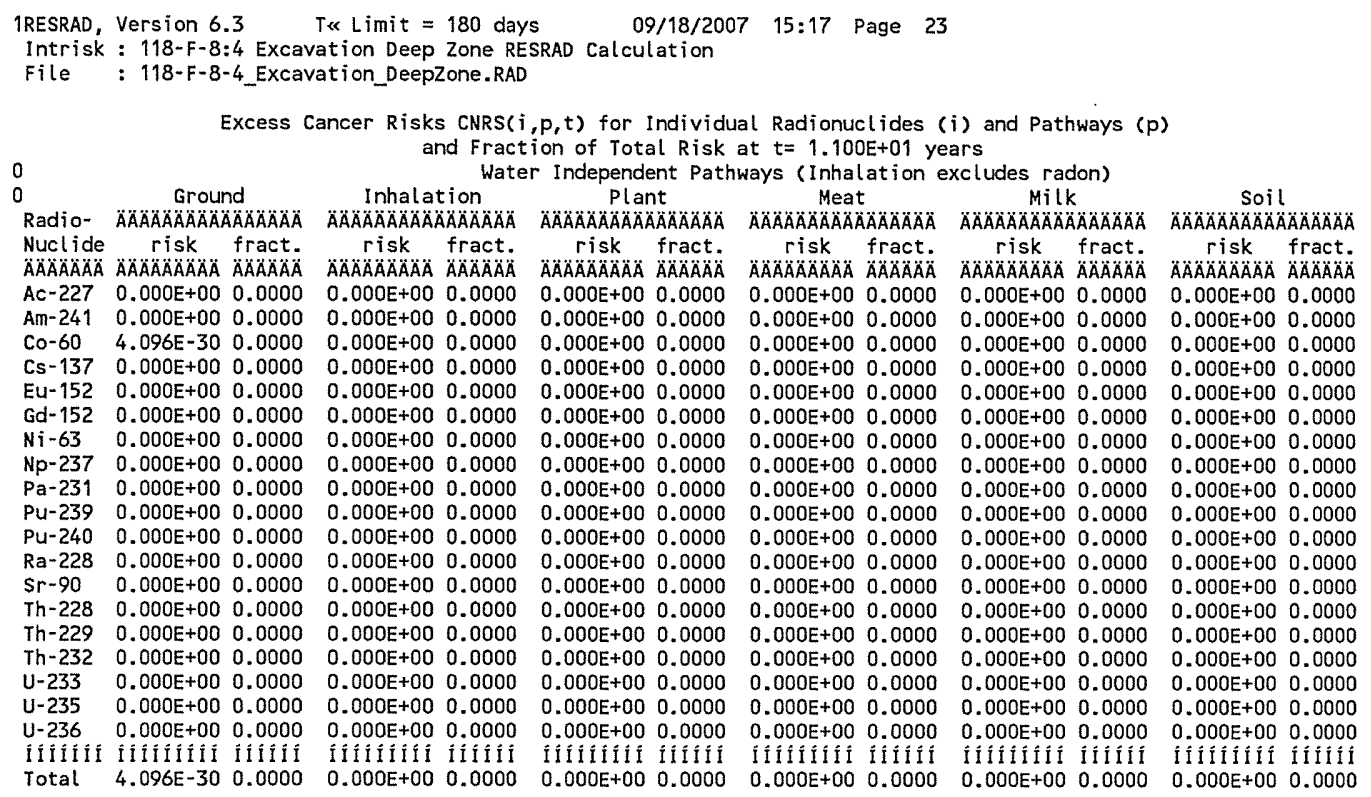

\footnotetext{
Attachment
} 6 


\section{ATTACHMENT 6}

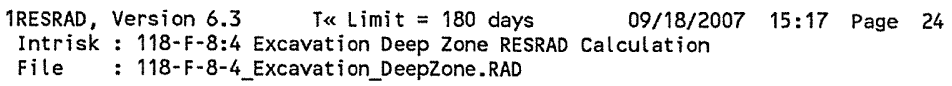

Water Dependent Pathways

\begin{tabular}{|c|c|c|c|c|c|c|c|c|c|c|c|c|}
\hline \multirow[b]{2}{*}{$\begin{array}{l}\text { Nuclide } \\
\text { ÄÄÄÄÄÄÄ }\end{array}$} & \multicolumn{2}{|c|}{ Water } & \multicolumn{2}{|c|}{ Fish } & \multicolumn{2}{|c|}{ Plant } & \multicolumn{2}{|c|}{$\begin{array}{c}\text { Meat } \\
\text { ÄÄÄÄAAAAAAOAOÄÄ }\end{array}$} & \multicolumn{2}{|c|}{ Milk } & \multicolumn{2}{|c|}{$\begin{array}{r}\text { All Pathways** } \\
\text { ALAAAOAOAOAOA }\end{array}$} \\
\hline & $\begin{array}{c}r i s k \\
A \cap A O A O A O A O A O\end{array}$ & $\begin{array}{l}\text { fract. } \\
\ddot{A} A \ddot{A} \ddot{A} \ddot{A} \ddot{A}\end{array}$ & 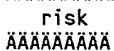 & $\begin{array}{l}\text { fract. } \\
\qquad A \cap A C A B A\end{array}$ & $\begin{array}{l}\text { risk } \\
\triangle A O A B A B A B A\end{array}$ & 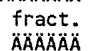 & $A \ddot{A}$ & $\begin{array}{l}\text { fract. } \\
\text { ÄÄÄÄÄ }\end{array}$ & $\begin{array}{l}\text { risk } \\
\text { rAAAAAÄ }\end{array}$ & $\begin{array}{l}\text { fract. } \\
\text { ÄAABAÄ }\end{array}$ & risk & fract. \\
\hline$A C-227$ & & & & 0.0000 & & 0.0000 & & & & & & \\
\hline & $0 E+00$ & 000 & $D O E+0 O$ & 0.0000 & $0.000 E \div 00$ & 0.0000 & $000 E+00$ & 0.0000 & $0.000 E+00$ & 0.0000 & $=00$ & 0.0000 \\
\hline & & & & & & 10 & 11 & 10 & & & & 0002 \\
\hline & & & & & & & & & & & & \\
\hline & $0 E+00$ & & $D E+00$ & 0.0000 & & & & 00 & & 0.0000 & & \\
\hline & 0.0 & 0.0 & $E+00$ & 0. & $+\infty 0$ & & +00 & & $0 E+00$ & & $=0$ & 0000 \\
\hline & & & & & & & & & & & & \\
\hline & & & +00 & & & & & 00 & & & & \\
\hline & 1.6 & & -20 & & & & & & -21 & & & 100 \\
\hline & 0.0 & & +00 & & 0 & & 00 & & .00 & & & \\
\hline & & & $+\infty$ & 0.0 & & & 0.0 & 0. & & 0. & & \\
\hline & 8.8 & & -23 & & & & & & 24 & 10 & 1. & 100 \\
\hline & 0. & & 08 & & & & & & & & & \\
\hline & & & -24 & 0.0 & & & & & & & & \\
\hline & 1.48 & & -21 & 0.0 & & & 22 & & -23 & & 1.8 & 0 \\
\hline & $7865-77$ & & -24 & & & & & & & & & \\
\hline & & & & & & & & & & & & \\
\hline & & & $E-15$ & 0. & $E-14$ & & -16 & & $E-15$ & o & 6.7 & 0.000 \\
\hline & & & -14 & & & & & & & & & \\
\hline & 111111 & & $1+1111+1 / 2$ & & & & & & & & & \\
\hline otal & $065 E-06$ & 0.7831 & $65 E-08$ & 0.0527 & $33 E-07$ & 0.1201 & $2.813 E-08$ & 0.0207 & $3.177 E-08$ & 0.0234 & -06 & 1.00 \\
\hline
\end{tabular}

** Sum of water independent ground, inhalation, plant, meat, milk, soil and water dependent water, fish, plant, meat, milk pathways

Excess Cancer Risks CNRS9 (irn, $i, t)$ and CNRS9W( $i r n, i, t)$ for Inhatation of Radon and its Decay Products at $t=1.100 E+01$ years

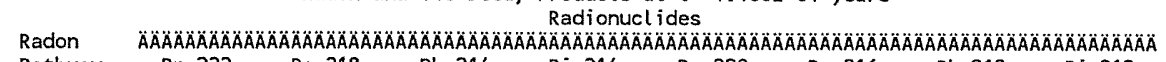
Pathway Rn-222 Po-218 Pb-214 $\quad$ Bi-214 $\quad$ Rn-220 Po-216 Pb-212 Bi-212

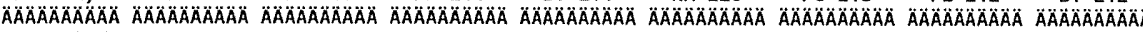
$\begin{array}{lllllllll}\text { Water-ind. } 0.000 E+00 & 0.000 E+00 & 0.000 E+00 & 0.000 E+00 & 0.000 E+00 & 0.000 E+00 & 0.000 E+00 & 0.000 E+00\end{array}$

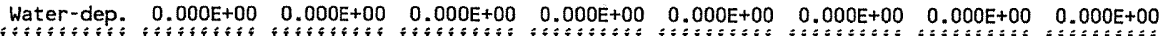

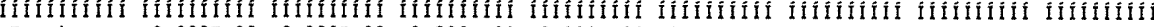

$\begin{array}{lllllllll}\text { Total } & 0.000 E+00 & 0.000 E+00 & 0.000 E+00 & 0.000 E+00 & 0.000 E+00 & 0.000 E+00 & 0.000 E+00 & 0.000 E+00\end{array}$

Water-ind. == Water-independent Water-dep. == Water-dependent

\begin{tabular}{|c|c|c|}
\hline \multirow{2}{*}{\multicolumn{3}{|c|}{$\begin{array}{ll}\text { Attachment } & 6 \\
\text { Oriainator: S.W Clark }\end{array}$}} \\
\hline & & \\
\hline Chk'd By & M. W. Perrott & Date \\
\hline Calc. No. & $0100 F-C A-V 0303$ & Rev \\
\hline
\end{tabular}




\section{ATTACHMENT 6}

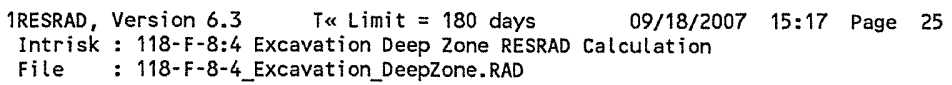

Total Excess Cancer Risk CNRSI $(i, p, t) * * *$ for Initially Existent Radionuclides (i) and Pathways $(p)$

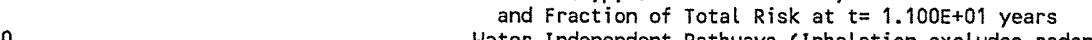

Total Excess Cancer Risk CNRSI $(i, p, t) * * *$ for Initially Existent Radionuclides $(i)$ and Pathways $(p)$ and Fraction of rotal Risk at $\mathrm{t}=1.100 \mathrm{E}+01$ years

Water Dependent Pathways

\begin{tabular}{|c|c|c|c|c|c|c|c|c|c|c|c|c|c|c|}
\hline \multirow[b]{2}{*}{$\begin{array}{l}\text { Nuclide } \\
\text { AAAAAAAA }\end{array}$} & \multicolumn{2}{|c|}{$\begin{array}{c}\text { Water } \\
\text { ÄAAAAAAAAAOAAÄÄÄ }\end{array}$} & \multicolumn{2}{|c|}{ 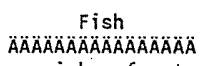 } & \multicolumn{2}{|c|}{$\begin{array}{c}\text { Radon } \\
\text { AAAAAAAAAAAAAAAAAOA }\end{array}$} & \multicolumn{2}{|c|}{ 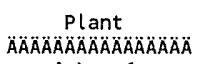 } & \multicolumn{2}{|c|}{$\begin{array}{c}\text { Meat } \\
\text { ÄÄÄÄÄÄÄÄÄÄÄÄÄA }\end{array}$} & \multicolumn{2}{|c|}{$\begin{array}{c}M i l k \\
\text { ÄÄÄAOAOAOAOAOAOAOAOA }\end{array}$} & \multicolumn{2}{|c|}{$\begin{array}{c}\text { All pathways } \\
\text { ÄÄAAAAAAAAAAAAA }\end{array}$} \\
\hline & $\ddot{A}$ & $\begin{array}{l}\text { fract. } \\
\text { ÄÄÄÄÄA }\end{array}$ & $\begin{array}{l}\text { risk } \\
\text { ÄÄÄÄÄA }\end{array}$ & $\begin{array}{l}\text { fract. } \\
\ddot{A} \ddot{A} \ddot{A} \ddot{A} \ddot{A} A\end{array}$ & risk & $\begin{array}{l}\text { fract. } \\
\triangle A A ̈ A A A A O A\end{array}$ & $\begin{array}{l}\text { risk } \\
\text { ÄÄÄÄÄÄ }\end{array}$ & $\begin{array}{l}\text { fract. } \\
\dddot{A} A A A A A B A\end{array}$ & 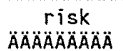 & & risk & & risk & \\
\hline 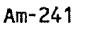 & & & & & & & & & & & & & & \\
\hline & & & & & & & & & & & & & & \\
\hline & & & & & & & & & & & & & & \\
\hline & & & & & & & & & & & & & & \\
\hline & & & & & & & & & & & & & & \\
\hline & & & & & & & & & & & & & & \\
\hline & & & & & & & & & & & & & & \\
\hline & & & & & & & & & & & & & & \\
\hline & & & & & & & & & & & & & & \\
\hline & & & 00 & & $E+00$ & & 0 & & -00 & & $=-0 c$ & 234 & & \\
\hline
\end{tabular}

***CNRSI $(i, p, t)$ includes contribution from decay daughter radionuclides

\begin{tabular}{|c|c|}
\hline Attachment & Sheet No. 2 \\
\hline Originator: S.W. Clark & Date \\
\hline M. W. Perrott & Date \\
\hline $0100 \mathrm{~F}-\mathrm{CA}-\mathrm{V} 0303$ & Rev. No. \\
\hline
\end{tabular}




\section{ATTACHMENT 6}

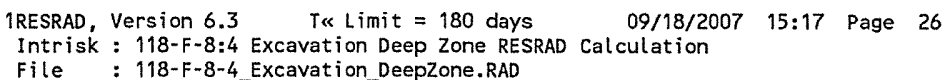

Water-ind. $==$ Water-independent $\quad$ Water-dep. $==$ Water-dependent

$\begin{array}{ll}\text { Attachment } & \frac{6}{\text { Sheet No. } 26 \text { of } 41} \\ \text { Originator: } \frac{\text { S.W. Clark }}{\text { M.W. Perrott }} & \text { Date } \\ \text { Chk'd By } & \text { Date } \\ \text { Calc. No. } \frac{\text { O100F-CA-V0303 }}{\text { Rev. No. _ }} & \end{array}$




\section{ATTACHMENT 6}

\begin{tabular}{|c|c|c|c|c|c|c|c|c|c|c|c|c|}
\hline \multirow[t]{2}{*}{$\begin{array}{l}\text { 1RESRAD, } \\
\text { Intrisk } \\
\text { File }\end{array}$} & $\begin{array}{l}\text { Version } \\
: 118-\mathrm{F}- \\
: 118-\mathrm{F}\end{array}$ & $\begin{array}{l}4 \text { EXC } \\
4 \_E x C\end{array}$ & $\begin{array}{l}\text { Limit } \\
\text { ion Dee } \\
\text { ion_Dee }\end{array}$ & $\begin{array}{l}180 \mathrm{dg} \\
\text { Zone } \mathrm{f} \\
\text { one. Rf }\end{array}$ & $\mathrm{DCal}$ & $\begin{array}{l}18 / 2 \\
\text { tion }\end{array}$ & $15: 17$ & ge & & & & \\
\hline & \multicolumn{12}{|c|}{$\begin{array}{c}\text { Excess Cancer Risks CNRS }(i, p, t) \text { for Individual Radionuclides }(i) \text { and Pathways }(p) \\
\text { and Fraction of Total Risk at } t=4.300 E+01 \text { years }\end{array}$} \\
\hline & Gro & & Inhalat & tion & Plar & & Meat & & Milk & & Soil & \\
\hline & ÄÄ & ÄÄÄÄ & $\triangle A A A A B$ & Ä̈̈̈ & $\triangle A \cap A \cap A O A O A ̈ A ̈ A$ & ÄÄ & $\triangle A \triangle A O A O A B$ & $\triangle A O A O A O A O A ̈$ & 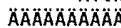 & $\ddot{A} \ddot{A} \ddot{A}$ & גם & 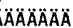 \\
\hline $\begin{array}{l}\text { Nucl ide } \\
\ddot{A} \ddot{A} \ddot{A} \ddot{A} A \ddot{A} \ddot{A}\end{array}$ & & 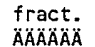 & & & & & $\ddot{\mathrm{A}}$ & $\begin{array}{l}\text { fract. } \\
\text { ÄÄÄÄÄA }\end{array}$ & risk & & risk & $\begin{array}{l}\text { fract. } \\
A B A A A B A\end{array}$ \\
\hline$A c-277$ & 0 & 0.0000 & $0 E+00$ & 0.0 & $0.000 E+00$ & 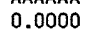 & & & & & & \\
\hline & & & & & & & & & & & & \\
\hline & & & & & & & & 0.0000 & & 0.0 & $+\infty 0$ & 1000 \\
\hline & & & & & & & & & & & & \\
\hline & & & & & -00 & & & 300 & & & & \\
\hline & & 0.0000 & 00 & 0.0 & +00 & 0.0 & & 0. & & 0.0 & & \\
\hline & & & & & & & & & & & & \\
\hline & & & & & & & & 00 & & & & \\
\hline & & & & & & & & 0 & & & & \\
\hline & & & & & & & & & & & & \\
\hline & & & & & 0 & & & & & & & \\
\hline & & 0.0 & +00 & & -00 & 0 & & 0.0 & & 0. & & \\
\hline & & & & & & & & & & & & \\
\hline Th & 0.00 & 0.0 & 0 & & 0.0 & & 0.0 & 0.0 & & & & \\
\hline & & & +00 & & & & & & & & & \\
\hline & & & & & 0.000 & & 0.0 & 0.0000 & +00 & 00 & 0.0 & 000 \\
\hline & +00 & 0.0 & $E+00$ & & $0.000 E+00$ & & $E+\infty$ & 0.0000 & $E+00$ & & $E+00$ & \\
\hline 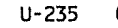 & & & & & & & & & & 0.0 & 0.0 & \\
\hline & & & & 00 & $0.000 \mathrm{E}+00$ & 0.0000 & 0.0 & 0.0000 & 0.0 & 0.0 & $0.000 E+00$ & 0.0000 \\
\hline & & & & & & & & & & & & \\
\hline & & & & & & & & & & & & \\
\hline
\end{tabular}

\begin{tabular}{|c|c|}
\hline Attachment & Sheet No. 27 of 41 \\
\hline Originator: S.W. Clark & Date \\
\hline Chk'd By M.W. Perrott & Date \\
\hline Calc. No. 0100 F-CA-V0303 & Rev. No. \\
\hline
\end{tabular}




\section{ATTACHMENT 6}

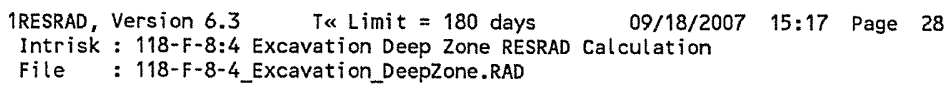

Water Dependent Pathways

\begin{tabular}{|c|c|c|c|c|c|c|c|c|c|c|c|c|}
\hline \multirow[b]{2}{*}{$\begin{array}{l}\text { Nuclide } \\
\triangle A A O A A B A A B A\end{array}$} & \multicolumn{2}{|c|}{ Water } & \multicolumn{2}{|c|}{ 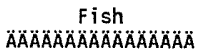 } & \multicolumn{2}{|c|}{ 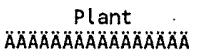 } & \multicolumn{2}{|c|}{$\begin{array}{c}\text { Meat } \\
\text { ÄĂÄÃÄÄÄÄÄÄÄÄ }\end{array}$} & \multicolumn{2}{|c|}{ 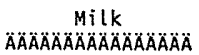 } & \multicolumn{2}{|c|}{$\begin{array}{r}\text { All Pathways** } \\
\text { ÄÄÄÄÄÄÄÄÄÄÄÄ }\end{array}$} \\
\hline & $\begin{array}{c}\text { risk } \\
\text { AAAAAAAÄÄ }\end{array}$ & $\begin{array}{l}\text { fract. } \\
\text { ÄÄÄÄÄÄ }\end{array}$ & $\begin{array}{c}\text { risk } \\
\triangle A \cap A O A B A O A O A ̈\end{array}$ & $\begin{array}{l}\text { fract. } \\
\triangle A B A B A B A\end{array}$ & $\begin{array}{c}\text { risk } \\
\triangle A \cap A ̈ A ̈ A ̈ A ̈ A ̈ A\end{array}$ & $\begin{array}{l}\text { fract. } \\
\text { ÄÄÄÄÄA }\end{array}$ & $\begin{array}{c}\text { risk } \\
\text { AAAAAAAAAA }\end{array}$ & 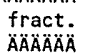 & $\begin{array}{l}\text { risk } \\
\text { AAAAAAAOA }\end{array}$ & 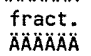 & risk & fract. \\
\hline$A c-227$ & & 0.0000 & & & & & & & & & & \\
\hline $1-241$ & $0 E+00$ & & $000 E+00$ & 0.0000 & $000 E+00$ & 0.0000 & $0 E+00$ & 0.0000 & $000 E+00$ & 0.0000 & & . \\
\hline & & & & & & 0. & & & & & & \\
\hline & & & & & & & & & & & & \\
\hline & $E+00$ & & +00 & & & & & & & & & \\
\hline & 0.0 & & & & & & & & $E+00$ & & & \\
\hline & & & & & & & & & & & & \\
\hline & & & & & & & & & & & & \\
\hline & 9.4 & & & & & & & & & & & 300 \\
\hline & 0.0 & & bo & & & & & & & & & \\
\hline & & & & & Do & & & & 00 & & & \\
\hline & & & & & & & & & & 0. & & 00 \\
\hline & & & & & & & & & & & & \\
\hline & & & -23 & & & & & & & & & \\
\hline & & & & & & & & & -22 & & & 200 \\
\hline & 2. & & & & & & & & & & & \\
\hline & -14 & & & & & & & & & & & \\
\hline & & & & & & 0.0000 & & 0.0 & $E-15$ & 0. & 1. & 0.000 \\
\hline & & & 3 & & & & & & & & & \\
\hline & & & & & & & & & & & & \\
\hline tat & $.202 E-06$ & .7796 & $.037 E-08$ & 0.0521 & $1.848 \mathrm{E}-07$ & 0.1199 & $3.153 \mathrm{E}-08$ & 0.0205 & 4.29 & 0.0279 & $E-06$ & 1.000 \\
\hline
\end{tabular}

** Sum of water independent ground, inhalation, plant, meat, milk, soil and water dependent water, fish, plant, meat, milk pathways

Excess Cancer Risks CNRS9 $(i r n, i, t)$ and CNRSPW(irn, $i, t$ ) for Inhalation of Radon and its Decay Products at $t=4.300 \mathrm{E}+01$ years

Radionucl $i d e s$

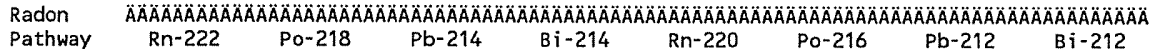
$\begin{array}{llllllll}\text { Pathway Rn-222 } & \mathrm{PO}-218 & \mathrm{~Pb}-214 & \mathrm{~B} i-214 & \mathrm{Rn}-220 & \mathrm{PO}-216 & \mathrm{~Pb}-212 & \mathrm{Bi}-212\end{array}$

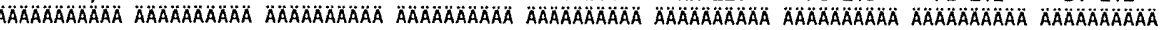
$\begin{array}{lllllllll}\text { Water }- \text { ind. } & 0.000 E+00 & 0.000 E+00 & 0.000 E+00 & 0.000 E+00 & 0.000 E+00 & 0.000 E+00 & 0.000 E+00 & 0.000 E+00\end{array}$

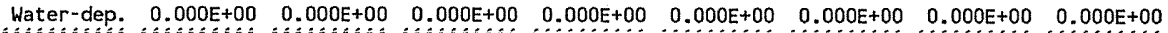

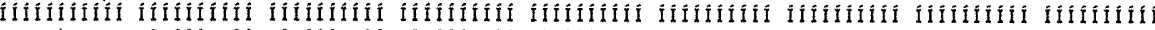

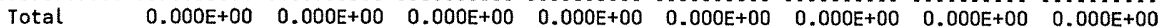

Water-ind. = Water-independent Water-dep. $=$ Water-dependent

\begin{tabular}{|c|c|}
\hline \multirow{2}{*}{\multicolumn{2}{|c|}{$\begin{array}{l}\text { Attachment } \frac{6}{\text { Originator: }} \text { S.W. Clark } \\
\text { Sheet No. } 28 \text { of } 41 \\
\text { Date }\end{array}$}} \\
\hline & \\
\hline M. W. Perrott & \\
\hline $0100 F-C A-V 0303$ & Rev \\
\hline
\end{tabular}




\section{ATTACHMENT 6}

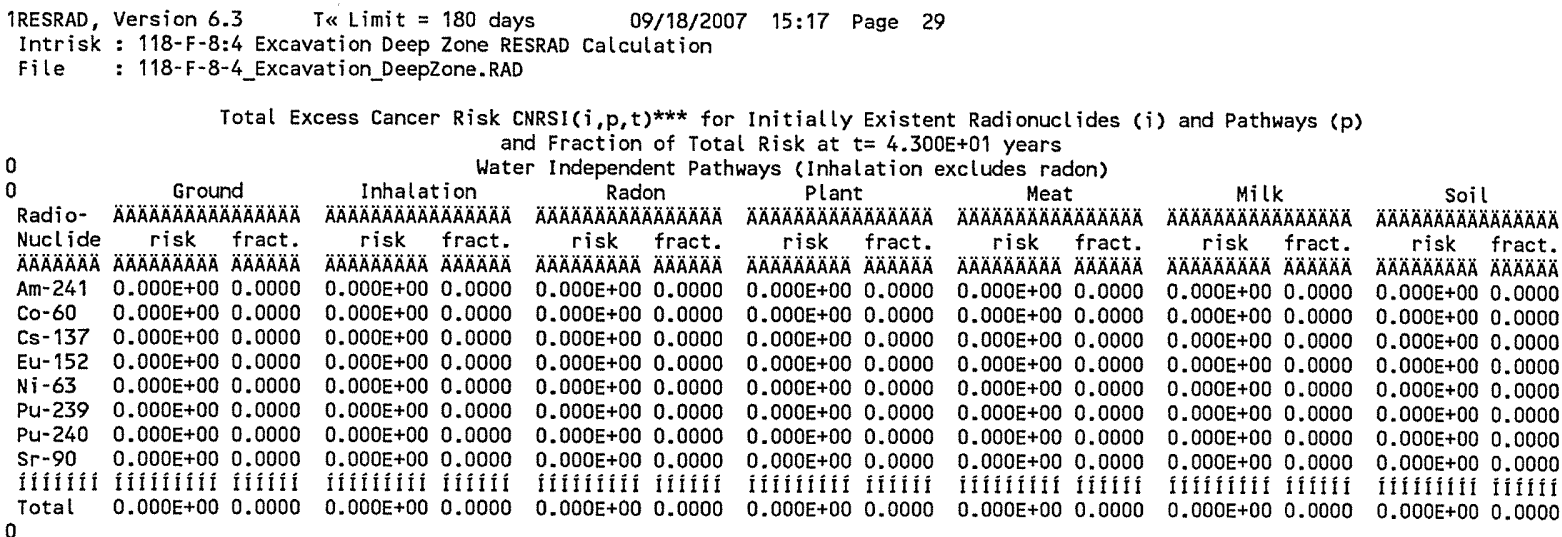

Total Excess Cancer Risk CNRSI $(i, p, t) * * *$ for Initially Existent Radionuclides $(i)$ and Pathways $(p)$ and Fraction of Total Risk at $t=4.300 E+01$ years

Water Dependent Pathways

\begin{tabular}{|c|c|c|c|c|c|c|c|c|c|c|c|c|c|c|}
\hline \multirow{2}{*}{$\begin{array}{l}\text { Radio- } \\
\text { Nucl ide } \\
\text { ÄAAAAAÄ }\end{array}$} & \multicolumn{2}{|c|}{ 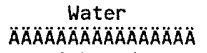 } & \multicolumn{2}{|c|}{$\begin{array}{c}\text { Fish } \\
\text { ÄÄÄÄAAAAAOAOAOAOA }\end{array}$} & \multicolumn{2}{|c|}{ 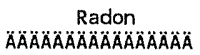 } & \multicolumn{2}{|c|}{ 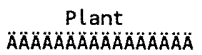 } & \multicolumn{2}{|c|}{$\begin{array}{c}\text { Meat } \\
\text { ÄAAAOAAAAAAAOAOAAA }\end{array}$} & \multicolumn{2}{|c|}{ 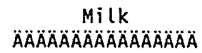 } & \multicolumn{2}{|c|}{$\begin{array}{c}\text { All pathways } \\
\text { AAAAAAAAAAAAAAAAAA }\end{array}$} \\
\hline & & $\begin{array}{l}\text { fract. } \\
\text { ÄAÄÄÄA }\end{array}$ & & & & & & fract & rick & fract. & risk & & risk & $\begin{array}{l}\text { fract. } \\
\text { AAAAAAAA }\end{array}$ \\
\hline & & & & & & & & & & & & & & \\
\hline & & & & & & & & & & & & & & \\
\hline & & & & & & & & & & & & & & \\
\hline & & & & & & & & & & & & & & \\
\hline & & & & & & & & & & & & & & \\
\hline & & & & & & & & & & & & & & \\
\hline & & & & & & & & & & & & & & \\
\hline & & & & & & & & & & & & & & \\
\hline & & & & & & & & & & & & & & \\
\hline & - & 0.7796 & $.05 / E-08$ & 0.0521 & 8.00 & & $E-U /$ & 0.1199 & $3.153 E-08$ & 0.0205 & $4.294 E-08$ & 0.0279 & $1.541 \mathrm{E}-06$ & 1.00 \\
\hline
\end{tabular}

***CNRSI $(i, p, t)$ includes contribution from decay daughter radionuclides

\begin{tabular}{|c|c|c|}
\hline \multirow{2}{*}{\multicolumn{3}{|c|}{$\begin{array}{l}\text { Attachment } \\
\text { Originator: S.W. Clark }\end{array}$}} \\
\hline & & \\
\hline Chk'd By & M.W. Perrott & Date \\
\hline Calc. No. & $0100 F-C A-V 0303$ & Rev. No. \\
\hline
\end{tabular}




\section{ATTACHMENT 6}

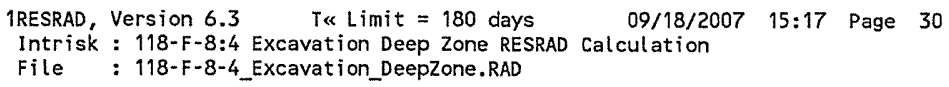

\begin{tabular}{|c|c|}
\hline Attachment & Sheet No. 30 of 41 \\
\hline Originator: $\overline{\mathrm{S} . \mathrm{W} \text {. Clark }}$ & Date \\
\hline M.W. Perrott & Date \\
\hline $0100 F-C A-V 0303$ & Rev. No. 0 \\
\hline
\end{tabular}




\section{ATTACHMENT 6}

\begin{tabular}{|c|c|c|c|c|c|c|c|c|c|c|c|c|}
\hline $\begin{array}{l}\text { 1RESRAD, } \\
\text { Intrisk } \\
\text { File }\end{array}$ & $\begin{array}{l}\text { Version } \\
: 118-F- \\
: 118-F-\end{array}$ & Exc & $\begin{array}{l}\text { ion Dee } \\
\text { ion_Dee }\end{array}$ & ne.RA & D Calc & tion & $r$ & ge & & & & \\
\hline 0 & & Lacess & Ris & $\begin{array}{l}\text { Fra } \\
\text { Wa }\end{array}$ & Indeper & & Inha & & & & & \\
\hline & & & Inhalat & tion & Plar & & Mea & & $\mathrm{Mill}$ & & $\because \cdots$ & \\
\hline & & & $A A A B A$ & $\ddot{A} A ̈ A ̈ A ̈$ & 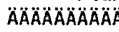 & $\triangle A ̈ A ̈ A ̈ A ̈$ & 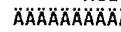 & ÄÄÄA & $\triangle A O A A A A A B$ & $\triangle O A O A O A O A$ & $\triangle A A A A A A A A$ & IAAA \\
\hline $\begin{array}{l}\text { Nuclide } \\
A A A A B A A A\end{array}$ & & $\begin{array}{l}\text { fract. } \\
\triangle A B A O A O A B\end{array}$ & $\begin{array}{l}k \\
\mathrm{~A} \ddot{A} \mathrm{~A} A\end{array}$ & & rick & & risk & & $\begin{array}{c}\text { risk } \\
A \cap A B A A A B A\end{array}$ & & 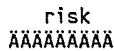 & act. \\
\hline$A C-227$ & $O O E+00$ & 0.0000 & & & $0.000 E+00$ & 0.0000 & $+\infty 0$ & 0.0000 & 0 . & 0.0 & +00 & .0000 \\
\hline & & 0.0000 & & & & & & & & & & 0000 \\
\hline Co & & 0.0000 & & 0 & 0. & & & & & & & \\
\hline & & & & & & & & & & & & \\
\hline & & 0.0000 & & & 00 & & & 0. & & 0. & & 0 \\
\hline Gd & & 0.0 & & & & & & & & & & \\
\hline & & 0.0 & & & 0.0 & & 0. & & & & & \\
\hline & & 0.0 & & & & & & & & 0. & & \\
\hline & & & & & & & & & & & & \\
\hline & & & & & 00 & & 0. & & & & & \\
\hline & & 0.00 & & & 00 & & & & & 0. & & 300 \\
\hline & & & & & & & & & & & & \\
\hline & & 0 & & & 0 & & & & & & & \\
\hline & & 0.00 & & & 0 & & & & & & & 000 \\
\hline & & & & & & & & & & & & 000 \\
\hline & & & & & & & & & & & & \\
\hline$u-2$ & +00 & 0.00 & +00 & 0.0 & $0.000 E+00$ & 0.0 & & & & & & 000 \\
\hline$u-2$ & & & & & 0.0 & 10 & $=00$ & 0.0000 & $0.000 \mathrm{E}$ & 0.0 & $0.000 \mathrm{E}+00$ & 0.0000 \\
\hline & & & & & & & & & & & & \\
\hline & & & & & & & & & & & & \\
\hline 10lab & $000 E+00$ & 0.0000 & $0.000 E+00$ & 0.0000 & $0.000 E+00$ & 0.0000 & $0.000 E+00$ & 0.0000 & $0.000 \mathrm{E}+00$ & 0.0000 & $0.000 \mathrm{E}+0$ & .00 \\
\hline
\end{tabular}

\begin{tabular}{|c|c|}
\hline Attachment & Sheet No. 31 of 41 \\
\hline Originator: S.W. Clark & Date \\
\hline M. W. Perrott & Date \\
\hline $0100 F-C A-V 0303$ & Rev. No. \\
\hline
\end{tabular}




\section{ATTACHMENT 6}

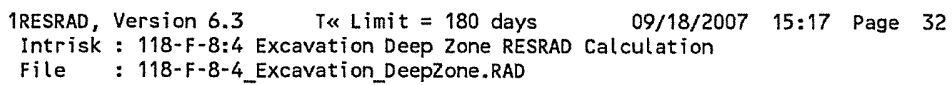

Water Dependent Pathways

\begin{tabular}{|c|c|c|c|c|c|c|c|c|c|c|c|c|}
\hline \multirow{2}{*}{ 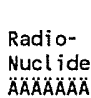 } & \multicolumn{2}{|c|}{ Water } & \multicolumn{2}{|c|}{$\begin{array}{c}\text { Fish } \\
\text { ÄAAAAAOAOAOAOAOAOÄ }\end{array}$} & \multicolumn{2}{|c|}{ 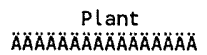 } & \multicolumn{2}{|c|}{$\begin{array}{c}\text { Meat } \\
\text { AAAAAAAOABAAAOAOAOÄ }\end{array}$} & \multicolumn{2}{|c|}{ Milk } & \multicolumn{2}{|c|}{$\begin{array}{r}\text { All Pathways** } \\
\text { AAAAAAAAAAAAAAAAA }\end{array}$} \\
\hline & AAA & $\begin{array}{l}\text { fract. } \\
\triangle A B A B A B A B\end{array}$ & 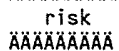 & $\begin{array}{l}\text { fract. } \\
\ddot{A} \ddot{A} \ddot{A} \ddot{A} \ddot{A} \ddot{A}\end{array}$ & $\begin{array}{c}\text { risk } \\
\text { ÄÄÄÄABAAÄ }\end{array}$ & $\begin{array}{l}\text { fract. } \\
\triangle A B A A A B A\end{array}$ & $\begin{array}{r}r i s k \\
\triangle A \cap A B A B A O A B A\end{array}$ & $\begin{array}{l}\text { fract. } \\
\text { ÄÄÄÄÄ }\end{array}$ & $\begin{array}{c}\text { risk } \\
A A A A A A B A\end{array}$ & $\begin{array}{l}\text { fract. } \\
\text { ÄAAAAA }\end{array}$ & $\begin{array}{c}\text { risk } \\
\text { AAAOAAÄ }\end{array}$ & $\begin{array}{l}\text { fract. } \\
A B A B A B A\end{array}$ \\
\hline & & & & & & & & & $4.990 \mathrm{E}-19$ & & & 100 \\
\hline & & & $+\infty$ & & & & & 0.0000 & $.000 E+00$ & 0.0000 & & 0.0000 \\
\hline & & & & & & 00 & & 0.0000 & $1.943 E-18$ & 0.0000 & & 0.0000 \\
\hline & & & & & & & & 0 & & & & \\
\hline & & & & & & & & & & & & \\
\hline & 0. & 0.0 & $0 E+00$ & & 0. & & & & +00 & & & 000 \\
\hline & 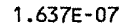 & & & & & & & & & & & \\
\hline & & & & & & & & & & & & \\
\hline & & & & & & & & & 20 & & & 000 \\
\hline & 0 & & +00 & & & & & & & & & \\
\hline & & & +00 & & & & & & & & & 0 \\
\hline & & & & 0. & & 0. & & 0. & $E-22$ & 0 & & 000 \\
\hline & & & & & & & & & & & & \\
\hline & & & & & & & & & $7 E-25$ & & & 000 \\
\hline & & 0.0 & & 0.0 & 4. & 0. & & & $77 E-21$ & & & 000 \\
\hline & & & & & & & & & & & & \\
\hline & & & & & & & & & & & & \\
\hline & 3.7 & 0 & $1.342 E-14$ & 0.0000 & 5.5 & 0.0000 & 3.5 & 0 & $5 E-14$ & 0. & 4. & 0.0000 \\
\hline & & & & & & & & & & & & \\
\hline & & & & & & & & & & & & \\
\hline & - ot & .10 & $2.8 / 6 E-08$ & 0.0455 & $=-08$ & 30.1185 & $-0 c+2$ & & -00 & & & 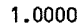 \\
\hline
\end{tabular}

** Sum of water independent ground, inhalation, plant, meat, milk, soil

and water dependent water, fish, plant, meat, milk pathways

0

Excess Cancer Risks CNRS9(irn, $i, t$ ) and CNRS9W(irn, $i, t$ ) for Inhalation of Radon and its Decay Products at $t=1.350 \mathrm{E}+02$ years

Radionuclides

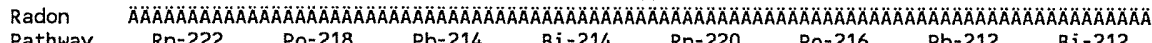

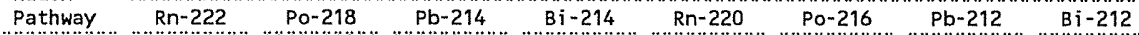

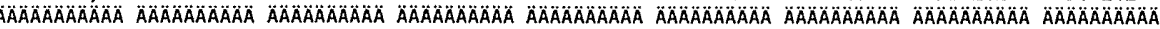

$\begin{array}{lllllllll}\text { Water }- \text { ind. } & 0.000 E+00 & 0.000 E+00 & 0.000 E+00 & 0.000 E+00 & 0.000 E+00 & 0.000 E+00 & 0.000 E+00 & 0.000 E+00\end{array}$

Water-dep. $\begin{array}{lllllllll}0.000 E+00 & 0.000 E+00 & 0.000 E+00 & 0.000 E+00 & 0.000 E+00 & 0.000 E+00 & 0.000 E+00 & 0.000 E+00\end{array}$

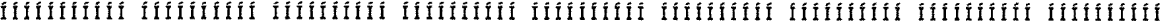

Total $0.000 \mathrm{E}+00 \quad 0.000 \mathrm{E}+00$ 0.000E+00 $0.000 \mathrm{E}+00$ 0.000E+00 $0.000 \mathrm{E}+00$ 0.000E+00 $0.000 \mathrm{E}+00$

Water-ind. = = Water-independent Water-dep. $==$ Water-dependent

\begin{tabular}{|c|c|}
\hline Attachment & Sheet No. 32 of 41 \\
\hline Originator: S.W. Clark & Date \\
\hline M. W. Perrott & Date \\
\hline 0100F-CA-V0303 & Rev. No. 0 \\
\hline
\end{tabular}




\section{ATTACHMENT 6}

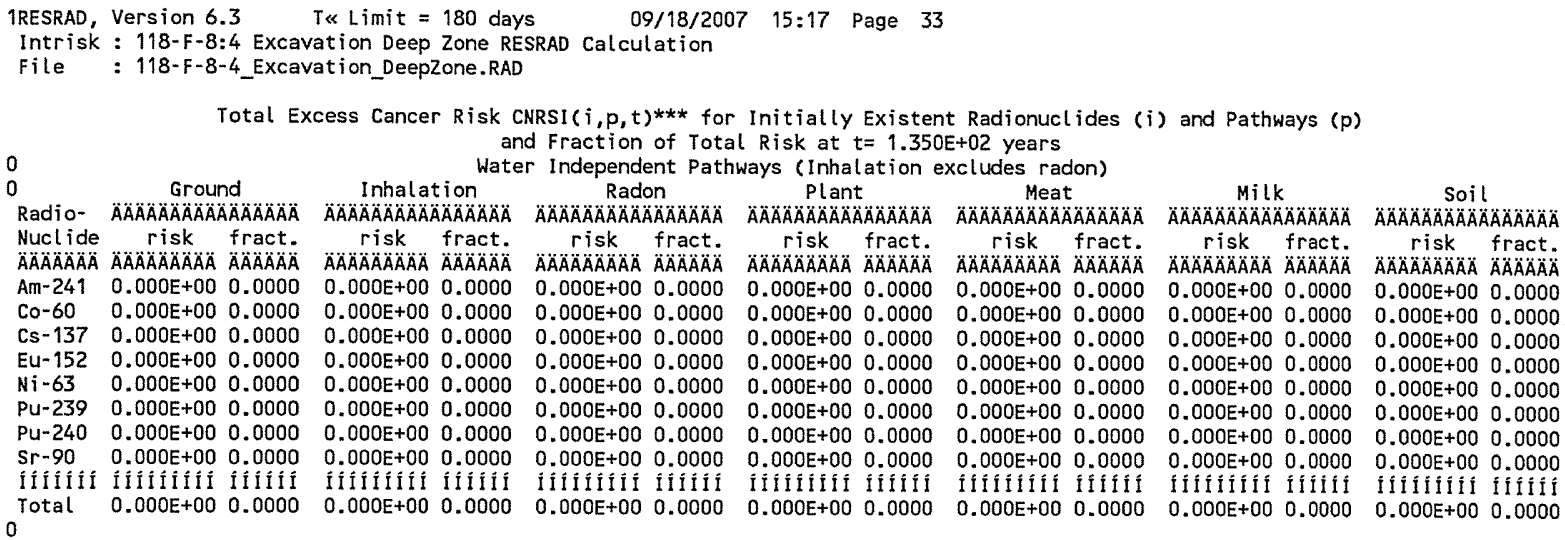

Total Excess Cancer Risk CNRSI $(i, p, t) * * *$ for Initially Existent Radionuclides (i) and Pathways ( $p$ ) and Fraction of Total Risk at $t=1.350 E+02$ years

Water Dependent Pathways

\begin{tabular}{|c|c|c|c|c|c|c|c|c|c|c|c|c|c|c|}
\hline \multirow[b]{2}{*}{$\begin{array}{l}\text { Nuclide } \\
\text { ÄÄÄÄÄÄÄA }\end{array}$} & \multicolumn{2}{|c|}{ Water } & \multicolumn{2}{|c|}{ Fish } & \multicolumn{2}{|c|}{ Radon } & \multicolumn{2}{|c|}{$\begin{array}{c}\text { Plant } \\
\text { ÄÄAOAAAOÄÄÄÄÄÄÄ }\end{array}$} & \multicolumn{2}{|c|}{ 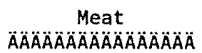 } & \multicolumn{2}{|c|}{$\begin{array}{c}M i l k \\
\text { ÄĀÄAAAAAAAAOAOAOÄ }\end{array}$} & \multicolumn{2}{|c|}{ 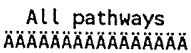 } \\
\hline & sk & $\begin{array}{l}\text { fract. } \\
\because A B A B A B A A\end{array}$ & sK & $\begin{array}{l}\text { fract. } \\
\ddot{A} A \ddot{A} A \ddot{A} \ddot{A}\end{array}$ & $\begin{array}{l}\text { risk } \\
\text { ÄÄAAAAAA }\end{array}$ & & 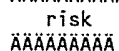 & $\begin{array}{l}\text { fract. } \\
\text { ÄÄÄÄÄ }\end{array}$ & risk & & risk & & $\begin{array}{l}\text { risk } \\
\text { rist }\end{array}$ & $\begin{array}{l}\text { fract. } \\
\text { fraAAA }\end{array}$ \\
\hline & & & & & & & & & & & & & & \\
\hline & & & & & & & & & & & & & & \\
\hline & 3. & & & & & & & & & & & & & \\
\hline & & & & & & & & & & & & & & \\
\hline & & & & & & & & & & & & & & \\
\hline & & & & & & & & & & & & & & \\
\hline & & & & & & & & & & & & & & \\
\hline & & & & & & & & & & & & & & \\
\hline & & & & & & & & & & & & & & \\
\hline & & & -08 & & E. $=000+00$ & & $481 t-08$ & & -08 & 0.0180 & $=-08$ & & $.315 E-07$ & \\
\hline
\end{tabular}

$* * * \operatorname{CNRSI}(i, p, t)$ includes contribution from decay daughter radionuclides

\begin{tabular}{|c|c|c|}
\hline \multirow{2}{*}{\multicolumn{2}{|c|}{ Attachment $\frac{6}{\mathrm{~S} \text { W Clark }}$}} & Sheet No. 33 \\
\hline & & Dat \\
\hline Chk'd By & M. W. Perrott & Date \\
\hline Calc. No. & $0100 \mathrm{~F}-\mathrm{CA}-\mathrm{V} 0303$ & $\bar{N}$ \\
\hline
\end{tabular}




\section{ATTACHMENT 6}

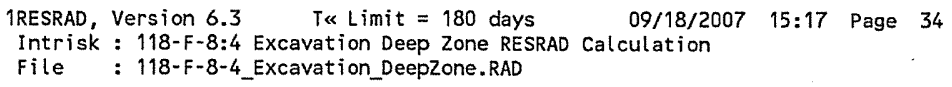




\section{ATTACHMENT 6}

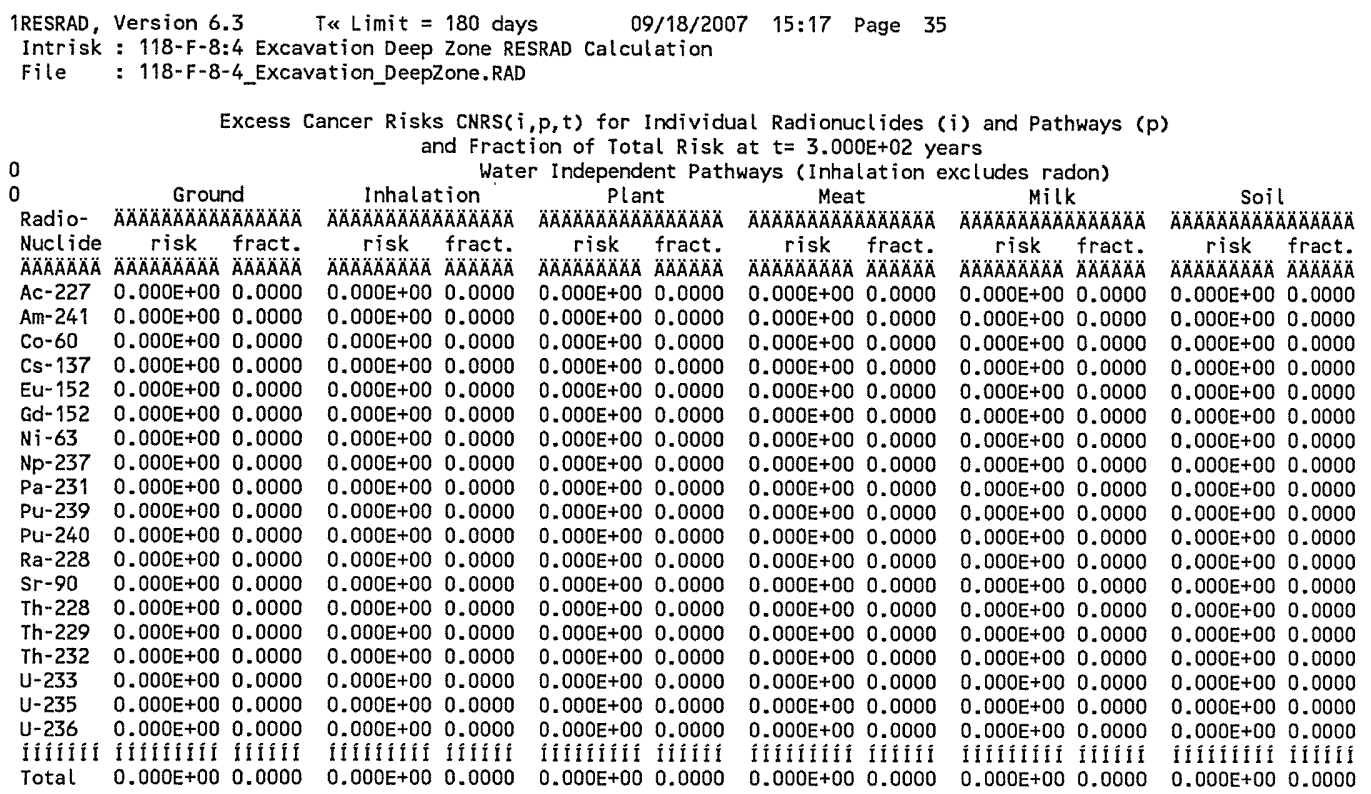




\section{ATTACHMENT 6}

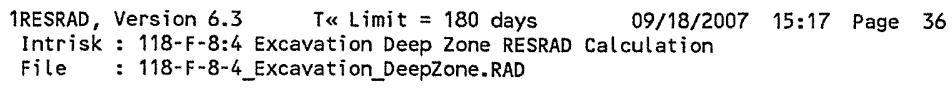

Water Dependent Pathways

\begin{tabular}{|c|c|c|c|c|c|c|c|c|c|c|c|c|}
\hline & $\begin{array}{r}\text { Water } \\
\text { AAAAAAAAAA }\end{array}$ & ÄÄ̈̈ & $\begin{array}{r}F i s \\
\text { ÄÄÄÄÄÄA }\end{array}$ & $\ddot{A} A ̈ A ̈ A ̈$ & 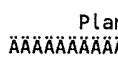 & $\triangle A O A O A O A O A$ & 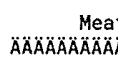 & $\triangle A \cap A ̈ A A A A ̈$ & $\begin{array}{r}M i l k \\
\triangle A O A A O A O A O A B A\end{array}$ & $\triangle A O A O A O A ̈$ & $\begin{array}{r}A l l P a \\
A A A A A A A A\end{array}$ & $\begin{array}{l}\text { hways*** } \\
A \cap A O A A A B A\end{array}$ \\
\hline $\begin{array}{l}\text { Nucl ide } \\
\triangle A \cap A B A A A B A\end{array}$ & $\begin{array}{c}\text { risk } \\
\triangle A A A O A B A A A B\end{array}$ & $\begin{array}{l}\text { fract. } \\
\ddot{A} \ddot{A} \ddot{A} \ddot{A} \ddot{A} \bar{A}\end{array}$ & 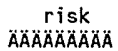 & $\begin{array}{l}\text { fract. } \\
\triangle A A A A A O A B\end{array}$ & $\begin{array}{c}r i s k \\
\triangle A O A ̈ A ̈ A ̈ A ̈ A ̈ A O A\end{array}$ & $\begin{array}{l}\text { fract. } \\
\triangle A O A B A O A B A\end{array}$ & $\begin{array}{c}r i s k \\
\dddot{A} A ̈ A \cap A ̈ A ̈ A ̈ A ̈ A B A\end{array}$ & 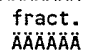 & 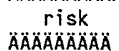 & $\begin{array}{l}\text { fract. } \\
\text { AAAAAAAA }\end{array}$ & $\begin{array}{c}\text { risk } \\
A B A A A A A B A\end{array}$ & \\
\hline-227 & $1.504 E-14$ & 0.0000 & $2.557 E-16$ & 0.0000 & $2.230 \mathrm{E}-15$ & 0.0000 & $1.019 \mathrm{E}-18$ & 0.0000 & $2.431 \mathrm{E}-18$ & 0.0000 & $1.753 E-14$ & 0.0000 \\
\hline 1 & $30 E+00$ & 1000 & $J O E+00$ & 0.0000 & $000 E+00$ & 0.0000 & $10 E+00$ & 0.0000 & $0.000 E+00$ & 0.0000 & $0.000 E+00$ & 0.0000 \\
\hline & & & & & 5 & & & & 27 & 300 & 25 & 00 \\
\hline & $7 E-09$ & 0.0116 & & 0.0060 & $4 E-10$ & 0.0016 & $8 E-10$ & 0.0009 & & 007 & & \\
\hline 2 & $0.000 E+00$ & 0.0000 & $0.000 E+00$ & 0.0000 & $.000 E+00$ & 0.0000 & $0.000 E+00$ & 0.0000 & $0.000 E+00$ & 0.0000 & $0.000 \mathrm{E}+00$ & .0000 \\
\hline & $0 E+00$ & 0.0 & 0.0 & 0.0000 & $D E+00$ & 0.0000 & $D E+00$ & 0.0 & $E+00$ & 300 & 0. & 300 \\
\hline & & 0.6620 & & & & 0.1054 & & 0.0 & & & 1. & \\
\hline-2 & $0.000 \mathrm{E}+00$ & 0.0000 & $0.000 E+00$ & 0.0 & $0.000 E+00$ & 0.0000 & $E+00$ & & & 000 & -00 & 300 \\
\hline-2 & $2.568 E-15$ & 0.0000 & $E-17$ & 0.0000 & $3.732 E-16$ & 0.0 & $E-17$ & 0. & -19 & 00 & & . \\
\hline & $+\infty 0$ & & 0. & 0.0000 & $0.000 E+00$ & 0.0000 & $E+00$ & 0. & $+\infty$ & 00 & 0. & $O C$ \\
\hline & $0.000 E+00$ & & & & $0.000 E+00$ & 0.0000 & $O E+00$ & 0.0 & 00 & 000 & 0. & 0.0000 \\
\hline $1-228$ & $1.786 \mathrm{E}-1$ & 0.0000 & 3.1 & 0. & $.739 E-20$ & 0.0 & -22 & & & & & \\
\hline & $8.188 E-09$ & 0.0 & $E-10$ & 0.0010 & $.271 E-09$ & 0.0 & -10 & 0. & 10 & 09 & & 66 \\
\hline & 1.7 & & 6.4 & 0.0 & 2.82 & 0.0000 & $8.435 E-24$ & 0.0 & $E-24$ & 0.0000 & 2. & .0000 \\
\hline & $2.273 E-16$ & & 7.5 & 0. & -17 & & $E-20$ & & & & & 0.0000 \\
\hline & 5 & 0.00 & $E-22$ & & $352 E-22$ & 0.0 & $E-24$ & 0. & 3. & & & \\
\hline & $632 E-13$ & 0.0000 & $9.452 E-16$ & 0.0000 & $3.927 E-14$ & 0.0000 & $2.522 E-16$ & 0.0000 & $1.285 E-15$ & 0.0 & $3.050 \mathrm{E}-1$ & 0000 \\
\hline & & & זורד & & & & & & & & & .0001 \\
\hline & & & & & L & & $E-14$ & & & & & \\
\hline & IIIIIIIIII & & IIIIIIIIII & & filififit & Ílíl & IIIIIIIII & & Ît & & IIIIIIIII & IIIIII \\
\hline & $1.069 \mathrm{E}-07$ & 0.7298 & $.021 E-05$ & 0.0275 & $.694 \mathrm{E}-08$ & 0.1157 & $748 E-09$ & 0.0 & $1.685 \mathrm{E}-08$ & 0.1 & 4 E- 07 & 1.0000 \\
\hline
\end{tabular}

** Sum of water independent ground, inhalation, plant, meat, milk, soil

0 and water dependent water, fish, plant, meat, milk pathways Excess Cancer Risks CNRS9(irn, $i, t)$ and CNRS $W(i r n, i, t)$ for Inhalation of Radon and its Decay Products at $t=3.000 E+02$ years

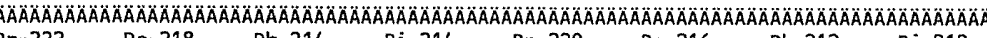
PaABA

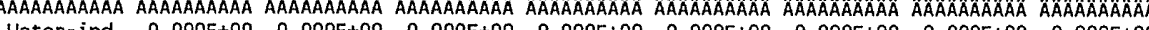

$\begin{array}{lllllllll}\text { water }- \text { ind. } & 0.000 E+00 & 0.000 E+00 & 0.000 E+00 & 0.000 E+00 & 0.000 E+00 & 0.000 E+00 & 0.000 E+00 & 0.000 E+00\end{array}$ Water-dep. $0.000 \mathrm{E}+00 \quad 0.000 \mathrm{E}+00 \quad 0.000 \mathrm{E}+00 \quad 0.000 \mathrm{E}+00 \quad 0.000 \mathrm{E}+00 \quad 0.000 \mathrm{E}+00 \quad 0.000 \mathrm{E}+00 \quad 0.000 \mathrm{E}+00$

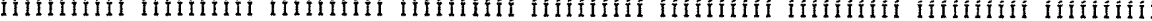

Total $\quad 0.000 E+00 \quad 0.000 E+00 \quad 0.000 E+00 \quad 0.000 E+00 \quad 0.000 E+00 \quad 0.000 E+00 \quad 0.000 E+00 \quad 0.000 E+00$

Water - ind. $==$ Water-independent $\quad$ Water-dep. $==$ Water-dependent

\begin{tabular}{|c|c|}
\hline Attachment & Sheet No. 36 of 41 \\
\hline Originator: S.W. Clark & Dat \\
\hline Chk'd By M.W. Perrott & Date \\
\hline Calc. No. $\quad 0100 \mathrm{~F}-\mathrm{CA}-\mathrm{V} 0303$ & Rev. No. \\
\hline
\end{tabular}




\section{ATTACHMENT 6}

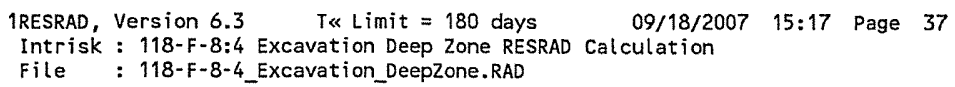

Total Excess Cancer Risk CNRSI $(i, p, t) * * *$ for Initially Existent Radionuclides (i) and Pathways ( $p$ )

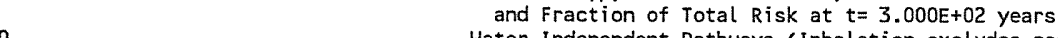

Total Excess Cancer Risk CNRSI $(i, p, t) * * *$ for Initially Existent Radionuclides (i) and Pathways $(p)$ and Fraction of Total Risk at $t=3.000 E+02$ years

Water Dependent Pathways

\begin{tabular}{|c|c|c|c|c|c|c|c|c|c|c|c|c|}
\hline & $\begin{array}{l}\text { Water } \\
\text { ÄÄÄÄÄÄÄÄÄÄÄÄÄA }\end{array}$ & $\begin{array}{c}\text { Fish } \\
\triangle A \cap A A A O A B A O A O A O A O A O A O A\end{array}$ & $\begin{array}{r}\text { Rad } \\
\triangle A \cap A A A A A B A B\end{array}$ & $\triangle A \cap A O A O A B A$ & $\begin{array}{r}\text { Plan } \\
\text { A }\end{array}$ & 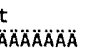 & $\begin{array}{r}\text { Mea } \\
\text { ÄAAAAAAÄÄ }\end{array}$ & A & $\begin{array}{r}M i l k \\
\text { ÄÄÄAAÄÄÄ }\end{array}$ & $\triangle A \cap A ̈ A ̈ A \cap A ̈$ & $\begin{aligned} \text { All path } \\
A O A A A B A A\end{aligned}$ & 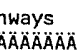 \\
\hline $\begin{array}{l}\text { גclide } \\
\text { Ü̈ÄÄÄÄ }\end{array}$ & 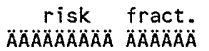 & $\begin{array}{l}\text { risk fract. } \\
\triangle A B A ̈ A B A O A A O A A A O A\end{array}$ & risk & & risk & & risk & & rick & & risk & \\
\hline & & & & & & & & & & & & \\
\hline & & & & & & & & & & & & \\
\hline & 1.6 & 00. & & & & & & & & & & \\
\hline & 00 & & & & & & & & & & & \\
\hline & & & & & & & & & & & & \\
\hline & 0 & & & & & & & & & & & \\
\hline & & & & & & & & & & & & \\
\hline & & & & & & & & & & & & \\
\hline & & & & & & & & & & & & \\
\hline t. & 298 & -090.0275 & $0.000 E+00$ & 0.0000 & $E-08$ & 157 & $:-09$ & 119 & -08 & 157 & 07 & \\
\hline
\end{tabular}

*** CNRSI $(i, p, t)$ includes contribution from decay daughter radionuclides

\begin{tabular}{|c|c|}
\hline Attachment & Sheet No. $\underline{37}$ of $\underline{41}$ \\
\hline Originator: S.W. Clark & Date \\
\hline Chk'd By M.W. Perrott & Date \\
\hline Calc. No. $\quad 0100 F-C A-V 0303$ & Rev. No. $\quad 0$ \\
\hline
\end{tabular}




\section{ATTACHMENT 6}

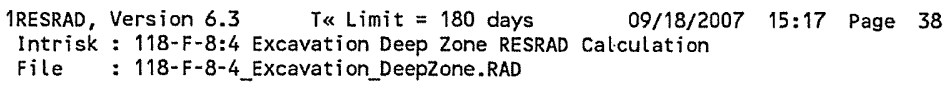
Radionuclides

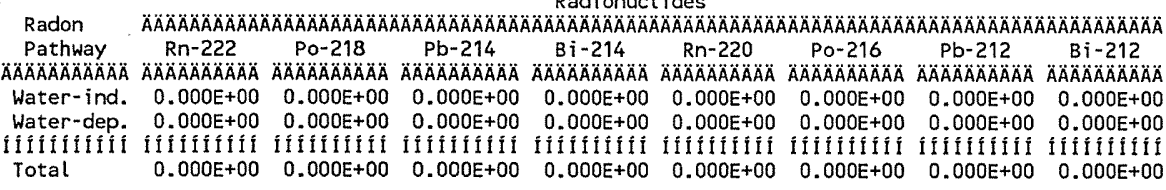

\begin{tabular}{|c|c|}
\hline Attachment & Sheet No. 38 of 41 \\
\hline Originator: S.W. Clark & Date \\
\hline Chk'd By M.W. Perrott & Date \\
\hline $0100 F-C A-V 0303$ & Rev. No. 0 \\
\hline
\end{tabular}




\section{ATTACHMENT 6}

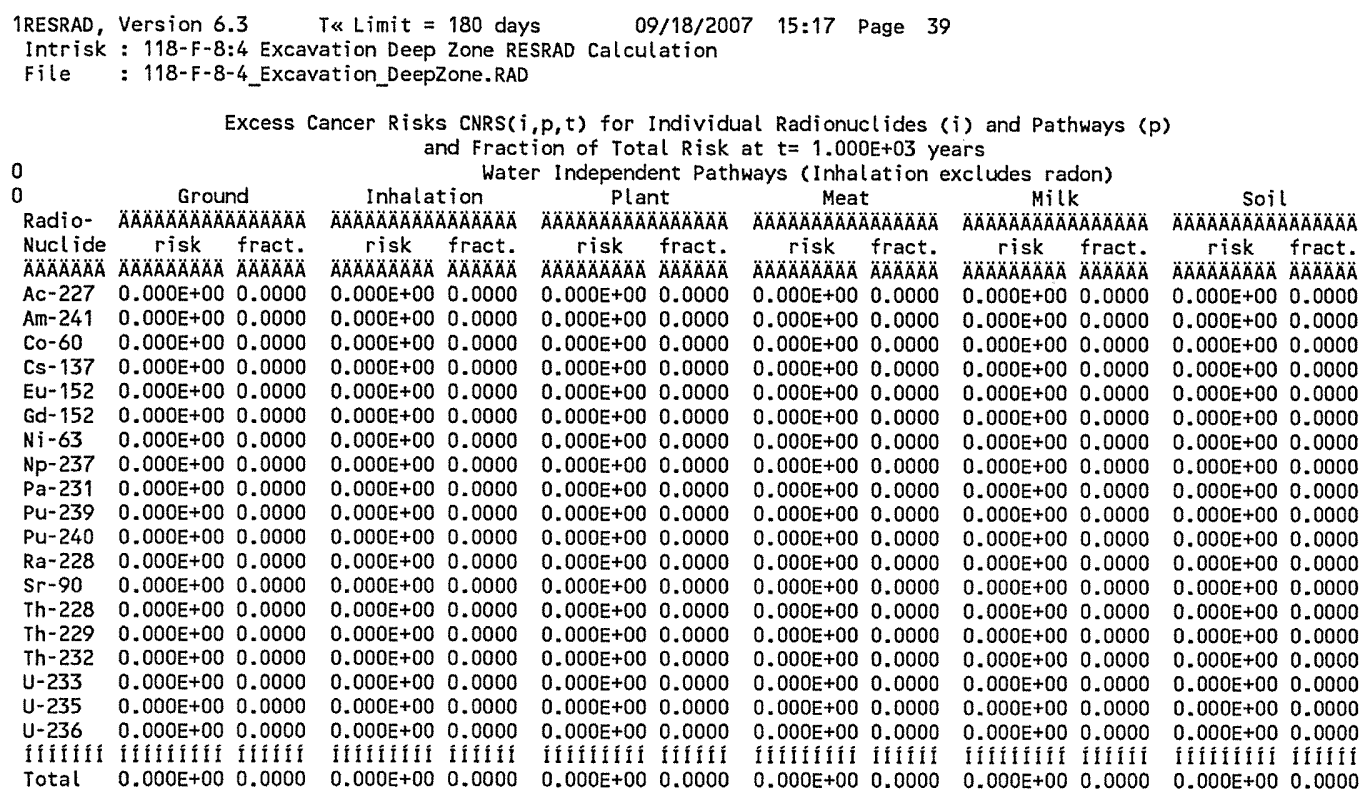

Attachment 6 Sheet No. $\underline{39}$ of 41 Date Date 


\section{ATTACHMENT 6}

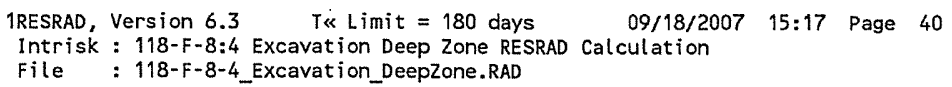

Water Dependent Pathways

\begin{tabular}{|c|c|c|c|c|c|c|c|c|c|c|c|c|}
\hline \multirow[b]{2}{*}{$\begin{array}{l}\text { Nuclide } \\
\text { ÄÄÄÄÄÄA }\end{array}$} & \multicolumn{2}{|c|}{ 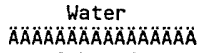 } & \multicolumn{2}{|c|}{$\begin{array}{c}\text { Fish } \\
\text { ÄÄAAAAAAAAAAAOÄÄ }\end{array}$} & \multicolumn{2}{|c|}{$\begin{array}{c}\text { Plant } \\
\text { ÄÄÄAOAOAAAAAAAOAOA }\end{array}$} & \multicolumn{2}{|c|}{ 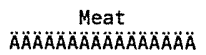 } & \multicolumn{2}{|c|}{$\begin{array}{c}\text { Milk } \\
\text { AAAAAAAAAAAAAAAAA }\end{array}$} & \multicolumn{2}{|c|}{$\begin{array}{r}\text { All Pathways** } \\
\text { ÄÄÄAÄÄÄÄÄÄÄÄ }\end{array}$} \\
\hline & $\begin{array}{c}r i s k \\
A \cap A ̈ A ̈ A O A B A B\end{array}$ & $\begin{array}{l}\text { fract. } \\
\text { ÄÄÄÄÄ }\end{array}$ & $\begin{array}{c}r i s k \\
A A \cap A ̈ A \cap A ̈ A ̈ A\end{array}$ & $\begin{array}{l}\text { fract. } \\
\triangle A \cap A B A B A B A\end{array}$ & $\begin{array}{c}\text { risk } \\
\text { AAAOÄÄÄÄA }\end{array}$ & $\begin{array}{l}\text { fract. } \\
\text { ÄÄÄAAA }\end{array}$ & $\begin{array}{c}\text { risk } \\
A \ddot{A} \ddot{A} \ddot{A} \ddot{A} A\end{array}$ & 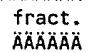 & $\begin{array}{r}r i s k \\
A B A O A O A B A\end{array}$ & $\begin{array}{l}\text { fract. } \\
\text { ÄAAAAAAB }\end{array}$ & $\begin{array}{r}\text { risk } \\
A A B A A B A\end{array}$ & $\begin{array}{l}\text { fract. } \\
\triangle A B A A A B A\end{array}$ \\
\hline$A c-227$ & & 0.0001 & & & & & & & 1.73 & & $1.250 \mathrm{E}-13$ & \\
\hline & $0 E+00$ & 000 & $\mathrm{OE}+00$ & 000 & $D E+00$ & 0.0000 & $E+00$ & 0.0000 & $+\infty$ & 0000 & $000 E+00$ & 0000 \\
\hline & 10 & & $+\infty 0$ & & 100 & bo & 00 & 00 & & & & \\
\hline & & & & & & & & & & & & \\
\hline & 10 & & $E+00$ & & & & & & & & & \\
\hline & 0 & & $+\infty$ & & +00 & & & & & & +00 & \\
\hline & & & & & & & & & & & & \\
\hline & & & & & & & & & & & & \\
\hline & 1 & & & & & & & & & & & \\
\hline & & & +00 & & & & & & & & & \\
\hline & & & +00 & & 0.0 & 0. & & 0. & & & & \\
\hline & & & & & & & & & & & & \\
\hline & & & & & 17 & & & & & & & \\
\hline & & & 4.0 & & & & & & & & & \\
\hline & & & & & & & & & & & -15 & \\
\hline & & & & & & & & & & & & \\
\hline & & & & & & & & & & & & \\
\hline$u-2$ & & & & & $E-12$ & 0.0018 & 9. & 0.0 & 4.95 & & $E-11$ & 0.0135 \\
\hline & & & & & & & & & & & & \\
\hline & & & & & & & & & & & & \\
\hline Total & $389 \mathrm{E}-10$ & 02 & $.775 \mathrm{E}-11$ & 0.0206 & $010 E-10$ & 0.1170 & 8.44 & 0.0098 & $9.705 \mathrm{E}-11$ & 0.1124 & $.631 E-10$ & 1.00 \\
\hline
\end{tabular}

** Sum of water independent ground, inhalation, plant, meat, milk, soil

and water dependent water, fish, plant, meat, milk pathways

Excess Cancer Risks CNRS9(irn, $i, t$ ) and CNRSOW(irn, $i, t)$ for Inhalation of

Radon and its Decay Products at $t=1.000 E+03$ years

$0 \quad$ Radionucl ides

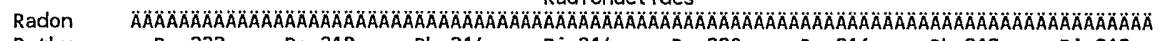

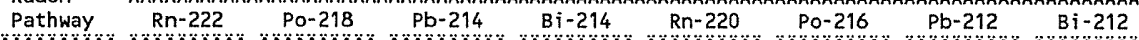

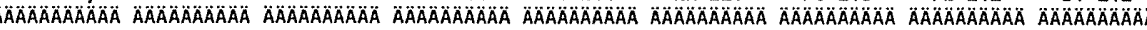

$\begin{array}{lllllllll}\text { Water }-i \text { ind. } & 0.000 E+00 & 0.000 E+00 & 0.000 E+00 & 0.000 E+00 & 0.000 E+00 & 0.000 E+00 & 0.000 E+00 & 0.000 E+00\end{array}$ Water-dep: $\begin{array}{llllllll}0.000 E+00 & 0.000 E+00 & 0.000 E+00 & 0.000 E+00 & 0.000 E+00 & 0.000 E+00 & 0.000 E+00 & 0.000 E+00\end{array}$

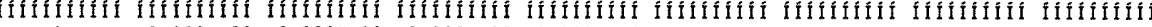

Total $\quad 0.000 E+00 \quad 0.000 E+00 \quad 0.000 E+00 \quad 0.000 E+00 \quad 0.000 E+00 \quad 0.000 E+00 \quad 0.000 E+00 \quad 0.000 E+00$

Water-ind. == Water-independent Water-dep. $==$ Water-dependent

\begin{tabular}{|c|c|}
\hline \multirow{2}{*}{\multicolumn{2}{|c|}{$\begin{array}{ll}\text { Attachment } & 6 \\
\text { Originator: S.W. Clark } & \text { Sheet No. } 40 \text { of } 41 \\
\text { Date }\end{array}$}} \\
\hline & \\
\hline M.W. Perrott & \\
\hline Calc. No. & 0 \\
\hline
\end{tabular}




\section{ATTACHMENT 6}

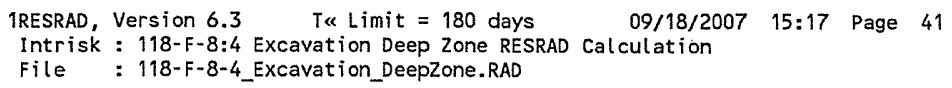

Total Excess Cancer Risk CNRSI $(i, p, t) * * *$ for Initially Existent Radionuclides (i) and Pathways ( $p$ ) and Fraction of Total Risk at $t=1.000 E+03$ years

Water Dependent Pathways

\begin{tabular}{|c|c|c|c|c|c|c|c|c|c|c|c|c|c|c|}
\hline \multirow{2}{*}{$\begin{array}{l}\text { Radio- } \\
\text { Nucl ide } \\
\text { ÄÄÄÄÄÄÄ }\end{array}$} & \multicolumn{2}{|c|}{$\begin{array}{c}\text { Water } \\
\text { ÄÄÄÄÄÄÄÄÄÄÄÄÄÄÄA }\end{array}$} & \multicolumn{2}{|c|}{$\begin{array}{c}\text { Fish } \\
\text { ĀÄÄÄÄÄÄÄÄÄĀÄ }\end{array}$} & \multicolumn{2}{|c|}{ 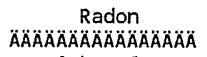 } & \multicolumn{2}{|c|}{$\begin{array}{c}\text { Plant } \\
\text { ALAAAAAAÄAAAOAAAOÄ }\end{array}$} & \multicolumn{2}{|c|}{$\begin{array}{c}\text { Meat } \\
\text { ÄÄÄAAAAAAAAÄÄÄA }\end{array}$} & \multicolumn{2}{|c|}{$\begin{array}{c}M i l k \\
\text { AOAAAAAAAAAAAOAOA }\end{array}$} & \multicolumn{2}{|c|}{$\begin{array}{c}\text { All pathways } \\
\text { AAAAAAAAAAAAABAAAAAA }\end{array}$} \\
\hline & risk & 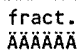 & risk & $\begin{array}{l}\text { fract. } \\
\ddot{A} \ddot{A} \ddot{A} \ddot{A} \ddot{A}\end{array}$ & sk & $\begin{array}{l}\text { fract. } \\
\triangle A O A O A B A B A\end{array}$ & 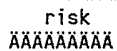 & & rit & 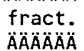 & risk & fract. & risk & \\
\hline 21 & & & & & & & & & & & & & & \\
\hline & & & & & & & & & & & & & & \\
\hline & & & & & & & & & & & & & & \\
\hline & & & & & & & & & & & & & & \\
\hline & & & & & & & & & & & & & & \\
\hline & & & & & & & & & & & & & & \\
\hline & & & & & & & & & & & & & & \\
\hline & & & & & & & & & & & & & & \\
\hline & & & & & & & & & & & & & & \\
\hline & & 0.7402 & $1.775 \mathrm{E}-11$ & 0.0206 & $O O E+O O$ & 0.0000 & $1.010 E-10$ & 1170 & $8.447 \mathrm{E}-12$ & 0.0098 & $9.705 \mathrm{E}-11$ & 0.1124 & $8.631 \mathrm{E}-10$ & \\
\hline
\end{tabular}

***CNRSI $(i, p, t)$ includes contribution from decay daughter radionuclides

\begin{tabular}{|c|c|}
\hline Attachment & Sheet No. $\underline{41}$ of 41 \\
\hline Originator: S.W. Clark & Date \\
\hline M.W. Perrott & Date \\
\hline 0100F-CA-V0303 & Rev No. \\
\hline
\end{tabular}




\section{ATTACHMENT 7}

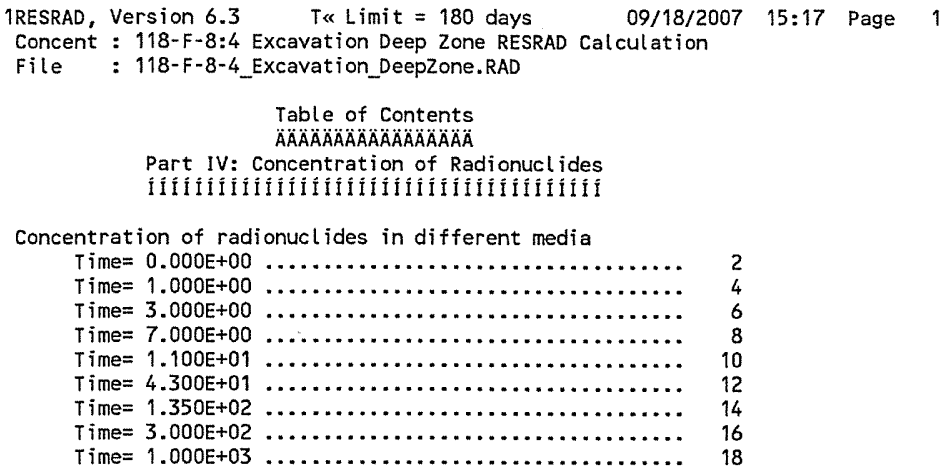




\section{ATTACHMENT 7}

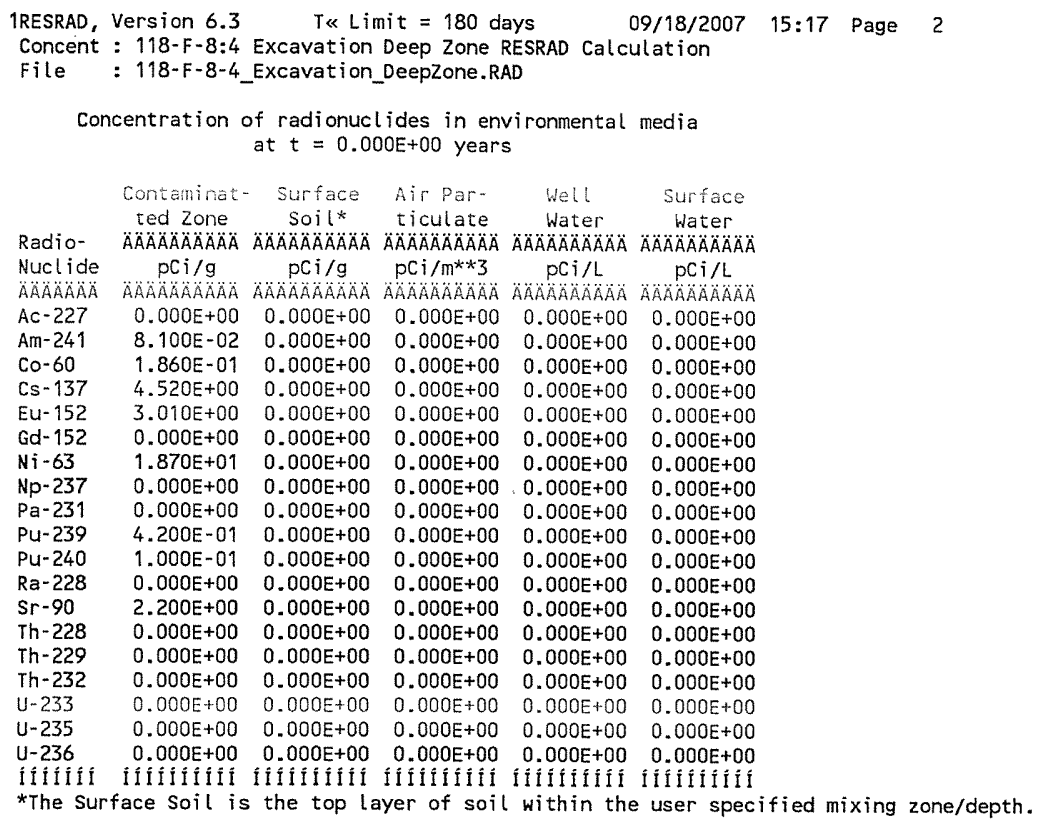

Concentrations in the media occurring in pathways that are suppressed are calculated using the current input parameters, i.e. using parameters appearing in the input screen when the pathways are active.

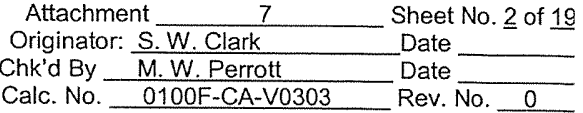




\section{ATTACHMENT 7}

\begin{tabular}{|c|c|c|c|c|c|c|c|c|c|}
\hline $\begin{array}{l}\text { 1RESRAD, } \\
\text { Concent } \\
\text { File }\end{array}$ & $\begin{array}{l}\text { Version } 6.3 \\
: 118-F-8: 4 \\
: 118-F-8-4\end{array}$ & $\begin{array}{l}\text { Te Lin } \\
\text { Excavation } \\
\text { Excavation }\end{array}$ & $\begin{array}{l}\text { mit }=180 \mathrm{da} \\
\text { Deep Zone } \\
\text { Deepzone. RA }\end{array}$ & $\begin{array}{l}\text { ays } \\
\text { RESRAD Calc } \\
\text { AD }\end{array}$ & $\begin{array}{l}09 / 18 / 2007 \\
\text { culation }\end{array}$ & 15:17 Page & 3 & & \\
\hline & & & :on & $\begin{array}{l}\text { f radion } \\
t=0.0\end{array}$ & $\begin{array}{l}\text { nuclides } \\
000 E+00 \text { ye }\end{array}$ & $m$ & & & \\
\hline & $\begin{array}{c}\text { Drinking } \\
\text { Water }\end{array}$ & $\begin{array}{l}\text { Nonleafy } \\
\text { Vegetable }\end{array}$ & $\begin{array}{c}\text { Leafy } \\
\text { Vegetable }\end{array}$ & $\begin{array}{l}\text { Fodder } \\
\text { Meat }\end{array}$ & $\begin{array}{l}\text { Fodder } \\
\text { Milk }\end{array}$ & Meat & Milk & Fish & Crustacea \\
\hline di & $\triangle A \cap A \cap A \cap A O A O A$ & $\triangle A A B A A B A A$ & 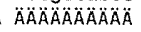 & $\triangle A \cap A B A O A O A O A$ & 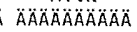 & 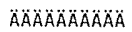 & ÄAAAAÄÄÄÄ & 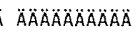 & $\triangle A O A A B A A A A A$ \\
\hline & $\mathrm{pCi} / \mathrm{L}$ & $\mathrm{pCi} / \mathrm{kg}$ & $\mathrm{pCi} / \mathrm{kg}$ & & & $\mathrm{pCl} / \mathrm{kg}$ & & & \\
\hline 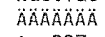 & $\triangle A \cap A \cap A A A B A A A$ & $\triangle A A A A A A A A A A$ & $\triangle \triangle A \triangle A B A O A \triangle A O A O A$ & 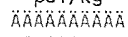 & $\triangle A A D A A A A B A A A$ & AAAMAAAXAOA & AAAAOAAAAAOA & $A A B A$ & BAAAAAAAAA \\
\hline$A C-227$ & $0.000 E+00$ & $0.000 E+00$ & $0.000 E+00$ & $0.000 E+00$ & $0.000 E+00$ & $0.000 E+00$ & $0.000 \mathrm{E}+00$ & $0.000 E+00$ & $0.000 E+00$ \\
\hline$A m-241$ & $0.000 E+00$ & $0.000 E+00$ & $0.000 E+00$ & $0.000 E+00$ & $0.000 E+00$ & $0.000 E+00$ & $0.000 E+00$ & $0.000 E+00$ & $0.000 E+00$ \\
\hline Co- 60 & $0.000 E+00$ & $0.000 \mathrm{E}+00$ & $0.000 E+00$ & $0.000 E+00$ & $0.000 E+00$ & $0.000 E+00$ & $0.000 E+00$ & $0.000 E+00$ & $0.000 E+00$ \\
\hline Cs -137 & $0.000 E+00$ & $0.000 E+00$ & $0.000 E+00$ & $0.000 E+00$ & $0.000 E+00$ & $0.000 E+00$ & $0.000 E+00$ & $0.000 E+00$ & $0.000 E+00$ \\
\hline Eu-152 & $0.000 E+00$ & $0.000 E+00$ & $0.000 E+00$ & $0.000 E+00$ & $0.000 E+00$ & $0.000 E+00$ & $0.000 E+00$ & $0.000 E+00$ & $0.000 E+00$ \\
\hline Gd- 152 & $0.000 E+00$ & $0.000 E+00$ & $0.000 E+00$ & $0.000 E+00$ & $0.000 E+00$ & $0.000 \mathrm{E}+00$ & $0.000 E+00$ & $0.000 E+00$ & $0.000 E+00$ \\
\hline $\mathrm{Ni}-63$ & $0.000 \mathrm{E}+00$ & $0.000 E+00$ & $0.000 \mathrm{E}+00$ & $0.000 E+00$ & $0.000 \mathrm{E}+00$ & $0.000 E+00$ & $0.000 E+00$ & $0.000 \mathrm{E}+00$ & $0.000 E+00$ \\
\hline $\mathrm{Np}-237$ & $0.000 \mathrm{E}+00$ & $0.000 E+00$ & $0.000 \mathrm{E}+00$ & $0.000 E+00$ & $0.000 E+00$ & $0.000 \mathrm{E}+00$ & $0.000 E+00$ & $0.000 E+00$ & $0.000 E+00$ \\
\hline Pa-231 & $0.000 E+00$ & $0.000 E+00$ & $0.000 E+00$ & $0.000 E+00$ & $0.000 E+00$ & $0.000 E+00$ & $0.000 E+00$ & $0.000 \mathrm{E}+00$ & $0.000 E+00$ \\
\hline Pu-239 & $0.000 E+00$ & $0.000 E+00$ & $0.000 E+00$ & $0.000 E+00$ & $0.000 E+00$ & $0.000 E+00$ & $0.000 E+00$ & $0.000 E+00$ & $0.000 E+00$ \\
\hline Pu-240 & $0.000 E+00$ & $0.000 E+00$ & $0.000 E+00$ & $0.000 E+00$ & $0.000 E+00$ & $0.000 E+00$ & $0.000 E+00$ & $0.000 E+00$ & $0.000 E+00$ \\
\hline$R a-228$ & $0.000 E+00$ & $0.000 E+00$ & $0.000 E+00$ & $0.000 E+00$ & $0.000 \mathrm{E}+00$ & $0.000 E+00$ & $0.000 \mathrm{E}+00$ & $0.000 \mathrm{E}+00$ & $0.000 E+00$ \\
\hline $\mathrm{sr}-90$ & $0.000 E+00$ & $0.000 E+00$ & $0.000 E+00$ & $0.000 E+00$ & $0.000 E+00$ & $0.000 E+00$ & $0.000 E+00$ & $0.000 \mathrm{E}+00$ & $0.000 E+00$ \\
\hline Th-228 & $0.000 E+00$ & $0.000 E+00$ & $0.000 E+00$ & $0.000 \mathrm{E}+00$ & $0.000 E+00$ & $0.000 \mathrm{E}+00$ & $0.000 \mathrm{E}+00$ & $0.000 E+00$ & $0.000 E+00$ \\
\hline Th-229 & $0.000 E+00$ & $0.000 E+00$ & $0.000 E+00$ & $0.000 E+00$ & $0.000 E+00$ & $0.000 \mathrm{E}+00$ & $0.000 \mathrm{E}+00$ & $0.000 E+00$ & $0.000 E+00$ \\
\hline Th-232 & $0.000 E+00$ & $0.000 E+00$ & $0.000 E+00$ & $0.000 E+00$ & $0.000 \mathrm{E}+00$ & $0.000 E+00$ & $0.000 \mathrm{E}+00$ & $0.000 E+00$ & $0.000 E+00$ \\
\hline$U-233$ & $0.000 E+00$ & $0.000 E+00$ & $0.000 E+00$ & $0.000 E+00$ & $0.000 E+00$ & $0.000 E+00$ & $0.000 E \div 00$ & $0.000 \mathrm{E}+00$ & $0.000 E+00$ \\
\hline$u-235$ & $0.000 E+00$ & $0.000 E+00$ & $0.000 E+00$ & $0.000 E+00$ & $0.000 E+00$ & $0.000 E+00$ & $0.000 E+00$ & $0.000 E+00$ & $0.000 E+00$ \\
\hline$U-236$ & $0.000 \mathrm{E}+00$ & $0.000 \mathrm{E}+00$ & $0.000 \mathrm{E}+00$ & $0.000 E+00$ & $0.000 E+00$ & $0.000 E+00$ & $0.000 E+00$ & $0.000 \mathrm{E}+00$ & $0.000 E+00$ \\
\hline & & & & & & & & & IIIII \\
\hline & & & & & & & . & $\ldots$ & 2010 \\
\hline
\end{tabular}

Concentrations in the media occurring in pathways that are suppressed are calculated using the current input parameters, i.e. using parameters appearing in the input screen when the pathways are active.

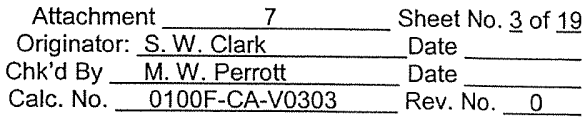




\section{ATTACHMENT 7}

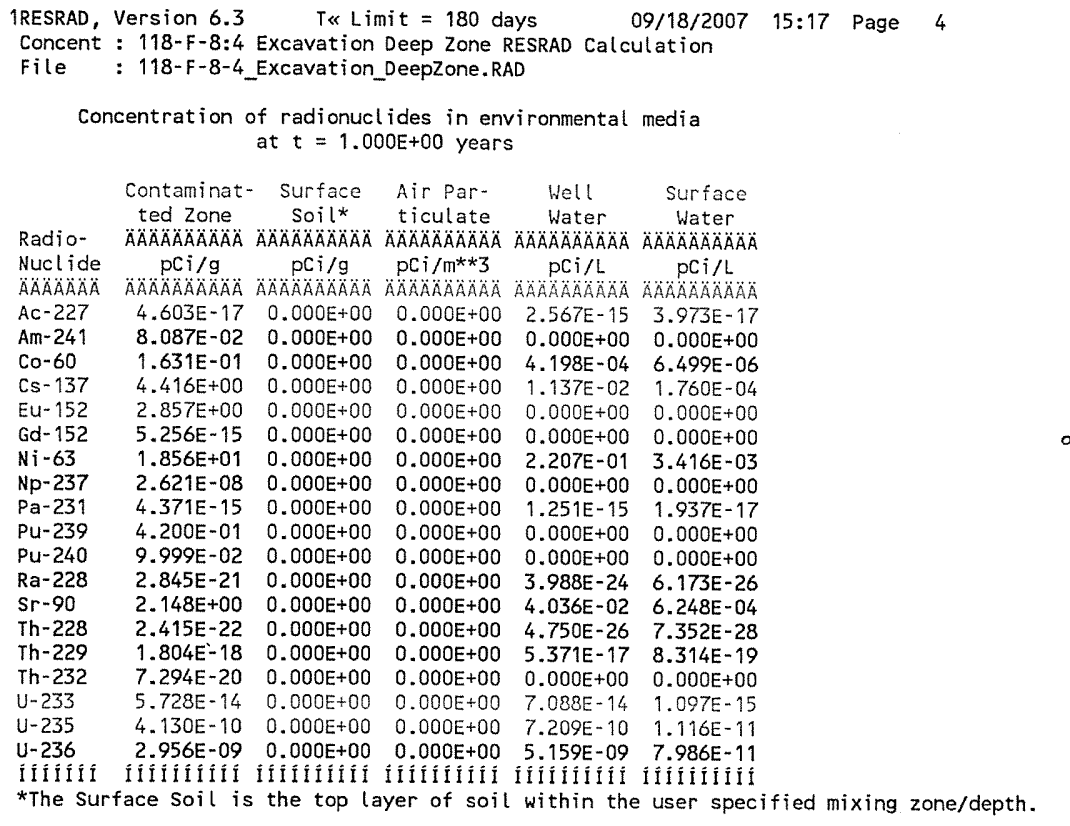

Concentrations in the media occurring in pathways that are suppressed are calculated using the current input parameters, i.e. using parameters appearing in the input screen when the pathways are active.

\begin{tabular}{|c|c|}
\hline Attachment & Sheet No 4 of 19 \\
\hline Originator: S.W. Clark & Date \\
\hline Chk'd By M. W. Perrott & Date \\
\hline $0100 F-C A-V 0303$ & Rev. No. \\
\hline
\end{tabular}




\section{ATTACHMENT 7}

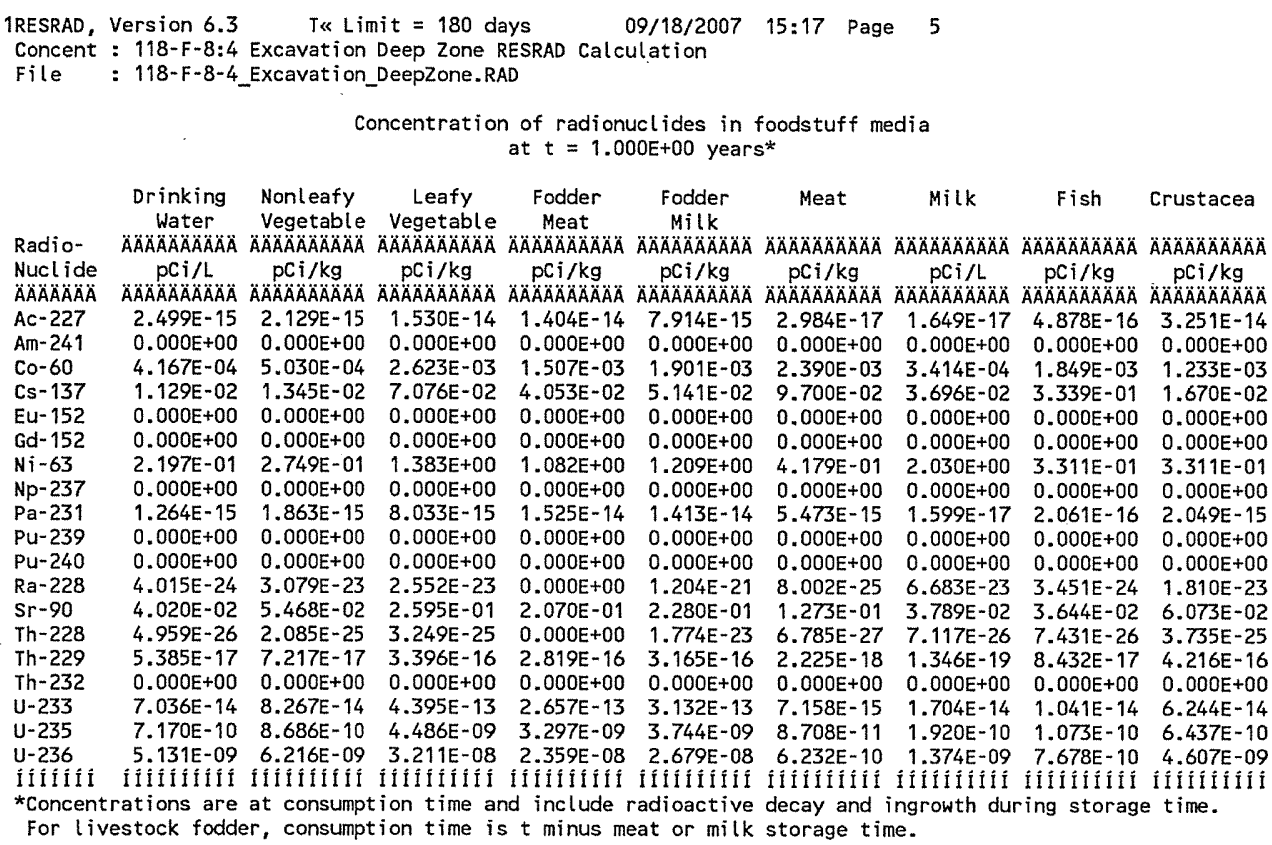

Concentrations in the media occurring in pathways that are suppressed are calculated using the current input parameters, i.e. using parameters appearing in the input screen when the pathways are active.

\begin{tabular}{|c|c|c|}
\hline \multirow{2}{*}{\multicolumn{2}{|c|}{ Attachment $\frac{7}{W}$}} & \multirow{2}{*}{$\begin{array}{l}\text { Sheet No. } \underline{5} \text { of } 19 \\
\text { Date }\end{array}$} \\
\hline & S. W. Clark & \\
\hline Chk'd By. & U. W. Perrott & Date \\
\hline Calc. No. & 0100F-CA-V0303 & Rev. No. \\
\hline
\end{tabular}


CVP-2007-00004

Rev. 0

\section{ATTACHMENT 7}

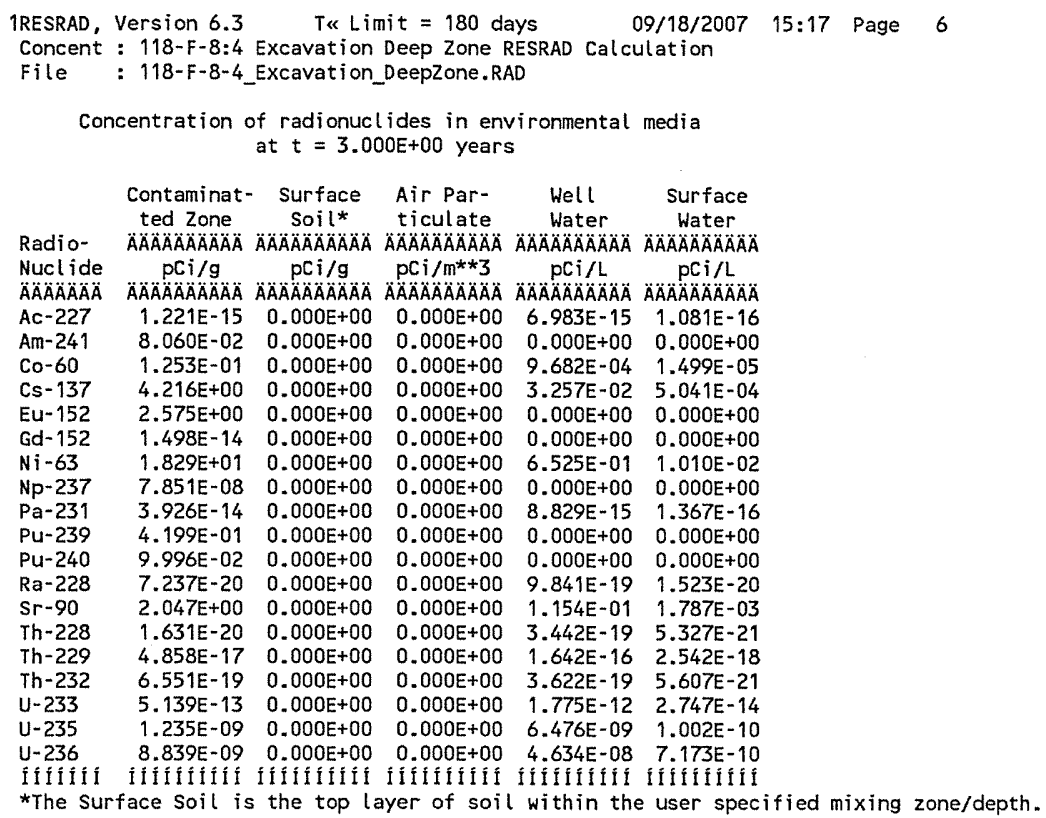

Concentrations in the media occurring in pathways that are suppressed are calculated using the current input parameters, i.e. Using parameters appearing in the input screen when the pathways are active.

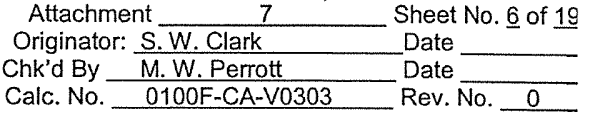


ATTACHMENT 7

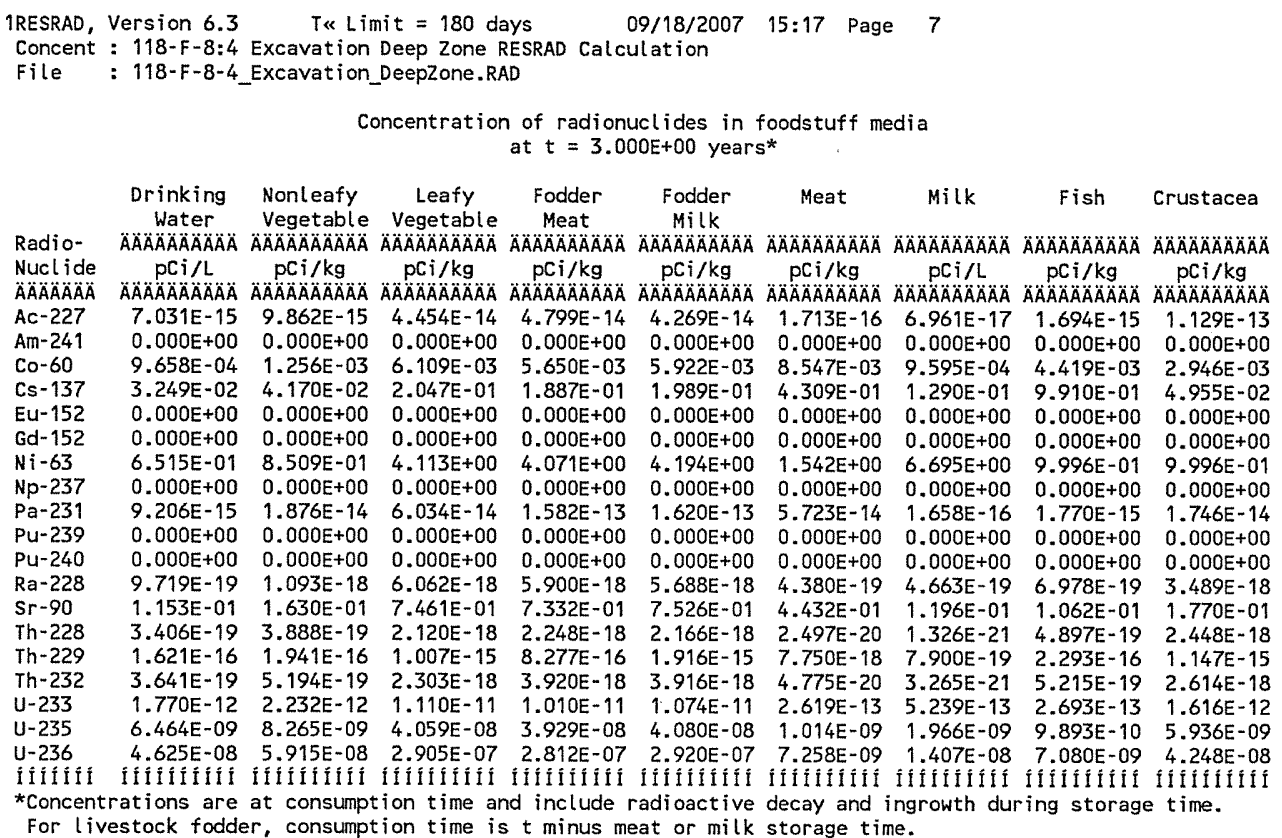

Concentrations in the media occurring in pathways that are suppressed are calculated using the current input parameters, i.e. using parameters appearing in the input screen when the pathways are active.

\begin{tabular}{|c|c|}
\hline Attachment & Sheet No. I of 19 \\
\hline Originator: S. W. Clark & Date \\
\hline M. W. Perrott & Date \\
\hline $0100 \mathrm{~F}-\mathrm{CA}-\mathrm{V} 0303$ & Rev. No. $\quad 0$ \\
\hline
\end{tabular}




\section{ATTACHMENT 7}

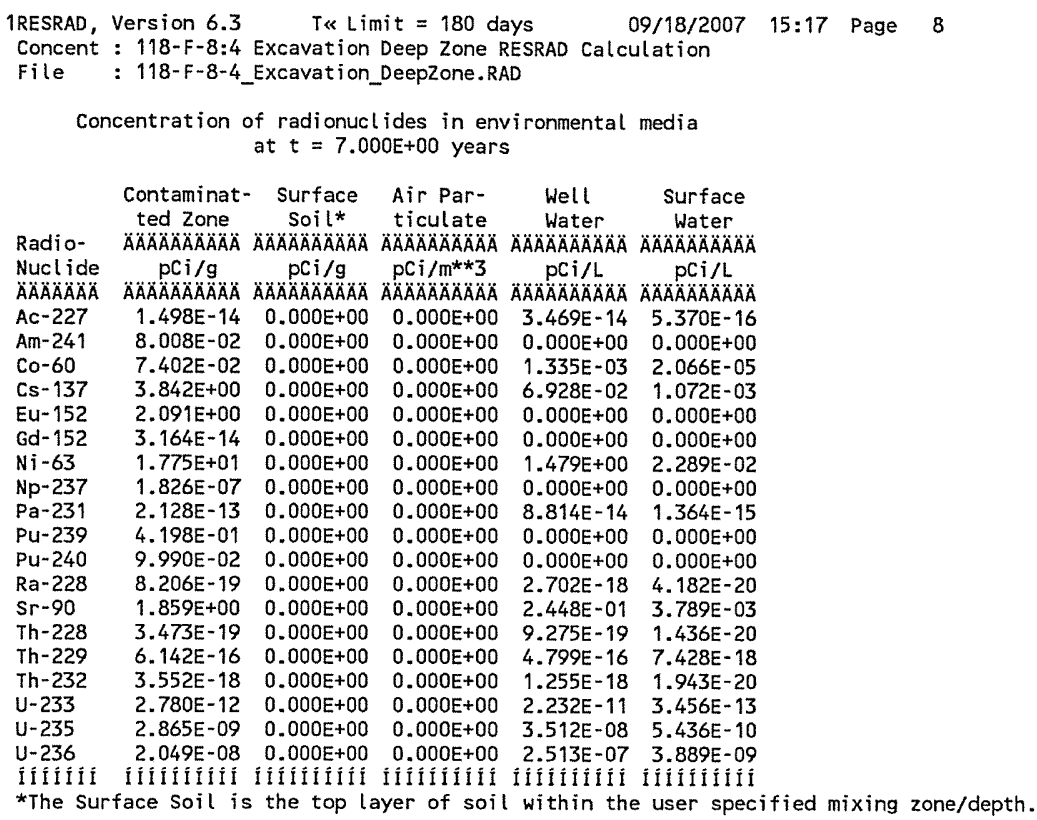

Concentrations in the media occurring in pathways that are suppressed are calculated using the current input parameters, i.e. using parameters appearing in the input screen when the pathways are active.

$\begin{array}{ll}\text { Attachment } \frac{7}{1} & \text { Sheet No. } 8 \text { of } 19 \\ \text { Originator: } \frac{\text { S.W. Clark }}{\text { M.W. Perrott }} & \text { Date } \\ \text { Chk'd By } & \text { Date } \\ \text { Calc. No. } & \text { Rev. No. NoOF-CA-V0303 } 0\end{array}$


ATTACHMENT 7

\begin{tabular}{|c|c|c|c|c|c|c|c|c|c|}
\hline $\begin{array}{l}\text { IRESRAD, } \\
\text { Concent } \\
\text { File }\end{array}$ & $\begin{array}{l}\text { Version } 6.3 \\
: 118-F-8: 4 \\
: 118-F-8-4\end{array}$ & $\begin{array}{l}\text { Te Lin } \\
\text { Excavation } \\
\text { Excavation }\end{array}$ & $\begin{array}{l}\text { mit }=180 \mathrm{da} \\
\text { Deep Zone } \\
\text { DeepZone. RA }\end{array}$ & $\begin{array}{l}\text { days } \\
\text { RESRAD } 0 \\
\text { RAD }\end{array}$ & $\begin{array}{l}09 / 18 / 2007 \\
\text { sulation }\end{array}$ & 15:17 Pag & 9 & & \\
\hline & & & oncentrat & $\begin{array}{l}\text { on of radi } \\
\text { at } t=7\end{array}$ & $\begin{array}{l}\text { lides i } \\
E+00 \text { ye }\end{array}$ & $f$ & & & \\
\hline & $\begin{array}{c}\text { Drinking } \\
\text { Water }\end{array}$ & $\begin{array}{l}\text { Nonleafy } \\
\text { Vegetable }\end{array}$ & $\begin{array}{c}\text { Leafy } \\
\text { Vegetable }\end{array}$ & $\begin{array}{l}\text { Fodder } \\
\text { Meat }\end{array}$ & $\begin{array}{l}\text { Fodder } \\
\text { Milk }\end{array}$ & Meat & Milk & Fish & Crustacea \\
\hline Radio & 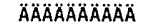 & $\triangle A A A A A A A A A A A$ & $\triangle A A A A O A A A O A O A ̈$ & $\triangle \triangle A A A A A A A A A O A O A$ & $\triangle A A O A A A A A A B A ̈ A ̈ A$ & $\triangle A A A A A A A A A B$ & ÄÄÄÄÄÄÄÄÄ & $\triangle A A A A A A A A A A$ & $\triangle A A A A A A A A C A B$ \\
\hline & & $\mathrm{pC}$ & & 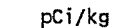 & & $\mathrm{pCi} / \mathrm{kg}$ & $\mathrm{pCi} / \mathrm{L}$ & $\mathrm{pCi} / \mathrm{kg}$ & $\mathrm{pCi} / \mathrm{kg}$ \\
\hline$\ddot{A A ̈}$ & $\triangle A A A A A A A A A A A$ & $\triangle A O A A A A A A A A A A$ & $\triangle A A A A A A A A A A A$ & 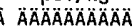 & 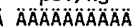 & $\triangle A A A A B A O A O A O A O A$ & 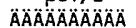 & 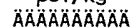 & $\triangle \triangle A \cap A ̈ A ̈$ \\
\hline $\mathrm{Ac}$ & $3.442 E-14$ & $4.048 E-14$ & $2.149 \mathrm{E}-13$ & $1.998 E-13$ & $1.873 E-13$ & $1.033 \mathrm{E}-15$ & $3.153 E-16$ & $7.616 E-15$ & $5.073 E-13$ \\
\hline$A m-241$ & $.000 E+00$ & $0.000 E+00$ & $0.000 E+00$ & $0.000 E+00$ & $0.000 \mathrm{E}+00$ & $0.000 E+00$ & $0.000 E+00$ & $0.000 E+00$ & $0.000 \mathrm{E}+00$ \\
\hline $\mathrm{CO}-$ & 1.3 & 1.76 & $8.447 E-03$ & $8.647 \mathrm{E}-03$ & $8.772 E-03$ & $1.298 \mathrm{E}-02$ & $1.391 \mathrm{E}-03$ & $6.153 \mathrm{E}-03$ & $4.102 E-03$ \\
\hline Cs & 12 & 29.063 & 4.36 & 1 4.456E-01 & $4.546 E-01$ & $1.009 E+00$ & $2.885 E-01$ & $2.129 \mathrm{E}+00$ & $1.065 \mathrm{E}-01$ \\
\hline Eu-152 & $0.000 \mathrm{E}+00$ & $0.000 E+00$ & $0.000 E+00$ & $0.000 \mathrm{E}+00$ & $0.000 E+00$ & $0.000 E+00$ & $0.000 E+00$ & $0.000 E+00$ & $0.000 E+00$ \\
\hline $\mathrm{Gd}$ & $0.000 E+00$ & $0.000 E+00$ & $0.000 E+00$ & $0.000 E+00$ & $0.000 E+00$ & $0.000 E+00$ & $0.000 E+00$ & $0 E+00$ & $0.000 E+00$ \\
\hline $\mathrm{Ni}$ & $1.478 E+00$ & $1.953 \mathrm{E}+00$ & $9.337 \mathrm{E}+00$ & $9.789 E+00$ & $9.907 \mathrm{E}+00$ & $3.692 \mathrm{E}+00$ & $1.562 E+01$ & $2.279 E+00$ & $2.279 E+00$ \\
\hline$N p-237$ & $0.000 E+00$ & $0.000 E+00$ & $0.000 E+00$ & $0.000 E+00$ & $0.000 E+00$ & $0.000 E+00$ & $0.000 E+00$ & $0.000 E+00$ & $0.000 E+00$ \\
\hline $\mathrm{Pa}-$ & $8.997 E-14$ & $1.517 E-13$ & $5.780 E-13$ & $1.164 E-12$ & $1.178 \mathrm{E}-12$ & $4.240 E-13$ & $1.036 E-15$ & $E-14$ & $\mathrm{BE}-13$ \\
\hline Pu- & $0.000 E+00$ & $0.000 E+00$ & $0.000 E+00$ & $0.000 \mathrm{E}+00$ & $0.000 E+00$ & $0.000 E+00$ & $0.000 \mathrm{E}+00$ & $0.000 E+00$ & $0.000 E+00$ \\
\hline Pu-240 & $0.000 \mathrm{E}+00$ & $0.000 \mathrm{E}+00$ & $0.000 E+00$ & $0.000 E+00$ & $0.000 E+00$ & $0.000 \mathrm{E}+00$ & $0.000 E+00$ & $0.000 E+00$ & $0.000 E+00$ \\
\hline $\mathrm{Ra}-228$ & $2.706 E-18$ & $3.694 E-18$ & $1.712 E-17$ & $E-17$ & $3 E-17$ & $1.490 E-18$ & $1.560 E-18$ & $2.119 E-18$ & $1.060 \mathrm{E}-17$ \\
\hline $\mathrm{Sr}-90$ & $2.447 \mathrm{E}-01$ & 1 $3.498 \mathrm{E}-01$ & $1.585 E+00$ & $1.640 \mathrm{E}+00$ & $1.657 \mathrm{E}+00$ & $9.880 \mathrm{E}-01$ & $2.605 E-01$ & $2.265 \mathrm{E}-01$ & $E-01$ \\
\hline & $9.311 \mathrm{E}-19$ & $1.292 E-18$ & $5.881 E-18$ & $37.435 E-18$ & $7.674 E-18$ & $8.369 E-20$ & $4.401 E-21$ & $1.461 \mathrm{E}-18$ & $7.303 E-18$ \\
\hline & $4.823 E-16$ & $6.913 E-16$ & $3.050 E-15$ & $4.727 E$ & 5.26 & $5.327 E-17$ & $3.623 E-18$ & $7.147 \mathrm{E}-16$ & $3.580 E-15$ \\
\hline Th & $1.290 \mathrm{E}-18$ & $2.338 E-18$ & $8.339 E-18$ & $1.868 \mathrm{E}-17$ & $1.958 \mathrm{E}-17$ & $2.475 E-19$ & $1.710 \mathrm{E}-20$ & $1.994 \mathrm{E}-18$ & $1.000 \mathrm{E}-17$ \\
\hline U-233 & $2.230 E-11$ & $2.877 E-11$ & $1.401 E-10$ & $1.419 E-10$ & $1.453 E-10$ & $3.651 \mathrm{E}-12$ & $6.932 E-12$ & $3.425 \mathrm{E}-12$ & $2.055 \mathrm{E}-11$ \\
\hline U-235 & $3.509 E-08$ & $4.555 E-08$ & $2.206 \mathrm{E}-07$ & $2.294 E-07$ & $2.330 \mathrm{E}-07$ & $5.892 E-09$ & $1.106 \mathrm{E}-08$ & $5.405 \mathrm{E}-09$ & $3.243 \mathrm{E}-08$ \\
\hline U. 236 & $2.511 \mathrm{E}$ & 07 & 1.5 & $1.642 \mathrm{E}-06$ & $1.668 \mathrm{E}-06$ & $4.216 \mathrm{E}-08$ & $7.911 E-08$ & $3.868 E-08$ & 2.321E-07 \\
\hline 5. & 20 & 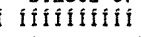 & $\cdots$ & $\because 4$ & & & YOTL U & fífilitín & fil \\
\hline & & & & & & & & & \\
\hline
\end{tabular}

Concentrations in the media occurring in pathways that are suppressed are calculated using the current input parameters, i.e. using parameters appearing in the input screen when the pathways are active.

$\begin{array}{ll}\text { Attachment } \frac{7}{1} & \text { Sheet No. } 9 \text { of } 19 \\ \text { Originator: } \frac{\text { S.W. Clark }}{\text { M.W. Perrott }} & \text { Date } \\ \text { Chk'd By } & \text { Date } \\ \text { Calc. No. } & \text { Rev. No. } 0100 \text { F-CA-V0303 }\end{array}$




\section{ATTACHMENT 7}

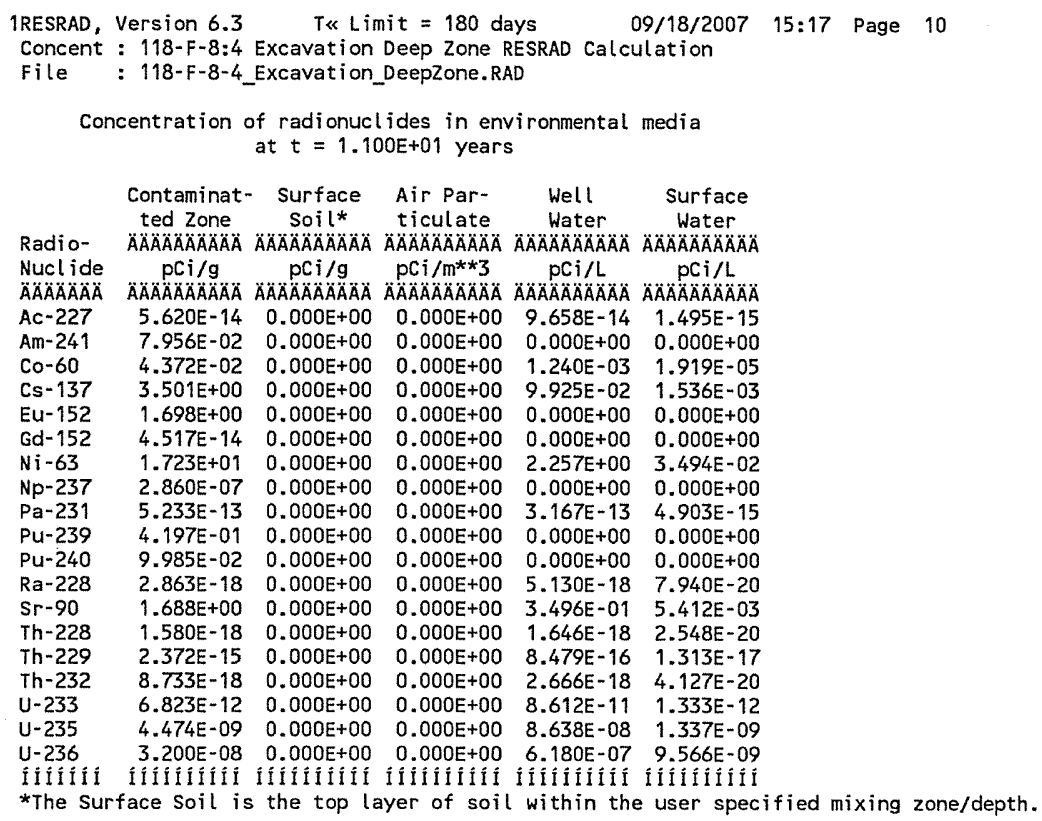

Concentrations in the media occurring in pathways that are suppressed are calculated using. the current input parameters, i.e. using parameters appearing in the input screen when the pathways are active.

\begin{tabular}{|c|c|}
\hline Attachment & Sheet No. 10 of 19 \\
\hline Originator: S.W. Clark & Date \\
\hline M. W. Perrott & Date \\
\hline $0100 \mathrm{~F}-\mathrm{CA}-\mathrm{V} 0303$ & Rev, No. 0 \\
\hline
\end{tabular}




\section{ATTACHMENT 7}

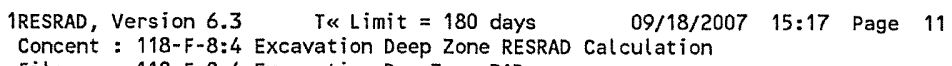

Drinking Nonleafy Leafy Fodder Fodder Meat Milk Fish Crustacea

Radio- W A Nuclide $\mathrm{pCi} / \mathrm{L} \quad \mathrm{pCi} / \mathrm{kg} \quad \mathrm{pCi} / \mathrm{kg} \quad \mathrm{pCi} / \mathrm{kg} \quad \mathrm{pCi} / \mathrm{kg} \quad \mathrm{pCi} / \mathrm{kg} \quad \mathrm{pCi} / \mathrm{L} \quad \mathrm{pCi} / \mathrm{kg} \quad \mathrm{pCi} / \mathrm{kg}$

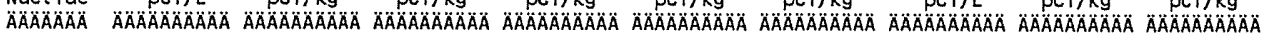
$\begin{array}{llllllllll}\text { AC-227 } & 9.617 \mathrm{E}-14 & 1.208 \mathrm{E}-13 & 6.026 \mathrm{E}-13 & 6.011 \mathrm{E}-13 & 6.916 \mathrm{E}-13 & 3.184 \mathrm{E}-15 & 1.067 \mathrm{E}-15 & 2.178 \mathrm{E}-14 & 1.450 \mathrm{E}-12\end{array}$ $\begin{array}{llllllllll}A m-241 & 0.000 E+00 & 0.000 E+00 & 0.000 E+00 & 0.000 E+00 & 0.000 E+00 & 0.000 E+00 & 0.000 E+00 & 0.000 E+00 & 0.000 E+00\end{array}$ $\begin{array}{llllllllll}\mathrm{C}-60 & 1.239 \mathrm{E}-03 & 1.652 \mathrm{E}-03 & 7.850 \mathrm{E}-03 & 8.246 \mathrm{E}-03 & 8.298 \mathrm{E}-03 & 1.236 \mathrm{E}-02 & 1.309 \mathrm{E}-03 & 5.729 \mathrm{E}-03 & 3.819 \mathrm{E}-03\end{array}$ $\begin{array}{lllllllllll}\text { Cs-137 } & 9.918 E-02 & 1.306 E-01 & 6.260 E-01 & 6.556 E-01 & 6.635 E-01 & 1.482 E+00 & 4.188 E-01 & 3.058 E+00 & 1.529 E-01\end{array}$ $\begin{array}{lllllllllll}\text { Eu-152 } & 0.000 E+00 & 0.000 E+00 & 0.000 E+00 & 0.000 E+00 & 0.000 E+00 & 0.000 E+00 & 0.000 E+00 & 0.000 E+00 & 0.000 E+00\end{array}$ $\begin{array}{lllllllllll}\mathrm{Gd}-152 & 0.000 E+00 & 0.000 E+00 & 0.000 E+00 & 0.000 E+00 & 0.000 E+00 & 0.000 E+00 & 0.000 E+00 & 0.000 E+00 & 0.000 E+00\end{array}$ $\begin{array}{llllllllll}\mathrm{Ni}-63 & 2.256 \mathrm{E}+00 & 2.991 \mathrm{E}+00 & 1.426 \mathrm{E}+01 & 1.518 \mathrm{E}+01 & 1.529 \mathrm{E}+01 & 5.717 \mathrm{E}+00 & 2.403 \mathrm{E}+01 & 3.484 \mathrm{E}+00 & 3.484 \mathrm{E}+00\end{array}$ $\mathrm{NP}-237 \quad 0.000 \mathrm{E}+00 \quad 0.000 \mathrm{E}+00 \quad 0.000 \mathrm{E}+00 \quad 0.000 \mathrm{E}+00 \quad 0.000 \mathrm{E}+00 \quad 0.000 \mathrm{E}+00$ O $0.000 \mathrm{E}+00 \quad 0.000 \mathrm{E}+00 \quad 0.000 \mathrm{E}+00$ Pa-231 $3.213 E-13 \quad 5.075 E-13 \quad 2.052 E-12$ 3.

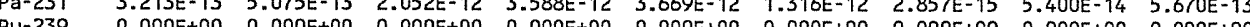
$\begin{array}{llllllllll}\mathrm{PU}-239 & 0.000 E+00 & 0.000 E+00 & 0.000 E+00 & 0.000 E+00 & 0.000 E+00 & 0.000 E+00 & 0.000 E+00 & 0.000 E+00 & 0.000 E+00\end{array}$ $\begin{array}{llllllllll}\mathrm{PU}-240 & 0.000 \mathrm{E}+00 & 0.000 \mathrm{E}+00 & 0.000 E+00 & 0.000 \mathrm{E}+00 & 0.000 \mathrm{E}+00 & 0.000 \mathrm{E}+00 & 0.000 \mathrm{E}+00 & 0.000 \mathrm{E}+00 & 0.000 \mathrm{E}+00\end{array}$ $\begin{array}{lllllllllll}\text { Ra-228 } & 5.145 \mathrm{E}-18 & 7.070 \mathrm{E}-18 & 3.259 \mathrm{E}-17 & 3.589 \mathrm{E}-17 & 3.576 \mathrm{E}-17 & 2.694 \mathrm{E}-18 & 2.792 \mathrm{E}-18 & 4.053 \mathrm{E}-18 & 2.027 \mathrm{E}-17\end{array}$ $\begin{array}{llllllllll}\mathrm{Sr}-90 & 3.495 \mathrm{E}-01 & 5.011 \mathrm{E}-01 & 2.264 \mathrm{E}+00 & 2.376 \mathrm{E}+00 & 2.390 \mathrm{E}+00 & 1.430 \mathrm{E}+00 & 3.747 \mathrm{E}-01 & 3.239 \mathrm{E}-01 & 5.398 \mathrm{E}-01\end{array}$ $\begin{array}{lllllllllll}\text { Th-228 } & 1.655 \mathrm{E}-18 & 2.325 \mathrm{E}-18 & 1.047 \mathrm{E}-17 & 1.267 \mathrm{E}-17 & 1.261 \mathrm{E}-17 & 1.460 \mathrm{E}-19 & 7.558 \mathrm{E}-21 & 2.616 \mathrm{E}-18 & 1.308 \mathrm{E}-17\end{array}$

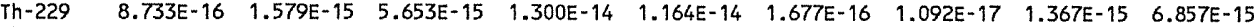

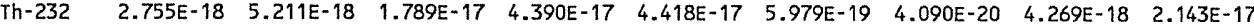
U-233 8.606E-11 $11118 E-10 \quad 5.411 E-10 \quad 5.646 E-10 \quad 5.725 E-10 \quad 1.449 E-11 \quad 2.715 E-11 \quad 1.326 E-11 \quad 7.957 E-111$

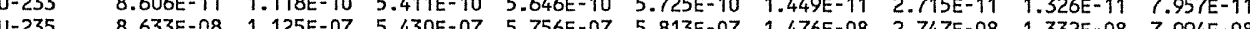

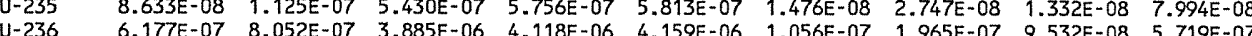

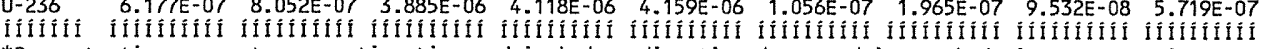
*Concentrations are at consumption time and include radioactive decay and ingrowth during storage time.

For livestock fodder, consumption time is $t$ minus meat or milk storage time.

Concentrations in the media occurring in pathways that are suppressed are calculated using the current input parameters, i.e. using parameters appearing in the input screen when the pathways are active.

\begin{tabular}{|c|c|}
\hline Attachment & et No. 11 of 19 \\
\hline Originator: S.W. Clark & Date \\
\hline M. W. Perrott & Date \\
\hline $0100 F-C A-V 0303$ & Rev. No. \\
\hline
\end{tabular}




\section{ATTACHMENT 7}

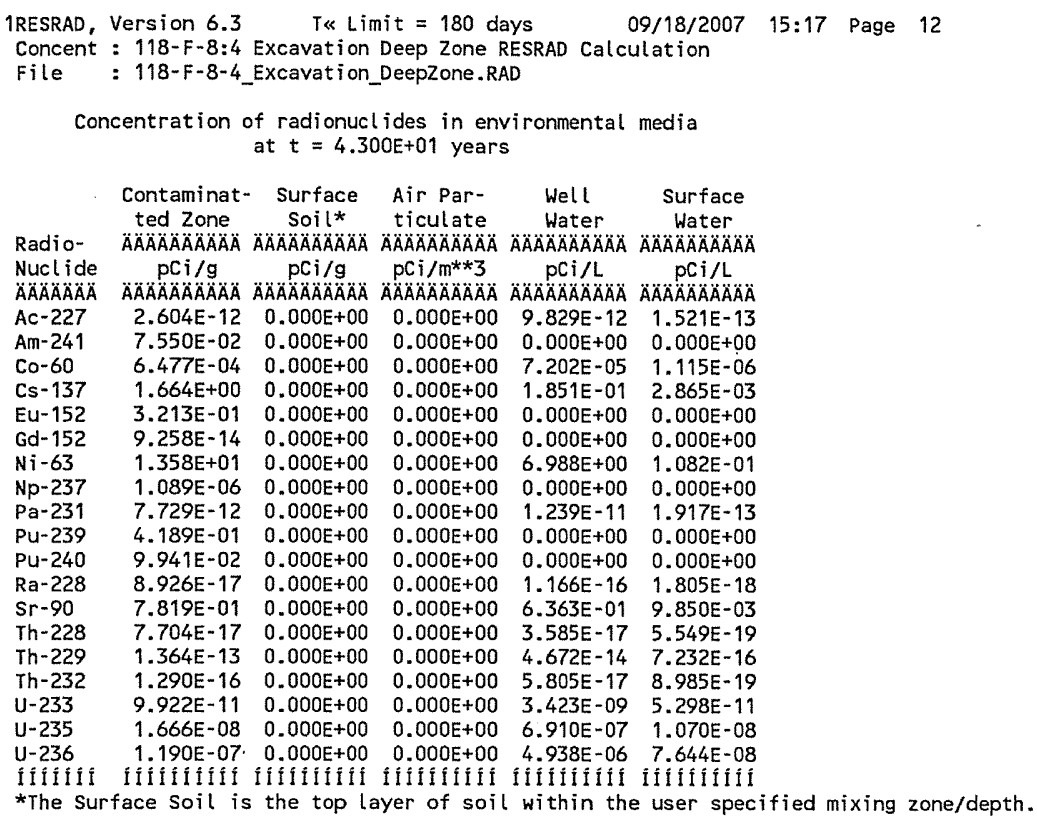

Concentrations in the media occurring in pathways that are suppressed are calculated using the current input parameters, i.e. using parameters appearing in the input screen when the pathways are active.

\begin{tabular}{|c|c|}
\hline Attachment & Sheet No. 12 of 19 \\
\hline Originator: $\mathrm{S.W}$. Clark & Date \\
\hline M. W. Perrott & Date \\
\hline Calc. No. $\quad 0100 \mathrm{~F}-\mathrm{CA}-\mathrm{V} 0303$ & Rev. No. 0 \\
\hline
\end{tabular}




\section{ATTACHMENT 7}

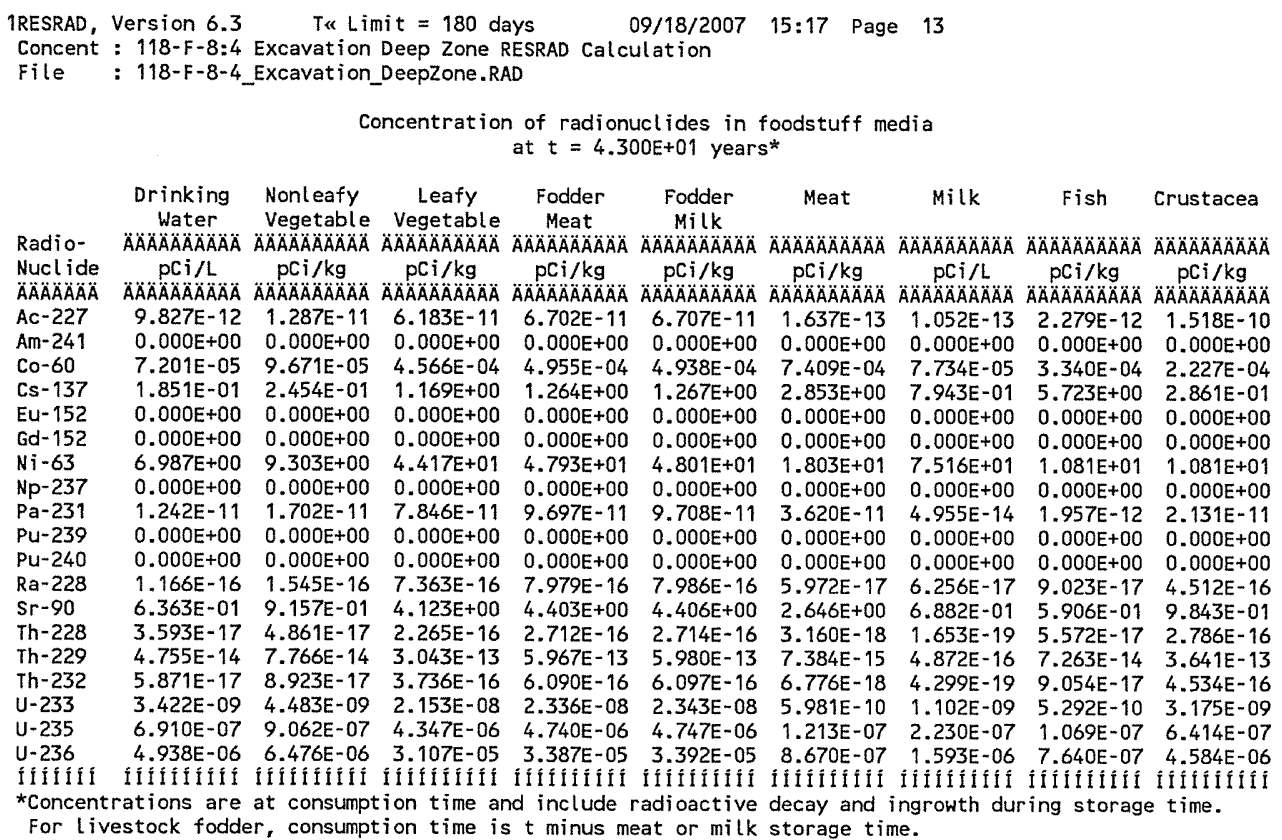

Concentrations in the media occurring in pathways that are suppressed are calculated using the current input parameters, i.e. using parameters appearing in the input screen when the pathways are active.

\begin{tabular}{|c|c|}
\hline Attachment & Sheet No. 13 of 19 \\
\hline Originator: S.W. Clark & Date \\
\hline Chk'd By M. W. Perrott & Date \\
\hline Calc. No. $0100 \mathrm{~F}-\mathrm{CA}-\mathrm{V} 0303$ & Rev. No. \\
\hline
\end{tabular}




\section{ATTACHMENT 7}

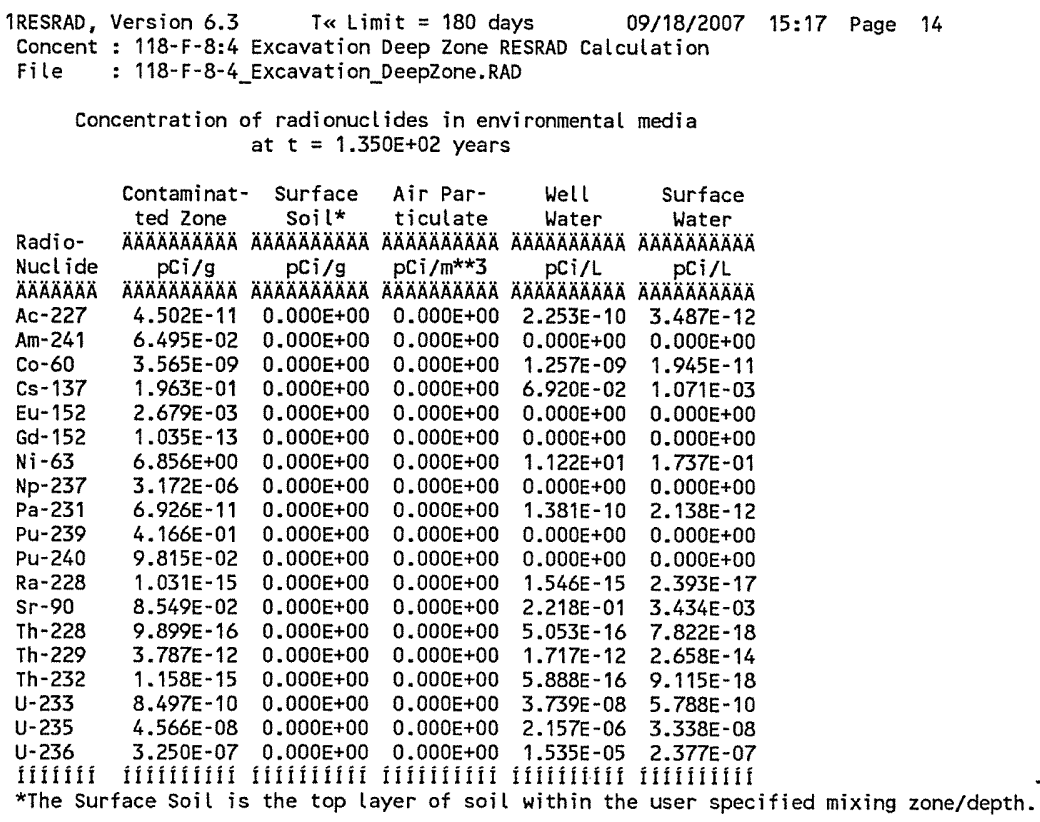

Concentrations in the media occurring in pathways that are suppressed are calculated using the current input parameters, i.e. using parameters appearing in the input screen when the pathways are active.

$\begin{array}{ll}\text { Attachment } \frac{7}{\text { S.W. Clark }} & \text { Sheet No. } 14 \text { of } 19 \\ \text { Originator: } & \text { Date } \\ \text { Chk'd By } & \text { Date } \\ \text { Calc. No. Perrott } & \text { Rev. No. } \quad 0\end{array}$




\section{ATTACHMENT 7}

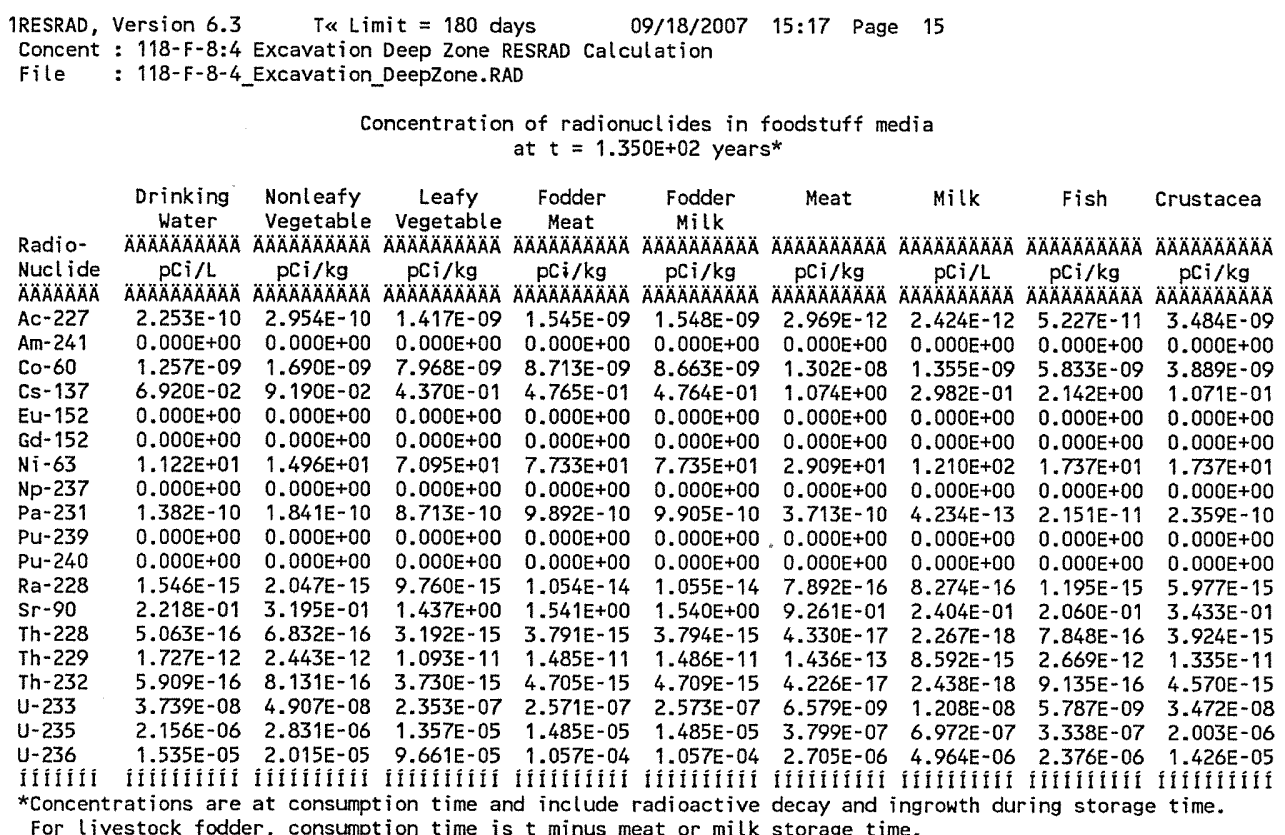

Concentrations in the media occurring in pathways that are suppressed are calculated using the current input parameters, i.e. using paraneters appearing in the input screen when the pathways are active.

\begin{tabular}{|c|c|}
\hline Attachment & Sheet No. 15 of 19 \\
\hline Originator: S.W. Clark & Date \\
\hline 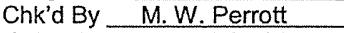 & Date \\
\hline Calc. No. $\quad 0100 F-C A-V 0303$ & Rev. No. 0 \\
\hline
\end{tabular}




\section{ATTACHMENT 7}

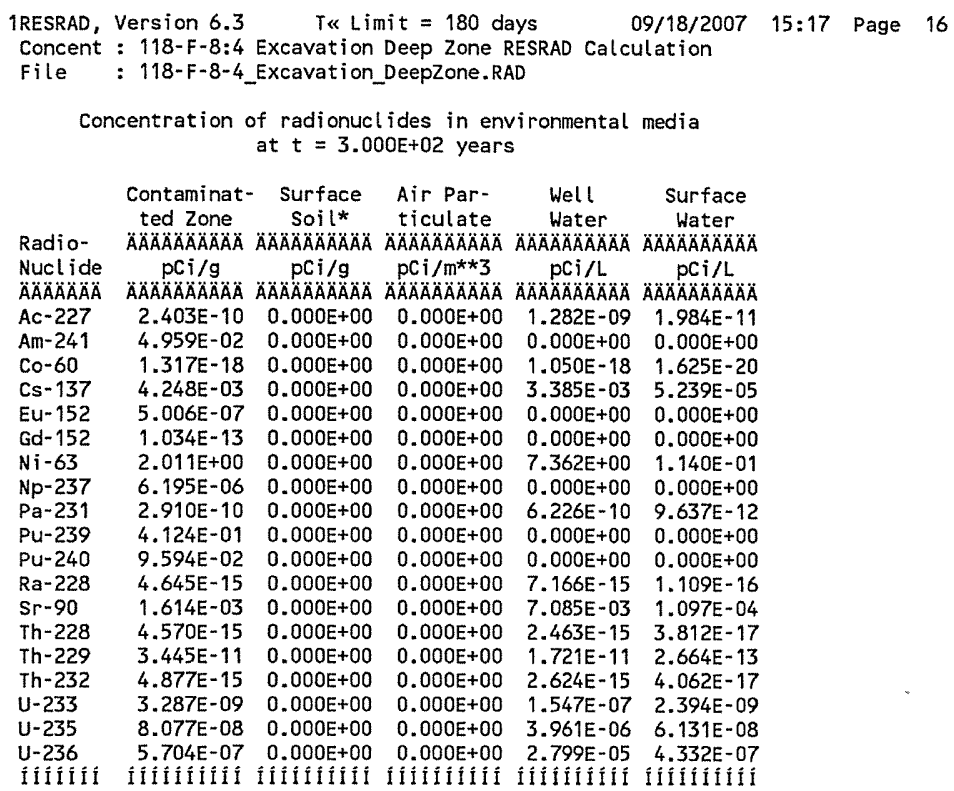

*The Surface Soil is the top layer of soil within the user specified mixing zone/depth.

Concentrations in the media occurring in pathways that are suppressed are calculated using the current input parameters, i.e. using parameters appearing in the input screen when the pathways are active.

\begin{tabular}{|c|c|}
\hline \multirow{2}{*}{\multicolumn{2}{|c|}{$\begin{array}{ll}\text { Attachment } & \frac{7}{\text { S.W. Clark }} \text { Sheet No. } 16 \text { of } 19 \\
\text { Originator: } & \text { Date }\end{array}$}} \\
\hline & \\
\hline M. W. Perrott & Date \\
\hline $0100 F-C A-V 0303$ & Rev. No. \\
\hline
\end{tabular}




\section{ATTACHMENT 7}

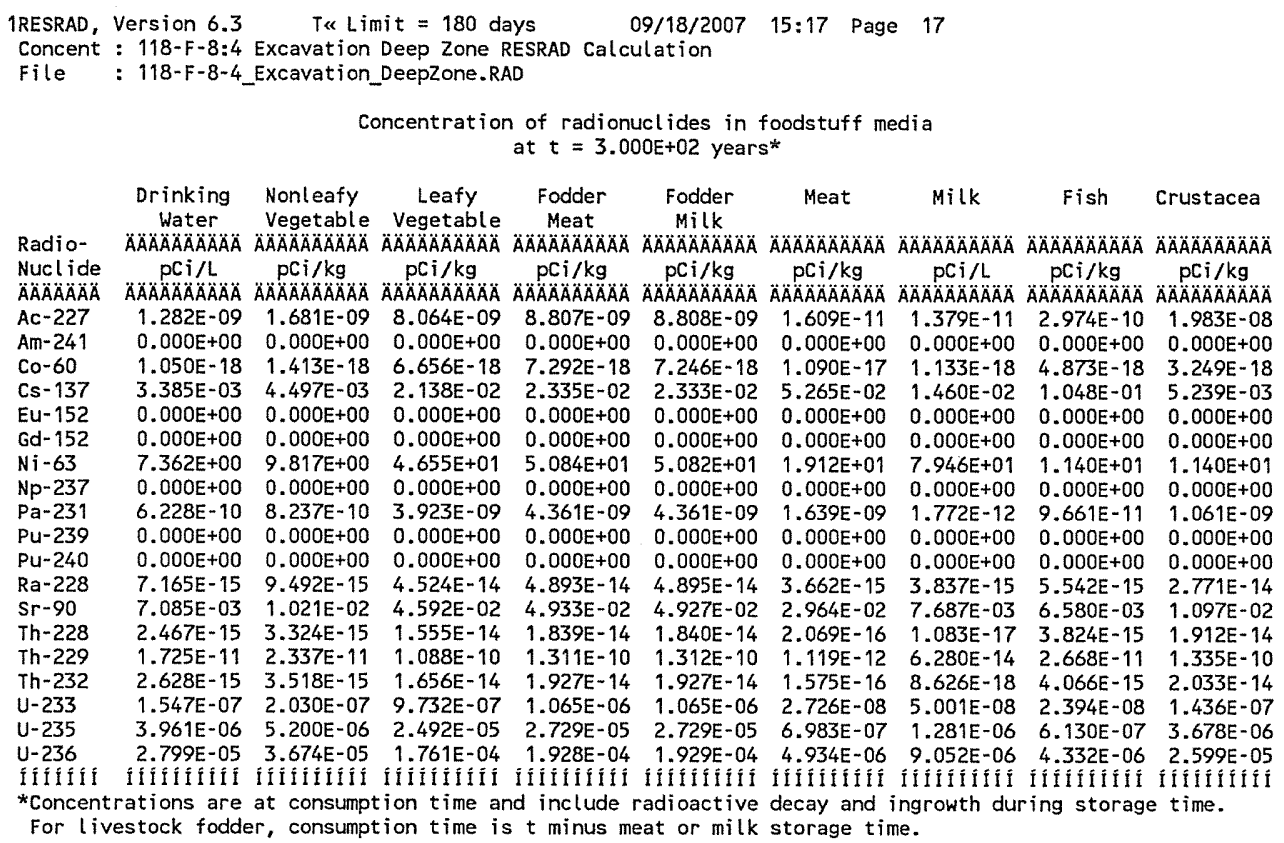

Concentrations in the media occurring in pathways that are suppressed are calculated using the current input parameters, i.e. using parameters appearing in the input screen when the pathways are active.

$\begin{array}{ll}\text { Attachment } \frac{7}{\text { S.W. Clark }} & \text { Sheet No. } 17 \text { of } 19 \\ \text { Originator: } & \text { Date } \\ \text { Chk'd By } \frac{\text { M.W. Perrott }}{0100 \text { F-CA-V0303 }} \text { Date } \\ \text { Calc. No. Nev. No. } \quad 0\end{array}$




\section{ATTACHMENT 7}

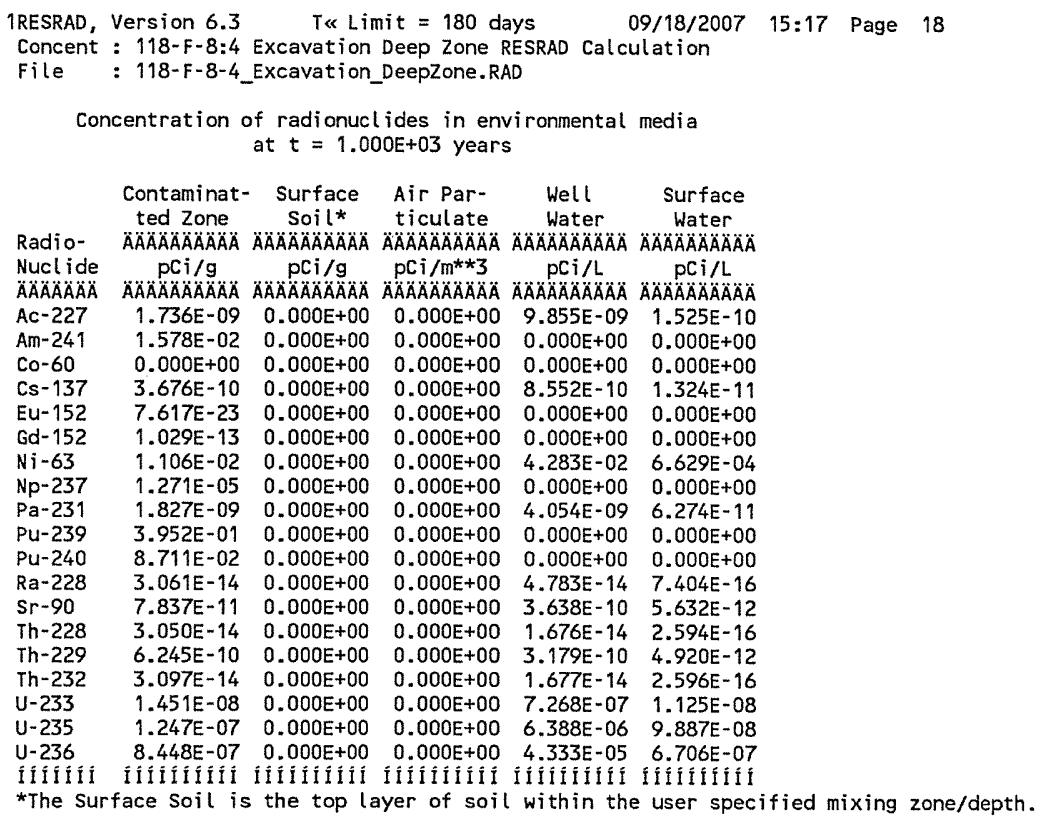

Concentrations in the media occurring in pathways that are suppressed are calculated using the current input parameters, i.e. using parameters appearing in the input screen when the pathways are active.

\begin{tabular}{|c|c|}
\hline \multirow{2}{*}{\multicolumn{2}{|c|}{$\begin{array}{ll}\text { Attachment } & 7 \\
\text { Originator: S.W. Slark } & \text { Sheet No. } 18 \text { of } 19 \\
\text { Date }\end{array}$}} \\
\hline & \\
\hline Chk'd By M. W. Perrott & Date \\
\hline $0100 F-C A-V 0303$ & Rev. No. \\
\hline
\end{tabular}




\section{ATTACHMENT 7}

\begin{tabular}{|c|c|c|c|c|c|c|c|c|c|}
\hline $\begin{array}{l}\text { 1RESRAD, } \\
\text { Concent } \\
\text { File }\end{array}$ & $\begin{array}{l}\text { Version } 6.3 \\
: 118-F-8: 4 \\
: 118-F-8-4\end{array}$ & $\begin{array}{l}\text { T\& Lin } \\
\text { Excavation } \\
\text { Excavation }\end{array}$ & $\begin{array}{l}\text { mit }=180 \mathrm{da} \\
\text { Deep Zone } R \\
\text { DeepZone.RA }\end{array}$ & $\begin{array}{l}\text { lays } \\
\text { RESRAD } \\
\text { AD }\end{array}$ & $\begin{array}{l}09 / 18 / 2007 \\
\text { culation }\end{array}$ & $15: 17$ & 19 & & \\
\hline & & & oncentrat & $\begin{array}{l}\text { n of radi } \\
\text { at } t=1\end{array}$ & $\begin{array}{l}\text { lides } i \\
E+03 \text { ye }\end{array}$ & 1 & & & \\
\hline & $\begin{array}{c}\text { Drinking } \\
\text { Water }\end{array}$ & $\begin{array}{l}\text { Nonleafy } \\
\text { Vegetable }\end{array}$ & $\begin{array}{c}\text { Leafy } \\
\text { Vegetable }\end{array}$ & $\begin{array}{l}\text { Fodder } \\
\text { Meat }\end{array}$ & $\begin{array}{l}\text { Fodder } \\
\text { Milk }\end{array}$ & Meat & Milk & Fish & Crustacea \\
\hline Radio & ÄÄÄÄĀÄÄÄ & 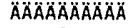 & ÄĂÄÄÄÄÄÄÄ & $\triangle A O A A A A A A A A B$ & $\triangle A A A A A A A A A A A$ & $\triangle A A B A A A A A A O A B$ & $\triangle A A A A A A A A A A ̈ A$ & $\triangle A O A A A O A O A O A ̈ A ̈$ & $\triangle A A A A A A O A O A ̈ A ̈$ \\
\hline & & & & & & $\mathrm{Ci} / \mathrm{kg}$ & & & \\
\hline & $\triangle A A A A A A A A A A B$ & 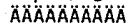 & AAAAAAOAAAAÄÄ & $\triangle \triangle A A A A A A A B A A B$ & - $\triangle A A A A A B A A A A A$ & $\triangle A O A O A A A A O A B$ & 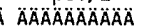 & $\ddot{A} \ddot{A} \ddot{A} A$ & $\ddot{A} \ddot{A} A \ddot{A}$ \\
\hline$A C-227$ & $9.854 \mathrm{E}-09$ & $1.293 \mathrm{E}-08$ & $6.200 E-08$ & $6.776 E-08$ & $36.776 E-08$ & $1.202 E-10$ & $1.061 \mathrm{E}-10$ & $2.287 E-09$ & $1.525 \mathrm{E}-07$ \\
\hline$A m-241$ & $0.000 \mathrm{E}+00$ & $0.000 \mathrm{E}+00$ & $0.000 \mathrm{E}+00$ & $0.000 \mathrm{E}+00$ & $0.000 E+00$ & $0.000 E+00$ & $0.000 E+00$ & $0.000 E+00$ & $0.000 E+00$ \\
\hline Co- 60 & $0.000 E+00$ & $0.000 E+00$ & $0.000 E+00$ & $0.000 \mathrm{E}+00$ & $0.000 \mathrm{E}+00$ & $0.000 E+00$ & $0.000 E+00$ & $0.000 E+00$ & $0.000 E+00$ \\
\hline Cs- & -10 & $1.137 E-09$ & $5.402 E-09$ & $5.909 E-09$ & 5.902E-09 & $1.332 E-08$ & $3 \quad 3.692 E-09$ & $2.648 E-08$ & $1.324 E-09$ \\
\hline Eu-1 & $0.000 E+00$ & $0.000 E+00$ & $0.000 E+00$ & $0.000 E+00$ & $0.000 E+00$ & $0.000 \mathrm{E}+00$ & $0.000 \mathrm{E}+00$ & $0.000 E+00$ & $0.000 E+00$ \\
\hline Gd- 15 & $0.000 E+00$ & $0.000 E+00$ & $0.000 E+00$ & $0.000 E+00$ & $0.000 E+00$ & $0.000 E+00$ & $0.000 E+00$ & $0.000 E+00$ & $0.000 \mathrm{E}+00$ \\
\hline $\mathrm{Ni}-63$ & $283 E-02$ & $5.710 E-02$ & $2.708 \mathrm{E}-01$ & $2.957 \mathrm{E}-01$ & $2.956 \mathrm{E}-01$ & $1.112 \mathrm{E}-01$ & $4.622 E-01$ & $6.629 \mathrm{E}-02$ & $6.629 E-02$ \\
\hline $\mathrm{Np}-23$ & $E+00$ & 0.00 & $0.000 E+00$ & $0.000 E+00$ & $0.000 E+00$ & $0.000 E+00$ & $0.000 E+00$ & $0.000 E+00$ & $0.000 \mathrm{E}+00$ \\
\hline $\mathrm{Pa}-231$ & $4.054 E-09$ & $5.342 E-09$ & $2.553 E-08$ & $2.806 E-08$ & $2.806 E-08$ & $1.056 \mathrm{E}-08$ & $1.108 E-11$ & $8 E-10$ & $4 E-09$ \\
\hline $\mathrm{Pu}-2$ & $0.000 E+00$ & $0.000 E+00$ & $0.000 E+00$ & $0.000 \mathrm{E}+00$ & $0.000 E+00$ & $0.000 E+00$ & $0.000 E+00$ & $0.000 \mathrm{E}+00$ & $0.000 E+00$ \\
\hline Pu-24 & $0.000 E+00$ & $0.000 E+00$ & $0.000 E+00$ & $0.000 E+00$ & $0.000 E+00$ & $0.000 E+00$ & $0.000 E+00$ & $0.000 E+00$ & $0.000 E+00$ \\
\hline $\mathrm{Ra}-228$ & $4.782 E-14$ & $6.336 E-14$ & $3.020 E-13$ & $3.268 \mathrm{E}-13$ & $3.268 E-13$ & $2.446 E-14$ & $2.562 E-14$ & $E-14$ & $E-13$ \\
\hline $\mathrm{Sr}-90$ & $3.638 E-10$ & $5.243 E-10$ & $2.358 \mathrm{E}-09$ & $2.533 E-09$ & $2.530 E-09$ & $1.522 \mathrm{E}-09$ & $3.947 \mathrm{E}-10$ & $3.379 E-10$ & $E-10$ \\
\hline Th- & $1.679 E-14$ & $2.260 \mathrm{E}-14$ & $1.058 E-13$ & $1.250 E-13$ & $1.250 E-13$ & $1.397 E-15$ & $7.316 E-17$ & $2.602 E-14$ & $1.301 E-13$ \\
\hline Th-229 & $3.181 \mathrm{E}-10$ & $4.208 E-10$ & $2.002 E-09$ & $2.250 E-09$ & $2.250 \mathrm{E}-09$ & $1.755 \mathrm{E}-$ & $9.340 E-13$ & $4.922 E-10$ & $2.461 \mathrm{E}-09$ \\
\hline Th. & $1.678 E-14$ & $2.212 E-14$ & $1.056 E-13$ & $1.174 \mathrm{E}-13$ & $1.174 E-13$ & $9.031 E-16$ & $4.761 E-17$ & $2.597 \mathrm{E}-14$ & $1.298 E-13$ \\
\hline u-233 & $7.268 \mathrm{E}-07$ & $9.542 E-07$ & $4.573 E-06$ & $5.009 \mathrm{E}-06$ & $5.009 E-06$ & $1.282 E-07$ & $2.351 E-07$ & $1.125 \mathrm{E}-07$ & $6.750 \mathrm{E}-07$ \\
\hline & $.388 E-06$ & $8.386 \mathrm{E}-06$ & $4.019 E-05$ & $4.403 E-05$ & $4.403 E-05$ & $1.127 \mathrm{E}-0$ & $2.066 \mathrm{E}-06$ & $9.887 \mathrm{E}-07$ & $5.932 E-06$ \\
\hline$U-$ & 4.3 & 5.6 & 2.72 & 2.9 & $E-04$ & $7.641 \mathrm{E}-$ & $1.401 \mathrm{E}-05$ & $6.706 \mathrm{E}-06$ & 4.024E-05 \\
\hline & 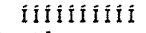 & 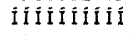 & 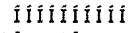 & & ífí & 1.0712 & $9072=0$ & İ & II I \\
\hline & & & & & & & & & \\
\hline
\end{tabular}

Concentrations in the media occurring in pathways that are suppressed are calculated using the current input parameters, i.e. using parameters appearing in the input screen when the pathways are active.

\begin{tabular}{|c|c|}
\hline \multirow{2}{*}{\multicolumn{2}{|c|}{$\begin{array}{ll}\text { Attachment } & 7 \\
\text { Originator: S.W. Clark } & \text { Sheet No. } 19 \text { of } 19 \\
\text { Date }\end{array}$}} \\
\hline & \\
\hline M. W. Perrott & \\
\hline $0100 F-C A-V 0303$ & Rev \\
\hline
\end{tabular}




\section{ATTACHMENT 8}

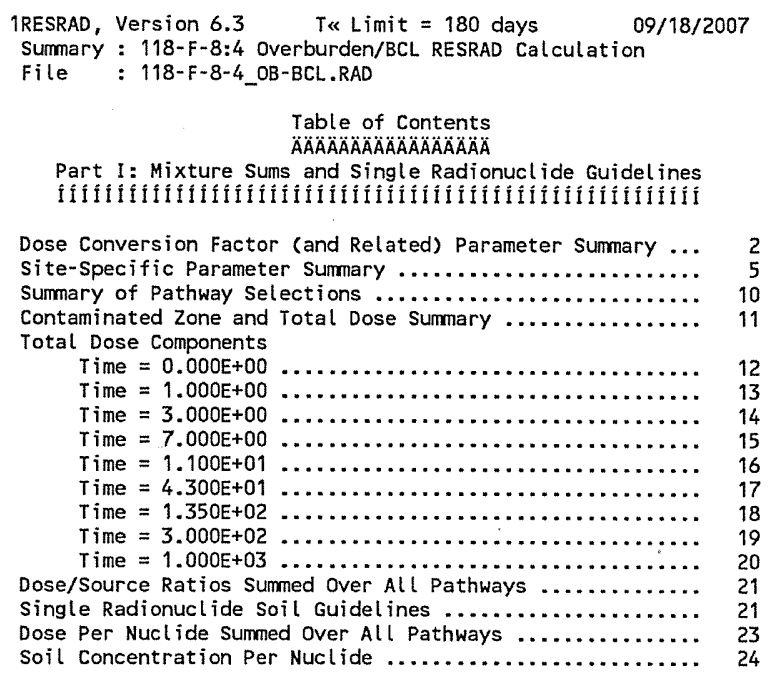

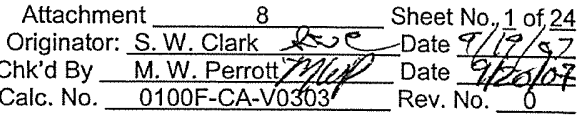




\section{ATTACHMENT 8}

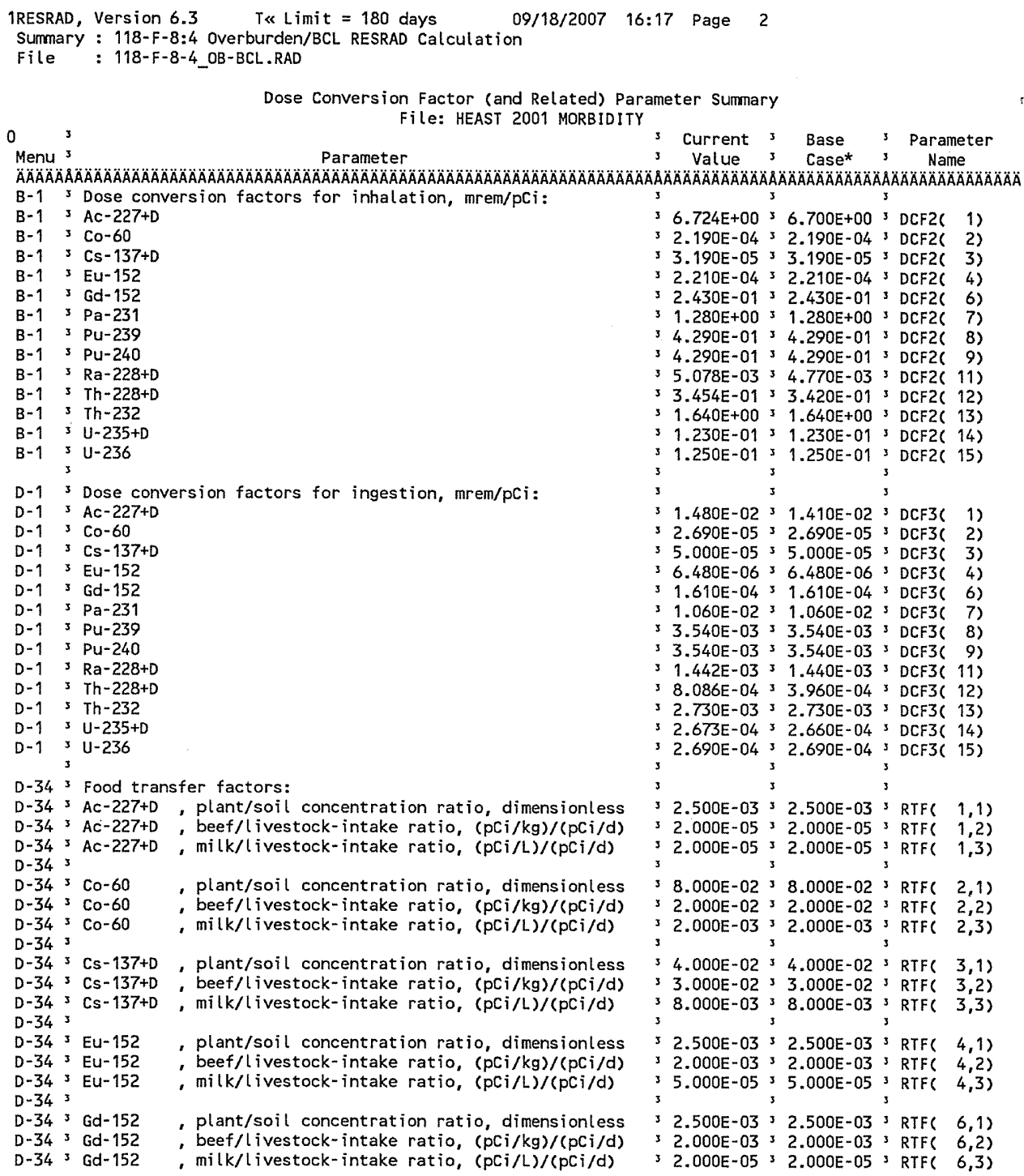

\begin{tabular}{|c|c|}
\hline Attachment & Sheet No. 2 of 24 \\
\hline Originator: S.W. Clark & Date \\
\hline Chk'd By M.W. Perrott & Date \\
\hline $0100 \mathrm{~F}-\mathrm{CA}-\mathrm{V} 0303$ & Rev. No. 0 \\
\hline
\end{tabular}




\section{ATTACHMENT 8}

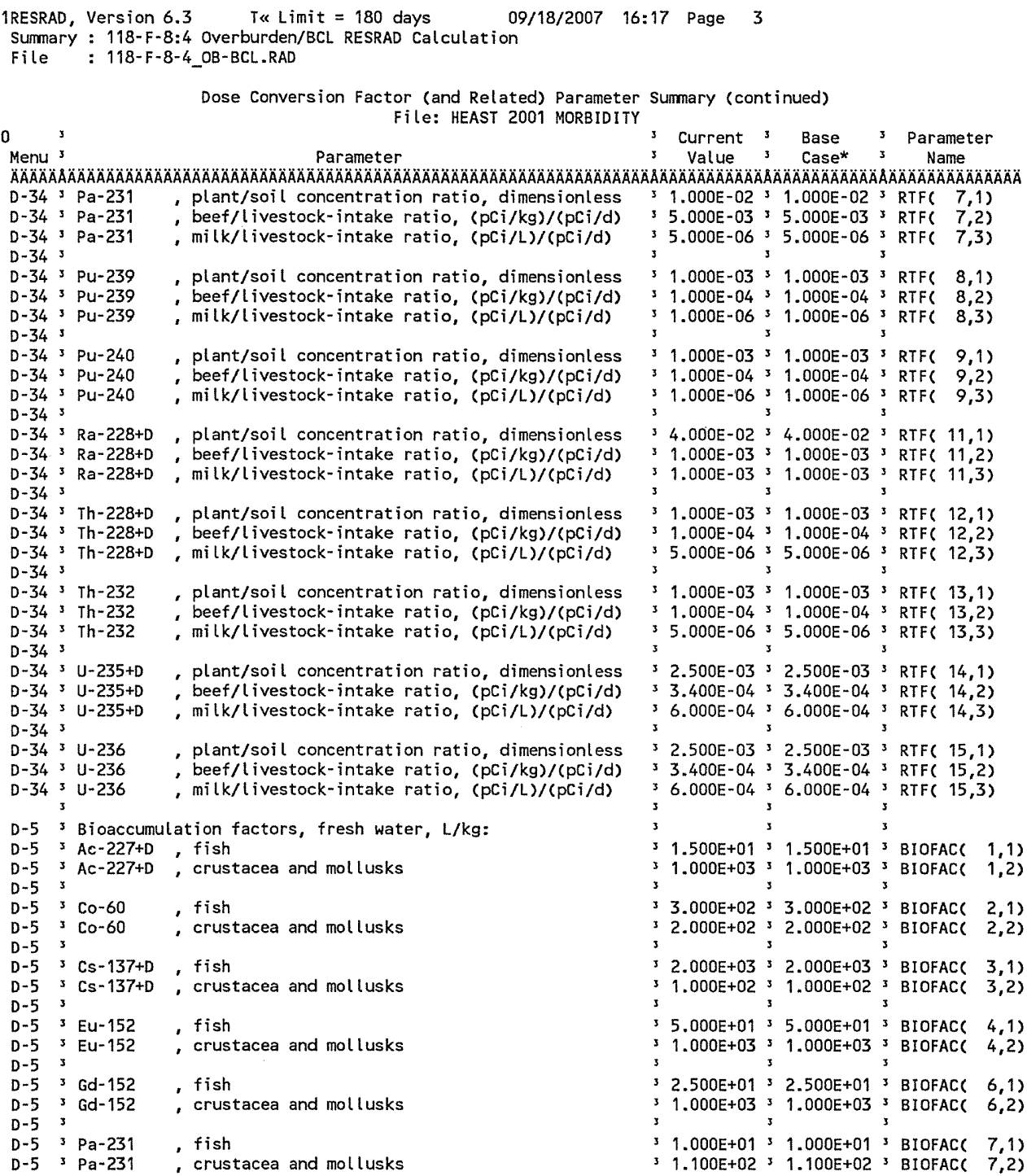




\section{ATTACHMENT 8}

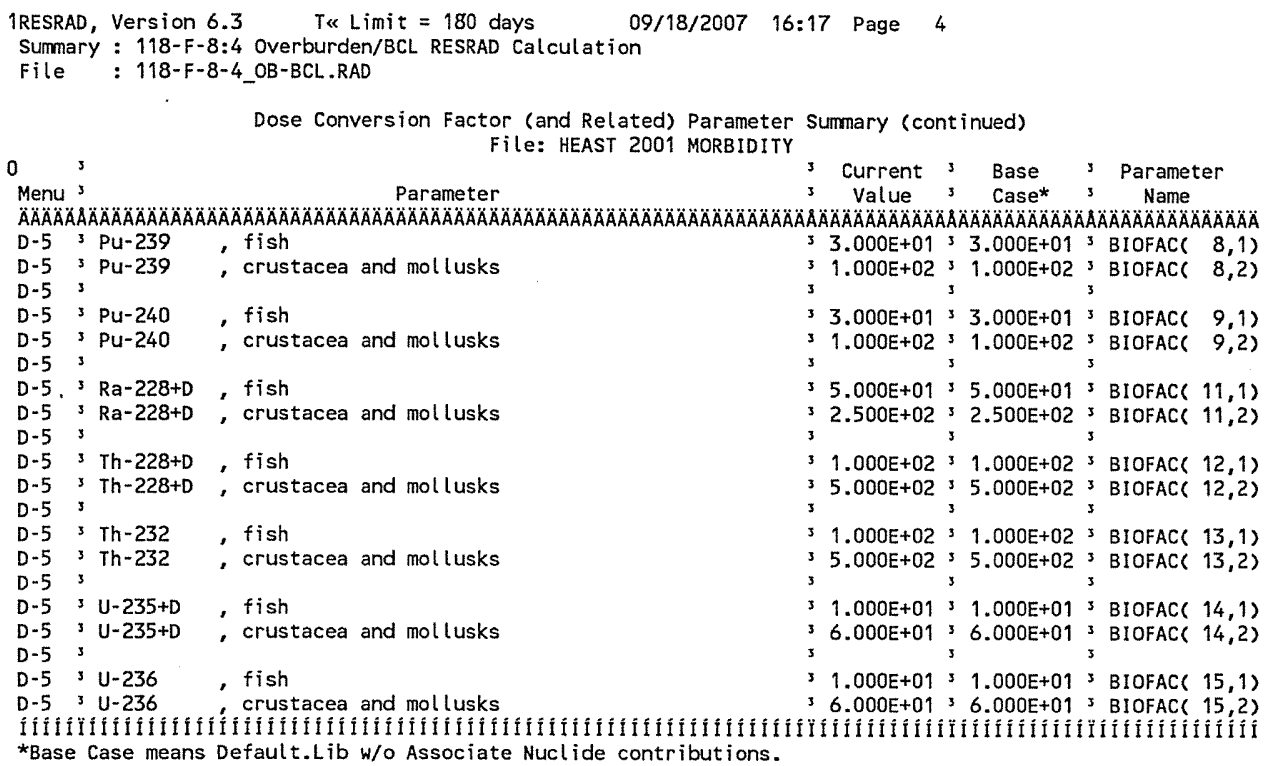

\begin{tabular}{|c|c|}
\hline Attachment & Sheet No. 4 of 24 \\
\hline Originator: S.W. Clark & Date \\
\hline M. W. Perrott & Date \\
\hline $0100 \mathrm{~F}-\mathrm{CA}-\mathrm{V} 0303$ & Rev, No. 0 \\
\hline
\end{tabular}




\section{ATTACHMENT 8}

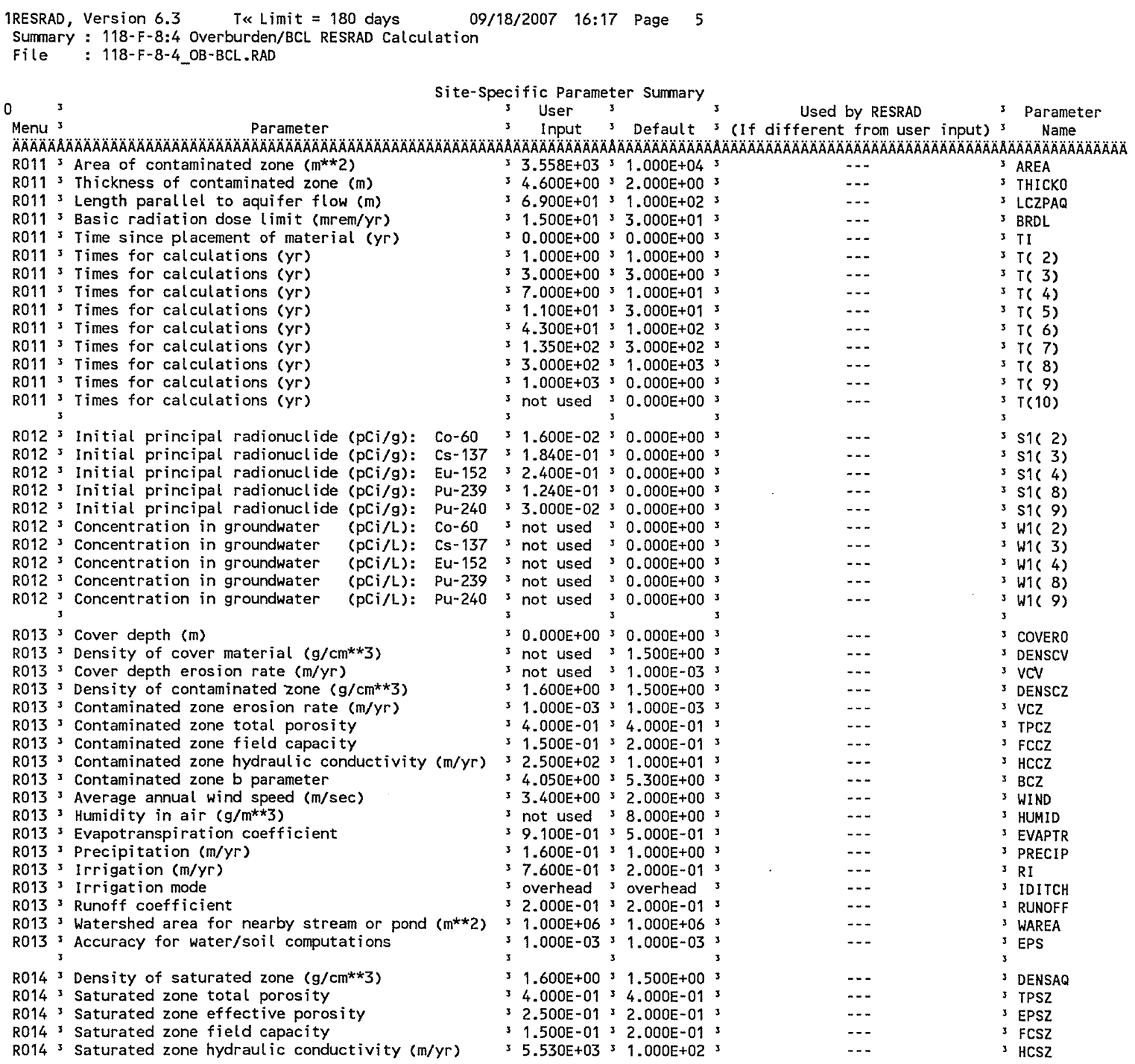

\begin{tabular}{|c|c|}
\hline \multirow{2}{*}{\multicolumn{2}{|c|}{$\begin{array}{cc}\text { Attachment } & 8 \\
\text { Originator: S.W. Clark } & \text { Sheet No. } \underline{5} \text { of } \underline{24}\end{array}$}} \\
\hline & \\
\hline M. W. Perrott & Date \\
\hline $0100 \mathrm{~F}-\mathrm{CA}-\mathrm{V} 0303$ & Rev \\
\hline
\end{tabular}




\section{ATTACHMENT 8}

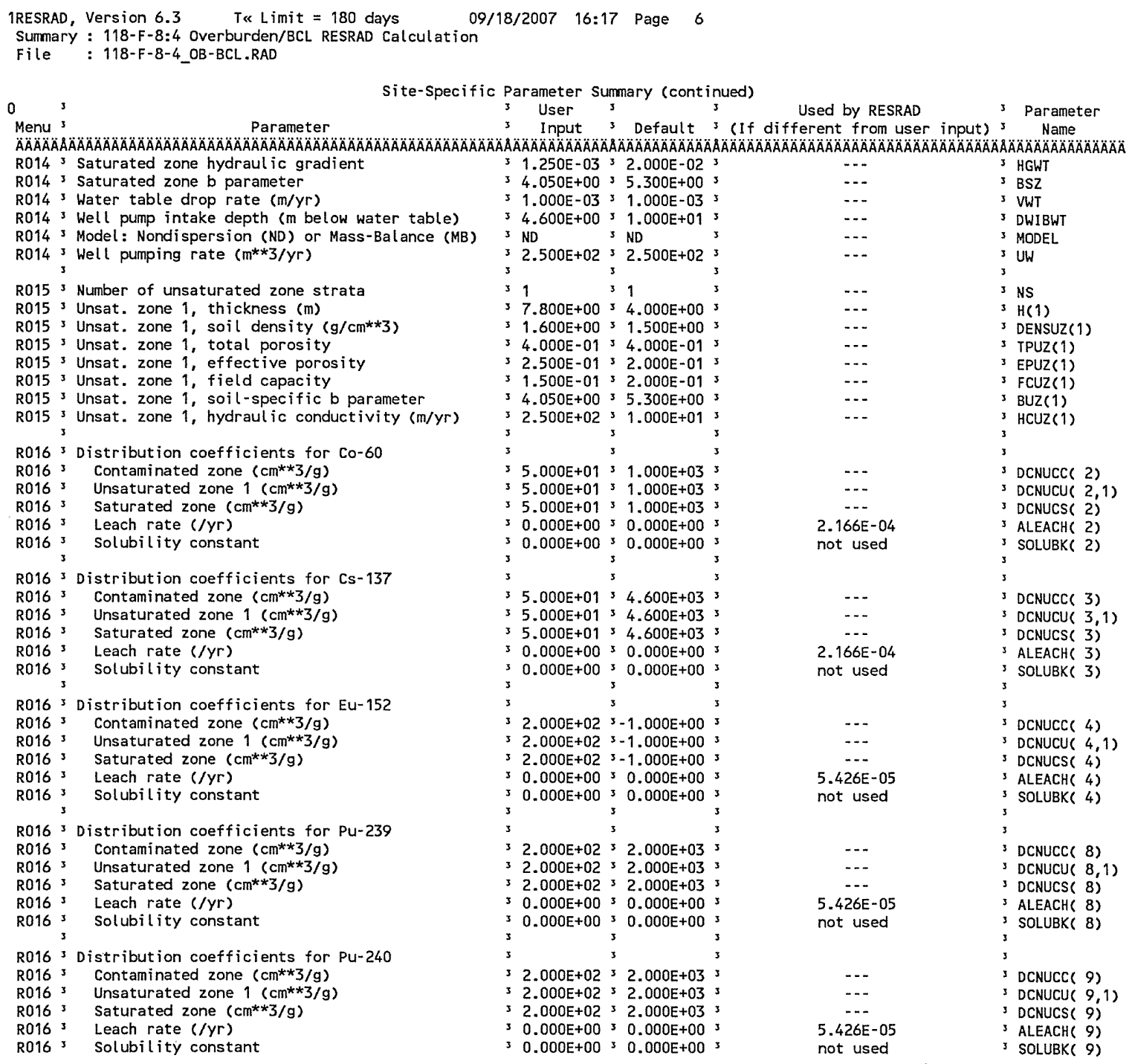

$\begin{array}{ll}\text { Attachment } \frac{8}{\text { S. Clark }} & \text { Sheet No. } \underline{6} \text { of } \underline{24} \\ \text { Originator: } & \text { Date } \\ \text { Chk'd By } \frac{\text { M.W. Perrott }}{0100 \text { F-CA-V0303 }} & \text { Date } \\ \text { Calc. No. Rev. No. } \quad 0\end{array}$




\section{ATTACHMENT 8}

1RESRAD, Version 6.3 T\& Limit $=180$ days $09 / 18 / 2007 \quad 16: 17$ Page 7

Summary : 118-F-8:4 Overburden/BCL RESRAD Calculation

File: $118-F-8-4$ OB-BCL.RAD

0

Si te-Specific Parameter Summary (continued)

(1)

Parameter

3 User 3

3

Used by RESRAD I Parameter R016 3 Distribution COef

Distribution coefficients for daughter Ac-227

R016 Contaminated zone $\left(\mathrm{cm}^{* * 3 / \mathrm{g}}\right)$

Unsaturated zone $1\left(\mathrm{~cm}^{* * 3 / \mathrm{g})}\right.$

R016 3 Saturated zone $\left(\mathrm{cm}^{* * 3 / g}\right)$

R016 3 Leach rate (/yr)

Solubility constant

R016 3 Distribution coefficients for daughter Gd-152

R016 ${ }^{3}$ Contaminated zone $\left(\mathrm{cm}^{* * 3 / \mathrm{g})}\right.$

R016 3 Unsaturated zone $1(\mathrm{~cm} * * 3 / \mathrm{g})$

R016 3 Saturated zone $(\mathrm{cm} * * 3 / \mathrm{g})$

R016 Leach rate (/yr)

3 Solubility constant

R016 3 Distribution coefficients for daughter Pa-231

R016 : Contaminated zone $\left(\mathrm{cm}^{* * 3 / \mathrm{g})}\right.$

R016:Unsaturated zone $1\left(\mathrm{~cm}^{* * 3 / g}\right)$

20163 Saturated zone $\left(\mathrm{cm}^{* * 3 / \mathrm{g})}\right.$

R016 3 Leach rate (/yr)

R016 Solubility constant

R016 Distribution coefficients for daughter Ra-228

$R 0163$ Contaminated zone $\left(\mathrm{cm}^{* * 3 / \mathrm{g})}\right.$
$R 016$ Unsaturated zone $1\left(\mathrm{~cm}^{* * 3} / \mathrm{g}\right)$

R016 3 Saturated zone $\left(\mathrm{cm}^{* * 3 / \mathrm{g})}\right.$

R016: Leach rate (/yr)

R016 Solubility constant

R016 3 Distribution coefficients for daughter Th-228

R016 3 Contaminated zone $\left(\mathrm{cm}^{* * 3 / \mathrm{g})}\right.$

R016:Unsaturated zone $1\left(\mathrm{~cm}^{* * 3} / \mathrm{g}\right)$

R016 3 Saturated zone $(\mathrm{cm} * * 3 / \mathrm{g})$

R016 3 Leach rate $(/ \mathrm{yr})$

R016 Solubility constant

R016 3 Distribution coefficients for daughter Th- 232

R016 3 Contaminated zone $\left(\mathrm{cm}^{* * 3 / \mathrm{g})}\right.$

R016 3 Unsaturated zone $1\left(\mathrm{~cm}^{* * 3 / \mathrm{g})}\right.$

R016 3 Saturated zone $\left(\mathrm{cm}^{* * 3 / \mathrm{g})}\right.$

R016 s Leach rate (/yr)

R016 Solubility constant

R016 3 Distribution coefficients for daughter U-235

R016 3 Contaminated zone $\left(\mathrm{cm}^{* * 3 / \mathrm{g})}\right.$

R016 3 Unsaturated zone $1\left(\mathrm{~cm}^{* * 3 / \mathrm{g})}\right.$

R016 3 Saturated zone $\left(\mathrm{cm}^{* * 3 / g}\right)$

R016 's Leach rate (/yr)

R016 $^{3}$ Solubility constant

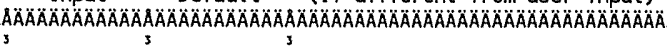

$32.000 E+01^{3} 2.000 E+01^{3}$ $3 \begin{array}{lll}2.000 E+013 & 2.000 E+013 \\ 3 & 2.000 E+013 & 2.000 E+013\end{array}$ $32.000 \mathrm{E}+0132.000 \mathrm{E}+013$

$30.000 E+0030.000 E+00$ s

$30.000 E+00^{3} 0.000 E+00^{3}$

$3-1.000 E+003-1.000 E+00 s$

$3-1.000 \mathrm{E}+00$ s- $-1.000 \mathrm{E}+003$

$3-1.000 E+003-1.000 E+003$

$30.000 \mathrm{E}+00^{3} 0.000 \mathrm{E}+00^{3}$

$30.000 E+00>0.000 E+00$ ?

3

$35.000 E+0135.000 E+013$

$5.000 E+01 \div 5.000 E+01$

$5.000 E+0135.000 E+013$

$0.000 \mathrm{E}+00^{3} 0.000 \mathrm{E}+00$,

$0.000 E+00^{3} 0.000 E+00^{3}$

3

$37.000 E+0137.000 E+013$

$37.000 E+0137.000 E+01$

$37.000 E+0137.000 E+013$

$30.000 \mathrm{E}+00^{3} 0.000 \mathrm{E}+00^{3}$

s $0.000 \mathrm{E}+00>0.000 \mathrm{E}+00$,

3

× $2.000 \mathrm{E}+02 \leq 6.000 \mathrm{E}+04$

, $3.000 \mathrm{E}+05^{3} 6.000 \mathrm{E}+0 \mathrm{C}^{3}$

$2.000 \mathrm{O}+02^{3} 6.000 \mathrm{C}+04^{3}$

2.000E+02 3 $6.000+04^{3}$

$\begin{array}{llll}0.000 E+003 & 0.000 E+00 ; \\ 3 & 0.000 E+003 & 0.000 E+00 ;\end{array}$

$2.000 E+02 \times 6.000 E+04$

$32.000 \mathrm{E}+0236.000 \mathrm{E}+043$

$32.000 \mathrm{E}+0236.000 \mathrm{E}+04^{3}$

$30.000 E+00>0.000 E+00$ ?

s $0.000 \mathrm{E}+0030.000 \mathrm{E}+00$ s

$2.000 E+00$ \& $5.000 E+01$

$32.000 E+00=5.000 E+01$

$32.000 E+0035.000 E+013$

\& $0.000 \mathrm{E}+00$ > $0.000 \mathrm{E}+00$;

$30.000 E+0030.000 E+003$

$\ldots$
$\cdots$
$5.397 \mathrm{E}-04$
not used

$8.249 \mathrm{E}+02$
$8.249 \mathrm{E}+02$

DCNUCC( 1$)$

DCNUCU $(1,1)$

DCNUCS( 1$)$

ALEACH( 1)

SOLUBK (1)

DCNUCC (6)

3 DCNUCU( 6,1)

DCNUCS( 6 )

3 ALEACH (6)

SOLUBK( 6)

-.. $\quad 3$ DCNUCC( 7$)$

-.. 3 DCNUCU( 7,1

-.. 3 DCNUCS( 7

2.166E-04 3 ALEACH( 7 )

not used 3 SOLUBK( 7 )

$\begin{array}{ll}-.- & 3 \\ \ldots- & 3 \\ -- & \text { DCNUCC (11) }\end{array}$

$\cdots$

1.549E-04 04 ALEACH(11)

not used 3 SOLUBK(11)

-.. 3 DCNUCC (12)

-.- $\operatorname{DCNUCU}(12,1)$

$5.426 \mathrm{E}-05$

not used

DCNUCS $(12)$

ALEACH( 12)

SOLUBK(12)

-.- $\quad 3$ DCNUCC (13)

-.. 3 DCNUCU $(13,1)$

3 DCNUCS(13)

I ALEACH(13)

S SOLUBK(13)

… 3 DCNUCC (14)

DCNUCU $(14,1)$

3 DCNUCS (14)

3 ALEACH (14)
Attachment 8 Sheet No. $\underline{7}$ of 24

riginator: S W. Clark

Chk'd By M.W. Perrott

Calc. No. 0100F-CA-V0303
Date

Date

Rev. No. 0 


\section{ATTACHMENT 8}

1RESRAD, Version $6.3 \quad T \ll$ Limit $=180$ days $09 / 18 / 2007 \quad 16: 17$ Page 8

Summary : 118-F-8:4 Overburden/BCL RESRAD Calculation

file: $118-F-8-4$ OB-BCL.RAD

Site-Specific Parameter Summary (cont inued)

Menu ${ }^{3}$

3 User 3 Used by RESRAD

3 Parameter

Menu ${ }^{3} \quad$ Parameter Input 3 Default 3 (If different from user input) 3 Name

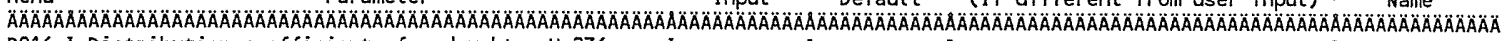
R016 ${ }^{3}$ Distribution coefficients for daughter U-236

R016 3 Contaminated zone $\left(\mathrm{cm}^{* * 3 / g)}\right.$

R016 Unsaturated zone $1\left(\mathrm{~cm}^{* * 3 / \mathrm{g}}\right)$

$R 016^{3}$ Saturated zone $\left(\mathrm{cm}^{* * 3 / \mathrm{g}}\right)$

R016 3 Leach rate $(/ \mathrm{yr})$

R016 3 Solubility constant

R017'3 Inhalation rate $\left(\mathrm{m}^{* * 3 / \mathrm{yr})}\right.$

R017 3 Mass loading for inhalation $\left(\mathrm{g} / \mathrm{m}^{* * 3}\right)$

R017 3 Exposure duration

R017' Shielding factor, inhalation

R017 Shielding factor, external gamma

R017 3 Fraction of time spent indoors

R017 3 Fraction of time spent outdoors (on site)

R017' Shape factor flag, external gamma

R017 3 Radii of shape factor array (used if FS = -1):

R017 3 Outer annular radius (m), ring 1:

$2017^{3}$ Outer annular radius (m), ring 2

$R_{017^{3}}$ Outer annular radius (m), ring 3

R017 ${ }^{3}$ Outer annular radius (m), ring 4:

$R 017^{3}$ Outer annular radius (m), ring 5:

$\mathrm{R}_{017^{3}}{ }^{3}$ Outer annular radius (m), ring 6 :

$\mathrm{R}_{017^{3}}{ }^{3}$ Outer annular radius (m), ring 7 :

R017 3 Outer annular radius (m), ring 8:

$\mathrm{R}_{017^{3}}$ Outer annular radius (m), ring 9

R017 3 Outer annular radius (m), ring 10:

$\mathrm{R}_{017^{3}}$ Outer annular radius (m), ring 11:

$32.000 E+00^{3} 5.000 E+01^{3}$

$2.000 E+00 ; 5.000 E+013$

$32.000 E+0035.000 E+013$

$0.000 E+00^{3} 0.000 E+00^{3}$

${ }^{3} 0.000 E+0030.000 E+003$

$37.300 E+03$; $8.400 E+033$

$1.000 E-0431.000 E-04^{3}$

$3.000 E+0133.000 E+013$

$4.000 E-0134.000 E-013$

8.000E-01 3 7.000E-013

${ }^{3} 6.000 \mathrm{E}-01^{3} 5.000 \mathrm{E}-01^{3}$

$32.000 E-0132.500 E-013$

$31.000 E+0031.000 E+00^{3}$

3 not used $35.000 E+013$

not used $37.071 E+013$

not used $30.000 E+00^{3}$

not used $30.000 E+00^{3}$

3 not used $30.000 E+003$

3 not used $30.000 E+00$ s

3 not used s $0.000 E+00^{3}$

3 not used s $0.000 E+00^{3}$

3 not used $30.000 E+00^{3}$

3 not used $30.000 E+00^{3}$

not used $0.000 E+00$

3 not used $30.000 E+003$

R017 3 Fractions of annular areas within AREA:

$\mathrm{R}^{017^{3}}$ Ring 1

R017'sing 2

R017 3 Ring 3

R017'3 Ring 4

R017 s Ring 5

R017 ${ }^{3}$ Ring 6

R017 Ring 7

R017 3 Ring 8

$\mathrm{R}^{2} \mathrm{R}^{3}$ Ring 9

R017'sing 10

R017: Ring 11

R017' Ring 12

R018 3 Fruits, vegetables and grain consumption (kg/yr) $31.100 \mathrm{E}+02=1.600 \mathrm{E}+02$

R018 3 Leafy vegetable consumption $(\mathrm{kg} / \mathrm{yr})$

R018 3 Milk consumption ( $L / y r$ )

R018' Meat and poultry consumption $(\mathrm{kg} / \mathrm{yr})$

R018 ${ }^{3}$ Fish consumption ( $\mathrm{kg} / \mathrm{yr}$ )

$\mathrm{R}^{2} 18^{3}$ Other seafood consumption ( $\mathrm{kg} / \mathrm{yr}$ )

$\mathrm{R} 0188^{3}$ Soil ingestion rate $(\mathrm{g} / \mathrm{yr})$

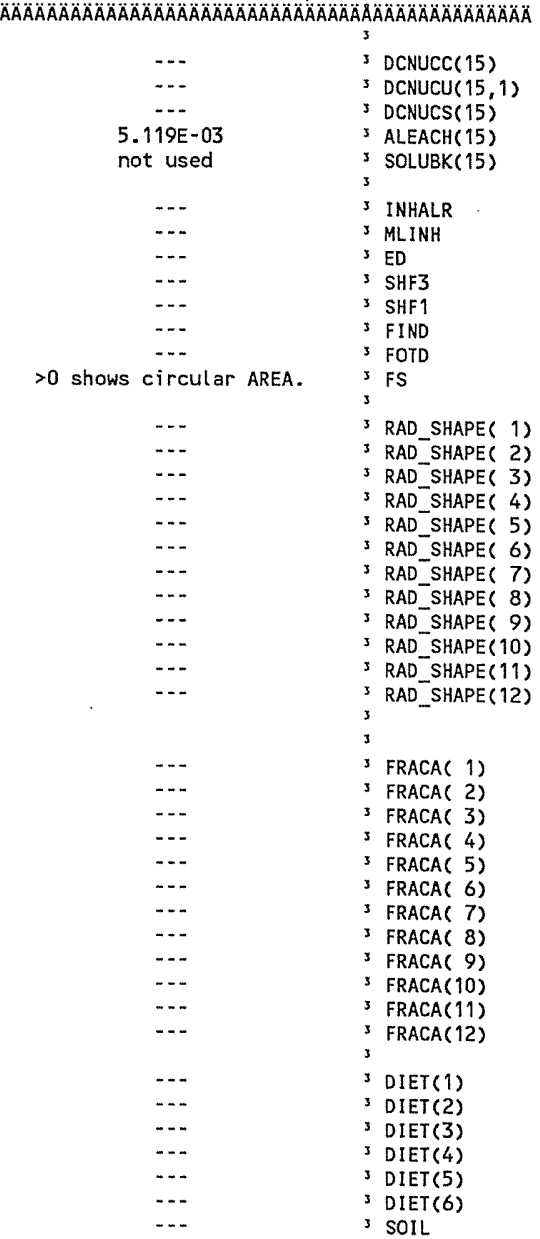

$\begin{array}{ll}\text { Attachment } \frac{8}{\text { S. Clark }} & \text { Sheet No. } 8 \text { of } \underline{24} \\ \text { Originator: } \frac{\text { S.W. }}{\text { M. W. Perrott }} & \text { Date } \\ \text { Chk'd By } & \text { Rev. No. o }\end{array}$




\section{ATTACHMENT 8}

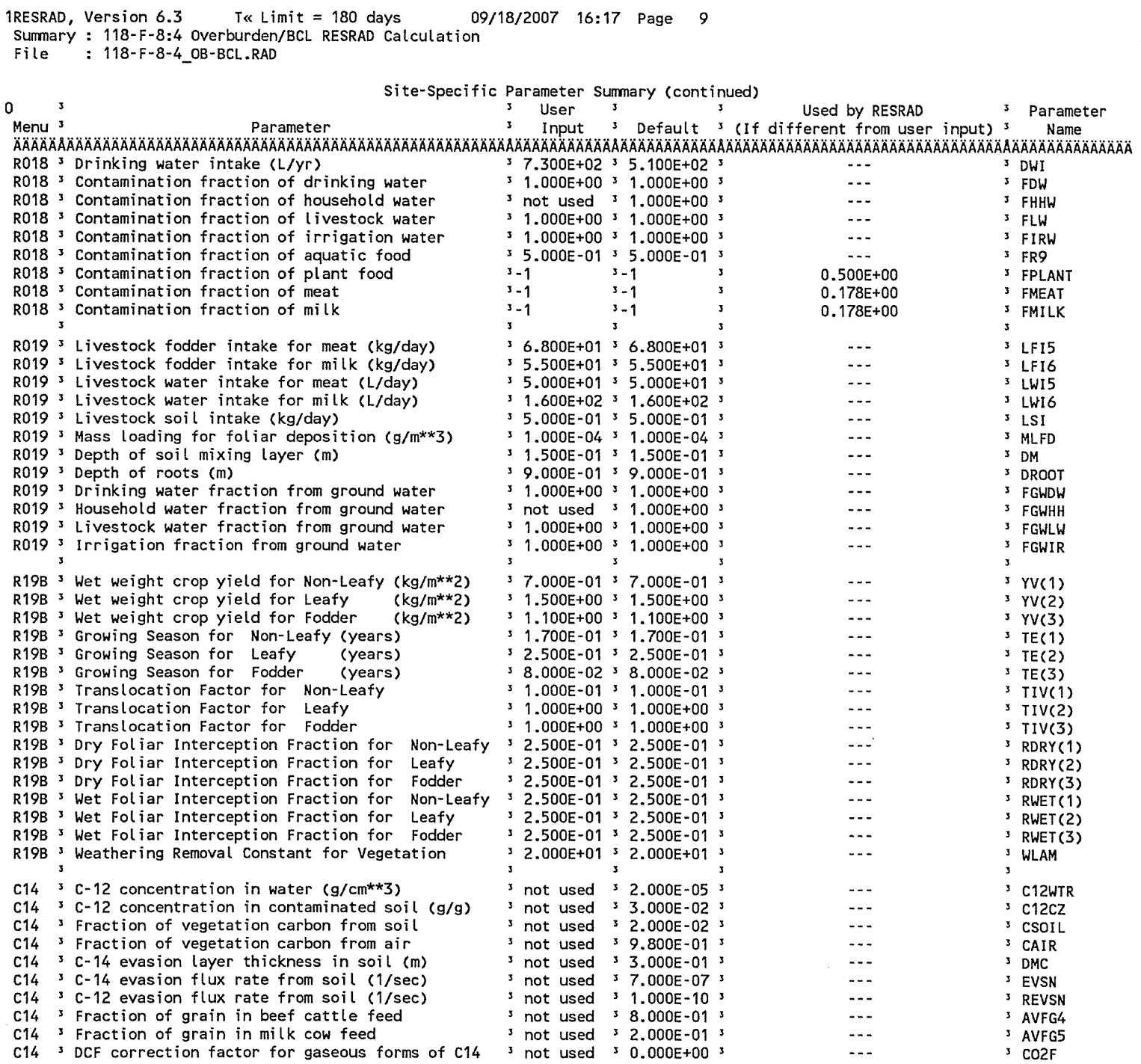

$\begin{array}{ll}\text { Attachment } \frac{8}{\text { W. Clark }} & \text { Sheet No. } \underline{9} \text { of } 24 \\ \text { Originator: } \frac{\text { S.Wate }}{\text { M.W. Perrott }} & \text { Date } \\ \text { Chk'd By } & \text { Rev. No. } 0 \\ \text { Calc. No. } \frac{0100 \text { F-CA-V0303 }}{01}\end{array}$




\section{ATTACHMENT 8}

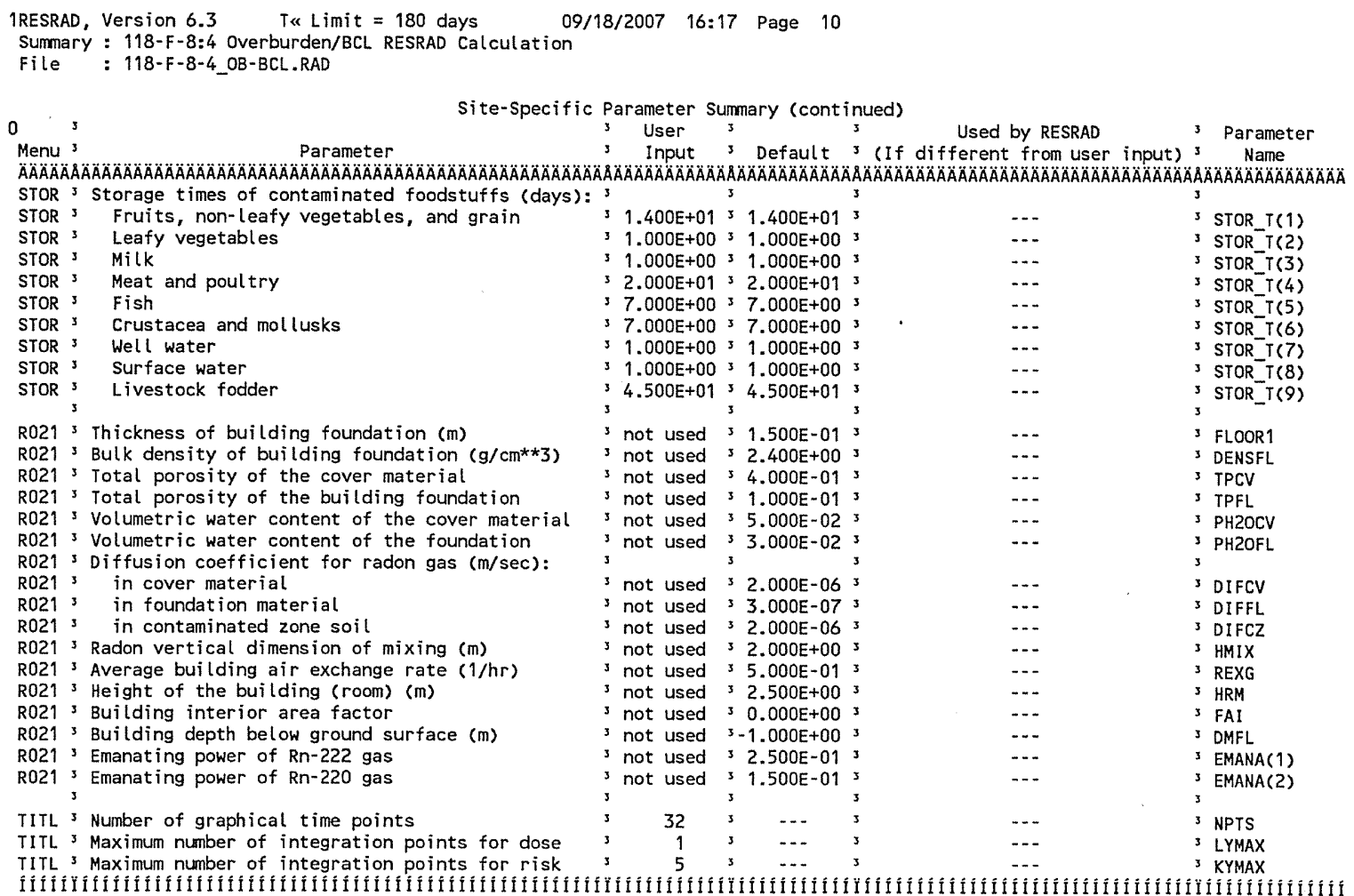

Summary of Pathway Selections

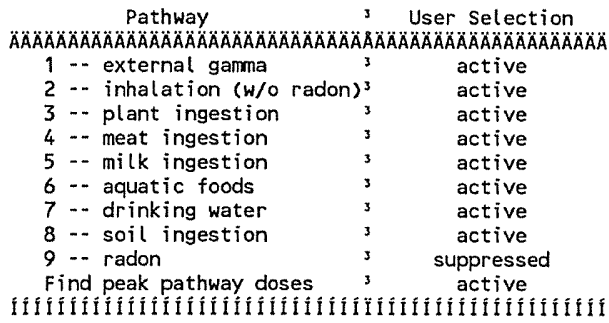

\begin{tabular}{|c|c|}
\hline \multirow{2}{*}{\multicolumn{2}{|c|}{$\begin{array}{l}\text { Attachment } \\
\text { Originator: S.W. Clark }\end{array}$}} \\
\hline & \\
\hline M.W. Perrott & Date \\
\hline $0100 \mathrm{~F}-\mathrm{CA}-\mathrm{V} 0303$ & Rev. No. \\
\hline
\end{tabular}




\section{ATTACHMENT 8}

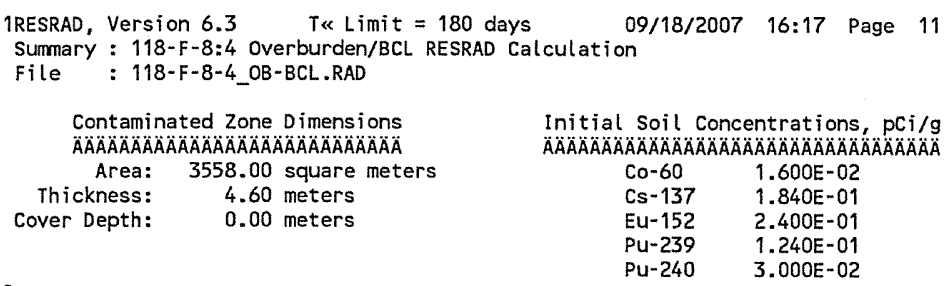

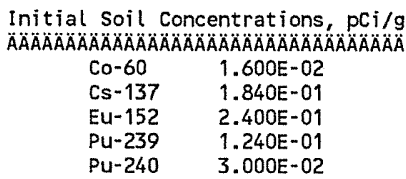

Total Dose TDOSE( $t$ ), mrem/yr Basic Radiation Dose Limit $=1.500 E+01 \mathrm{mrem} / \mathrm{yr}$

Total Mixture sum $M(t)=$ Fraction of Basic Dose limit Received at Time $(t)$

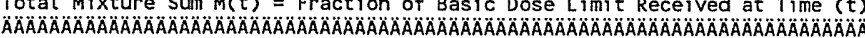

$t$ (years): $\begin{array}{ccccccccc}0.000 E+00 & 1.000 E+00 & 3.000 E+00 & 7.000 E+00 & 1.100 E+01 & 4.300 E+01 & 1.350 E+02 & 3.000 E+02 & 1.000 E+03\end{array}$

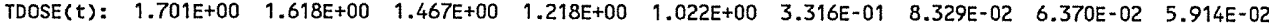
$M(t): \quad \begin{array}{lllllllll}134 E-01 & 1.078 E-01 & 9.779 E-02 & 8.117 E-02 & 6.811 E-02 & 2.210 E-02 & 5.552 E-03 & 4.247 E-03 & 3.943 E-03\end{array}$ OMaximum $\operatorname{TDOSE}(t): 1.701 \mathrm{E}+00 \mathrm{mrem} / \mathrm{yr}$ at $t=0.000 \mathrm{E}+00$ years

\begin{tabular}{|c|c|}
\hline \multirow{2}{*}{\multicolumn{2}{|c|}{$\begin{array}{ll}\text { Attachment } & 8 \\
\text { Originator: S.W. Clark } & \text { Sheet No. } 11 \text { of } 24 \\
\text { Date }\end{array}$}} \\
\hline & \\
\hline M. W. Perrott & Date \\
\hline $0100 \mathrm{~F}-\mathrm{CA}-\mathrm{V} 0303$ & Rev. No. \\
\hline
\end{tabular}




\section{ATTACHMENT 8}

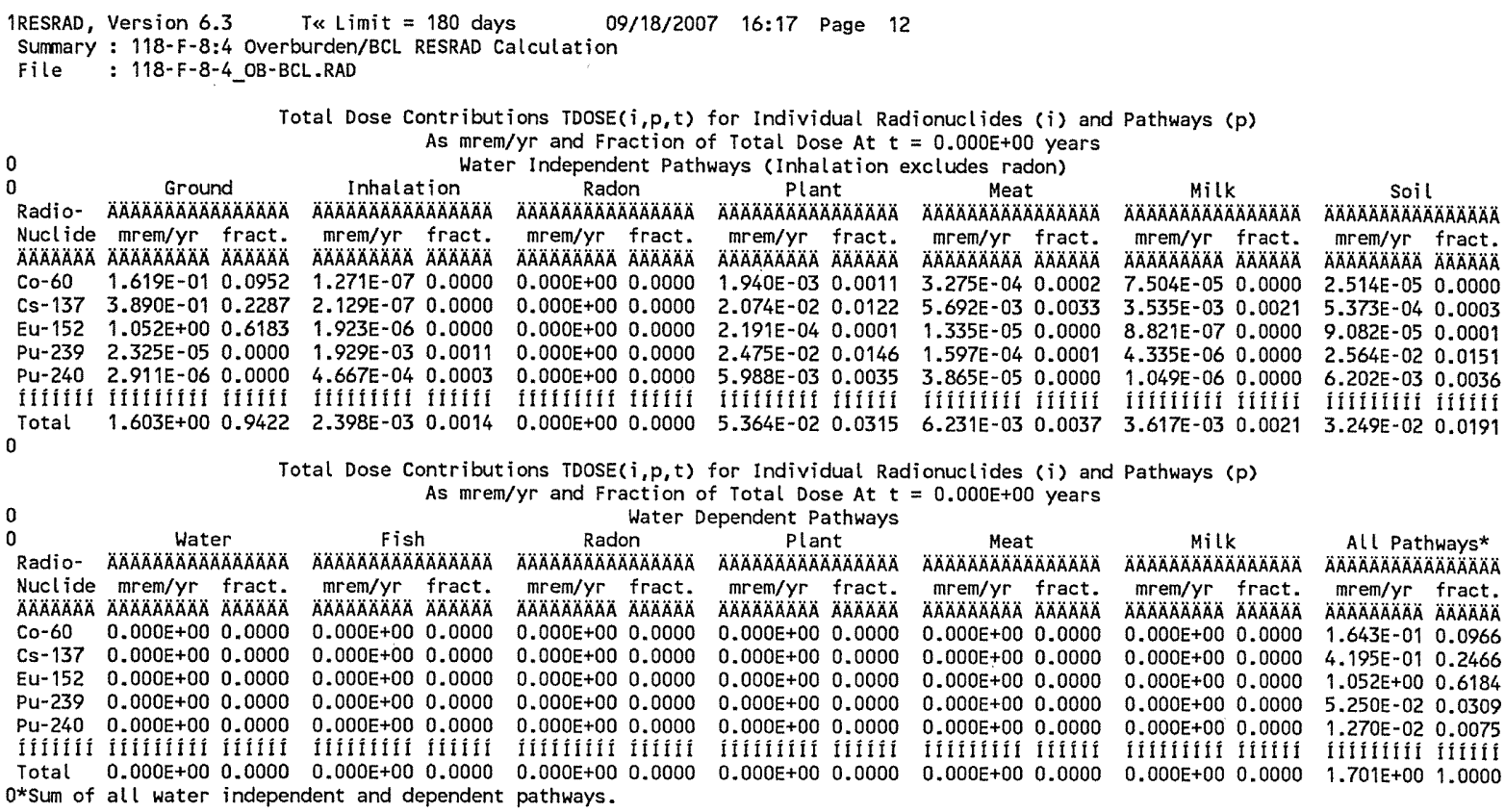

\begin{tabular}{|c|c|}
\hline Attachment & Sheet No. 12 of 24 \\
\hline Originator: $\mathrm{S.W}$. Clark & Date \\
\hline Chk'd By M.W. Perrott & Date \\
\hline Calc. No. $\quad 0100 \mathrm{~F}-\mathrm{CA}-\mathrm{V} 0303$ & Rev. No. 0 \\
\hline
\end{tabular}




\section{ATTACHMENT 8}

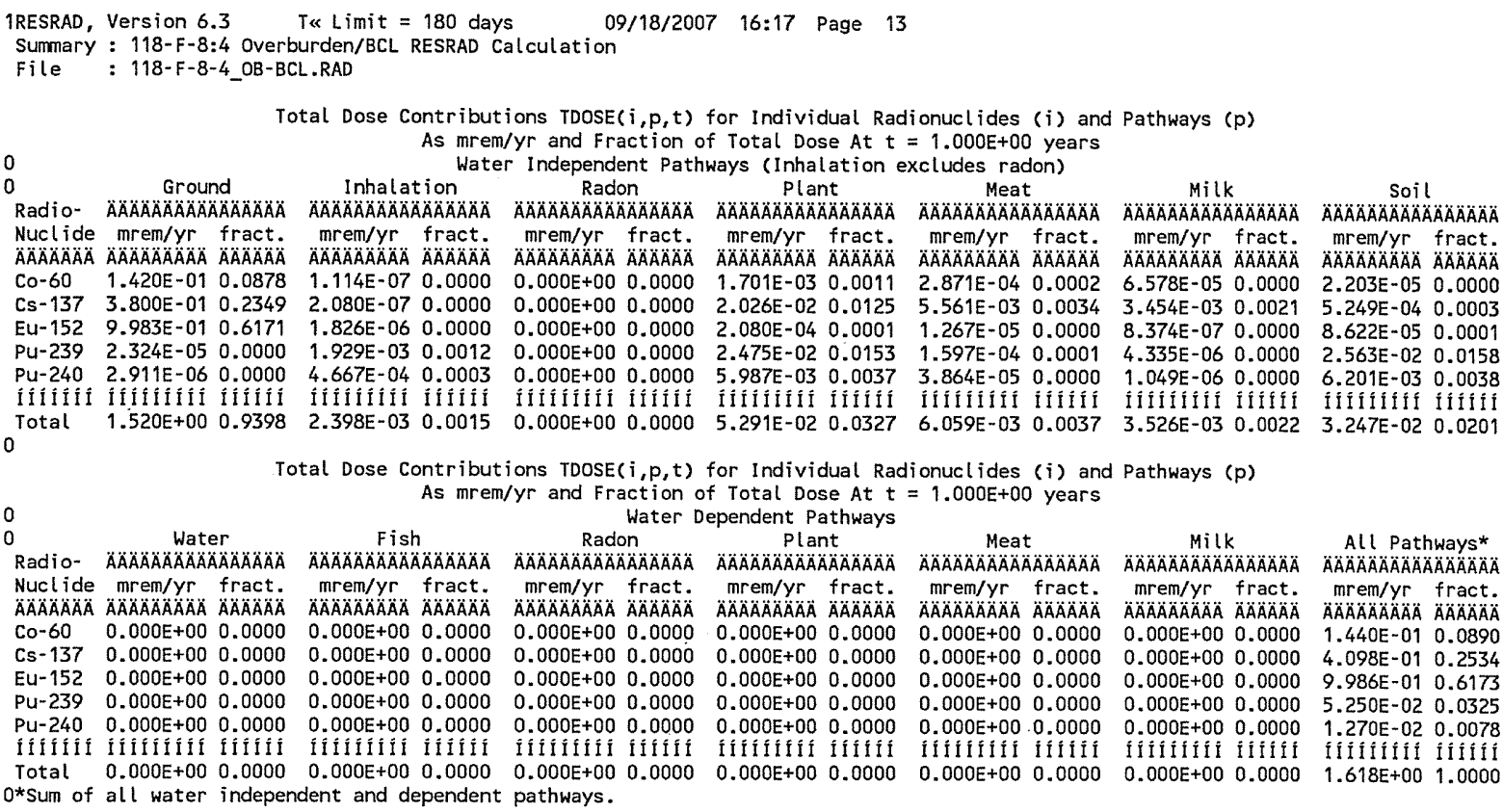

\begin{tabular}{|c|c|}
\hline Attachment & Sheet No. 13 of 24 \\
\hline Originator: S.W. Clark & Date \\
\hline Chk'd By M.W. Perrott & Date \\
\hline Calc. No. $\quad 0100 \mathrm{~F}-\mathrm{CA}-\mathrm{V} 0303$ & Rev. No. 0 \\
\hline
\end{tabular}




\section{ATTACHMENT 8}

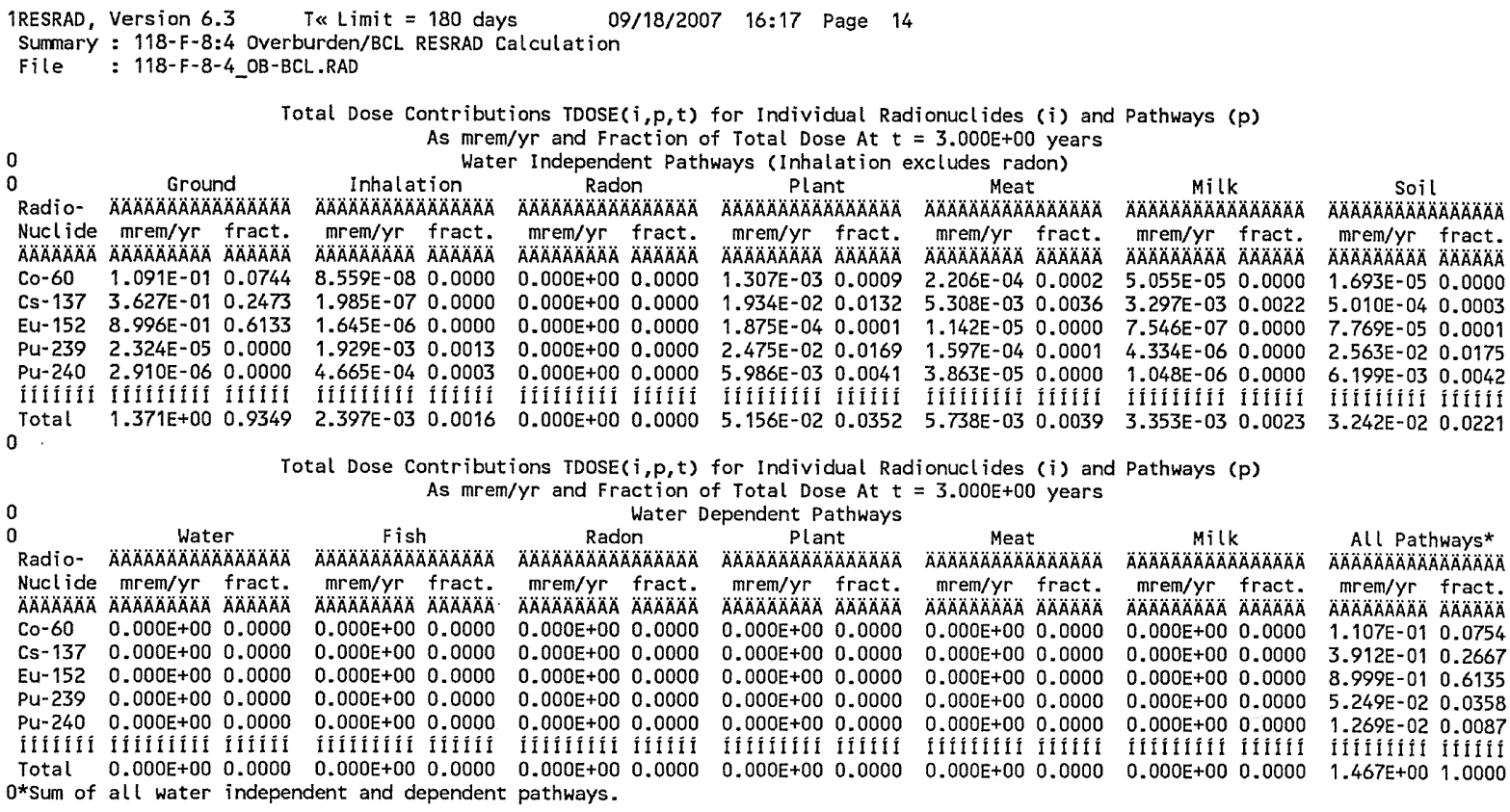

\begin{tabular}{|c|c|}
\hline Attachment & Sheet No. 14 of 24 \\
\hline Originator: S.W. Clark & Date \\
\hline M. W. Perrott & Date \\
\hline $0100 \mathrm{~F}-\mathrm{CA}-\mathrm{V} 0303$ & Rev. No. 0 \\
\hline
\end{tabular}




\section{ATTACHMENT 8}

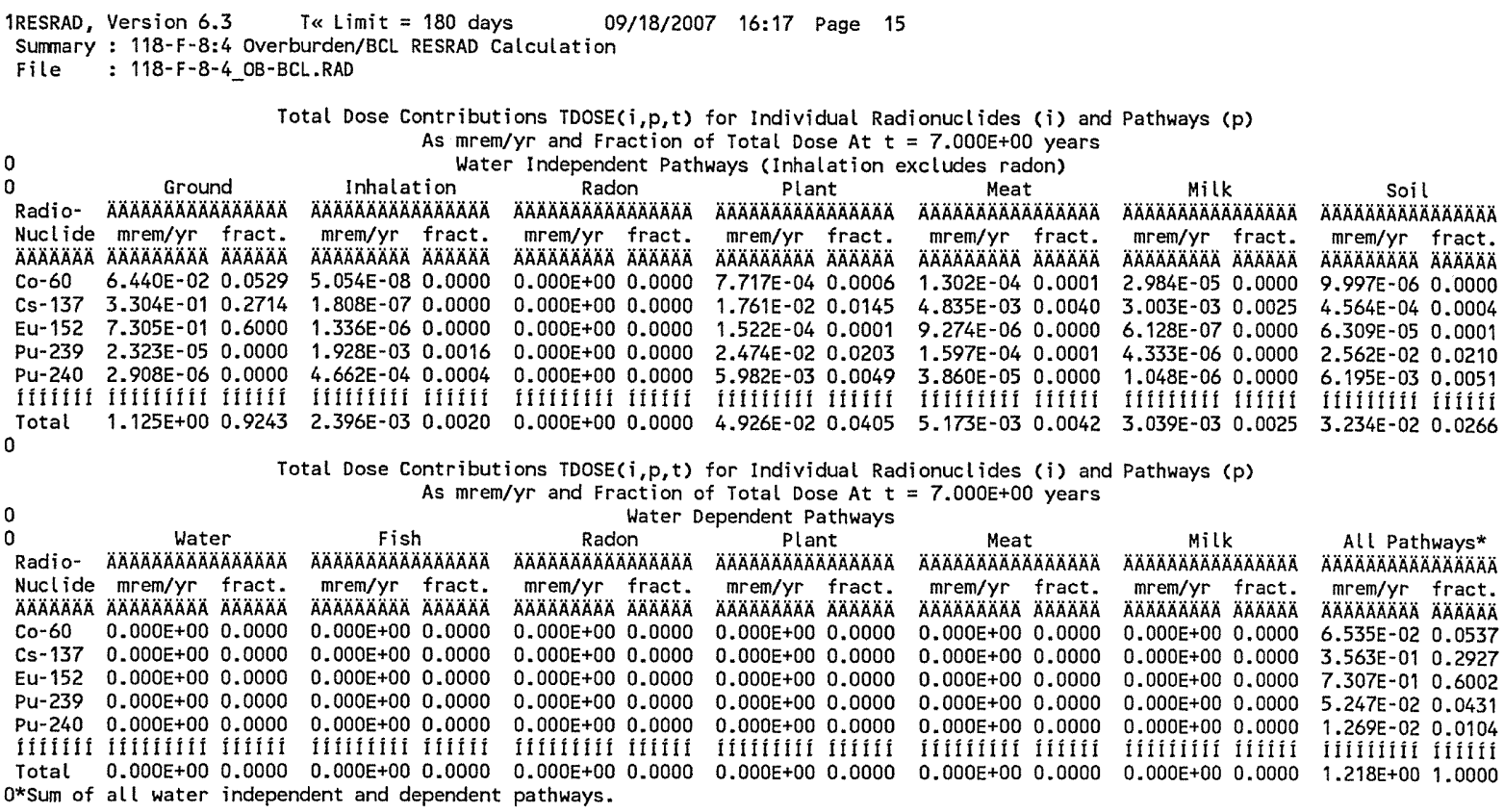

\begin{tabular}{|c|c|}
\hline Attachment & Sheet No. 15 of 24 \\
\hline Originator: S.W. Clark & Date \\
\hline M.W. Perrott & Date \\
\hline $0100 F-C A-V 0303$ & Rev. No. \\
\hline
\end{tabular}




\section{ATTACHMENT 8}

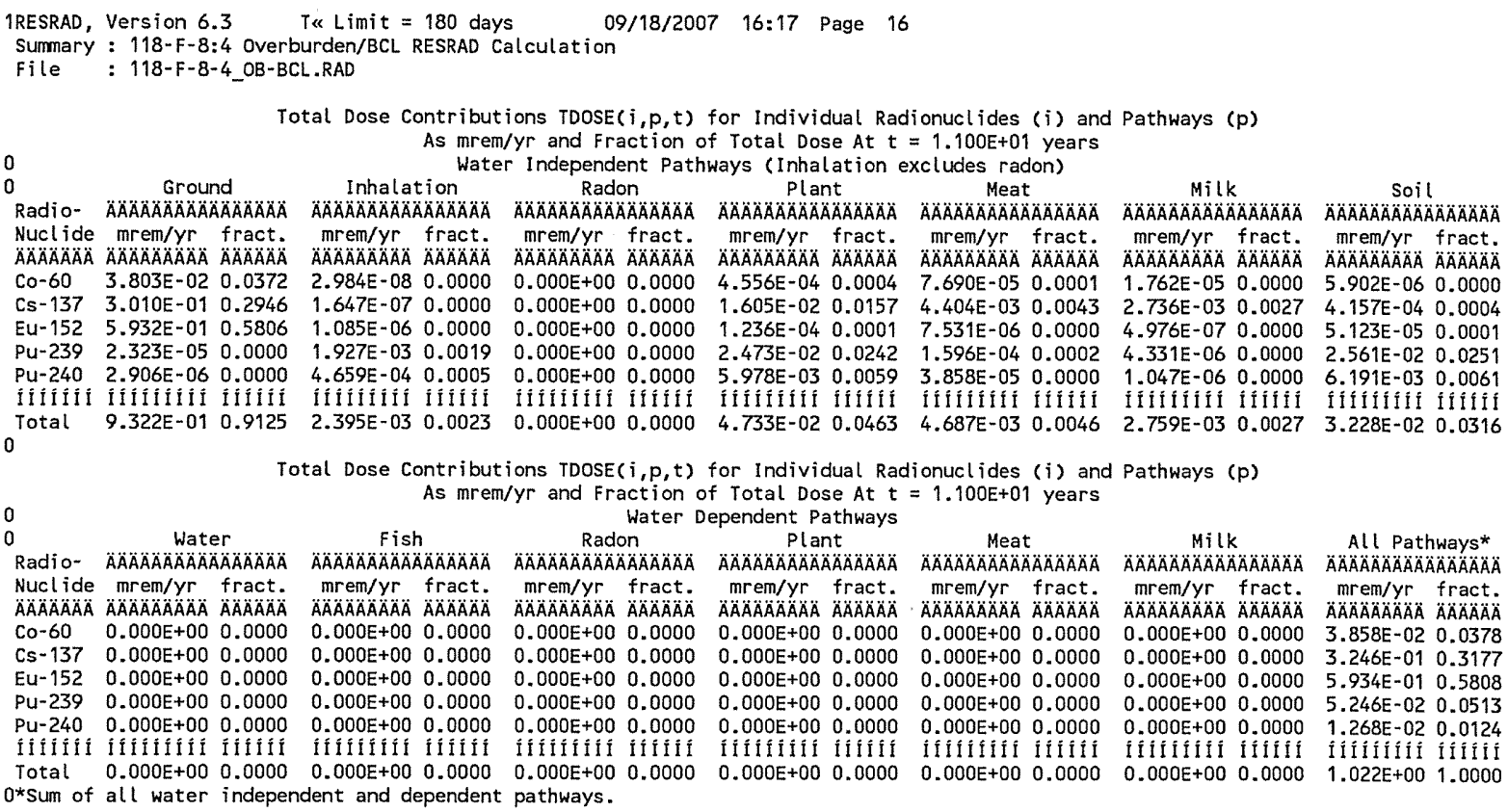

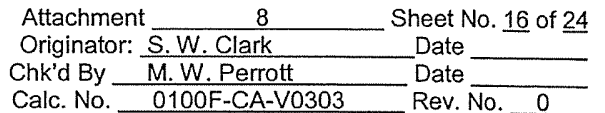




\section{ATTACHMENT 8}

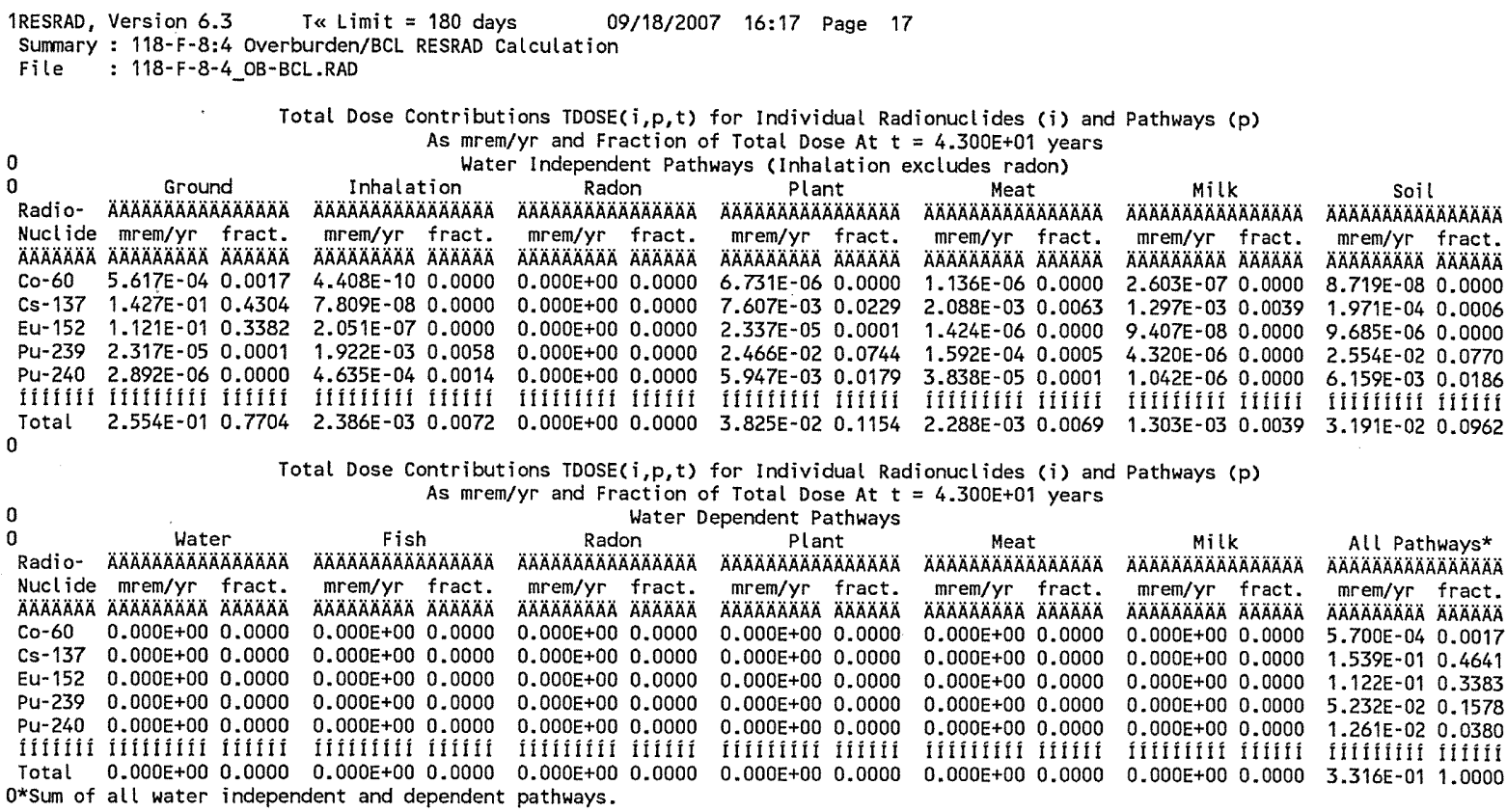

Total Dose Contributions $\operatorname{TDOSE}(i, p, t)$ for Individual Radionuclides (i) and Pathways ( $p$ )

\begin{tabular}{|c|c|}
\hline Attachment & Sheet No. 17 of 24 \\
\hline Originator: S.W. Clark & Date \\
\hline M. W. Perrott & Date \\
\hline Calc. No. $0100 \mathrm{~F}-\mathrm{CA}-\mathrm{V} 0303$ & Rev. No. 0 \\
\hline
\end{tabular}




\section{ATTACHMENT 8}

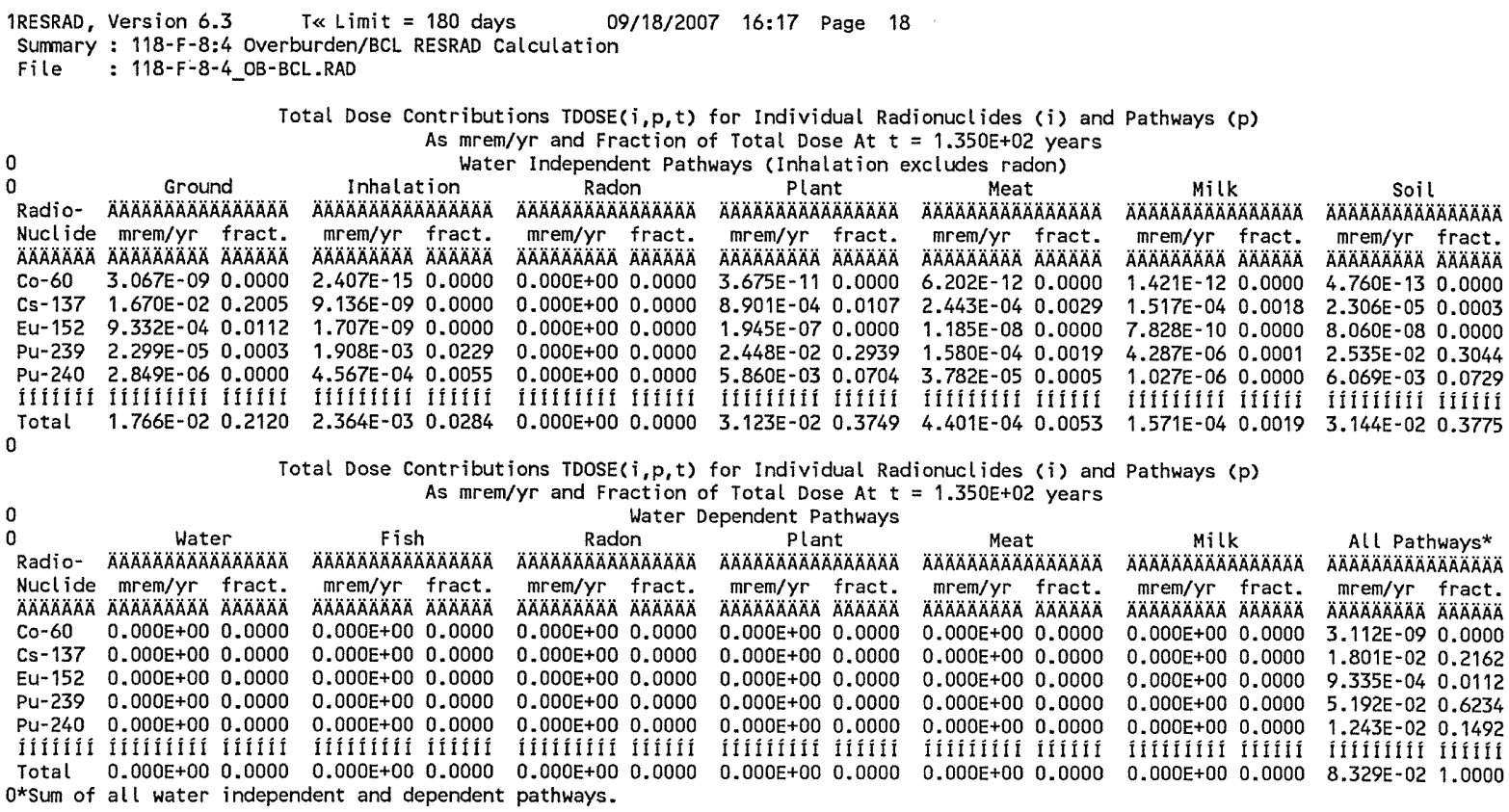




\section{ATTACHMENT 8}

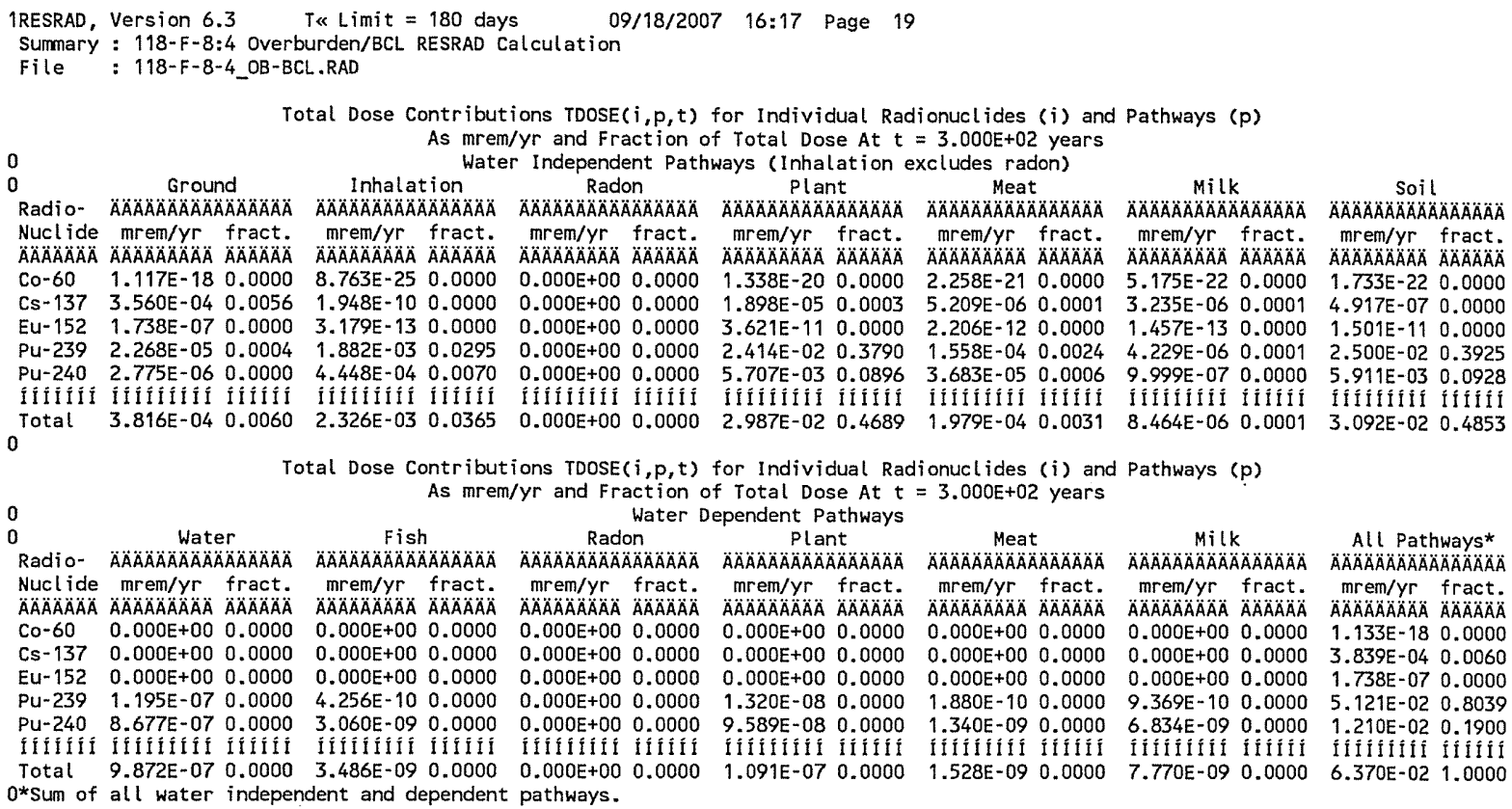

Total Dose Contributions $\operatorname{TDOSE}(i, p, t)$ for Individual Radionuclides (i) and Pathways ( $p$ ) As $\mathrm{mrem} / \mathrm{yr}$ and Fraction of Total Dose At $t=3.000 \mathrm{E}+02$ years

Total Dose Contributions $\operatorname{TDOSE}(i, p, t)$ for Individual Radionuclides (i) and Pathways (p) As mrem/yr and Fraction of Total Dose At $t=3.000 E+02$ years

\begin{tabular}{|c|c|}
\hline Attachment & Sheet No. 19 of 24 \\
\hline Originator: S.W. Clark & Date \\
\hline M.W. Perrott & Date \\
\hline $0100 \mathrm{~F}-\mathrm{CA}-\mathrm{V} 0303$ & Rev. No. 0 \\
\hline
\end{tabular}




\section{ATTACHMENT 8}

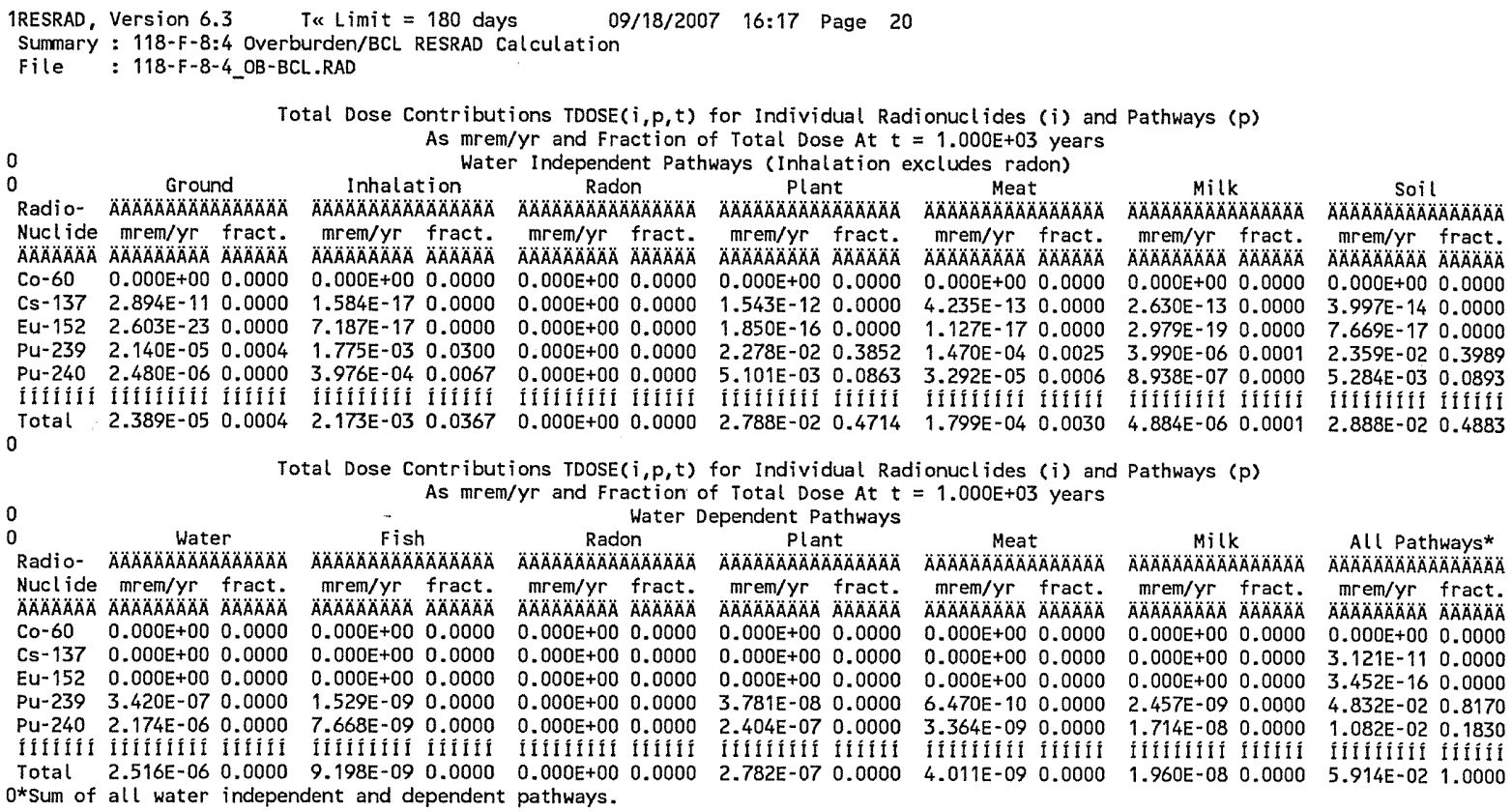

Total Dose Contributions TDOSE $(i, p, t)$ for Individual Radionuclides (i) and Pathways ( $p$ )

\begin{tabular}{|c|c|}
\hline Attachment & Sheet No. 20 of 24 \\
\hline Originator: $\overline{\text { S.W. Clark }}$ & Date \\
\hline M. W. Perrott & Date \\
\hline $0100 F-C A-V 0303$ & Rev. No. 0 \\
\hline
\end{tabular}




\section{ATTACHMENT 8}

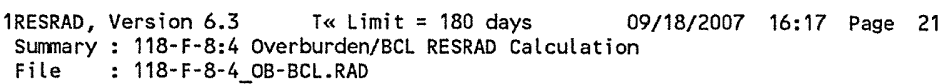

\begin{tabular}{|c|c|c|c|c|c|c|c|c|c|}
\hline $\begin{array}{l}\text { (i) } \\
(\mathbb{A} A \bar{A} A \bar{A} A \bar{A}\end{array}$ & $\begin{aligned} t= & 0.000 E+00 \\
& A A A A A A A A A A A\end{aligned}$ & $\begin{array}{l}1.000 E+00 \\
\triangle A O A O A O A A B A O A\end{array}$ & $\begin{array}{l}3.000 E+00 \\
\triangle A O A O A A O A O A O A\end{array}$ & $\begin{array}{l}7.000 E+00 \\
\triangle A A O A B A A A B A\end{array}$ & $\begin{array}{l}1.100 E+01 \\
\triangle A \cap A O A B A O A O A A A\end{array}$ & $\begin{array}{l}4.300 E+01 \\
\triangle . B A A A A A A A A\end{array}$ & $\begin{array}{l}1.350 E+02 \\
\triangle A B A O A O A O A A A\end{array}$ & $\begin{array}{l}3.000 E+02 \\
\triangle A O A O A O A O A O A O A\end{array}$ & $\begin{array}{l}1.000 E+03 \\
A A A B A A B A B A\end{array}$ \\
\hline & $1.461 E+00$ & $1.666 \mathrm{E}+00$ & $2.169 \mathrm{E}+00$ & $3.673 \mathrm{E}+00$ & $6.220 \mathrm{E}+00$ & $4.211 \mathrm{E}+02$ & $7.713 E+07$ & *1.132E+15 & $* 1.132 E+15$ \\
\hline 37 & $6.579 E+00$ & $6.734 E+00$ & $7.056 \mathrm{E}+00$ & $7.746 E+00$ & $8.503 E+00$ & $1.793 E+01$ & $1.533 E+02$ & $7.189 E+03$ & $8.843 E+10$ \\
\hline 152 & $3.422 E+00$ & $3.605 E+00$ & $4.001 E+00$ & $4.927 E+00$ & $6.067 E+00$ & $3.209 E+01$ & $3.856 E+03$ & $2.071 E+07$ & $* 1.765 E+14$ \\
\hline & $3.543 E+01$ & $3.543 E+01$ & $3.543 \mathrm{E}+01$ & $3.545 E+01$ & $3.546 \mathrm{E}+01$ & $3.555 \mathrm{E}+01$ & $3.583 E+01$ & $3.632 \mathrm{E}+01$ & $3.849 \mathrm{E}+01$ \\
\hline & $3.543 E+01$ & $3.544 E+01$ & $3.545 \mathrm{E}+01$ & $3.547 E+01$ & $3.550 E+01$ & $3.568 \mathrm{E}+01$ & $3.621 E+01$ & $3.718 \mathrm{E}+01$ & $4.158 \mathrm{E}+01$ \\
\hline & İ I I I I I I I I I I I & 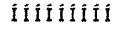 & ilitilifíli & lififintit & liffitity & fifififif & 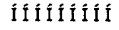 & ilitifity & ifififiti \\
\hline
\end{tabular}
Single Radionuclide Soil Guidelines $G(i, t)$ in $\mathrm{pC} i / g$ Basic Radiation Dose Limit $=1.500 \mathrm{E}+01 \mathrm{mrem} / \mathrm{yr}$

At specific activity limit

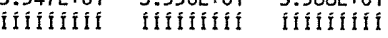




\section{ATTACHMENT 8}

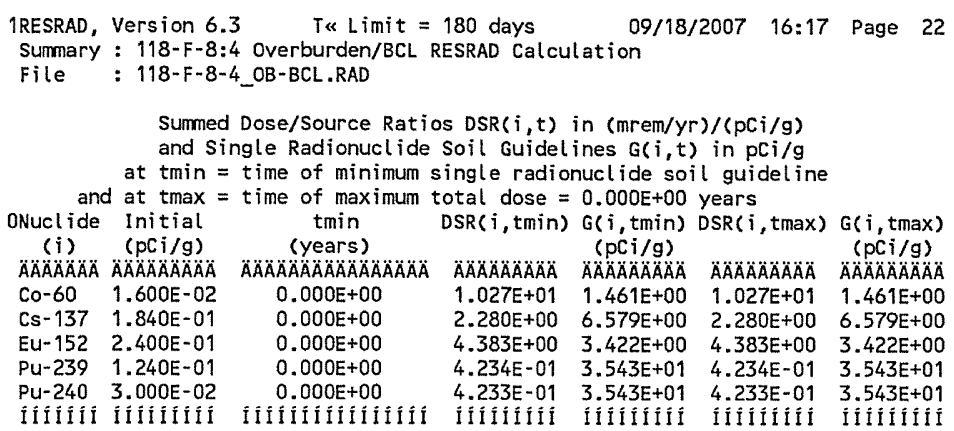




\section{ATTACHMENT 8}

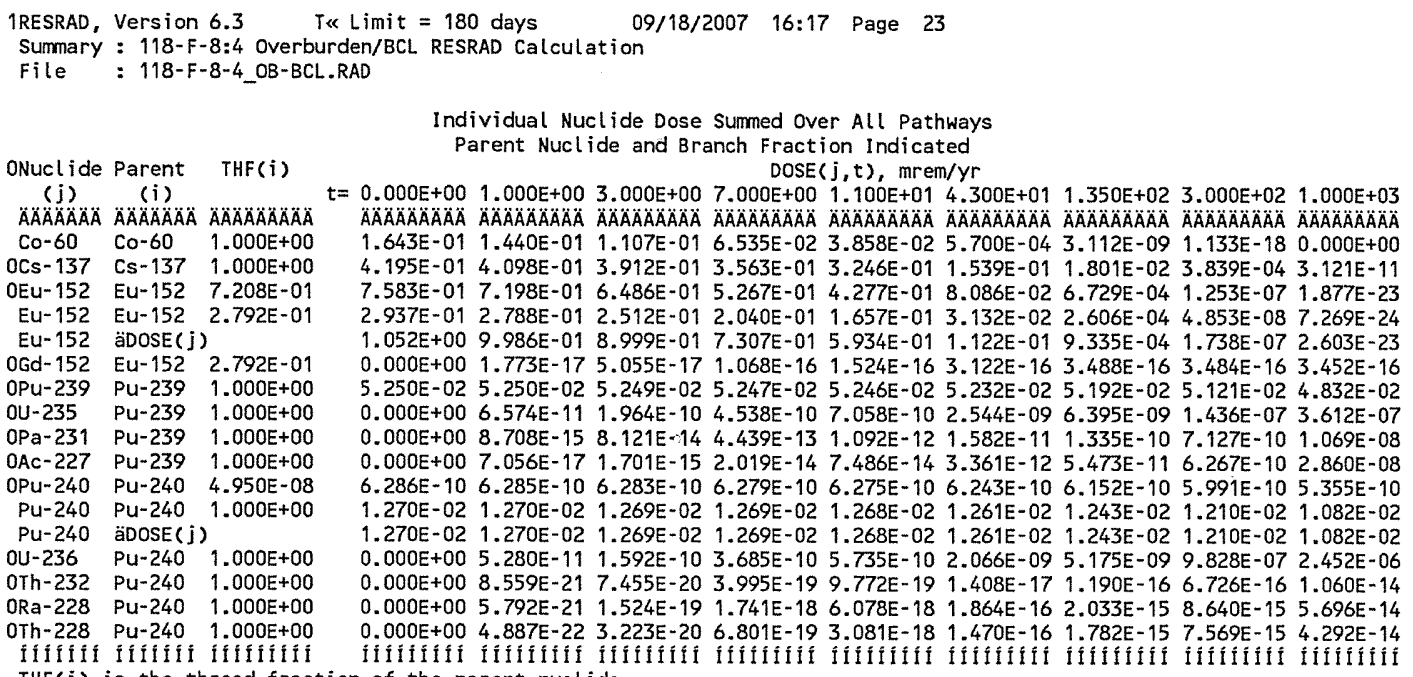

THF(i) is the thread fraction of the parent nuclide.

\begin{tabular}{|c|c|}
\hline \multirow{2}{*}{\multicolumn{2}{|c|}{$\begin{array}{ll}\text { Attachment } & 8 \\
\text { Originator: S.W. Slark } & \text { Sheet No. } 23 \text { of } 24 \\
\text { Date }\end{array}$}} \\
\hline & \\
\hline M. W. Perrott & Date \\
\hline $0100 F-C A-V 0303$ & 0 \\
\hline
\end{tabular}




\section{ATTACHMENT 8}

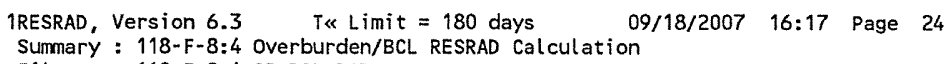

ONuclide Parent THF(i)

(j) (i)

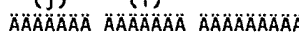

CO-60 CO-60 1.000E+00

OCs -137 Cs $-137 \quad 1.000 \mathrm{E}+00$

OEU-152 EU-152 7.208E-01

Eu-152 Eu-152 2.792E-01

Eu-152 äs(j):

OGd-152 Eu-152 2.792E-01

OPU-239 PU-239 $1.000 E+00$

OU-235 PU-239 1.000E+0O

OPa-231 PU-239 $1.000 \mathrm{E}+00$

$0 A C-227$ PU-239 1.000E+00

OPU-240 PU-240 4.950E-08

PU-240 PU-240 1.000E+00

Pu-240 äs(j):

OU -236 PU-240 $1.000 \mathrm{E}+00$

OTh-232 PU-240 $1.000 \mathrm{E}+00$

ORa-228 PU-240 1.000E+OO

OTh-228 PU-240 1.000E+00

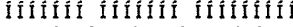

THF( $i$ ) is the thread fraction

ORESCALC.EXE execution time =

\section{Parent Nuclide and Branch Fraction Indicated
$S(j, t), \mathrm{pCi} / \mathrm{g}$ \\ Parent Nuclide and Branch Fraction Indicated}

$=0.000 E+001.000 E+003.000 E+007.000 E+00$

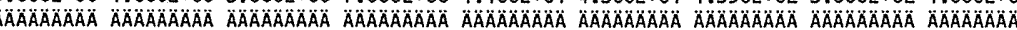

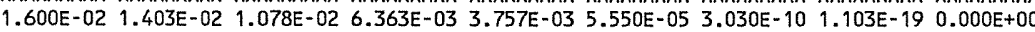
1.840E-01 1.798E-01 1.716E-01 1.563E-01 1.424E-01 6.750E-02 $7.897 \mathrm{E}-03 \quad 1.684 \mathrm{E}-04 \quad 1.369 \mathrm{E}-11$ $\begin{array}{lllllllll}1.730 E-01 & 1.642 E-01 & 1.480 E-01 & 1.202 E-01 & 9.758 E-02 & 1.845 E-02 & 1.535 E-04 & 2.858 E-08 & 4.281 E-24\end{array}$

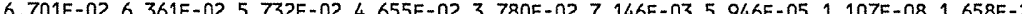
$2.400 E-012.278 E-012.053 E-011.667 E-0121.354 E-012.550 E-02 ~ 2.130 E-043.965 E-085.940 E-24$ $2.400 E-01$ 2.278E 01 2.053E-01 1.667E-01 1.354E-01 2.559E-02 $2.130 E-043.965 E-085.940 E-24$ $0.000 \mathrm{E}+00 \quad 4.191 \mathrm{E}-16 \quad 1.194 \mathrm{E}-15 \quad 2.523 \mathrm{E}-15 \quad 3.601 \mathrm{E}-15 \quad 7.378 \mathrm{E}-15 \quad 8.242 \mathrm{E}-15 \quad 8.231 \mathrm{E}-15 \quad 8.156 \mathrm{E}-15$ 1.240E-01 1.240E-01 1.240E-01 1.239E-01 1.239E-01 1.236E-01 1.226E-01 1.209E-01 1.141E-01 $0.000 \mathrm{E}+00 \quad 1.218 \mathrm{E}-10 \quad 3.635 \mathrm{E}-10 \quad 8.395 \mathrm{E}-10 \quad 1.306 \mathrm{E}-09 \quad 4.705 \mathrm{E}-09 \quad 1.183 \mathrm{E}-08$ 1.843E-08 2.217E-08 $0.000 \mathrm{E}+00 \quad 1.290 \mathrm{E}-15 \quad 1.156 \mathrm{E}-14 \quad 6.251 \mathrm{E}-14 \quad 1.533 \mathrm{E}-13 \quad 2.212 \mathrm{E}-12 \quad 1.866 \mathrm{E}-11 \quad 7.146 \mathrm{E}-11 \quad 3.552 \mathrm{E}-10$ $0.000 \mathrm{E}+00 \quad 1.358 \mathrm{E}-17 \quad 3.599 \mathrm{E}-16 \quad 4.405 \mathrm{E}-15 \quad 1.648 \mathrm{E}-14 \quad 7.487 \mathrm{E}-13 \quad 1.222 \mathrm{E}-11 \quad 5.945 \mathrm{E}-11 \quad 3.375 \mathrm{E}-10$ $1.485 \mathrm{E}-09 \quad 1.485 \mathrm{E}-09 \quad 1.484 \mathrm{E}-09 \quad 1.483 \mathrm{E}-09$ 1.482E-09 $1.475 \mathrm{E}-09 \quad 1.453 \mathrm{E}-09$ 1.415E-09 $1.265 \mathrm{E}-09$

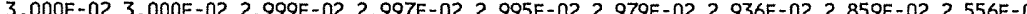

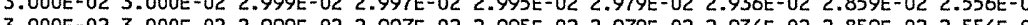
3.000E $0.000 E+00$ 8.857E-10 2.643E-09 $6.103 E-09$ 9.490E-09 3.415E-08 8.552E-08 1.321E-07 $1.515 E-07$ $0.000 E+00 \quad 2.187 E-20 \quad 1.961 E-19 \quad 1.060 E-18 \quad 2.600 E-18 \quad 3.757 E-17 \quad 3.179 E-16 \quad 1.226 E-15 \quad 6.298 E-15$

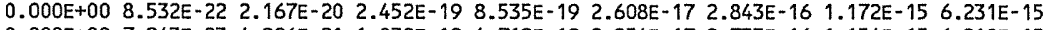
$0.000 E+00 \quad 7.243 E-23 \quad 4.886 E-21 \quad 1.038 E-19 \quad 4.712 E-19 \quad 2.254 E-17 \quad 2.733 E-16 \quad 1.154 E-15 \quad 6.210 E-15$

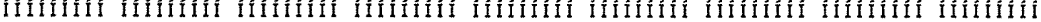
of the parent nuclide.

12.27 seconds

\begin{tabular}{|c|c|}
\hline Attachment & Sheet No. 24 of 24 \\
\hline Originator: S.W. Clark & Date \\
\hline Chk'd By M.W. Perrott & Date \\
\hline $0100 F-C A-V 0303$ & Rev. No. $\quad 0$ \\
\hline
\end{tabular}




\section{ATTACHMENT 9}

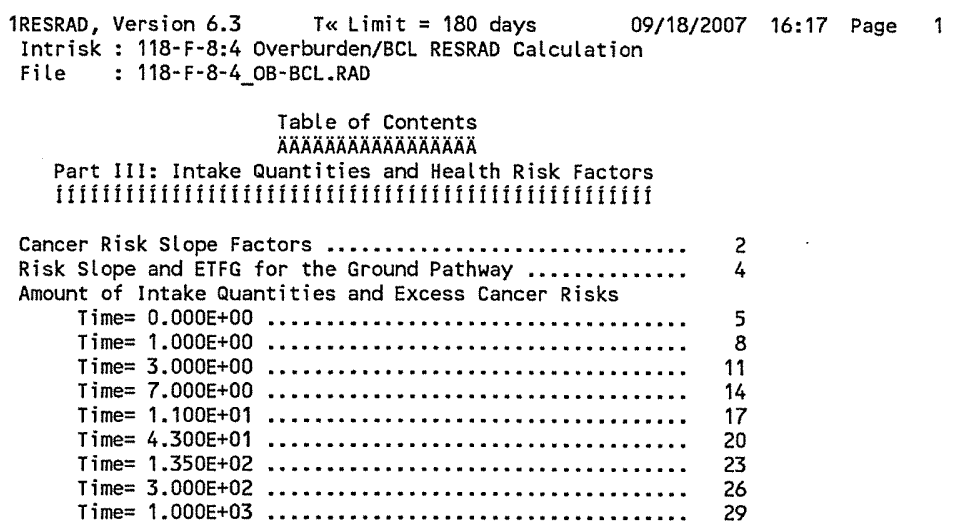




\section{ATTACHMENT 9}

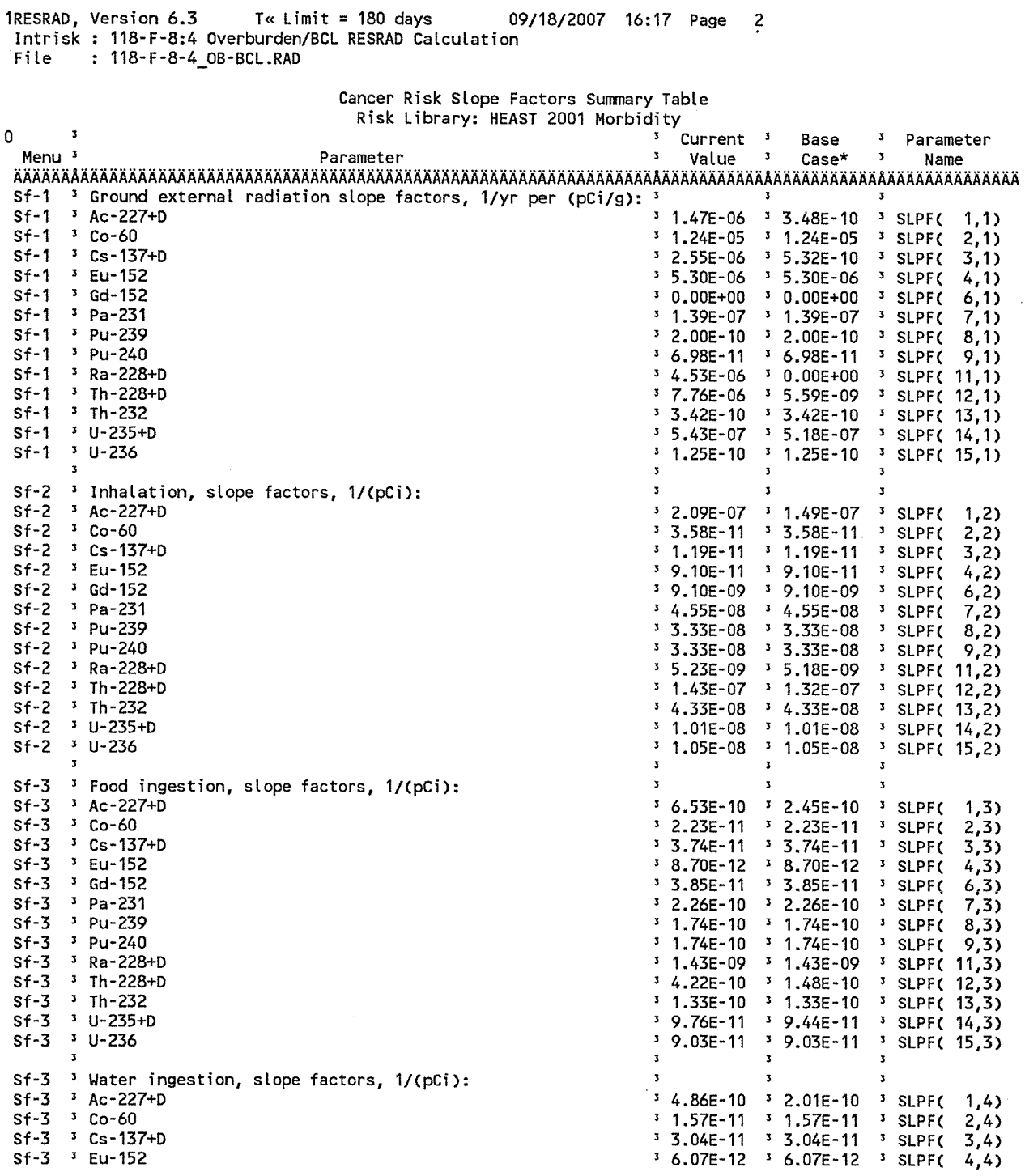

\begin{tabular}{ll} 
Attachment $\frac{9}{1}$ & Sheet No. 2 of 31 \\
Originator: $\frac{\text { S.W. Clark }}{\text { D. W. Perrott }}$ & Date \\
Chk'd By & Date \\
Calc. No. & O100F-CA-V0303 \\
\hline
\end{tabular}




\section{ATTACHMENT 9}

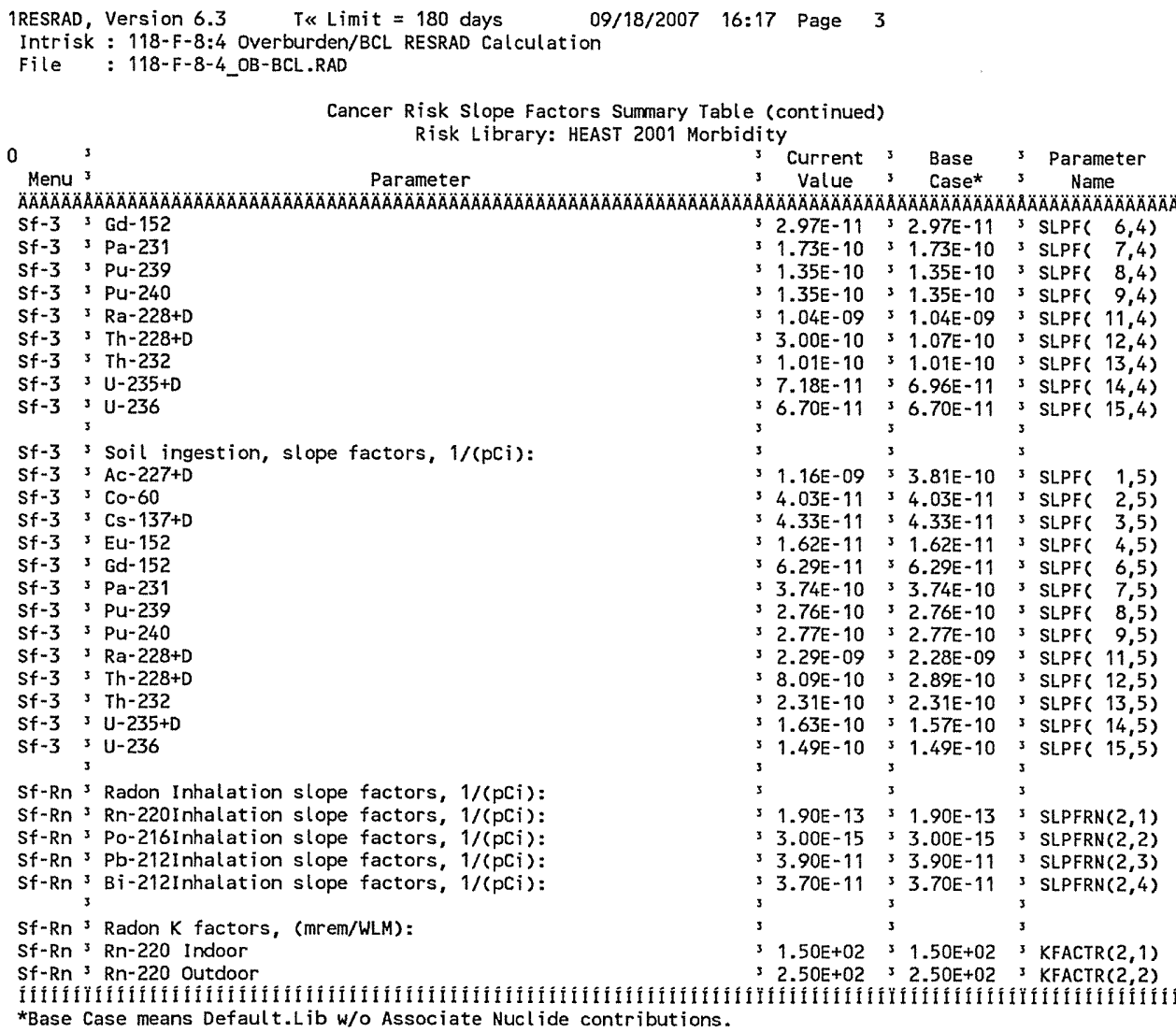

\begin{tabular}{|c|c|}
\hline \multicolumn{2}{|r|}{ Sheet No. $\underline{3}$ of $\underline{31}$} \\
\hline Originator: $\mathrm{S}, \mathrm{W}$. Clark & Date \\
\hline Chk'd By M.W. Perrott & Date \\
\hline Calc. No. 0100F-CA-V0303 & Rev. No. \\
\hline
\end{tabular}




\section{ATTACHMENT 9}

\begin{tabular}{|c|c|c|c|c|c|c|c|c|c|c|}
\hline $\begin{array}{l}\text { 1RESRAD, } \\
\text { Intrisk } \\
\text { File }\end{array}$ & $\begin{array}{l}\text { Version } 6.3 \\
: 118-F-8: 4 \\
: 118-F-8-4\end{array}$ & $\begin{array}{l}\text { Overburde } \\
\mathrm{OB}-\mathrm{BCL} . \mathrm{RA}\end{array}$ & IL RESRA & Calculation & $18 / 2007$ & S:17 Page & 4 & & & \\
\hline & slop & & & ET & & & (dimens & (2) & & \\
\hline & & & $0 \mathrm{~F}+0^{2}$ & $3.000 E+00$ & $7.000 E \div 00$ & $1.100 \mathrm{E}+\mathrm{C}$ & $4.300 E+01$ & $350 E+02$ & $3.000 E+02$ & $1.000 E+$ \\
\hline$\triangle A \cap A A A O A O A$ & $\triangle A A A B A A O A O A$ & $\triangle A A A A A A A A O A O$ & $A A O A A A O A B A O A$ & $\triangle A A A A A A A O A O A O A$ & $\triangle A \cap A B A B A O A O A B A B$ & 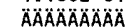 & $\triangle A A O A O A O A O A O A A$ & $\triangle A ̈ A ̈ A \cap A O A ̈ A ̈$ & & \\
\hline$A C-227$ & $3.480 E-10$ & $6.376 E-01$ & $6.376 \mathrm{E}-01$ & $6.376 E-01$ & $6.376 E-01$ & 6.3 & $6.376 E-01$ & $6.376 \mathrm{E}-01$ & $.376 \mathrm{E}-01$ & $3.376 E-01$ \\
\hline & & $6.248 E-01$ & $8 E-01$ & $6.248 \mathrm{E}-01$ & $6.248 \mathrm{E}-01$ & $6.248 \mathrm{E}-01$ & $6.248 \mathrm{E}-01$ & $6.248 \mathrm{E}-01$ & $6.248 E-01$ & $6.248 \mathrm{E}-01$ \\
\hline & & & & -01 & $E-01$ & $7 E-01$ & 6.197E-01 & $E-01$ & $E-01$ & $7 E-01$ \\
\hline & & & & 01 & -01 & & $E-01$ & -01 & -01 & \\
\hline & 8.8 & 6.2 & 6. & $6.216 E-01$ & 6E-01 & $6.216 \mathrm{E}-01$ & $6.216 E-01$ & $6 E-01$ & $16 E-01$ & $6 \mathrm{E}-01$ \\
\hline & & 6. & 6. & $=-01$ & $E-01$ & $6.242 \mathrm{E}-01$ & -01 & -01 & & $E-01$ \\
\hline & & & & & -01 & & & & & \\
\hline & & & 6. & 6.2 & $E-01$ & 6.2 & 6.2 & & & $E-01$ \\
\hline & 1 & -01 & 6. & $E-01$ & $E-01$ & $3 E-01$ & 6.3 & & -01 & $E-01$ \\
\hline & & & 0 & .00 & $+\infty$ & $0 E+00$ & 0.0 & & $+\infty 0$ & $E+00$ \\
\hline & & & $E-01$ & -01 & $=-01$ & 6. & 6. & 01 & -01 & $E-01$ \\
\hline & & & & & & & & & & $E=01$ \\
\hline & & & & & & & 6.3 & & & $E-01$ \\
\hline & & 6 & 6. & 01 & 6 & & & & & $E-01$ \\
\hline & & & & & +00 & & & & & $E+\infty$ \\
\hline & & & 6. & & -01 & 6. & 6.2 & & 01 & $E-01$ \\
\hline & & & & & & 6.2 & & & & $=-01$ \\
\hline & & & & & & & & & & E-01 \\
\hline & & & & & & 6.6 & 6. & & & $E-01$ \\
\hline & & & & & & & & & & $E-01$ \\
\hline & & & & & & 6.3 & 01 & & & $E-01$ \\
\hline $\mathrm{Ra}$ & & 0 & 0 & 0 & 0. & 0.0 & 0. & & & \\
\hline & & & 6. & & & & & & & -01 \\
\hline & & & & & & & & & & $E-01$ \\
\hline & & & & & & 6.3 & & & & \\
\hline & & 1 & & & & & & & & \\
\hline & & & 6. & 6.39 & $E-01$ & $6.391 E-01$ & $6.391 E-01$ & 01 & $E-01$ & $1 \mathrm{E}-01$ \\
\hline & & & & & -01 & $6.413 \mathrm{E}-$ & & & & $3 E-01$ \\
\hline & & & & & & & & & & \\
\hline & 1.76 & $6.268 \mathrm{E}-0$ & $6.268 \mathrm{E}-\mathrm{C}$ & $6.268 \mathrm{E}-01$ & $6.268 \mathrm{E}-01$ & $6.268 \mathrm{E}-01$ & $6.268 \mathrm{E}-01$ & $6.268 \mathrm{E}-01$ & $6.268 E-01$ & $3 E-01$ \\
\hline & & & & & & $6.344 E-01$ & $6.344 \mathrm{E}-01$ & $6.344 \mathrm{E}-01$ & & 6. \\
\hline & & & 0. & & & & & & & \\
\hline & fidifiti & fitfifiti & iffilitif & IIfIIIIIII & IIIIIIIIII & İî́îlíñ & IIIIIIIIII & IIIIIIIII & Ififlitifif & 1111 \\
\hline
\end{tabular}

\begin{tabular}{|c|c|}
\hline \multirow{2}{*}{\multicolumn{2}{|c|}{$\begin{array}{c}\text { Attachment } \\
\text { Originator: S. W. Clark }\end{array}$}} \\
\hline & \\
\hline Chk'd By M.W. Perrott & Date \\
\hline $0100 F-C A-V 0303$ & Rev. No. 0 \\
\hline
\end{tabular}




\section{ATTACHMENT 9}

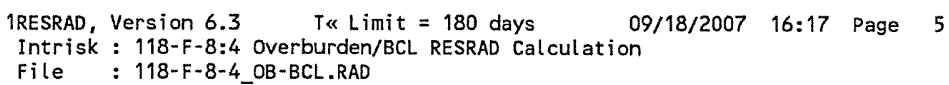

Water-ind. = = Water-independent Water-dep. == Water-dependent

$\begin{array}{ll}\text { Attachment } \frac{9}{\text { S. W. Clark }} & \text { Sheet No. } 5 \text { of } 31 \\ \text { Originator: } \frac{\text { S. Wate }}{\text { M.W. Perrott }} & \text { Date } \\ \text { Chk'd By } & \text { Rev. No. } 0 \\ \text { Calc. No. } & \text { O100F-CA-V0303 }\end{array}$




\section{ATTACHMENT 9}

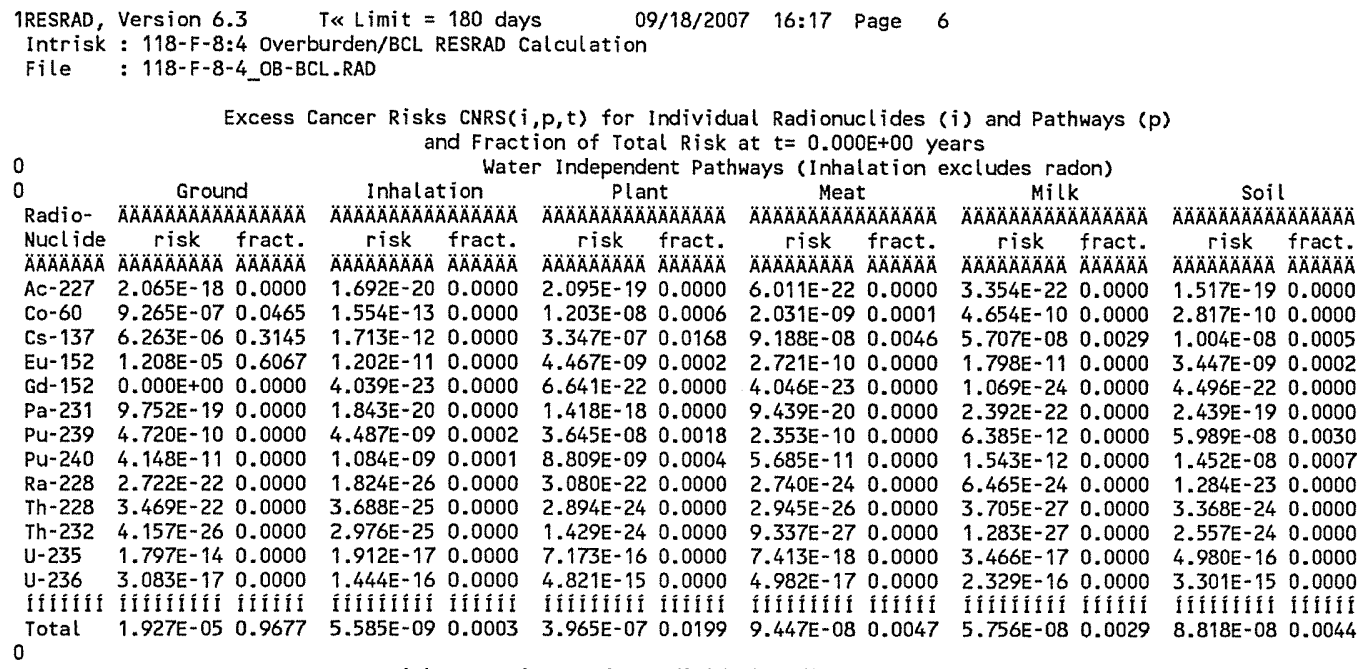

Excess Cancer Risks CNRS( $i, p, t)$ for Individual Radionuclides (i) and Pathways ( $p$ ) and Fraction of Total Risk at $t=0.000 E+00$ years

Water Dependent Pathways

\begin{tabular}{|c|c|c|c|c|c|c|c|c|c|c|c|}
\hline \multirow{2}{*}{ 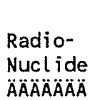 } & \multicolumn{2}{|c|}{ 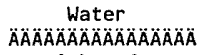 } & \multicolumn{2}{|c|}{ 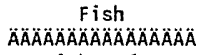 } & \multicolumn{2}{|c|}{$\begin{array}{c}P l \text { lant } \\
\text { ÄÄAAAAAAAAOAAOAOA }\end{array}$} & \multicolumn{2}{|c|}{$\begin{array}{c}\text { Meat } \\
\text { ÄAAAAAAAAAAAAAAÄÄ }\end{array}$} & \multicolumn{2}{|c|}{ 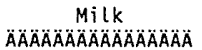 } & $\begin{array}{r}\text { All Pathways } \\
\text { A* } \\
\text { AAAAAAAAAAAAAAA }\end{array}$ \\
\hline & $\begin{array}{c}\text { risk } \\
A \cap A B A O A O A O A B\end{array}$ & $\begin{array}{l}\text { fract. } \\
\text { ÄÄÄÄÄÄ }\end{array}$ & $\begin{array}{c}r i s k \\
\triangle A A O A O A O A O A O A\end{array}$ & $\begin{array}{l}\text { fract. } \\
\ddot{A} \ddot{A} \ddot{A} \ddot{A} \ddot{A} \dot{A}\end{array}$ & $\begin{aligned} \text { risk } \\
\triangle A A A A B A A A\end{aligned}$ & $\begin{array}{l}\text { fract. } \\
\ddot{A} A \ddot{A} A \ddot{A} \ddot{A} \bar{A}\end{array}$ & 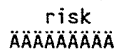 & $\begin{array}{l}\text { fract. } \\
\mathscr{A} \ddot{A} \ddot{A} \ddot{A} \ddot{A} A\end{array}$ & $\begin{array}{r}r i s k \\
A \cap A B A B A O A B\end{array}$ & $\begin{array}{l}\text { fract. } \\
\because A B A A A B A B\end{array}$ & 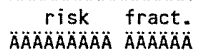 \\
\hline 227 & $0.000 E+00$ & 0.0000 & $0.000 E+00$ & 0.0000 & $0.000 E+00$ & 0.0000 & & .0000 & $00 E+00$ & 000 & 0000 \\
\hline & $0.000 E+00$ & 0 & $.000 E+00$ & 0.0000 & $00 E+00$ & 00 & $E+\infty$ & 00 & $E+00$ & & \\
\hline & & & $O E+\infty O$ & & & & & & & & \\
\hline & $0.000 E+00$ & 0.0000 & $0.000 E+00$ & & $E+00$ & 0.0000 & & 000 & 100 & & 1 \\
\hline & $0.000 \mathrm{E}+$ & & $E+00$ & & $=+\infty$ & & & & & & \\
\hline & $0.000 \mathrm{E}$ & 0.0 & $E+00$ & 0. & 0. & & 0 & & 00 & & \\
\hline & $0.000 \mathrm{E}$ & & 0. & & 0. & 0 & 0 & 0 & 0 & bo & \\
\hline & 0.00 & & $+\infty$ & & & & & & & & \\
\hline & $0.000 E+00$ & 0. & $E+00$ & 0. & 0. & & 0. & & $\mathrm{DE}+0 \mathrm{C}$ & & 22 \\
\hline Th- & $0.000 E+00$ & 0.0000 & $0.000 E+00$ & 0.0000 & $0.000 \mathrm{E}+00$ & 0.0 & 0.0 & 0. & $0.000 E+00$ & 00 & -22 \\
\hline & 0 & & & & & & & & & & \\
\hline & $0.000 \mathrm{E}+$ & & $+\infty$ & & 0.0 & & 0. & & & & \\
\hline & $0.000 E+00$ & 0.0000 & $0.000 E+00$ & 0.0000 & 0. & & & & & & 000 \\
\hline & & & & & & & & & & & \\
\hline $10 \mathrm{t}$ & $0.000 E+00$ & 0.0000 & $0.000 \mathrm{E}+00$ & 0.0000 & 0. & & 0.00 & 00 & $0.000 \mathrm{E}+$ & 0.0000 & $1.991 \mathrm{E}-05 \quad 1.000$ \\
\hline
\end{tabular}

** Sum of water independent ground, inhalation, plant, meat, milk, soil

and water dependent water, fish, plant, meat, milk pathways

\begin{tabular}{|c|c|}
\hline Attachment & Sheet No. 6 of 31 \\
\hline Originator: S. W. Clark & Date \\
\hline Chk'd By M. W. Perrott & Date \\
\hline Calc. No. $0100 \mathrm{~F}-\mathrm{CA}-\mathrm{V} 0303$ & Rev. No. 0 \\
\hline
\end{tabular}




\section{ATTACHMENT 9}

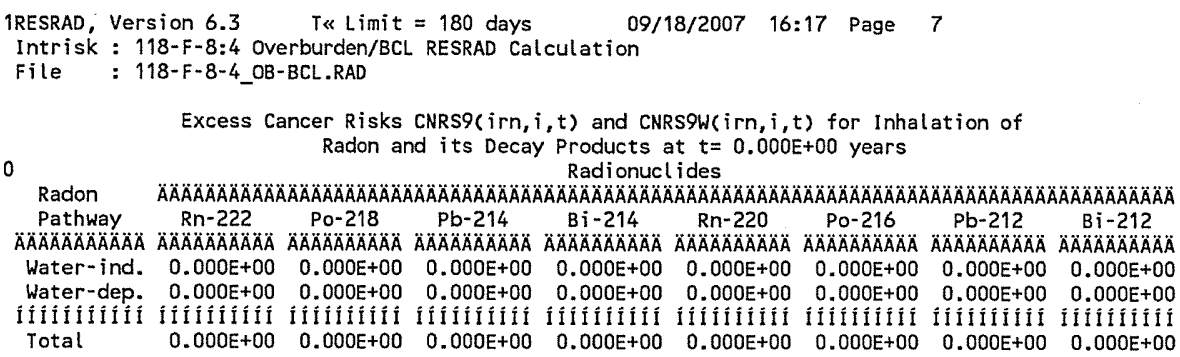

Total Excess Cancer Risk CNRSI $(i, p, t) * * *$ for Initially Existent Radionuclides ( $i$ ) and Pathways $(p)$ and Fraction of Total Risk at $t=0.000 E+00$ years

Water Dependent Pathways

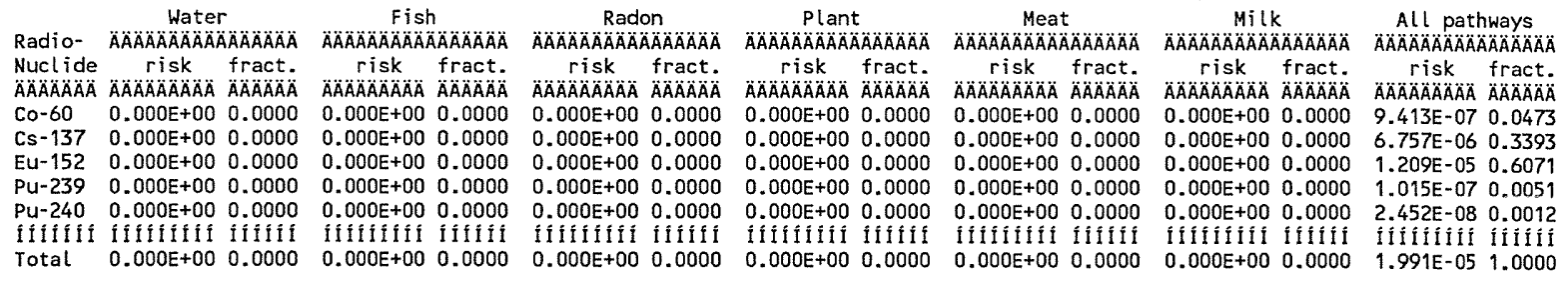

$* * * \operatorname{CNRSI}(i, p, t)$ includes contribution from decay daughter radionuclides

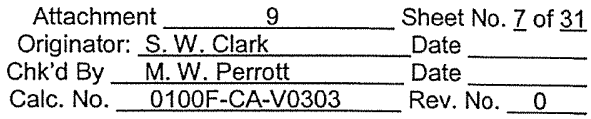




\section{ATTACHMENT 9}

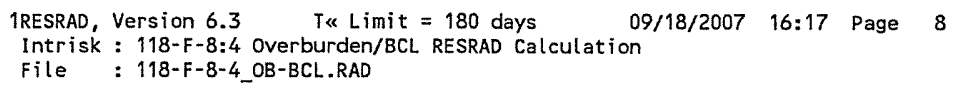

\begin{tabular}{|c|c|}
\hline \multirow{2}{*}{\multicolumn{2}{|c|}{$\begin{array}{r}\text { Attachment } \frac{9}{\text { Originator: S. W. Clark }}\end{array}$}} \\
\hline & \\
\hline M. W. Perrott & Date \\
\hline $0100 \mathrm{~F}-\mathrm{CA}-\mathrm{V} 0303$ & Rev. No. \\
\hline
\end{tabular}




\section{ATTACHMENT 9}

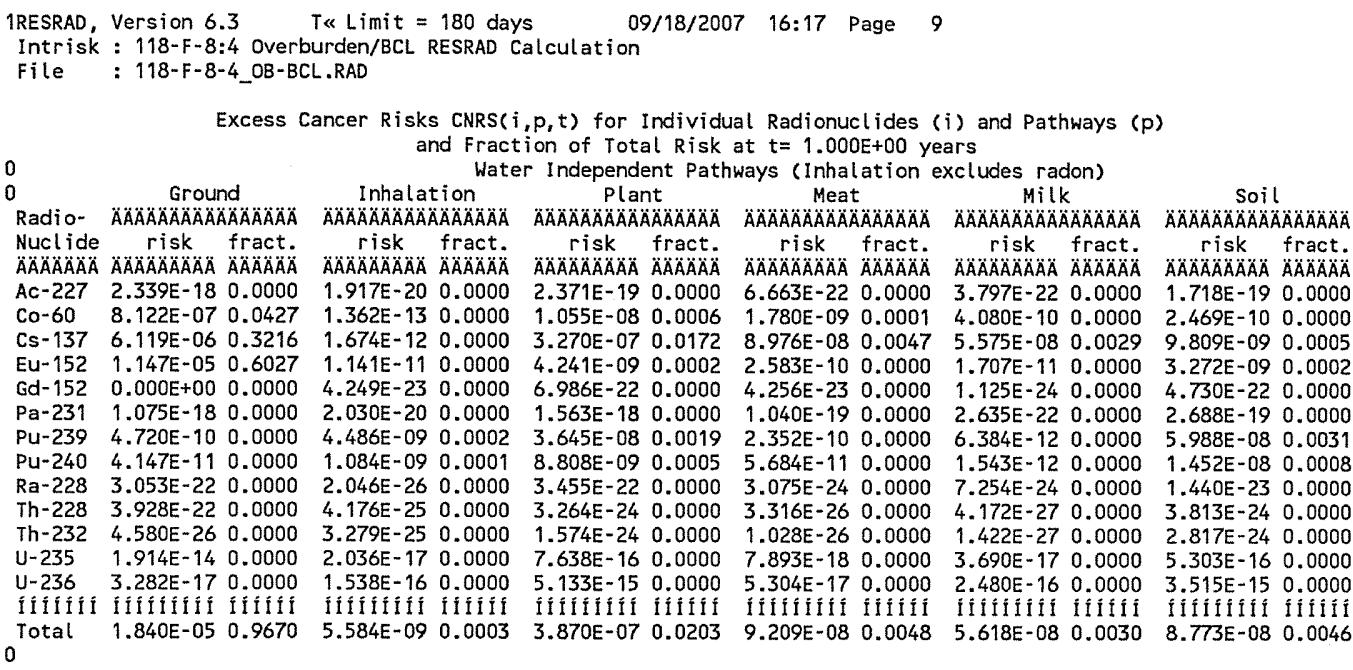

Water Dependent Pathways

\begin{tabular}{|c|c|c|c|c|c|c|c|c|c|c|c|c|}
\hline \multirow[b]{2}{*}{$\begin{array}{l}\text { Nucl ide } \\
\text { ÄAAAAAAOA }\end{array}$} & \multicolumn{2}{|c|}{ Water } & \multicolumn{2}{|c|}{$\begin{array}{c}\text { Fish } \\
\text { ÄAAAAAAAAAOAOAOAOA }\end{array}$} & \multicolumn{2}{|c|}{$\begin{array}{c}\text { Plant } \\
\text { ÄÄÄÄÄÄÄÄÄÄÄÄA }\end{array}$} & \multicolumn{2}{|c|}{$\begin{array}{c}\text { Meat } \\
\text { ÄAAAAAAAAAAAAOAAA }\end{array}$} & \multicolumn{2}{|c|}{$\begin{array}{c}\text { Milk } \\
\text { ÄÄÄAAAAAAOAOAOÄA }\end{array}$} & \multicolumn{2}{|c|}{ 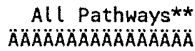 } \\
\hline & $\begin{array}{c}\text { risk } \\
A \cap A A A A B A B A O A\end{array}$ & 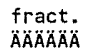 & $\begin{array}{c}\text { risk } \\
A A A B A B A A B\end{array}$ & $\begin{array}{l}\text { fract. } \\
\ddot{A} \ddot{A} \ddot{A} \ddot{A} \ddot{A} \bar{A}\end{array}$ & $\begin{array}{c}r i s k \\
\triangle A O A O A B A ̈ A B A ̈\end{array}$ & $\begin{array}{l}\text { fract. } \\
\because \ddot{A B A ̈ A ̈ A ̈ A}\end{array}$ & $\begin{array}{c}r i s k \\
\triangle A ̈ A ̈ A ̈ A ̈ A ̈ A ̈ A ̈ A ̈\end{array}$ & $\begin{array}{l}\text { fract. } \\
\ddot{A} \ddot{A} \ddot{A} \ddot{A} \bar{A} \bar{A}\end{array}$ & $\begin{array}{c}r i s k \\
\triangle A B A A A A A B A\end{array}$ & 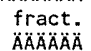 & risk & fract. \\
\hline$A C-227$ & & 0.0000 & & & $0.000 E+00$ & 0.0000 & & 000 & & & & \\
\hline & $O O E+0 O$ & 0. & $D O E+0 O$ & 100 & $D O E+00$ & 000 & $D O 0 E+00$ & 000 & $.000 E+00$ & 0.0000 & & \\
\hline & & & & & & & & & & & & \\
\hline & & & & & & & & & & & & \\
\hline & 0.0 & & & & 00 & & & & .00 & & & . \\
\hline & 0. & & -00 & & & & & & & & & \\
\hline & & & 00 & & & 0. & 0.00 & & & & & \\
\hline & & & & & & & 0.00 & 100 & $E+00$ & 0. & & 13 \\
\hline & 0. & & +00 & & jo & & 0 & & & & & \\
\hline & 0.0 & & +00 & & 00 & & 0.000 & & +00 & & 22 & \\
\hline Th & 0.00 & & $.000 E+00$ & & $0.000 E+00$ & 0.0 & $0.000 \mathrm{E}+00$ & 00 & $E+\infty$ & 0. & & 1000 \\
\hline & & & & & & & & & & & & \\
\hline & & & & & & & & & & & & \\
\hline & fíf & & & & & & fiffit & & lifififi & & filifit & 111111 \\
\hline & $.000 E+00$ & 0.0000 & $.000 E+00$ & 0.0000 & $.000 E+00$ & 0.0000 & $0.000 E+00$ & 0.0000 & $0.000 E+00$ & 0.0000 & $13 E-05$ & 1.000 \\
\hline
\end{tabular}

** Sum of water independent ground, inhalation, plant, meat, milk, soil

and water dependent water, fish, plant, meat, milk pathways

\begin{tabular}{|c|c|}
\hline Attachment & Sheet No. $\underline{9}$ of 31 \\
\hline Originator: S. W. Clark & Date \\
\hline M.W. Perrott & Date \\
\hline $0100 F-C A-V 0303$ & Rev. No \\
\hline
\end{tabular}




\section{ATTACHMENT 9}

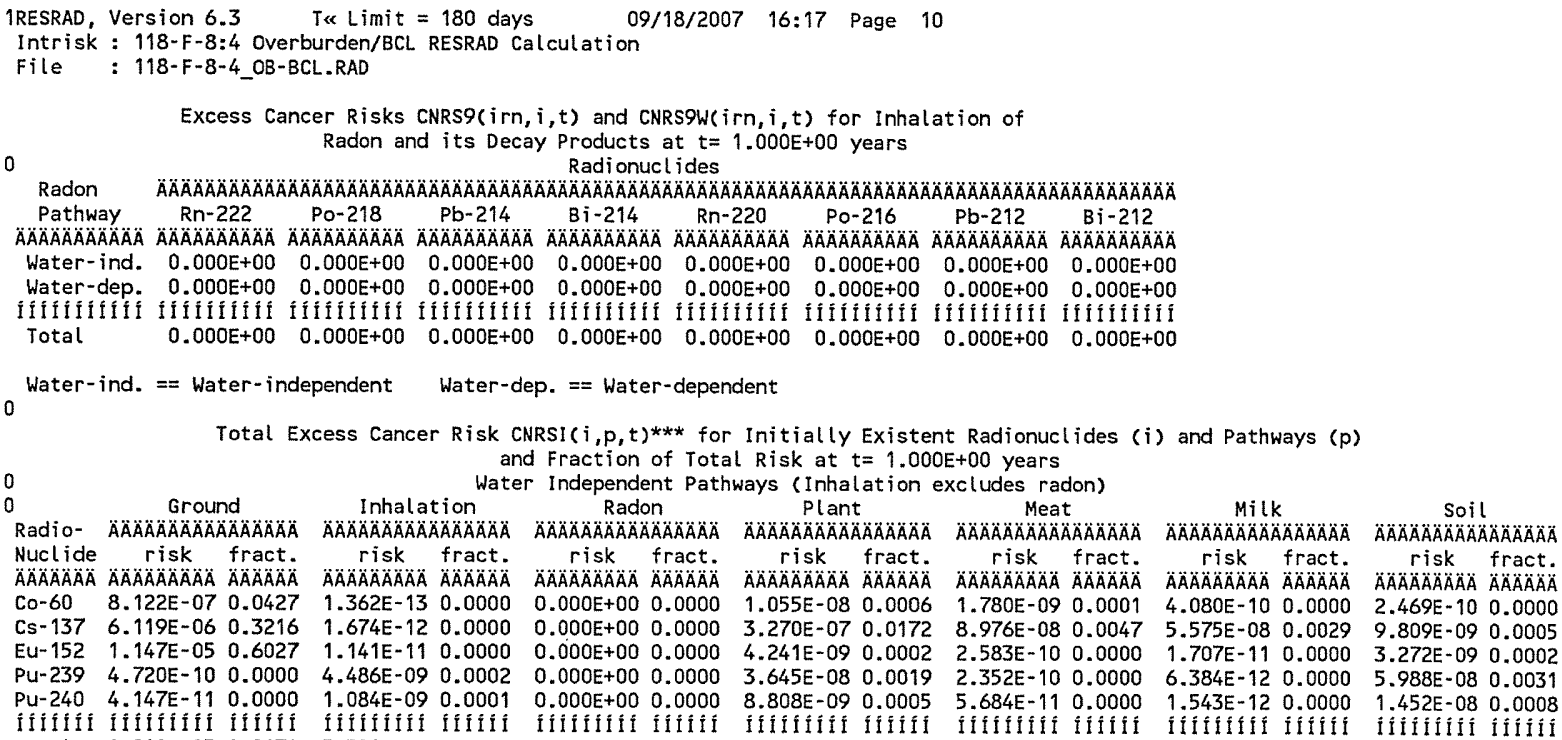

Total Excess Cancer Risk CNRSI $(i, p, t)^{* * *}$ for Initially Existent Radionuclides (i) and Pathways $(p)$ and Fraction of Total Risk at $t=1.000 E+00$ years

Water Dependent Pathways

\begin{tabular}{|c|c|c|c|c|c|c|c|c|c|c|c|c|c|c|}
\hline \multirow{2}{*}{$\begin{array}{l}\text { Radio- } \\
\text { Nucl ide } \\
\text { ÄÄÄÄÄA }\end{array}$} & \multicolumn{2}{|c|}{$\begin{array}{c}\text { Water } \\
\text { ÄÄÄÄÄAAAOAOAOÄÄ }\end{array}$} & \multicolumn{2}{|c|}{ 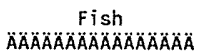 } & \multicolumn{2}{|c|}{ 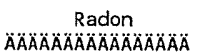 } & \multicolumn{2}{|c|}{$\begin{array}{c}\text { Plant } \\
\text { ALAAAAOAOÄÄAOAOAAAOA }\end{array}$} & \multicolumn{2}{|c|}{$\begin{array}{c}\text { Meat } \\
\text { ÄAAAAAAAAAAAAOAOAOA }\end{array}$} & \multicolumn{2}{|c|}{ 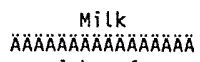 } & \multicolumn{2}{|c|}{$\begin{array}{c}\text { All pathways } \\
\text { AAAOAAAAAAAAAAAABA }\end{array}$} \\
\hline & & & risk & $\begin{array}{l}\text { fract. } \\
A A A A A A B A\end{array}$ & $\begin{array}{c}\text { risk } \\
\triangle A A A A A A A B\end{array}$ & $\begin{array}{l}\text { fract. } \\
\text { ÄÄÄÄÄ }\end{array}$ & rick & & risk & & $\begin{array}{c}\text { risk } \\
\text { ÄAAAOAAAOA }\end{array}$ & & risk & \\
\hline & & & & & & & & & & & & & & \\
\hline & & & & & & & & & & & & & & \\
\hline & & & & & & & & & & & & & & \\
\hline & & & & & & & & & & & & & & \\
\hline & & & & & & & & & & & & & & \\
\hline & & & & & & & & & & & & & & \\
\hline & ח000 & 10 & 0 & 0 & $E+00$ & 10 & 0 & 0 & +00 & 0.0000 & $0.000 E+00$ & 0.0000 & & \\
\hline
\end{tabular}

***CNRSI $(i, p, t)$ includes contribution from decay daughter radionuclides

\begin{tabular}{|c|c|}
\hline Attachment & Sh \\
\hline Originator: S.W. Clark & $\mathrm{Da}$ \\
\hline M. W. Perrott & Date \\
\hline $0100 \mathrm{~F}-\mathrm{CA}-\mathrm{V} 0303$ & Rev. No. 0 \\
\hline
\end{tabular}




\section{ATTACHMENT 9}

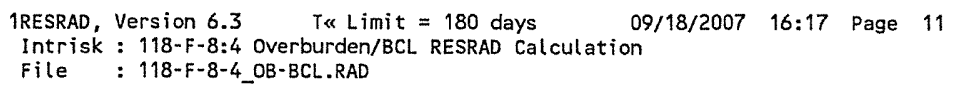

Water-ind. $==$ Water-independent Water-dep. $==$ Water-dependent

\begin{tabular}{|c|c|}
\hline Attachment & Sheet No. 11 of 31 \\
\hline Originator: S.W. Clark & Date \\
\hline Chk'd By M.W. Perrott & Date \\
\hline Calc. No. $0100 \mathrm{~F}-\mathrm{CA}-\mathrm{V} 0303$ & Rev. No. \\
\hline
\end{tabular}




\section{ATTACHMENT 9}

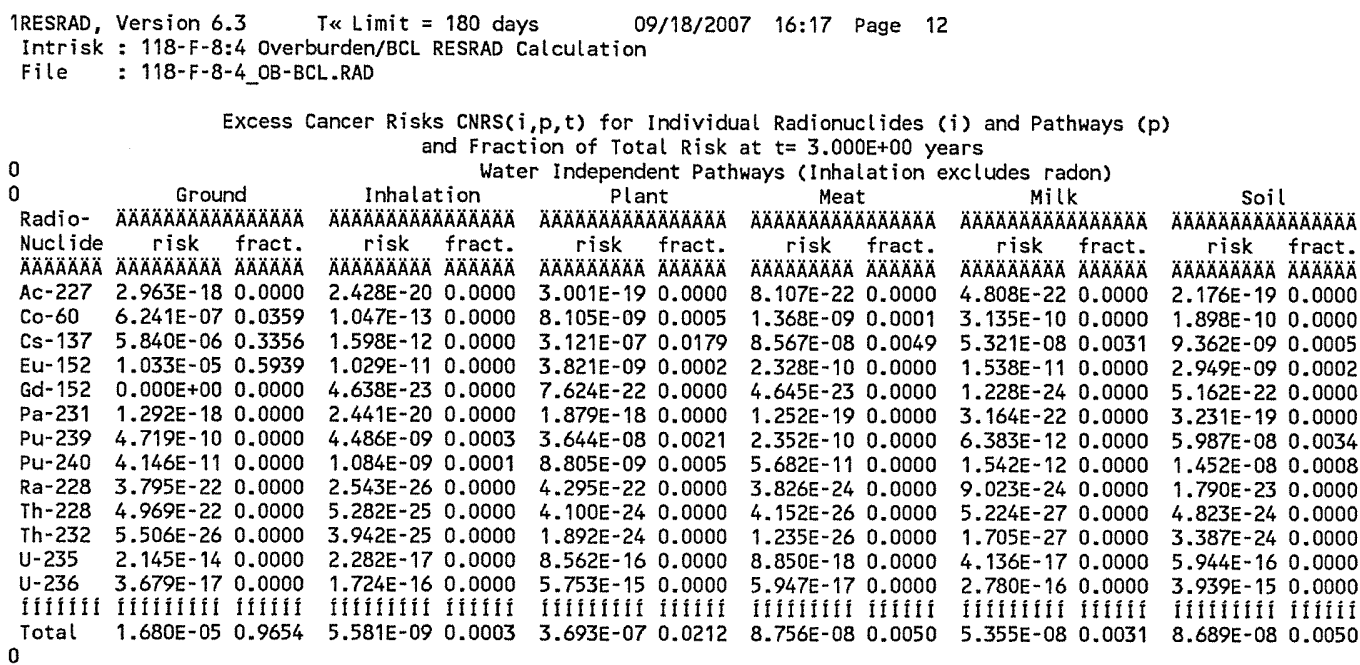

Excess Cancer Risks CNRS $(i, p, t)$ for Individual Radionuclides (i) and Pathways $(p)$ and Fraction of Total Risk at $t=3.000 E+00$ years

Water Dependent Pathways

\begin{tabular}{|c|c|c|c|c|c|c|c|c|c|c|c|c|}
\hline \multirow[b]{2}{*}{$\begin{array}{l}\text { Nuclide } \\
\text { AAAAAAAAABA }\end{array}$} & \multicolumn{2}{|c|}{ 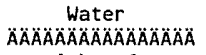 } & \multicolumn{2}{|c|}{ 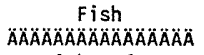 } & \multicolumn{2}{|c|}{ 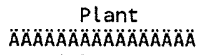 } & \multicolumn{2}{|c|}{$\begin{array}{c}\text { Meat } \\
\text { ÄÄÄÄÄÄÄÄÄÄÄÄ }\end{array}$} & \multicolumn{2}{|c|}{$\begin{array}{c}\text { Milk } \\
\text { ÄÄAAAAAAAAAAAÄA }\end{array}$} & \multicolumn{2}{|c|}{ 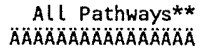 } \\
\hline & $\begin{array}{c}\text { risk } \\
\triangle A A A A A A A A\end{array}$ & $\begin{array}{l}\text { fract: } \\
\text { ÄÄÄÄÄA }\end{array}$ & $\begin{array}{c}\text { risk } \\
\text { ÄÄÄÄÄÄÄA }\end{array}$ & $\begin{array}{l}\text { fract. } \\
\text { ÄÄÄÄÄÄ }\end{array}$ & $\begin{array}{c}\text { risk } \\
\text { ÄÄÄÄÄÄÄ }\end{array}$ & $\begin{array}{l}\text { fract. } \\
\text { ÄÄÄÄÄA }\end{array}$ & $\begin{array}{c}\text { risk } \\
\triangle A \cap A ̈ A ̈ A ̈ A ̈ A ̈ A\end{array}$ & $\begin{array}{l}\text { fract. } \\
\text { AिAAAÄ }\end{array}$ & $\begin{array}{c}\text { risk } \\
\text { ÄÄÄÄÄÄẢ }\end{array}$ & $\begin{array}{l}\text { fract. } \\
\triangle A ̈ A ̈ A ̈ A ̈ A\end{array}$ & $\begin{array}{c}\text { risk } \\
\triangle A A A A A A A A B A\end{array}$ & $\begin{array}{l}\text { fract. } \\
\text { ÄAAÄÄA }\end{array}$ \\
\hline$A C-227$ & $0.000 E+00$ & 0.0000 & $0.000 E+00$ & 0.0000 & $0.000 E+00$ & 0.0000 & & 0.0000 & $0.000 E+00$ & & & \\
\hline-60 & $O E+00$ & 0.0000 & $\mathrm{E}+00$ & 000 & $\mathrm{E}+00$ & 0.0000 & $10 E+00$ & 000 & $0 E+00$ & 100 & & \\
\hline & .00 & & $+\infty 0$ & & & & +00 & & & & & \\
\hline Eu- & $0.000 E+00$ & 0.0000 & $.000 E+00$ & 0.0000 & $E+00$ & 0.0000 & 00 & 00 & +00 & 00 & & \\
\hline & $E+00$ & 0.0 & +00 & & $+\infty$ & & & & 00 & & & \\
\hline & $E+00$ & & +00 & & 0 & & & & & & & \\
\hline & $0.000 E+00$ & & 30 & & 0 . & 0. & 0. & 0. & jo & 00 & & \\
\hline & $r$ & & +00 & & & & & & & & & \\
\hline & 0 & 0.0 & +00 & & bo & & 0 & & $E+00$ & 0 & & \\
\hline Th-228 & $0.000 E+00$ & 0.0 & $0.000 \mathrm{E}+00$ & 0.0 & 0.0 & 0.0 & 0.0 & 0.0 & $0.000 E+00$ & 0.0000 & & \\
\hline & OOOE+Oח & & $000 E+00$ & & & & & & $0.000 E+00$ & 0.0000 & 5. & 0.000 \\
\hline & & & $+\infty$ & & 0.0 & & & & 0.00 & & & \\
\hline & & 00 & 0 & & & 0. & & & & & & \\
\hline & & & & & & & & & & & & \\
\hline Total & $000 E+00$ & .0000 & $E+00$ & 0.0 & 0. & 0.0000 & 0.000 & 0.0000 & $0.000 E+00$ & 0.00 & $1.740 \mathrm{E}-05$ & 1000 \\
\hline
\end{tabular}

** Sum of water independent ground, inhalation, plant, meat, milk, soil

and water dependent water, fish, plant, meat, milk pathways

\begin{tabular}{|c|c|}
\hline \multirow{2}{*}{\multicolumn{2}{|c|}{$\begin{array}{ll}\text { Attachment } & 9 \\
\text { Originator: S.W. Clark } & \text { Sheet No. } 12 \text { of } 31 \\
\text { Date }\end{array}$}} \\
\hline & \\
\hline M. W. Perrott & Date \\
\hline $0100 F-C A-V 0303$ & No. \\
\hline
\end{tabular}




\section{ATTACHMENT 9}

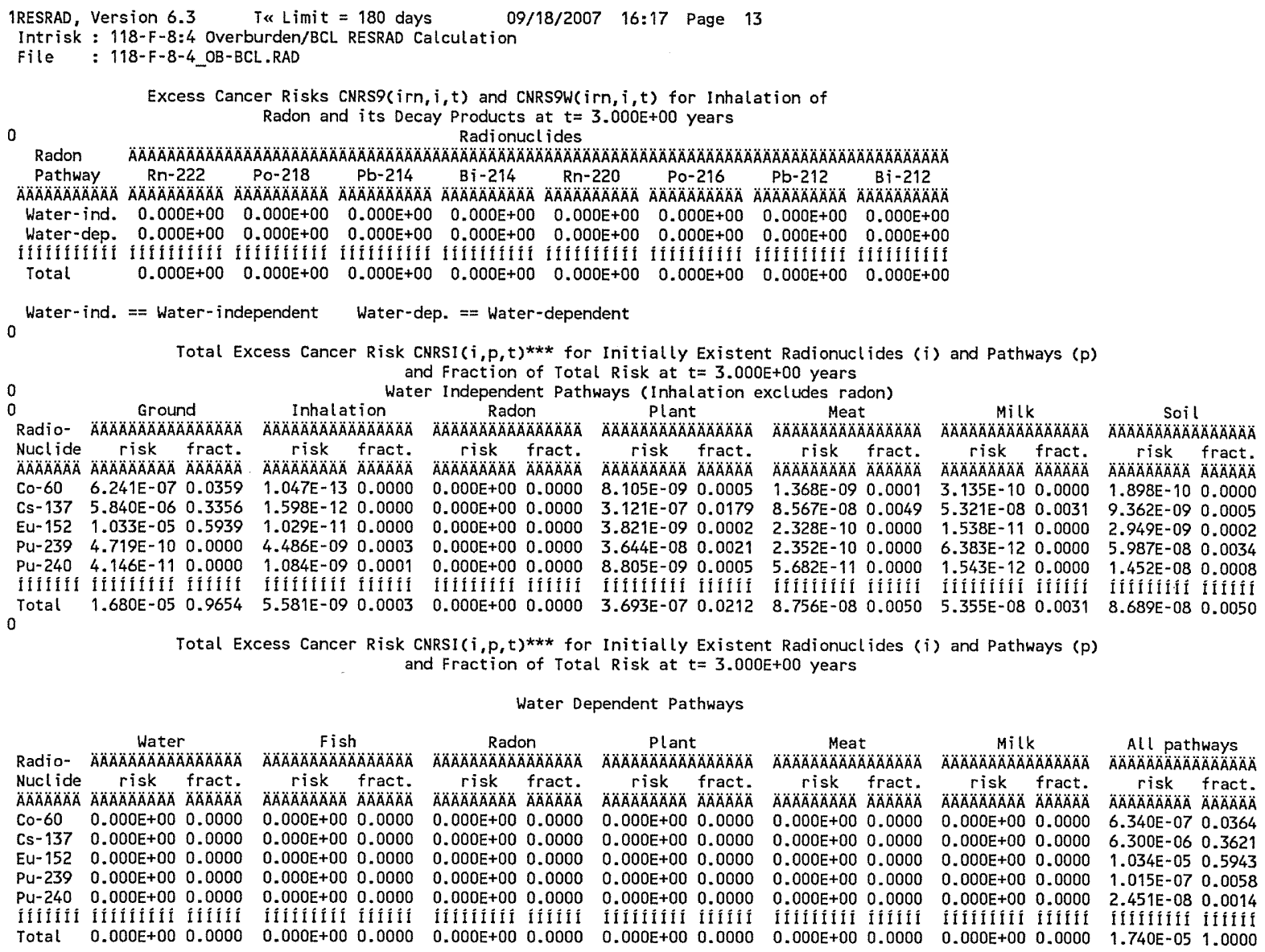

Total Excess Cancer Risk CNRSI $(i, p, t)^{* * *}$ for Initially Existent Radionuclides $(i)$ and Pathways $(p)$ and Fraction of Total Risk at $t=3.000 E+00$ years

Water Dependent Pathways

\begin{tabular}{|c|c|c|c|c|c|c|c|c|c|c|c|c|c|c|}
\hline \multirow{2}{*}{$\begin{array}{l}\text { Radio- } \\
\text { Nucl ide } \\
A A A A A A A A\end{array}$} & \multicolumn{2}{|c|}{$\begin{array}{c}\text { Water } \\
\text { ÄÄÄAÄAAAAAAAOAOAO }\end{array}$} & \multicolumn{2}{|c|}{ 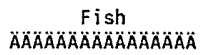 } & \multicolumn{2}{|c|}{$\begin{array}{c}\text { Radon } \\
\text { ÁÄÄAAAAAAAOAOAOAOA }\end{array}$} & \multicolumn{2}{|c|}{ 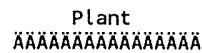 } & \multicolumn{2}{|c|}{$\begin{array}{c}\text { Meat } \\
\text { AAAAAAAAAAOAOAOÄÄÄÄ }\end{array}$} & \multicolumn{2}{|c|}{$\begin{array}{c}M i l k \\
\text { AAAAOAAABAOAOAOAOAOA }\end{array}$} & \multicolumn{2}{|c|}{$\begin{array}{c}\text { All pathways } \\
\text { ACAAAAAAAAAAAAAAA }\end{array}$} \\
\hline & & & risk & & risk & $\begin{array}{l}\text { fract. } \\
\triangle A \cap A ̈ A A A A\end{array}$ & risk & & rick & & risk & & risk & \\
\hline & & & & & & & & & & & & & & \\
\hline & & & & & & & & & & & & & & \\
\hline & & & & & & & & & & & & & & \\
\hline & & & & & & & & & & & & & & \\
\hline & & & & & & & & & & & & & & \\
\hline & & & & & & & & & & & & & & \\
\hline & & & & 00 & No & & Tou & & $U E+00$ & 00 & $300 E+00$ & 0.0000 & $40 E-05$ & \\
\hline
\end{tabular}

***CNRSI $(i, p, t)$ includes contribution from decay daughter radionuclides

$\begin{array}{ll}\text { Attachment } \frac{9}{\text { S.W. Clark }} & \text { Sheet No. } 13 \text { of } 31 \\ \text { Originator: } & \text { Date } \\ \text { Chk'd By } & \text { Date } \\ \text { Calc. No. W. Perrott } & \text { Rev. No. } \quad \text { O100F-CA-V0303 }\end{array}$




\section{ATTACHMENT 9}

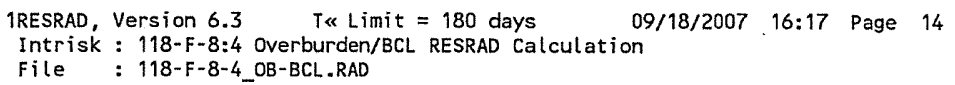

Water - ind. $==$ Water-independent Water-dep. $==$ Water-dependent

\begin{tabular}{|c|c|}
\hline Attachment & $\mathrm{SH}$ \\
\hline Originator: S.W. Clark & Date \\
\hline M. W. Perrott & Date \\
\hline $0100 \mathrm{~F}-\mathrm{CA}-\mathrm{V} 0303$ & No. 0 \\
\hline
\end{tabular}




\section{ATTACHMENT 9}

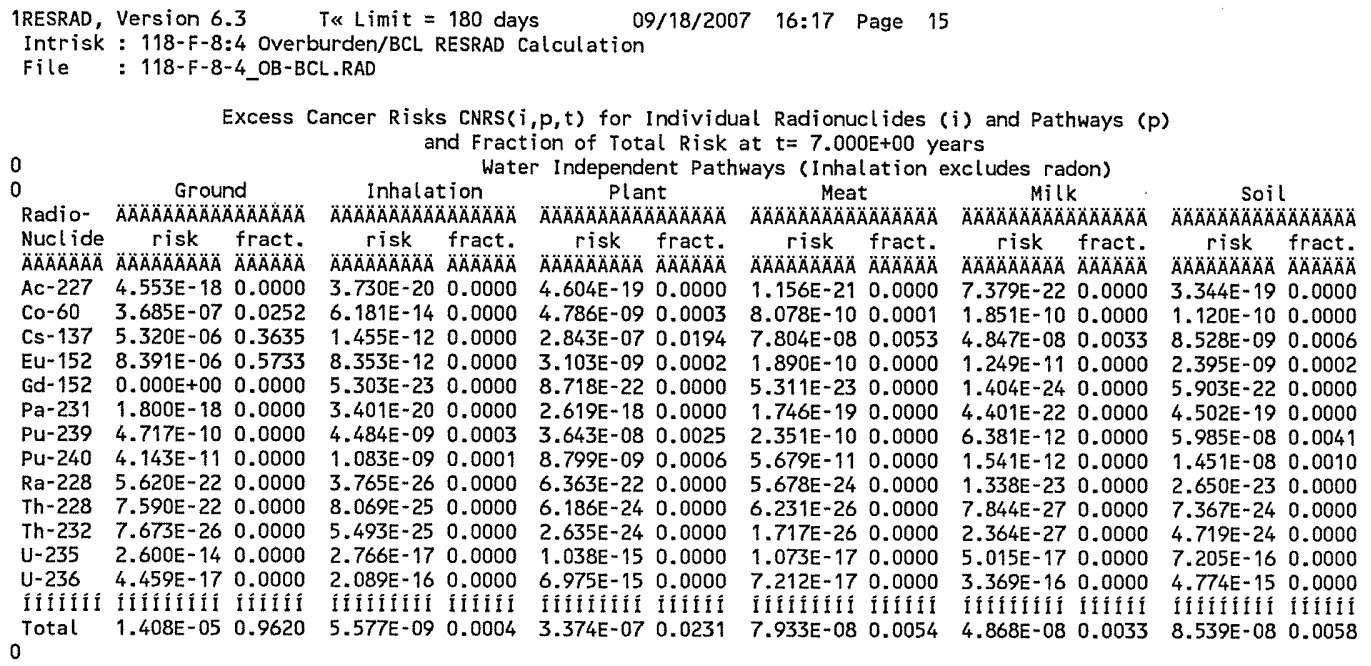

Excess Cancer Risks CNRS $(i, p, t)$ for Individual Radionuclides (i) and Pathways $(p)$ and Fraction of Total Risk at $t=7.000 \mathrm{E}+00$ years

Water Dependent Pathways

\begin{tabular}{|c|c|c|c|c|c|c|c|c|c|c|}
\hline & $\begin{array}{r}\text { Water } \\
\text { ÄAAAAAAAO }\end{array}$ & A A ÄÄÄÄA & $\begin{aligned} & \text { Fish } \\
& \text { ÄAAAOAAAOA }\end{aligned}$ & A & 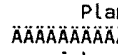 & $\begin{array}{l}n t \\
\ddot{A} A ̈ A ̈ A ̈ A ̈ A ̈ A\end{array}$ & $\begin{array}{r}\text { Mea } \\
\triangle A \cap A ̈ A ̈ A ̈ A ̈ A ̈ A ̈\end{array}$ & t & 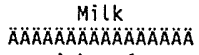 & 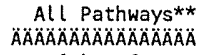 \\
\hline $\begin{array}{l}\text { Nuclide } \\
\text { ÄÄAAAÄÄ }\end{array}$ & $\begin{array}{c}\text { risk } \\
\text { ÄÄÄÄÄÄ }\end{array}$ & $\begin{array}{l}\text { fract. } \\
\text { ÄABAAAA }\end{array}$ & $\begin{array}{c}\text { risk } \\
A A B A O A O A O A ̈\end{array}$ & $\begin{array}{l}\text { fract. } \\
A ̈ A ̈ A ̈ A ̈ A\end{array}$ & $\begin{array}{c}\text { risk } \\
\text { ÄÄÄÄÄÄÄ }\end{array}$ & $\begin{array}{l}\text { fract. } \\
\not A A O A O A A A\end{array}$ & $\begin{array}{c}\text { risk } \\
A A O A A A A A B A\end{array}$ & $\begin{array}{l}\text { fract. } \\
\ddot{A} \ddot{A} \ddot{A} \ddot{A} \ddot{A}\end{array}$ & 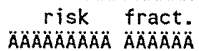 & 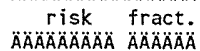 \\
\hline$c-227$ & $0.000 \mathrm{E}+00$ & 0.0000 & & & $0.000 \mathrm{E}+00$ & & $0.000 E+00$ & & $0.000 E+000$ & \\
\hline & $0.000 E+00$ & 0.0000 & $000 E+00$ & 0.0000 & $0.000 E+00$ & 0.0000 & $0.000 E+00$ & 0.0000 & $0.000 E+00 \quad 0.0000$ & -070.0256 \\
\hline & $+\infty$ & & $E+00$ & & $+\infty$ & & $O E+00$ & 0. & DOE +000 & 060 \\
\hline & 0.0 & 0.0 & $E+00$ & 0.0 & & & $=00$ & 0.0000 & $0.000 E+00 \quad 0.0000$ & זונדות \\
\hline 152 & $0.000 E+00$ & 0.0000 & $.000 E+00$ & 0.0000 & $0.000 E+00$ & 0.0000 & $0.000 E+00$ & 0.0000 & $O E+00 \quad 0$ & 000 \\
\hline & $0.000 E+00$ & 0.0 & $D E+00$ & 0. & $+\infty$ & & $+\infty 0$ & & 1000 & 000 \\
\hline & 0.0 & 0.0 & $E+00$ & 0.0000 & 0.0 & $0.6+2$ & 0.0 & 0.0 & $0.000 E+000$. & 1. \\
\hline & $0.000 E+00$ & & $0 E+00$ & & $+\infty$ & 0.0 & $0.000 E+00$ & 0.0000 & $0.000 E+000$. & 080. \\
\hline & $+\infty$ & 0.0 & $E+00$ & 0.0 & .00 & & 00 & & 000 & \\
\hline & $E+\infty$ & 0.0 & $000 E+00$ & 0.0000 & $+\infty 0$ & 0 & $0.000 E+00$ & 00 & $0.000 E+000.0$ & \\
\hline Th- & +00 & 0.0 & $0.000 E+00$ & 0.0000 & $0.000 E+00$ & 0.0 & $0.000 E+00$ & 0.0 & $0.000 E+00 \quad 0.0000$ & -240 \\
\hline & $000 \mathrm{E}+00$ & & $0 E+00$ & & & & & & & \\
\hline & & & & & & & & & & \\
\hline & & & & & & & İíílííli & & IIIIIIIII Î́ & 1111111 \\
\hline tal & $.000 E+00$ & 0.0000 & $.000 E+00$ & 0.0000 & $0.000 E+00$ & 0.0000 & $0.000 E+00$ & 0.0000 & $0.000 E+00 \quad 0.0000$ & E-05 $\quad 1.0000$ \\
\hline
\end{tabular}

** Sum of water independent ground, inhalation, plant, meat, milk, soil

and water dependent water, fish, plant, meat, milk pathways

\begin{tabular}{|c|c|}
\hline Attachment & Sheet No. 1 \\
\hline Originator: S.W. Clark & Date \\
\hline M. W. Perrott & Date \\
\hline $0100 \mathrm{~F}-\mathrm{CA}-\mathrm{V} 0303$ & Rev. No. \\
\hline
\end{tabular}




\section{ATTACHMENT 9}

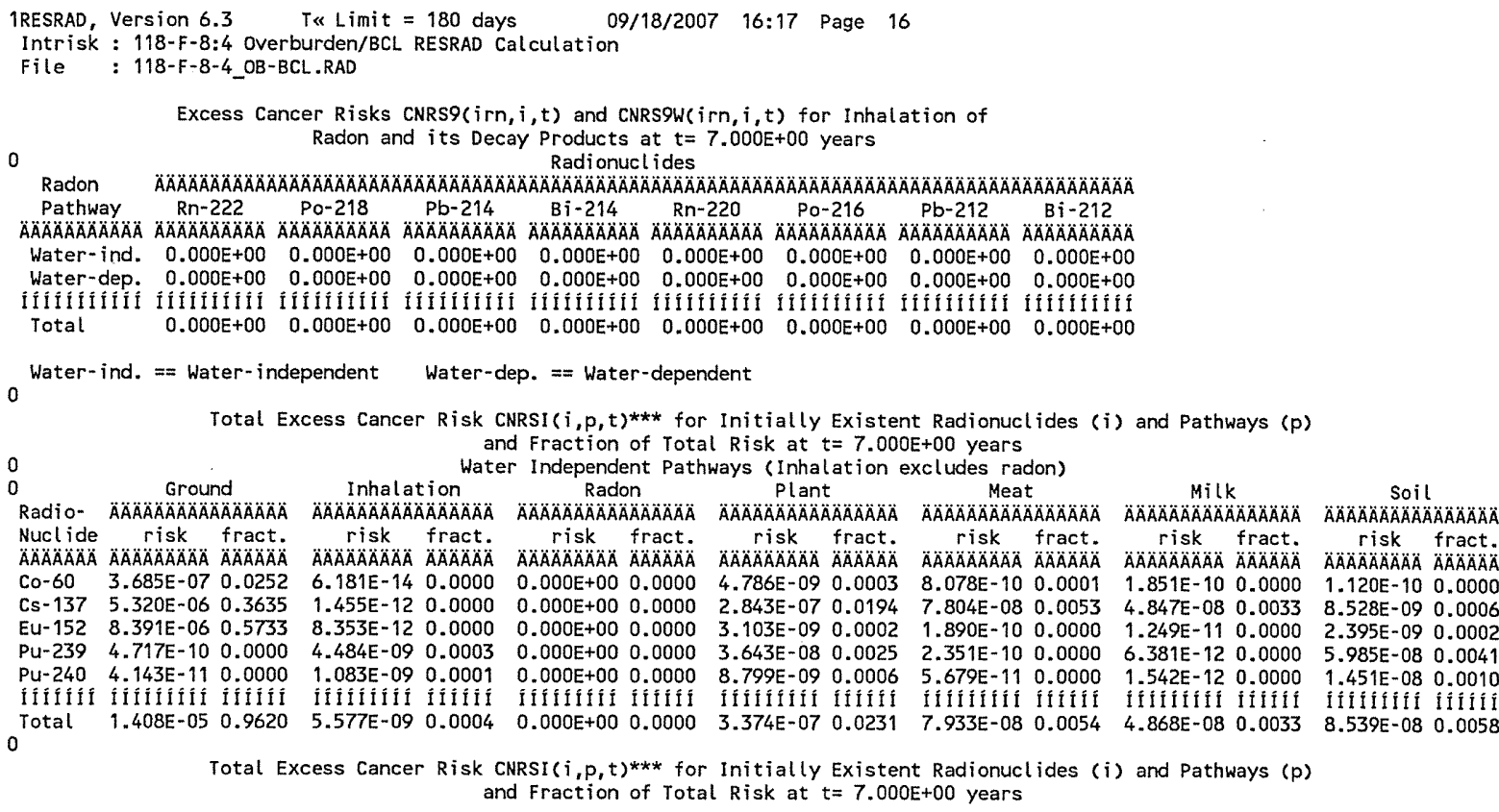

Water Dependent Pathways

\begin{tabular}{|c|c|c|c|c|c|c|c|c|c|c|c|c|c|}
\hline \multirow{2}{*}{$\begin{array}{l}\text { Radio- } \\
\text { Nucl ide } \\
\text { ÄÄÄÄÄÄÄA }\end{array}$} & \multicolumn{2}{|c|}{$\begin{array}{c}\text { Water } \\
\text { ÄÄÄÄÄÄÄÄÄÄÄÄĂ }\end{array}$} & \multicolumn{2}{|c|}{ 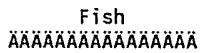 } & \multicolumn{2}{|c|}{ 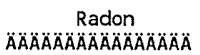 } & \multicolumn{2}{|c|}{ 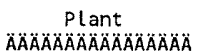 } & \multicolumn{2}{|c|}{$\begin{array}{c}\text { Meat } \\
\text { AAAAAAOAAOAAAAAAAOAOA }\end{array}$} & \multicolumn{2}{|c|}{$\begin{array}{c}\text { Milk } \\
\text { AOAOAAAAAAAOAOAOA }\end{array}$} & $\begin{array}{c}\text { All pathways } \\
\text { AAAAAAAAOAAAAOAOAOAAA }\end{array}$ \\
\hline & $\begin{array}{c}\text { risk } \\
\dddot{\text { A }} \text { ÄÄÄÄÄÄ }\end{array}$ & $\begin{array}{l}\text { fract. } \\
\text { ÄÄÄÄÄ̈ }\end{array}$ & 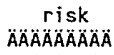 & $\begin{array}{l}\text { fract. } \\
\text { ÄÄÄÄÄA }\end{array}$ & $\begin{array}{c}\text { risk } \\
\text { ĀAAAAAAAAA }\end{array}$ & $\begin{array}{l}\text { fract. } \\
\text { ÄÄÄÄÄÄ }\end{array}$ & $\begin{array}{c}\text { risk } \\
\text { ÄAAÄÄÄÄÄ }\end{array}$ & $\begin{array}{l}\text { fract. } \\
\text { ÄÄÄÄÄA }\end{array}$ & $\begin{array}{c}\text { risk } \\
A A A A A A A B A\end{array}$ & $\begin{array}{l}\text { fract. } \\
\text { ÄÄÄÄÄA }\end{array}$ & $\begin{array}{c}\text { risk } \\
\text { ÄÄÄÄÄÄÄÄA }\end{array}$ & $\begin{array}{l}\text { fract. } \\
\text { ÄÄÄÄÄ }\end{array}$ & $\begin{array}{r}\text { risk fract. } \\
\text { AAAAAAAAAO AAAAAAA }\end{array}$ \\
\hline Co- 60 & $0.000 E+00$ & 0.0000 & $0.000 E+00$ & 0.0000 & $0.000 \mathrm{E}+00$ & 0.0000 & $0.000 E+00$ & 0.0000 & $0.000 E+00$ & 0.0000 & $0.000 \mathrm{E}+00$ & 0.0000 & $3.744 E-070.0256$ \\
\hline 137 & $0.000 E+00$ & 0.0000 & $0.000 E+00$ & 0.0000 & $0.000 E+00$ & 0.0000 & $0.000 E+00$ & 0.0000 & $0.000 E+00$ & 0.0000 & $0.000 E+00$ & 0.0000 & 3921 \\
\hline Eu-152 & $0.000 E+00$ & & $0.000 E+00$ & 0.0000 & $0.000 E+00$ & 0.0000 & $0.000 E+00$ & 0.0000 & $0.000 E+00$ & 0.0000 & $0.000 E+00$ & 0.0000 & .5737 \\
\hline 239 & $0.000 \mathrm{E}+00$ & 0.0000 & $0.000 \mathrm{E}+00$ & 0.0000 & $0.000 \mathrm{E}+00$ & 0.0000 & $0.000 E+00$ & 0.0000 & $0.000 E+00$ & 0.0000 & $0.000 E+00$ & 0.0000 & $15 E-07 \quad 0.0069$ \\
\hline 240 & $0.000 E+00$ & 0.0000 & $0.000 E+00$ & 0.0000 & $0.000 E+00$ & 0.0000 & $0.000 E+00$ & 0.0000 & $0.000 E+00$ & 0.0000 & $0.000 E+00$ & 0.0000 & 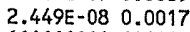 \\
\hline & iffit & & fifif & fliffi & IIIfífifî & Iffifi & ffífifiti & Iffifí & fî́îlîtíl & IIIIIII & IIIHIIIII & IIIIII & IIIIII IIIIfi \\
\hline Total & $0.000 E+00$ & 0.0000 & $0.000 E+00$ & 0.0000 & $0.000 E+00$ & 0.0000 & $0.000 E+00$ & 0.0000 & $0.000 E+00$ & 0.0000 & $0.000 \mathrm{E}+00$ & 0.0000 & $1.464 \mathrm{E}-05 \quad 1.0000$ \\
\hline
\end{tabular}

$* * * \operatorname{CNRSI}(i, p, t)$ includes contribution from decay daughter radionuclides

$\begin{array}{ll}\text { Attachment } \frac{9}{\text { S.W. Clark }} & \text { Sheet No. } 16 \text { of } 31 \\ \text { Originator: } & \text { Sate } \\ \text { Chk'd By } \frac{\text { M. W. Perrott }}{\text { Date }} \\ \text { Calc. No. } \quad \text { O100F-CA-V0303 Rev. No. _ o }\end{array}$




\section{ATTACHMENT 9}

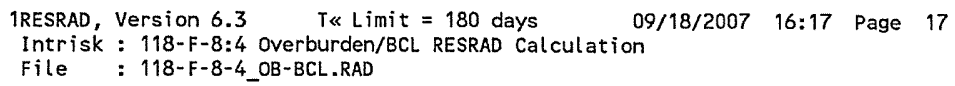

Water-ind. = Water-independent Water-dep. $==$ Water-dependent

\begin{tabular}{|c|c|}
\hline Attachment & Sheet No. 17 of 31 \\
\hline Originator: S.W. Clark & Date \\
\hline M.W. Perrott & Date \\
\hline 0100F-CA-V0303 & Rev \\
\hline
\end{tabular}


CVP-2007-00004

Rev. 0

\section{ATTACHMENT 9}

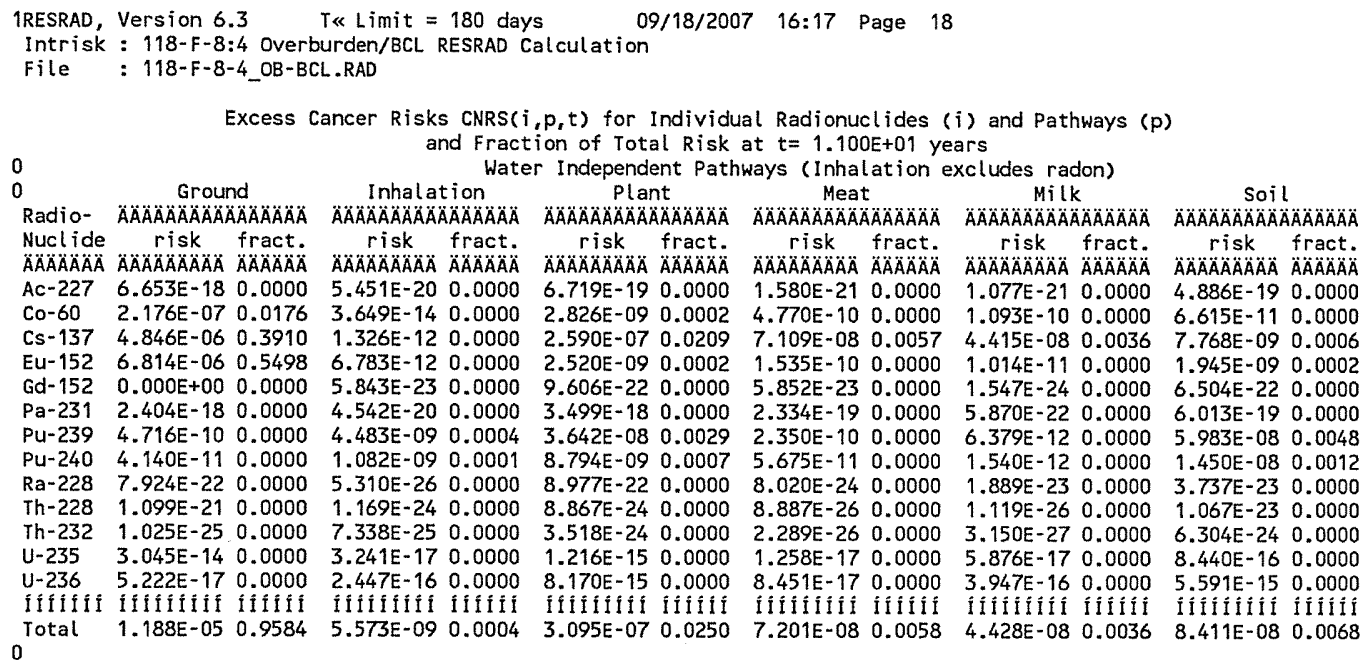

\begin{tabular}{|c|c|}
\hline Attachment & Sheet No. \\
\hline Originator: S.W. Clark & Date \\
\hline M. W. Perrott & Date \\
\hline $0100 F-C A-V 0303$ & Rev. No. \\
\hline
\end{tabular}




\section{ATTACHMENT 9}

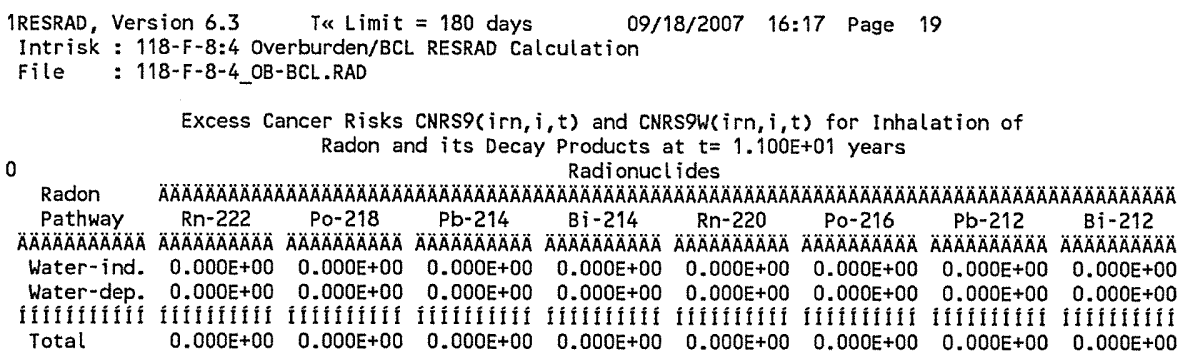

Total Excess Cancer Risk CNRSI $i, p, t)^{* * *}$ for Initially Existent Radionuclides $(i)$ and Pathways $(p)$ and Fraction of Total Risk at $t=1.100 E+01$ years

Water Dependent Pathways

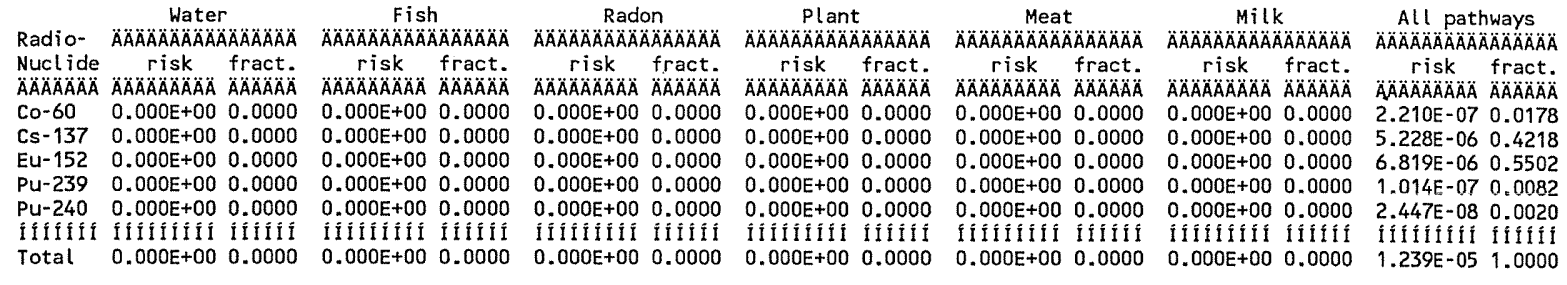

*** $\operatorname{CNRSI}(i, p, t)$ includes contribution from decay daughter radionuclides

\begin{tabular}{|c|c|}
\hline Attachment & Sheet No. 19 \\
\hline Originator: S.W. Clark & Date \\
\hline Chk'd By M. W. Perrott & Date \\
\hline Calc. No. $\quad 0100 \mathrm{~F}-\mathrm{CA}-\mathrm{V} 0303$ & Rev. No. $\quad 0$ \\
\hline
\end{tabular}




\section{ATTACHMENT 9}

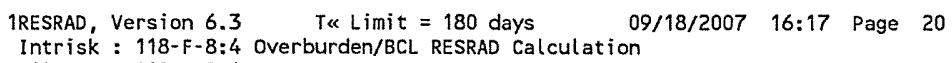

$\begin{array}{ll}\text { Attachment } \frac{9}{\text { S.W. Clark }} & \text { Sheet No. } 20 \text { of } \underline{31} \\ \text { Originator: } & \text { Date } \\ \text { Chk'd By } & \text { Date } \\ \text { Calc. No. W. Perrott } & \text { Rev. No. } 0\end{array}$




\section{ATTACHMENT 9}

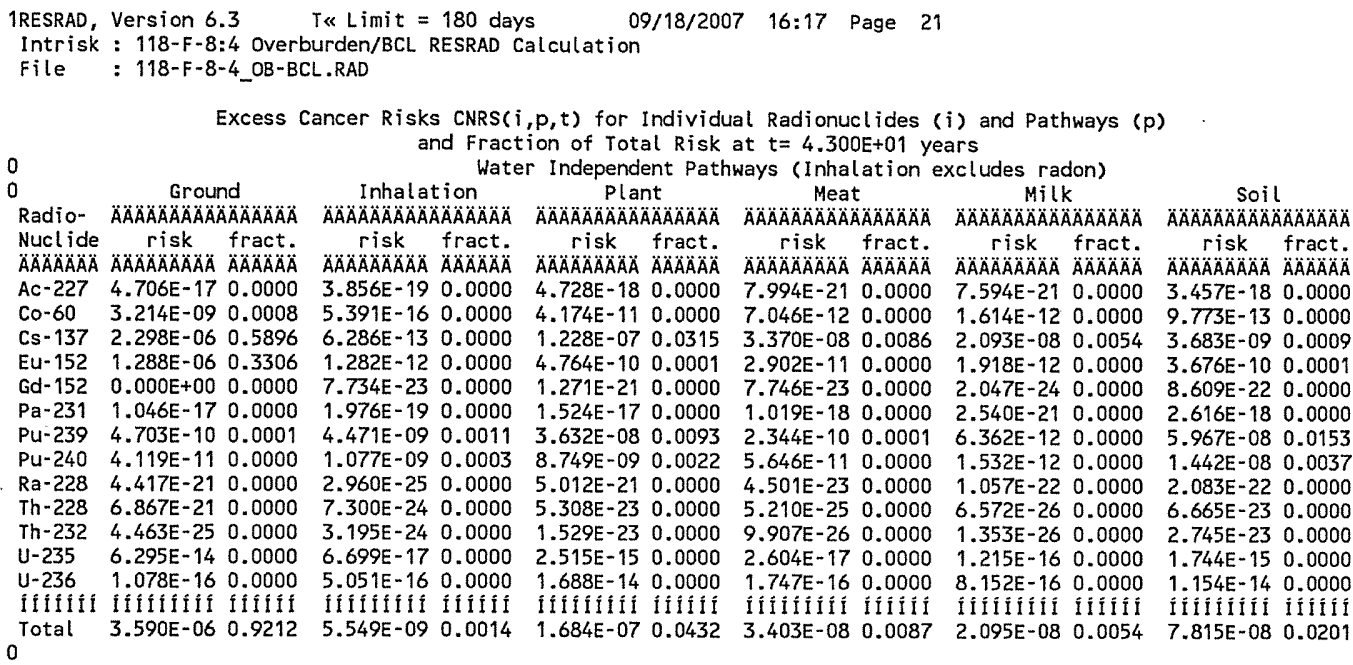

Excess Cancer Risks CNRS $(i, p, t)$ for Individual Radionuclides (i) and Pathways $(p)$ and Fraction of Total Risk at $t=4.300 E+01$ years

Water Dependent Pathways

\begin{tabular}{|c|c|c|c|c|c|c|c|c|c|c|c|c|}
\hline \multirow[b]{2}{*}{$\begin{array}{l}\text { Nuclide } \\
\text { ÄÄÄÄAOAOA }\end{array}$} & \multicolumn{2}{|c|}{ Water } & \multicolumn{2}{|c|}{$\begin{array}{c}F i s h \\
\triangle A \cap A O A O A O A A O A O A O A O A O A O A\end{array}$} & \multicolumn{2}{|c|}{ 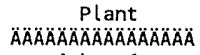 } & \multicolumn{2}{|c|}{ 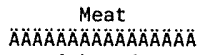 } & \multicolumn{2}{|c|}{$\begin{array}{c}\text { Milk } \\
\text { ÄAAAAAAAAAAAAOAOAOA }\end{array}$} & \multicolumn{2}{|c|}{ 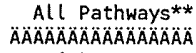 } \\
\hline & $\begin{array}{c}\text { risk } \\
A A B A A A A A O A\end{array}$ & $\begin{array}{l}\text { fract. } \\
\triangle A A B A A A B A\end{array}$ & $\begin{array}{c}\text { risk } \\
\triangle A O A A A A A O A\end{array}$ & $\begin{array}{l}\text { fract. } \\
\dddot{A} A \ddot{A} A \ddot{A} A \ddot{A}\end{array}$ & $\begin{array}{c}\text { risk } \\
\text { AAAAAAAAOA }\end{array}$ & $\begin{array}{l}\text { fract. } \\
A B A A B A B A\end{array}$ & $\begin{array}{c}\text { risk } \\
\text { AAAAAAAAAA }\end{array}$ & $\begin{array}{l}\text { fract. } \\
\not A \not ̈ A ̈ A ̈ A ̈\end{array}$ & $\begin{array}{r}\text { risk } \\
\triangle A \cap A O A O A O A O\end{array}$ & 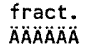 & $\begin{array}{c}\text { risk } \\
\text { AAAAAOAAAAOAB }\end{array}$ & $\begin{array}{l}\text { fract. } \\
\triangle A A B A B A B\end{array}$ \\
\hline$A C-227$ & $0.000 \mathrm{E}+00$ & 0.0000 & $0.000 E+00$ & 0.0000 & & 0.0000 & $0.000 \mathrm{E}+00$ & & & & & \\
\hline & $0.000 E+00$ & 0.0000 & $0.000 E+00$ & 0.0000 & $0.000 E+00$ & 0.0000 & $0.000 E+00$ & 0.0000 & $0.000 E+00$ & 0.0000 & $=-09$ & .0008 \\
\hline & $0.000 E+00$ & 0.0000 & $0 E+00$ & & & & & & & & & 31 \\
\hline & 0. & & 0.0 & & & 0.0000 & & 0.0000 & 0.000 & .0000 & 06 & \\
\hline & $0.000 \mathrm{E}+00$ & & 0.0 & & $0.000 E+00$ & 0.0000 & $0.000 E+00$ & 0.0000 & $0.000 E+00$ & 300 & & 000 \\
\hline & 0.000 & 0.0000 & $+\infty$ & & 0. & 00 & $+\infty$ & 00 & -00 & & & 000 \\
\hline & 0. & 0.00 & 0.08 & 0. & 0. & 0. & 0 & Do & 00 & bo & & \\
\hline & & & & & +00 & 0.0000 & $0.000 E+00$ & 0.0000 & 0. & 000 & .08 & 0.0062 \\
\hline 228 & $0.000 \mathrm{E}+00$ & 0. & $E+00$ & & 0. & & 0 & & & & & \\
\hline & $=00$ & & $0.000 E+00$ & & 0.0 & & $+\infty$ & 00 & $30 E+00$ & & & \\
\hline$h-232$ & $0.000 E+00$ & & $0.000 \mathrm{E}+00$ & 0.0 & $0.000 E+00$ & 0.0000 & $0.000 E+00$ & 0. & $0.000 \mathrm{E}+00$ & 100 & & 0.0000 \\
\hline & & & $0.000 \mathrm{E}+0$ & & & & & & & & & \\
\hline & & & & & & & & & & & & \\
\hline & & & If fiffi & & & & iffififi & & Iftififit & & IIIIfIIIII & ilififi \\
\hline Total & $0.000 E+00$ & 0.0000 & $0.000 \mathrm{E}+00$ & 0.0000 & $0.000 E+00$ & 0.0000 & $0.000 E+00$ & 0.0000 & $0.000 E+00$ & 0.0000 & $3.897 \mathrm{E}-06$ & 1.0000 \\
\hline
\end{tabular}

** Sum of water independent ground, inhalation, plant, meat, milk, soil and water dependent water, fish, plant, meat, milk pathways

$\begin{array}{ll}\text { Attachment } \frac{9}{\text { S.W. Clark }} & \text { Sheet No. } 21 \text { of } 31 \\ \text { Originator: } & \text { Date } \\ \text { Chk'd By } \frac{\text { M.W. Perrott }}{\text { Date }} & \\ \text { Calc. No. } & \text { Rev. No. } 0\end{array}$




\section{ATTACHMENT 9}

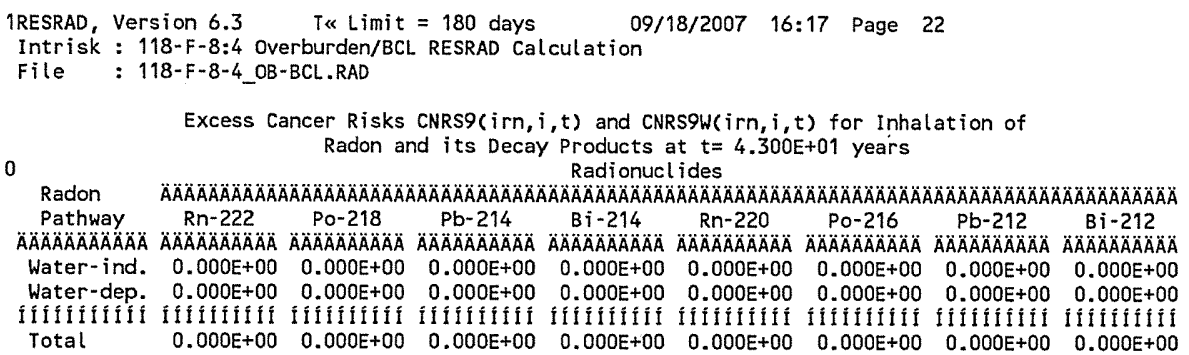

Total Excess Cancer Risk CNRSI $(i, p, t) * * *$ for Initially Existent Radionuclides (i) and Pathways ( $p$ ) and Fraction of Total Risk at $t=4.300 E+01$ years

Water Dependent Pathways

\begin{tabular}{|c|c|c|c|c|c|c|c|c|c|c|c|c|c|c|}
\hline & 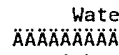 & $A \cap A B A \cap A ̈ A ̈$ & $\begin{array}{r}F \ddot{I} \\
\triangle A \cap A ̈ A ̈ A ̈ A ̈ A ̈\end{array}$ & 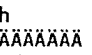 & 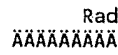 & $\triangle A O A O A B A A A$ & $\begin{array}{r}\text { Plan } \\
\triangle A ̈ A ̈ A O A B A A O A\end{array}$ & $\triangle A A O A A A O A O A$ & $\begin{array}{r}\text { Mea } \\
A A A B A A A ́\end{array}$ & t & $\begin{array}{r}M i L \\
A B A A A ̈ A B A\end{array}$ & $A \cap A A A B$ & $\begin{array}{r}\text { All pat } \\
A \cap A B A A B A A\end{array}$ & $\begin{array}{l}\text { vays } \\
A \mathscr{A} A \cap A B A O A\end{array}$ \\
\hline$\triangle A A A A A B$ & risk & $\begin{array}{l}\text { fract. } \\
\text { ÄÄÄÄÄA }\end{array}$ & risk & fract. & risk & $\begin{array}{l}\text { fract. } \\
\qquad A \cap A B A A B\end{array}$ & risk & $\begin{array}{l}\text { fract. } \\
\text { ÄÄABAAA }\end{array}$ & $\begin{array}{l}\text { risk } \\
\text { ÄÄÄÄÄÄA }\end{array}$ & $\begin{array}{l}\text { fract. } \\
\text { ÄÄÄAAAA }\end{array}$ & $\begin{array}{l}\text { risk } \\
\text { ÄÄÄAAAAA }\end{array}$ & t. & risk & \\
\hline & .00 & & 0 & & & & & & & & & & & \\
\hline & & & & & & & & & & & & & & \\
\hline & & & & & & & & & & & & & & \\
\hline & & & & & & & 00 & & & & & & & \\
\hline & & & & & & & & & & & & & & \\
\hline & & & & & & & & & & & & & & \\
\hline & & 00 & $E+00-2$ & & & & OnOF+0 & & 00 & & $+\infty$ & & & \\
\hline
\end{tabular}

***CNRSI $(i, p, t)$ includes contribution from decay daughter radionuclides

\begin{tabular}{|c|c|}
\hline Attachment & Sheet No. 22 of 31 \\
\hline Originator: S.W. Clark & Date \\
\hline M. W. Perrott & Date \\
\hline $0100 F-C A-V 0303$ & Rev. No. 0 \\
\hline
\end{tabular}




\section{ATTACHMENT 9}

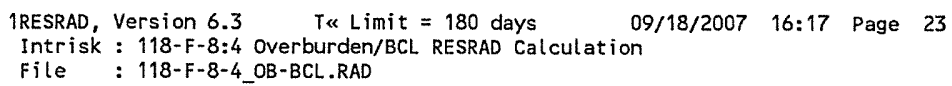

Water-ind. == Water-independent Water-dep. $==$ Water-dependent

\begin{tabular}{|c|c|}
\hline Attachment & Sheet No. 23 \\
\hline Originator: S.W. Clark & _Date \\
\hline M. W. Perrott & Date \\
\hline Calc. No. $\quad 0100 F-C A-V 0303$ & Rev. No. \\
\hline
\end{tabular}




\section{ATTACHMENT 9}

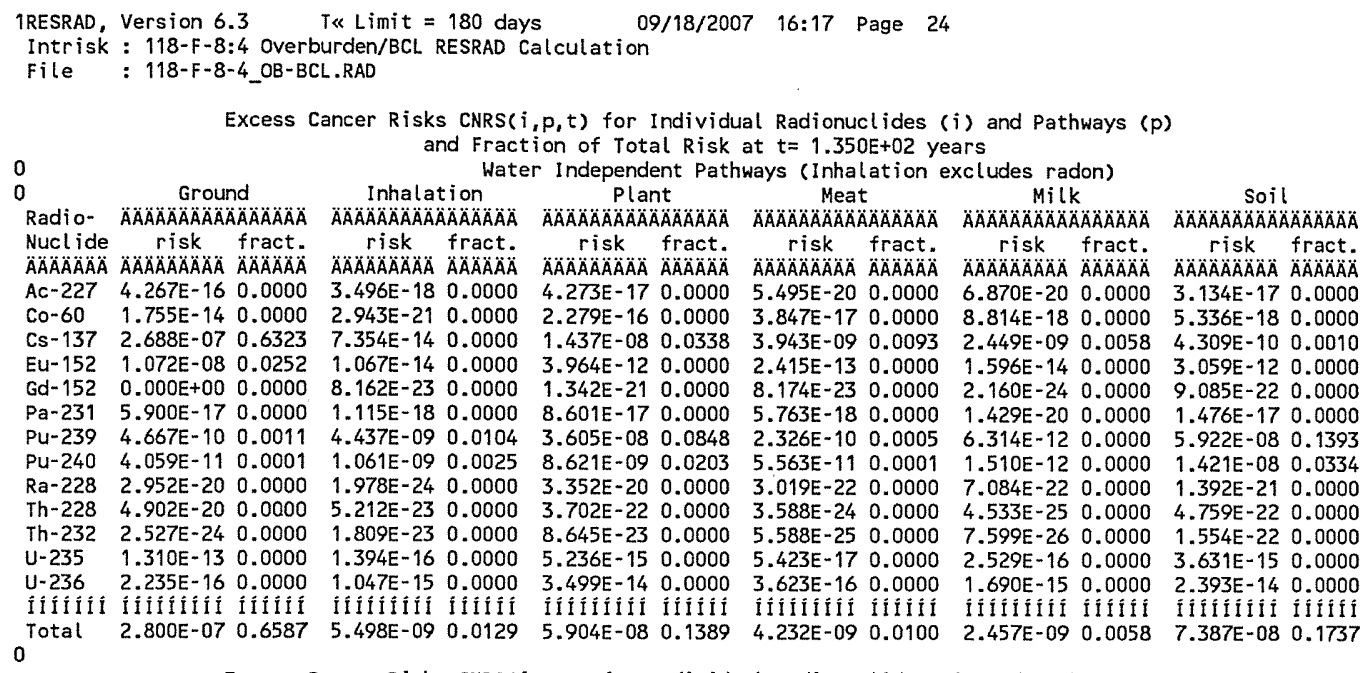

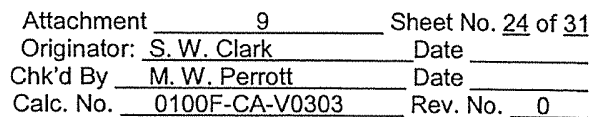




\section{ATTACHMENT 9}

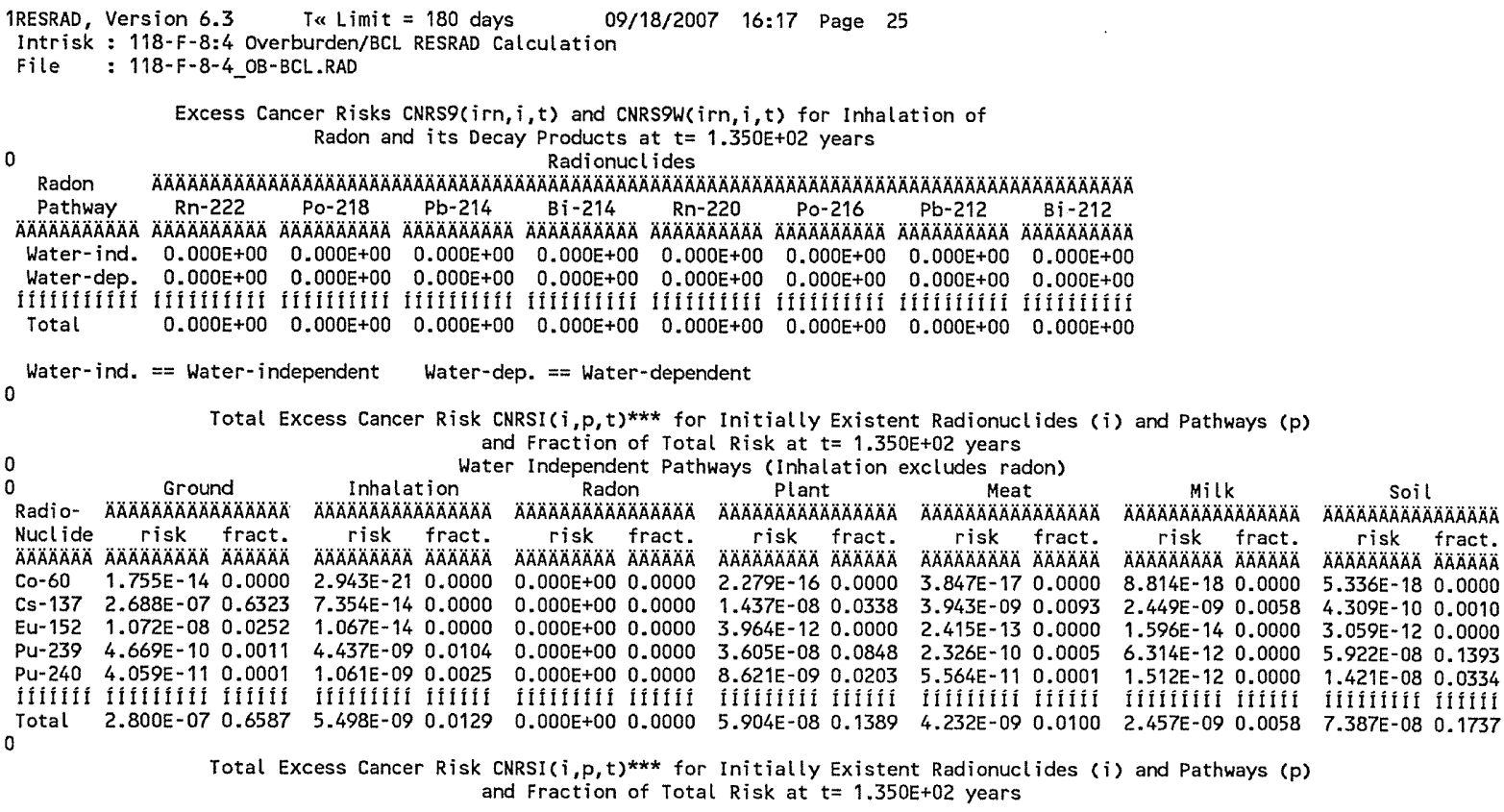

Water Dependent Pathways

\begin{tabular}{|c|c|c|c|c|c|c|c|c|c|c|c|c|c|c|}
\hline \multirow{2}{*}{$\begin{array}{l}\text { Radio- } \\
\text { Nucl ide } \\
\text { ÄÄÄÄÄÄ }\end{array}$} & \multicolumn{2}{|c|}{$\begin{array}{c}\text { Water } \\
\text { ÄÄÄÄÄÄÄÄÄÄÄÄÄÄA }\end{array}$} & \multicolumn{2}{|c|}{ 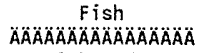 } & \multicolumn{2}{|c|}{$\begin{array}{c}\text { Radon } \\
\text { ÄÄÄÄÄÄÄÄÄÄÄÄÄÄA }\end{array}$} & \multicolumn{2}{|c|}{ 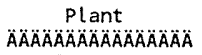 } & \multicolumn{2}{|c|}{$\begin{array}{c}\text { Meat } \\
\text { AAAOAAOAAAAOAAABA }\end{array}$} & \multicolumn{2}{|c|}{ 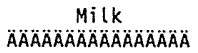 } & \multicolumn{2}{|c|}{$\begin{array}{c}\text { All pathways } \\
\text { AAAAAAAAAAAAAAAAAA }\end{array}$} \\
\hline & 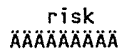 & $\begin{array}{l}\text { fract. } \\
\triangle A A O A O A B A\end{array}$ & $\begin{array}{c}\text { risk } \\
\text { ÄÄÄÄAOAOAOAB }\end{array}$ & $\begin{array}{l}\text { fract. } \\
\ddot{A} A \ddot{A} \ddot{A} \ddot{A} \ddot{A}\end{array}$ & $\begin{array}{c}\text { risk } \\
\triangle A A A B A B A A A\end{array}$ & $\begin{array}{l}\text { fract. } \\
\qquad A A A B A B A\end{array}$ & $\begin{array}{c}\text { risk } \\
\dddot{A} A \bar{A} \triangle A B A B A B A\end{array}$ & $\begin{array}{l}\text { fract. } \\
\text { ÄÄÄÄÄÄA }\end{array}$ & $\begin{array}{c}r i s k \\
\text { A AAAAAAAAA }\end{array}$ & $\begin{array}{l}\text { fract. } \\
\text { AAAAAA }\end{array}$ & $\begin{array}{c}\text { risk } \\
\text { A AAAAAAAB }\end{array}$ & $\begin{array}{l}\text { fract. } \\
\text { ÄÄÄÄA }\end{array}$ & $\begin{array}{c}\text { risk } \\
\text { ÄAAOAOABA }\end{array}$ & fract. \\
\hline Co-60 & $0.000 E+00$ & 0.0000 & $0.000 E+00$ & 0.0000 & $0.000 E+00$ & 0.0000 & $0.000 E+00$ & 0.0000 & $0.000 E+00$ & 0.0000 & $0.000 \mathrm{E}+00$ & 0.0000 & $1.783 E-14$ & .0000 \\
\hline Cs -137 & $.000 E+00$ & 0.0000 & $0.000 E+00$ & 0.0000 & $0.000 \mathrm{E}+00$ & 0.0000 & $0.000 E+00$ & 0.0000 & $0.000 E+00$ & 0.0000 & $0.000 E+00$ & 0.0000 & $2.900 E-07$ & 0.6822 \\
\hline Eu- 152 & $0.000 E+00$ & 0.0000 & $0.000 \mathrm{E}+00$ & 0.0000 & $0.000 E+00$ & 0.0000 & $0.000 E+00$ & 0000 & $0.000 E+00$ & 0.0000 & $0.000 E+00$ & .0000 & 1.073 & 025 \\
\hline Pu-239 & $0.000 E+00$ & 0.0000 & $0.000 E+00$ & 0.0000 & $0.000 E+00$ & 0.0000 & $0.000 E+00$ & 0.0000 & $0.000 E+00$ & 0.0000 & $0.000 E+00$ & 0.0000 & $04 \mathrm{E}$ & 2362 \\
\hline & & & 0 & & $0.000 E+0$ & 0.0000 & $0.000 \mathrm{E}+00$ & 0.0000 & $0.000 E+00$ & 0.0000 & $0.000 E+00$ & 0.0000 & & 556 \\
\hline & 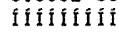 & II Í & & & Ii & if i & $\hat{I} \hat{I}$ & & & & & & & \\
\hline Total & DOE +00 & 0.0000 & $0.000 E+00$ & 0.0000 & $0.000 E+00$ & 0.0000 & $0.000 E+00$ & 0.0000 & $0.000 E+00$ & 0.0000 & $0.000 E+00$ & 0.0000 & 4.251E-07 & 1.0000 \\
\hline
\end{tabular}

***CNRSI $(i, p, t)$ includes contribution from decay daughter radionuclides

\begin{tabular}{|c|c|}
\hline Attachment & She \\
\hline Originator: S.W. Clark & Dat \\
\hline M. W. Perrott & Date \\
\hline $0100 F-C A-V 0303$ & Rev. No. 0 \\
\hline
\end{tabular}


CVP-2007-00004

Rev. 0

\section{ATTACHMENT 9}

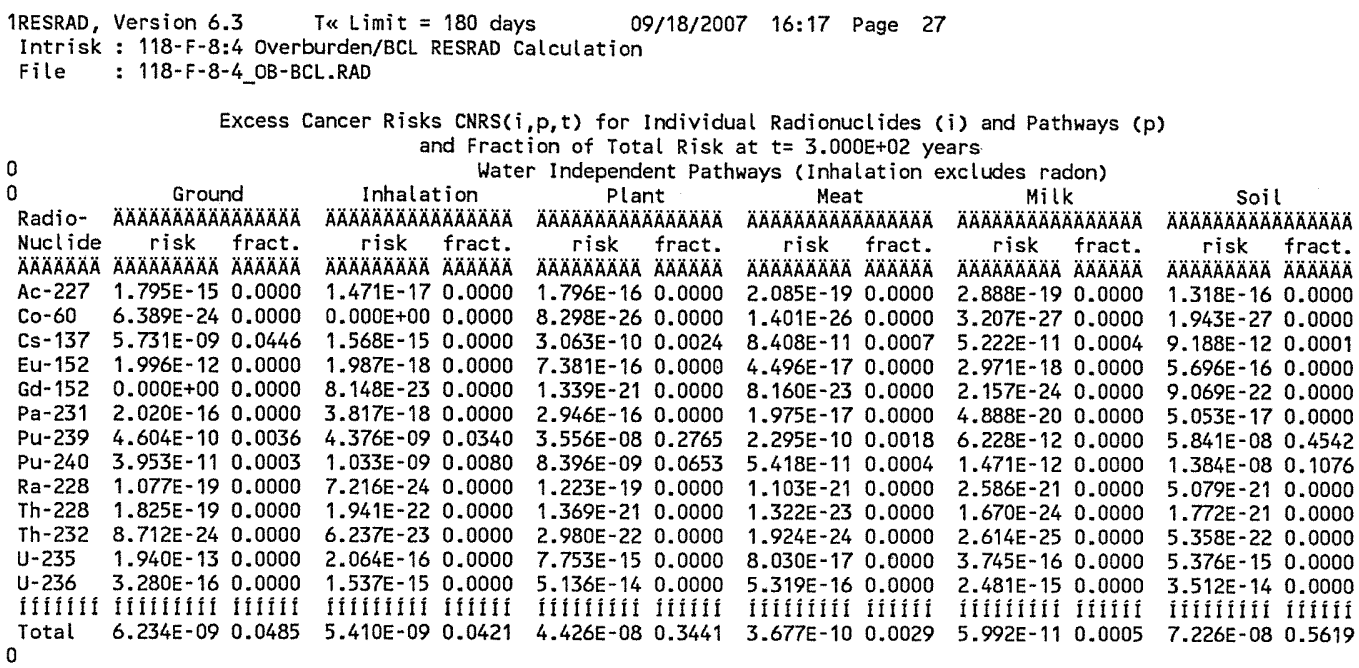

Excess Cancer Risks CNRS( $i, p, t)$ for Individual Radionuclides (i) and Pathways $(p)$ and Fraction of Total Risk at $t=3.000 E+02$ years

Water Dependent Pathways

\begin{tabular}{|c|c|c|c|c|c|c|c|c|c|c|c|c|}
\hline \multirow{2}{*}{$\begin{array}{l}\text { Radio- } \\
\text { Nucl lide } \\
\ddot{A} \ddot{A} \ddot{A} \ddot{A} \ddot{A} A \ddot{A}\end{array}$} & \multicolumn{2}{|c|}{$\begin{array}{c}\text { Water } \\
\text { AAAOAOAOAOAOAOÄÄÄÄ }\end{array}$} & \multicolumn{2}{|c|}{$\begin{array}{c}\text { Fish } \\
\triangle A O A A O A O A B A O A B A O A O A O A\end{array}$} & \multicolumn{2}{|c|}{$\begin{array}{c}\text { Plant } \\
\text { AOAOAOAAOAAOAOAOAOAOA }\end{array}$} & \multicolumn{2}{|c|}{ 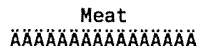 } & \multicolumn{2}{|c|}{$\begin{array}{c}\text { Milk } \\
\text { AAAAAAAAOAOAOAOA }\end{array}$} & \multicolumn{2}{|c|}{$\begin{array}{l}\text { All Pathways** } \\
A A B A A A A B A B A B A A B A\end{array}$} \\
\hline & $\begin{array}{c}\text { risk } \\
\text { AिAAAAAAA }\end{array}$ & 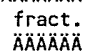 & $\begin{array}{c}\text { risk } \\
\text { AAAAAAAABA }\end{array}$ & $\begin{array}{l}\text { fract. } \\
\triangle A B A A A A A\end{array}$ & $\begin{array}{c}\text { risk } \\
\text { AAAAAAAAAÄA }\end{array}$ & fract. & $\begin{array}{c}\text { risk } \\
\text { AAAAAAAAAA }\end{array}$ & 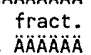 & $\begin{array}{c}\text { risk } \\
\text { AAAAAAAAAOA }\end{array}$ & $\begin{array}{l}\text { fract. } \\
\text { ÄÄÄÄÄ }\end{array}$ & $\begin{array}{c}r i s k \\
\text { ÄÄAAAAAAAOA }\end{array}$ & $\begin{array}{l}\text { fract. } \\
\text { ÄÄÄÄ̈ }\end{array}$ \\
\hline$c-227$ & $4.897 E-16$ & 0.0000 & $1.104 E-17$ & 0.0000 & $7.262 E-17$ & 0.0000 & $7.845 E-20$ & 0.0000 & $1.722 E-19$ & 0.0000 & & 0.0000 \\
\hline Co-t & $0.000 \mathrm{E}+00$ & 0.0000 & $0 E+00$ & 0.0000 & $0 E+00$ & 000 & $+\infty 0$ & 000 & $0 E+00$ & 00 & & \\
\hline & & & & & & & & & & & & \\
\hline Eu- & $0.000 \mathrm{E}+00$ & 0.0000 & $0.000 E+00$ & 1000 & $O E+00$ & 0000 & & 000 & 100 & 000 & 12 & 000 \\
\hline & $E+\infty 0$ & 0. & 0 & & 0 & & & & 00 & & & \\
\hline & & 0 & 0.0 & 0. & & 10 & & & & & & \\
\hline & 0.00 & & 0 & & & 0 & & 0 & & 10 & & 701 \\
\hline & $E+00$ & & & & & & & & & & & \\
\hline & 7. & & & 00 & -21 & Do & 23 & 00 & $267 E-22$ & & & \\
\hline Th-228 & 6.6 & 0. & 3.2 & 0.0 & 1.0 & 0.0 & 7 & & 1. & & 1. & 00 \\
\hline & & & רדי & & & & & & & & 9 & 000 \\
\hline & & & & & & & & & & & & \\
\hline & & & & & & & & & & & & \\
\hline & & & & & & & & & & & & \\
\hline Total & $1 E-12$ & & $4.075 E-14$ & 0.0000 & 1. & 0.0 & 1. & 0.0000 & 9. & 000 & $6 E-07$ & 4 \\
\hline
\end{tabular}

** Sum of water independent ground, inhalation, plant, meat, milk, soil

and water dependent water, fish, plant, meat, milk pathways

\begin{tabular}{|c|c|}
\hline Attachment & Sheet No. 27 of 31 \\
\hline Originator: S.W. Clark & Date \\
\hline Chk'd By M.W. Perrott & Date \\
\hline Calc. No. $0100 \mathrm{~F}$-CA-V0303 & Rev. No. \\
\hline
\end{tabular}


CVP-2007-00004

Rev. 0

\section{ATTACHMENT 9}

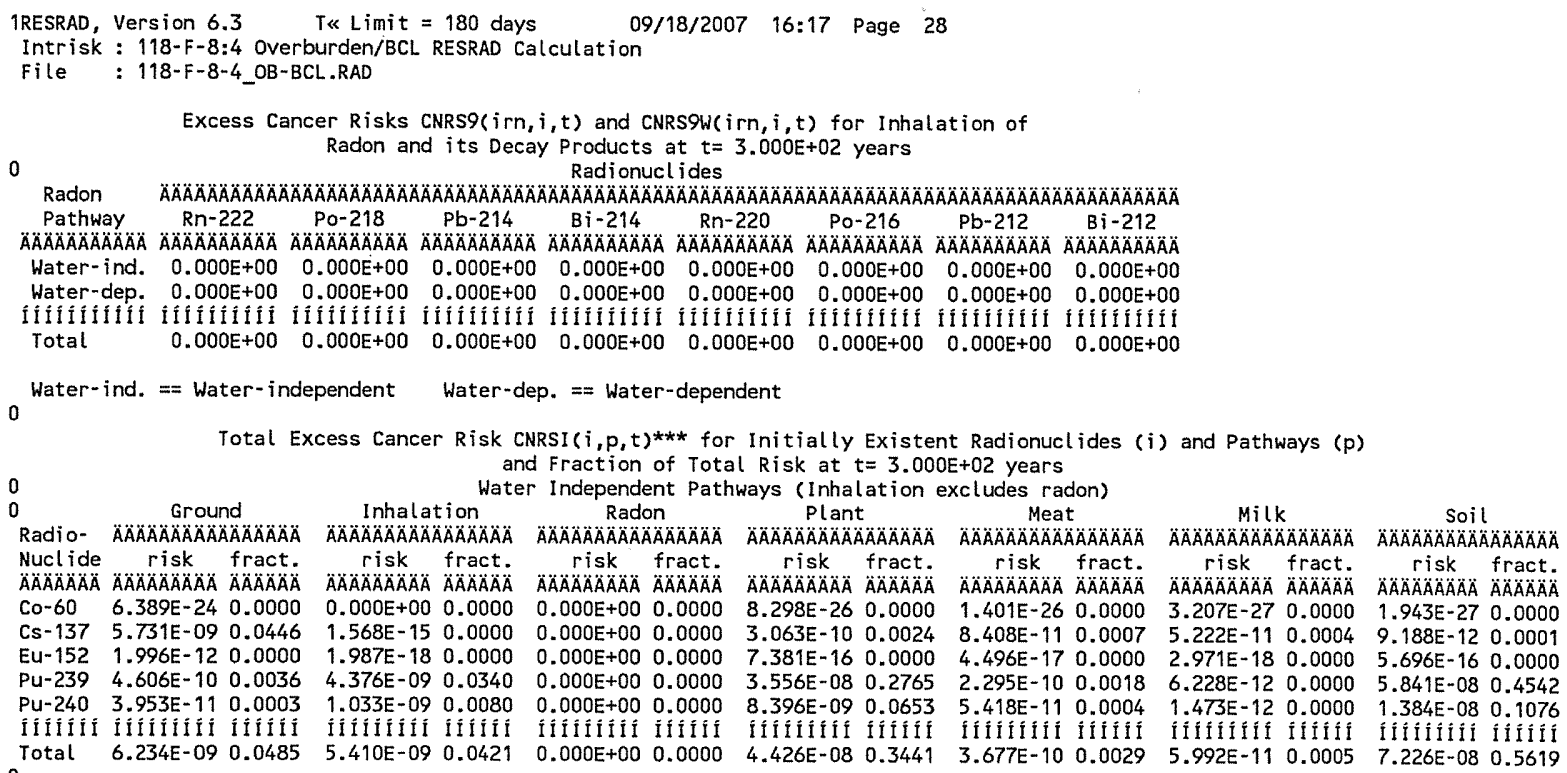

Water Dependent Pathways

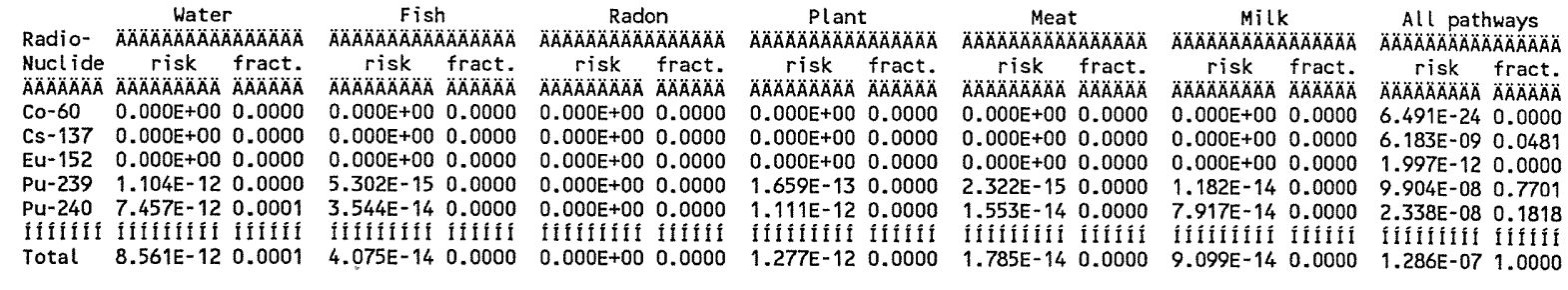

$* * *$ CNRSI $(i, p, t)$ includes contribution from decay daughter radionuclides

\begin{tabular}{|c|c|}
\hline Attachment & Sheet No. \\
\hline Originator: S.W. Clark & Date \\
\hline M.W. Perrott & Date \\
\hline Calc. No. $0100 F-C A-V 0303$ & Rev. No. \\
\hline
\end{tabular}




\section{ATTACHMENT 9}

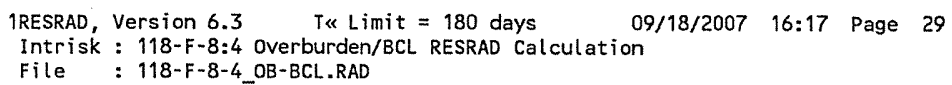

Water-ind. = Water-independent Water-dep. $==$ Water-dependent

\begin{tabular}{|c|c|}
\hline \multirow{2}{*}{\multicolumn{2}{|c|}{$\begin{array}{l}\text { Attachment } \frac{9}{\text { Originator: S.W. Clark }} \text { Sheet No. } 29 \text { of } 31 \\
\text { Date }\end{array}$}} \\
\hline & \\
\hline M. W. Perrott & \\
\hline $0100 F-C A-V 0303$ & Rev \\
\hline
\end{tabular}




\section{ATTACHMENT 9}

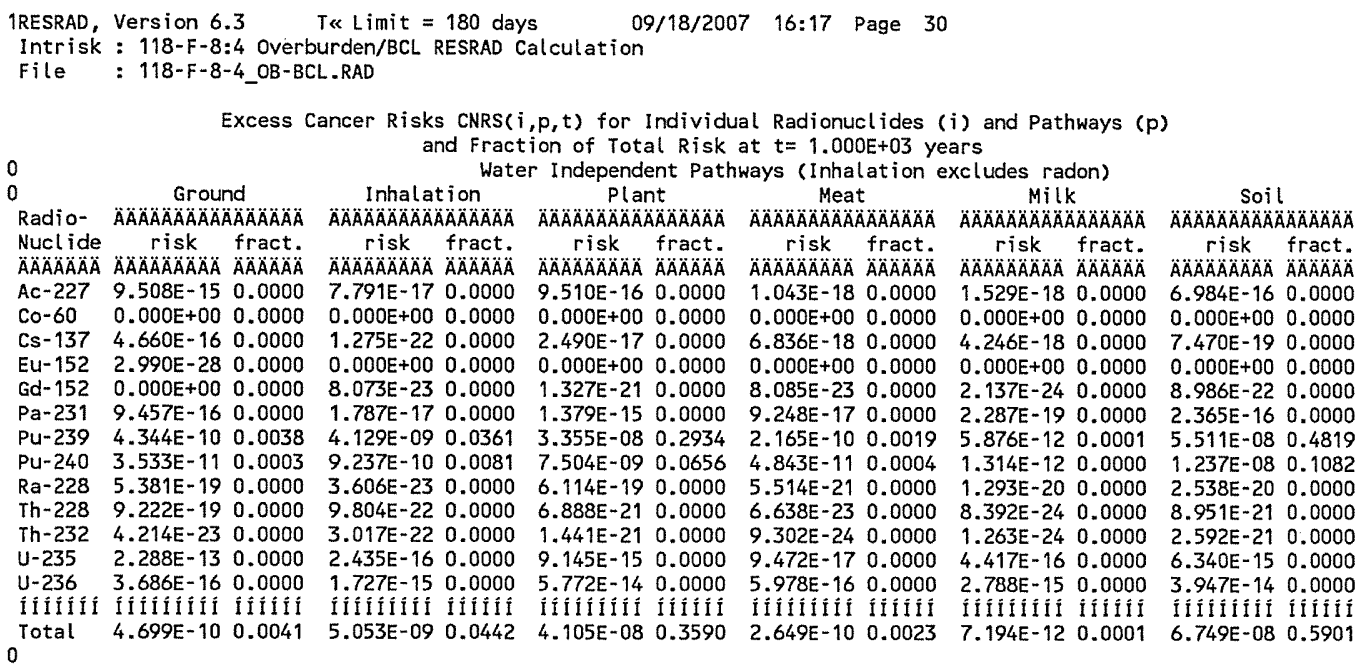

Water Dependent Pathways

\begin{tabular}{|c|c|c|c|c|c|c|c|c|c|c|c|}
\hline \multirow{2}{*}{$\begin{array}{l}\text { Radio- } \\
\text { Nuclide } \\
\ddot{A B A ̈ A ̈ A ̈ A ̈ A ̈}\end{array}$} & \multicolumn{2}{|c|}{$\begin{array}{c}\text { Water } \\
\text { ÄÄAAAAÄÄÄÄÄÄÄA }\end{array}$} & \multicolumn{2}{|c|}{$\begin{array}{c}\text { Fish } \\
\text { AAAAAAAAAAAOAAAOAOA }\end{array}$} & \multicolumn{2}{|c|}{$\begin{array}{c}\text { Plant } \\
\text { AAAAAAAAAAAAAAAA }\end{array}$} & \multicolumn{2}{|c|}{$\begin{array}{c}\text { Meat } \\
\text { AAÄAAAAAAÄÄAAAOÄÄ }\end{array}$} & \multicolumn{2}{|c|}{ 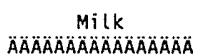 } & $\begin{array}{r}\text { All Pathways** } \\
\text { ÄÄÄÄÄÄÄÄÄÄÄÄAA }\end{array}$ \\
\hline & $\begin{array}{c}\text { risk } \\
\text { AAAAAAAOAOA }\end{array}$ & $\begin{array}{l}\text { fract. } \\
\text { ÄÄÄÄA }\end{array}$ & $\begin{array}{c}\text { risk } \\
\text { AAAAAAAAAÄ }\end{array}$ & $\begin{array}{l}\text { fract. } \\
A B A B A B A\end{array}$ & $\begin{array}{c}\text { risk } \\
\text { AAAAAAAAAA }\end{array}$ & $\begin{array}{l}\text { fract. } \\
A B A B A B A\end{array}$ & $\begin{array}{c}\text { risk } \\
\text { AAAAAAAAAA }\end{array}$ & $\begin{array}{l}\text { fract. } \\
\text { ÄÄÄÄÄ̈ }\end{array}$ & $\begin{array}{c}\text { risk } \\
\text { AAAAAAAOÄ }\end{array}$ & $\begin{array}{l}\text { fract. } \\
A B A ̈ A B A B\end{array}$ & $\begin{array}{l}\text { risk fract. } \\
\text { AAAAAAAAOA AAAAAA }\end{array}$ \\
\hline$c-227$ & $2.426 E-14$ & 0.0000 & $5.471 E-16$ & 0.0000 & $3.600 \mathrm{E}-15$ & 0.0000 & $3.492 E-18$ & 0.0000 & $8.546 \mathrm{E}-18$ & 0.0000 & $3.966 \mathrm{E}-14 \quad 0.0000$ \\
\hline & $O O E+00$ & 0 & $0 E+00$ & 1000 & $00 E+00$ & 000 & $O E+00$ & 00 & 00 & 000 & 000 \\
\hline & & & & & $E+\infty$ & & & & & & \\
\hline & $0.000 E+00$ & 0.0000 & $O E+00$ & 000 & $0.000 E+00$ & 0.0000 & $\mathrm{OOE}+00$ & & .00 & 1000 & 000 \\
\hline & $+\infty 0$ & 0 & $+\infty$ & & 30 & & 30 & & & & \\
\hline & & 0.0000 & & 0 & $E-16$ & 0 & & & & & \\
\hline & 0. & & & & 0 & 0 & 30 & & & 00 & \\
\hline & & & & & 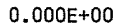 & & & & & & \\
\hline & 9 & 100 & -21 & 00 & $E-20$ & 0 & & & & 00 & 18 \\
\hline Th-228 & $19 E-20$ & 0.0000 & & 0. & $3.855 \mathrm{E}-21$ & 0.0000 & 2. & 00 & & 100 & 000 \\
\hline & & & & & & & & & & & \\
\hline & & & & & $E-13$ & & & & & & \\
\hline & & & & & & 0.0 & 3 & & & & 002 \\
\hline & & & & & & & & & & & \\
\hline otat & $1 E-11$ & & -10 & & $E-12$ & 0.0 & 3.9 & & 1.98 & 000 & -071. \\
\hline
\end{tabular}

** Sum of water independent ground, inhalation, plant, meat, milk, soil

and water dependent water, fish, plant, meat, milk pathways

\begin{tabular}{|c|c|}
\hline Attachment & Sheet No. $\underline{30}$ of 31 \\
\hline Originator: S.W. Clark & Date \\
\hline M. W. Perrott & Date \\
\hline 0100F-CA-V0303 & Rev. No. $\quad 0$ \\
\hline
\end{tabular}




\section{ATTACHMENT 9}

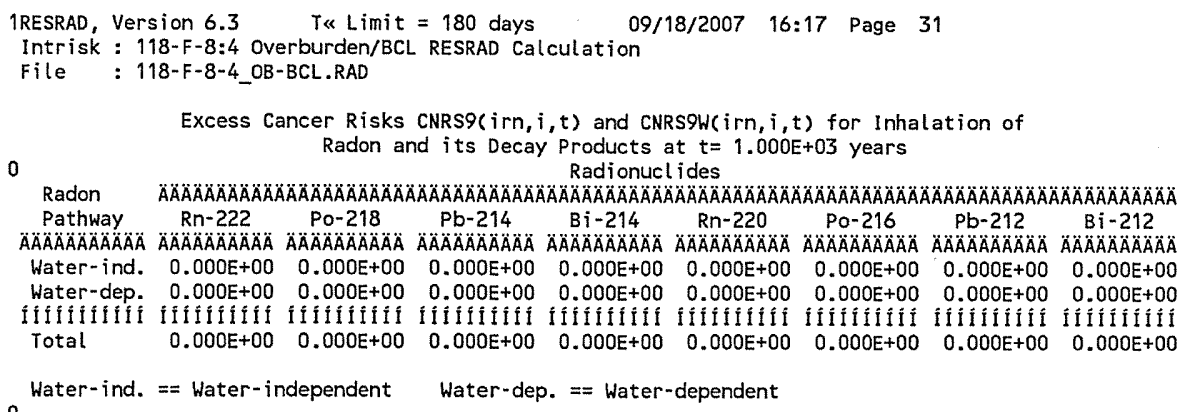

Total Excess Cancer Risk CNRSI $(i, p, t)^{* * *}$ for Initially Existent Radionuclides (i) and Pathways (p) and Fraction of Total Risk at $t=1.000 E+03$ years

Water Dependent Pathways

\begin{tabular}{|c|c|c|c|c|c|c|c|c|c|c|c|}
\hline & $\begin{array}{l}\text { Water } \\
\text { WaÄÄÄÄÄÄÄÄÄÄÄÄA }\end{array}$ & $\begin{array}{c}\text { Fish } \\
\text { AäÄÄÄÄÄÄÄÄÄÄÄA }\end{array}$ & $\begin{array}{r}\text { Rad } \\
\text { RäÄÄÄÄ }\end{array}$ & $\triangle A O A O A B A$ & 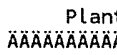 & $\triangle A \cap A A A B A$ & $\begin{array}{r}M e \\
\text { AAARAÄÄ }\end{array}$ & ÄÄÄÄÄ & 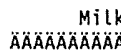 & Ä̈̈ÄÄÄ & $\begin{array}{c}\text { All pathways } \\
A A B A A A B A A A A A A A\end{array}$ \\
\hline$\triangle A B A O A O A$ & 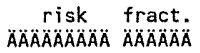 & $\begin{array}{r}\text { risk fract. } \\
\text { ÄÄAAAOA ÄAAAAAOA }\end{array}$ & $\begin{array}{c}\text { risk } \\
\text { AAAAAAAAAAA }\end{array}$ & $\begin{array}{l}\text { fract. } \\
A \cap A ̈ A ̈ A ̈ A B A\end{array}$ & $\begin{array}{c}\text { risk } \\
\text { AAAAAAAAÄ }\end{array}$ & $\begin{array}{l}\text { fract. } \\
\text { ÄAAAAOAB }\end{array}$ & $\begin{array}{l}\text { risk } \\
A \not ̈ A ̈ A O A O A B\end{array}$ & fra & $\begin{array}{l}\text { risk } \\
A A ̈ A B A B A B\end{array}$ & $\begin{array}{l}\text { fract. } \\
A B A A O A B A B\end{array}$ & risk \\
\hline & 000.0000 & +000.0 & $+\infty$ & & & & & & $m$ & & \\
\hline & & +000 & & & & & & & & & \\
\hline & & & & & & & & & & & \\
\hline & & 140 & 0 & & & & & & & & \\
\hline & & & & & & & & & & & \\
\hline & & & & & & & & & & & \\
\hline (1) & -11 & -14 & 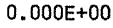 & & & & 14 & & -13 & & 071. \\
\hline
\end{tabular}

*** $\operatorname{CNRSI}(i, p, t)$ includes contribution from decay daughter radionuclides

$\begin{array}{ll}\text { Attachment } & \frac{9}{\text { Sheet No. } 31 \text { of } 31} \\ \text { Originator: } \frac{\text { S.W. Clark }}{\text { M. W. Perrott }} & \text { Date } \\ \text { Chk'd By } & \text { Date } \\ \text { Calc. No. } & \text { Rev. No. } 0\end{array}$




\title{
ATTACHMENT 10
}

\author{
1RESRAD, Version 6.3 T« Limit $=180$ days $\quad 09 / 18 / 2007 \quad 16: 17$ Page 1 \\ Concent : 118-F-8:4 Overburden/BCL RESRAD Calculation \\ File : 118-F-8-4_OB-BCL.RAD \\ Table of Contents

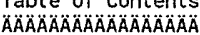 \\ Part IV: Concentration of Radionuclides

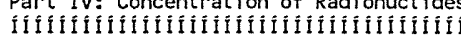 \\ Concentration of radionuclides in different media

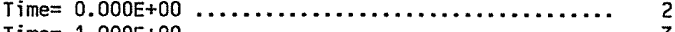

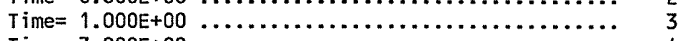

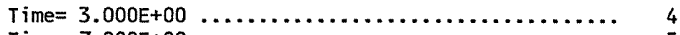

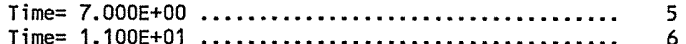

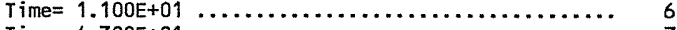

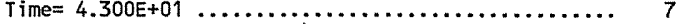

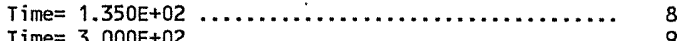 \\ Time $=1.000 \mathrm{E}+03 \ldots \ldots \ldots \ldots \ldots \ldots \ldots \ldots \ldots \ldots \ldots, 10$
}

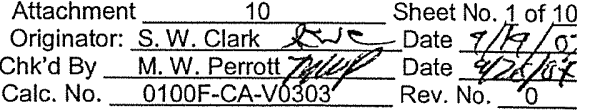


CVP-2007-00004

Rev. 0

\section{ATTACHMENT 10}

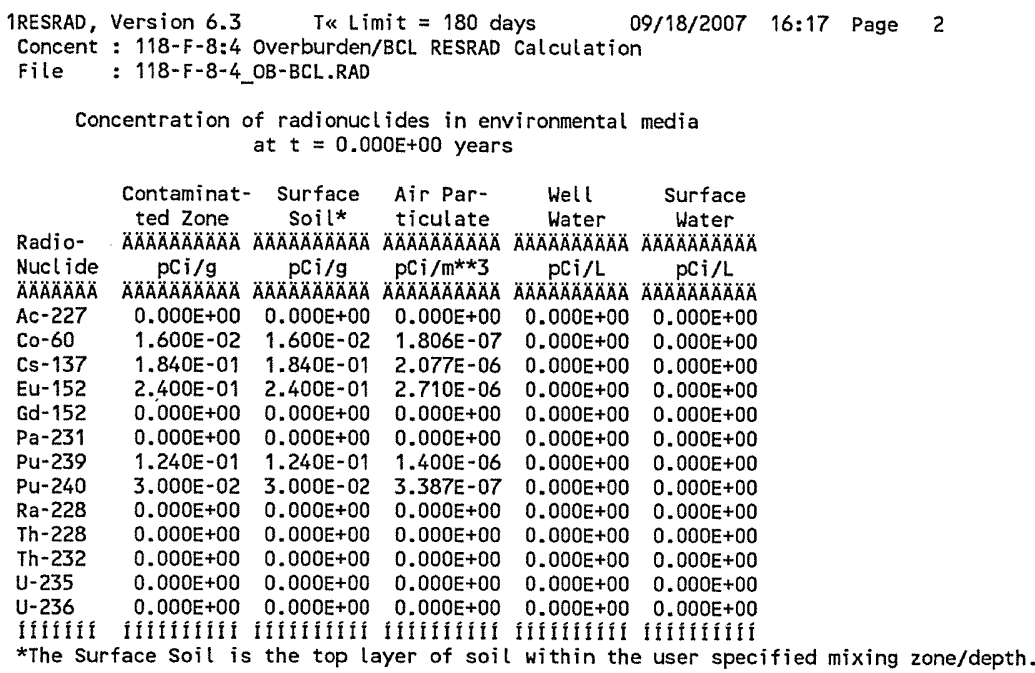

Concentrations in the media occurring in pathways that are suppressed are calculated using the current input parameters, i.e. using parameters appearing in the input screen when the pathways are active.

Concentration of radionuclides in foodstuff media at $\mathrm{t}=0.000 \mathrm{E}+00$ years*

Drinking Nonleafy Leafy Fodder Fodder Meat Milk Fish Crustacea

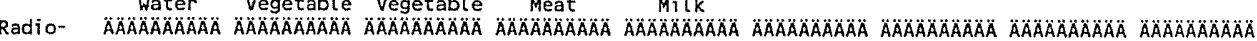
Nuclide $\mathrm{pCi} / \mathrm{L} p \mathrm{pCi} / \mathrm{kg} \mathrm{pCi} / \mathrm{kg} p C i / \mathrm{kg} p C i / \mathrm{kg} p C i / \mathrm{kg} p \mathrm{pCi} / \mathrm{L} p \mathrm{pCi} / \mathrm{kg} p \mathrm{pCi} / \mathrm{kg}$

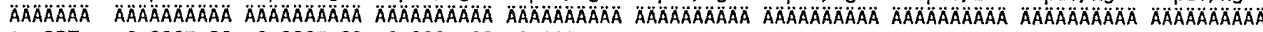
$\begin{array}{lllllllllll}A C-227 & 0.000 E+00 & 0.000 E+00 & 0.000 E+00 & 0.000 E+00 & 0.000 E+00 & 0.000 E+00 & 0.000 E+00 & 0.000 E+00 & 0.000 E+00\end{array}$ $\begin{array}{llllllllll}\mathrm{CO}-60 & 0.000 \mathrm{E}+00 & 1.280 \mathrm{E}+00 & 1.280 \mathrm{E}+00 & 1.280 \mathrm{E}+00 & 1.280 \mathrm{E}+00 & 1.901 \mathrm{E}+00 & 1.568 \mathrm{E}-01 & 0.000 \mathrm{E}+00 & 0.000 \mathrm{E}+00\end{array}$ $\begin{array}{llllllllll}\text { CS-137 } & 0.000 E+00 & 7.360 E+00 & 7.361 E+00 & 7.361 E+00 & 7.361 E+00 & 1.778 E+01 & 3.975 E+00 & 0.000 E+00 & 0.000 E+00\end{array}$ $\begin{array}{lllllllllll}\text { Eu-152 } & 0.000 E+00 & 6.001 E-01 & 6.007 E-01 & 6.008 E-01 & 6.008 E-01 & 3.217 E-01 & 7.652 E-03 & 0.000 E+00 & 0.000 E+00\end{array}$ $\begin{array}{lllllllllll}\mathrm{Gd}-152 & 0.000 \mathrm{E}+00 & 0.000 \mathrm{E}+00 & 0.000 \mathrm{E}+00 & 0.000 \mathrm{E}+00 & 0.000 \mathrm{E}+00 & 0.000 \mathrm{E}+00 & 0.000 \mathrm{E}+00 & 0.000 \mathrm{E}+00 & 0.000 \mathrm{E}+00\end{array}$ $\begin{array}{llllllllll}\mathrm{Pa}-231 & 0.000 \mathrm{E}+00 & 0.000 \mathrm{E}+00 & 0.000 \mathrm{E}+00 & 0.000 \mathrm{E}+00 & 0.000 \mathrm{E}+00 & 0.000 \mathrm{E}+00 & 0.000 \mathrm{E}+00 & 0.000 \mathrm{E}+00 & 0.000 \mathrm{E}+00\end{array}$ PU-239 $0.000 E+001.241 E-011.244 E-01 \quad 1.244 E-01 \quad 1.244 E-017.046 E-03 \quad 6.884 E-05 \quad 0.000 E+00 \quad 0.000 E+00$ PU-240 $0.000 E+003.002 E-023.009 E-023.010 E-02$ 3.010E-02 $1.705 E-03$ $1.666 E-050.000 E+00$ $0.000 E+00$ $\begin{array}{llllllllll}\text { PU-240 } & 0.000 E+00 & 3.002 E-02 & 3.009 E-02 & 3.010 E-02 & 3.010 E-02 & 1.705 E-03 & 1.666 E-05 & 0.000 E+00 & 0.000 E+00\end{array}$ $\begin{array}{llllllllll}R a-228 & 0.000 E+00 & 0.000 E+00 & 0.000 E+00 & 0.000 E+00 & 0.000 E+00 & 0.000 E+00 & 0.000 E+00 & 0.000 E+00 & 0.000 E+00 \\ T h-228 & 0.000 E+00 & 0.000 E+00 & 0.000 E+00 & 0.000 E+00 & 0.000 E+00 & 0.000 E+00 & 0.000 E+00 & 0.000 E+00 & 0.000 E+00\end{array}$ $\begin{array}{llllllllll}T h-228 & 0.000 E+00 & 0.000 E+00 & 0.000 E+00 & 0.000 E+00 & 0.000 E+00 & 0.000 E+00 & 0.000 E+00 & 0.000 E+00 & 0.000 E+00\end{array}$ $\begin{array}{llllllllll}\text { Th }-232 & 0.000 E+00 & 0.000 E+00 & 0.000 E+00 & 0.000 E+00 & 0.000 E+00 & 0.000 E+00 & 0.000 E+00 & 0.000 E+00 & 0.000 E+00\end{array}$ $\begin{array}{llllllllll}U-235 & 0.000 E+00 & 0.000 E+00 & 0.000 E+00 & 0.000 E+00 & 0.000 E+00 & 0.000 E+00 & 0.000 E+00 & 0.000 E+00 & 0.000 E+00\end{array}$ $\begin{array}{llllllllll}U-236 & 0.000 E+00 & 0.000 E+00 & 0.000 E+00 & 0.000 E+00 & 0.000 E+00 & 0.000 E+00 & 0.000 E+00 & 0.000 E+00 & 0.000 E+00\end{array}$

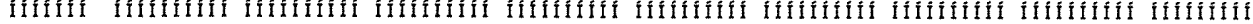

*Concentrations are at consumption time and include radioactive decay and ingrowth during storage time.

For livestock fodder, consumption time is $t$ minus meat or milk storage time.

Concentrations in the media occurring in pathways that are suppressed are calculated using the current input parameters, i.e. using parameters appearing in the input screen when the pathways are active.

\begin{tabular}{|c|c|}
\hline Attachment & Sheet No. 2 of 10 \\
\hline Originator: S. W. Clark & Date \\
\hline Chk'd By M.W. Perrott & Date \\
\hline $0100 \mathrm{~F}-\mathrm{CA}-\mathrm{V} 0303$ & Rev. No. 0 \\
\hline
\end{tabular}




\section{ATTACHMENT 10}

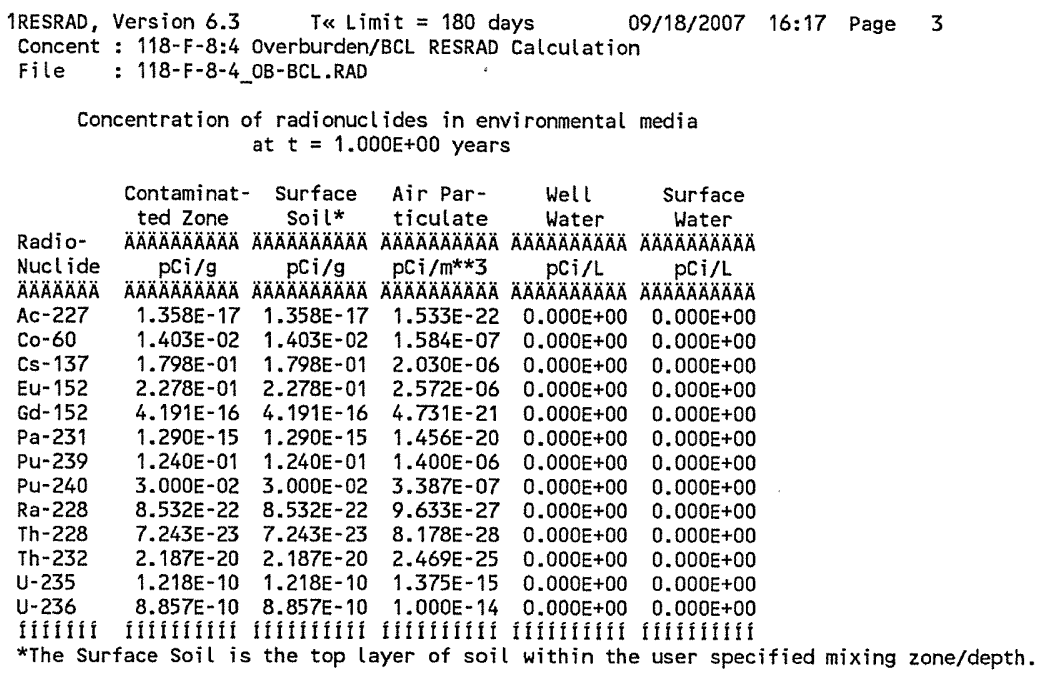

Concentrations in the media occurring in pathways that are suppressed are calculated using the current input parameters, i.e. using parameters appearing in the input screen when the pathways are active.

Concentration of radionuclides in foodstuff media at $\mathrm{t}=1.000 \mathrm{E}+00$ years*

\begin{tabular}{|c|c|c|c|c|c|c|c|c|c|}
\hline & $\begin{array}{c}\text { Drinking } \\
\text { Water }\end{array}$ & $\begin{array}{l}\text { Nonleafy } \\
\text { Vegetable }\end{array}$ & $\begin{array}{c}\text { Leafy } \\
\text { Vegetable }\end{array}$ & $\begin{array}{c}\text { Fodder } \\
\text { Meat }\end{array}$ & $\begin{array}{l}\text { Fodder } \\
\text { Milk }\end{array}$ & Meat & Milk & Fish & Crustacea \\
\hline & 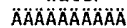 & $\triangle A \cap A O A O A O A A O A B A$ & 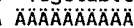 & $\triangle A \cap A \cap A ̈ A A A A O A O A ̈$ & 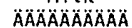 & $\triangle A \cap A B A B A A B A O A$ & $\triangle \triangle A B A O A B A A A A B A$ & $\triangle \triangle A A A B A A B A A B A$ & $\triangle \triangle A B A A B A A A A B$ \\
\hline & & $\mathrm{pCi} / \mathrm{kg}$ & $p c$ & $\mathrm{pci} / \mathrm{kg}$ & $\mathrm{pCi} / \mathrm{kg}$ & $\mathrm{pci} / \mathrm{kg}$ & $\mathrm{pCi} / \mathrm{L}$ & $\mathrm{pCi} / \mathrm{kg}$ & $\mathrm{pCi} / \mathrm{kg}$ \\
\hline & $\triangle A Q A A A A A A A C A O A$ & $\triangle A A A A A A A A A O A B$ & 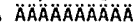 & $\triangle A A A A A A A A A A O A$ & $\triangle A Q A A A A A A A B A B$ & $\triangle \triangle A A A A A A A A A B$ & $\triangle A A A A A A A O A B A$ & $\triangle \triangle A A A A A A A A A A A ̈$ & $\triangle A O A A A A A A A A O A$ \\
\hline 277 & $0.000 E+00$ & $4.490 E-17$ & $3.484 \mathrm{E}-17$ & $5.430 E-17$ & $6.262 \mathrm{E}-17$ & $1.080 \mathrm{E}-17$ & $2.042 E-19$ & $0.000 E+00$ & $0.000 E+00$ \\
\hline & 0.0 & $=00$ & $+\infty$ & $0 E+00$ & $1.123 E+00$ & $6 E+00$ & 1.37 & $0.000 E+00$ & $0.000 E+00$ \\
\hline & 0.0 & $E+00$ & 7.1 & $E+\infty$ & $E+00$ & +01 & +00 & $0.000 E+00$ & $0.000 \mathrm{E}+00$ \\
\hline & $+\infty$ & 5.6 & 5.7 & -01 & $E-01$ & & 7. & $E+00$ & $E+00$ \\
\hline & $0.000 E+00$ & 1.0 & 1.0 & $E-16$ & $6 E-15$ & 5.6 & 5.36 & $0.000 E+00$ & $0 E+00$ \\
\hline & 0.0 & 1.2 & & -15 & $E-14$ & & 8.7 & $0.000 E \div 00$ & $\mathrm{EE}+00$ \\
\hline & 0.0 & 1.2 & 1.2 & $E-01$ & $1.244 \mathrm{E}-01$ & -03 & 6.8 & $E+00$ & $E+00$ \\
\hline & $0.000 E+00$ & 3.0 & 3.0 & $E-02$ & $E-02$ & -03 & 1.6 & $E+00$ & $E+00$ \\
\hline & 0.0 & 3.0 & 3.3 & -20 & $8 E-20$ & $E-21$ & $E-21$ & $\mathrm{DE}+00$ & $E+\infty$ \\
\hline & $0.000 E+00$ & 4.8 & 1.0 & $E-22$ & $E-21$ & & & $E+00$ & $E+00$ \\
\hline Th- & $0.000 E+00$ & $2.430 \mathrm{E}-20$ & 2.2 & $E-20$ & $3 E-20$ & $E-21$ & 1.0 & $E+\infty$ & $E+\infty$ \\
\hline & $0.000 \mathrm{E}+00$ & $2.976 E-10$ & $3.044 E-10$ & $2.659 \mathrm{E}-10$ & $2.817 E-10$ & $2.610 E-11$ & $4.574 \mathrm{E}-11$ & $0.000 \mathrm{E}+00$ & $0.000 E+00$ \\
\hline & $0.000 E+00$ & $2.164 \mathrm{E}-09$ & $2.213 E-09$ & & $2.048 \mathrm{E}-09$ & 1.8 & $3.326 \mathrm{E}-10$ & $0.000 E+00$ & $0.000 \mathrm{E}+00$ \\
\hline & & & & & & 6 & & 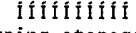 & 0 \\
\hline
\end{tabular}

For at consumption time and include radioactive decay and ingrowth during storage time.

For livestock fodder, consumption time is $t$ minus meat or milk storage time.

Concentrations in the media occurring in pathways that are suppressed are calculated using the current input parameters, i.e. using parameters appearing in the input screen when the pathways are active.

$\begin{array}{ll}\text { Attachment } \frac{10}{\text { S.W. Clark }} & \text { Sheet No. } 3 \text { of } 10 \\ \text { Originator: } & \text { Date } \\ \text { Chk'd By } & \text { Date } \\ \text { Calc. No. N. Perrott } & \text { Rev. No. } 0\end{array}$




\section{ATTACHMENT 10}

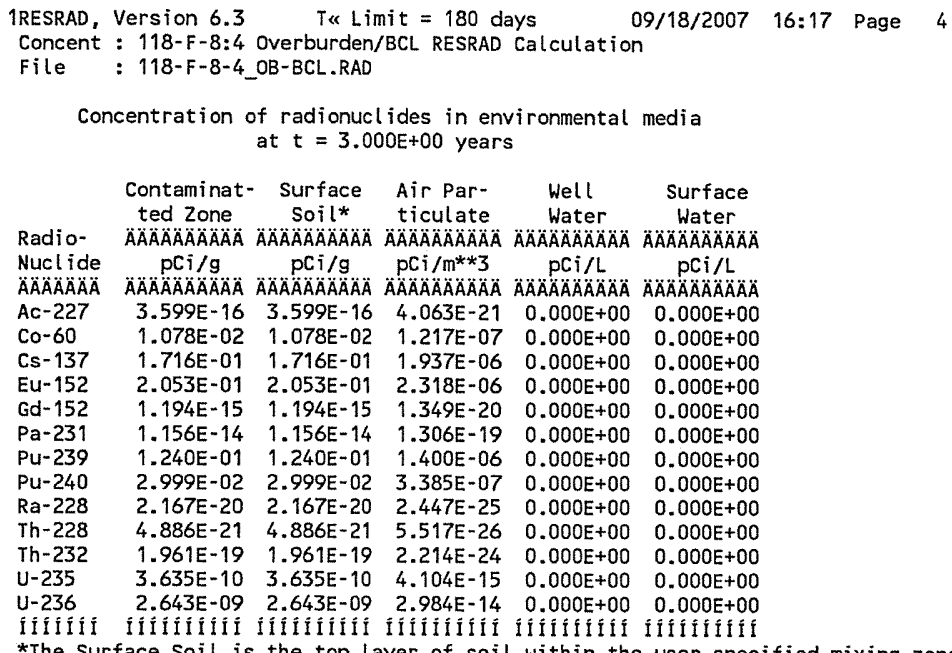

Concentrations in the media occurring in pathways that are suppressed are calculated using the current input parameters, i.e. using parameters appearing in the input screen when the pathways are active.

\begin{tabular}{|c|c|}
\hline Attachment & Sheet No. 4 of 10 \\
\hline Originator: S.W. Clark & Date \\
\hline Chk'd By M.W. Perrott & Date \\
\hline Calc. No. $0100 \mathrm{~F}-\mathrm{CA}-\mathrm{V} 0303$ & Rev. No. 0 \\
\hline
\end{tabular}




\section{ATTACHMENT 10}

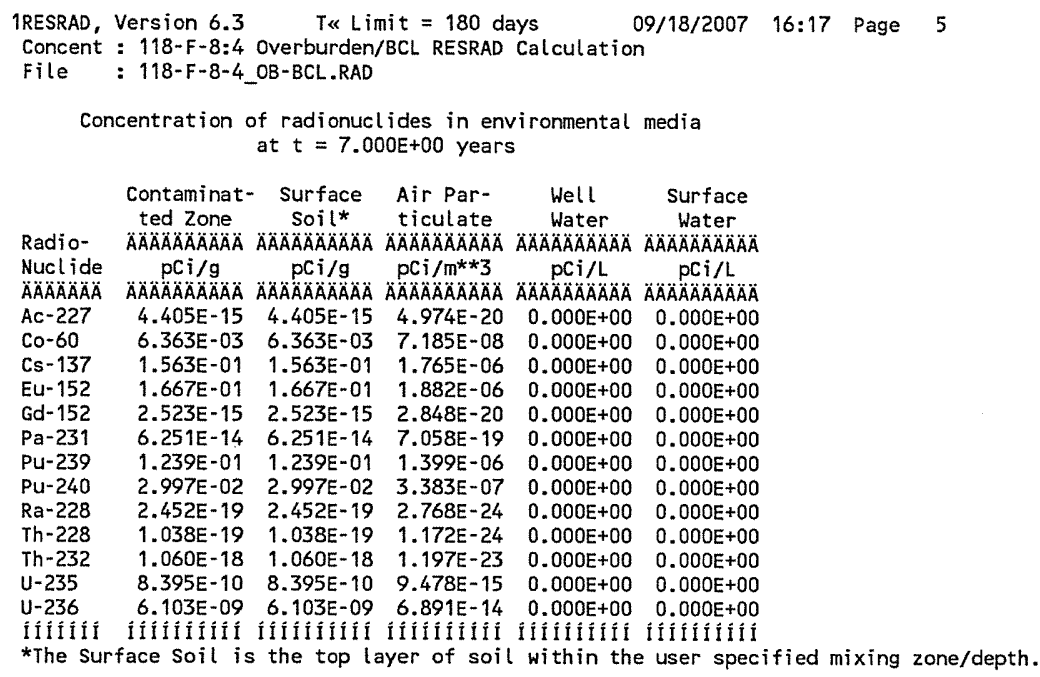

Concentrations in the media occurring in pathways that are suppressed are calculated using the current input parameters, i.e. using parameters appearing in the input screen when the pathways are active.

\begin{tabular}{|c|c|c|c|c|c|c|c|c|c|}
\hline \multirow{5}{*}{$\begin{array}{l}\text { Radio- } \\
\text { Nuclide }\end{array}$} & \multicolumn{7}{|c|}{$\begin{array}{c}\text { Concentration of radionuclides in foodstuff media } \\
\text { at } t=7.000 E+00 \text { years }^{*}\end{array}$} & \multirow[b]{2}{*}{ Fish } & \multirow[b]{2}{*}{ Crustacea } \\
\hline & $\begin{array}{l}\text { Drinking } \\
\text { Water }\end{array}$ & $\begin{array}{l}\text { Nonleafy } \\
\text { Vegetable }\end{array}$ & $\begin{array}{l}\text { Leafy } \\
\text { Vegetable }\end{array}$ & $\begin{array}{l}\text { Fodder } \\
\text { Meat }\end{array}$ & $\begin{array}{l}\text { Fodder } \\
\text { Milk }\end{array}$ & Meat & Milk & & \\
\hline & $\triangle A O A A A A A A A B A$ & $A A A A A A A A A A A A$ & 1 AAOAAAAAAAAOA & ÄAAAAAAAAÄÄ & $\triangle A \cap A \cap A B A A A A B A$ & 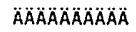 & 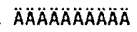 & $\triangle A A A A A A B A A A$ & 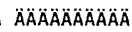 \\
\hline & $\mathrm{pCi} / \mathrm{L}$ & $\mathrm{pCi} / \mathrm{kg}$ & $\mathrm{pci} / \mathrm{kg}$ & $\mathrm{pCi} / \mathrm{kg}$ & $\mathrm{pCi} / \mathrm{kg}$ & $\mathrm{pCi} / \mathrm{kg}$ & $\mathrm{pCi} / \mathrm{L}$ & $\mathrm{pCi} / \mathrm{kg}$ & $\mathrm{pCi} / \mathrm{kg}$ \\
\hline & $\triangle A A A A A A A A A B$ & $\triangle A A A A A A A A A A B$ & AAAAAAAAAAAAAA & $\triangle A A B A O A O A A A A A A A$ & $\triangle A A A A A B A A O A A B A$ & 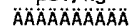 & 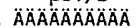 & A $\triangle A O A O A O A O A O A O A B$ & ÄÄÄAAAOAOÄ \\
\hline$c-227$ & $0.000 E+00$ & $1.158 E-14$ & 1.107E-14 & $1.252 E-14$ & - $1.279 E-14$ & $6.831 \mathrm{E}-16$ & $5.809 E-17$ & $0.000 E+00$ & $0.000 E+00$ \\
\hline $0-60$ & $0.000 E+00$ & $5.091 \mathrm{E}-01$ & $5.091 E-01$ & $5.128 E-01$ & $5.093 \mathrm{E}-01$ & $7.560 \mathrm{E}-01$ & $6.236 \mathrm{E}-02$ & $0.000 \mathrm{E}+00$ & $0.000 E+00$ \\
\hline 37 & $0.000 E+00$ & $6.252 \mathrm{E}+00$ & $6.252 E+00$ & $6.260 \mathrm{E}+00$ & $6.252 E+00$ & $1.510 E+01$ & $3.376 E+00$ & $0.000 E+00$ & $0.000 E+00$ \\
\hline 52 & $0.000 E+00$ & 4.169E-01 & 4. $173 \mathrm{E}-01$ & $4.185 E-01$ & $4.174 E-01$ & $2.235 E-01$ & $5.315 E-03$ & $0.000 E+00$ & $0.000 E+00$ \\
\hline$G d-152$ & $0.000 E+00$ & $6.309 E-15$ & $6.314 E-15$ & $6.274 E-15$ & $6.313 E-15$ & $3.382 E-15$ & $3.219 E-17$ & $0.000 E+00$ & $0.000 E+00$ \\
\hline$a-231$ & $0.000 \mathrm{E}+00$ & $6.200 \mathrm{E}-13$ & $6.249 E-13$ & $5.995 E-13$ & $6.085 E-13$ & $3.579 E-13$ & $3.421 E-16$ & $0.000 E+00$ & $0.000 E+00$ \\
\hline Pu-239 & $0.000 E+00$ & $1.240 E-01$ & $1.243 E-01$ & $1.243 E-01$ & $1.243 E-01$ & $7.042 E-03$ & $6.880 E-05$ & $0.000 E+00$ & $0.000 E+00$ \\
\hline Pu- 240 & $0.000 E+00$ & $2.998 \mathrm{E}-02$ & $\quad 3.005 E-02$ & $3.006 \mathrm{E}-02$ & $3.006 \mathrm{E}-02$ & 1.703E-03 & $1.664 E-05$ & $0.000 E+00$ & $0.000 E+00$ \\
\hline Ra-228 & $0.000 E+00$ & $9.618 E-18$ & $9.795 E-18$ & $9.006 \mathrm{E}-18$ & $9.200 E-18$ & 7.279E- 19 & $6.282 E-19$ & $0.000 E+00$ & $0.000 E+00$ \\
\hline 28 & $0.000 E+00$ & $2.335 E-19$ & $1.136 E-19$ & $4.869 E-19$ & $4.978 E-19$ & $2.254 \mathrm{E}-20$ & $1.019 E-21$ & $0.000 E+00$ & $0.000 E+00$ \\
\hline Th-232 & $0.000 E+00$ & $1.078 E-18$ & $1.065 E-18$ & $1.101 \mathrm{E}-18$ & $1.117 E-18$ & $6.340 E-20$ & $3.270 E-21$ & $0.000 E+00$ & $0.000 E+00$ \\
\hline $4-235$ & $0.000 E+00$ & $2.093 \mathrm{E}-09$ & $2.101 E-09$ & $2.064 E-09$ & 2.079E-09 & $1.897 E-10$ & $3.204 \mathrm{E}-10$ & $0.000 E+00$ & $0.000 E+00$ \\
\hline 36 & $0.000 E+00$ & $1.521 E-08$ & 1.527E-08 & $1.501 \mathrm{E}-08$ & $1.512 E-08$ & $1.379 E-09$ & 2.329E-09 & $0.000 E+00$ & $0.000 \mathrm{E}+00$ \\
\hline & İİííííí & 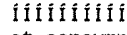 & 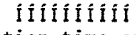 & $\ldots \ldots$ & 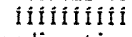 & 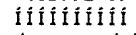 & 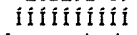 & 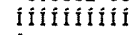 & 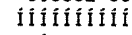 \\
\hline
\end{tabular}

For livestock fodder, consumption time is $t$ minus meat or milk storage time.

Concentrations in the media occurring in pathways that are suppressed are calculated using the current input parameters, i.e. using parameters appearing in the input screen when the pathways are active.

$\begin{array}{ll}\text { Attachment } \frac{10}{\text { S.W. Clark }} & \text { Sheet No. } \underline{5} \text { of } 1 \mathrm{C} \\ \text { Originator: } & \text { Date } \\ \text { Chk'd By } & \text { Date } \\ \text { Calc. No. Ne. Perrott } & \text { Rev. No. } 0\end{array}$




\section{ATTACHMENT 10}

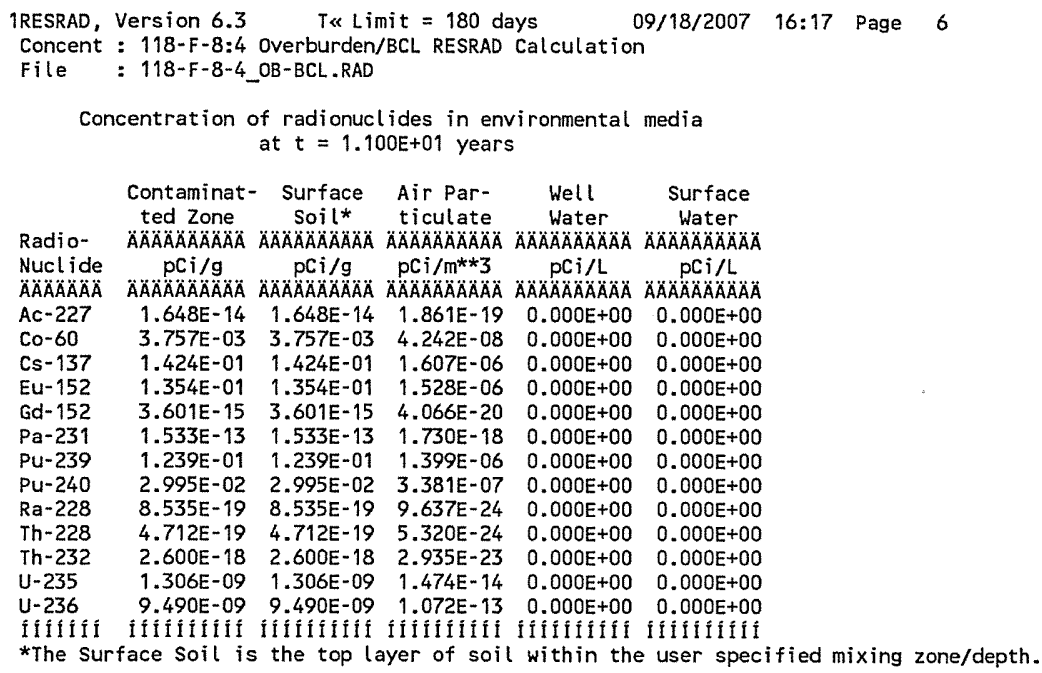

Concentrations in the media occurring in pathways that are suppressed are calculated using the current input parameters, i.e. using parameters appearing in the input screen when the pathways are active.

Concentration of radionuclides in foodstuff media at $\mathrm{t}=1.100 \mathrm{E}+01$ years*

Drinking Nonleafy Leafy Fodder Fodder Meat Milk Fish Crustacea

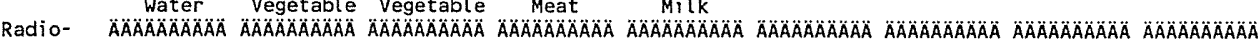

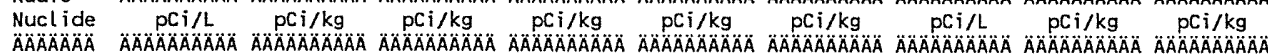

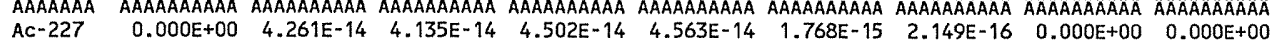

$\begin{array}{llllllllll}\mathrm{A}-227 & 0.000 \mathrm{E}+00 & 3.006 \mathrm{E}-01 & 3.006 \mathrm{E}-01 & 3.028 \mathrm{E}-01 & 3.007 \mathrm{E}-01 & 4.464 \mathrm{E}-01 & 3.682 \mathrm{E}-02 & 0.000 \mathrm{E}+00 & 0.000 \mathrm{E}+00\end{array}$

$\begin{array}{llllllllll}\text { Cs-137 } & 0.000 E+00 & 5.695 E+00 & 5.695 E+00 & 5.703 E+00 & 5.696 E+00 & 1.375 E+01 & 3.075 E+00 & 0.000 E+00 & 0.000 E+00\end{array}$

$\begin{array}{lllllllllll}\text { EU-152 } & 0.000 E+00 & 3.385 E-01 & 3.388 E-01 & 3.398 E-01 & 3.389 E-01 & 1.815 E-01 & 4.316 E-03 & 0.000 E+00 & 0.000 E+00\end{array}$

$\begin{array}{llllllllll}\text { Gd-152 } & 0.000 E+00 & 9.006 E-15 & 9.014 E-15 & 8.982 E-15 & 9.013 E-15 & 4.827 E-15 & 4.594 E-17 & 0.000 E+00 & 0.000 E+00\end{array}$

$\begin{array}{lllllllllll}\mathrm{Pa}-231 & 0.000 \mathrm{E}+00 & 1.525 \mathrm{E}-12 & 1.532 \mathrm{E}-12 & 1.493 \mathrm{E}-12 & 1.507 \mathrm{E}-12 & 8.872 \mathrm{E}-13 & 8.262 \mathrm{E}-16 & 0.000 \mathrm{E}+00 & 0.000 \mathrm{E}+00\end{array}$

Pu-239 $\quad 0.000 E+00 \quad 1.240 E-01 \quad 1.243 E-01 \quad 1.243 E-01 \quad 1.243 E-01 \quad 7.040 E-03 \quad 6.878 E-05 \quad 0.000 E+00 \quad 0.000 E+00$

PU 240 O.000E+00 $2.997 E-0233004 E-023.004 E-023.004 E-02 \quad 1.702 E-03 \quad 1.663 E-050.000 E+000.000 E+00$

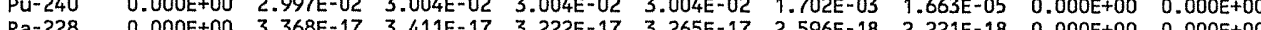

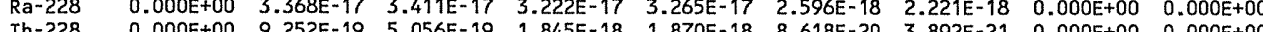

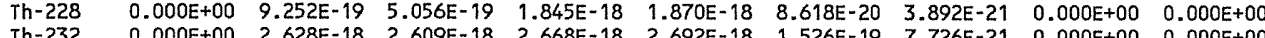

$\begin{array}{cccccccccc}\text { Th-232 } & 0.000 E+00 & 2.628 E-18 & 2.609 E-18 & 2.668 E-18 & 2.692 E-18 & 1.526 E-19 & 7.726 E-21 & 0.000 E+00 & 0.000 E+00\end{array}$

$\begin{array}{llllllllll}\mathrm{U}-235 & 0.000 \mathrm{E}+00 & 3.258 \mathrm{E}-09 & 3.267 \mathrm{E}-09 & 3.232 \mathrm{E}-09 & 3.247 \mathrm{E}-09 & 2.960 \mathrm{E}-10 & 4.987 \mathrm{E}-10 & 0.000 \mathrm{E}+00 & 0.000 \mathrm{E}+00\end{array}$

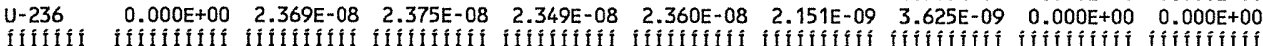

*Concentrations are at consumption time and include radioactive decay and ingrowth during storage time.

For livestock fodder, consumption time is $t$ minus meat or milk storage time.

Concentrations in the media occurring in pathways that are suppressed are calculated using the current input parameters, i.e. using parameters appearing in the input screen when the pathways are active.

$\begin{array}{ll}\text { Attachment } \frac{10}{\text { S.W. Clark }} & \text { Sheet No. } 6 \text { of } 10 \\ \text { Originator: } & \text { Date } \\ \text { Chk'd By } & \text { Date } \\ \text { Calc. No. Perrott } & \text { Rev. No. }\end{array}$




\section{ATTACHMENT 10}

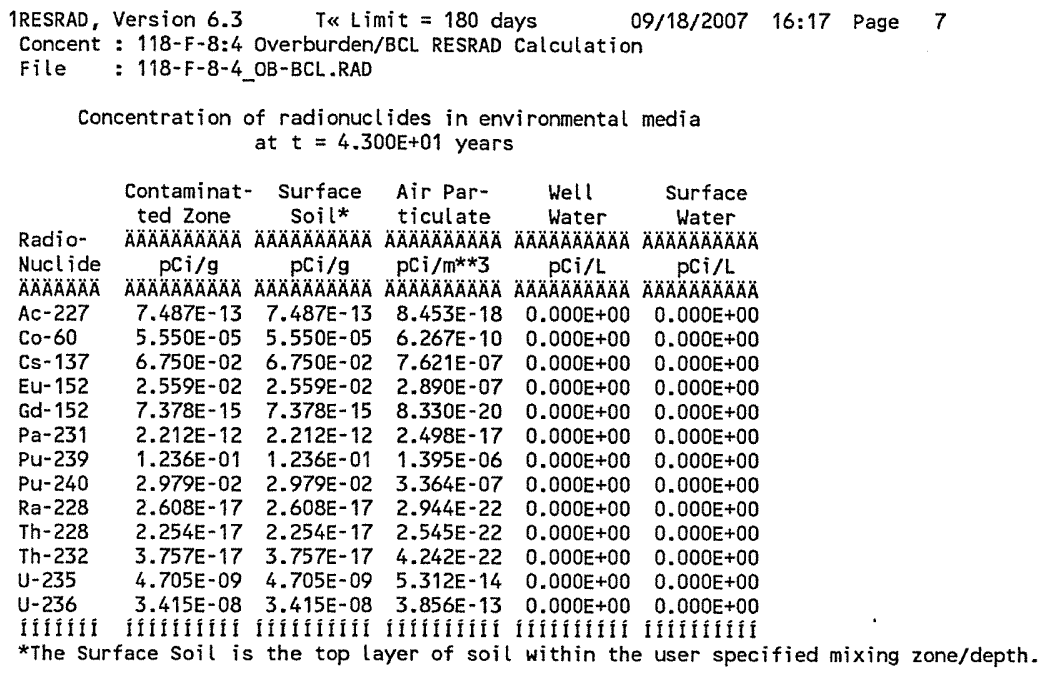

Concentrations in the media occurring in pathways that are suppressed are calculated using the current input parameters, i.e. using parameters appearing in the input screen when the pathways are active.

\begin{tabular}{|c|c|c|c|c|c|c|c|c|c|}
\hline \multicolumn{10}{|c|}{$\begin{array}{c}\text { Concentration of radionuclides in foodstuff media } \\
\text { at } t=4.300 E+01 \text { years* }\end{array}$} \\
\hline & $\begin{array}{l}\text { Drinking } \\
\text { Water }\end{array}$ & $\begin{array}{l}\text { Nonleafy } \\
\text { Vegetable }\end{array}$ & $\begin{array}{l}\text { Leafy } \\
\text { Vegetable }\end{array}$ & $\begin{array}{l}\text { Fodder } \\
\text { Meat }\end{array}$ & $\begin{array}{l}\text { Fodder } \\
\text { Milk }\end{array}$ & Meat & Milk & Fish & Crustacea \\
\hline adio & 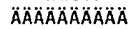 & 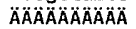 & $\triangle A A A O A A A A A B A A$ & 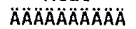 & $\triangle A B A O A ̈ A ̈ A ̈ A B A ̈ A ̈$ & $\triangle A O A O A O A O A O A O A ̈ A$ & $\triangle A \cap A ̈ A \cap A ̈ A O A O A Z A ̈$ & $\triangle A \triangle A A O A O A O A O A O A ̈$ & $\triangle \triangle A A A A B A B A B A A O A$ \\
\hline $\begin{array}{l}\text { Nucl ide } \\
\text { ABABÄÄ }\end{array}$ & $\begin{array}{c}\text { PCi/L } \\
\text { AAAAAOAOAA }\end{array}$ & $\begin{array}{c}\mathrm{pCi} / \mathrm{kg} \\
A B A B A B A\end{array}$ & $\begin{array}{c}p C i / k g \\
0 A B A\end{array}$ & $\begin{array}{c}\mathrm{pCi} / \mathrm{kg} \\
\end{array}$ & $\mathrm{pCi} / \mathrm{kg}$ & $\mathrm{pCi} / \mathrm{kg}$ & $\begin{array}{c}p C i / L \\
000\end{array}$ & $\mathrm{pci} / \mathrm{kg}$ & $\mathrm{pCi} / \mathrm{kg}$ \\
\hline$A C-227$ & $0.000 E+00$ & $1.892 E-12$ & $1.875 \mathrm{E}-12$ & $1.932 E-12$ & $1.939 E-12$ & $3.270 E-14$ & $9.618 \mathrm{E}-15$ & $0,00 \mathrm{E}+00$ & 1 0 OOE+OO \\
\hline & $0.000 E+00$ & $4.440 \mathrm{E}-03$ & $4.440 E-03$ & 4.473E-03 & $4.442 \mathrm{E}-03$ & $6.594 E-03$ & $5.440 E-04$ & $0.000 E+00$ & $0.000 E+00$ \\
\hline Cs-137 & $0.000 E+00$ & $2.700 E+00$ & $2.700 E+00$ & $2.704 \mathrm{E}+00$ & $2.700 E+00$ & $6.521 E+00$ & $1.458 \mathrm{E}+00$ & $0.000 E+00$ & $0.000 E+00$ \\
\hline Eu-152 & $0.000 E+00$ & $6.400 E-02$ & $6.406 \mathrm{E}-02$ & $6.425 \mathrm{E}-02$ & $6.408 \mathrm{E}-02$ & $3.431 \mathrm{E}-02$ & $8.160 E-04$ & $0.000 E+00$ & $0.000 \mathrm{E}+00$ \\
\hline$G d-152$ & $0.000 E+00$ & $1.845 E-14$ & $1.847 \mathrm{E}-14$ & $1.846 E-14$ & $1.847 \mathrm{E}-14$ & $9.890 E-15$ & $9.410 \mathrm{E}-17$ & $0.000 E+00$ & $0.000 E+00$ \\
\hline $\mathrm{Pa}-231$ & $0.000 E+00$ & $2.210 E-11$ & $2.213 E-11$ & $2.199 E-11$ & $2.204 E-11$ & $1.299 E-11$ & $1.169 \mathrm{E}-14$ & $0.000 E+00$ & $0.000 \mathrm{E}+00$ \\
\hline Pu- 239 & $0.000 E+00$ & $1.236 E-01$ & $1.239 \mathrm{E}-01$ & $1.240 E-01$ & $1.240 E-01$ & 7.021E-03 & $6.860 \mathrm{E}-05$ & $0.000 E+00$ & $0.000 E+00$ \\
\hline Pu-240 & $0.000 E+00$ & $2.981 E-02$ & $2.988 E-02$ & $2.989 \mathrm{E}-02$ & $2.989 \mathrm{E}-02$ & $1.693 \mathrm{E}-03$ & $1.654 \mathrm{E}-05$ & $0.000 E+00$ & $0.000 E+00$ \\
\hline $\mathrm{Ra}-228$ & $0.000 E+00$ & $1.036 E-15$ & $1.043 \mathrm{E}-15$ & $1.019 E-15$ & $1.022 E-15$ & $8.174 \mathrm{E}-17$ & $6.920 E-17$ & $0.000 \mathrm{E}+00$ & $0.000 E+00$ \\
\hline Th-228 & $0.000 E+00$ & $3.652 E-17$ & $2.361 \mathrm{E}-17$ & $6.620 \mathrm{E}-17$ & $6.638 \mathrm{E}-17$ & $3.154 \mathrm{E}-18$ & $1.431 \mathrm{E}-19$ & $0.000 E+00$ & $0.000 E+00$ \\
\hline Th-232 & $0.000 E+00$ & $3.769 E-17$ & $3.769 E-17$ & $3.791 E-17$ & $3.800 E-17$ & $2.153 \mathrm{E}-18$ & $1.061 \mathrm{E}-19$ & $0.000 E+00$ & $0.000 E+00$ \\
\hline$u-235$ & $0.000 E+00$ & $1.176 E-08$ & $1.178 \mathrm{E}-08$ & $1.175 E-08$ & $1.176 \mathrm{E}-08$ & $1.071 E-09$ & $1.799 \mathrm{E}-09$ & $0.000 E+00$ & $0.000 E+00$ \\
\hline & $0.000 E+00$ & $8.537 \mathrm{E}-08$ & $8.548 \mathrm{E}-08$ & $8.529 E-08$ & $8.538 \mathrm{E}-08$ & 7.774E-09 & $1.306 \mathrm{E}-08$ & $0.000 E+00$ & $0.000 \mathrm{E}+00$ \\
\hline & & & & & & & & & ÎIIIIIIIII \\
\hline
\end{tabular}

For livestock fodder, consumption time is $t$ minus meat or milk storage time.

Concentrations in the media occurring in pathways that are suppressed are calculated using the current input parameters, i.e. using parameters appearing in the input screen when the pathways are active.

\begin{tabular}{|c|c|}
\hline Attachment & Sheet No. 7 of 10 \\
\hline Originator: S.W. Clark & Date \\
\hline Chk'd By M.W. Perrott & Date \\
\hline $0100 F-C A-V 0303$ & Rev. No. \\
\hline
\end{tabular}




\section{ATTACHMENT 10}

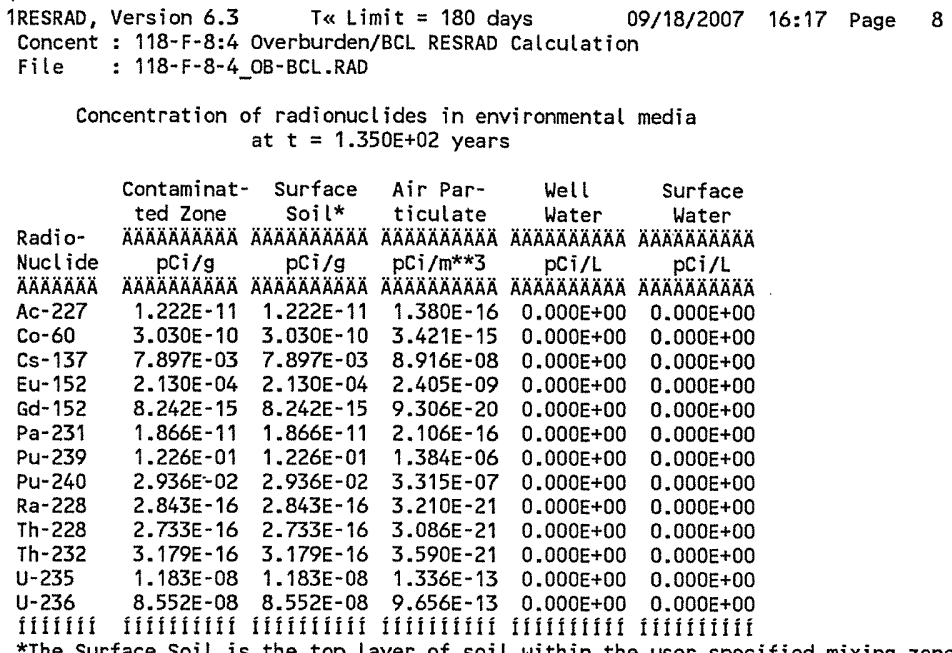

Concentrations in the media occurring in pathways that are suppressed are calculated using the current input parameters, i.e. using parameters appearing in the input screen when the pathways are active.

Concentration of radionuclides in foodstuff media at $\mathrm{t}=1.350 \mathrm{E}+02$ years*

\begin{tabular}{|c|c|c|c|c|c|c|c|c|c|}
\hline & $\begin{array}{c}\text { Drinking } \\
\text { Water }\end{array}$ & $\begin{array}{l}\text { Nonleafy } \\
\text { Vegetable }\end{array}$ & $\begin{array}{c}\text { Leafy } \\
\text { Vegetable }\end{array}$ & $\begin{array}{l}\text { Fodder } \\
\text { Meat }\end{array}$ & $\begin{array}{l}\text { Fodder } \\
\text { Milk }\end{array}$ & Meat & Milk & Fish & Crustacea \\
\hline dio & $\triangle A \cap A ̈ A \cap A \cap A \cap A \cap A ̈ A ̈$ & $\triangle A \cap A \cap A A A A A A A A A$ & $\triangle A \cap A ̈ A A A A A B A B A$ & $\triangle A \cap A A A B A A A O A A$ & $\triangle A A A A A A A A O A O A$ & $\triangle A A A A O A A A A O A ̈ A$ & ӒÄÄAAAAÄÄ & $\triangle A \triangle A O A A A O A O A$ & ÄÄÄÄÄÄÄÄÄ \\
\hline & $p C i / L$ & $\mathrm{pCi} / \mathrm{kg}$ & nci & & & & & & \\
\hline & A $A A A A A A A O A ̈$ & $\triangle A A A A A A A A B A ̈$ & 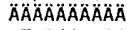 & ÄAAAAAAOAOAOA & $\triangle A A A A A A A A A O A O A$ & $\triangle A A A A A A A A A A A A$ & 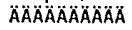 & 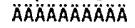 & $\triangle A O A A A A A A A A A A B$ \\
\hline$c-227$ & $0.000 E+00$ & $3.074 E-11$ & $3.061 \mathrm{E}-11$ & $3.112 E-11$ & $3.115 E-11$ & $3.557 E-13$ & $1.565 E-13$ & $0.000 E+00$ & $0.000 E+00$ \\
\hline $0-60$ & $0.000 E+00$ & $2.424 E-08$ & $2.424 E-08$ & $2.442 E-08$ & $2.425 E-08$ & $3.600 \mathrm{E}-08$ & $2.970 E-09$ & $0.000 E+00$ & $0.000 E+00$ \\
\hline s-137 & $0.000 E+00$ & $3.159 E-01$ & $3.159 \mathrm{E}-01$ & $3.163 E-01$ & 3.159E-01 & $7.630 \mathrm{E}-01$ & $1.706 \mathrm{E}-01$ & $0.000 E+00$ & $0.000 E+00$ \\
\hline Eu-152 & $0.000 E+00$ & $5.326 E-04$ & $5.331 E-04$ & 5.347E-04 & $5.332 E-04$ & $2.855 E-04$ & $6.791 E-06$ & $0.000 E+00$ & $0.000 E+00$ \\
\hline Gd-152 & $0.000 E+00$ & $2.061 \mathrm{E}-14$ & $2.063 E-14$ & $2.063 E-14$ & $2.063 E-14$ & $1.105 E-14$ & $1.051 E-16$ & $0.000 E+00$ & $0.000 E+00$ \\
\hline $\mathrm{Pa}-23$ & $0.000 E+00$ & $1.865 \mathrm{E}-10$ & $1.866 \mathrm{E}-10$ & $1.863 E-10$ & $1.864 E-10$ & $1.099 E-10$ & $9.816 E-14$ & $0.000 E+00$ & $0.000 E+00$ \\
\hline Pu-239 & $0.000 E+00$ & 1.22 & $E-01$ & $1.230 E-01$ & $1.230 E-01$ & $6.967 E-03$ & $6.807 E-05$ & $0.000 E+00$ & $0.000 E+00$ \\
\hline Pu- 240 & $0.000 E+00$ & $2.938 E-02$ & $2.944 E-02$ & $2.945 \mathrm{E}-02$ & $2.945 \mathrm{E}-02$ & $1.668 \mathrm{E}-03$ & $1.630 \mathrm{E}-05$ & $0.000 E+00$ & $0.000 E+00$ \\
\hline $\mathrm{Ra}-22$ & $0.000 \mathrm{E}+00$ & $1.131 \mathrm{E}-14$ & $1.137 E-14$ & $1.118 E-14$ & $1.119 E-14$ & $8.965 E-16$ & $7.573 E-16$ & $0.000 E+00$ & $0.000 E+00$ \\
\hline Th-228 & $0.000 E+00$ & $4.260 E-16$ & $2.851 E-16$ & $7.532 E-16$ & $7.538 E-16$ & $3.608 E-17$ & $1.641 \mathrm{E}-18$ & $0.000 E+00$ & $0.000 E+00$ \\
\hline Th-232 & $0.000 E+00$ & $3.184 E-16$ & $3.189 E-16$ & $3.195 E-16$ & $3.197 E-16$ & $1.811 \mathrm{E}-17$ & $8.872 E-19$ & $0.000 \mathrm{E}+00$ & $0.000 E+00$ \\
\hline$U-235$ & $0.000 E+00$ & $2.958 \mathrm{E}-08$ & $2.961 E-08$ & $2.960 \mathrm{E}-08$ & $2.961 E-08$ & $2.695 E-09$ & $4.526 \mathrm{E}-09$ & $0.000 E+00$ & $0.000 E+00$ \\
\hline & $0 E+00$ & 2. $139 \mathrm{E}-07$ & $2.141 \mathrm{E}-07$ & $2.140 \mathrm{E}-07$ & 2.141E-07 & $1.949 \mathrm{E}-08$ & $3.272 E-08$ & $0.000 E+00$ & $0.000 E+00$ \\
\hline & & & & & & & & & İíîî́tî \\
\hline
\end{tabular}

Concentrations in the media occurring in pathways that are suppressed are calculated using the current input parameters, i.e. using parameters appearing in the input screen when the pathways are active.

\begin{tabular}{|c|c|}
\hline Attachment & Sheet No \\
\hline Originator: S.W. Clark & Date \\
\hline M.W. Perrott & Date \\
\hline $0100 F-C A-V 0303$ & Rev. No. \\
\hline
\end{tabular}




\section{ATTACHMENT 10}

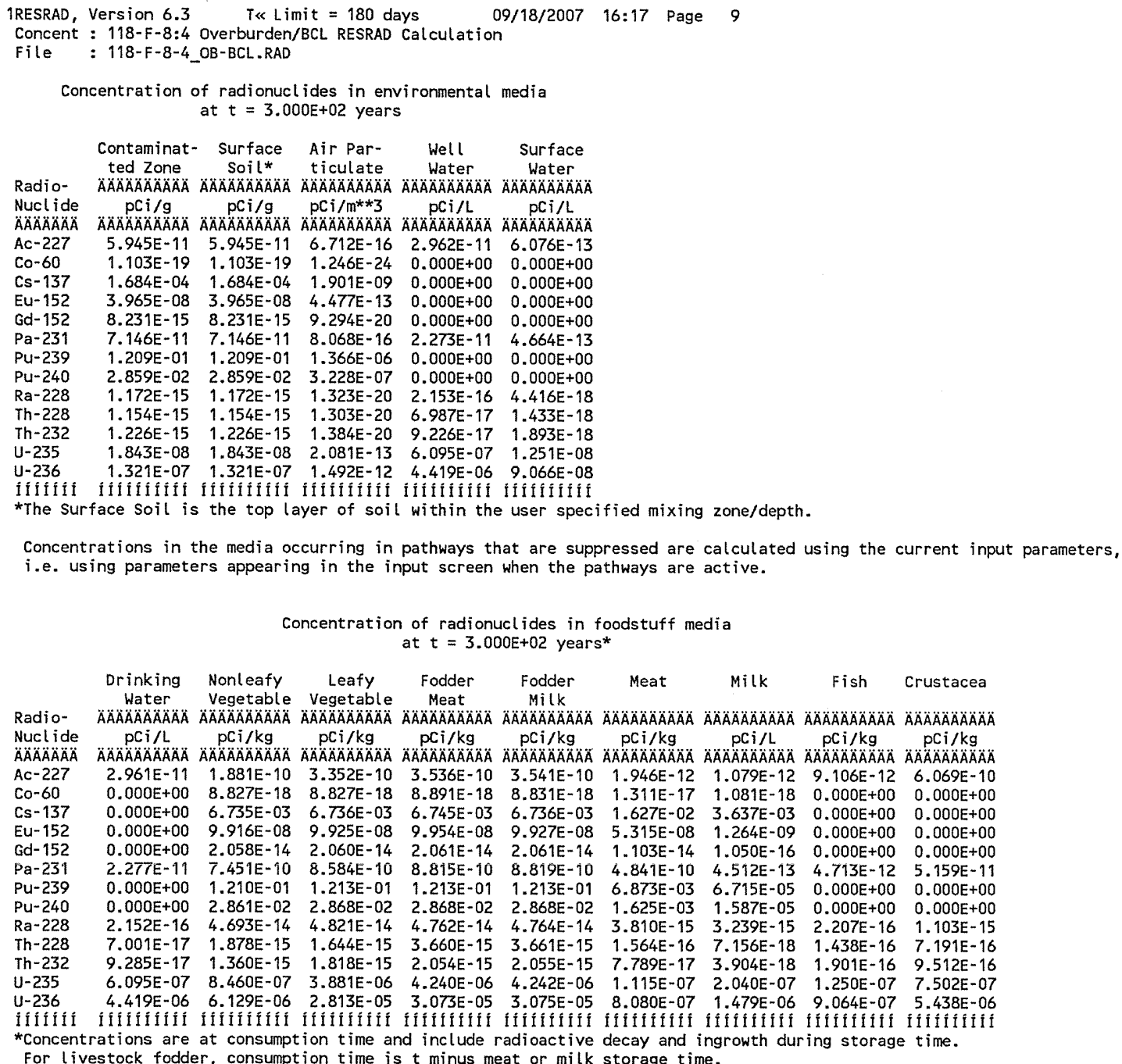

Concentrations in the media occurring in pathways that are suppressed are calculated using the current input parameters, i.e. using parameters appearing in the input screen when the pathways are active.

\begin{tabular}{|c|c|}
\hline Attachment & t $N$ \\
\hline Originator: S.W. Clark & Dat \\
\hline M. W. Perrott & Date \\
\hline $0100 \mathrm{~F}-\mathrm{CA}-\mathrm{V} 0303$ & Rev. No. \\
\hline
\end{tabular}




\section{ATTACHMENT 10}

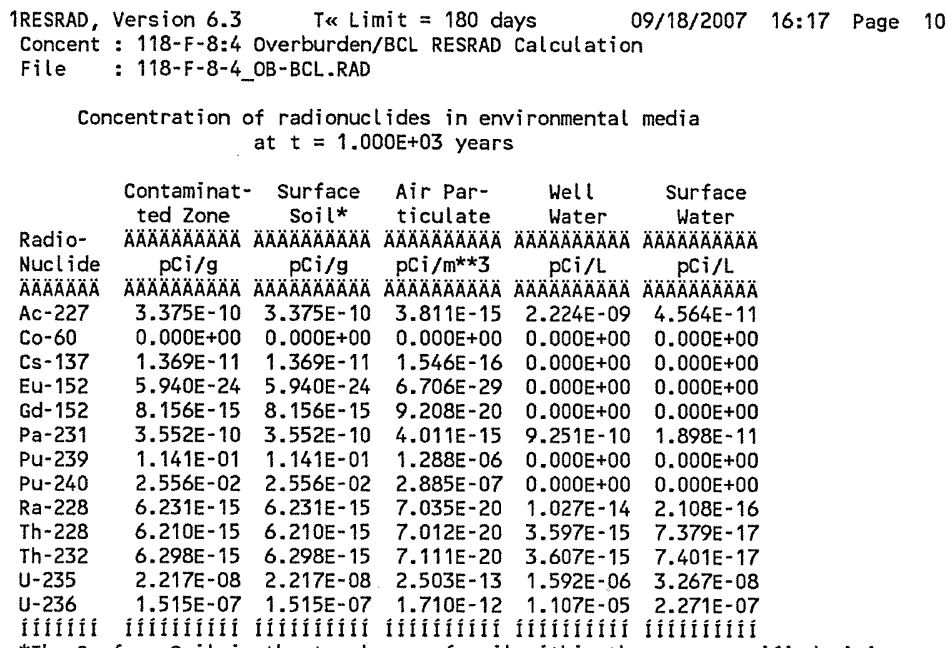

Concentrations in the media occurring in pathways that are suppressed are calculated using the current input parameters, i.e. using parameters appearing in the input screen when the pathways are active.

Concentration of radionuclides in foodstuff media at $\mathrm{t}=1.000 \mathrm{E}+03$ years*

\begin{tabular}{|c|c|c|c|c|c|c|c|c|c|}
\hline & $\begin{array}{c}\text { Drinking } \\
\text { Water }\end{array}$ & $\begin{array}{l}\text { Nonleafy } \\
\text { Vegetable }\end{array}$ & $\begin{array}{c}\text { Leafy } \\
\text { Vegetable }\end{array}$ & $\begin{array}{l}\text { Fodder } \\
\text { Meat }\end{array}$ & $\begin{array}{l}\text { Fodder } \\
\text { Milk }\end{array}$ & Meat & Milk & Fish & Crustacea \\
\hline dio- & $\triangle A A A A A A A A A B A$ & AÄÄÄAAAOAAÄ & $\triangle A A A A O A A A A O A$ & AAAAAAAAAAAA & $\triangle A A O A A A A A O A O A$ & ÄÄÄAAAAOÄÄ & $\triangle A A \triangle A O A Z A ̈ A ̈ A ̈ A ̈$ & $\triangle A O A A O A A O A O A O A O A$ & $\triangle A A A A A A A A A B A$ \\
\hline & $p C i / L$ & $\mathrm{pci} / \mathrm{kg}$ & $\mathrm{pCi} / \mathrm{kg}$ & $\mathrm{pCi} / \mathrm{kg}$ & $\mathrm{pci} / \mathrm{kg}$ & $\mathrm{pCi} / \mathrm{kg}$ & $\mathrm{pCi} / \mathrm{L}$ & $\mathrm{pCi} / \mathrm{kg}$ & $\mathrm{pCi} / \mathrm{kg}$ \\
\hline ÄÄÄÄÄÄ & $\triangle A A A A A A A B A ̈ A ̈$ & AAÄÄÄAOÄÄ & ÄAAÄÄÄAÄÄ & $\triangle A A A A A A A A A A B$ & $\triangle A A A A A A A A B A O A$ & $\triangle A O A A A A A A A A A A B$ & $\triangle A A A A A A A A O A O A$ & $\triangle A A A A A A B A A A O A ̈$ & $\triangle A O A A A A A A O A A$ \\
\hline$A C-227$ & $2.224 E-09$ & $3.765 E-09$ & $1.484 E-08$ & $1.615 E-08$ & $1.615 E-08$ & $3.536 E-11$ & $2.826 E-11$ & $6.842 E-10$ & $4.561 E-08$ \\
\hline & $0.000 E+00$ & $0.000 E+00$ & $0.000 E+00$ & $0.000 E+00$ & $0.000 \mathrm{E}+00$ & $0.000 E+00$ & $0.000 E+00$ & $0.000 E+00$ & $0.000 E+00$ \\
\hline Cs- 137 & $0.000 E+00$ & $5.476 E-10$ & $5.476 E-10$ & $5.483 E-10$ & $5.477 \mathrm{E}-10$ & $1.323 \mathrm{E}-09$ & $2.957 E-10$ & $0.000 E+00$ & $0.000 E+00$ \\
\hline Eu- 152 & $0.000 E+00$ & $1.485 E-23$ & $1.487 E-23$ & $1.491 \mathrm{E}-23$ & $1.487 E-23$ & $7.962 \mathrm{E}-24$ & $1.894 \mathrm{E}-25$ & $0.000 E+00$ & $0.000 E+00$ \\
\hline Gd-152 & $0.000 E+00$ & $2.039 \mathrm{E}-14$ & $2.041 E-14$ & $2.042 E-14$ & $2.042 E-14$ & $1.093 E-14$ & $1.040 \mathrm{E}-16$ & $0.000 E+00$ & $0.000 E+00$ \\
\hline 231 & $9.252 E-10$ & $4.772 E-09$ & $9.380 \mathrm{E}-09$ & $9.958 E-09$ & $9.959 E-09$ & 4.505E-09 & $4.397 \mathrm{E}-12$ & $1.899 \mathrm{E}-10$ & $2.088 \mathrm{E}-09$ \\
\hline & $0.000 E+00$ & $1.142 E-01$ & $1.145 \mathrm{E}-01$ & $1.145 \mathrm{E}-01$ & $1.145 E-01$ & $6.484 E-03$ & $6.335 E-05$ & $0.000 E+00$ & $0.000 E+00$ \\
\hline $1-240$ & $0.000 E+00$ & $2.557 E-02$ & $2.563 \mathrm{E}-02$ & $2.564 E-02$ & $2.564 E-02$ & $1.452 E-03$ & $1.419 E-05$ & $0.000 E+00$ & $0.000 E+00$ \\
\hline 228 & $1.027 E-14$ & $2.617 E-13$ & $3.140 E-13$ & $3.158 \mathrm{E}-13$ & $3.158 E-13$ & $2.494 E-14$ & $2.212 E-14$ & $1.053 E-14$ & $5.266 \mathrm{E}-14$ \\
\hline Th-228 & $3.603 E-15$ & $1.441 \mathrm{E}-14$ & $2.918 E-14$ & $4.358 E-14$ & $4.359 E-14$ & $1.104 E-15$ & $5.230 E-17$ & $7.401 E-15$ & $3.700 E-14$ \\
\hline & $3.609 E-15$ & $1.107 \mathrm{E}-14$ & $2.903 E-14$ & $3.165 E-14$ & $3.165 E-14$ & $5.535 E-16$ & $2.783 E-17$ & $7.403 E-15$ & $3.702 \mathrm{E}-14$ \\
\hline & $1.592 E-06$ & $2.146 \mathrm{E}-06$ & $1.008 \mathrm{E}-05$ & $1.103 E-05$ & $1.103 E-05$ & $2.859 E-07$ & $5.236 \mathrm{E}-07$ & $3.267 \mathrm{E}-07$ & $1.960 \mathrm{E}-06$ \\
\hline & $1.107 E-05$ & $1.491 \mathrm{E}-05$ & $7.004 \mathrm{E}-05$ & $7.670 E-05$ & $7.670 E-05$ & $1.987 E-06$ & $3.639 E-06$ & $2.271 E-06$ & $1.363 \mathrm{E}-05$ \\
\hline & & & & & & & & & 80. \\
\hline
\end{tabular}

For livestock fodder, consumption time is $t$ minus meat or milk storage time.

Concentrations in the media occurring in pathways that are suppressed are calculated using the current input parameters, i.e. using parameters appearing in the input screen when the pathways are active.

\begin{tabular}{|c|c|}
\hline Attachment & Sheet No. 10 of 10 \\
\hline Originator: S.W. Clark & Date \\
\hline Chk'd By M.W. Perrott & Date \\
\hline Calc. No. $0100 \mathrm{~F}-\mathrm{CA}-\mathrm{V} 0303$ & Rev. No. \\
\hline
\end{tabular}




\section{CALCULATION COVER SHEET}

Project Title: 100-F Field Remediation

Job No. 14655

Area: $100-\mathrm{F}$

Discipline: Environmental

${ }^{*}$ Calculation No: 0100F-CA-V0294

Subject: 118-F-8:4 105-F Fuel Storage Basin Cleanup Verification 95\% UCL Calculation

Computer Program: Excel

Program No: Excel 2003

The attached calculations have been generated to document compliance with established cleanup levels. These calculations should be used in conjunction with other relevant documents in the administrative record.

Committed Calculation 区

Preliminary

Superseded

Voided

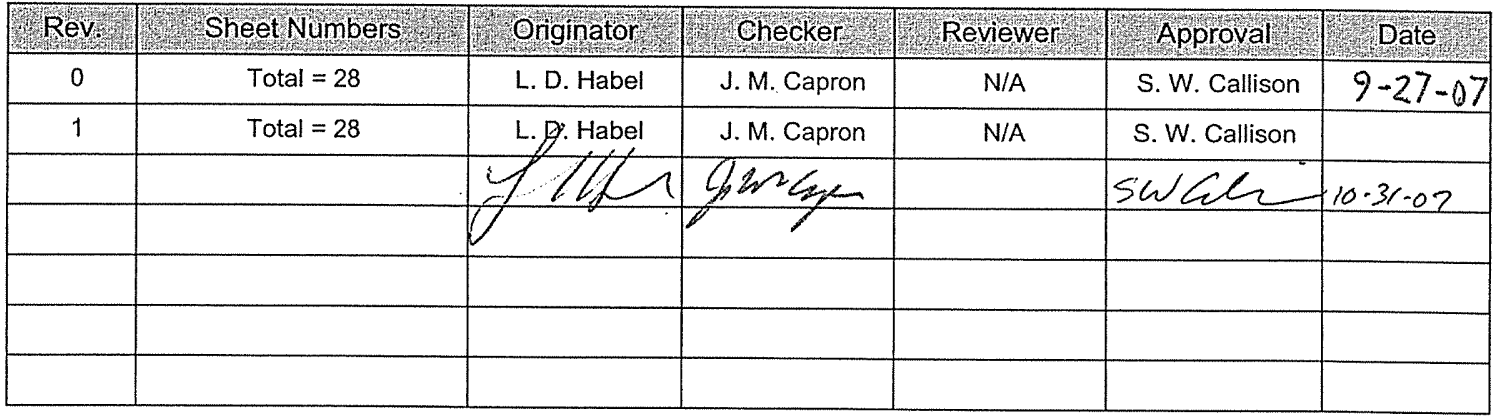

b ga

SUMMARY OF REVISION

\begin{tabular}{|l|l|}
\hline 1 & $\begin{array}{l}\text { Corrected RPLcalculations, sheets 18, } 19 \text { and } 20 . \text { Added flags from 3rd party validation for SDG K0816. } \\
\text { Affected sheets } 9 \text { and } 10 \text { and attachment } 1 \text { sheets } 1,3,4 \text { and } 6 .\end{array}$ \\
\hline & \\
\hline
\end{tabular}




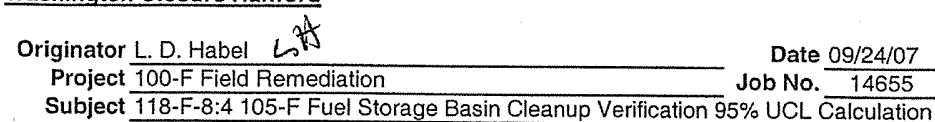

Summary

1 Purpose:

2 Calculate the 95\% upper confidence limit (UCL) values to evaluate compliance with cleanup standards for the subject site. Also, 3 perform the Washington Administrative Code (WAC) 173-340-740(7)(e) 3-part test for each nonradioactive contaminant of concern (COC) and calculate the relative percent difference (RPD) for primary-duplicate and primary-split sample pairs, as necessary.

\section{Table of Contents:}

Sheets 1 to 4 - Summary

Sheets 5 to 8 - 118-F-8:4 Excavation Shallow Zone Statistical Calculations

Sheets 9 to 12 - 118-F-8:4 Excavation Deep Zone Statistical Calculations

Sheets 13 to $16-118-F-8: 4$ Overburden/BCL Material Statistical Calculations

Sheet 17 - Ecology Software (MTCAStat) Results (Overburden/BCL Material)

Sheets 18 to 20 -Split/Duplicate Analysis

Attachment 1 - 118-F-8:4 Verification Sampling Results (7 sheets)

Given/References:

1) Sample Results (Attachment 1).

2) Background values and remedial action goals (RAGs) are taken from DOE-RL (2005), DOE-RL (2001), and Ecology (2005).

3) $\mathrm{BHI}, 2004$, Cleanup Verification Package for the 118-F-8:1, 105-F Reactor Below-Grade Structures and Underlying Soils; the 118-F-8:3, 105-F Fuel Storage Basin Underlying Soils; and the 100-F-10 French Drain, CVP-2003-00017, Rev. 0, Bechtel Hanford, Inc., Richland, Washington.

4) DOE-RL, 1996, Hanford Site Background: Part 2, Soil Background for Radionuclides, DOE/RL-96-12, Rev. 0, U.S. Department of Energy, Richland Operations Office, Richland, Washington.

5) DOE-RL, 2000, Sampling and Analysis Plan (SAP) for the 105-Phase IV Fuel Storage Basin, DOE/RL-2000-54, Rev. O, U.S. Department of Energy, Richland Operations Office, Richland, Washington.

6) DOE-RL, 2001, Hanford Site Background: Part 1, Soil Background for Nonradioactive Analytes, DOE/RL-92-24, Rev. 4, U.S. Department of Energy, Richland Operations Office, Richland, Washington.

7) DOE-RL, 2005, Remedial Design Report/Remedial Action Work Plan for the 100 Area (RDR/RAWP), DOE/RL-96-17, Rev. 5, U.S. Department of Energy, Richland Operations Office, Richland, Washington.

8) Ecology, 1992, Statistical Guidance for Ecology Site Managers, Publication \#92-54, Washington Department of Ecology, Olympia, Washington.

9) Ecology, 1993, Statistical Guidance for Ecology Site Managers, Supplement S-6, Analyzing Site or Background Data with Below-detection Limit or Below-PQL Values (Censored Data Sets), Publication \#92-54, Washington Department of Ecology, Olympia, Washington.

10) EPA, 1994b, USEPA Contract Laboratory Program National Functional Guidelines for Inorganic Data Review, EPA 540/R-94/013. U.S. Environmental Protection Agency, Washington, D.C.

11) WAC 173-340, 1996, "Model Toxic Control Act - Cleanup," Washington Administrative Code.

Solution:

Calculation methodology is described in Ecology Pub. \#92-54 (Ecology 1992, 1993), below, and in the RDR/RAWP (DOE-RL 2005). Use data from attached worksheets to perform the $95 \%$ UCL calculation for each analyte, the

WAC 173-340-740(7)(e) 3-part test for nonradionuclides, and the RPD calculations.

Calculation Description:

The subject calculations were performed on data from soil verification samples from the subject waste site. The data were entered into an EXCEL 2003 spreadsheet and calculations performed by using the built-in spreadsheet functions and/or creating formulae within the cells. The statistical evaluation of data for use in accordance with the RDR/RAWP (DOE-RL 2005) is documented by this calculation. Duplicate and split RPD results are used in evaluation of data quality within the CVP for this site. 


\section{Washington Closure Hanford}

Originator L. D. Habel L L

Project 100-F Field Remediation

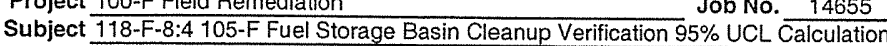

CALCULATION SHEET

UCL Methodology:

118-F-8:4 is a portion of the 118-F-8:3 waste site that was deferred for remediation (BHI 2004). The COC list for $118-\mathrm{F}-8: 4$ is the same as 118-F-8:3.

The COCs are: Am-241, Ba-133, C-14, Co-60, Cs-137, Eu-152, Eu-154, Eu-155, H-3, Ni-63, Pu-238, Pu-239/240, Sr-90, U-234, U-235, U-238, Cr+6, Hg, Pb, Ba, and PCBs (DOE-RL 2000).

Selenium was among other analytes included in the analysis for Barium and Lead. Since it was detected in one of the samples it

has been included in the data analysis presented herein.

For radionuclides, the reported value is used when calculating the $95 \%$ UCL, including results less than the minimum detectable activity (MDA). When the laboratory does not report a value below the MDA, $1 / 2$ of the MDA is used in the calculation. For nonradionuclides reported as being undetected (signified by a " $U$ " in the qualifier column), $1 / 2$ the detection limit is used for calculation of the statistics (Ecology 1993): For the statistical evaluation of duplicate sample pairs, the samples are averaged before being included in the data set, after adjustments for censored data as described above.

For nonradionuclide small data sets $(n<10)$ and all radionuclide data sets, statistical calculations are performed assuming nonparametric distribution, without further testing for distributional form. For non-radionuclide data sets of ten or greater, distributional testing and calculation of the 95\% UCL is done using Ecology's MTCAStat 3.0 software (Ecology 1993). Due to differences in addressing censored data between the RDR/RAWP (DOE-RL 2005b) and MTCAStat coding and due to a limitation in the MTCAStat coding (no direct capability to address variable quantitation limits within a data set), substitutions for censored data are performed before software input and the resulting input set is treated as uncensored. The input set to the MTCAStat software is provided under the heading "Statistical Computation Input Data" on the datasheets contained herein.

All calculations for Americium-241 and uranium isotopes were performed using data from isotope-specific analyses rather than data resulting from gamma spectroscopy.

The WAC 173-340-740(7)(e) 3-part test is performed for non-radionuclide analytes only and determines if:

1) the $95 \%$ UCL exceeds the most stringent cleanup limit for each COC,

2) greater than $10 \%$ of the raw data exceed the most stringent cleanup limit for each COC

3) the maximum value of the raw data set exceeds two times the most stringent cleanup limit for each COC.

The RPD values are evaluated for analytes detected in a primary-duplicate or primary-split sample pair for the purposes of data quality assessment within the CVP. The RPD is calculated when both the primary value and the duplicate value for a given analyte are above detection limits and are greater than 5 times the target detection limit (TDL). The RPD calculations use the following formula:

$$
\mathrm{RPD}=[|\mathrm{M}-\mathrm{S}| /((\mathrm{M}+\mathrm{S}) / 2)]^{*} 100
$$

where, $\quad M=$ main sample value

$$
S=\text { split (or duplicate) sample value }
$$

For quality assurance/quality control (QAVC) split and duplicate RPD calculations, a value less than $30 \%$ indicates the data compare favorably. For regulatory splits, a threshold of $35 \%$ is used (EPA 1994b). If the RPD is greater than $30 \%$ (or $35 \%$ for regulatory split data), further investigation regarding the usability of the data is performed. To assist in the identification of anomalous sample pairs, when an analyte is detected in the primary or duplicate/split sample, but was quantified at less than 5 times the TDL in one or both samples, an additional parameter is evaluated. In this case, if the difference between the primary and duplicate/split results exceed a control limit of 2 times the TDL, further assessment regarding the usability of the data is performed. Additional discussion is provided in the data quality assessment section of the applicable CVP, as necessary.

Remarks:

Initial results (J15574) indicated that the stockpiled soil in area $\mathrm{A} 1$ of the overburden/BCL decision unit had detections of carbon-14 in excess of the remedial action goal. The contaminated soil was removed and the sample was retaken. The sample (J15690) was analyzed for carbon-14, strer-tond and tritium. Carbon-14 results replaced the initial $\mathrm{J15574}$ results in statistical calculations. 


\section{Washington Closure Hanford}

CALCULATION SHEET

Originator L.D. Habel LO

Project 100-F Field Remediation

Date 09/24/07

Caic. No. 0100F-CA-V0294

Job No. 14655

Rev. No.

Subject $118-\mathrm{F}-8: 4$ 105-F Fuel Storage Basin Cleanup Verification 95\% UCL Calculation

Checked J.M. Capron tore Date

Sheet No.

0

$09 / 24 / 07$

1 Summary (continued)

3 Conclusion:

4 The results presented in the summary tables that follow are for use in RESidual RADioactivity modeling and the CVP for this site.

6

8 Excavation Shallow Zone - WAC 173-340 3-Part Test for most stringent RAG:

9 Final Statistical Value $>$ Cleanup Limit? YES

$10>10 \%$ above Cleanup Limit? YES

11 Any sample $>2 x$ Cleanup Limit?

NO

13 Because of the "yes" answer to the 3-part test for lead and mercury,

14 additional evaluation of the attainment of cleanup criteria will be

15 performed.

16

17 Excavation Deep Zone - WAC 173-340 3-Part Test for most stringent RAG:

18 Final Statistical Value > Cleanup Limit? YES

$19>10 \%$ above Cleanup Limit? YES

20 Any sample $>2 \times$ Cleanup Limit? YES

21

22 Because of the "yes" answer to the 3-part test for Aroclor-1260,

23 additional evaluation of the attainment of cleanup criteria will be

24 performed.

25

26 OB/BCL - WAC 173-340 3-Part Test for most stringent RAG:

27 Final Statistical Value $>$ Cleanup Limit? NO

$28>10 \%$ above Cleanup Limit? NO

29 Any sample $>2 x$ Cleanup Limit? NO

31 All data sets evaluated meet the 3-part test criteria when compared

32 to the most stringent cleanup limit. 
Originator L.D. Habel h

Project 100 -F Field Remediation

lation

Project $\frac{100-F \text { Field Remediation }}{118-F-8: 4105-F \text { Fuel Storage Basin Cleanup Verification } 95 \% \text { UCL Calculation }}$

1 Summary (continued)

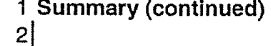

Results Summary ( $95 \%$ UCL or Maximum as appropriate)

\begin{tabular}{|c|c|c|c|c|c|c|c|c|}
\hline 3 & Analyte & $\begin{array}{c}\text { Excavation } \\
\text { Shallow Zone }\end{array}$ & $\mathbf{Q}$ & $\begin{array}{l}\text { Excavation } \\
\text { Deep Zone }\end{array}$ & $Q$ & $\mathrm{OB}-\mathrm{BCL}$ & $Q$ & Units \\
\hline 4 & Americium-241 & 0.110 & $\mathrm{U}$ & 0.081 & & 0.027 & $\mathrm{U}$ & $\mathrm{pCi} / \mathrm{g}$ \\
\hline 5 & Barium-133 & 0.020 & $\mathrm{U}$ & 0.022 & $\mathrm{U}$ & 0.018 & $\mathrm{U}$ & $\mathrm{pCi} / \mathrm{g}$ \\
\hline 6 & Carbon-14 & -0.325 & $\mathrm{U}$ & 0.070 & $\mathrm{U}$ & -0.7 & $\mathrm{U}$ & $\mathrm{pCi} / \mathrm{g}$ \\
\hline 7 & Cesium-137 & 0.281 & & 4.52 & & 0.184 & & $\mathrm{pCi} / \mathrm{g}$ \\
\hline 8 & Cobalt-60 & 0.019 & $U$ & 0.186 & & 0.016 & & $\mathrm{pCi} / \mathrm{g}$ \\
\hline 9 & Europium -152 & 0.160 & & 3.01 & & 0.240 & & $\mathrm{pCi} / \mathrm{g}$ \\
\hline 10 & Europium-154 & 0.061 & $\mathrm{U}$ & 0.181 & $U$ & 0.050 & $\mathrm{U}$ & $\mathrm{pCi} / \mathrm{g}$ \\
\hline 11 & Europium-155 & 0.050 & $\mathrm{U}$ & 0.057 & $\mathrm{U}$ & 0.044 & $\mathrm{U}$ & $\mathrm{pCi} / \mathrm{g}$ \\
\hline 12 & Nickel-63 & 1.26 & $\mathrm{U}$ & 18.7 & & 0.637 & $\mathrm{U}$ & $\mathrm{pCi} / \mathrm{g}$ \\
\hline 13 & Plutonium-238 & 0.023 & $U$ & 0.056 & U & 0.008 & $\mathrm{U}$ & $\mathrm{pCi} / \mathrm{g}$ \\
\hline 14 & Plutonium-239/240 & 0.063 & $\mathrm{U}$ & 0.520 & & 0.154 & & $\mathrm{pCi} / \mathrm{g}$ \\
\hline 15 & Strontium-90 & 0.151 & $U$ & 2.20 & & 0.036 & $\mathrm{U}$ & $\mathrm{pCi} / \mathrm{g}$ \\
\hline 16 & Tritium & 2.85 & $\mathrm{U}$ & 1.38 & $\mathrm{U}$ & 0.857 & $\mathrm{U}$ & $\mathrm{pCi} / \mathrm{g}$ \\
\hline 17 & Uranium-233/234 & $0(<B G)$ & & $0(<B G)$ & & $0(<B G)$ & & $\mathrm{mg} / \mathrm{kg}$ \\
\hline 18 & Uranium-235 & $0(<B G)$ & & $0(<B G)$ & $\mathrm{U}$ & $0(<B G)$ & & $\mathrm{mg} / \mathrm{kg}$ \\
\hline 19 & Uranium-238 & $0(<B G)$ & & $0(<B G)$ & & $D(<B G)$ & & $\mathrm{mg} / \mathrm{kg}$ \\
\hline 20 & Barium & 70.1 & & 52.6 & & 67.8 & & $\mathrm{mg} / \mathrm{kg}$ \\
\hline 21 & Hexavalent Chromium & 0.26 & & 0.26 & & 0.27 & & $\mathrm{mg} / \mathrm{kg}$ \\
\hline 22 & Lead & 9.0 & & 5.5 & & 5.5 & & $\mathrm{mg} / \mathrm{kg}$ \\
\hline 23 & Mercury & 0.41 & & 0.05 & & 0.57 & & $\mathrm{mg} / \mathrm{kg}$ \\
\hline 24 & Selenium & 1.5 & & $\overline{-}$ & $\mathrm{U}$ & - & $\mathrm{U}$ & $\mathrm{mg} / \mathrm{kg}$ \\
\hline 25 & Aroclor-1016 & 14 & U & 14 & U & 16 & $\mathrm{U}$ & $\mathrm{ug} / \mathrm{kg}$ \\
\hline 26 & Aroclor-1221 & 14 & $\mathrm{U}$ & 14 & U & 16 & $\mathrm{U}$ & $\mathrm{ug} / \mathrm{kg}$ \\
\hline 27 & Aroclor-1232 & 14 & U & 14 & U & 16 & U & $\mathrm{ug} / \mathrm{kg}$ \\
\hline 28 & Aroclor-1242 & 14 & U & 14 & $\mathrm{U}$ & 16 & $U$ & $\mathrm{ug} / \mathrm{kg}$ \\
\hline 29 & Aroclor-1248 & 14 & $\mathrm{U}$ & 14 & U & 16 & U & $\mathrm{ug} / \mathrm{kg}$ \\
\hline 30 & Aroclor-1254 & 14 & $\mathrm{U}$ & 14 & $\mathrm{U}$ & 16 & & $\mathrm{ug} / \mathrm{kg}$ \\
\hline 31 & Aroclor -1260 & 14 & $\mathrm{U}$ & 49 & & $\therefore 16$ & $\mathrm{U}$ & $\mathrm{ug} / \mathrm{kg}$ \\
\hline
\end{tabular}

33

34 Abbreviations/Acronyms:

$35 \mathrm{BCL}=$ below cleanup levels

$36 \mathrm{BG}=$ background

$37 \mathrm{COC}=$ contaminant of concern

$38 \mathrm{COPC}=$ contaminant of potential concern

39 CVP = Cleanup Verification Package

$40 \mathrm{GW}=$ groundwater

$41 \mathrm{MDA}=$ minimum detectable activity

$42 \mathrm{MTCA}=$ Model Toxics Control Act

$43 \mathrm{NA}=$ not applicable

$44 \mathrm{OB}=$ overburden

$45 \mathrm{PQL}=$ practical quantitation limit

46
Calc. No. 0100F-CA-V0294 Rev. No. 0 Checked J.M. Capron /4ser $\subset$ Date $09 / 24 / 07$

Sheet No. 4 of 20
Date $\quad 09 / 24 / 07$ 
CVP-2007-00004

Rev. 0

C-216 
Washington Closure Hanford

Originator L.D. Habel LH

Subject 118-F-8:4 105-F Fuel Storage Basin Cleanup Verification 95\% UCL Calculation
CALCULATION SHEET

Date $\frac{09 / 24 / 07}{1405}$
Job No. 14655
Rev. No. $\frac{0}{\text { Date }}$

(a)

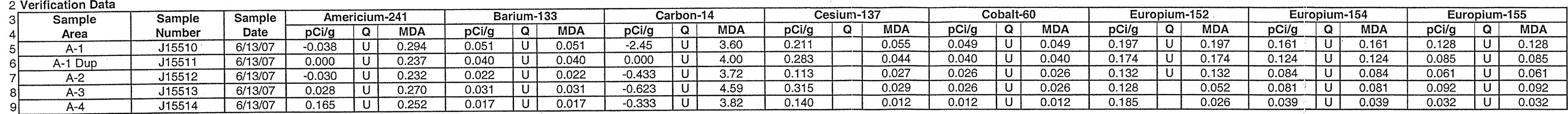

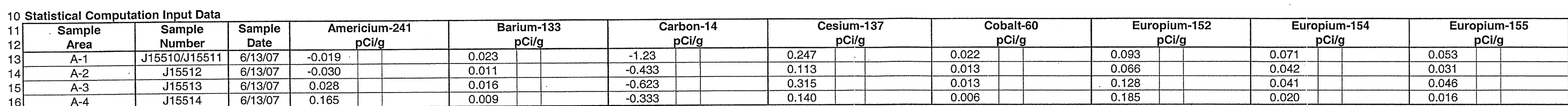

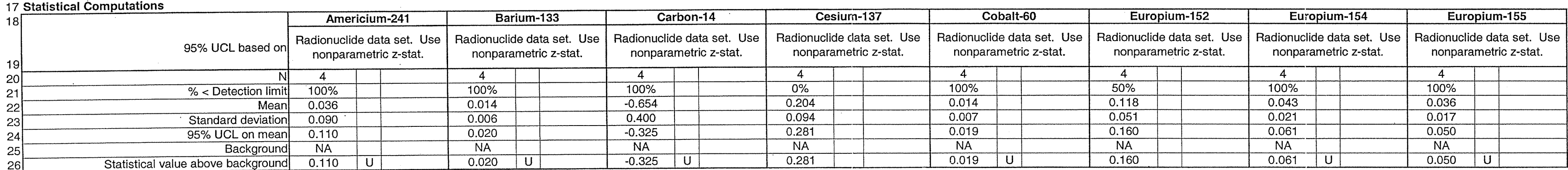


Originator L.D. Habel LAt

Project
Subject
100-F Field Remediation
$118-\mathrm{F}-8: 4$ 105-Fuel Storage Basin Cleanup Verification 95\% UCL Calculation
CALCULATION SHEET

$$
\begin{aligned}
\text { Date } & 09 / 24 / 07 \\
\text { Job No. } & 14655
\end{aligned}
$$

$\begin{aligned} \text { Rev. No. } & \frac{0}{\text { Date }} \\ \text { Sheet No. } & \frac{09 / 24 / 07}{6 \text { of } 20}\end{aligned}$

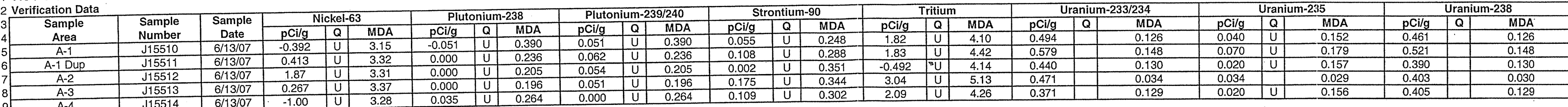

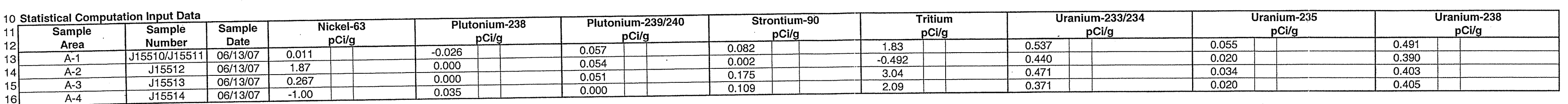

17 Statistical Computations

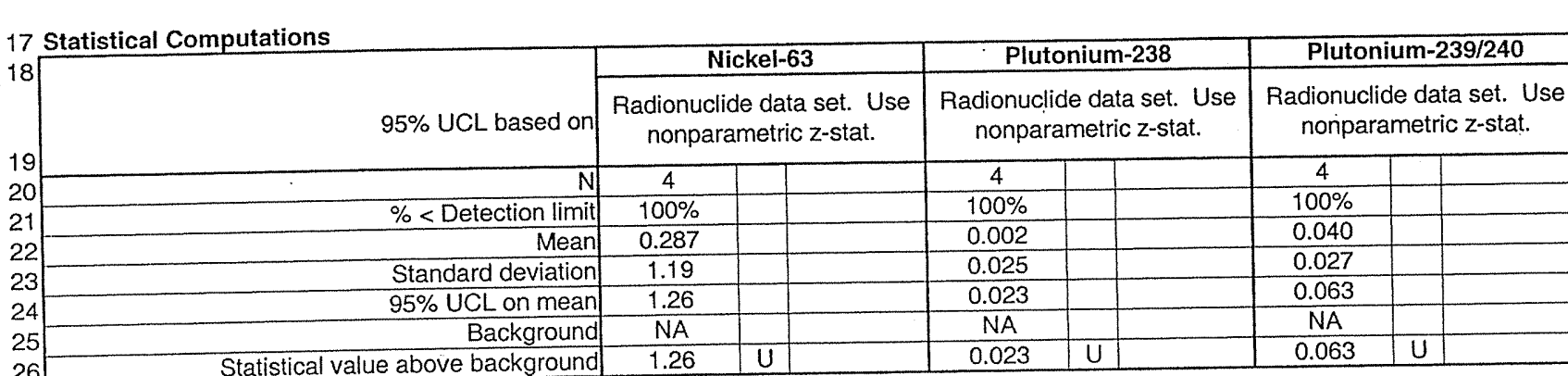

\begin{tabular}{|c|c|c|}
\hline \multicolumn{2}{|c|}{ Strontium-90 } \\
\hline $\begin{array}{c}\text { Radionuclide data set. Use } \\
\text { nonparametric z-stat. }\end{array}$ \\
\hline 4 \\
\hline $100 \%$ & & \\
\hline 0.092 & & \\
\hline 0.072 & & \\
\hline 0.151 & & \\
\hline NA & & \\
\hline 0.151 & U & \\
\hline
\end{tabular}

\begin{tabular}{|c|c|c|c|}
\hline \multirow{2}{*}{\multicolumn{2}{|c|}{\begin{tabular}{|c|} 
Tritium \\
$\begin{array}{c}\text { Radionuclited data set. Use } \\
\text { nonporametric z-stat. }\end{array}$
\end{tabular}}} & \multicolumn{2}{|c|}{ Uranium-233/234 } \\
\hline & & $\begin{array}{r}\text { Radio } \\
\text { nor }\end{array}$ & $\begin{array}{l}\text { clide data set. Uss } \\
\text { arametric } z \text {-stat. }\end{array}$ \\
\hline 4 & & 4 & \\
\hline$\frac{100 \%}{162}$ & & $\frac{0 \%}{0.045}$ & \\
\hline & & 0.069 & \\
\hline 2.85 & & 0.511 & \\
\hline$\frac{N A}{285}$ & 11 & $\frac{1.1}{0<B G)}$ & \\
\hline
\end{tabular}

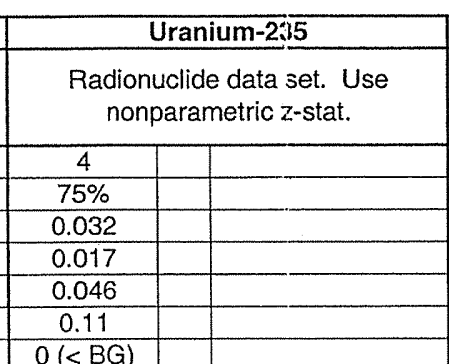

Uranium-238 Radionuclide data set. Use
nonparametric z z-stat.

0.42
\begin{tabular}{rrr} 
Background & $\mathrm{NA}$ & \\
\hline Slatistical value above background & 1.26 & $\mathrm{U}$ \\
\hline
\end{tabular} 
$\underset{\text { Originator L.D. Habel }}{\text { Project }}$ 100-F Field Remed

Project 100-F Field Remediation
CALCULATION SHEET

1 118-F-8:4 Excavation Shallow Zone Statistical Calculations
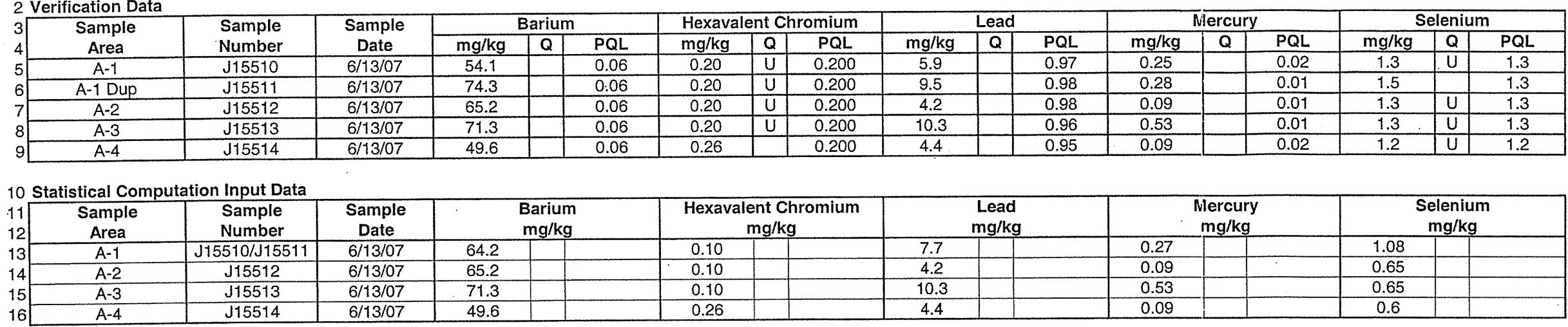

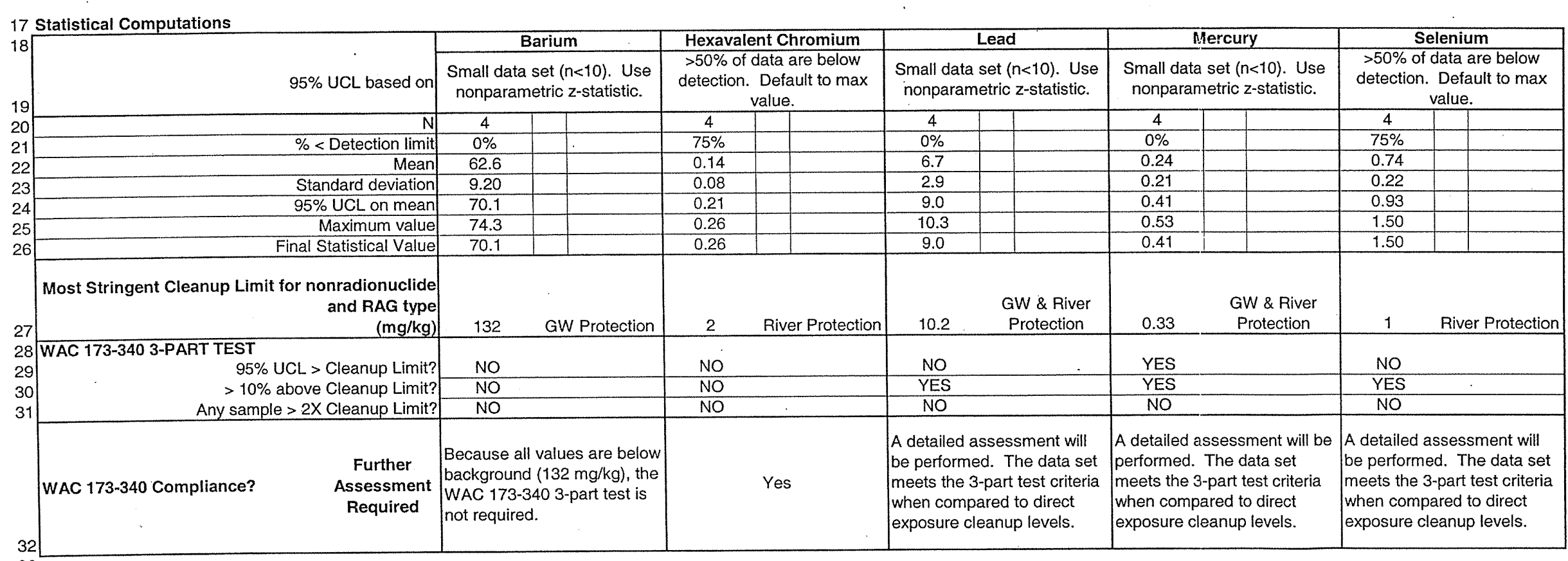




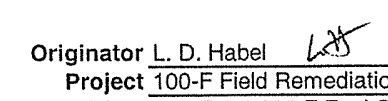

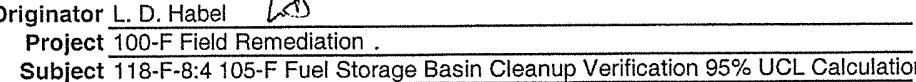

118-F-8:4 Excavation Shallow Zone Statistical Calculations
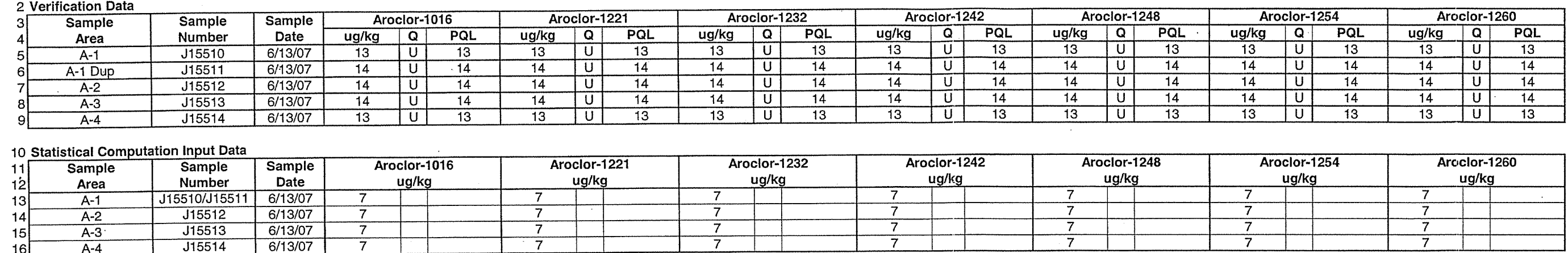

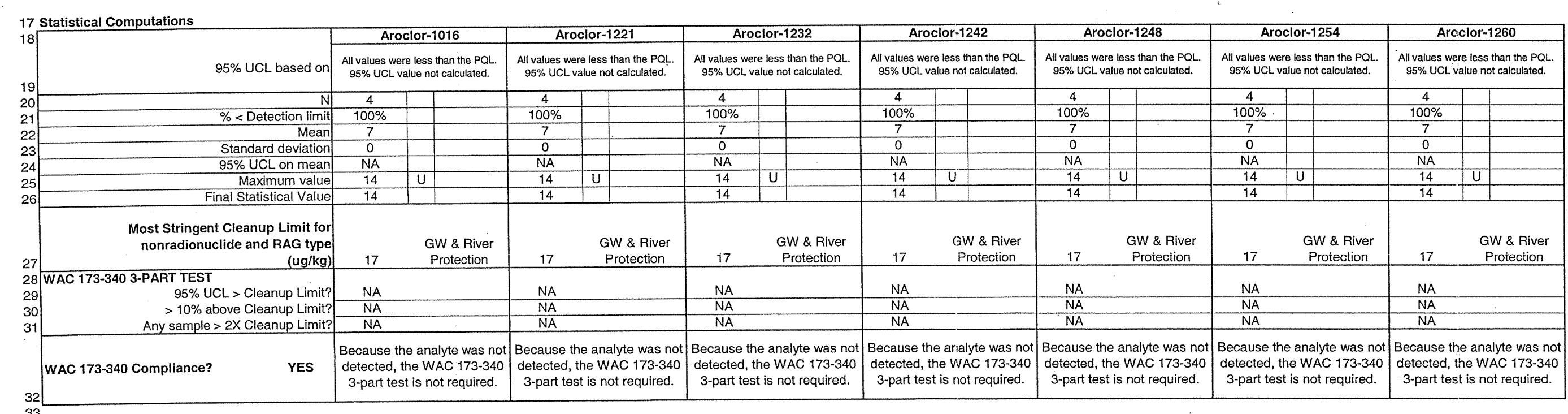


Washington Closure Hanford

Originator L. D. Habel

Project to-0.F Field Aemediation
Subject 118--F-8:4 105-F Fuel Storage Basin Cleanup Verification 95\% UCL Calculation
CALCULATION SHEET

Job №. $\frac{10 / 24 / 07}{14655}$

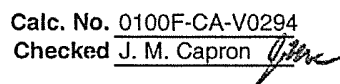

Rev. No. $\frac{1}{10 / 2407}$

118-F-8:4 Excavation Deep Zone Statistical Calculations

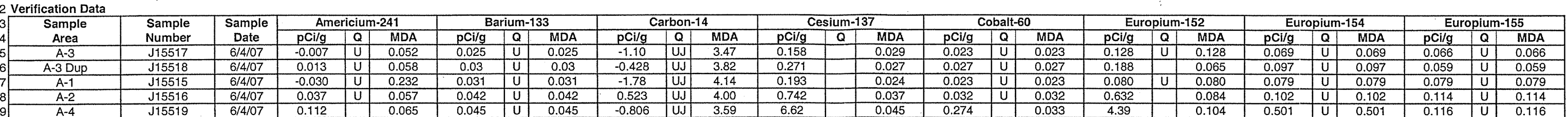

10 Statistical Computation Input Da

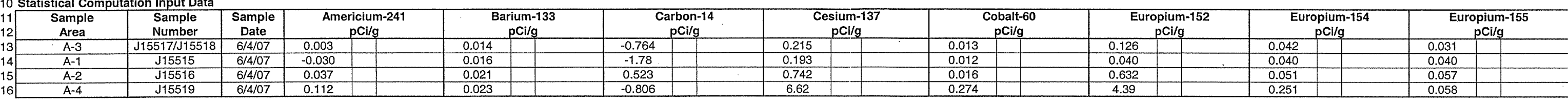

17 Statistical Computations

\begin{tabular}{|l|l}
18 & \\
20 & \\
21 & \\
22 & \\
23 & \\
25 & \\
26 &
\end{tabular}

\begin{tabular}{l|l|l|l|l} 
& \\
\hline & Americium-241 & Barium-133 & Carbon-14 & Cesiurn-137
\end{tabular}

Cobalt-60

inium-155 \begin{tabular}{c|c}
$\begin{array}{c}\text { Radionuclide data set. Use } \\
\text { nonparametric z-stat. }\end{array}$ & $\begin{array}{c}\text { Radionuclide data set. Use } \\
\text { nonparametric } z \text {-stat. }\end{array}$ \\
\hline
\end{tabular} \begin{tabular}{c|c|c|c|}
$\begin{array}{c}\text { Radionuclide data set. Use } \\
\text { nonparametric z-stat. }\end{array}$ & $\begin{array}{c}\text { Radionuclide data set. Use } \\
\text { nonparametric z-stat. }\end{array}$ & $\begin{array}{c}\text { Radionuclide data set. Use } \\
\text { nonparametric z-stat. }\end{array}$ & $\begin{array}{c}\text { Radionuclide data set. Use } \\
\text { nonparametric z-stat. }\end{array}$ \\
\hline
\end{tabular} $\%<$ Detection lim \begin{tabular}{|c|c|c|c|c|c|c|c|}
\hline 4 & 4 & & 4 & & 4 & \\
$75 \%$ & & $100 \%$ & & $100 \%$ & & $0 \%$ & \\
\hline 0.031 & & 0.018 & & -0.707 & & 1.94 & \\
\hline
\end{tabular} \begin{tabular}{c|c|c|}
\hline 4 & \\
$75 \%$ & & \\
0.079 & &
\end{tabular} \begin{tabular}{|c|c|c|c|}
\multicolumn{2}{|c|}{ nonparametric z-stat. } & \multicolumn{2}{|c|}{$\begin{array}{l}\text { Radionuclide datata set. } \\
\text { nonparametric z-stat. }\end{array}$} \\
\hline 4 & 4 \\
\hline $100 \%$ & & $100 \%$ & \\
\hline
\end{tabular} 
Washington Closure Hanford

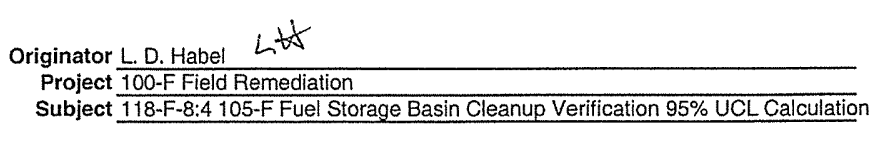

CALCULATION SHEET

Jab No. $\frac{10 / 24107}{14655}$

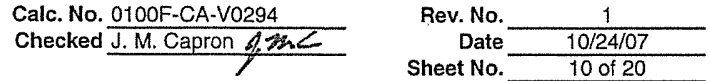

1 118-F-8:4 Excavation Deep Zone Statistical Calculation

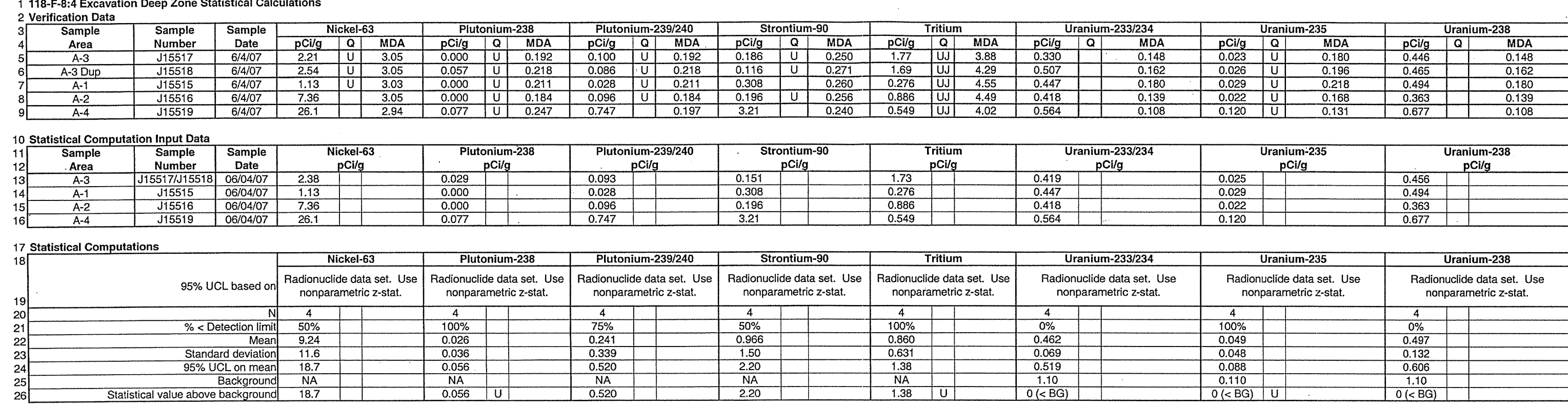




\section{Washington Closure Hanford}

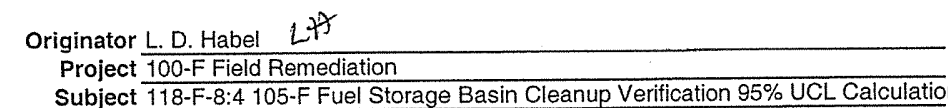

CALCULATION SHEE

Job No.

118--F-8: Excavation Deep Zone Statistical Calculations
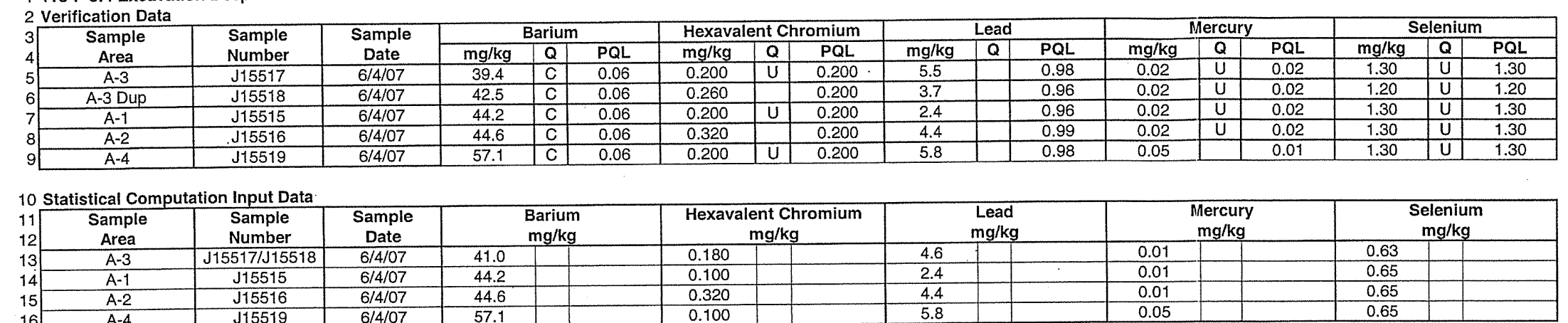

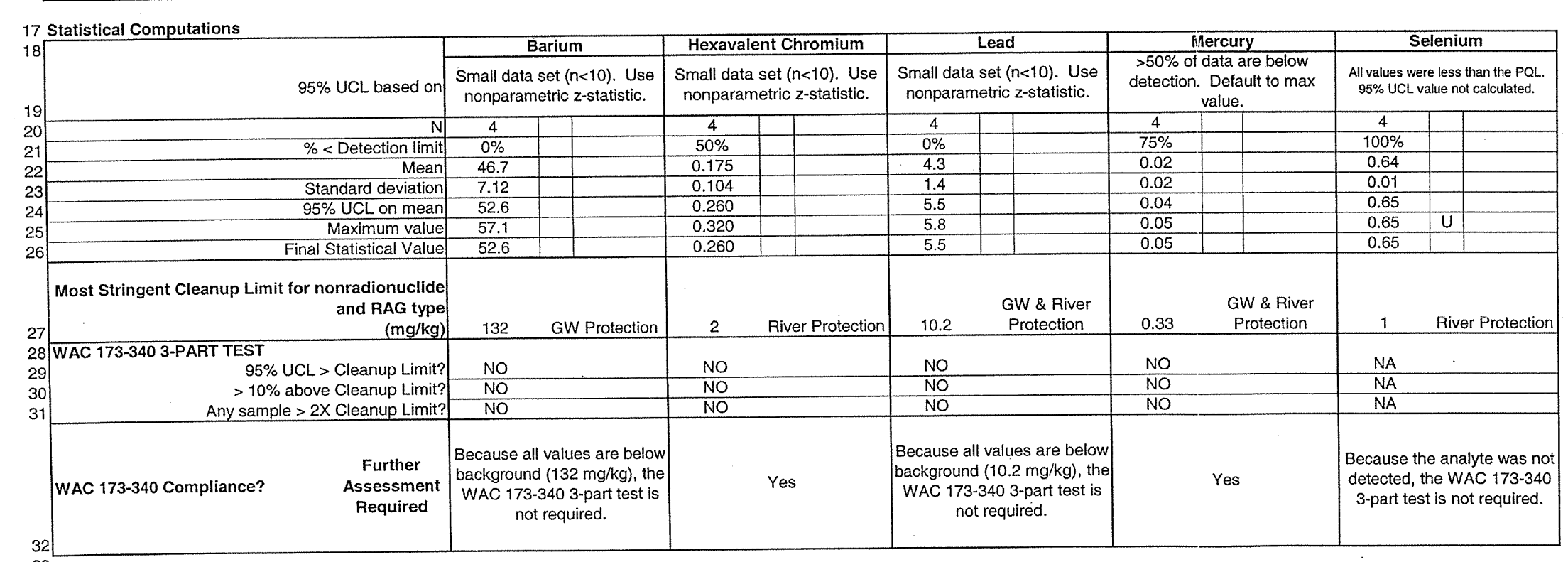


1118--8:-4 Excavation Deep Zone Statistical Calculations
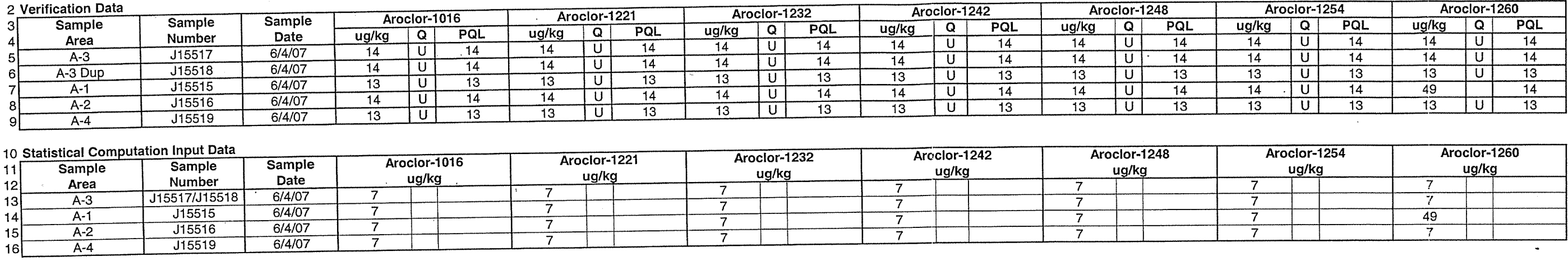

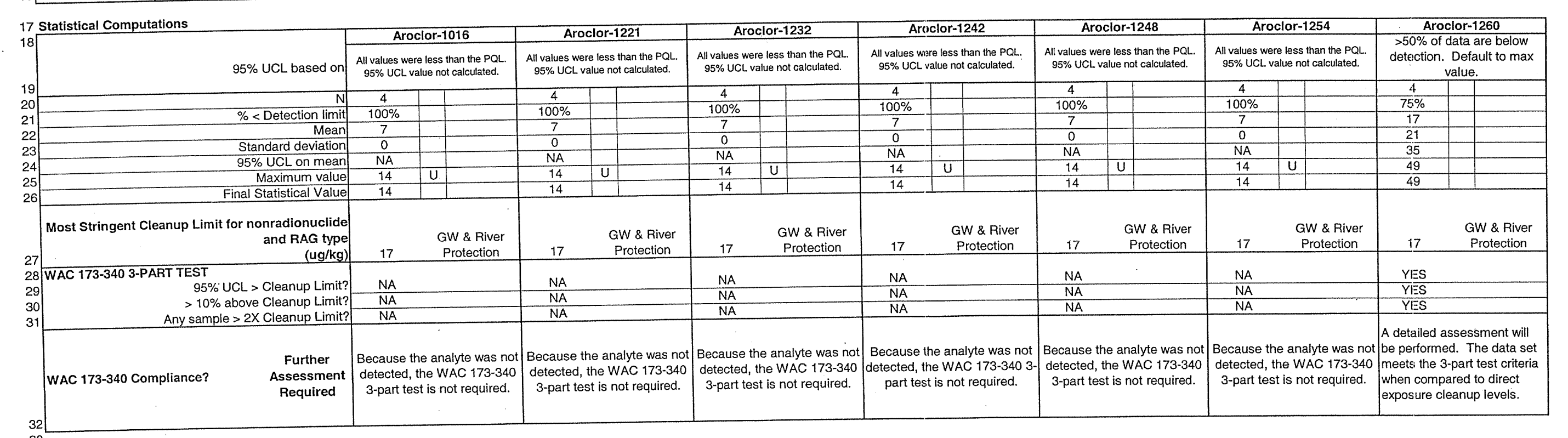


Washington Closure Hanford

$\underset{\text { Originator } L \text { D. Habel } \angle A}{\text { Project } 100-\text { F Field Reme }}$

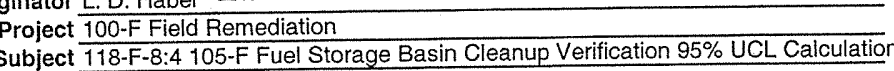

CALCUI.ATION SHEET
Job №. $\frac{09 / 24 / 07}{14655}$

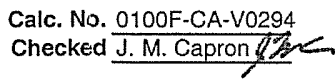
Rev. No. $\frac{0}{\text { Date }}$
Sheet No. $\frac{0912407}{130 \text { f } 20}$

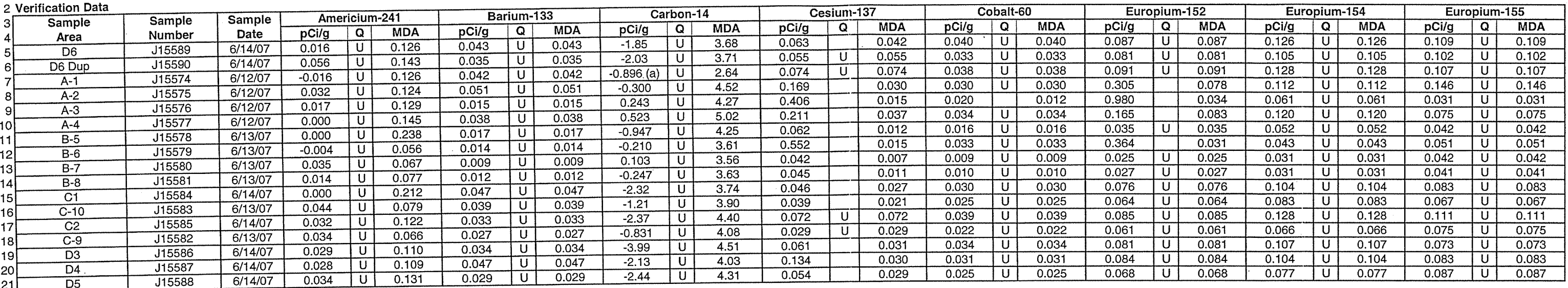

22 Statistical Computation Input Data
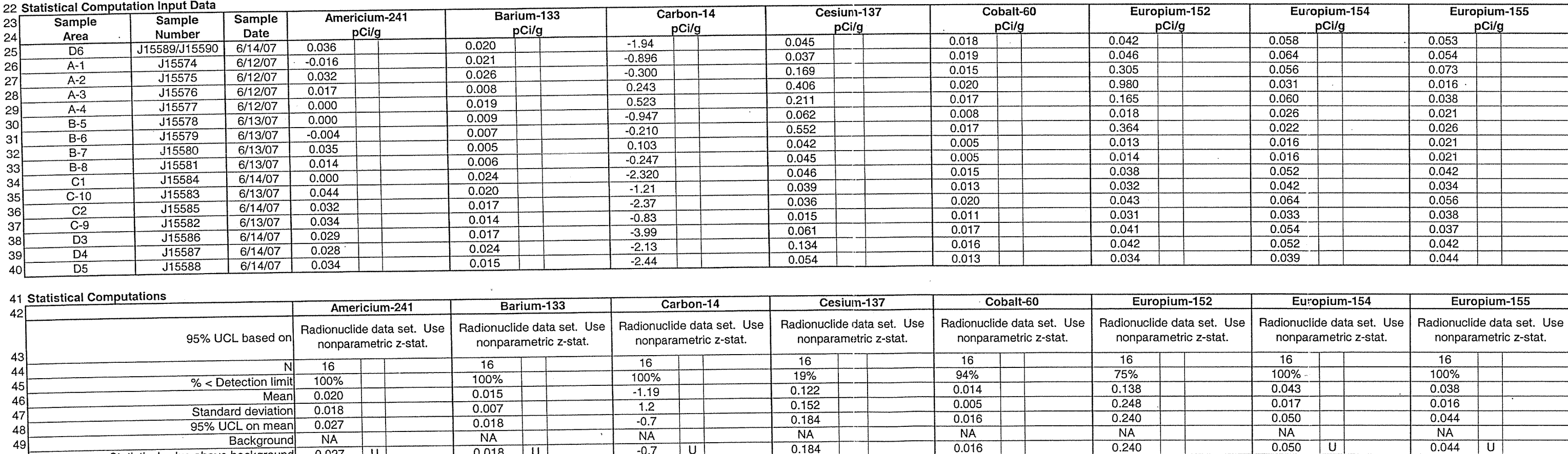

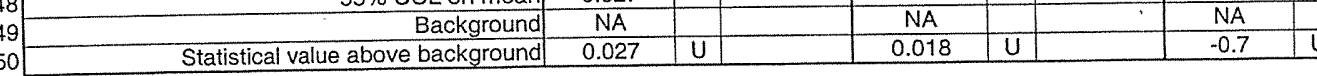

a) Intitial results (J15574) indicated that the stockpiled soil in area A-1 had detections of Carbon-14 in excess of the remedilal action goal. The contaminated 


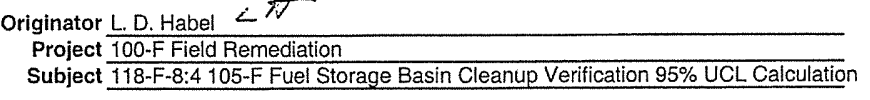

CALCULATION SHEET
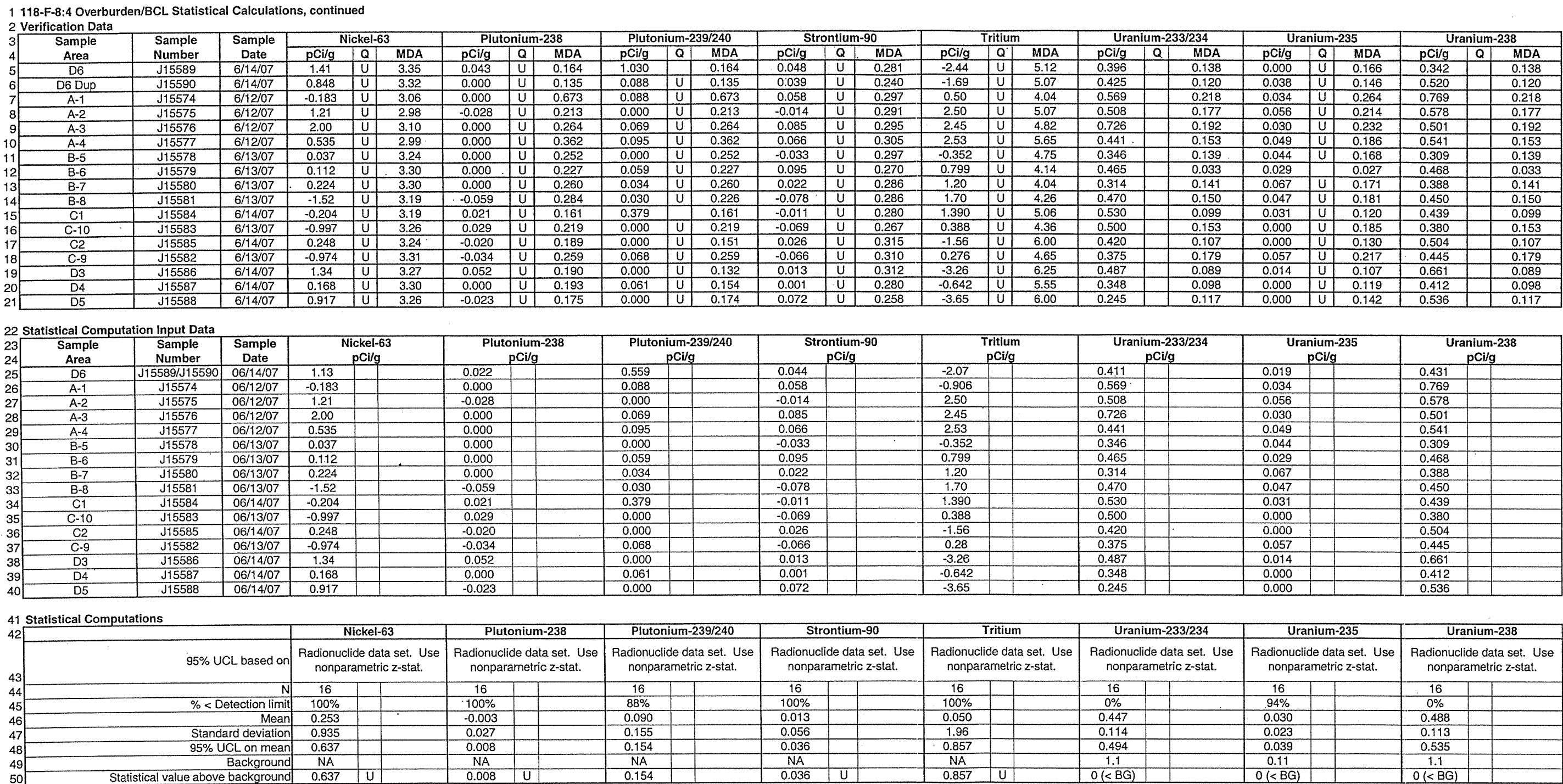
Originator L. D. Habel Ltt

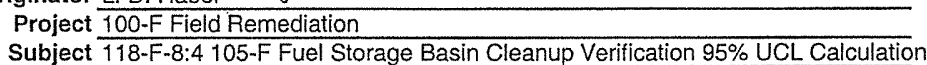

CALCULATION SHEET

Date
Job No.

118-F-8:4 Overburden/BCL Statistical Calculations
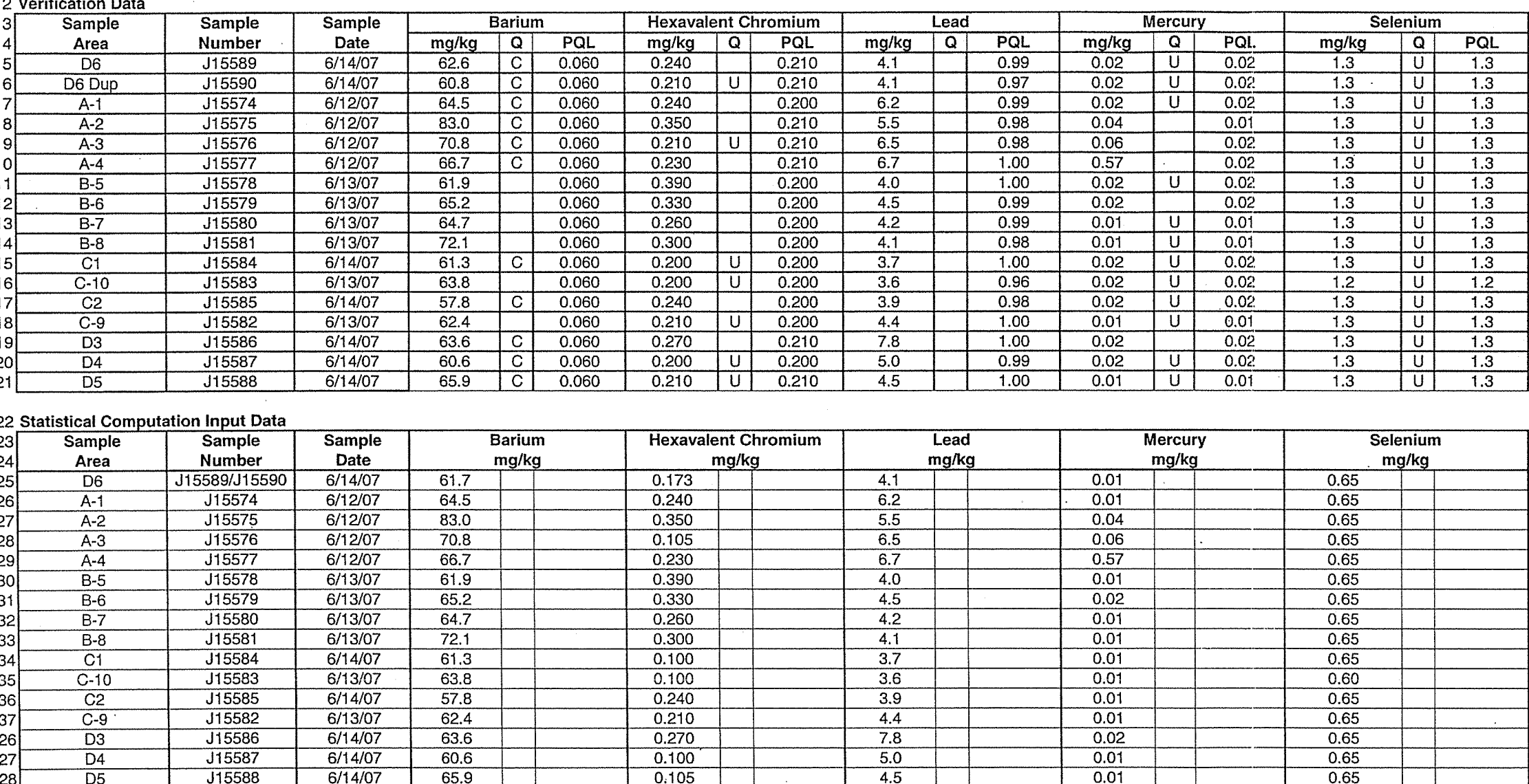

9 Statistical Computations

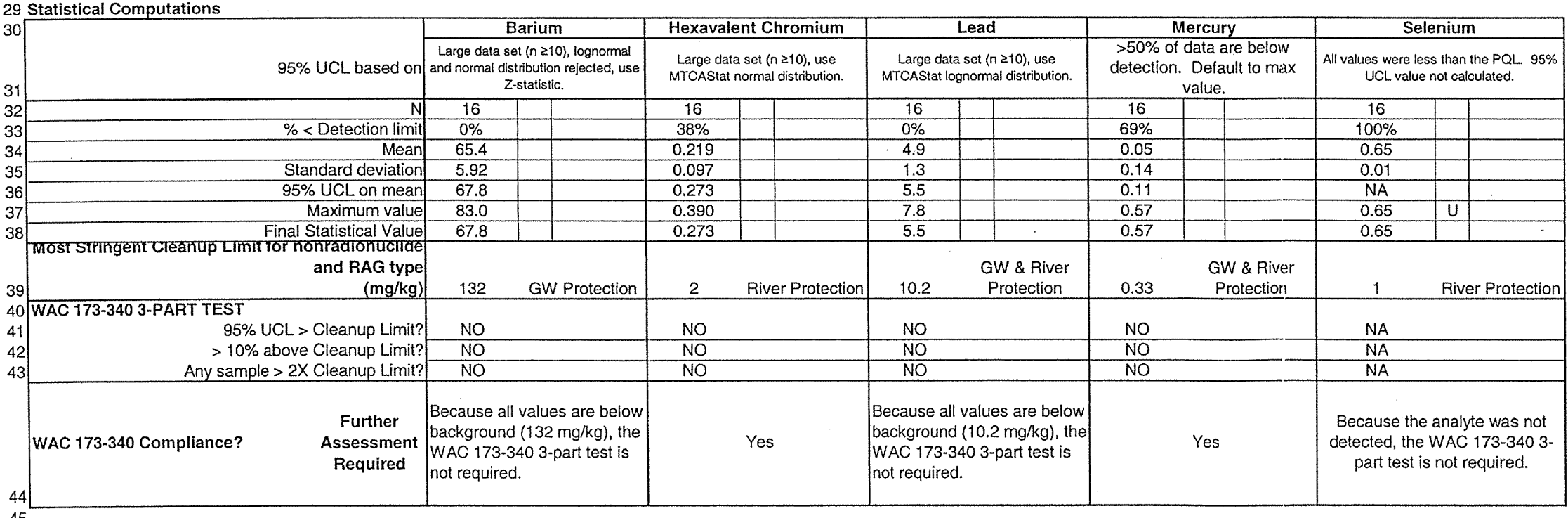


Washington Closure Hanford

Originator LD. Habel $L 4$

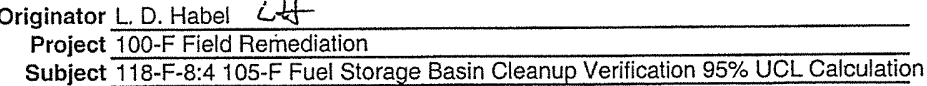

CALCULATION SHEET

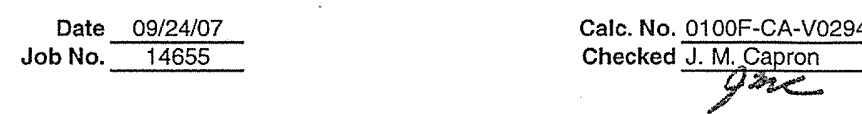

$\begin{aligned} & \text { Rev. No. } \frac{0}{\text { Date }} \\ & 099 / 24 / 07 \\ & 160+20\end{aligned}$

\section{118-F-8:4 Overburden/BCL Statistical Calculations}

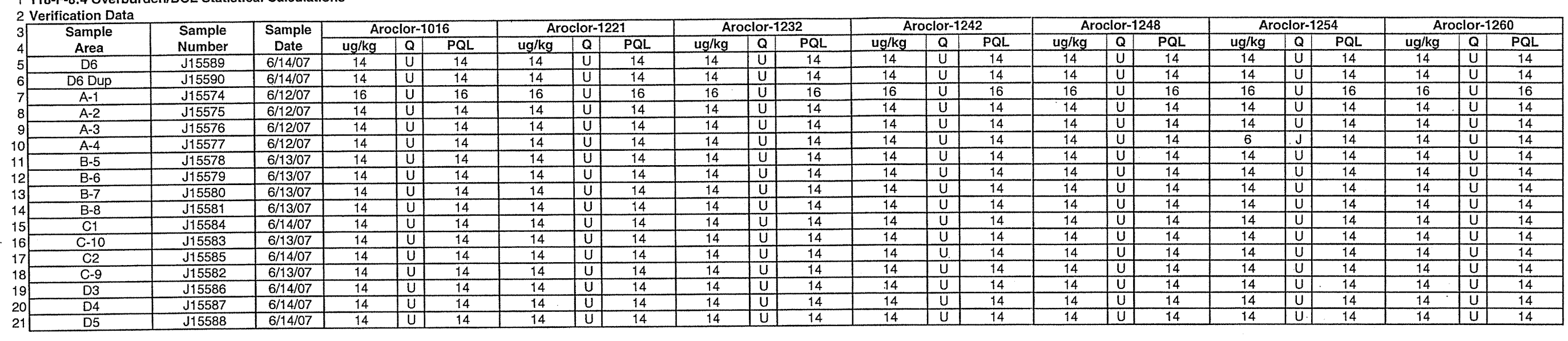

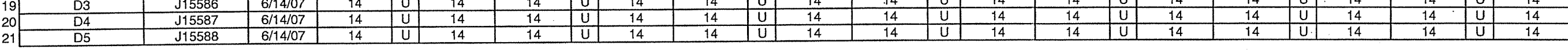

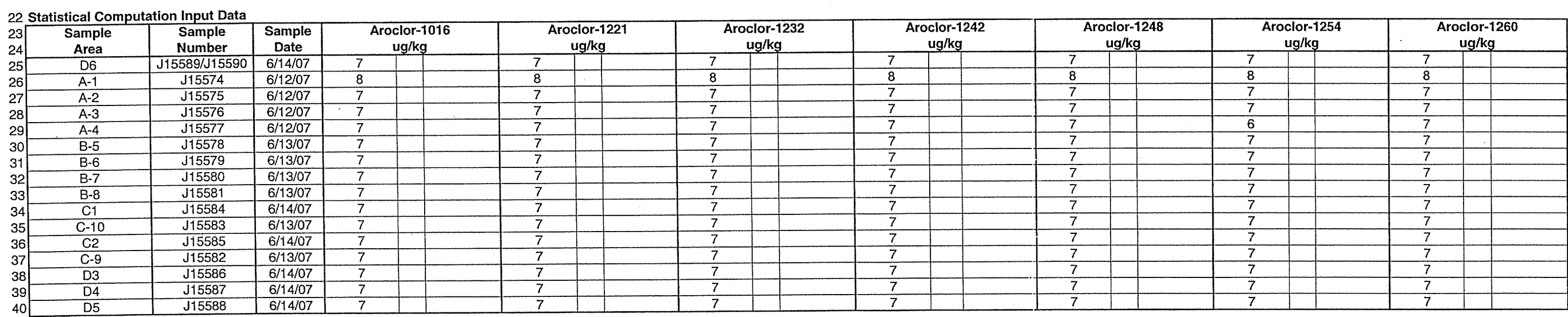

\begin{tabular}{|c|c|c|c|c|c|c|c|c|c|c|c|c|c|c|c|}
\hline \multirow{3}{*}{\multicolumn{2}{|c|}{ 95\% UCL based on }} & \multirow{2}{*}{\multicolumn{2}{|c|}{ 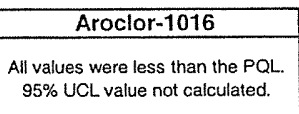 }} & \multirow{2}{*}{\multicolumn{2}{|c|}{ 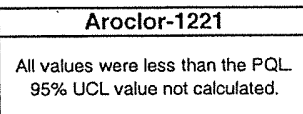 }} & \multirow{2}{*}{\multicolumn{2}{|c|}{ 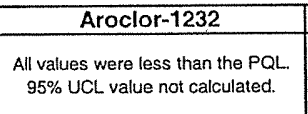 }} & \multirow{2}{*}{\multicolumn{2}{|c|}{ 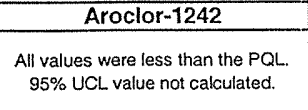 }} & \multirow{2}{*}{\multicolumn{2}{|c|}{ 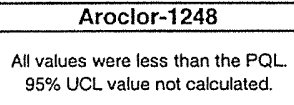 }} & \multirow{2}{*}{\multicolumn{2}{|c|}{ 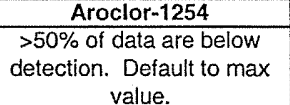 }} & \multirow{2}{*}{\multicolumn{2}{|c|}{ 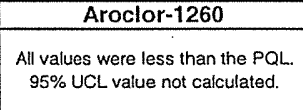 }} \\
\hline & & & & & & & & & & & & & & & \\
\hline & & $\frac{16}{100 \%}$ & & \begin{tabular}{|l|l|}
16 & \\
$100 \%$ \\
\end{tabular} & & $\frac{16}{100 \%}$ & $\square$ & $\begin{array}{l}16 \\
100 \% \\
\end{array}$ & & $\begin{array}{c}16 \\
100 \% \\
\end{array}$ & & $\begin{array}{c}\frac{16}{94 \%} \\
94 \%\end{array}$ & & $\begin{array}{l}16 \\
100 \% \\
\end{array}$ & \\
\hline & $\begin{array}{l}\text { Mean } \\
\text { deviation }\end{array}$ & $\frac{7}{0}$ & - & $\frac{7}{7}$ & & $\frac{7}{0}$ & E & 7 & $\square$ & $\frac{7}{0}$ & 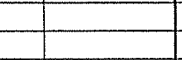 & $\frac{7}{0}$ & & $\frac{7}{0}$ & $\exists$ \\
\hline & nam & NA & & NA & & NA & 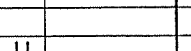 & NA & H. & NA & & 7 & & $\mathrm{NA}$ & \\
\hline & $\begin{array}{c}\text { Maximum detected value } \\
\text { Final Statistical value }\end{array}$ & $\frac{16}{16}$ & U & \begin{tabular}{l|l}
16 & $U$ \\
16 & \\
\end{tabular} & 4 & $\frac{16}{16}$ & 4 & $\frac{16}{16}$ & 0 & $\frac{16}{16}$ & 0 & $\frac{16}{16}$ & & $\frac{16}{16}$ & 0 \\
\hline & $\begin{array}{r}\text { Most Stringent Cleanup Limit for } \\
\text { nonradionuclide and RAG } \\
\text { (ugpheg }\end{array}$ & & & & & 17 & & 17 & & 17 & & 17 & & 17 & rotection \\
\hline & & & & NA & & & & & & & & NO & & & \\
\hline & & NA & & $\frac{N A}{N A}$ & & $\frac{N A}{N A}$ & & $\frac{N A}{N A}$ & & NA & & $\begin{array}{l}\text { NO } \\
\text { NO }\end{array}$ & & $\frac{N A}{N A}$ & \\
\hline & YES & $\begin{array}{l}\text { Because } \\
\text { detected } \\
\text { s-artit }\end{array}$ & $\begin{array}{l}\text { alyte was not } \\
\text { laA c } 173-340 \\
\text { lot required. }\end{array}$ & $\begin{array}{l}\text { Because the an, } \\
\text { detected, the W } \\
3 \text {-part test is n. }\end{array}$ & $\begin{array}{l}\text { analyte was not } \\
\text { WAA 173-340 } \\
\text { s not required. }\end{array}$ & \begin{tabular}{|l} 
Because th \\
deteleted $t$ \\
3-part tes
\end{tabular} & $\begin{array}{l}2 \text { analyte was not } \\
\text { le WhC } 137-340 \\
\text { is not required. }\end{array}$ & $\begin{array}{l}\text { Because th } \\
\text { detected th } \\
\text { part test }\end{array}$ & $\begin{array}{l}\text { le analyte was not } \\
\text { ewAC } 173-340 . \\
\text { is not required. }\end{array}$ & $\begin{array}{l}\text { Because the } \\
\text { deteceld, th } \\
3 \text {-part test }\end{array}$ & is not required. & & Ye & & \\
\hline
\end{tabular}




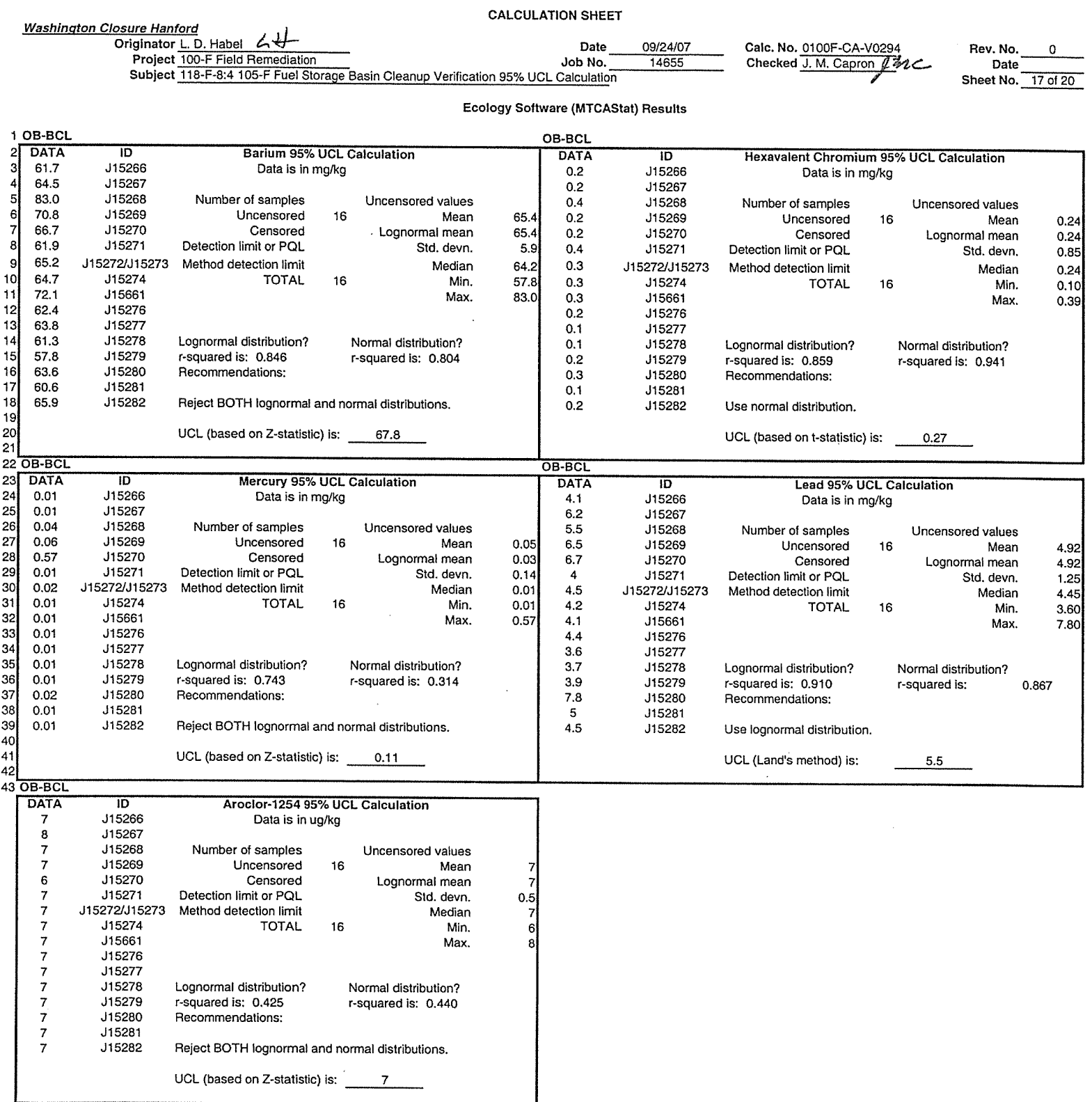


CALCULATION SHEET

Washington Closure Hanford

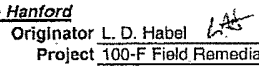

Subject $118-F-8.4$ - 105-F Fuel Storage Basin Cleanup Verification $95 \%$ UCL Calculation

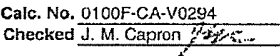

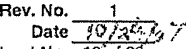

1 Splitrouplicate Analysis, Excavation Shallow Zone

\begin{tabular}{|c|c|c|c|c|c|c|c|c|c|c|c|c|c|c|c|c|c|c|c|c|c|c|c|c|}
\hline \multirow{3}{*}{$\begin{array}{c}\text { Spluouplicale Ana } \\
\text { Sample } \\
\text { Area } \\
\text { SzA-1 }\end{array}$} & \multirow{2}{*}{$\begin{array}{l}\text { Sis, Exple } \\
\text { Sumber } \\
\text { Number }\end{array}$} & \multirow{2}{*}{$\frac{\frac{0}{\text { Sample }}}{\text { Date }}$} & \multicolumn{3}{|c|}{ Cesium-137 } & \multicolumn{2}{|c|}{ Potassium -40} & \multicolumn{2}{|c|}{ Radium-226 } & \multicolumn{3}{|c|}{ Radium-228 } & \multicolumn{3}{|c|}{ Thorium-228 } & \multicolumn{3}{|c|}{ Thorium-232 } & \multicolumn{3}{|c|}{ Uranium-233/234 } & \multicolumn{3}{|c|}{ Uranium-238 } \\
\hline & & & pCig & a & MDA & $\mathrm{pCl/g}$ & \begin{tabular}{l|l} 
a & MDA \\
\end{tabular} & $\mathrm{pCl} / \mathrm{g}$ & \begin{tabular}{|l|l|}
0 & MDA \\
\end{tabular} & $\mathrm{pCl} / \mathrm{g}$ & 0 & MDA & $\mathrm{pcilg}$ & & $\mathrm{MDA}$ & $\mathrm{pCl} / \mathrm{g}$ & a & MDA & $\mathrm{pclig}$ & $a$ & & & 0 & MDA \\
\hline & & $6 / 13 / 07$ & 0.211 & & 0.055 & 14.8 & 0.417 & 0.538 & 0.092 & 0.688 & & 0.179 & 0.881 & & 0.085 & 0.688 & & 0.179 & 0.494 & & 0.126 & 0.461 & & 0.126 \\
\hline SZA-1 Dup & J15511 & $6 / 13 / 27$ & 0.283 & & 0.044 & 14.3 & 0.362 & 0.456 & 0.059 & 0.694 & & 0.143 & 0.732 & & 0.042 & 0.694 & & 0.143 & 0.579 & & 0.148 & 0.521 & & \\
\hline SZA-1 Split & $\mathrm{J15520}$ & $6 / 13 / 07$ & 0.252 & & 0.019 & & & & $=$ & $=$ & & 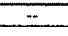 & & & $\sim$ & $=$ & & & 0.718 & & 0.033 & 0.771 & & \\
\hline \multirow{2}{*}{\multicolumn{3}{|c|}{ 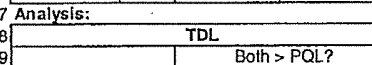 }} & \multicolumn{3}{|c|}{0.1} & \multirow{2}{*}{\multicolumn{2}{|c|}{400}} & \multirow{2}{*}{\multicolumn{2}{|c|}{0.1}} & \multicolumn{3}{|c|}{0.2} & \multicolumn{3}{|c|}{1} & \multirow{2}{*}{\multicolumn{3}{|c|}{1}} & & \\
\hline & & & \multirow{2}{*}{\multicolumn{3}{|c|}{$\begin{array}{c}\text { Yes (continue) } \\
\text { No-evaluate difference }\end{array}$}} & \multirow{2}{*}{\multicolumn{2}{|c|}{$\begin{array}{l}\text { Yes (continue) } \\
\text { No- evaluate difference }\end{array}$}} & & & \multirow{2}{*}{\multicolumn{3}{|c|}{ No-evaluate difierence }} & & contir & & & & & \multirow{2}{*}{\multicolumn{3}{|c|}{ No-evaluate difference }} & \multirow{2}{*}{\multicolumn{3}{|c|}{$\begin{array}{l}\text { Yes (continue) } \\
\text { No: evaluate difference }\end{array}$}} \\
\hline \multirow{2}{*}{ Duplicate Analysis } & \multicolumn{2}{|c|}{$\begin{array}{l}\text { Both }>\text { POL? } \\
\text { Both }>5 \times T D L ?\end{array}$} & & & & & & \multicolumn{2}{|c|}{ No - evaluate difierence } & & & & \multicolumn{3}{|c|}{ No-evaluate difference } & \multicolumn{3}{|c|}{ No- evaluate difference } & & & & & & \\
\hline & \multirow{2}{*}{\multicolumn{2}{|c|}{ Difference $>2 \times T D$ ? }} & & accep & & & cceplable & & accaptable & & accept & & No:- & accept & table & No- & accepi & & No- & & & & 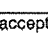 & \\
\hline & & & & Conti & & & & & & & & & & & & & & & & & & & $\frac{n+1}{n+1}$ & \\
\hline Split Analysis & & XTOL? & No-eva & uate o & lifterence & & & & & & & & & & & & & & No-eval & & ifference & No-eval & uated & Ifferen \\
\hline & $\frac{F}{\text { Differenc }}$ & Li? & No- & $\overrightarrow{a c c}$ & table & & & & & & & & & & & & & & No- & & table & No- & accept & able \\
\hline
\end{tabular}
17 Splitifouplicate Analysis, Excavation Shallow Zone (continued)

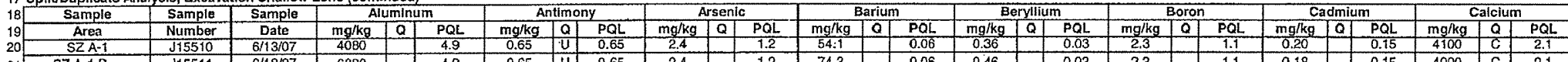

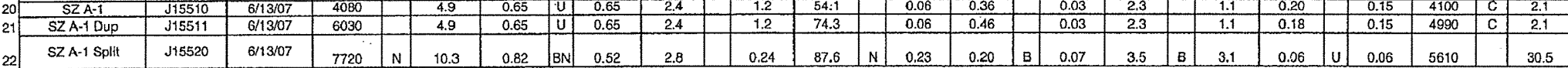

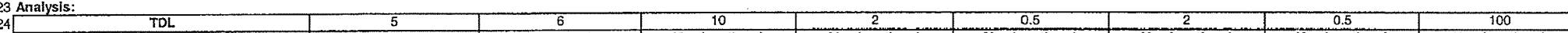

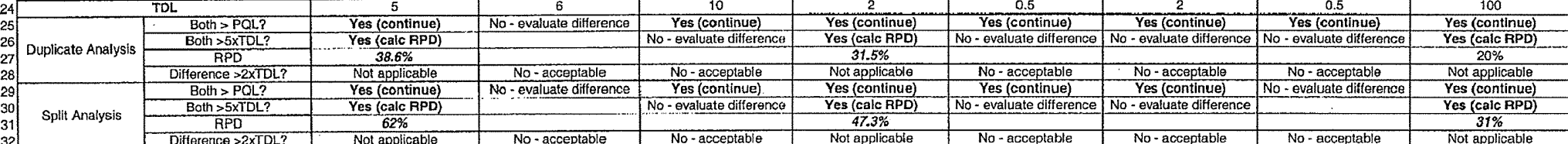
3 Splitibuplicate Analysis, Excavation Shallow Zone (contlnued)

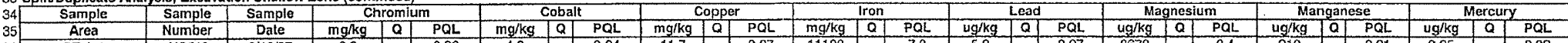

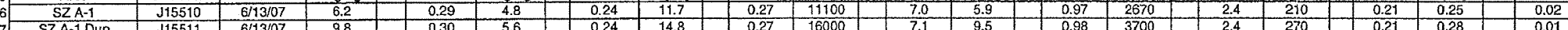

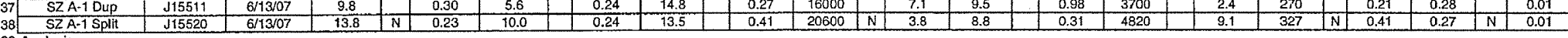

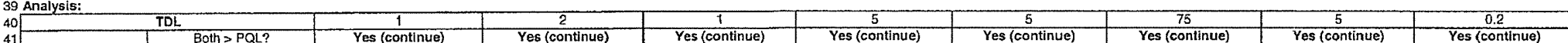

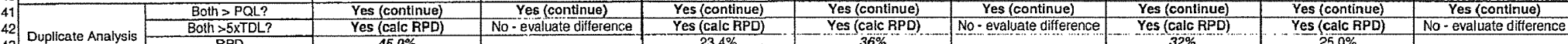

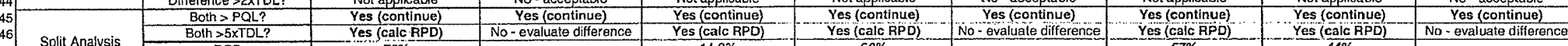
48

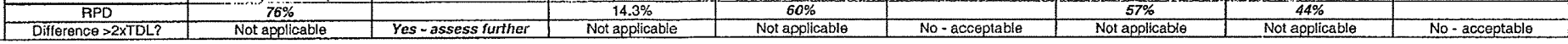
49 Splitrouplicate Analysis, Excavation Shallow Zone (continued)

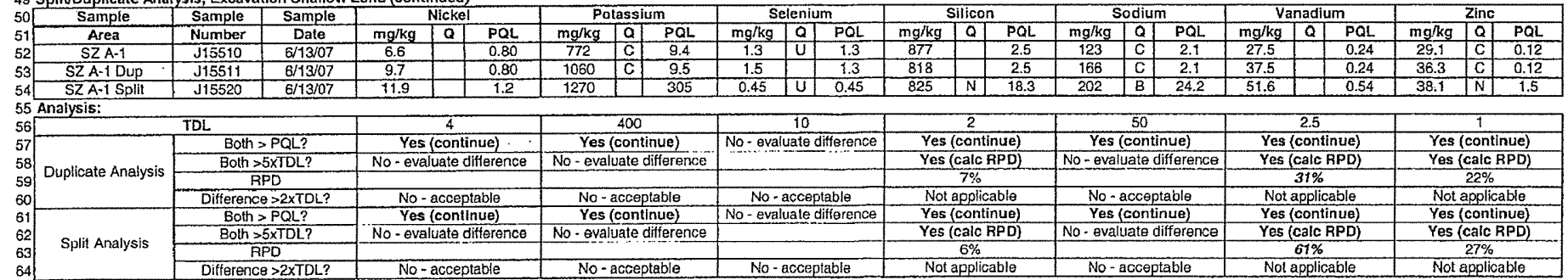




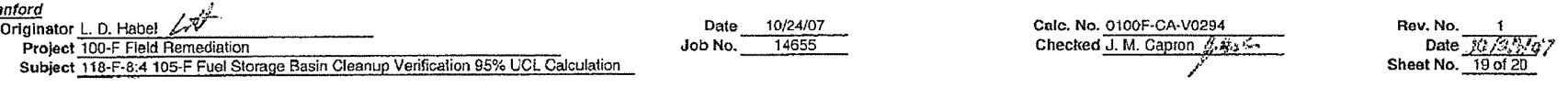

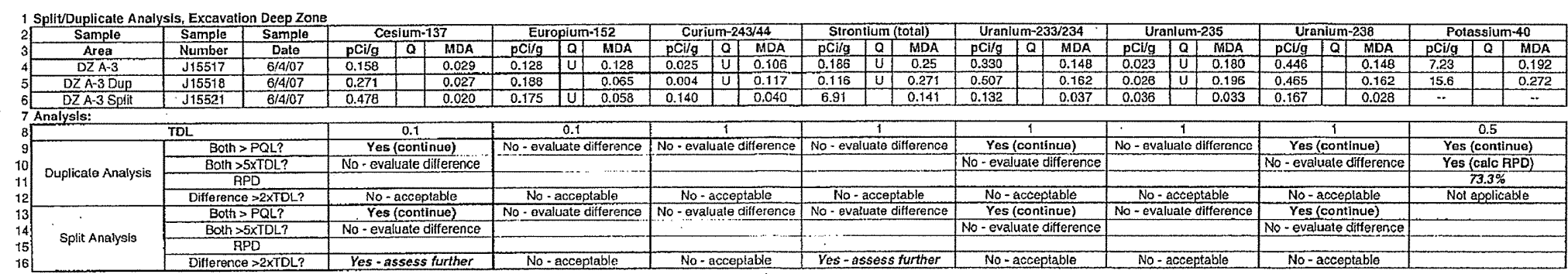

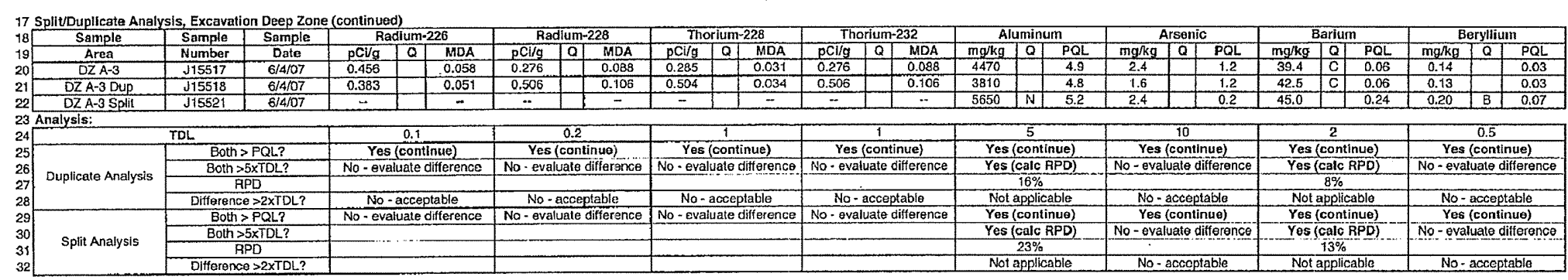

33 SplivDupilicate Analysis, Excavation Deep Zone (continued)

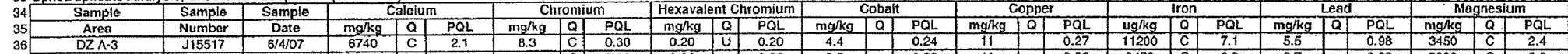

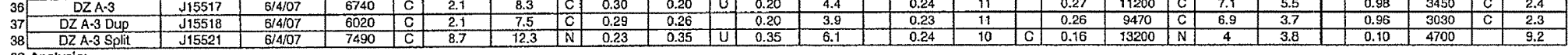

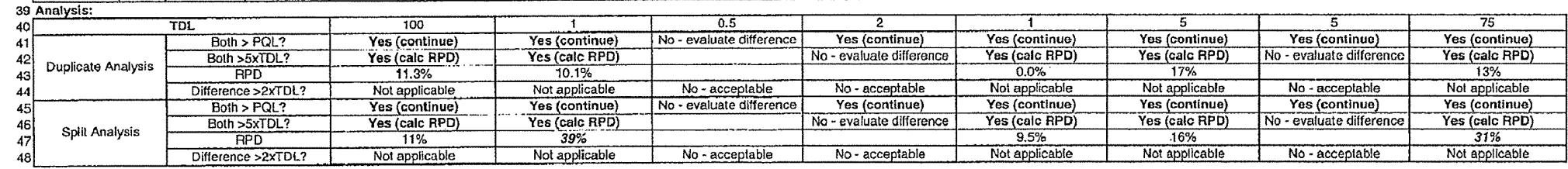
49 Splitruplicate Analysis, Excavation Deep Zone (continued)

\begin{tabular}{|c|c|c|c|c|c|c|c|c|c|c|c|c|c|c|c|c|c|c|c|c|c|c|c|c|}
\hline \multirow{3}{*}{ 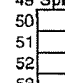 } & \multirow{2}{*}{$\begin{array}{c}\text { plit/Duplicate Anais } \\
\text { Sample } \\
\text { Area } \\
\end{array}$} & \multirow{3}{*}{$\begin{array}{l}\text { Saimple } \\
\text { Number } \\
\text { J15557 } \\
\end{array}$} & \multirow{2}{*}{$\begin{array}{l}\frac{1}{\text { Sample }} \\
\text { Date } \\
6 / 4107\end{array}$} & \multicolumn{3}{|c|}{ Manganese } & \multicolumn{3}{|c|}{ Mercury } & \multicolumn{3}{|c|}{ Molybdenum } & \multicolumn{2}{|c|}{ Nicke! } & \multicolumn{2}{|c|}{ Potassium } & \multicolumn{3}{|c|}{ Silicon } & \multicolumn{2}{|c|}{ Vanadium } & \multicolumn{3}{|c|}{ Zinc } \\
\hline & & & & $\frac{\mathrm{mg} / \mathrm{kg}}{236}$ & a) & $\frac{P O L}{021}$ & $\frac{\mathrm{mg} / \mathrm{kg}}{0.02}$ & $\left|\frac{a}{b}\right|$ & $\frac{P O L}{0.02}$ & \begin{tabular}{|l|l|l}
$\mathrm{mg} / \mathrm{kg}$ \\
0.48
\end{tabular} & & $\frac{P O L}{0.48}$ & $\frac{\mathrm{mg} / \mathrm{kg}}{8.5}$ & \begin{tabular}{l|l}
0 & $P Q L$ \\
0 & 0.8
\end{tabular} & $\frac{m g / k g}{554}$ & \begin{tabular}{|l|l}
$a$ & $P Q L$ \\
$C$ & 9.5
\end{tabular} & $\frac{\mathrm{mgkg}}{959}$ & $\mid \begin{array}{l}a \\
c\end{array}$ & $\frac{P Q L}{2.6}$ & $\frac{\mathrm{mg} / \mathrm{kg}}{26.9}$ & $\begin{array}{l}2 \\
\end{array}$ & $\frac{\mathrm{mglkg}}{30.1}$ & $\left|\begin{array}{l}a \\
c\end{array}\right|$ & $\frac{P Q L}{0.12}$ \\
\hline & $\frac{\text { D2A-3 }}{D Z \text { A } 3 \text { Dup }}$ & & $\frac{64 / 407}{6 / 4 / 07}$ & $\frac{236}{212}$ & & $\begin{array}{l}0.21 \\
0.20 \\
\end{array}$ & $\begin{array}{l}0.02 \\
0.02 \\
\end{array}$ & it & $\frac{0.02}{0.02}$ & 0.48 & & 0.048 & $\begin{array}{l}8.5 \\
8.3 \\
\end{array}$ & 0.78 & \begin{tabular}{|l}
493 \\
\end{tabular} & 9.3 & $\frac{1090}{1070}$ & $\mid$ & 2.5 & $\frac{2.9}{21.0}$ & 0.23 & 29.0 & +4 & \\
\hline & $\mathrm{DZA} \cdot 3$ Split & J15521 & 6/4/07 & 244 & $\mathrm{~N}$ & $0.23+2$ & 0.0 & 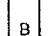 & 0.01 & $0.91 \quad-1$ & $|N|$ & 0.91 & 11.5 & & 735 & 937 & 500 & $C N \mid$ & 1.1 & 20 & & 70 & $c$ & 4 \\
\hline \multirow{2}{*}{\multicolumn{4}{|c|}{${ }^{A n a l y s i s: ~} \quad T_{\text {Both }}$}} & \multirow{2}{*}{\multicolumn{3}{|c|}{5}} & \multirow{2}{*}{\multicolumn{3}{|c|}{$\frac{0.2}{0-\text { evaluale difference }}$}} & \multirow{2}{*}{\multicolumn{3}{|c|}{$\frac{5}{0 . \text { evaluate difference }}$}} & \multicolumn{2}{|r|}{4} & \multicolumn{2}{|r|}{400} & \multirow{2}{*}{\multicolumn{3}{|c|}{2}} & \multicolumn{2}{|r|}{2.5} & \\
\hline & & & & & & & & & & & & & \multirow{2}{*}{\multicolumn{2}{|c|}{$\begin{array}{c}\text { Yes continue) } \\
\text { No - } \theta \text { valuate difference }\end{array}$}} & \multirow{2}{*}{\multicolumn{2}{|c|}{ No-evaluate difference }} & & & & & continue) & & & \\
\hline & \multirow{2}{*}{ Duplicate Analysis } & \multicolumn{2}{|l|}{$\begin{array}{l}\text { Both } \\
\text { Both }\end{array}$} & \multicolumn{3}{|c|}{$\begin{array}{l}\text { Yes Scontinue) } \\
\text { Yes (calc RPD) }\end{array}$} & \multicolumn{3}{|c|}{ evaluale une } & \multicolumn{3}{|c|}{ - ..... } & & & & & \multicolumn{3}{|c|}{ Yes (calc $\mathrm{APO})$} & \multicolumn{2}{|c|}{ Yes (calc RPD) } & & $\begin{array}{l}\frac{1 \text { calc } F}{3706} \\
3706\end{array}$ & \\
\hline & & Diflerenci & TDL? & & applicic & & No: & accep & lable & No & - & able & No & & & & & tap & & & & & applice & \\
\hline & & Both: & L? & & - & & No-eva & iluale o & Iference & vo-evalu & uate difi & fiterence & & & & & & & & & & & & \\
\hline & Analysis & Both > & & & $\frac{\text { (calc f }}{3 \%}$ & & & & & & & & No-evalu & Jate difference & & sate differes & & S & & & & & $\frac{10 F}{\%}$ & \\
\hline & & Differench & TDI? & & 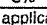 & & & accep & tabis & & pta & & & table & No-a & Ecceptabie & & & & & cable & & & \\
\hline
\end{tabular}




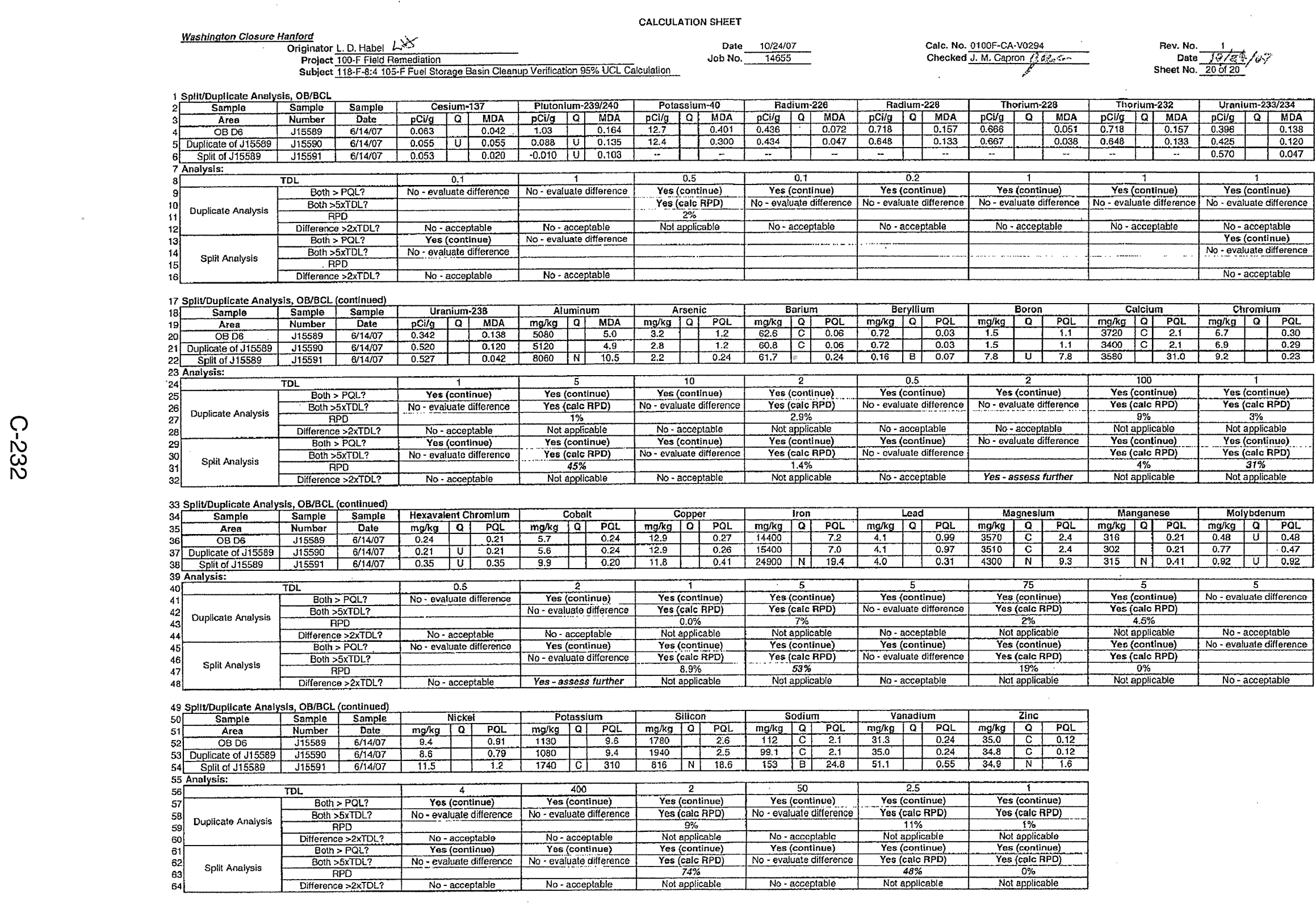


Attachment 1. 118-F-8:4 Verification Sampling Results.

\begin{tabular}{|c|c|c|c|c|c|c|c|c|c|c|c|c|c|c|c|c|c|}
\hline \multirow{2}{*}{$\begin{array}{c}\text { Sample } \\
\text { Location } \\
\end{array}$} & \multirow{2}{*}{$\begin{array}{c}\text { HEIS } \\
\text { Number }\end{array}$} & \multirow{2}{*}{$\begin{array}{c}\text { Sample } \\
\text { Date }\end{array}$} & \multicolumn{3}{|c|}{ Americium-241 } & \multicolumn{3}{|c|}{ Americium-241 (GEA) } & \multicolumn{3}{|c|}{ Barium-133 } & \multicolumn{3}{|c|}{ Carbon-14 } & \multicolumn{3}{|c|}{ Cesium-137 } \\
\hline & & & $\mathrm{pCi} / \mathrm{g}$ & $Q$ & MDA & $\mathrm{pCi} / \mathrm{g}$ & $Q$ & MDA & $\mathrm{pCi} / \mathrm{g}$ & $Q$ & MDA & $\mathrm{pCi} / \mathrm{g}$ & $Q$ & $\mathrm{MDA}$ & $\mathrm{pCi} / \mathrm{g}$ & $Q$ & $\mathrm{MDA}$ \\
\hline SZ A-1 & $\mathrm{J} 15510$ & $6 / 13 / 07$ & -0.038 & $\mathrm{U}$ & 0.294 & 0.329 & $\mathrm{U}$ & 0.329 & 0.051 & $\mathrm{U}$ & 0.051 & -2.45 & $\mathrm{U}$ & 3.60 & 0.211 & & 0.055 \\
\hline SZ A-1 Dup & $\mathrm{J15511}$ & $6 / 13 / 07$ & 0.000 & $\mathrm{U}$ & 0.237 & 0.047 & $\mathrm{U}$ & 0.047 & 0.040 & $\mathrm{U}$ & 0.040 & 0.000 & $\mathrm{U}$ & 4.00 & 0.283 & & 0.044 \\
\hline SZ A-1 Split & $\mathrm{J} 15520$ & $6 / 13 / 07$ & 0.022 & $\mathrm{U}$ & 0.053 & - & & - & 0.004 & $\mathrm{U}$ & 0.019 & 0.041 & $\mathrm{U}$ & 0.771 & 0.252 & & 0.019 \\
\hline $\mathrm{SZA-2}$ & $\mathrm{J} 15512$ & $6 / 13 / 07$ & -0.030 & $\mathrm{U}$ & 0.232 & 0.040 & $\mathrm{U}$ & 0.040 & 0.022 & $\mathrm{U}$ & 0.022 & -0.433 & $\mathrm{U}$ & 3.72 & 0.113 & & 0.027 \\
\hline $\mathrm{SZA-3}$ & $\mathrm{J} 15513$ & $6 / 13 / 07$ & 0.028 & $\mathrm{U}$ & 0.270 & 0.159 & $\mathrm{U}$ & 0.159 & 0.031 & $\mathrm{U}$ & 0.031 & -0.623 & $\mathrm{U}$ & 4.59 & 0.315 & & 0.029 \\
\hline $\mathrm{SZA-4}$ & $\mathrm{J} 15514$ & $6 / 13 / 07$ & 0.165 & $\mathrm{U}$ & 0.252 & 0.037 & $\mathrm{U}$ & 0.037 & 0.017 & $\mathrm{U}$ & 0.017 & -0.333 & $\mathrm{U}$ & 3.82 & 0.140 & & 0.012 \\
\hline DZA-1 & $\mathrm{J} 15515$ & $6 / 4 / 07$ & -0.030 & $\mathrm{U}$ & 0.232 & 0.142 & $\mathrm{U}$ & 0.142 & 0.031 & $\mathrm{U}$ & 0.031 & -1.78 & UJ & 4.14 & 0.193 & & 0.024 \\
\hline $\mathrm{DZA-2}$ & $\mathrm{J} 15516$ & $6 / 4 / 07$ & 0.037 & $\mathrm{U}$ & 0.057 & 0.196 & $\mathrm{U}$ & 0.196 & 0.042 & $\mathrm{U}$ & 0.042 & 0.523 & UJ & 4.00 & 0.742 & & 0.037 \\
\hline $\mathrm{DZA-3}$ & $\mathrm{J} 15517$ & $6 / 4 / 07$ & -0.007 & $\mathrm{U}$ & 0.052 & 0.042 & $\mathrm{U}$ & 0.042 & 0.025 & $\mathrm{U}$ & 0.025 & -1.10 & UI & 3.47 & 0.158 & & 0.029 \\
\hline DZ A-3 Dup & J15518 & $6 / 4 / 07$ & 0.013 & $\mathrm{U}$ & 0.058 & 0.031 & $\mathrm{U}$ & 0.031 & 0.030 & $\mathrm{U}$ & 0.030 & -0.428 & UJ & 3.82 & 0.271 & & 0.027 \\
\hline DZ A-3 Split & J15521 & $6 / 4 / 07$ & 0.030 & $\mathrm{U}$ & 0.047 & - & & - & 0.003 & $\mathrm{U}$ & 0.019 & -0.505 & $\mathrm{U}$ & 0.854 & 0.478 & & 0.020 \\
\hline $\mathrm{DZA}-4$ & J15519 & $6 / 4 / 07$ & 0.112 & & 0.065 & 0.069 & $\mathrm{U}$ & 0.069 & 0.045 & $\mathrm{U}$ & 0.045 & -0.806 & UJ & 3.59 & 6.62 & & 0.045 \\
\hline $\mathrm{OBA}-1$ & $\mathrm{~J} 15574$ & $6 / 12 / 07$ & -0.016 & $\mathrm{U}$ & 0.126 & 0.280 & $\mathrm{U}$ & 0.280 & 0.042 & $\mathrm{U}$ & 0.042 & 76.1 & & 4.96 & 0.074 & $\mathrm{U}$ & 0.074 \\
\hline $\mathrm{OBA}-1$ & $\mathrm{~J} 15690$ & $6 / 22 / 07$ & - & & - & - & & - & - & & $\ldots$ & -0.896 & U & 2.64 & - & & - \\
\hline $\mathrm{OB} \mathrm{A-2}$ & $\mathrm{J} 15575$ & $6 / 12 / 07$ & 0.032 & $\mathrm{U}$ & 0.124 & 0.106 & $\mathrm{U}$ & 0.106 & 0.051 & $\mathrm{U}$ & 0.051 & -0.300 & $\mathrm{U}$ & 4.52 & 0.169 & & 0.030 \\
\hline $\mathrm{OBA}-3$ & $\mathrm{~J} 15576$ & $6 / 12 / 07$ & 0.017 & $\mathrm{U}$ & 0.129 & 0.015 & $\mathrm{U}$ & 0.015 & 0.015 & $\mathrm{U}$ & 0.015 & 0.243 & $\mathrm{U}$ & 4.27 & 0.406 & & 0.015 \\
\hline $\mathrm{OB} \mathrm{A-4}$ & $\mathrm{J} 15577$ & $6 / 12 / 07$ & 0.000 & $\mathrm{U}$ & 0.145 & 0.040 & $\mathrm{U}$ & 0.040 & 0.038 & $\mathrm{U}$ & 0.038 & 0.523 & $\mathrm{U}$ & 5.02 & 0.211 & & 0.037 \\
\hline OB B-5 & $\mathrm{J} 15578$ & $6 / 13 / 07$ & 0.000 & $\mathrm{U}$ & 0.238 & 0.114 & $\mathrm{U}$ & 0.114 & 0.017 & $\mathrm{U}$ & 0.017 & -0.947 & $\mathrm{U}$ & 4.25 & 0.062 & & 0.012 \\
\hline OB B-6 & $\mathrm{J} 15579$ & $6 / 13 / 07$ & -0.004 & $\mathrm{U}$ & 0.056 & 0.016 & $\mathrm{U}$ & 0.016 & 0.014 & $\mathrm{U}$ & 0.014 & -0.210 & $\mathrm{U}$ & 3.61 & 0.552 & & 0.015 \\
\hline OB B-7 & $\mathrm{J} 15580$ & $6 / 13 / 07$ & 0.035 & $\mathrm{U}$ & 0.067 & 0.017 & $\mathrm{U}$ & 0.017 & 0.009 & $\mathrm{U}$ & 0.009 & 0.103 & $\mathrm{U}$ & 3.56 & 0.042 & & 0.007 \\
\hline OB B-8 & J15581 & $6 / 13 / 07$ & 0.014 & $\mathrm{U}$ & 0.077 & 0.061 & $\mathrm{U}$ & 0.061 & 0.012 & $\mathrm{U}$ & 0.012 & -0.247 & $\mathrm{U}$ & 3.63 & 0.045 & & 0.011 \\
\hline $\mathrm{OBCl}$ & $\mathrm{J} 15584$ & $4 / 07$ & 0.000 & $\mathrm{U}$ & 0.212 & 0.099 & $\mathrm{U}$ & 0.099 & 0.047 & $\mathrm{U}$ & 0.047 & -2.32 & $\mathrm{U}$ & 3.74 & 0.046 & & 0.027 \\
\hline $\mathrm{OBC}-10$ & $\mathrm{~J} 15583$ & 107 & 0.044 & $\mathrm{U}$ & 0.079 & 0.080 & $\mathrm{U}$ & 0.080 & 0.039 & $\mathrm{U}$ & 0.039 & -1.21 & $\mathrm{U}$ & 3.90 & 0.039 & & 0.021 \\
\hline $\mathrm{OBC} 2$ & $\mathrm{~J} 1$ & $6 / 14 / 07$ & 0.032 & $\mathrm{U}$ & 0.122 & 0.246 & $\mathrm{U}$ & 0.246 & 0.033 & $\mathrm{U}$ & 0.033 & -2.37 & $\mathrm{U}$ & 4.40 & 0.072 & $\mathrm{U}$ & 0.072 \\
\hline OB C-9 & 82 & $6 / 13 / 07$ & 0.034 & $\mathrm{U}$ & 0.066 & 0.134 & $\mathrm{U}$ & 0.134 & 0.027 & $\mathrm{U}$ & 0.027 & -0.831 & $\mathrm{U}$ & 4.08 & 0.029 & $\mathrm{U}$ & 0.029 \\
\hline $\mathrm{OB} \mathrm{D} 3$ & $\mathrm{J1}$ & $6 / 14 / 07$ & 0.029 & $\mathrm{U}$ & 0.110 & 0.040 & $\mathrm{U}$ & 0.040 & 0.034 & $\mathrm{U}$ & 0.034 & -3.99 & $\mathrm{U}$ & 4.51 & 0.061 & & 0.031 \\
\hline $\mathrm{OB} \mathrm{D4}$ & $\mathrm{J} 15587$ & $6 / 14 / 07$ & 0.028 & $\mathrm{U}$ & 0.109 & 0.100 & $\mathrm{U}$ & 0.100 & 0.047 & $\mathrm{U}$ & 0.047 & -2.13 & $\mathrm{U}$ & 4.03 & 0.134 & & 0.030 \\
\hline OB D5 & $\mathrm{J} 15588$ & $6 / 14 / 07$ & 0.034 & $\mathrm{U}$ & 0.131 & 0.152 & $\mathrm{U}$ & 0.152 & 0.029 & $\mathrm{U}$ & 0.029 & -2.44 & $\mathrm{U}$ & 4.31 & 0.054 & & 0.029 \\
\hline OB D6 & $\mathrm{J} 15589$ & $6 / 14 / 07$ & 0.016 & $\mathrm{U}$ & 0.126 & 0.281 & $\mathrm{U}$ & 0.281 & 0.043 & $\mathrm{U}$ & 0.043 & -1.85 & $\mathrm{U}$ & 3.68 & 0.063 & & 0.042 \\
\hline OB D6 Dup & $\mathrm{J} 15590$ & $6 / 14 / 07$ & 0.056 & $\mathrm{U}$ & 0.143 & 0.041 & $\mathrm{U}$ & 0.041 & 0.035 & $\mathrm{U}$ & 0.035 & -2.03 & $\mathrm{U}$ & 3.71 & 0.055 & $\mathrm{U}$ & 0.055 \\
\hline OB D6 Split & $\mathrm{J} 15591$ & $6 / 14 / 07$ & 0.034 & $\mathrm{U}$ & 0.053 & $\cdots$ & & - & 0.000 & $\mathrm{U}$ & 0.017 & -0.225 & $\mathrm{U}$ & 0.835 & 0.053 & & 0.020 \\
\hline
\end{tabular}

\begin{tabular}{|c|c|c|c|c|c|c|c|c|c|c|c|c|c|c|c|c|c|}
\hline \multirow{2}{*}{$\begin{array}{c}\text { Sample } \\
\text { Location } \\
\end{array}$} & \multirow{2}{*}{$\begin{array}{c}\text { HEIS } \\
\text { Number }\end{array}$} & \multirow{2}{*}{$\begin{array}{c}\text { Sample } \\
\text { Date }\end{array}$} & \multicolumn{3}{|c|}{ Cobalt-60 } & \multicolumn{3}{|c|}{ Curium-242 } & \multicolumn{3}{|c|}{ Curium-243/44 } & \multicolumn{3}{|c|}{ Europium-152 } & \multicolumn{3}{|c|}{ Europium-154 } \\
\hline & & & $\mathrm{pCi} / \mathrm{g}$ & $Q$ & MDA & $\mathrm{pCi} / \mathrm{g}$ & $Q$ & $\mathrm{MDA}$ & $\mathrm{pCi} / \mathrm{g}$ & $Q$ & MDA & $\mathrm{pCi} / \mathrm{g}$ & Q & $\mathrm{MDA}$ & $\mathrm{pCi} / \mathrm{g}$ & $Q$ & MDA \\
\hline $\mathrm{SZA-1}$ & $\mathrm{J} 15510$ & $6 / 13 / 07$ & 0.049 & $\mathrm{U}$ & 0.049 & 0.000 & $\mathrm{U}$ & 0.304 & -0.038 & $\mathrm{U}$ & 0.368 & 0.197 & $\mathrm{U}$ & 0.197 & 0.161 & $\mathrm{U}$ & 0.161 \\
\hline SZ A-1 Dup & $\mathrm{J} 15511$ & $6 / 13 / 07$ & 0.040 & $\mathrm{U}$ & 0.040 & -0.032 & $\mathrm{U}$ & 0.246 & 0.000 & $\mathrm{U}$ & 0.382 & 0.174 & $\mathrm{U}$ & 0.174 & 0.124 & $\mathrm{U}$ & 0.124 \\
\hline SZA-1 Split & $\mathrm{J} 15520$ & $6 / 13 / 07$ & 0.009 & $\mathrm{U}$ & 0.020 & - & & - & 0.000 & $\mathrm{U}$ & 0.053 & 0.129 & $\mathrm{U}$ & 0.053 & 0.021 & $\mathrm{U}$ & 0.067 \\
\hline $\mathrm{SZA}-2$ & $\mathrm{~J} 15512$ & $6 / 13 / 07$ & 0.026 & $\mathrm{U}$ & 0.026 & 0.000 & $\mathrm{U}$ & 0.240 & 0.000 & $\mathrm{U}$ & 0.290 & 0.132 & $\mathrm{U}$ & 0.132 & 0.084 & $\mathrm{U}$ & 0.084 \\
\hline $\mathrm{SZA-3}$ & $\mathrm{J} 15513$ & $6 / 13 / 07$ & 0.026 & $\mathrm{U}$ & 0.026 & 0.000 & $\mathrm{U}$ & 0.224 & 0.028 & U & 0.348 & 0.128 & & 0.052 & 0.081 & $\mathrm{U}$ & 0.081 \\
\hline $\mathrm{SZA}-4$ & $\mathrm{~J} 15514$ & $6 / 13 / 07$ & 0.012 & $\mathrm{U}$ & 0.012 & 0.000 & $\mathrm{U}$ & 0.261 & 0.033 & $\mathrm{U}$ & 0.406 & 0.185 & & 0.026 & 0.039 & $\mathrm{U}$ & 0.039 \\
\hline $\mathrm{DZA}-1$ & $\mathrm{~J} 15515$ & $6 / 4 / 07$ & 0.023 & $\mathrm{U}$ & 0.023 & 0.032 & UI & 0.241 & -0.030 & $\mathrm{U}$ & 0.291 & 0.080 & $\mathrm{U}$ & 0.080 & 0.079 & $\mathrm{U}$ & 0.079 \\
\hline $\mathrm{DZA}-2$ & $\mathrm{J15516}$ & $6 / 4 / 07$ & 0.032 & $\mathrm{U}$ & 0.032 & -0.007 & $\mathrm{UJ}$ & 0.059 & -0.037 & $\mathrm{U}$ & 0.107 & 0.632 & & 0.084 & 0.102 & $\mathrm{U}$ & 0.102 \\
\hline $\mathrm{DZA}-3$ & $\mathrm{~J} 15517$ & $6 / 4 / 07$ & 0.023 & $\mathrm{U}$ & 0.023 & 0.008 & UJ & 0.057 & 0.025 & $\mathrm{U}$ & 0.106 & 0.128 & $\mathrm{U}$ & 0.128 & 0.069 & $\mathrm{U}$ & 0.069 \\
\hline DZ A-3 Dup & $\mathrm{J} 15518$ & $6 / 4 / 07$ & 0.027 & $\mathrm{U}$ & 0.027 & 0.027 & $\mathrm{UJ}$ & 0.055 & 0.004 & $\mathrm{U}$ & 0.117 & 0.188 & & 0.065 & 0.097 & $\mathrm{U}$ & 0.097 \\
\hline DZ A-3 Split & $\mathrm{J} 15521$ & $6 / 4 / 07$ & 0.015 & $\mathrm{U}$ & 0.024 & - & & - & 0.140 & & 0.040 & 0.175 & $\mathrm{U}$ & .058 & 0.024 & $\mathrm{U}$ & 0.075 \\
\hline $\mathrm{DZA}-4$ & $\mathrm{~J} 15519$ & $6 / 4 / 07$ & 0.274 & & 0.033 & 0.000 & UJ & 0.051 & 0.031 & $\mathrm{U}$ & 0.094 & 4.39 & & 0.104 & 0.501 & $\mathrm{U}$ & 0.501 \\
\hline $\mathrm{OB} \mathrm{A-1}$ & $\mathrm{J15574}$ & $6 / 12 / 07$ & 0.038 & $\mathrm{U}$ & 0.038 & 0.000 & $\mathrm{U}$ & 0.130 & -0.016 & $\mathrm{U}$ & 0.158 & 0.091 & $\mathrm{U}$ & 0.091 & 0.128 & $\mathrm{U}$ & 0.128 \\
\hline $\mathrm{OB} \mathrm{A-2}$ & $\mathrm{J} 15575$ & $6 / 12 / 07$ & 0.030 & $\mathrm{U}$ & 0.030 & 0.000 & $\mathrm{U}$ & 0.128 & 0.049 & $\mathrm{U}$ & 0.124 & 0.305 & & 0.078 & 0.112 & $\mathrm{U}$ & 0.112 \\
\hline $\mathrm{OB} \mathrm{A-3}$ & $\mathrm{J} 15576$ & $6 / 12 / 07$ & 0.020 & & 0.012 & 0.017 & $\mathrm{U}$ & 0.133 & 0.051 & $U$ & 0.129 & 0.980 & & 0.034 & 0.061 & $\mathrm{U}$ & 0.061 \\
\hline $\mathrm{OBA}-4$ & $\mathrm{~J} 15577$ & $6 / 12 / 07$ & 0.034 & $\mathrm{U}$ & 0.034 & 0.000 & $U$ & 0.149 & 0.000 & $\mathrm{U}$ & 0.234 & 0.165 & & 0.083 & 0.120 & $\mathrm{U}$ & 0.120 \\
\hline OB B-5 & $\mathrm{J} 15578$ & $6 / 13 / 07$ & 0.016 & $\mathrm{U}$ & 0.016 & 0.032 & $\mathrm{U}$ & 0.246 & 0.031 & $\mathrm{U}$ & 0.298 & 0.035 & $\mathrm{U}$ & 0.035 & 0.052 & $\mathrm{U}$ & 0.052 \\
\hline $\mathrm{OB} \mathrm{B}-6$ & $\mathrm{~J} 15579$ & $6 / 13 / 07$ & 0.033 & $\mathrm{U}$ & 0.033 & 0.004 & $\mathrm{U}$ & 0.031 & -0.012 & $\mathrm{U}$ & 0.060 & 0.364 & & 0.031 & 0.043 & $\mathrm{U}$ & 0.043 \\
\hline OB B-7 & $\mathrm{J} 15580$ & $6 / 13 / 07$ & 0.009 & $\mathrm{U}$ & 0.009 & -0.005 & $\mathrm{U}$ & 0.057 & 0.015 & $\mathrm{U}$ & 0.096 & 0.025 & $\mathrm{U}$ & 0.025 & 0.031 & $\mathrm{U}$ & 0.031 \\
\hline $\mathrm{OBB}-8$ & $\mathrm{~J} 15581$ & $6 / 13 / 07$ & 0.010 & $\mathrm{U}$ & 0.010 & -0.019 & $\mathrm{U}$ & 0.067 & -0.009 & $\mathrm{U}$ & 0.129 & 0.027 & $\mathrm{U}$ & 0.027 & 0.031 & $\mathrm{U}$ & 0.031 \\
\hline $\mathrm{OBC} 1$ & $J 15584$ & $6 / 14 / 07$ & 0.030 & $\mathrm{U}$ & 0.030 & 0.029 & $\mathrm{U}$ & 0.220 & -0.028 & $\mathrm{U}$ & 0.266 & 0.076 & $\mathrm{U}$ & 0.076 & 0.104 & $\mathrm{U}$ & 0.104 \\
\hline OB C-10 & $\mathrm{J} 15583$ & $6 / 13 / 07$ & 0.025 & $\mathrm{U}$ & 0.025 & -0.012 & $\mathrm{U}$ & 0.059 & -0.040 & $\mathrm{U}$ & 0.117 & 0.064 & $\mathrm{U}$ & 0.064 & 0.083 & $\mathrm{U}$ & 0.083 \\
\hline $\mathrm{OBC} 2$ & $\mathrm{~J} 15585$ & $6 / 14 / 07$ & 0.039 & $\mathrm{U}$ & 0.039 & 0.000 & $\mathrm{U}$ & 0.127 & 0.032 & $\mathrm{U}$ & 0.122 & 0.085 & $\mathrm{U}$ & 0.085 & 0.128 & $\mathrm{U}$ & 0.128 \\
\hline $\mathrm{OBC}-9$ & $\mathrm{~J} 15582$ & $6 / 13 / 07$ & 0.022 & $\mathrm{U}$ & 0.022 & 0.025 & $\mathrm{U}$ & 0.056 & 0.078 & $\mathrm{U}$ & 0.109 & 0.061 & $\mathrm{U}$ & 0.061 & 0.066 & $\mathrm{U}$ & 0.066 \\
\hline $\mathrm{OBD} 3$ & $\mathrm{~J} 15586$ & $6 / 14 / 07$ & 0.034 & $\mathrm{U}$ & 0.034 & 0.000 & $\mathrm{U}$ & 0.114 & 0.043 & $\mathrm{U}$ & 0.137 & 0.081 & $\mathrm{U}$ & 0.081 & 0.107 & $\mathrm{U}$ & 0.107 \\
\hline OB D4 & $\mathrm{J} 15587$ & $6 / 14 / 07$ & 0.031 & $\mathrm{U}$ & 0.031 & 0.000 & $\mathrm{U}$ & 0.113 & -0.014 & $\mathrm{U}$ & 0.191 & 0.084 & $\mathrm{U}$ & 0.084 & 0.104 & $\mathrm{U}$ & 0.104 \\
\hline OB D5 & $\mathrm{J} 15588$ & $6 / 14 / 07$ & 0.025 & $\mathrm{U}$ & 0.025 & 0.000 & $\mathrm{U}$ & 0.136 & -0.017 & $\mathrm{U}$ & 0.131 & 0.068 & $\mathrm{U}$ & 0.068 & 0.077 & $\mathrm{U}$ & 0.077 \\
\hline OB D6 & $J 15589$ & $6 / 14 / 07$ & 0.040 & $\mathrm{U}$ & 0.040 & 0.017 & $\mathrm{U}$ & 0.130 & 0.066 & $\bar{U}$ & 0.126 & 0.087 & $\mathrm{U}$ & 0.087 & 0.126 & $\mathrm{U}$ & 0.126 \\
\hline OB D6 Dup & $\mathrm{J} 15590$ & $6 / 14 / 07$ & 0.033 & $\mathrm{U}$ & 0.033 & 0.000 & $\mathrm{U}$ & 0.149 & -0.019 & $\mathrm{U}$ & 0.143 & 0.081 & $\mathrm{U}$ & 0.081 & 0.105 & $\mathrm{U}$ & 0.105 \\
\hline OB D6 Split & $\mathrm{J} 15591$ & $6 / 14 / 07$ & 0.004 & $\mathrm{U}$ & 0.022 & - & & - & 0.028 & $\mathrm{U}$ & 0.045 & -0.016 & $\mathrm{U}$ & 0.042 & -0.022 & $\mathrm{U}$ & 0.068 \\
\hline
\end{tabular}

Note: Data qualified with B, C, D and/or J, are considered acceptable values. $\mathrm{GEA}=$ gamma energy analysis

HEIS = Hanford Environmental Information System $Q=$ qualifier

$M D A=$ minimum detectable activity
$\mathrm{PQL}=$ practical quantitation limit

$\mathrm{U}=$ uncletected

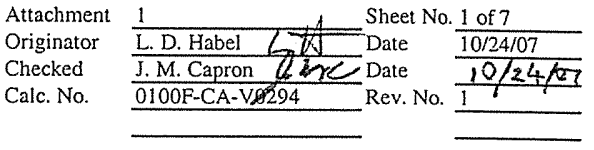




\begin{tabular}{|c|c|c|c|c|c|c|c|c|c|c|c|c|c|c|c|c|c|}
\hline \multirow{2}{*}{$\begin{array}{c}\text { Sample } \\
\text { Location }\end{array}$} & \multirow{2}{*}{$\begin{array}{c}\text { HEIS } \\
\text { Number }\end{array}$} & \multirow{2}{*}{$\begin{array}{c}\text { Sample } \\
\text { Date }\end{array}$} & \multicolumn{3}{|c|}{ Europium-155 } & \multicolumn{3}{|c|}{ Nickel-63 } & \multicolumn{3}{|c|}{ Plutonium-238 } & \multicolumn{3}{|c|}{ Plutonium-239/240 } & \multicolumn{3}{|c|}{ Potassium-40 } \\
\hline & & & $\mathrm{pCi} / \mathrm{g}$ & $\bar{Q}$ & MDA & $\mathrm{pCi} / \mathrm{g}$ & $Q$ & $\mathrm{MDA}$ & $\mathrm{pCi} / \mathrm{g}$ & $\bar{Q}$ & MDA & $\mathrm{pCi} / \mathrm{g}$ & Q & $\mathrm{MDA}$ & $\mathrm{pCi} / \mathrm{g}$ & Q & $\mathrm{MOA}$ \\
\hline SZA-1 & $\mathrm{J} 15510$ & $6 / 13 / 07$ & 0.128 & $\mathrm{U}$ & 0.128 & -0.392 & $\mathrm{U}$ & 3.15 & -0.051 & $\mathrm{U}$ & 0.390 & 0.051 & $\mathrm{U}$ & 0.390 & 14.6 & & 0.417 \\
\hline SZA-1 Dup & $\mathrm{J} 15511$ & $6 / 13 / 07$ & 0.085 & $\mathrm{U}$ & 0.085 & 0.413 & $\mathrm{U}$ & 3.32 & 0.000 & $\mathrm{U}$ & 0.236 & 0.062 & $\mathrm{U}$ & 0.236 & 14.3 & & 0.362 \\
\hline SZ A-1 Split & $\mathrm{J} 15520$ & $6 / 13 / 07$ & 0.050 & $\mathrm{U}$ & 0.048 & 5.10 & $\mathrm{U}$ & 5.45 & 0.000 & $\bar{U}$ & 0.074 & 0.034 & $\mathrm{U}$ & 0.107 & - & & \\
\hline SZA-2 & $\mathrm{J} 15512$ & $6 / 13 / 07$ & 0.061 & $\mathrm{U}$ & 0.061 & 1.87 & U & 3.31 & 0.000 & $\mathrm{U}$ & 0.205 & 0.054 & U & 0.205 & 15.3 & & 0.212 \\
\hline SZA-3 & $\mathrm{J} 15513$ & $6 / 13 / 07$ & 0.092 & $\mathrm{U}$ & 0.092 & 0.267 & $\mathrm{U}$ & 3.37 & 0.000 & $\mathrm{U}$ & 0.196 & 0.051 & $\mathrm{u}$ & 0.196 & 14.6 & & 0.274 \\
\hline $\mathrm{SZA-4}$ & $\mathrm{J} 15514$ & $6 / 13 / 07$ & 0.032 & $\mathrm{U}$ & 0.032 & -1.00 & $\mathrm{U}$ & 3.28 & 0.035 & $\bar{U}$ & 0.264 & 0 & $\mathrm{u}$ & 0.264 & 15.1 & & 0.111 \\
\hline DZA-1 & $\mathrm{J} 15515$ & $6 / 4 / 07$ & 0.079 & $\mathrm{U}$ & 0.079 & 1.13 & $\mathrm{U}$ & 3.03 & 0.000 & $\mathrm{U}$ & 0.211 & 0.028 & $\mathrm{U}$ & 0.211 & 13.6 & & 0.275 \\
\hline DZA-2 & $\mathrm{J} 15516$ & $6 / 4 / 07$ & 0.114 & $\mathrm{U}$ & 0.114 & 7.36 & & 3.05 & 0.000 & $\mathrm{U}$ & 0.184 & 0.096 & $u$ & 0.184 & 13.9 & & 0.309 \\
\hline $\mathrm{DZA}-3$ & $J 15517$ & 6/4/07 & 0.066 & $\mathrm{U}$ & 0.066 & 2.21 & $\mathrm{U}$ & 3.05 & 0.000 & $\mathrm{U}$ & 0.192 & 0.100 & $\mathrm{U}$ & 0.192 & 7.23 & & 0.192 \\
\hline DZ A-3 Dup & $J 15518$ & $6 / 4 / 07$ & 0.059 & $\mathrm{U}$ & 0.059 & 2.54 & $\mathrm{U}$ & 3.05 & 0.057 & $\mathrm{U}$ & 0.218 & 0.086 & U & 0.218 & 15.6 & & 0.272 \\
\hline DZ A-3 Split & $\mathrm{J} 15521$ & $6 / 4 / 07$ & 0.020 & $\mathrm{U}$ & 0.046 & 4.31 & $\mathrm{U}$ & 5.80 & -0.006 & $\mathrm{U}$ & 0.085 & 0.027 & $\mathrm{U}$ & 0.072 & - & & - \\
\hline DZA-4. & $\mathrm{J} 15519$ & $6 / 4 / 07$ & 0.116 & $\mathrm{U}$ & 0.116 & 26.1 & & 2.94 & 0.077 & $\mathrm{U}$ & 0.247 & 0.747 & & 0.197 & 14.4 & & 0.273 \\
\hline $\mathrm{OB} \mathrm{A-1}$ & $\mathrm{J} 15574$ & $6 / 12 / 07$ & 0.107 & $\mathrm{U}$ & 0.107 & -0.183 & $\mathrm{U}$ & 3.06 & 0.000 & $\mathrm{U}$ & 0.673 & 0.088 & U & 0.673 & 15.2 & & 0.367 \\
\hline $\mathrm{OB} \mathrm{A-2}$ & $J 15575$ & $6 / 12 / 07$ & 0.146 & $\mathrm{U}$ & 0.146 & 1.21 & $\mathrm{U}$ & 2.98 & -0.028 & $\mathrm{U}$ & 0.213 & 0.000 & $\mathrm{U}$ & 0.213 & 14.0 & & 0.289 \\
\hline $\mathrm{OB} \mathrm{A-3}$ & $\mathrm{J} 15576$ & $6 / 12 / 07$ & 0.031 & $\mathrm{U}$ & 0.031 & 2.00 & $\mathrm{U}$ & 3.10 & 0.000 & $\mathrm{U}$ & 0.264 & 0.069 & U & 0.264 & 12.9 & & 0.113 \\
\hline $\mathrm{OB} \mathrm{A-4}$ & $\mathrm{J} 15577$ & $6 / 12 / 07$ & 0.075 & $\mathrm{U}$ & 0.075 & 0.535 & $U$ & 2.99 & 0.000 & U & 0.362 & 0.095 & $\mathrm{U}$ & 0.362 & 14.2 & & 0.348 \\
\hline $\mathrm{OB} \mathrm{B}-5$ & $\mathrm{~J} 15578$ & $6 / 13 / 07$ & 0.042 & $\mathrm{U}$ & 0.042 & 0.037 & $\mathrm{U}$ & 3.24 & 0.000 & $\mathrm{U}$ & 0.252 & 0.000 & 0 & 0.252 & 14.0 & & 0.146 \\
\hline $\mathrm{OB} \mathrm{B}-6$ & $\mathrm{~J} 15579$ & $6 / 13 / 07$ & 0.051 & $\mathrm{U}$ & 0.051 & 0.112 & $\mathrm{U}$ & 3.30 & 0.000 & $\mathrm{U}$ & 0.227 & 0.059 & u & 0.227 & 13.2 & & 0.118 \\
\hline OB B-7 & $\mathrm{J15580}$ & $6 / 13 / 07$ & 0.042 & $\mathrm{U}$ & 0.042 & 0.224 & $\mathrm{U}$ & 3.30 & 0.000 & $\mathrm{U}$ & 0.260 & 0.034 & U & 0.260 & 13.8 & & 0.087 \\
\hline $\mathrm{OB} \mathrm{B}-8$ & $\mathrm{~J} 15581$ & $6 / 13 / 07$ & 0.041 & $\mathrm{U}$ & 0.041 & -1.520 & $\mathrm{U}$ & 3.19 & -0.059 & $\mathrm{U}$ & 0.284 & 0.030 & $\mathrm{U}$ & 0.226 & 13.9 & & 0.106 \\
\hline $\mathrm{OBC1}$ & $\checkmark 15584$ & $6 / 14 / 07$ & 0.083 & $\mathrm{U}$ & 0.083 & -0.204 & $\mathrm{U}$ & 3.19 & 0.021 & $\mathrm{U}$ & 0.161 & 0.379 & & 0.161 & 13.2 & & 0.257 \\
\hline $\mathrm{OBC}-10$ & $\mathrm{~J} 15583$ & $6 / 13 / 07$ & 0.067 & $\mathrm{U}$ & 0.067 & -0.997 & $\mathrm{U}$ & 3.26 & 0.029 & $\mathrm{U}$ & 0.219 & 0.000 & $\mathrm{U}$ & 0.219 & 12.8 & & 0.211 \\
\hline $\mathrm{OBC2}$ & $\mathrm{J} 15585$ & $6 / 14 / 07$ & 0.111 & U & 0.111 & 0.248 & $\mathrm{U}$ & 3.24 & -0.020 & $\mathrm{U}$ & 0.189 & 0.000 & uे & 0.151 & 14.0 & & 0.417 \\
\hline $\mathrm{OBC}-9$ & $\mathrm{~J} 15582$ & $6 / 13 / 07$ & 0.075 & $\mathrm{U}$ & 0.075 & -0.974 & $\mathrm{U}$ & 3.31 & -0.034 & $\mathrm{U}$ & 0.259 & 0.068 & $\mathrm{u}$ & 0.259 & 13.6 & & 0.204 \\
\hline OB D3 & $\mathrm{J} 15586$ & $6 / 14 / 07$ & 0.073 & $\mathrm{U}$ & 0.073 & 1.34 & $\mathrm{U}$ & 3.27 & 0.052 & $\mathrm{U}$ & 0.190 & 0.000 & 0 & 0.132 & 13.2 & & 0.283 \\
\hline $\mathrm{OBD} 4$ & $\mathrm{~J} 15587$ & $6 / 14 / 07$ & 0.083 & $\mathrm{U}$ & 0.083 & 0.168 & $\mathrm{U}$ & 3.30 & 0.000 & $\mathrm{U}$ & 0.193 & 0.061 & $\mathrm{U}$ & 0.154 & 13.5 & & 0.266 \\
\hline OB DS & J15588 & $6 / 14 / 07$ & 0.087 & $\mathrm{U}$ & 0.087 & 0.917 & $\mathrm{U}$ & 3.26 & -0.023 & $\mathrm{U}$ & 0.175 & 0.000 & u & 0.174 & 13.6 & & 0.214 \\
\hline OB D6 & J15589 & $6 / 14 / 07$ & 0.109 & $\mathrm{U}$ & 0.109 & 1.41 & U & 3.35 & 0.043 & $\mathrm{U}$ & 0.164 & 1.03 & & 0.164 & 12.7 & & 0.401 \\
\hline OB D6 Dup & $\mathrm{J} 15590$ & $6 / 14 / 07$ & 0.102 & $\mathrm{U}$ & 0.102 & 0.848 & $\mathrm{U}$ & 3.32 & 0.000 & $\mathrm{U}$ & 0.135 & 0.088 & 0 & 0.135 & 12.4 & & 0.300 \\
\hline OB D6 Split & 315591 & $6 / 14 / 07$ & 0.043 & $\mathrm{U}$ & 0.049 & 1.42 & $\mathrm{U}$ & 5.49 & 0.016 & $\bar{U}$ & 0.078 & -0.010 & $\mathrm{U}$ & 0.103 & 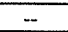 & & \\
\hline
\end{tabular}

\begin{tabular}{|c|c|c|c|c|c|c|c|c|c|c|c|c|c|c|c|c|c|}
\hline \multirow{2}{*}{$\begin{array}{c}\text { Sample } \\
\text { Location }\end{array}$} & \multirow{2}{*}{$\begin{array}{c}\text { HEIS } \\
\text { Number }\end{array}$} & \multirow{2}{*}{$\begin{array}{c}\text { Sample } \\
\text { Date }\end{array}$} & \multicolumn{3}{|c|}{ Radium-226 } & \multicolumn{3}{|c|}{ Radium-228 } & \multicolumn{3}{|c|}{ Silver-108 metastable } & \multicolumn{3}{|c|}{ Thorium-228 GEA } & \multicolumn{3}{|c|}{ Thorium-232 GEA } \\
\hline & & & $\mathrm{pCi} / \mathrm{g}$ & Q & $\mathrm{MDA}$ & $\mathrm{pCi} / \mathrm{g}$ & $Q$ & MDA & $\mathrm{pCi} / \mathrm{g}$ & $Q$ & MDA & $\mathrm{pCi} / \mathrm{g}$ & Q & $\mathrm{MDA}$ & $\mathrm{pCi} / \mathrm{g}$ & $Q$ & $\mathrm{MDA}$ \\
\hline $\mathrm{SZA-1}$ & $\mathrm{J} 15510$ & & 0.538 & & 0.092 & 0.688 & & 0.179 & 0.030 & $\mathrm{U}$ & 0.030 & 0.881 & & 0.085 & 0.688 & & 0.179 \\
\hline \begin{tabular}{|l} 
SZA-1 Dup \\
\end{tabular} & $\mathrm{J} 15511$ & $6 / 13 / 07$ & 0.456 & & 0.059 & 0.694 & & 0.143 & 0.030 & $\mathrm{U}$ & 0.030 & 0.732 & & 0.042 & 0.694 & & 0.143 \\
\hline \begin{tabular}{|l} 
SZ A-1 Split \\
\end{tabular} & $\mathrm{J15520}$ & $6 / 13 / 07$ & - & & - & - & & - & -0.012 & $\mathrm{U}$ & 0.013 & - & & - & & & 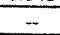 \\
\hline SZA-2 & $\mathrm{J} 15512$ & $6 / 13 / 07$ & 0.389 & & 0.046 & 0.584 & & 0.122 & 0.017 & U & 0.017 & 0.563 & & 0.029 & 0.584 & & 0.122 \\
\hline SZA-3 & $\mathrm{J} 15513$ & $6 / 13 / 07$ & 0.497 & & 0.051 & 0.645 & & 0.115 & 0.019 & $\mathrm{U}$ & 0.019 & 0.659 & & 0.036 & 0.645 & & 0.115 \\
\hline SZA-4 & $\mathrm{J} 15514$ & $6 / 13 / 07$ & 0.371 & & 0.018 & 0.623 & & 0.046 & \begin{tabular}{|l|l|}
0.008 \\
\end{tabular} & $\mathrm{U}$ & 0.008 & 0.670 & & 0.021 & 0.623 & & 0.046 \\
\hline $\mathrm{DZA-1}$ & $\mathrm{J} 15515$ & $6 / 4 / 07$ & 0.426 & & 0.042 & 0.479 & & 0.091 & 0.018 & $\bar{U}$ & 0.018 & 0.632 & & 0.040 & 0.479 & & 0.091 \\
\hline $\mathrm{DZA}-2$ & $\$ 15516$ & $6 / 4 / 07$ & 0.398 & & 0.060 & 0.702 & & 0.137 & 0.026 & $\mathrm{U}$ & 0.026 & 0.796 & & 0.062 & 0.702 & & 0.137 \\
\hline DZA-3 & $J 15517$ & $6 / 4 / 07$ & 0.456 & & 0.058 & 0.276 & & 0.088 & \begin{tabular}{|l|}
0.018 \\
\end{tabular} & $\bar{U}$ & 0.018 & 0.285 & & 0.031 & 0.276 & & 0.088 \\
\hline DZ A-3 Dup & $\mathrm{J} 15518$ & $6 / 4 / 07$ & 0.383 & & 0.051 & 0.506 & & 0.106 & \begin{tabular}{|l|}
0.019 \\
\end{tabular} & $\mathrm{U}$ & 0.019 & 0.504 & & 0.034 & 0.506 & & 0.106 \\
\hline DZA-3 Split & J15521 & $6 / 4 / 07$ & $=$ & & - & $=$ & & - & -0.004 & $\mathrm{U}$ & 0.015 & - & & - & 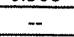 & & 0.20 \\
\hline $\mathrm{DZA-4}$ & $\mathrm{J} 15519$ & $6 / 4 / 07$ & 0.480 & & 0.072 & 0.744 & & 0.160 & \begin{tabular}{|l|}
0.037 \\
\end{tabular} & $\mathrm{U}$ & 0.037 & 0.732 & & 0.050 & 0.744 & & 0.160 \\
\hline OB A-1 & $\mathrm{J} 15574$ & $6 / 12 / 07$ & 0.553 & & 0.078 & 0.914 & & 0.172 & \begin{tabular}{|l|}
0.027 \\
\end{tabular} & $\mathrm{U}$ & 0.027 & 0.755 & & 0.049 & 0.914 & & 0.172 \\
\hline $\mathrm{OB} \mathrm{A-2}$ & $\mathrm{J} 15575$ & $6 / 12 / 07$ & 0.488 & & 0.055 & 0.738 & & 0.117 & 0.024 & $\overline{\mathrm{U}}$ & 0.024 & 0.856 & & 0.060 & 0.738 & & 0.117 \\
\hline $\mathrm{OBA}-3$ & $\mathrm{~J} 15576$ & $6 / 12 / 07$ & 0.429 & & 0.026 & 0.596 & & 0.054 & \begin{tabular}{|l|l|}
0.010 \\
\end{tabular} & $\mathrm{U}$ & 0.010 & 0.610 & & 0.018 & 0.596 & & 0.054 \\
\hline $\mathrm{OBA}-4$ & $\mathrm{~J} 15577$ & $6 / 12 / 07$ & 0.450 & & 0.058 & 0.694 & & 0.125 & \begin{tabular}{|l|}
0.023 \\
\end{tabular} & $\mathrm{U}$ & 0.023 & 0.742 & & 0.042 & 0.694 & & 0.125 \\
\hline OB B-5 & $\mathrm{J} 15578$ & $6 / 13 / 07$ & 0.460 & & 0.028 & 0.691 & & 0.060 & \begin{tabular}{|l|}
0.010 \\
\end{tabular} & $\mathrm{U}$ & 0.010 & 0.646 & & 0.019 & 0.691 & & 0.060 \\
\hline $\mathrm{OB} \mathrm{B-6}$ & $\mathrm{J} 15579$ & $6 / 13 / 07$ & 0.446 & & 0.022 & 0.622 & & 0.051 & 0.010 & $\mathrm{U}$ & 0.010 & 0.664 & & 0.016 & 0.622 & & 0.051 \\
\hline OB B-7 & $\mathrm{J} 15580$ & $6 / 13 / 07$ & 0.467 & & 0.018 & 0.681 & & 0.038 & $\begin{array}{l}0.007 \\
\end{array}$ & $\bar{U}$ & 0.007 & 0.687 & & 0.011 & 0.681 & & 0.038 \\
\hline $\mathrm{OBB}-8$ & $\mathrm{~J} 15581$ & $6 / 13 / 07$ & 0.462 & & 0.021 & 0.693 & & 0.044 & 0.007 & $\mathrm{U}$ & 0.007 & 0.682 & & 0.014 & 0.693 & & 0.044 \\
\hline $\mathrm{OBCl}$ & $\mathrm{J} 15584$ & $6 / 14 / 07$ & 0.405 & & 0.050 & 0.619 & & 0.109 & 0.022 & $\mathrm{U}$ & 0.022 & 0.786 & & 0.050 & 0.619 & & 0.109 \\
\hline $\mathrm{OBC}-10$ & $\mathrm{~J} 15583$ & $6 / 13 / 07$ & 0.445 & & 0.038 & 0.666 & & 0.084 & 0.017 & U & 0.017 & 0.811 & & 0.043 & 0.666 & & 0.084 \\
\hline $\mathrm{OBC2}$ & $\mathrm{J} 15585$ & $6 / 14 / 07$ & 0.486 & & 0.069 & 0.711 & & 0.156 & 0.026 & $\mathrm{U}$ & 0.026 & 0.671 & & 0.048 & 0.711 & & 0.156 \\
\hline $\mathrm{OBC}-9$ & $\mathrm{~J} 15582$ & $6 / 13 / 07$ & 0.420 & & 0.045 & 0.672 & & 0.106 & 0.016 & $\mathrm{U}$ & 0.016 & 0.652 & & 0.030 & 0.672 & & 0.106 \\
\hline $\mathrm{OBD} 3$ & $J 15586$ & $6 / 14 / 07$ & 0.468 & & 0.057 & 0.724 & & 0.109 & 0.023 & $\mathrm{U}$ & 0.023 & 0.655 & & 0.038 & 0.724 & & 0.109 \\
\hline $\mathrm{OBD} 4$ & $\mathrm{~J} 15587$ & $6 / 14 / 07$ & 0.472 & & 0.048 & 0.686 & & 0.097 & 0.022 & $\mathrm{U}$ & 0.022 & 0.830 & & 0.051 & 0.686 & & 0.097 \\
\hline OB D5 & $\mathrm{J} 15588$ & $6 / 14 / 07$ & 0.415 & & 0.048 & 0.678 & & 0.120 & 0.017 & $\mathrm{U}$ & 0.017 & 0.660 & & 0.035 & 0.678 & & 0.120 \\
\hline $\mathrm{OBD}$ & $J 15589$ & $6 / 14 / 07$ & 0.436 & & 0.072 & 0.718 & & 0.157 & 0.025 & U & 0.025 & 0.666 & & 0.051 & 0.718 & & 0.157 \\
\hline OB D6 Dup & $\mathrm{J} 15590$ & $6 / 14 / 07$ & 0.434 & & 0.047 & 0.648 & & 0.133 & 0.023 & $\mathrm{U}$ & 0.023 & 0.667 & & 0.038 & 0.648 & & 0.133 \\
\hline OB D6 Split & $\mathrm{J} 15591$ & $6 / 14 / 07$ & - & & 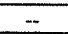 & $\ldots$ & & - & -0.002 & $\mathrm{U}$ & 0.014 & 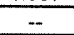 & & 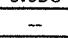 & - & & $-\cdots$ \\
\hline
\end{tabular}

Note: Data qualified with $B, C, D$ and/or J, are considered acceptable values.

GEA = gamma energy analysis

$\begin{array}{ll}\text { HEIS = Hanford Environmental Information System } & Q=\text { qualifier } \\ & U=\text { undetected }\end{array}$
Attachment 1

Originator L.D. Habel L Date

Checked J.M. Capron Date

Calc. No. $0100 \mathrm{~F}-\mathrm{CA}-\mathrm{V} 0294$ Rev. No. 0 


\begin{tabular}{|c|c|c|c|c|c|c|c|c|c|c|c|c|c|c|c|c|c|}
\hline \multirow{2}{*}{$\begin{array}{c}\text { Sample } \\
\text { Location }\end{array}$} & \multirow{2}{*}{$\begin{array}{c}\text { HEIS } \\
\text { Number }\end{array}$} & \multirow{2}{*}{$\begin{array}{c}\text { Sample } \\
\text { Date }\end{array}$} & \multicolumn{3}{|c|}{ Strontium (total) } & \multicolumn{3}{|c|}{ Tritium } & \multicolumn{3}{|c|}{ Uranium-233/234 } & \multicolumn{3}{|c|}{ Uranium-235 } & \multicolumn{3}{|c|}{ Uranium-235 GEA } \\
\hline & & & $\mathrm{pCi} / \mathrm{g}$ & $Q$ & MDA & $\mathrm{pCi} / \mathrm{g}$ & $Q$ & MDA & $\mathrm{pCi} / \mathrm{g}$ & $Q$ & MDA & $\mathrm{pCi} / \mathrm{g}$ & $\mathrm{Q}$ & $\mathrm{MDA}$ & $\mathrm{pCi} / \mathrm{g}$ & $Q$ & $\mathrm{MDA}$ \\
\hline $\mathrm{SZA-1}$ & $\mathrm{J} 15510$ & $6 / 13 / 07$ & 0.055 & $\mathrm{U}$ & 0.248 & 1.82 & $\mathrm{U}$ & 4.10 & 0.494 & & 0.126 & 0.040 & $\mathrm{U}$ & 0.152 & 0.182 & $\mathrm{U}$ & 0.182 \\
\hline SZ A-1 Dup & $\mathrm{J} 15511$ & $6 / 13 / 07$ & 0.108 & $\mathrm{U}$ & 0.288 & 1.83 & U & 4.42 & 0.579 & & 0.148 & 0.070 & $\mathrm{U}$ & 0.179 & 0.141 & $\mathrm{U}$ & 0.141 \\
\hline SZ A-1 Split & $\mathrm{J} 15520$ & $6 / 13 / 07$ & 0.151 & $\mathrm{U}$ & 0.155 & 0.003 & U & 0.018 & 0.718 & & 0.033 & 0.014 & $\mathrm{U}$ & 0.033 & - & & $\ldots$ \\
\hline SZ A-2 & $\mathrm{J} 15512$ & $6 / 13 / 07$ & 0.002 & $\mathrm{U}$ & 0.351 & -0.492 & $\mathrm{U}$ & 4.14 & 0.440 & & 0.130 & 0.020 & $\mathrm{U}$ & 0.157 & 0.099 & $\mathrm{U}$ & 0.099 \\
\hline $\mathrm{SZA-3}$ & $\mathrm{J} 15513$ & $6 / 13 / 07$ & 0.175 & $\mathrm{U}$ & 0.344 & 3.04 & $\mathrm{U}$ & 5.13 & 0.471 & & 0.034 & 0.034 & & 0.029 & 0.120 & $\mathrm{U}$ & 0.120 \\
\hline$S Z A-4$ & $\mathrm{~J} 15514$ & $6 / 13 / 07$ & 0.109 & $\mathrm{U}$ & 0.302 & 2.09 & $\mathrm{U}$ & 4.26 & 0.371 & & 0.129 & 0.020 & $\mathrm{U}$ & 0.156 & 0.049 & $\mathrm{U}$ & 0.049 \\
\hline DZ A-1 & $\mathrm{J} 15515$ & $6 / 4 / 07$ & 0.308 & & 0.260 & 0.276 & UJ & 4.55 & 0.447 & & 0.180 & 0.029 & $\bar{U}$ & 0.218 & 0.105 & $\mathrm{U}$ & 0.105 \\
\hline $\mathrm{DZA-2}$ & $\mathrm{J15516}$ & $6 / 4 / 07$ & 0.196 & $\mathrm{U}$ & 0.256 & 0.886 & UJ & 4.49 & 0.418 & & 0.139 & 0.022 & $U$ & 0.168 & 0.149 & $\mathrm{U}$ & 0.149 \\
\hline DZA-3 & $\mathrm{J} 15517$ & $6 / 4 / 07$ & 0.186 & $\mathrm{U}$ & 0.250 & 1.77 & UJ & 3.88 & 0.330 & & 0.148 & 0.023 & $\mathrm{U}$ & 0.180 & 0.098 & $\mathrm{U}$ & 0.098 \\
\hline DZ A-3 Dup & $\mathrm{J} 15518$ & $6 / 4 / 07$ & 0.116 & $\mathrm{U}$ & 0.271 & 1.69 & UJ & 4.29 & 0.507 & & 0.162 & 0.026 & U & 0.196 & 0.110 & $\mathrm{U}$ & 0.110 \\
\hline DZ A-3 Split & $\mathrm{J} 15521$ & $6 / 4 / 07$ & 6.91 & & 0.141 & -0.008 & U & 0.049 & 0.132 & & 0.037 & 0.036 & & 0.033 & - & & - \\
\hline $\mathrm{DZA}-4$ & $\mathrm{~J} 15519$ & $6 / 4 / 07$ & 3.21 & & 0.240 & 0.549 & UJ & 4.02 & 0.564 & & 0.108 & 0.120 & U & 0.131 & 0.161 & $\mathrm{U}$ & 0.161 \\
\hline $\mathrm{OBA}-1$ & $\mathrm{~J} 15574$ & $6 / 12 / 07$ & 0.058 & $\mathrm{U}$ & 0.297 & 0.495 & $\mathrm{U}$ & 5.52 & 0.569 & & 0.218 & 0.034 & $\mathrm{U}$ & 0.264 & 0.162 & $\mathrm{U}$ & 0.162 \\
\hline $\mathrm{OBA}$ A-1 & $\mathrm{J} 15690$ & $6 / 22 / 07$ & - & & $\ldots$ & -0.906 & U & 4.04 & - & & - & $\ldots$ & & - & - & & - \\
\hline $\mathrm{OBA}-2$ & $\mathrm{~J} 15575$ & $6 / 12 / 07$ & -0.014 & $\mathrm{U}$ & 0.291 & 2.50 & U & 5.07 & 0.508 & & 0.177 & 0.056 & $\mathrm{U}$ & 0.214 & 0.137 & $\mathrm{U}$ & 0.137 \\
\hline $\mathrm{OBA}-3$ & $\mathrm{~J} 15576$ & $6 / 12 / 07$ & 0.085 & $\mathrm{U}$ & 0.295 & 2.45 & $\mathrm{U}$ & 4.82 & 0.726 & & 0.192 & 0.030 & $\mathrm{U}$ & 0.232 & 0.055 & $\mathrm{U}$ & 0.055 \\
\hline $\mathrm{OBA}-4$ & $\mathrm{~J} 15577$ & $6 / 12 / 07$ & 0.066 & $\mathrm{U}$ & 0.305 & 2.53 & $\mathrm{U}$ & 5.65 & 0.441 & & 0.153 & 0.049 & $\mathrm{U}$ & 0.186 & 0.139 & $\mathrm{U}$ & 0.139 \\
\hline OB B-5 & $\mathrm{J} 15578$ & $6 / 13 / 07$ & -0.033 & $\mathrm{U}$ & 0.297 & -0.352 & $\mathrm{U}$ & 4.75 & 0.346 & & 0.139 & 0.044 & $\mathrm{U}$ & 0.168 & 0.064 & $\mathrm{U}$ & 0.064 \\
\hline $\mathrm{OB} \mathrm{B-6}$ & $\mathrm{J} 15579$ & $6 / 13 / 07$ & 0.095 & $\mathrm{U}$ & 0.270 & 0.799 & U & 4.14 & 0.465 & & 0.033 & 0.029 & & 0.027 & 0.050 & $\mathrm{U}$ & 0.050 \\
\hline OB B-7 & $\mathrm{J} 15580$ & $6 / 13 / 07$ & 0.022 & $\mathrm{U}$ & 0.286 & 1.20 & $\mathrm{U}$ & 4.04 & 0.314 & & 0.141 & 0.067 & $\mathrm{U}$ & 0.171 & 0.054 & $\mathrm{U}$ & 0.054 \\
\hline OB B-8 & $\mathrm{J} 15581$ & $6 / 13 / 07$ & -0.078 & $\mathrm{U}$ & 0.286 & 1.70 & $\mathrm{U}$ & 4.26 & 0.470 & & 0.150 & 0.047 & $\mathrm{U}$ & 0.181 & 0.042 & $\mathrm{U}$ & 0.042 \\
\hline $\mathrm{OBCl}$ & $\mathrm{J} 15584$ & $6 / 14 / 07$ & -0.011 & $\mathrm{U}$ & 0.280 & 1.39 & $\mathrm{U}$ & 5.06 & 0.530 & & 0.099 & 0.031 & $\mathrm{U}$ & 0.120 & 0.131 & $\mathrm{U}$ & 0.131 \\
\hline OB C-10 & $\mathrm{J} 15583$ & $6 / 13 / 07$ & -0.069 & $\mathrm{U}$ & 0.267 & 0.388 & $\mathrm{U}$ & 4.36 & 0.500 & & 0.153 & 0.000 & $\mathrm{U}$ & 0.185 & 0.104 & $\mathrm{U}$ & 0.104 \\
\hline $\mathrm{OBC2}$ & $\mathrm{J} 15585$ & $6 / 14 / 07$ & 0.026 & $\mathrm{U}$ & 0.315 & -1.560 & $\mathrm{U}$ & 6.00 & 0.420 & & 0.107 & 0.000 & $\mathrm{U}$ & 0.130 & 0.144 & U & 0.144 \\
\hline $\mathrm{OBC}-9$ & $\mathrm{~J} 15582$ & $6 / 13 / 07$ & -0.066 & $\mathrm{U}$ & 0.310 & 0.276 & U & 4.65 & 0.375 & & 0.179 & 0.057 & $\mathrm{U}$ & 0.217 & 0.102 & $\mathrm{U}$ & 0.102 \\
\hline $\mathrm{OB} \mathrm{D3}$ & $\mathrm{J} 15586$ & $6 / 14 / 07$ & 0.013 & $\mathrm{U}$ & 0.312 & -3.260 & $\mathrm{U}$ & 6.25 & 0.487 & & 0.089 & 0.014 & $\mathrm{U}$ & 0.107 & 0.122 & U & 0.122 \\
\hline OB D4 & $\mathrm{J} 15587$ & $6 / 14 / 07$ & 0.001 & $\mathrm{U}$ & 0.280 & -0.642 & $\mathrm{U}$ & 5.55 & 0.348 & & 0.098 & 0.000 & $\mathrm{U}$ & 0.119 & 0.130 & U & 0.130 \\
\hline OB D5 & $\mathrm{J} 15588$ & $6 / 14 / 07$ & 0.072 & $\mathrm{U}$ & 0.258 & -3.650 & $\mathrm{U}$ & 6.00 & 0.245 & & 0.117 & 0.000 & $\mathrm{U}$ & 0.142 & 0.119 & $\mathrm{U}$ & 0.119 \\
\hline OB D6 & $\mathrm{J} 15589$ & $6 / 14 / 07$ & 0.048 & $\mathrm{U}$ & 0.281 & -2.440 & $\mathrm{U}$ & 5.12 & 0.396 & & 0.138 & 0.000 & $\mathrm{U}$ & 0.166 & 0.161 & U & 0.161 \\
\hline OB D6 Dup & $\mathrm{J} 15590$ & $6 / 14 / 07$ & 0.039 & $\mathrm{U}$ & 0.240 & -1.690 & $\mathrm{U}$ & 5.07 & 0.425 & & 0.120 & 0.038 & U & 0.146 & 0.124 & $\mathrm{U}$ & 0.124 \\
\hline OB D6 Split & $J 15591$ & $6 / 14 / 07$ & 0.008 & $\mathrm{U}$ & 0.179 & 0.005 & $\mathrm{U}$ & 0.020 & 0.570 & & 0.047 & -0.003 & U & 0.042 & - & & $\ldots$ \\
\hline
\end{tabular}

\begin{tabular}{|c|c|c|c|c|c|c|c|c|}
\hline \multirow{2}{*}{$\begin{array}{c}\text { Sample } \\
\text { Location }\end{array}$} & \multirow{2}{*}{$\begin{array}{c}\text { HEIS } \\
\text { Number }\end{array}$} & \multirow{2}{*}{$\begin{array}{c}\text { Sample } \\
\text { Date }\end{array}$} & \multicolumn{3}{|c|}{ Uranium-238 } & \multicolumn{3}{|c|}{ Uranium-238 GEA } \\
\hline & & & $\mathrm{pCi} / \mathrm{g}$ & $\mathbf{Q}$ & $\mathrm{MDA}$ & $\mathrm{pCi} / \mathrm{g}$ & $\mathbf{Q}$ & MDA \\
\hline $\mathrm{SZA-1}$ & $\mathrm{J} 15510$ & $6 / 13 / 07$ & 0.461 & & 0.126 & 5.13 & $\mathrm{U}$ & 5.13 \\
\hline SZ A-1 Dup & J15511 & $6 / 13 / 07$ & 0.521 & & 0.148 & 4.47 & $\mathrm{U}$ & 4.47 \\
\hline SZA-1 Split & $\mathrm{J} 15520$ & $6 / 13 / 07$ & 0.771 & & 0.033 & - & & - \\
\hline $\mathrm{SZA-2}$ & $\mathrm{J} 15512$ & $6 / 13 / 07$ & 0.390 & & 0.130 & 2.92 & $\mathrm{U}$ & 2.92 \\
\hline $\mathrm{SZA-3}$ & $\mathrm{J} 15513$ & $6 / 13 / 07$ & 0.403 & & 0.030 & 2.96 & $\mathrm{U}$ & 2.96 \\
\hline SZ A-4 & $\mathrm{J} 15514$ & $6 / 13 / 07$ & 0.405 & & 0.129 & 1.35 & $\mathrm{U}$ & 1.35 \\
\hline $\mathrm{DZA}-1$ & $\mathrm{~J} 15515$ & $6 / 4 / 07$ & 0.494 & & 0.180 & 2.62 & $\mathrm{U}$ & 2.62 \\
\hline $\mathrm{DZ} A-2$ & $\mathrm{~J} 15516$ & $6 / 4 / 07$ & 0.363 & & 0.139 & 3.56 & $\mathrm{U}$ & 3.56 \\
\hline $\mathrm{DZA}-3$ & $\mathrm{~J} 15517$ & $6 / 4 / 07$ & 0.446 & & 0.148 & 2.88 & $\mathrm{U}$ & 2.88 \\
\hline DZ A-3 Dup & $\mathrm{J} 15518$ & $6 / 4 / 07$ & 0.465 & & 0.162 & 3.40 & $\mathrm{U}$ & 3.40 \\
\hline DZA-3 Split & $\mathrm{J} 15521$ & $6 / 4 / 07$ & 0.167 & & 0.028 & - & & - \\
\hline $\mathrm{DZA-4}$ & $J 15519$ & $6 / 4 / 07$ & 0.677 & & 0.108 & 4.08 & $\mathrm{U}$ & 4.08 \\
\hline $\mathrm{OBA}-1$ & $\mathrm{J15574}$ & $6 / 12 / 07$ & 0.769 & & 0.218 & 4.66 & $\mathrm{U}$ & 4.66 \\
\hline $\mathrm{OBA}-2$ & $\mathrm{J15575}$ & $6 / 12 / 07$ & 0.578 & & 0.177 & 3.69 & $\mathrm{U}$ & 3.69 \\
\hline $\mathrm{OBA}-3$ & $\mathrm{~J} 15576$ & $6 / 12 / 07$ & 0.501 & & 0.192 & 1.63 & $\mathrm{U}$ & 1.63 \\
\hline $\mathrm{OB} \mathrm{A}-4$ & $\mathrm{~J} 15577$ & $6 / 12 / 07$ & 0.541 & & 0.153 & 4.20 & $\mathrm{U}$ & 4.20 \\
\hline $\mathrm{OB} \mathrm{B-5}$ & $\mathrm{J} 15578$ & $6 / 13 / 07$ & 0.309 & & 0.139 & 1.72 & $\mathrm{U}$ & 1.72 \\
\hline $\mathrm{OB} \mathrm{B-6}$ & $\mathrm{J} 15579$ & $6 / 13 / 07$ & 0.468 & & 0.033 & 1.53 & $\mathrm{U}$ & 1.53 \\
\hline OB B-7 & $\mathrm{J} 15580$ & $6 / 13 / 07$ & 0.388 & & 0.141 & 1.36 & $\mathrm{U}$ & 1.36 \\
\hline $\mathrm{OBB}-8$ & $\mathrm{~J} 15581$ & $6 / 13 / 07$ & 0.450 & & 0.150 & 1.18 & $\mathrm{U}$ & 1.18 \\
\hline $\mathrm{OBCl}$ & $\mathrm{J} 15584$ & $6 / 14 / 07$ & 0.439 & & 0.099 & 3.42 & $\mathrm{U}$ & 3.42 \\
\hline $\mathrm{OBC}-10$ & 315583 & $6 / 13 / 07$ & 0.380 & & 0.153 & 2.83 & $\mathrm{U}$ & 2.83 \\
\hline $\mathrm{OBC2}$ & $\mathrm{J} 15585$ & $6 / 14 / 07$ & 0.504 & & 0.107 & 4.98 & $\mathrm{U}$ & 4.98 \\
\hline OB C-9 & 515582 & $6 / 13 / 07$ & 0.445 & & 0.179 & 2.52 & $\mathrm{U}$ & 2.52 \\
\hline $\mathrm{OB} \mathrm{D3}$ & $J 15586$ & $6 / 14 / 07$ & 0.661 & & 0.089 & 3.78 & $\mathrm{U}$ & 3.78 \\
\hline OB D4 & $\mathrm{J} 15587$ & $6 / 14 / 07$ & 0.412 & & 0.098 & 3.59 & $\mathrm{U}$ & 3.59 \\
\hline OB D5 & $\mathrm{J} 15588$ & $6 / 14 / 07$ & 0.536 & & 0.117 & 2.72 & $\mathrm{U}$ & 2.72 \\
\hline $\mathrm{OB} \mathrm{D6}$ & J15589 & $6 / 14 / 07$ & 0.342 & & 0.138 & 4.43 & $\mathrm{U}$ & 4.43 \\
\hline OB D6 Dup & $\mathrm{J15590}$ & $6 / 14 / 07$ & 0.520 & & 0.120 & 3.74 & $\mathrm{U}$ & 3.74 \\
\hline OB D6 Split & $\mathrm{J} 15591$ & $6 / 14 / 07$ & 0.527 & & 0.042 & - & & - \\
\hline
\end{tabular}

Note: Data qualified with B, C, D and/or J, are considered acceptable values. $\mathrm{GEA}=$ gamma energy analysis

HEIS $=$ Hanford Environmental Information System $\mathrm{MDA}=$ minimum detectable activity
$\mathrm{PQL}=$ practical quantitation limit

$Q=$ qualifier

$Q=$ qualifier
$U=$ undetected
Attachment 1

Originator L. D. Habel LA Date $\frac{10 / 24 / 07}{100}$

Checked J.M.Capron Date 


\begin{tabular}{|c|c|c|c|c|c|c|c|c|c|c|c|c|c|c|c|c|c|}
\hline \multirow{2}{*}{$\begin{array}{c}\text { Sample } \\
\text { Location }\end{array}$} & \multirow{2}{*}{$\begin{array}{c}\text { HEIS } \\
\text { Number }\end{array}$} & \multirow{2}{*}{$\begin{array}{c}\text { Sample } \\
\text { Date }\end{array}$} & \multicolumn{3}{|c|}{ Aluminum } & \multicolumn{3}{|c|}{ Antimony } & \multicolumn{3}{|c|}{ Arsenic } & \multicolumn{3}{|c|}{ Barium } & \multicolumn{3}{|c|}{ Beryllium } \\
\hline & & & $\mathrm{mg} / \mathrm{kg}$ & $Q$ & $\mathrm{PQL}$ & $\mathrm{mg} / \mathrm{kg}$ & $Q$ & PQL & $\mathrm{mg} / \mathrm{kg}$ & $Q$ & $\mathrm{PQL}$ & $\mathrm{mg} / \mathrm{kg}$ & Q & PQL & $\mathrm{mg} / \mathrm{kg}$ & $Q$ & PQL \\
\hline $\mathrm{SZA-1}$ & $\mathrm{J} 15510$ & $6 / 13 / 07$ & 4080 & & 4.9 & 0.65 & $\mathrm{U}$ & 0.65 & 2.4 & & 1.2 & 54.1 & & 0.06 & 0.36 & & 0.03 \\
\hline SZA-1 Dup & $\mathrm{J} 15511$ & $6 / 13 / 07$ & 6030 & & 4.9 & 0.65 & U & 0.65 & 2.4 & & 1.2 & 74.3 & & 0.06 & 0.46 & & 0.03 \\
\hline SZA-1 Split & $\mathrm{J} 15520$ & $6 / 13 / 07$ & 7720 & $\mathrm{~N}$ & 10.3 & 0.82 & $\mathrm{BN}$ & 0.52 & 2.8 & & 0.2 & 87.6 & $\mathrm{~N}$ & 0.23 & 0.20 & $\mathrm{~B}$ & 0.07 \\
\hline $\mathrm{SZ} \mathrm{A-2}$ & $\mathrm{J} 15512$ & $6 / 13 / 07$ & 5080 & & 4.9 & 0.66 & $\mathrm{U}$ & 0.66 & 2.0 & & 1.2 & 65.2 & & 0.06 & 0.37 & & 0.03 \\
\hline $\mathrm{SZA}-3$ & $\mathrm{~J} 15513$ & $6 / 13 / 07$ & 6130 & & 4.8 & 0.64 & U & 0.64 & 2.6 & & 1.2 & 71.3 & & 0.06 & 0.46 & & 0.03 \\
\hline $\mathrm{SZA-4}$ & $\mathrm{J} 15514$ & $6 / 13 / 07$ & 5400 & & 4.8 & 0.64 & $\mathrm{U}$ & 0.64 & 1.2 & $\mathrm{U}$ & 1.2 & 49.6 & & 0.06 & 0.36 & & 0.03 \\
\hline $\mathrm{DZA}-1$ & $\mathrm{~J} 15515$ & $6 / 4 / 07$ & 4590 & & 4.8 & 0.64 & UJ & 0.64 & 1.8 & & 1.2 & 44.2 & C & 0.06 & 0.11 & & 0.03 \\
\hline $\mathrm{DZ} A-2$ & $\mathrm{~J} 15516$ & $6 / 4 / 07$ & 4730 & & 5.0 & 0.66 & UI & 0.66 & 2.0 & & 1.2 & 44.6 & C & 0.06 & 0.13 & & 0.03 \\
\hline $\mathrm{DZA}-3$ & $\mathrm{~J} 15517$ & $6 / 4 / 07$ & 4470 & & 4.9 & 0.65 & UI & 0.65 & 2.4 & & 1.2 & 39.4 & C & 0.06 & 0.14 & & 0.03 \\
\hline DZ A-3 Dup & $\mathrm{J} 15518$ & $6 / 4 / 07$ & 3810 & & 4.8 & 0.64 & UI & 0.64 & 1.6 & & 1.2 & 42.5 & C & 0.06 & 0.13 & & 0.03 \\
\hline DZ A-3 Split & $\mathrm{J} 15521$ & $6 / 4 / 07$ & 5650 & $\mathrm{~N}$ & 5.2 & 0.52 & UN & 0.52 & 2.4 & & 0.2 & 45.0 & & 0.24 & 0.20 & $\mathrm{~B}$ & 0.07 \\
\hline $\mathrm{DZ} \mathrm{A-4}$ & $\mathrm{J} 15519$ & $6 / 4 / 07$ & 4610 & & 5.0 & 0.66 & UJ & 0.66 & 1.5 & & 1.2 & 57.1 & C & 0.06 & 0.10 & & 0.03 \\
\hline $\mathrm{OBA}-1$ & $\mathrm{~J} 15574$ & $6 / 12 / 07$ & 5770 & & 5.0 & 0.66 & $\mathrm{U}$ & 0.66 & 1.3 & & 1.2 & 64.5 & C & 0.06 & 0.03 & $\mathrm{U}$ & 0.03 \\
\hline $\mathrm{OBA}-2$ & $\mathrm{~J} 15575$ & $6 / 12 / 07$ & 6330 & & 5.0 & 0.66 & $\mathrm{U}$ & 0.66 & 1.7 & & 1.2 & 83.0 & C & 0.06 & 0.03 & $\mathrm{U}$ & 0.03 \\
\hline $\mathrm{OB} \mathrm{A-3}$ & $\mathrm{J} 15576$ & $6 / 12 / 07$ & 5200 & & 4.9 & 0.65 & $\mathrm{U}$ & 0.65 & 1.6 & & 1.2 & 70.8 & C & 0.06 & 0.03 & $\mathrm{U}$ & 0.03 \\
\hline $\mathrm{OB} \mathrm{A-4}$ & $\mathrm{J} 15577$ & $6 / 12 / 07$ & 5540 & & 5.1 & 0.67 & $\mathrm{U}$ & 0.67 & 1.3 & $\mathrm{U}$ & 1.3 & 66.7 & C & 0.06 & 0.03 & $\mathrm{U}$ & 0.03 \\
\hline $\mathrm{OBB}-5$ & $\mathrm{~J} 15578$ & $6 / 13 / 07$ & 6230 & & 5.0 & 0.67 & $\mathrm{U}$ & 0.67 & 2.8 & & 1.2 & 61.9 & & 0.06 & 0.58 & & 0.03 \\
\hline OB B-6 & $\mathrm{J} 15579$ & $6 / 13 / 07$ & 5580 & & 5.0 & 0.66 & $\mathrm{U}$ & 0.66 & 2.6 & & 1.2 & 65.2 & & 0.06 & 0.57 & & 0.03 \\
\hline $\mathrm{OB} \mathrm{B}-7$ & $\mathrm{~J} 15580$ & $6 / 13 / 07$ & 5730 & & 5.0 & 0.66 & $\mathrm{U}$ & 0.66 & 2.4 & & 1.2 & 64.7 & & 0.06 & 0.58 & & 0.03 \\
\hline OB B-8 & $\mathrm{J} 15581$ & $6 / 13 / 07$ & 6510 & & 5.0 & 0.66 & $\mathrm{U}$ & 0.66 & 2.0 & & 1.2 & 72.1 & & 0.06 & 0.61 & & 0.03 \\
\hline $\mathrm{OBC1}$ & $\mathrm{J} 15584$ & $6 / 14 / 07$ & 5610 & & 5.0 & 0.67 & $\mathrm{U}$ & 0.67 & 2.9 & & 1.2 & 61.3 & C & 0.06 & 0.79 & & 0.03 \\
\hline $\mathrm{OBC}-10$ & $\mathrm{~J} 15583$ & $6 / 13 / 07$ & 6240 & & 4.8 & 0.64 & $\mathrm{U}$ & 0.64 & 2.1 & & 1.2 & 63.8 & & 0.06 & 0.53 & & 0.03 \\
\hline $\mathrm{OBC} 2$ & $\mathrm{~J} 15585$ & $6 / 14 / 07$ & 6010 & & 4.9 & 0.65 & $\mathrm{U}$ & 0.65 & 3.4 & & 1.2 & 57.8 & C & 0.06 & 0.85 & & 0.03 \\
\hline $\mathrm{OBC}-9$ & $\mathrm{~J} 15582$ & $6 / 13 / 07$ & 6260 & & 5.1 & 0.68 & $\mathrm{U}$ & 0.68 & 2.9 & & 1.3 & 62.4 & & 0.06 & 0.61 & & 0.03 \\
\hline OB D3 & $\mathrm{J} 15586$ & $6 / 14 / 07$ & 6100 & & 5.1 & 0.68 & $\mathrm{U}$ & 0.68 & 3.3 & & 1.3 & 63.6 & C & 0.06 & 0.81 & & 0.03 \\
\hline OB D4 & $\mathrm{J15587}$ & $6 / 14 / 07$ & 5040 & & 5.0 & 0.66 & $\mathrm{U}$ & 0.66 & 2.9 & & 1.2 & 60.6 & C & 0.06 & 0.77 & & 0.03 \\
\hline OB D5 & 315588 & $6 / 14 / 07$ & 5780 & & 5.0 & 0.67 & $\mathrm{U}$ & 0.67 & 3.5 & & 1.2 & 65.9 & C & 0.06 & 0.69 & & 0.03 \\
\hline OB D6 & J15589 & $6 / 14 / 07$ & 5080 & & 5.0 & 0.66 & $\mathrm{U}$ & 0.66 & 3.2 & & 1.2 & 62.6 & C & 0.06 & 0.72 & & 0.03 \\
\hline OB D6 Dup & $\mathrm{J} 15590$ & $6 / 14 / 07$ & 5120 & & 4.9 & 0.65 & $\mathrm{U}$ & 0.65 & 2.8 & & 1.2 & 60.8 & C & 0.06 & 0.72 & & 0.03 \\
\hline OB D6 Split & 515591 & $6 / 14 / 07$ & 8060 & $\mathrm{~N}$ & 10.5 & 0.53 & UN & 0.53 & 2.2 & & 0.2 & 61.7 & & 0.24 & 0.16 & $\mathrm{~B}$ & 0.07 \\
\hline
\end{tabular}

\begin{tabular}{|c|c|c|c|c|c|c|c|c|c|c|c|c|c|c|c|c|c|}
\hline \multirow{2}{*}{$\begin{array}{c}\text { Sample } \\
\text { Location }\end{array}$} & \multirow{2}{*}{$\begin{array}{c}\text { HEIS } \\
\text { Number }\end{array}$} & \multirow{2}{*}{$\begin{array}{c}\text { Sample } \\
\text { Date }\end{array}$} & \multicolumn{3}{|c|}{ Boron } & \multicolumn{3}{|c|}{ Cadmium } & \multicolumn{3}{|c|}{ Calcium } & \multicolumn{3}{|c|}{ Chromium (total) } & \multicolumn{3}{|c|}{ Hexavalent } \\
\hline & & & $\mathrm{mg} / \mathrm{kg}$ & $Q$ & $\mathrm{PQL}$ & $\mathrm{mg} / \mathrm{kg}$ & $Q$ & $\mathrm{PQL}$ & $\mathrm{mg} / \mathrm{kg}$ & $\mathbf{Q}$ & PQL & $\mathrm{mg} / \mathrm{kg}$ & $Q$ & PQL & $\mathrm{mg} / \mathrm{kg}$ & $Q$ & $\mathrm{PQL}$ \\
\hline $\mathrm{SZA-1}$ & $\mathrm{J15510}$ & $6 / 13 / 07$ & 2.3 & & 1.1 & 0.20 & & 0.15 & 4100 & $\mathrm{C}$ & 2.1 & 6.2 & & 0.29 & 0.20 & $\mathrm{U}$ & 0.20 \\
\hline SZ A-1 Dup & J15511 & $6 / 13 / 07$ & 2.3 & & 1.1 & 0.18 & & 0.15 & 4990 & C & 2.1 & 9.8 & & 0.30 & 0.20 & $\mathrm{U}$ & 0.20 \\
\hline SZA-1 Split & $J 15520$ & $6 / 13 / 07$ & 3.5 & $\mathrm{~B}$ & 3.1 & 0.06 & $\mathrm{U}$ & 0.06 & 5610 & & 30.5 & 13.8 & $\mathrm{~N}$ & 0.23 & 0.35 & $\bar{U}$ & 0.35 \\
\hline $\mathrm{SZA-2}$ & $\mathrm{J} 15512$ & $6 / 13 / 07$ & 1.7 & & 1.1 & 0.28 & & 0.15 & 5940 & C & 2.1 & 9.5 & & 0.30 & 0.20 & $\bar{U}$ & 0.20 \\
\hline $\mathrm{SZA-3}$ & $\mathrm{J} 15513$ & $6 / 13 / 07$ & 2.5 & & 1.0 & 0.26 & & 0.15 & 5170 & C & 2.1 & 10.1 & & 0.29 & 0.20 & $\mathrm{U}$ & 0.20 \\
\hline $\mathrm{SZ} \mathrm{A-4}$ & $\mathrm{J15514}$ & $6 / 13 / 07$ & 1.0 & $\mathrm{U}$ & 1.0 & 0.25 & & 0.14 & 5380 & $\mathrm{C}$ & 2.1 & 8.9 & & 0.29 & 0.26 & & 0.20 \\
\hline DZA-1 & $\mathrm{J} 15515$ & $6 / 4 / 07$ & 1.0 & $\mathrm{U}$ & 1.0 & 0.15 & $\mathrm{U}$ & 0.15 & 6250 & $\mathrm{C}$ & 2.1 & 12.8 & C & 0.29 & 0.20 & $\mathrm{U}$ & 0.20 \\
\hline DZ A-2 & $\mathrm{J15516}$ & $6 / 4 / 07$ & 1.1 & $\mathrm{U}$ & 1.1 & 0.15 & $\mathrm{U}$ & 0.15 & 6490 & $\mathrm{C}$ & 2.1 & 8.4 & C & 0.30 & 0.32 & & 0.20 \\
\hline $\mathrm{DZA}-3$ & $\mathrm{~J} 15517$ & $6 / 4 / 07$ & 1.1 & $\mathrm{U}$ & 1.1 & 0.15 & $\mathrm{U}$ & 0.15 & 6740 & C & 2.1 & 8.3 & C & 0.30 & 0.20 & $\mathrm{U}$ & 0.20 \\
\hline DZ A-3 Dup & $\mathrm{J} 15518$ & $6 / 4 / 07$ & 1.0 & $\mathrm{U}$ & 1.0 & 0.14 & $\mathrm{U}$ & 0.14 & 6020 & C & 2.1 & 7.5 & C & 0.29 & 0.26 & & 0.20 \\
\hline DZ A-3 Split & $\mathrm{J} 15521$ & $6 / 4 / 07$ & 1.5 & $\mathrm{U}$ & 1.5 & 0.06 & $\mathrm{U}$ & 0.06 & 7490 & C & 8.7 & 12.3 & $\mathrm{~N}$ & 0.23 & 0.35 & $\mathrm{U}$ & 0.35 \\
\hline $\mathrm{DZ} \mathrm{A}-4$ & J15519 & $6 / 4 / 07$ & 1.5 & & 1.1 & 0.15 & $\mathrm{U}$ & 0.15 & 5630 & C & 2.1 & 7.9 & C & 0.30 & 0.20 & $\mathrm{U}$ & 0.20 \\
\hline $\mathrm{OB} \mathrm{A-1}$ & $\mathrm{J} 15574$ & $6 / 12 / 07$ & 1.8 & & 1.1 & 0.15 & $\mathrm{U}$ & 0.15 & 4460 & C & 2.1 & 9.0 & C & 0.30 & 0.24 & & 0.20 \\
\hline $\mathrm{OB} \mathrm{A}-2$ & $\mathrm{J15575}$ & $6 / 12 / 07$ & 3.7 & & 1.1 & 0.15 & $\mathrm{U}$ & 0.15 & 5900 & C & 2.1 & 9.5 & C & 0.30 & 0.35 & & 0.21 \\
\hline $\mathrm{OB} \mathrm{A}-3$ & $\mathrm{~J} 15576$ & $6 / 12 / 07$ & 1.7 & & 1.1 & 0.15 & $\mathrm{U}$ & 0.15 & 4960 & C & 2.1 & 8.7 & C & 0.30 & 0.21 & $\mathrm{U}$ & 0.21 \\
\hline $\mathrm{OB} \mathrm{A-4}$ & $\mathrm{J} 15577$ & $6 / 12 / 07$ & 1.8 & & 1.1 & 0.15 & $\mathrm{U}$ & 0.15 & 4610 & C & 2.2 & 8.6 & C & 0.31 & 0.23 & & 0.21 \\
\hline $\mathrm{OB} \mathrm{B-5}$ & $\mathrm{J} 15578$ & $6 / 13 / 07$ & 1.5 & & 1.1 & 0.25 & & 0.15 & 3590 & C & 2.2 & 7.7 & & 0.30 & 0.39 & & 0.20 \\
\hline $\mathrm{OB} \mathrm{B-6}$ & $J 15579$ & $6 / 13 / 07$ & 1.1 & $\bar{U}$ & 1.1 & 0.29 & & 0.15 & 3690 & C & 2.1 & 7.2 & & 0.30 & 0.33 & & 0.20 \\
\hline $\mathrm{OBB}-7$ & J15580 & $6 / 13 / 07$ & 1.1 & $\mathrm{U}$ & 1.1 & 0.27 & & 0.15 & 3490 & $\mathrm{C}$ & 2.1 & 7.2 & & 0.30 & 0.26 & & 0.20 \\
\hline $\mathrm{OB} \mathrm{B-8}$ & $\mathrm{J} 15581$ & $6 / 13 / 07$ & 1.1 & $\mathrm{U}$ & 1.1 & 0.25 & & 0.15 & 3650 & C & 2.1 & 8.2 & & 0.30 & 0.30 & & 0.20 \\
\hline $\mathrm{OBCl}$ & $\mathrm{J} 15584$ & $6 / 14 / 07$ & 1.6 & & 1.1 & 0.15 & $\mathrm{U}$ & 0.15 & 3860 & C & 2.2 & 7.4 & & 0.30 & 0.20 & $\mathrm{U}$ & 0.20 \\
\hline $\mathrm{OB} \mathrm{C}-10$ & $\mathrm{~J} 15583$ & $6 / 13 / 07$ & 1.3 & & 1.0 & 0.35 & & 0.13 & 3380 & C & 2.1 & 7.6 & & 0.29 & 0.20 & $\mathrm{U}$ & 0.20 \\
\hline $\mathrm{OBC2}$ & $\mathrm{J} 15585$ & $6 / 14 / 07$ & 1.2 & & 1.1 & 0.15 & $\mathrm{U}$ & 0.15 & 3820 & C & 2.1 & 8.2 & & 0.30 & 0.24 & & 0.20 \\
\hline $\mathrm{OBC}-9$ & J15582 & $6 / 13 / 07$ & 1.1 & $\mathrm{U}$ & 1.1 & 0.26 & & 0.15 & 3690 & C & 2.2 & 8.2 & & 0.31 & 0.21 & $\bar{U}$ & 0.20 \\
\hline $\mathrm{OBD} 3$ & $\mathrm{~J} 15586$ & $6 / 14 / 07$ & 2.3 & & 1.1 & 0.15 & $\mathrm{U}$ & 0.15 & 4220 & C & 2.2 & 8.5 & & 0.31 & 0.27 & & 0.21 \\
\hline OB D4 & $\mathrm{J} 15587$ & $6 / 14 / 07$ & 2.0 & & 1.1 & 0.15 & U & 0.15 & 3650 & C & 2.1 & 7.2 & & 0.30 & 0.20 & $\mathrm{U}$ & 0.20 \\
\hline OBD5 & $\mathrm{J} 15588$ & $6 / 14 / 07$ & 2.3 & & 1.1 & 0.15 & $\mathrm{U}$ & 0.15 & 3670 & C & 2.2 & 7.5 & & 0.30 & 0.21 & $\mathrm{U}$ & 0.21 \\
\hline OB D6 & $\mathrm{J} 15589$ & $6 / 14 / 07$ & 1.5 & & 1.1 & 0.15 & $U$ & 0.15 & 3720 & C & 2.1 & 6.7 & & 0.30 & 0.24 & & 0.21 \\
\hline OB D6 Dup & $\mathrm{J} 15590$ & $6 / 14 / 07$ & 1.5 & & 1.1 & 0.15 & U & 0.15 & 3400 & C & 2.1 & 6.9 & & 0.29 & 0.21 & $\mathrm{U}$ & 0.21 \\
\hline OB D6 Split & $\mathrm{J} 15591$ & $6 / 14 / 07$ & 7.8 & $\mathrm{U}$ & 7.8 & 0.06 & UN & 0.06 & 3580 & & 31.0 & 9.2 & & 0.23 & 0.350 & $\mathrm{U}$ & 0.350 \\
\hline
\end{tabular}

Note: Data qualified with B, C, D and/or J, are considered acceptable values.

$\mathrm{GEA}=$ gamma energy analysis $\quad \mathrm{PQL}=$ practical quantitation limit

HEIS = Hanford Environmental Information System $Q=$ qualifier

$\mathrm{MDA}=$ minimum detectable activity $\quad \mathrm{U}=$ undetected

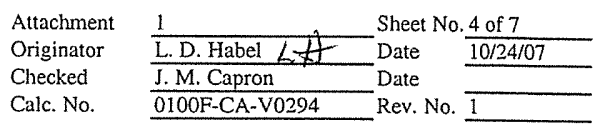




\begin{tabular}{|c|c|c|c|c|c|c|c|c|c|c|c|c|c|c|c|c|c|}
\hline \multirow{2}{*}{$\begin{array}{c}\text { Sample } \\
\text { Location }\end{array}$} & \multirow{2}{*}{$\begin{array}{c}\text { HEIS } \\
\text { Number } \\
\end{array}$} & \multirow{2}{*}{$\begin{array}{c}\text { Sample } \\
\text { Date }\end{array}$} & \multicolumn{3}{|c|}{ Cobalt } & \multicolumn{3}{|c|}{ Copper } & \multicolumn{3}{|c|}{ Iron } & \multicolumn{3}{|c|}{ Lead } & \multicolumn{3}{|c|}{ Magnesium } \\
\hline & & & $\mathrm{mg} / \mathrm{kg}$ & $Q$ & $\mathrm{PQL}$ & $\mathrm{mg} / \mathrm{kg}$ & Q & PQL & $\mathrm{mg} / \mathrm{kg}$ & $Q$ & PQL & $\mathrm{mg} / \mathrm{kg}$ & Q & $\mathrm{PQL}$ & $\mathrm{mg} / \mathrm{kg}$ & $Q$ & $\mathrm{PQL}$ \\
\hline SZA-1 & 515510 & $6 / 13 / 07$ & 4.8 & & 0.24 & 11.7 & & 0.27 & 11100 & & 7.0 & 5.9 & & 0.97 & 2670 & & 2.4 \\
\hline SZ A-1 Dup & $\mathrm{J} 15511$ & $6 / 13 / 07$ & 5.6 & & 0.24 & 14.8 & & 0.27 & 16000 & & 7.1 & 9.5 & & 0.98 & 3700 & & 2.4 \\
\hline SZ A-1 Split & $\mathrm{J} 15520$ & $6 / 13 / 07$ & 10.0 & & 0.24 & 13.5 & & 0.41 & 20600 & $\mathrm{~N}$ & 3.8 & 8.8 & & 0.31 & 4820 & & 9.1 \\
\hline $\mathrm{SZA}-2$ & $\mathrm{~J} 15512$ & $6 / 13 / 07$ & 4.3 & & 0.24 & 11.5 & & 0.27 & 11600 & & 7.1 & 4.2 & & 0.98 & 3530 & & 2.4 \\
\hline $\mathrm{SZA-3}$ & $\mathrm{J} 15513$ & $6 / 13 / 07$ & 5.7 & & 0.23 & 14.1 & & 0.26 & 15800 & & 7.0 & 10.3 & & 0.96 & 3870 & & 2.4 \\
\hline $\mathrm{SZA-4}$ & $\mathrm{J} 15514$ & $6 / 13 / 07$ & 4.7 & & 0.23 & 11.9 & & 0.26 & 12500 & & 6.9 & 4.4 & & 0.95 & 3830 & & 2.3 \\
\hline DZ A-1 & $\mathrm{J} 15515$ & $6 / 4 / 07$ & 4.5 & & 0.23 & 10.1 & & 0.26 & 11300 & $\mathrm{C}$ & 7.0 & 2.4 & & 0.96 & 3800 & $\mathrm{C}$ & 2.4 \\
\hline $\mathrm{DZA}-2$ & $\mathrm{~J} 15516$ & $6 / 4 / 07$ & 5.3 & & 0.24 & 12.5 & & 0.27 & 13000 & $\mathrm{C}$ & 7.1 & 4.4 & & 0.99 & 3620 & $\mathrm{C}$ & 2.4 \\
\hline DZA-3 & $\mathrm{J} 15517$ & $6 / 4 / 07$ & 4.4 & & 0.24 & 11.0 & & 0.27 & 11200 & $\mathrm{C}$ & 7.1 & 5.5 & & 0.98 & 3450 & $\mathrm{C}$ & 2.4 \\
\hline DZ A-3 Dup & $\mathrm{J} 15518$ & $6 / 4 / 07$ & 3.9 & & 0.23 & 11.0 & & 0.26 & 9470 & $\mathrm{C}$ & 6.9 & 3.7 & & 0.96 & 3030 & $\mathrm{C}$ & 2.3 \\
\hline DZA-3 Split & $\mathrm{J} 15521$ & $6 / 4 / 07$ & 6.1 & & 0.24 & 10.0 & $\mathrm{C}$ & 0.16 & 13200 & $N$ & 3.8 & 3.8 & & 0.10 & 4700 & & 9.2 \\
\hline $\mathrm{DZA}-4$ & $\mathrm{~J} 15519$ & $6 / 4 / 07$ & 5.4 & & 0.24 & 12.3 & & 0.27 & 13000 & $\mathrm{C}$ & 7.1 & 5.8 & & 0.98 & 3370 & $\mathrm{C}$ & 2.4 \\
\hline $\mathrm{OB} A-1$ & $\mathrm{~J} 15574$ & $6 / 12 / 07$ & 5.6 & & 0.24 & 13.0 & & 0.27 & 13700 & $\mathrm{C}$ & 7.1 & 6.2 & & 0.99 & 3550 & $\mathrm{C}$ & 2.4 \\
\hline $\mathrm{OBA}-2$ & $\mathrm{~J} 15575$ & $6 / 12 / 07$ & 5.9 & & 0.24 & 14.3 & & 0.27 & 14900 & $\mathrm{C}$ & 7.1 & 5.5 & & 0.98 & 3870 & $\mathrm{C}$ & 2.4 \\
\hline $\mathrm{OB} \mathrm{A-3}$ & $\mathrm{J15576}$ & $6 / 12 / 07$ & 5.5 & & 0.24 & 14.0 & & 0.27 & 13300 & $\mathrm{C}$ & 7.1 & 6.5 & & 0.98 & 3520 & $\mathrm{C}$ & 2.4 \\
\hline $\mathrm{OB} \mathrm{A-4}$ & $\mathrm{J} 15577$ & $6 / 12 / 07$ & 6.3 & & 0.24 & 12.8 & & 0.27 & 16600 & $\mathrm{C}$ & 7.3 & 6.7 & & 1.00 & 3690 & $\mathrm{C}$ & 2.5 \\
\hline OB B-5 & $\mathrm{J} 15578$ & $6 / 13 / 07$ & 6.4 & & 0.24 & 14.2 & & 0.27 & 18100 & & 7.3 & 4.0 & & 1.00 & 3840 & & 2.5 \\
\hline OB B-6 & $\mathrm{J} 15579$ & $6 / 13 / 07$ & 6.6 & & 0.24 & 14.3 & & 0.27 & 17300 & & 7.2 & 4.5 & & 0.99 & 3730 & & 2.4 \\
\hline $\mathrm{OB} \mathrm{B-7}$ & $\mathrm{J} 15580$ & $6 / 13 / 07$ & 6.5 & & 0.24 & 13.8 & & 0.27 & 17900 & & 7.1 & 4.2 & & 0.99 & 3790 & & 2.4 \\
\hline $\mathrm{OB} \mathrm{B}-8$ & $\mathrm{~J} 15581$ & $6 / 13 / 07$ & 6.7 & & 0.24 & 14.4 & & 0.27 & 19000 & & 7.1 & 4.1 & & 0.98 & 4060 & & 2.4 \\
\hline $\mathrm{OBCl}$ & J15584 & $6 / 14 / 07$ & 5.6 & & 0.24 & 13.4 & & 0.27 & 16300 & & 7.3 & 3.7 & & 1.00 & 3670 & $\mathrm{C}$ & 2.5 \\
\hline $\mathrm{OB} C-10$ & $\mathrm{~J} 15583$ & $6 / 13 / 07$ & 6.1 & & 0.23 & 14.7 & & 0.26 & 17300 & & 6.9 & 3.6 & & 0.96 & 3850 & & 2.3 \\
\hline $\mathrm{OBC} 2$ & $\mathrm{~J} 15585$ & $6 / 14 / 07$ & 6.0 & & 0.24 & 13.6 & & 0.27 & 17800 & & 7.1 & 3.9 & & 0.98 & 3820 & $\mathrm{C}$ & 2.4 \\
\hline $\mathrm{OB} \mathrm{C}-9$ & $\mathrm{~J} 15582$ & $6 / 13 / 07$ & 6.4 & & 0.25 & 14.4 & & 0.28 & 18200 & & 7.4 & 4.4 & & 1.00 & 3930 & & 2.5 \\
\hline OB D3 & $\mathrm{J15586}$ & $6 / 14 / 07$ & 5.8 & & 0.26 & 13.7 & & 0.28 & 17000 & & 7.4 & 7.8 & & 1.00 & 3880 & $\mathrm{C}$ & 2.5 \\
\hline $\mathrm{OBD} 4$ & J15587 & $6 / 14 / 07$ & 5.3 & & 0.24 & 12.6 & & 0.27 & 14000 & & 7.1 & 5.0 & & 0.99 & 3370 & $\mathrm{C}$ & 2.4 \\
\hline OBD5 & $\mathrm{J} 15588$ & $6 / 14 / 07$ & 6.3 & & 0.24 & 13.2 & & 0.27 & 17400 & & 7.3 & 4.5 & & 1.00 & 3970 & $\mathrm{C}$ & 2.5 \\
\hline OB D6 & 515589 & $6 / 14 / 07$ & 5.7 & & 0.24 & 12.9 & & 0.27 & 14400 & & 7.2 & 4.1 & & 0.99 & 3570 & $\mathrm{C}$ & 2.4 \\
\hline OB D6 Dup & $\mathrm{J15590}$ & $6 / 14 / 07$ & 5.6 & & 0.24 & 12.9 & & 0.26 & 15400 & & 7.0 & 4.1 & & 0.97 & 3510 & $\mathrm{C}$ & 2.4 \\
\hline OB D6 Split & J15591 & $6 / 14 / 07$ & 9.9 & & 0.20 & 11.8 & & 0.41 & 24900 & $\mathrm{~N}$ & 19.4 & 4.0 & & 0.31 & 4300 & $\mathrm{~N}$ & 9.3 \\
\hline
\end{tabular}

\begin{tabular}{|c|c|c|c|c|c|c|c|c|c|c|c|c|c|c|c|c|c|}
\hline \multirow{2}{*}{$\begin{array}{c}\text { Sample } \\
\text { Location }\end{array}$} & \multirow{2}{*}{$\begin{array}{c}\text { HEIS } \\
\text { Number }\end{array}$} & \multirow{2}{*}{$\begin{array}{c}\text { Sample } \\
\text { Date }\end{array}$} & \multicolumn{3}{|c|}{ Manganese } & \multicolumn{3}{|c|}{ Mercury } & \multicolumn{3}{|c|}{ Molybdenum } & \multicolumn{3}{|c|}{ Nickel } & \multicolumn{3}{|c|}{ Potassium } \\
\hline & & & $\mathrm{mg} / \mathrm{kg}$ & $Q$ & $\mathrm{PQL}$ & $\mathrm{mg} / \mathrm{kg}$ & $Q$ & PQL & $\mathrm{mg} / \mathrm{kg}$ & $Q$ & $\mathrm{PQL}$ & $\mathrm{mg} / \mathrm{kg}$ & Q & $\mathrm{PQL}$ & $\mathrm{mg} / \mathrm{kg}$ & $Q$ & PQL \\
\hline $\mathrm{SZA-1}$ & $\mathrm{J} 15510$ & $6 / 13 / 07$ & 210 & & 0.21 & 0.25 & & 0.02 & 0.47 & U & 0.47 & 6.6 & & 0.80 & 772 & $\mathrm{C}$ & 9.4 \\
\hline SZ A-1 Dup & $\mathrm{J} 15511$ & $6 / 13 / 07$ & 270 & & 0.21 & 0.28 & & 0.01 & 0.47 & $\mathrm{U}$ & 0.47 & 9.7 & & 0.80 & 1060 & $\mathrm{C}$ & 9.5 \\
\hline SZ A-1 Split & $\mathrm{J} 15520$ & $6 / 13 / 07$ & 327 & $\mathrm{~N}$ & 0.41 & 0.27 & $\mathrm{~N}$ & 0.01 & 0.91 & $\mathrm{U}$ & 0.91 & 11.9 & & 1.2 & 1270 & & 305 \\
\hline $\mathrm{SZ} \mathrm{A-2}$ & $\mathrm{J} 15512$ & $6 / 13 / 07$ & 251 & & 0.21 & 0.09 & & 0.01 & 0.48 & $\mathrm{U}$ & 0.48 & 8.3 & & 0.80 & 767 & $\mathrm{C}$ & 9.5 \\
\hline $\mathrm{SZA}-3$ & $\mathrm{~J} 15513$ & $6 / 13 / 07$ & 285 & & 0.20 & 0.53 & & 0.01 & 0.47 & $\mathrm{U}$ & 0.47 & 9.5 & & 0.79 & 1130 & $\mathrm{C}$ & 9.3 \\
\hline $\mathrm{SZ} \mathrm{A-4}$ & $\mathrm{J} 15514$ & $6 / 13 / 07$ & 259 & & 0.20 & 0.09 & & 0.02 & 0.46 & $\mathrm{U}$ & 0.46 & 9.6 & & 0.78 & 765 & $\mathrm{C}$ & 9.3 \\
\hline $\mathrm{DZA}-1$ & $\mathrm{~J} 15515$ & $6 / 4 / 07$ & 226 & & 0.20 & 0.02 & $\mathrm{U}$ & 0.02 & 0.47 & $\mathrm{U}$ & 0.47 & 10.6 & & 0.79 & 481 & $\mathrm{C}$ & 9.3 \\
\hline $\mathrm{DZA}-2$ & $\mathrm{~J} 15516$ & $6 / 4 / 07$ & 252 & & 0.21 & 0.02 & $\mathrm{U}$ & 0.02 & 0.48 & $\mathrm{U}$ & 0.48 & 9.1 & & 0.81 & 708 & $\mathrm{C}$ & 9.6 \\
\hline $\mathrm{DZA}-3$ & $\mathrm{~J} 15517$ & $6 / 4 / 07$ & 236 & & 0.21 & 0.02 & $\mathrm{U}$ & 0.02 & 0.48 & $\mathrm{U}$ & 0.48 & 8.5 & & 0.80 & 554 & $\mathrm{C}$ & 9.5 \\
\hline DZ A-3 Dup & $\mathrm{J} 15518$ & $6 / 4 / 07$ & 212 & & 0.20 & 0.02 & $\mathrm{U}$ & 0.02 & 0.51 & & 0.46 & 8.3 & & 0.78 & 493 & $\mathrm{C}$ & 9.3 \\
\hline DZ A-3 Split & $\mathrm{J} 15521$ & $6 / 4 / 07$ & 244 & $\mathrm{~N}$ & 0.22 & 0.02 & $\mathrm{~B}$ & 0.01 & 0.91 & UJN & 0.91 & 11.5 & & 1.2 & 735 & & 94 \\
\hline DZ A-4 & $\mathrm{J} 15519$ & $6 / 4 / 07$ & 249 & & 0.21 & 0.05 & & 0.01 & 0.48 & $\mathrm{U}$ & 0.48 & 9.2 & & 0.81 & 745 & $C$ & 9.5 \\
\hline $\mathrm{OBA}-1$ & $\mathrm{~J} 15574$ & $6 / 12 / 07$ & 257 & & 0.21 & 0.02 & $\mathrm{U}$ & 0.02 & 0.48 & $\mathrm{U}$ & 0.48 & 9.6 & & 0.81 & 1070 & & 9.6 \\
\hline $\mathrm{OBA}-2$ & $\mathrm{~J} 15575$ & $6 / 12 / 07$ & 283 & & 0.21 & 0.04 & & 0.01 & 0.56 & & 0.48 & 9.8 & & 0.81 & 1020 & & 9.5 \\
\hline $\mathrm{OBA}-3$ & $\mathrm{~J} 15576$ & $6 / 12 / 07$ & 264 & & 0.21 & 0.06 & & 0.02 & 0.47 & $\mathrm{U}$ & 0.47 & 9.1 & & 0.80 & 955 & & 9.5 \\
\hline $\mathrm{OB} \mathrm{A}-4$ & $\mathrm{~J} 15577$ & $6 / 12 / 07$ & 294 & & 0.21 & 0.57 & & 0.02 & 0.49 & $\mathrm{U}$ & 0.49 & 9.0 & & 0.82 & 1080 & & 9.8 \\
\hline OB B-5 & $\mathrm{J} 15578$ & $6 / 13 / 07$ & 322 & & 0.21 & 0.02 & $\mathrm{U}$ & 0.02 & 0.53 & & 0.49 & 9.1 & & 0.82 & 1230 & $\mathrm{C}$ & 9.7 \\
\hline $\mathrm{OB} \mathrm{B-6}$ & $\mathrm{J} 15579$ & $6 / 13 / 07$ & 331 & & 0.21 & 0.02 & & 0.02 & 0.48 & $\mathrm{U}$ & 0.48 & 8.9 & & 0.81 & 1190 & $\mathrm{C}$ & 9.6 \\
\hline $\mathrm{OB} \mathrm{B}-7$ & $\mathrm{~J} 15580$ & $6 / 13 / 07$ & 332 & & 0.21 & 0.01 & $\mathrm{U}$ & 0.01 & 0.48 & & 0.48 & 8.9 & & 0.81 & 1230 & C & 9.6 \\
\hline $\mathrm{OB} \mathrm{B-8}$ & $\mathrm{J} 15581$ & $6 / 13 / 07$ & 328 & & 0.21 & 0.01 & $\mathrm{U}$ & 0.01 & 0.53 & & 0.48 & 9.6 & & 0.81 & 1300 & $\mathrm{C}$ & 9.5 \\
\hline $\mathrm{OBCl}$ & $\mathrm{J} 15584$ & $6 / 14 / 07$ & 312 & & 0.21 & 0.02 & $\mathrm{U}$ & 0.02 & 0.81 & & 0.49 & 8.4 & & 0.82 & 1130 & & 9.7 \\
\hline $\mathrm{OBC}-10$ & $\mathrm{~J} 15583$ & $6 / 13 / 07$ & 305 & & 0.20 & 0.02 & $\mathrm{U}$ & 0.02 & 0.46 & & 0.46 & 9.2 & & 0.78 & 1250 & $\mathrm{C}$ & 9.3 \\
\hline $\mathrm{OBC} 2$ & $\mathrm{~J} 15585$ & $6 / 14 / 07$ & 326 & & 0.24 & 0.02 & $\mathrm{U}$ & 0.02 & 0.74 & & 0.47 & 9.6 & & 0.80 & 1160 & & $9.5^{\circ}$ \\
\hline OB C-9 & $\mathrm{J} 15582$ & $6 / 13 / 07$ & 325 & & 0.22 & 0.01 & $\mathrm{U}$ & 0.01 & 0.49 & $\mathrm{U}$ & 0.49 & 10.0 & & 0.83 & 1240 & $\mathrm{C}$ & 9.9 \\
\hline $\mathrm{OB} \mathrm{D3}$ & $\mathrm{J} 15586$ & $6 / 14 / 07$ & 299 & & 0.22 & 0.02 & & 0.02 & 0.49 & $\mathrm{U}$ & 0.49 & 9.8 & & 0.83 & 1150 & & 9.9 \\
\hline $\mathrm{OB} \mathrm{D4}$ & $\mathrm{J} 15587$ & $6 / 14 / 07$ & 286 & & 0.21 & 0.02 & $\mathrm{U}$ & 0.02 & 0.58 & & 0.48 & 9.1 & & 0.81 & 1060 & & 9.6 \\
\hline $\mathrm{OBD5}$ & $\mathrm{J15588}$ & $6 / 14 / 07$ & 336 & & 0.21 & 0.01 & $\mathrm{U}$ & 0.01 & 0.73 & & 0.49 & 9.4 & & 0.82 & 1210 & & 9.7 \\
\hline OB D6 & $\mathrm{J} 15589$ & $6 / 14 / 07$ & 316 & & 0.21 & 0.02 & $\mathrm{U}$ & 0.02 & 0.48 & $\mathrm{U}$ & 0.48 & 9.4 & & 0.91 & 1130 & & 9.6 \\
\hline OB D6 Dup & $\mathrm{J} 15590$ & $6 / 14 / 07$ & 302 & & 0.21 & 0.02 & $\mathrm{U}$ & 0.02 & 0.77 & & 0.47 & 8.6 & & 0.79 & 1080 & & 9.4 \\
\hline OB D6 Split & $\mathrm{J} 15591$ & $6 / 14 / 07$ & 315 & $\mathrm{~N}$ & 0.41 & 0.01 & $\mathrm{U}$ & 0.01 & 0.92 & $\mathrm{U}$ & 0.92 & 11.5 & & 1.2 & 1740 & $\mathrm{C}$ & 310 \\
\hline
\end{tabular}

Note: Data qualified with B, C, D and/or J, are considered acceptable values.

GEA = gamma energy analysis $\quad \mathrm{PQL}=$ practical quantitation limit

HEIS = Hanford Environmental Information System $Q=$ qualifier

$\mathrm{MDA}=$ minimum detectable activity $\quad \mathrm{U}=$ undetected

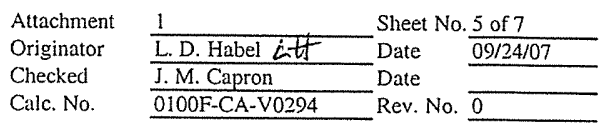


Attachment 1. 118-F-8:4 Verification Sampling Results.

\begin{tabular}{|c|c|c|c|c|c|c|c|c|c|c|c|c|c|c|c|c|c|}
\hline \multirow{2}{*}{$\begin{array}{c}\text { Sample } \\
\text { Location }\end{array}$} & \multirow{2}{*}{$\begin{array}{c}\text { HEIS } \\
\text { Number }\end{array}$} & \multirow{2}{*}{$\begin{array}{c}\text { Sample } \\
\text { Date }\end{array}$} & \multicolumn{3}{|c|}{ Selenium } & \multicolumn{3}{|c|}{ Silicon } & \multicolumn{3}{|c|}{ Silver } & \multicolumn{3}{|c|}{ Sodium } & \multicolumn{3}{|c|}{ Vanadium } \\
\hline & & & $\mathrm{mg} / \mathrm{kg}$ & $Q$ & PQL & $\mathrm{mg} / \mathrm{kg}$ & $Q$ & PQL & $\mathrm{mg} / \mathrm{kg}$ & $Q$ & $\mathrm{PQL}$ & $\mathrm{mg} / \mathrm{kg}$ & Q & PQL & $\mathrm{mg} / \mathrm{kg}$ & $Q$ & PQL \\
\hline $\mathrm{SZA-1}$ & $\$ 15510$ & $6 / 13 / 07$ & 1.3 & $\mathrm{U}$ & 1.3 & 877 & & 2.5 & 0.27 & U & 0.27 & 123 & C & 2.1 & 27.5 & & 0.24 \\
\hline SZ A-1 Dup & $\mathrm{J} 15511$ & $6 / 13 / 07$ & 1.5 & & 1.3 & 818 & & 2.5 & 0.27 & $\mathrm{U}$ & 0.27 & 166 & C & 2.1 & 37.5 & & 0.24 \\
\hline SZ A-1 Split & 515520 & $6 / 13 / 07$ & 0.5 & $\mathrm{U}$ & 0.5 & 825 & $\mathrm{~N}$ & 18.3 & 0.37 & $\mathrm{U}$ & 0.37 & 202 & \begin{tabular}{|l|}
$\mathrm{B}$ \\
\end{tabular} & 24.2 & 51.6 & & 0.54 \\
\hline $\mathrm{SZA-2}$ & $\mathrm{J} 15512$ & $6 / 13 / 07$ & 1.3 & $\mathrm{U}$ & 1.3 & 1050 & & 2.6 & 0.27 & $\mathrm{U}$ & 0.27 & 145 & C & 2.1 & 27.7 & & 0.24 \\
\hline $\mathrm{SZA-3}$ & $\mathrm{J} 15513$ & $6 / 13 / 07$ & 1.3 & $\mathrm{U}$ & 1.3 & 1190 & & 2.5 & 0.26 & $\mathrm{U}$ & 0.26 & 161 & C) & 2.0 & 36.5 & & 0.23 \\
\hline SZA-4 & $\mathrm{J} 15514$ & $6 / 13 / 07$ & 1.2 & $\mathrm{U}$ & 1.2 & 990 & & 2.5 & 0.26 & $\mathrm{U}$ & 0.26 & 140 & C & 2.0 & 31.2 & & 0.23 \\
\hline DZ A-1 & $\mathrm{J} 15515$ & $6 / 4 / 07$ & 1.3 & $\mathrm{U}$ & 1.3 & 942 & $\mathrm{CJ}$ & 2.5 & 0.26 & $\mathrm{U}$ & 0.26 & 106 & C & 2.0 & 25.4 & & 0.23 \\
\hline $\mathrm{DZA-2}$ & $\mathrm{J} 15516$ & $6 / 4 / 07$ & 1.3 & $\mathrm{U}$ & 1.3 & 1010 & CJ & 2.6 & 0.27 & $\mathrm{U}$ & 0.27 & 124 & C & 2.1 & 29.9 & & 0.24 \\
\hline $\mathrm{DZA}-3$ & $\mathrm{~J} 15517$ & $6 / 4 / 07$ & 1.3 & $\mathrm{U}$ & 1.3 & 959 & CJ & 2.6 & 0.27 & $\mathrm{U}$ & 0.27 & 124 & C & 2.1 & 26.9 & & 0.24 \\
\hline DZA-3 Dup & $\mathrm{J} 15518$ & $6 / 4 / 07$ & 1.2 & $\mathrm{U}$ & 1.2 & 1070 & $\mathrm{CJ}$ & 2.5 & 0.26 & $\mathrm{U}$ & 0.26 & 101 & C & 2.0 & 21.0 & & 0.23 \\
\hline DZ A-3 Split & $\mathrm{J15521}$ & $6 / 4 / 07$ & 0.5 & $\mathrm{U}$ & 0.5 & 500 & $\mathrm{CN}$ & 4.1 & 0.37 & $\mathrm{U}$ & 0.37 & 152 & & 5.9 & 32.0 & & 0.55 \\
\hline $\mathrm{DZ} A-4$ & $J 15519$ & $6 / 4 / 07$ & 1.3 & $\mathrm{U}$ & 1.3 & 1040 & CJ & 2.6 & 0.27 & $\mathrm{U}$ & 0.27 & 123 & C & 2.1 & 30.3 & & 0.24 \\
\hline OB A-1 & J15574 & $6 / 12 / 07$ & 1.3 & $\mathrm{U}$ & 1.3 & 1310 & C & 2.6 & 0.27 & $\mathrm{U}$ & 0.27 & 143 & C & 2.1 & 30.7 & & 0.24 \\
\hline $\mathrm{OBA}-2$ & $\mathrm{~J} 15575$ & $6 / 12 / 07$ & 1.3 & $\mathrm{U}$ & 1.3 & 1870 & $\mathrm{C}$ & 2.6 & 0.27 & $\mathrm{U}$ & 0.27 & 182 & C & 2.1 & 33.2 & & 0.24 \\
\hline $\mathrm{OBA}-3$ & $\mathrm{~J} 15576$ & $6 / 12 / 07$ & 1.3 & $\bar{U}$ & 1.3 & 1890 & C & 2.5 & 0.27 & $\mathrm{U}$ & 0.27 & 154 & C & 2.1 & 29.3 & & 0.24 \\
\hline $\mathrm{OB} \mathrm{A-4}$ & $\mathrm{J} 15577$ & $6 / 12 / 07$ & 1.3 & $\mathrm{U}$ & 1.3 & 1890 & C & 2.6 & 0.27 & $\mathrm{U}$ & 0.27 & 141 & C & 2.1 & 38.1 & & 0.24 \\
\hline OB B-5 & $\mathrm{J} 15578$ & $6 / 13 / 07$ & 1.3 & $\mathrm{U}$ & 1.3 & 1110 & & 2.6 & 0.27 & $\mathrm{U}$ & 0.27 & 143 & C & 2.1 & 44.4 & & 0.24 \\
\hline OB B-6 & $\mathrm{J15579}$ & $6 / 13 / 07$ & 1.3 & $\mathrm{U}$ & 1.3 & 1060 & & 2.6 & 0.27 & $\mathrm{U}$ & 0.27 & 114 & C & 2.1 & 40.5 & & 0.24 \\
\hline OB B-7 & $\mathrm{J} 15580$ & $6 / 13 / 07$ & 1.3 & $\mathrm{U}$ & 1.3 & 1140 & & 2.6 & 0.27 & $\mathrm{U}$ & 0.27 & 130 & C & 2.1 & 42.9 & & 0.24 \\
\hline OB B-8 & $\mathrm{J} 15581$ & $6 / 13 / 07$ & 1.3 & $\mathrm{U}$ & 1.3 & 1120 & & 2.6 & 0.27 & $\mathrm{U}$ & 0.27 & 156 & C & 2.1 & 45.7 & & 0.24 \\
\hline $\mathrm{OBCl}$ & $\mathrm{J} 15584$ & $6 / 14 / 07$ & 1.3 & $\mathrm{U}$ & 1.3 & 1550 & & 2.6 & 0.27 & $\mathrm{U}$ & 0.27 & 127 & C & 2.1 & 38.4 & & 0.24 \\
\hline $\mathrm{OB} \mathrm{C}-10$ & $\mathrm{~J} 15583$ & $6 / 13 / 07$ & 1.2 & $\mathrm{U}$ & 1.2 & 1060 & & 2.5 & 0.26 & $\mathrm{U}$ & 0.26 & 153 & C & 2.0 & 42.9 & & 0.23 \\
\hline $\mathrm{OBC} 2$ & J15585 & $6 / 14 / 07$ & 1.3 & $\mathrm{U}$ & 1.3 & 1580 & & 2.5 & 0.27 & $\mathrm{U}$ & 0.27 & 128 & C & 2.1 & 44.2 & & 0.24 \\
\hline OB C-9 & $\mathrm{J} 15582$ & $6 / 13 / 07$ & 1.3 & $\mathrm{U}$ & 1.3 & 1260 & & 2.7 & 0.28 & $\mathrm{U}$ & 0.28 & 148 & C & 2.2 & 44.1 & & 0.25 \\
\hline OB D3 & $\mathrm{J} 15586$ & $6 / 14 / 07$ & 1.3 & $\mathrm{U}$ & 1.3 & 1520 & & 2.7 & 0.28 & $\mathrm{U}$ & 0.28 & 140 & C & 2.2 & 40.3 & & 0.25 \\
\hline OB D4 & $\mathrm{J} 15587$ & $6 / 14 / 07$ & 1.3 & $\mathrm{U}$ & 1.3 & 1530 & & 2.6 & 0.27 & U & 0.27 & 116 & C & 2.1 & 31.9 & & 0.24 \\
\hline OBD5 & $\mathrm{J} 15588$ & $6 / 14 / 07$ & 1.3 & $\mathrm{U}$ & 1.3 & 1540 & & 2.6 & 0.27 & $\mathrm{U}$ & 0.27 & 123 & C & 2.1 & 39.0 & & 0.24 \\
\hline OB D6 & $\mathrm{J} 15589$ & $6 / 14 / 07$ & 1.3 & $\overline{\mathrm{U}}$ & 1.3 & 1780 & & 2.6 & 0.27 & $\mathrm{U}$ & 0.27 & 112 & C & 2.1 & 31.3 & & 0.24 \\
\hline OB D6 Dup & $\mathrm{J} 15590$ & $6 / 14 / 07$ & 1.3 & $\mathrm{U}$ & 1.3 & 1940 & & 2.5 & 0.26 & $\mathrm{U}$ & 0.26 & 99 & C & 2.1 & 35.0 & & 0.24 \\
\hline OB D6 Split & J15591 & $6 / 14 / 07$ & 0.5 & UN & 0.5 & 816 & $\mathrm{~N}$ & 18.6 & 0.38 & UN & 0.38 & 153 & B & 24.8 & 51.1 & & 0.55 \\
\hline
\end{tabular}

\begin{tabular}{|c|c|c|c|c|c|}
\hline Sample & HEIS & Sample & \multicolumn{3}{|c|}{ Zinc } \\
\cline { 4 - 6 } Location & Number & Date & mg/kg & Q & PQL \\
\hline SZ A-1 & J15510 & $6 / 13 / 07$ & 29.1 & $\mathrm{C}$ & 0.12 \\
\hline SZ A-1 Dup & $\mathrm{J} 15511$ & $6 / 13 / 07$ & 36.3 & $\mathrm{C}$ & 0.12 \\
\hline SZ A-1 Split & $\mathrm{J} 15520$ & $6 / 13 / 07$ & 38.1 & $\mathrm{~N}$ & 1.50 \\
\hline SZ A-2 & $\mathrm{J} 15512$ & $6 / 13 / 07$ & 30.9 & $\mathrm{C}$ & 0.12 \\
\hline SZ A-3 & $\mathrm{J} 15513$ & $6 / 13 / 07$ & 37.1 & $\mathrm{C}$ & 0.12 \\
\hline SZ A-4 & $\mathrm{J} 15514$ & $6 / 13 / 07$ & 30.8 & $\mathrm{C}$ & 0.12 \\
\hline DZ A-1 & $\mathrm{J} 15515$ & $6 / 4 / 07$ & 28.5 & $\mathrm{C}$ & 0.12 \\
\hline DZ A-2 & $\mathrm{J} 15516$ & $6 / 4 / 07$ & 34.0 & $\mathrm{C}$ & 0.12 \\
\hline DZ A-3 & $\mathrm{J} 15517$ & $6 / 4 / 07$ & 30.1 & $\mathrm{C}$ & 0.12 \\
\hline DZ A-3 Dup & $\mathrm{J} 15518$ & $6 / 4 / 07$ & 29.0 & $\mathrm{C}$ & 0.12 \\
\hline DZ A-3 Split & $\mathrm{J} 15521$ & $6 / 4 / 07$ & 27.0 & $\mathrm{C}$ & 1.40 \\
\hline DZ A-4 & $\mathrm{J} 15519$ & $6 / 4 / 07$ & 32.2 & $\mathrm{C}$ & 0.12 \\
\hline OB A-1 & $\mathrm{J} 15574$ & $6 / 12 / 07$ & 34.3 & $\mathrm{C}$ & 0.12 \\
\hline OB A-2 & $\mathrm{J} 15575$ & $6 / 12 / 07$ & 34.8 & $\mathrm{C}$ & 0.12 \\
\hline OB A-3 & $\mathrm{J} 15576$ & $6 / 12 / 07$ & 38.1 & $\mathrm{C}$ & 0.12 \\
\hline OB A-4 & $\mathrm{J} 15577$ & $6 / 12 / 07$ & 36.8 & $\mathrm{C}$ & 0.12 \\
\hline OB B-5 & $\mathrm{J} 15578$ & $6 / 13 / 07$ & 39.2 & $\mathrm{C}$ & 0.12 \\
\hline OB B-6 & $\mathrm{J} 15579$ & $6 / 13 / 07$ & 38.3 & $\mathrm{C}$ & 0.12 \\
\hline OB B-7 & $\mathrm{J} 15580$ & $6 / 13 / 07$ & 38.8 & $\mathrm{C}$ & 0.12 \\
\hline OB B-8 & $\mathrm{J} 15581$ & $6 / 13 / 07$ & 40.5 & $\mathrm{C}$ & 0.12 \\
\hline OB C1 & $\mathrm{J} 15584$ & $6 / 14 / 07$ & 36.8 & $\mathrm{C}$ & 0.12 \\
\hline OB C-10 & $\mathrm{J} 15583$ & $6 / 13 / 07$ & 37.2 & $\mathrm{C}$ & 0.12 \\
\hline OB C2 & $\mathrm{J} 15585$ & $6 / 14 / 07$ & 38.3 & $\mathrm{C}$ & 0.12 \\
\hline OB C-9 & $\mathrm{J} 15582$ & $6 / 13 / 07$ & 38.9 & $\mathrm{C}$ & 0.12 \\
\hline OB D3 & $\mathrm{J} 15586$ & $6 / 14 / 07$ & 38.9 & $\mathrm{C}$ & 0.12 \\
\hline OB D4 & $\mathrm{J} 15587$ & $6 / 14 / 07$ & 34.3 & $\mathrm{C}$ & 0.12 \\
\hline OB D5 & $\mathrm{J} 15588$ & $6 / 14 / 07$ & 38.4 & $\mathrm{C}$ & 0.12 \\
\hline OB D6 & $\mathrm{J} 15589$ & $6 / 14 / 07$ & 35.0 & $\mathrm{C}$ & 0.12 \\
\hline OB D6 Dup & $\mathrm{J} 15590$ & $6 / 14 / 07$ & 34.8 & $\mathrm{C}$ & 0.12 \\
\hline OB D6 Split & $\mathrm{J} 15591$ & $6 / 14 / 07$ & 34.9 & $\mathrm{~N}$ & 1.60 \\
\hline & & & & & \\
\hline
\end{tabular}

Note: Data qualified with B, C, D and/or J, are considered acceptable values. $\mathrm{GEA}=$ gamma energy analysis $\quad \mathrm{PQL}=$ practical quantitation limit HEIS = Hanford Environmental Information System $Q=$ qualifier $\mathrm{MDA}=$ minimum detectable activity $\quad \mathrm{U}=$ undetected

$\begin{array}{lll}\text { Attachment } & 1 & \text { Sheet No. } 6 \text { of } 7 \\ \text { Originator } & \text { L.D. Habel Ed Date } & 10 / 24 / 07 \\ \text { Checked } & \text { J.M. Capron } & \\ \text { Calc. No. } & \text { D100F-CA-V0294 Rev. No. }\end{array}$




\begin{tabular}{|c|c|c|c|c|c|c|c|c|c|c|c|c|c|c|c|c|c|}
\hline \multirow{2}{*}{$\begin{array}{c}\text { Sample } \\
\text { Location }\end{array}$} & \multirow{2}{*}{$\begin{array}{c}\text { HEIS } \\
\text { Number }\end{array}$} & \multirow{2}{*}{$\begin{array}{c}\text { Sample } \\
\text { Date }\end{array}$} & \multicolumn{3}{|c|}{ Aroclor-1016 } & \multicolumn{3}{|c|}{ Aroclor -1221} & \multicolumn{3}{|c|}{ Aroclor 1232} & \multicolumn{3}{|c|}{ Aroclor -1242} & \multicolumn{3}{|c|}{ Aroclor-1248 } \\
\hline & & & ug/kg & $Q$ & $\mathrm{PQL}$ & $\mathrm{ug} / \mathrm{kg}$ & $Q$ & PQL & ug/kg & $Q$ & PQL & $\mathrm{ug} / \mathrm{kg}$ & Q & $\mathrm{PQL}$ & $\mathrm{ug} / \mathrm{kg}$ & $Q$ & $\mathrm{PQL}$ \\
\hline $\mathrm{SZA-1}$ & $\mathrm{J} 15510$ & $6 / 13 / 07$ & 13 & $\mathrm{U}$ & 13 & 13 & Uे & 13 & 13 & Uे & 13 & 13 & U & 13 & 13 & $\vec{U}$ & 13 \\
\hline SZ A-1 Dup & $J 15511$ & $6 / 13 / 07$ & 14 & $\mathrm{U}$ & 14 & 14 & $\mathrm{U}$ & 14 & 14 & $\mathrm{U}$ & 14 & 14 & $\mathrm{U}$ & 14 & 14 & $\mathrm{U}$ & 14 \\
\hline SZ A-1 Split & $\mathrm{J} 15520$ & $6 / 13 / 07$ & 6 & $\mathrm{U}$ & 6 & 6 & $\mathrm{U}$ & 6 & 6 & $\mathrm{U}$ & 6 & 6 & U & 6 & 6 & $\bar{U}$ & 6 \\
\hline SZA-2 & $\mathrm{J} 15512$ & $6 / 13 / 07$ & 14 & $\mathrm{U}$ & 14 & 14 & $\mathrm{U}$ & 14 & 14 & $\mathrm{U}$ & 14 & 14 & $\mathrm{U}$ & 14 & 14 & $\mathrm{U}$ & 14 \\
\hline $\mathrm{SZA}-3$ & $\mathrm{~J} 15513$ & $6 / 13 / 07$ & 14 & $\mathrm{U}$ & 14 & 14 & $\mathrm{U}$ & 14 & 14 & $\mathrm{U}$ & 14 & 14 & $\mathrm{U}$ & 14 & 14 & $\bar{U}$ & 14 \\
\hline $\mathrm{SZA-4}$ & $\mathrm{J} 15514$ & $6 / 13 / 07$ & 13 & $\mathrm{U}$ & 13 & 13 & $\mathrm{U}$ & 13 & 13 & $\mathrm{U}$ & 13 & 13 & $\mathrm{U}$ & 13 & 13 & $\bar{U}$ & 13 \\
\hline DZA-1 & $\mathrm{J15515}$ & $6 / 4 / 07$ & 13 & $\mathrm{U}$ & 13 & 13 & $\mathrm{U}$ & 13 & 13 & $\mathrm{U}$ & 13 & 13 & $\mathrm{U}$ & 13 & 13 & $\mathrm{U}$ & 13 \\
\hline DZ A-2 & $\mathrm{J15516}$ & $6 / 4 / 07$ & 14 & $\mathrm{U}$ & 14 & 14 & $\mathrm{U}$ & 14 & 14 & $\mathrm{U}$ & 14 & 14 & $\mathrm{U}$ & 14 & 14 & $\mathrm{U}$ & 14 \\
\hline $\mathrm{DZA}-3$ & $\mathrm{~J} 15517$ & $6 / 4 / 07$ & 14 & $\mathrm{U}$ & 14 & 14 & $\mathrm{U}$ & 14 & 14 & $\mathrm{U}$ & 14 & 14 & U & 14 & 14 & $\mathrm{U}$ & 14 \\
\hline DZA-3 Dup & $\mathrm{J} 15518$ & $6 / 4 / 07$ & 14 & $\mathrm{U}$ & - 14 & 14 & $\mathrm{U}$ & 14 & 14 & $\mathrm{U}$ & 14 & 14 & $\mathrm{U}$ & 14 & 14 & $\mathrm{U}$ & 14 \\
\hline DZ A-3 Split & $\mathrm{J} 15521$ & $6 / 4 / 07$ & 6 & $\mathrm{U}$ & 6 & 6 & $\mathrm{U}$ & 6 & 6 & $\mathrm{U}$ & 6 & 6 & U & 6 & 6 & $\mathrm{U}$ & 6 \\
\hline $\mathrm{DZA}-4$ & $\mathrm{~J} 15519$ & $6 / 4 / 07$ & 13 & $\mathrm{U}$ & 13 & 13 & $\mathrm{U}$ & 13 & 13 & $\mathrm{U}$ & 13 & 13 & U & 13 & 13 & $\mathrm{U}$ & 13 \\
\hline $\mathrm{OBA}-1$ & $\mathrm{J15574}$ & $6 / 12 / 07$ & 16 & $\mathrm{U}$ & 16 & 16 & $\mathrm{U}$ & 16 & 16 & $\mathrm{U}$ & 16 & 16 & U & 16 & 16 & $\mathrm{U}$ & 16 \\
\hline $\mathrm{OBA}-2$ & 115575 & $6 / 12 / 07$ & 14 & $\mathrm{U}$ & 14 & 14 & $\mathrm{U}$ & 14 & 14 & U & 14 & 14 & U & 14 & 14 & $\mathrm{U}$ & 14 \\
\hline $\mathrm{OBA}-3$ & $\mathrm{J15576}$ & $6 / 12 / 07$ & 14 & $\mathrm{U}$ & 14 & 14 & $\mathrm{U}$ & 14 & 14 & $\mathrm{U}$ & 14 & 14 & U & 14 & 14 & $\overline{\mathrm{U}}$ & 14 \\
\hline $\mathrm{OBA}-4$ & J15577 & $6 / 12 / 07$ & 14 & $\mathrm{U}$ & 14 & 14 & $\mathrm{U}$ & 14 & 14 & $\mathrm{U}$ & 14 & 14 & $\mathrm{U}$ & 14 & 14 & $\mathrm{U}$ & 14 \\
\hline $\mathrm{OB} \mathrm{B-5}$ & $\mathrm{J} 15578$ & $6 / 13 / 07$ & 14 & $\mathrm{U}$ & 14 & 14 & $\mathrm{U}$ & 14 & 14 & $\mathrm{U}$ & 14 & 14 & U & 14 & 14 & $\mathrm{U}$ & 14 \\
\hline $\mathrm{OB} \mathrm{B-6}$ & $\mathrm{J} 15579$ & $6 / 13 / 07$ & 14 & $\mathrm{U}$ & 14 & 14 & U & 14 & 14 & $\mathrm{U}$ & 14 & 14 & U & 14 & 14 & $\mathrm{U}$ & 14 \\
\hline OB B-7 & $\mathrm{J} 15580$ & $6 / 13 / 07$ & 14 & $\mathrm{U}$ & 14 & 14 & $\mathrm{U}$ & 14 & 14 & $\mathrm{U}$ & 14 & 14 & $\mathrm{U}$ & 14 & 14 & $\mathrm{U}$ & 14 \\
\hline $\mathrm{OB} \mathrm{B-8}$ & $\mathrm{J} 15581$ & $6 / 13 / 07$ & 14 & $\mathrm{U}$ & 14 & 14 & $\mathrm{U}$ & 14 & 14 & $\mathrm{U}$ & 14 & 14 & $\mathrm{U}$ & 14 & 14 & $\mathrm{U}$ & 14 \\
\hline $\mathrm{OBCl}$ & $\mathrm{J} 15584$ & $6 / 14 / 07$ & 14 & $\mathrm{U}$ & 14 & 14 & $\mathrm{U}$ & 14 & 14 & U & 14 & 14 & U & 14 & 14 & $\mathrm{U}$ & 14 \\
\hline $\mathrm{OBC}-10$ & $\mathrm{~J} 15583$ & $6 / 13 / 07$ & 14 & $\mathrm{U}$ & 14 & 14 & $\mathrm{U}$ & 14 & 14 & $\mathrm{U}$ & 14 & 14 & U & 14 & 14 & $\mathrm{U}$ & 14 \\
\hline $\mathrm{OBC2}$ & $\mathrm{J} 15585$ & $6 / 14 / 07$ & 14 & $\mathrm{U}$ & 14 & 14 & $\mathrm{U}$ & 14 & 14 & $\mathrm{U}$ & 14 & 14 & $\mathrm{U}$ & 14 & 14 & $\mathrm{U}$ & 14 \\
\hline $\mathrm{OBC}-9$ & $\mathrm{~J} 15582$ & $6 / 13 / 07$ & 14 & $\mathrm{U}$ & 14 & 14 & $\mathrm{U}$ & 14 & 14 & $\mathrm{U}$ & 14 & 14 & U & 14 & 14 & $\mathrm{U}$ & 14 \\
\hline OB D3 & J15586 & $6 / 14 / 07$ & 14 & $\mathrm{U}$ & 14 & 14 & $\mathrm{U}$ & 14 & 14 & $\mathrm{U}$ & 14 & 14 & U & 14 & 14 & $\mathrm{U}$ & 14 \\
\hline OBD4 & $\mathrm{J} 15587$ & $6 / 14 / 07$ & 14 & $\mathrm{U}$ & 14 & 14 & $\mathrm{U}$ & 14 & 14 & $\mathrm{U}$ & 14 & 14 & $\mathrm{U}$ & 14 & 14 & $\mathrm{U}$ & 14 \\
\hline OB D5 & $\mathrm{J} 15588$ & $6 / 14 / 07$ & 14 & $\mathrm{U}$ & 14 & 14 & $\mathrm{U}$ & 14 & 14 & $\mathrm{U}$ & 14 & 14 & $\mathrm{U}$ & 14 & 14 & $\mathrm{U}$ & 14 \\
\hline OB D6 & J15589 & $6 / 14 / 07$ & 14 & $\mathrm{U}$ & 14 & 14 & U & 14 & 14 & $\mathrm{U}$ & 14 & 14 & $\mathrm{U}$ & 14 & 14 & $\mathrm{U}$ & 14 \\
\hline OB D6 Dup & $\mathrm{J15590}$ & $6 / 14 / 07$ & 14 & $\mathrm{U}$ & 14 & 14 & $\mathrm{U}$ & 14 & 14 & $\mathrm{U}$ & 14 & 14 & $\mathrm{U}$ & 14 & 14 & $\mathrm{U}$ & 14 \\
\hline OB D6 Split & Y15591 & $6 / 14 / 07$ & 6 & $\mathrm{U}$ & 6 & 6 & $\mathrm{U}$ & 6 & 6 & $\mathrm{U}$ & 6 & 6 & $\mathrm{U}$ & 6 & 6 & $\mathrm{U}$ & 6 \\
\hline
\end{tabular}

\begin{tabular}{|c|c|c|c|c|c|c|c|c|}
\hline Sample & HEIS & Sample & \multicolumn{3}{|c|}{ Aroclor-1254 } & \multicolumn{3}{|c|}{ Aroclor-1260 } \\
\cline { 5 - 9 } Location & Number & Date & ug/kg & Q & PQL & ug/kg & Q & PQL \\
\hline SZ A-1 & J15510 & $6 / 13 / 07$ & 13 & $\mathrm{U}$ & 13 & 13 & $\mathrm{U}$ & 13 \\
\hline SZ A-1 Dup & $\mathrm{J} 15511$ & $6 / 13 / 07$ & 14 & $\mathrm{U}$ & 14 & 14 & $\mathrm{U}$ & 14 \\
\hline SZ A-1 Split & $\mathrm{J} 15520$ & $6 / 13 / 07$ & 7 & $\mathrm{U}$ & 7 & 7 & $\mathrm{U}$ & 7 \\
\hline SZ A-2 & $\mathrm{J} 15512$ & $6 / 13 / 07$ & 14 & $\mathrm{U}$ & 14 & 14 & $\mathrm{U}$ & 14 \\
\hline SZ A-3 & $\mathrm{J} 15513$ & $6 / 13 / 07$ & 14 & $\mathrm{U}$ & 14 & 14 & $\mathrm{U}$ & 14 \\
\hline SZ A-4 & $\mathrm{J} 15514$ & $6 / 13 / 07$ & 13 & $\mathrm{U}$ & 13 & 13 & $\mathrm{U}$ & 13 \\
\hline DZ A-1 & $\mathrm{J} 15515$ & $6 / 4 / 07$ & 13 & $\mathrm{U}$ & 13 & 13 & $\mathrm{U}$ & 13 \\
\hline DZ A-2 & $\mathrm{J} 15516$ & $6 / 4 / 07$ & 14 & $\mathrm{U}$ & 14 & 49 & & 14 \\
\hline DZ A-3 & $\mathrm{J} 15517$ & $6 / 4 / 07$ & 14 & $\mathrm{U}$ & 14 & 14 & $\mathrm{U}$ & 14 \\
\hline DZ A-3 Dup & $\mathrm{J} 15518$ & $6 / 4 / 07$ & 14 & $\mathrm{U}$ & 14 & 14 & $\mathrm{U}$ & 14 \\
\hline DZ A-3 Split & $\mathrm{J} 15521$ & $6 / 4 / 07$ & 7 & $\mathrm{U}$ & 7 & 7 & $\mathrm{U}$ & 7 \\
\hline DZ A-4 & $\mathrm{J} 15519$ & $6 / 4 / 07$ & 13 & $\mathrm{U}$ & 13 & 13 & $\mathrm{U}$ & 13 \\
\hline OB A-1 & $\mathrm{J} 15574$ & $6 / 12 / 07$ & 16 & $\mathrm{U}$ & 16 & 16 & $\mathrm{U}$ & 16 \\
\hline OB A-2 & $\mathrm{J} 15575$ & $6 / 12 / 07$ & 14 & $\mathrm{U}$ & 14 & 14 & $\mathrm{U}$ & 14 \\
\hline OB A-3 & $\mathrm{J} 15576$ & $6 / 12 / 07$ & 14 & $\mathrm{U}$ & 14 & 14 & $\mathrm{U}$ & 14 \\
\hline OB A-4 & $\mathrm{J} 15577$ & $6 / 12 / 07$ & 6 & $\mathrm{~J}$ & 14 & 14 & $\mathrm{U}$ & 14 \\
\hline OB B-5 & $\mathrm{J} 15578$ & $6 / 13 / 07$ & 14 & $\mathrm{U}$ & 14 & 14 & $\mathrm{U}$ & 14 \\
\hline OB B-6 & $\mathrm{J} 15579$ & $6 / 13 / 07$ & 14 & $\mathrm{U}$ & 14 & 14 & $\mathrm{U}$ & 14 \\
\hline OB B-7 & $\mathrm{J} 15580$ & $6 / 13 / 07$ & 14 & $\mathrm{U}$ & 14 & 14 & $\mathrm{U}$ & 14 \\
\hline OB B-8 & $\mathrm{J} 15581$ & $6 / 13 / 07$ & 14 & $\mathrm{U}$ & 14 & 14 & $\mathrm{U}$ & 14 \\
\hline OB C1 & $\mathrm{J} 15584$ & $6 / 14 / 07$ & 14 & $\mathrm{U}$ & 14 & 14 & $\mathrm{U}$ & 14 \\
\hline OB C-10 & $\mathrm{J} 15583$ & $6 / 13 / 07$ & 14 & $\mathrm{U}$ & 14 & 14 & $\mathrm{U}$ & 14 \\
\hline OB C2 & $\mathrm{J} 15585$ & $6 / 14 / 07$ & 14 & $\mathrm{U}$ & 14 & 14 & $\mathrm{U}$ & 14 \\
\hline OB C-9 & $\mathrm{J} 15582$ & $6 / 13 / 07$ & 14 & $\mathrm{U}$ & 14 & 14 & $\mathrm{U}$ & 14 \\
\hline OB D3 & $\mathrm{J} 15586$ & $6 / 14 / 07$ & 14 & $\mathrm{U}$ & 14 & 14 & $\mathrm{U}$ & 14 \\
\hline OB D4 & $\mathrm{J} 15587$ & $6 / 14 / 07$ & 14 & $\mathrm{U}$ & 14 & 14 & $\mathrm{U}$ & 14 \\
\hline OB D5 & $\mathrm{J} 15588$ & $6 / 14 / 07$ & 14 & $\mathrm{U}$ & 14 & 14 & $\mathrm{U}$ & 14 \\
\hline OB D6 & $\mathrm{J} 15589$ & $6 / 14 / 07$ & 14 & $\mathrm{U}$ & 14 & 14 & $\mathrm{U}$ & 14 \\
\hline OB D6 Dup & $\mathrm{J} 15590$ & $6 / 14 / 07$ & 14 & $\mathrm{U}$ & 14 & 14 & $\mathrm{U}$ & 14 \\
\hline OB D6 Split & $\mathrm{J} 15591$ & $6 / 14 / 07$ & 7 & $\mathrm{U}$ & 7 & 7 & $\mathrm{U}$ & 7 \\
\hline
\end{tabular}

Note: Data qualified with $B, C, D$ and/or J, are considered acceptable values.

$\mathrm{B}=$ Estimated result. Result is less than $\mathrm{RL} \quad \mathrm{PQL}=$ practical quantitation limil

$D=$ Result obtained from the analysis of dilution $Q=$ qualifier

$\mathrm{GEA}=$ gamma energy analysis $\quad \mathrm{RL}=$ reporting limi

HEIS = Hanford Environmental Information System $\mathrm{U}=$ undetected

$\mathrm{MDA}=$ minimum detectable activity

$\mathrm{N}=$ spike sample is outside of control limits

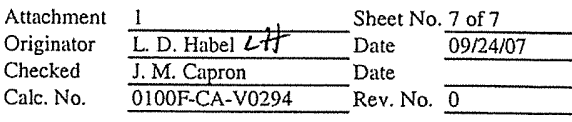




\section{CALCULATION COVER SHEET}

Area: $100-\mathrm{F}$

Discipline: Environmental ${ }^{*}$ Calculation No: 0100F-CA-V0296

Subject: 118-F-8:4 Hazard Quotient and Carcinogenic Risk Calculations

Computer Program: Excel

Program No: Excel 2003

The attached calculations have been generated to document compliance with established cleanup levels. These calculations should be used in conjunction with other relevant documents in the administrative record.

Committed Calculation $\square$

Preliminary

Superseded

Voided

\begin{tabular}{|c|c|c|c|c|c|c|}
\hline Rev & Sheet Numbers & Originator & Ghecker trits & Reviewer & Approval & Bate \\
\hline 0 & Total $=4$ & L. D., Habel & J.M. Capron & N/A & S. W. Callison & \\
\hline & & & & & swCe & 9.27 .07 \\
\hline & & & & & & \\
\hline & & & & & & \\
\hline & & & & & & \\
\hline & & & & & & \\
\hline & & & & & & \\
\hline
\end{tabular}

SUMMARY OF REVISION

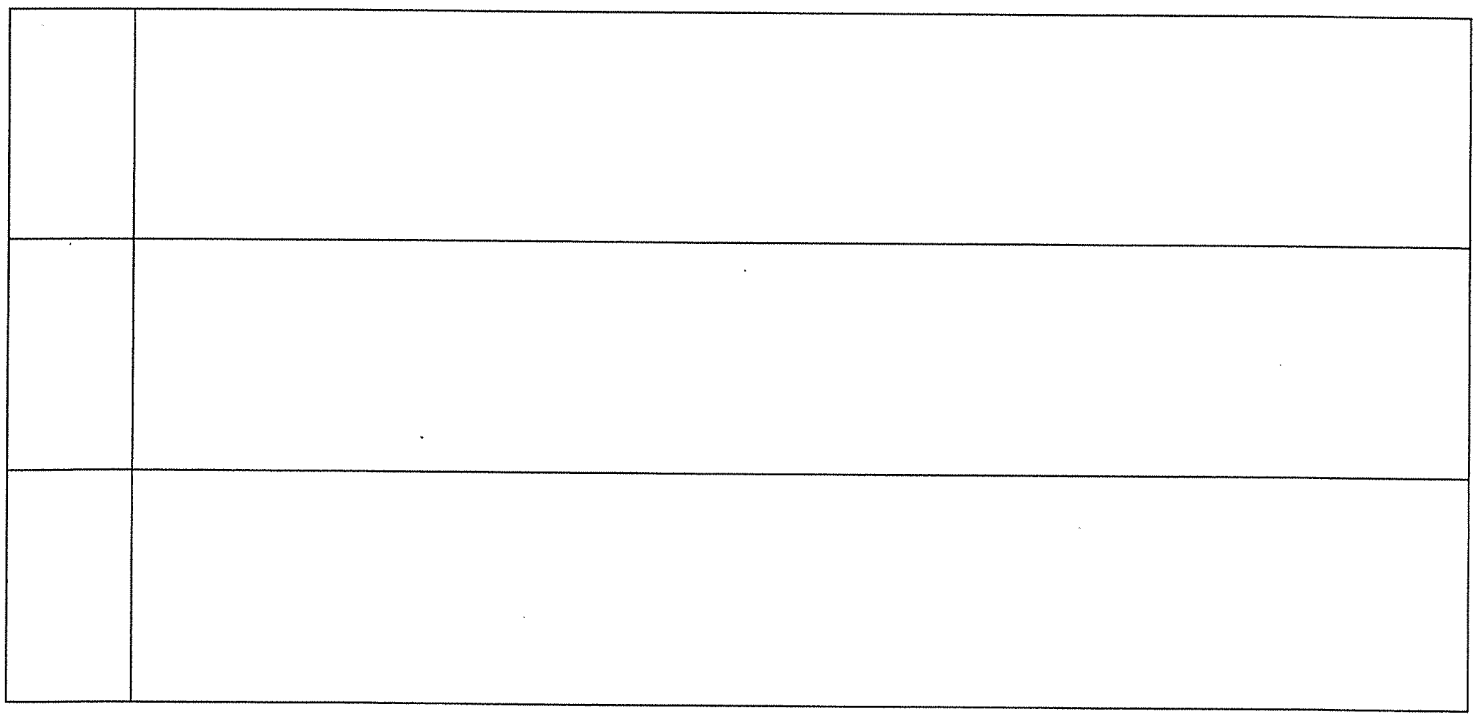


Washington Closure Hanford CALCULATION SHEET

\begin{tabular}{|c|c|c|c|c|c|c|c|}
\hline Originator: & L.D. Habel & Date: & $9 / 24 / 07$ & Calc. No.: & $0100 \mathrm{~F}-\mathrm{CA}-\mathrm{V} 0296$ & Rev.: & 0 \\
\hline Project: & 100-F Field Remediation & Job No: & 14655 & Checked: & J. M. Capron C/ & Date: & $9 / 24 / 07$ \\
\hline Subject: & \multicolumn{5}{|c|}{ 118-F-8:4 Hazard Quotient and Carcinogenic Risk Calculations } & \multicolumn{2}{|c|}{ Sheet No. 1 of 3} \\
\hline
\end{tabular}

\section{PURPOSE:}

Provide documentation to support the calculation of the hazard quotient (HQ) and carcinogenic (excess cancer) risk values for the 118-F-8:4 Fuel Storage Basin site remedial action. In accordance with the remedial action goals (RAGs) in the remedial design report/remedial action work plan (RDR/RAWP) (DOE-RL 2005), the following criteria must be met:

1) An $\mathrm{HQ}$ of $<1.0$ for all individual noncarcinogens

2) A cumulative HQ of $<1.0$ for noncarcinogens

3) An excess cancer risk of $<1 \times 10^{-6}$ for individual carcinogens

4) A cumulative excess cancer risk of $<1 \times 10^{-5}$ for carcinogens.

\section{GIVEN/REFERENCES:}

1) DOE-RL, 2005, Remedial Design Report/Remedial Action Work Plan for the 100 Areas, DOE/RL-96-17, Rev. 5, U.S. Department of Energy, Richland Operations Office, Richland, Washington.

2) WAC 173-340, "Model Toxics Control Act - Cleanup," Washington Administrative Code, 1996.

3) WCH, 2007, 118-F-8:4 105-F Fuel Storage Basin Cleanup Verification 95\% UCL Calculation, 0100F-CA-V0294, Washington Closure Hanford, Richland, Washington.

\section{SOLUTION:}

1) Calculate an HQ for each noncarcinogenic constituent detected above background and compare it to the individual HQ of $<1.0$ (DOE-RL 2005).

2) Sum the HQs and compare to the cumulative HQ criterion of $<1.0$.

3) Calculate an excess cancer risk value for each carcinogenic constituent detected above background and compare it to the individual excess cancer risk criterion of $<1 \times 10^{-6}$ (DOE-RL 2005).

4) Sum the excess cancer risk values and compare to the cumulative cancer risk criterion of $<1 \times 10^{-5}$. 
Washington Closure Hanford CALCULATION SHEET

\begin{tabular}{|r|l|r|r|r|l|c|c|}
\hline Originator: & L.D. Habel L & Date: & $9 / 24 / 07$ & Calc. No.: & 0100F-CA-V0296 & Rev.: & 0 \\
\hline Project: & $100-$ F Field Remediation & Job No: & 14655 & Checked: & J. M. Capron $9 / 20$ C & Date: & $9 / 24 / 07$ \\
\hline Subject: & $118-$ F-8:4 Hazard Quotient and Carcinogenic Risk Calculations & & Sheet No. 2 of 3 \\
\hline
\end{tabular}

\section{METHODOLOGY:}

Hazard quotient and carcinogenic risk calculations were conservatively calculated for the entire 118-F8:4 waste site using the maximum quantified value for each analyte in all decision units. WCH (2007). Of the nonradionuclide contaminants of concern $(\mathrm{COC})$ mercury and selenium were the only analytes that required the HQ and risk calculations because they were quantified above background. Additionally, hexavalent chromium, aroclor-1254, and aroclor-1260 required the HQ and risk calculations because these COCs were detected and a Washington State or Hanford Site background value is not available. All other site nonradionuclide COCs were not detected or were quantified below background levels. An example of the HQ and risk calculations is presented below:

1) For example, the maximum statistical result for hexavalent chromium $(0.27 \mathrm{mg} / \mathrm{kg})$, divided by the noncarcinogenic RAG value of $240 \mathrm{mg} / \mathrm{kg}$ (calculated in accordance with the noncarcinogenic toxic effects WAC 173-340-740[3]), is $1.1 \times 10^{-3}$. Comparing this value, and all other individual values, to the requirement of $<1.0$, this criterion is met.

2) After the HQ calculations are completed for the appropriate analytes, the cumulative HQ is obtained by summing the individual values. (To avoid errors due to intermediate rounding, the individual HQ values prior to rounding are used for this calculation.) The sum of the HQ values is $3.9 \times 10^{-2}$. Comparing this values to the requirement of $<1.0$, this criterion is met.

3) To calculate the excess cancer risk, the maximum statistical value is divided by the carcinogenic RAG value, then multiplied by $1 \times 10^{-6}$. For example, the maximum value for aroclor- 1254 is $0.016 \mathrm{mg} / \mathrm{kg}$; divided by $0.5 \mathrm{mg} / \mathrm{kg}$, and multiplied as indicated, is $3.2 \times 10^{-8}$. Comparing this value to the requirement of $<1 \times 10^{-6}$, this criterion is met.

4) After these calculations are completed for the carcinogenic analytes, the cumulative excess cancer risk is obtained by summing the individual values. The sum of the excess cancer risk values is $2.6 \times 10^{-7}$. Comparing this value to the requirement of $<1 \times 10^{-5}$, this criterion is met.

\section{RESULTS:}

1) List individual noncarcinogens and corresponding HQs $>1.0$ : None

2) List the cumulative noncarcinogenic $\mathrm{HQ}>1.0$ : None

3) List individual carcinogens and corresponding excess cancer risk $>1 \times 10^{-6}$ : None

4) List the cumulative excess cancer risk for carcinogens $>1 \times 10^{-5}$ : None.

Table 1 shows the results of the calculation. 
Washington Closure Hanford CALCULATION SHEET

Originator: $\mid$ L.D. Habel Lit

Project: $100-\mathrm{F}$ Field Remediation

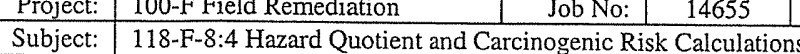

\begin{tabular}{l|l|l} 
Calc. No.: & $0100 \mathrm{~F}-\mathrm{CA}-\mathrm{V} 0296$ \\
\hline Check: & J.M.Capron
\end{tabular} Checked: J. M. Capron yeec

\begin{tabular}{|r|r|}
\hline Rev.: & 0 \\
\hline Date: & $9 / 24 / 07$ \\
\hline \multicolumn{2}{|c|}{ Sheet No. 3 of 3}
\end{tabular}

1

2

Table 1. Hazard Quotient and Excess Cancer Risk Results for the 118-F-8:4 Waste Site.

\begin{tabular}{|l|c|c|c|c|c|}
\hline Contaminants of Potential Concern & $\begin{array}{c}\text { Statistical Value } \\
(\mathbf{m g} / \mathbf{k g})\end{array}$ & $\begin{array}{c}\text { Noncarcinogen } \\
\mathbf{R A G}^{\mathbf{b}} \\
(\mathbf{m g} / \mathbf{k g})\end{array}$ & $\begin{array}{c}\text { Hazard } \\
\text { Quotient }\end{array}$ & $\begin{array}{c}\text { Carcinogen } \\
\mathbf{R A G}^{\mathbf{b}} \\
(\mathbf{m g} / \mathbf{k g})\end{array}$ & $\begin{array}{c}\text { Carcinogen } \\
\text { Risk }\end{array}$ \\
\hline Metals & & & & & \\
\hline Chromium, hexavalent &
\end{tabular}

Notes:

$\mathrm{RAG}=$ remedial action goal

$--=$ not applicable

${ }^{a}=$ From Calculation No. 0100F-CA-V0294 (WCH 2007).

$\mathrm{b}=$ Value obtained from Washington Administrative Code (WAC) 173-340-740(3), Method B, 1996, unless otherwise noted.

${ }^{c}=$ Value for the carcinogen RAG calculated based on the inhalation exposure pathway (WAC) 173-340-750(3), 1996.

6 CONCLUSION:

7

8 This calculation demonstrates that the 118-F-8:4 waste site meets the requirements for the hazard 9 quotients and carcinogenic (excess cancer) risk as identified in the RDR/RAWP (DOE-RL 2005). 


\section{CALCULATION COVER SHEET}

Project Title: Field Remediation

Job No. 14655

Area: $\quad 100-\mathrm{F}$

Discipline: Environmental क्येट 19107 *Calculation No: 0100ßB-CA-V0304

118-F-8:4 105-F Fuel Storage Basin Comparison to Drinking Water Standards (MCL) Subject: Calculation Brief

Computer Program: RESRAD

Program No: Version 6.3

The attached calculations have been generated to document compliance with established cleanup levels. These calculations should be used in conjunction with other relevant documents in the administrative record.

Committed Calculation $\bigotimes$

Preliminary

Superseded

Voided

\begin{tabular}{|c|c|c|c|c|c|c|}
\hline Rev & Sheet Numbers & Originator & Checker & Reviewer & Approval & Date \\
\hline 0 & $\begin{array}{l}\text { Cover }-1 \mathrm{pg} \\
\text { Calculations }-3 \mathrm{pg} \\
\text { Total } \quad-4 \text { pages }\end{array}$ & $\begin{array}{l}\& \leftarrow, \text { elong } \\
\text { S. W. Clark }\end{array}$ & $\begin{array}{l}\text { Hifw } \\
\text { M. W. Perrott }\end{array}$ & N/A & SW Cld & $9-25.07$ \\
\hline & & & & & & \\
\hline & & & & & & \\
\hline & & & & & & \\
\hline & & & & & & \\
\hline
\end{tabular}

SUMMARY OF REVISION

\begin{tabular}{|l|l|}
\hline & \\
& \\
\hline & \\
\hline & \\
\hline
\end{tabular}




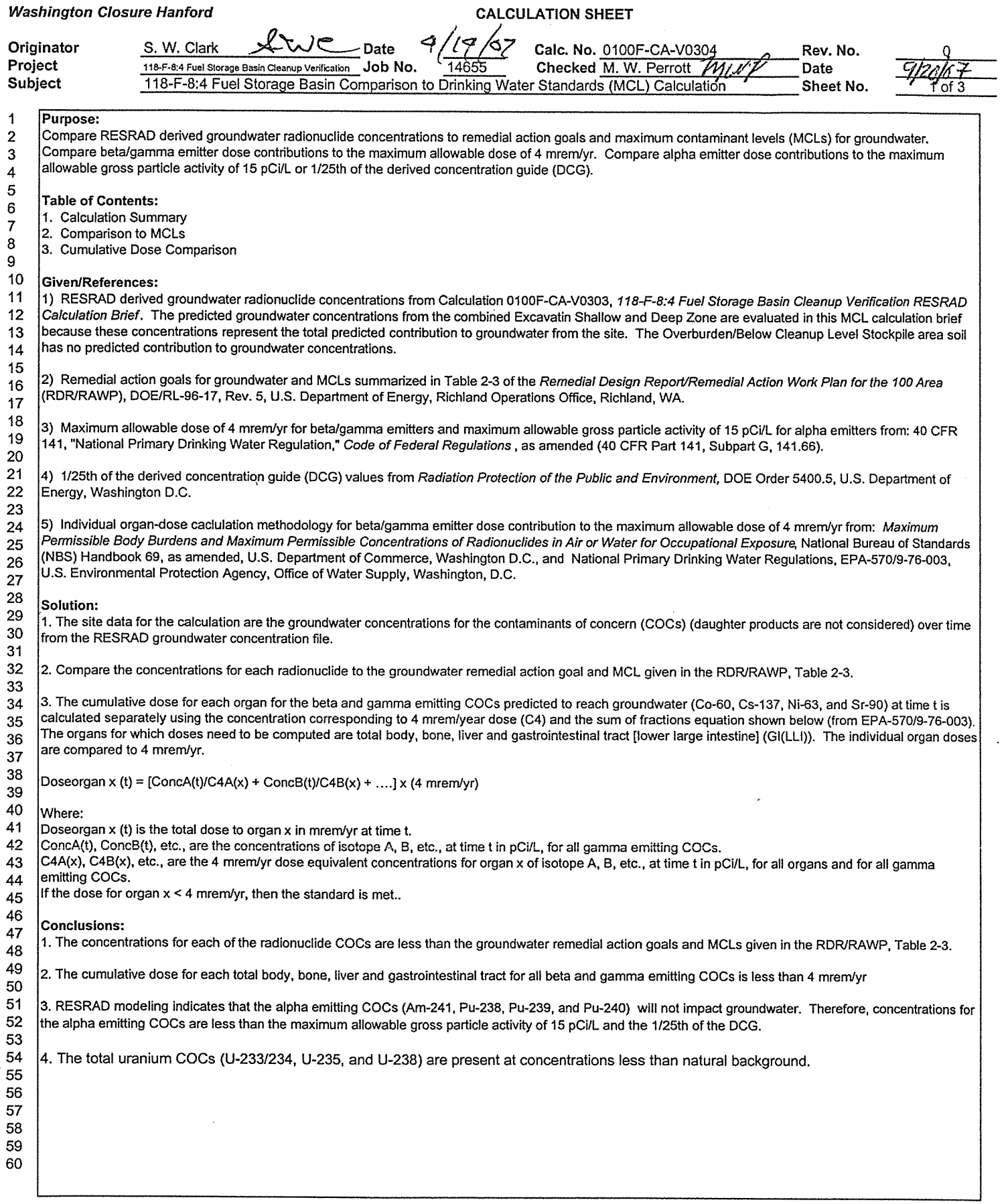




\section{Washington Closure Hanford}

CALCULATION SHEET

\begin{tabular}{|c|c|c|c|c|c|c|c|c|c|c|c|c|c|}
\hline $\begin{array}{l}\text { Originator } \\
\text { Project } \\
\text { Subject }\end{array}$ & \multicolumn{6}{|c|}{ 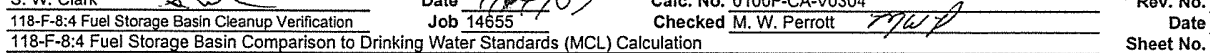 } & \multicolumn{6}{|c|}{$\frac{\text { S.W. Clark }}{118-F-8: 4 \text { Fuel Storage 8asin Cleanup Verification }} \quad$ Date $\frac{9 / 107}{14655} \quad$ Calc. No. 0100F-CA-V0304 } & \multirow[t]{2}{*}{$\begin{array}{r}\text { Rov. No. } \\
\text { Date } \\
\text { Sheet No. }\end{array}$} \\
\hline able of Con & sons of & axi & ne & on of & $\operatorname{la}$ & & & & & & & & \\
\hline Radionuclide & \begin{tabular}{|c|c|c|c|} 
Time (years \\
0
\end{tabular} & 1 & 3 & 7 & 11 & 43 & 135 & 300 & 1000 & MCL & $\begin{array}{l}\text { Exceeds } \\
\text { MCL? }\end{array}$ & $\begin{array}{l}\text { Peak } \\
\text { Conc. }\end{array}$ & $\begin{array}{c}\text { Year of Peak } \\
\text { Conc. }\end{array}$ \\
\hline Co-60 & 0 & $4.20 \mathrm{E}-04$ & $9.68 \mathrm{E}-04$ & $1.34 \mathrm{E}-03$ & $1.24 \mathrm{E}-03$ & $7.20 \mathrm{E}-05$ & $1.26 \mathrm{E}-09$ & $1.05 \mathrm{E}-18$ & 0 & 100 & No & $1.34 \mathrm{E}-03$ & 7 \\
\hline Cs-137 & 0 & $1.14 \mathrm{E}-02$ & $3.26 \mathrm{E}-02$ & $6.93 \mathrm{E}-02$ & $9.93 \mathrm{E}-02$ & $1.85 \mathrm{E}-01$ & $6.92 \mathrm{E}-02$ & $3.39 \mathrm{E}-03$ & $8.55 E-10$ & 60 & No & $1.85 E-01$ & 43 \\
\hline $\mathrm{Ni}-63$ & 0 & $2.21 \mathrm{E}-01$ & $6.53 \mathrm{E}-01$ & $1.48 \mathrm{E}+00$ & $2.26 \mathrm{E}+00$ & $6.99 \mathrm{E}+00$ & $1.12 \mathrm{E}+01$ & $7.36 \mathrm{E}+00$ & $4.28 \mathrm{E}-02$ & 50 & No & $1.12 E+01$ & 135 \\
\hline$S r-90$ & 0 & $4.04 \mathrm{E}-02$ & $1.15 E-01$ & $2.45 E-01$ & $3.50 \mathrm{E}-01$ & $6.36 \mathrm{E}-01$ & $2.22 \mathrm{E}-01$ & $7.09 \mathrm{E}-03$ & $3.64 \mathrm{E}-10$ & 8 & No & $6.36 \mathrm{E}-01$ & 43 \\
\hline
\end{tabular}

118-F-8-4 MCL (0100F-CA-V0304)

Comparison to MCLs 


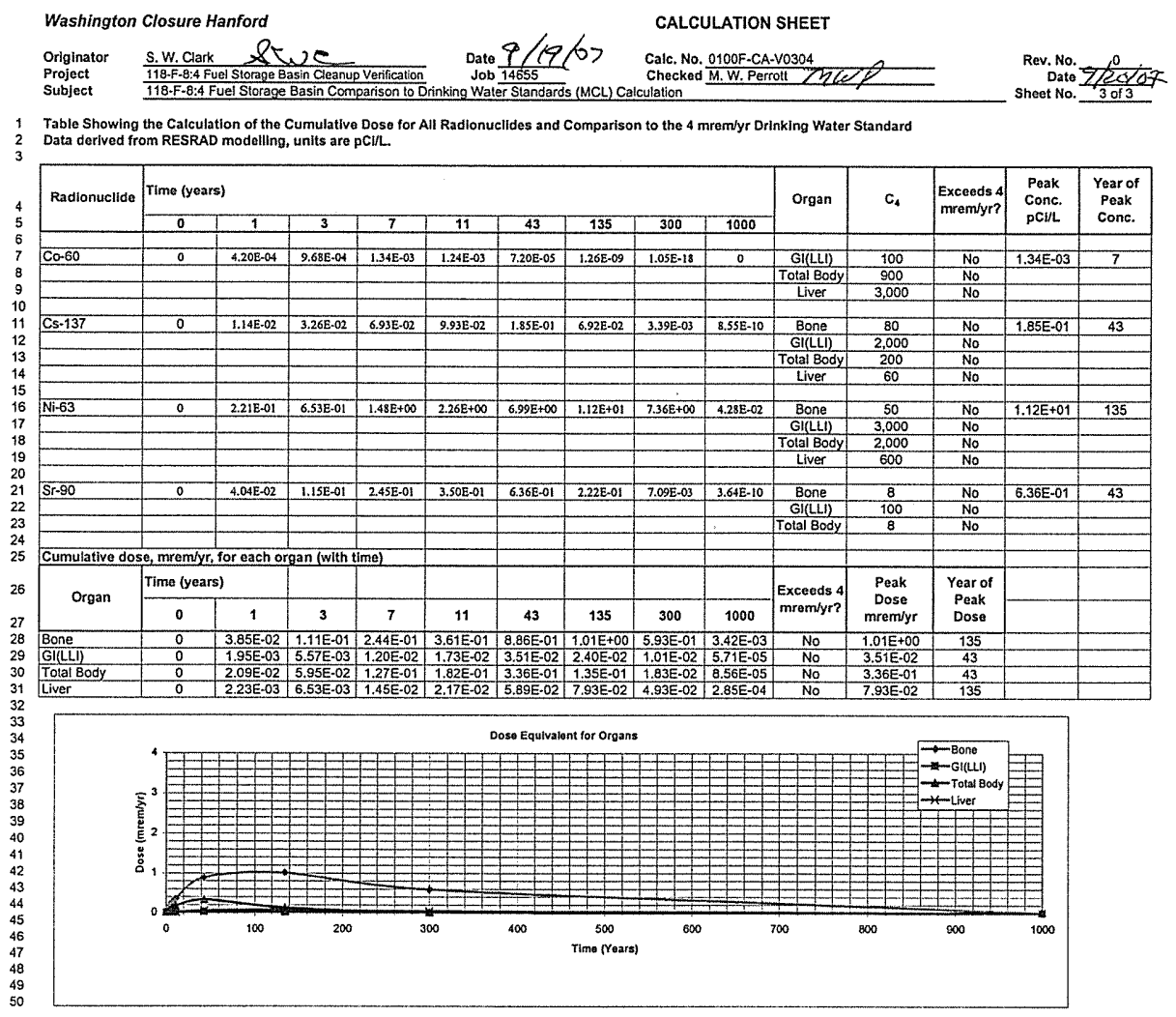




\section{CALCULATION COVER SHEET}

Project Title: 118-F-8:4 Fuel Storage Basin Sample Design

Job No. 14655

Area: $\quad 100-\mathrm{F}$

Discipline: Environmental Engineering

${ }^{*}$ Calculation No: 0100F-CA-V0307

Subject: $\quad$ Shallow and Deep Zone, Overburden Sampling Plan

\begin{tabular}{lll} 
& $\begin{array}{l}\text { Excel, Autodesk World, } \\
\text { and Autodesk Land } \\
\text { Map }\end{array}$ Program No: $\quad \begin{array}{l}\text { Excel 2003, World R2, and Land Map } \\
2004\end{array}$ \\
\hline
\end{tabular}

The attached calculations have been generated to document compliance with established cleanup levels. These calculations should be used in conjunction with other relevant documents in the administrative record.

Committed Calculation $\mathbb{X} \quad$ Preliminary $\square \quad$ Superseded $\square \quad$ Voided

\begin{tabular}{|c|c|c|c|c|c|c|}
\hline ReV & Sheet Numbers & Originator & 4 & sevewer & Aproval & bate \\
\hline 0 & Total $=8 \mathrm{Shts}$ & $\begin{array}{l}4 \sum_{G . C r u z} \\
q-26-07\end{array}$ & $\begin{array}{l}\text { Mes } \\
\text { c.A.Bentz } \\
9 / 2 \geqslant 87\end{array}$ & N/A & $\begin{array}{l}\text { Sw.W. Callison } \\
\text { S. }\end{array}$ & $10-2-07$ \\
\hline & & & & & & \\
\hline & & & & & & \\
\hline & & & & & & \\
\hline
\end{tabular}

SUMMARY OF REVISION

\begin{tabular}{|l|l|}
\hline & \\
\hline & \\
& \\
\hline & \\
\hline
\end{tabular}




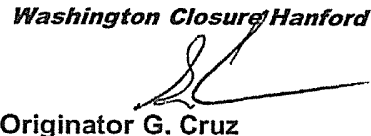

CALCULATION SHEET

Date 9/26/2007

Calc. No. 0100F-CA-V0307 Rev. No. 0

$\begin{array}{llll}\text { Project } & 118-F-8: 4 \text { Fuel Storage Basin Sample Design Job No. } 14655 \text { Checked } & 08 & \text { Date } 9 / 27 / 07 \\ \text { Subject } & \text { Shallow and Deep Zone, Overburden Sampling Plan } & 1 \text { of } 2\end{array}$

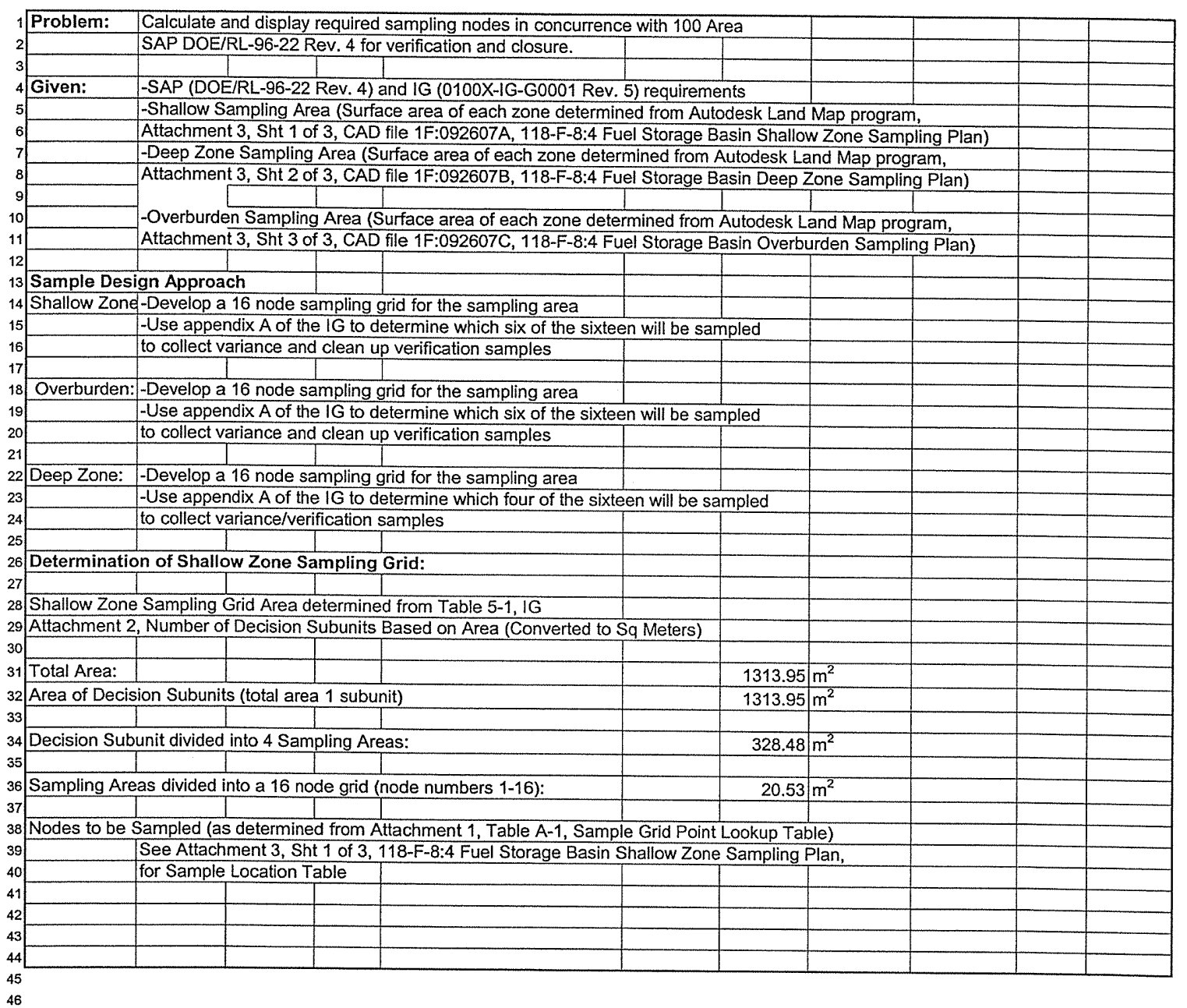




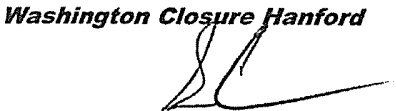

Originator G. Cruz

Date $9 / 26 / 2007$

CALCULATION SHEET
Calc. No. 0100F-CA-V0307

Job No. 14655

Subject Shallow and Deep Zone, Overburden Sampling Plan
Rev. No. 0

Checked QB Date $q / 27 / 07$

Sheet No. 2 of 2

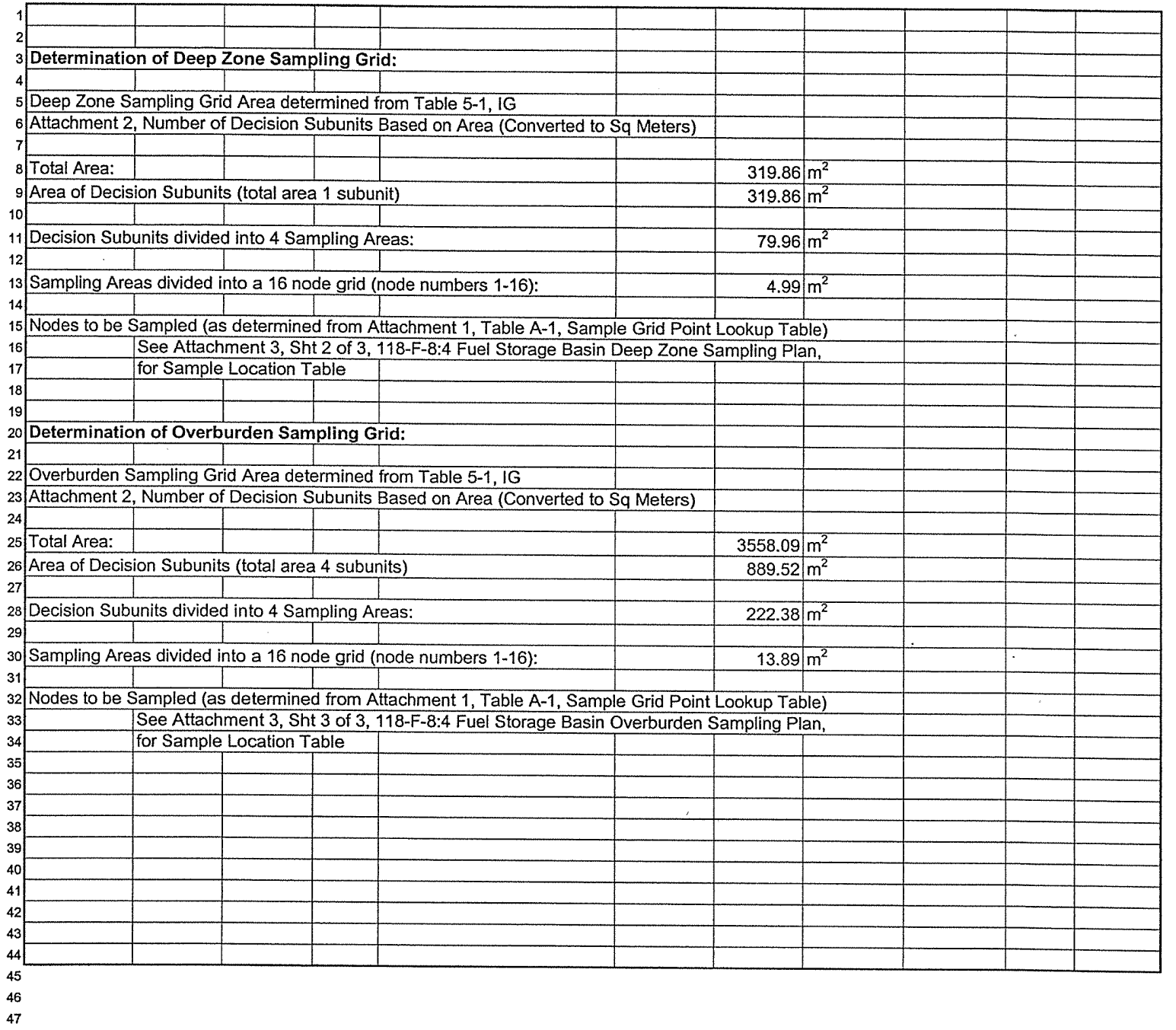




\begin{tabular}{l}
$\begin{array}{l}\text { Originator } \\
\text { Project } \\
\text { Subject }\end{array}$ \\
\cline { 2 - 3 } Shallow and Deep Zone, Overburden Sampling Plan
\end{tabular}

\section{ATTACHMENT}

${ }_{3}$ Sample Grid Point Lookup Table.

4

5

\begin{tabular}{|c|c|c|c|c|c|c|c|c|c|c|}
\hline Default Plan & $\begin{array}{l}\text { Sampling } \\
\text { Area 1 }\end{array}$ & $\begin{array}{c}\text { Sampling } \\
\text { Area 2 }\end{array}$ & $\begin{array}{c}\text { Sampling } \\
\text { Area } 3\end{array}$ & $\begin{array}{c}\text { Sampling } \\
\text { Area } 4\end{array}$ & $\begin{array}{c}\text { Sampling } \\
\text { Area } 5\end{array}$ & $\begin{array}{l}\text { Sampling } \\
\text { Area } 6\end{array}$ & $\begin{array}{l}\text { Sampling } \\
\text { Area } 7\end{array}$ & $\begin{array}{c}\text { Sampling } \\
\text { Area } 8\end{array}$ & $\begin{array}{c}\text { Sampling } \\
\text { Area } 9\end{array}$ & $\begin{array}{l}\text { Sampling } \\
\text { Area } 10\end{array}$ \\
\hline Variance $N$ erification & 3 & 6 & 1 & 4 & 5 & 1 & 3 & 3 & 4 & 16 \\
\hline Variance $N$ erification & 4 & 7 & 11 & 3 & 15 & 15 & 5 & 13 & 10 & 10 \\
\hline Variance $N$ erification & 16 & 3 & 2 & 7 & 7 & 10 & 11 & 4 & 3 & 14 \\
\hline \begin{tabular}{|l|} 
Variance/Nerification \\
\end{tabular} & 10 & 15 & 4 & 12 & 1 & 13 & 4 & 8 & 16 & 4 \\
\hline Variance & 2 & 14 & 5 & 9 & 13 & 12 & 8 & 2 & 14 & 8 \\
\hline Variance & 13 & 10 & 9 & 13 & 2 & 16 & 1 & 12 & 5 & 3 \\
\hline Not Sampling & 6 & 1 & 10 & 8 & 14 & 4 & 16 & 5 & 8 & 6 \\
\hline Not Sampling & 1 & 9 & 13 & 1 & 10 & 5 & 12 & 1 & 1 & 15 \\
\hline Not Sampling & 9 & 12 & 7 & 5 & 6 & 2 & 6 & 7 & 15 & 9 \\
\hline Not Sampling & 15 & 16 & 15 & 14 & 16 & 6 & 2 & 15 & 11 & 1 \\
\hline Not Sampling & 8 & 13 & 8 & 10 & 12 & 11 & 13 & 14 & 2 & 12 \\
\hline Not Sampling & 5 & 2 & 3 & 11 & 4 & 3 & 9 & 10 & 7 & 11 \\
\hline Not Sampling & 7 & 11 & 14 & 15 & 11 & 14 & 14 & 6 & 13 & 2 \\
\hline Not Sampling & 11 & 4 & 6 & 2 & 9 & 7 & 7 & 11 & 9 & 7 \\
\hline Not Sampling & 12 & 8 & 16 & 16 & 3 & 8 & 15 & 9 & 6 & 13 \\
\hline Not Sampling & 14 & 5 & 12 & 6 & 8 & 9 & 10 & 16 & 12 & 5 \\
\hline
\end{tabular}


Washington Clgsuye Hanford<smiles>CCCC[C@@H](O)CC(C)C</smiles>

Originator G. Cruz

Date $9 / 26 / 2007$

Calc. No. 0100F-CA-V0307 Rev. No. 0

Project 118-F-8:4 Fuel Storage Basin Sample Design Job No. 14655 Checked $\triangle 1 S$

Subject Shallow and Deep Zone, Overburden Sampling Plan Sheet No. 1 of 1

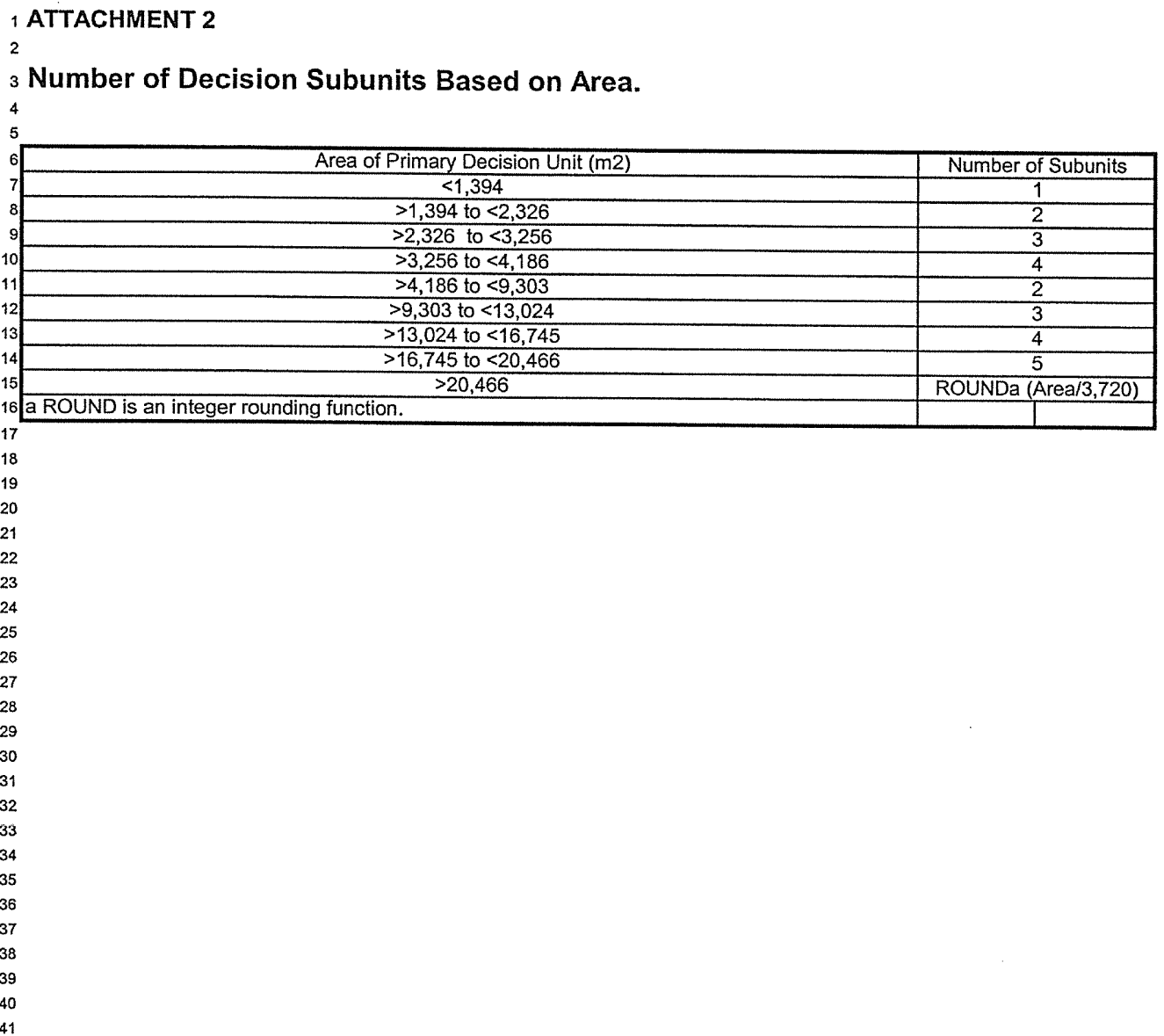




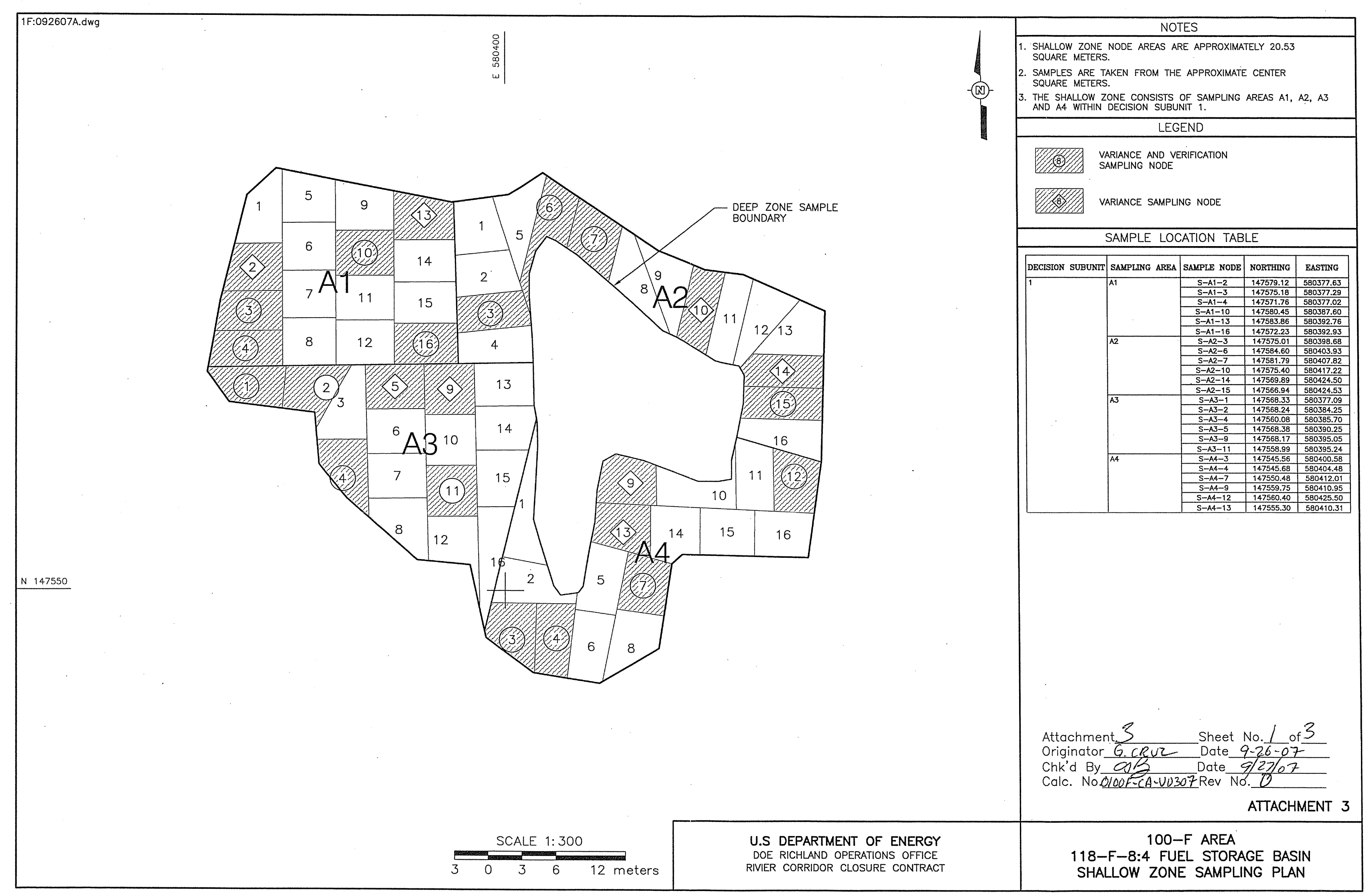




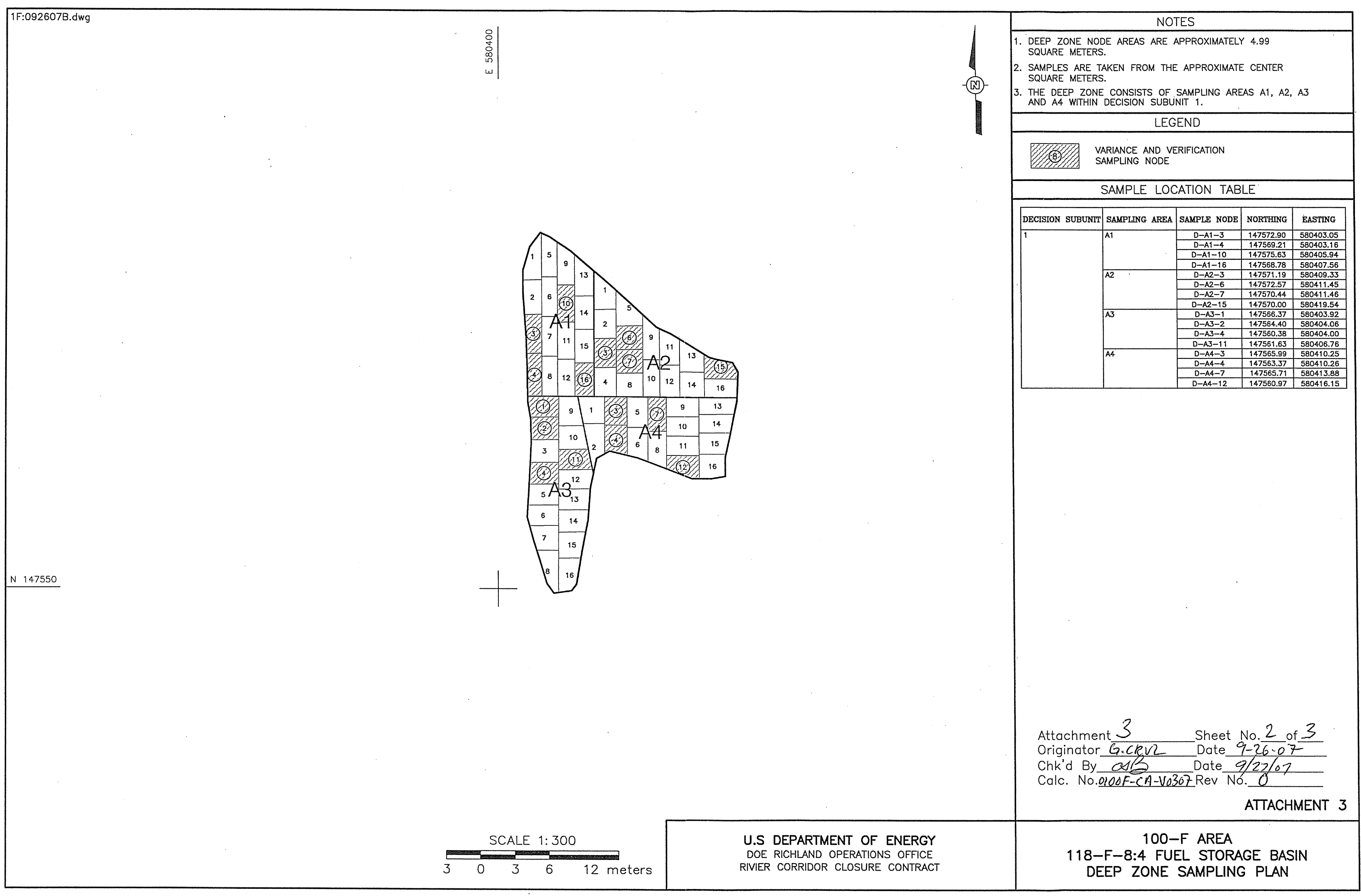




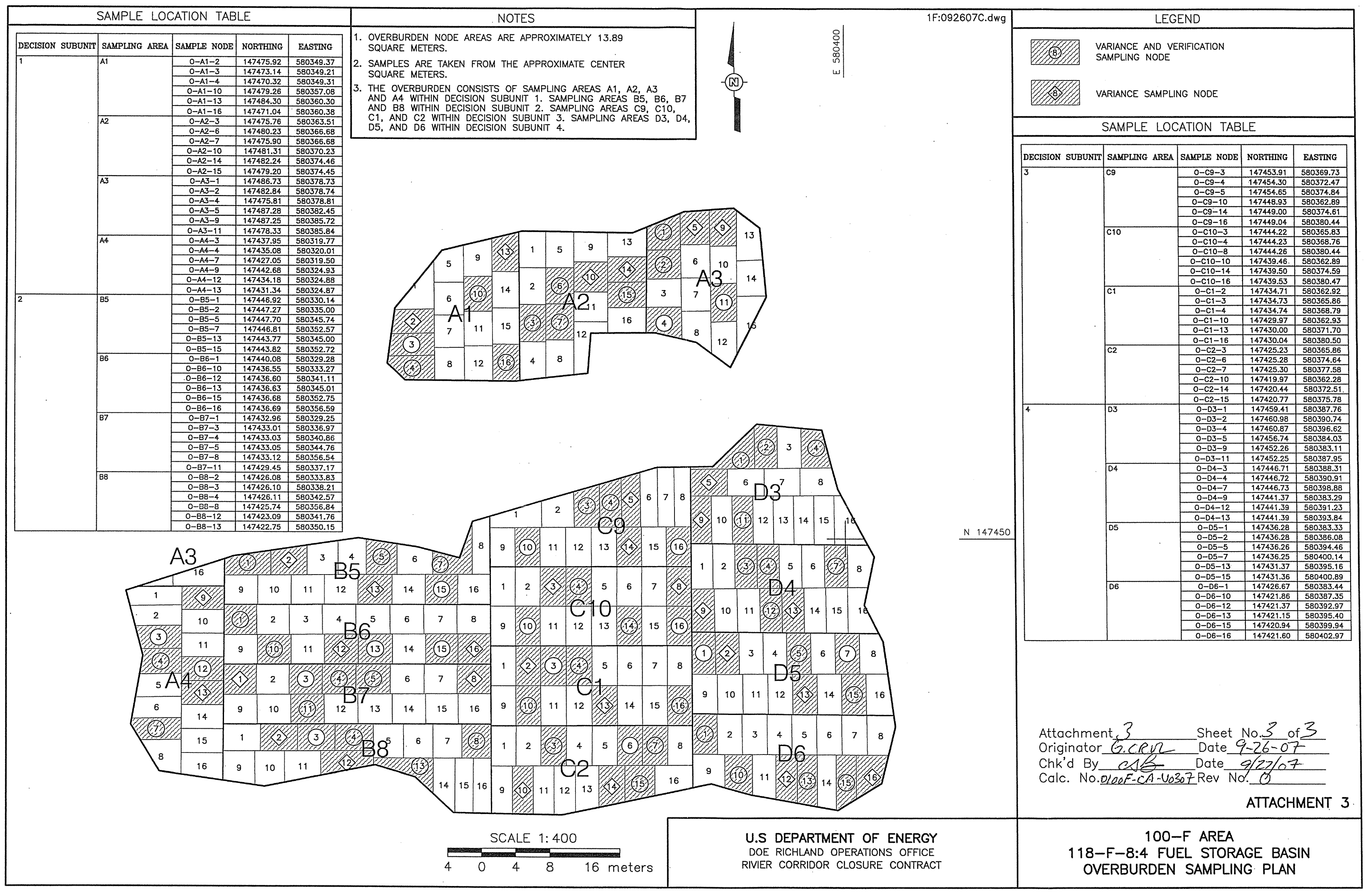




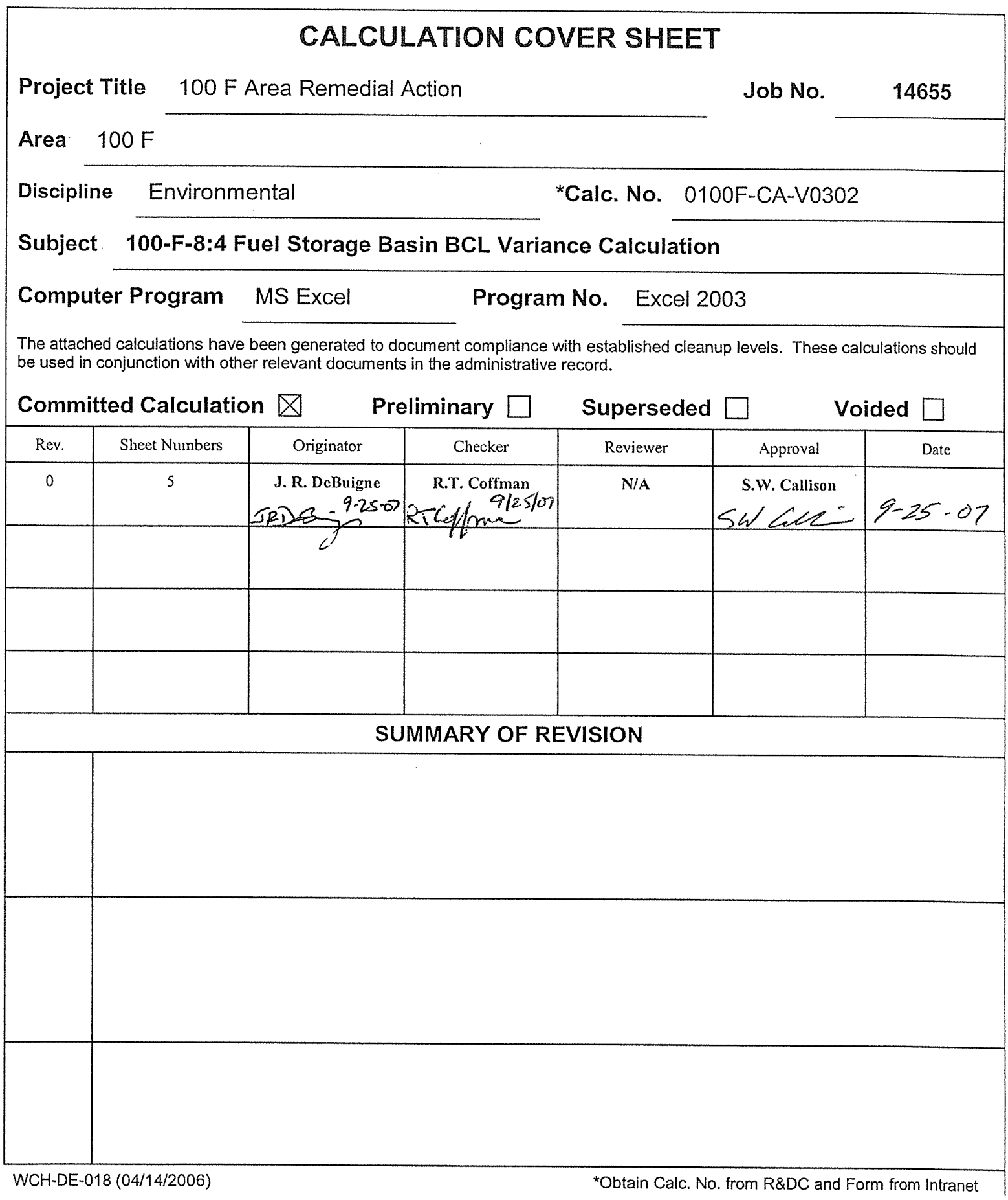




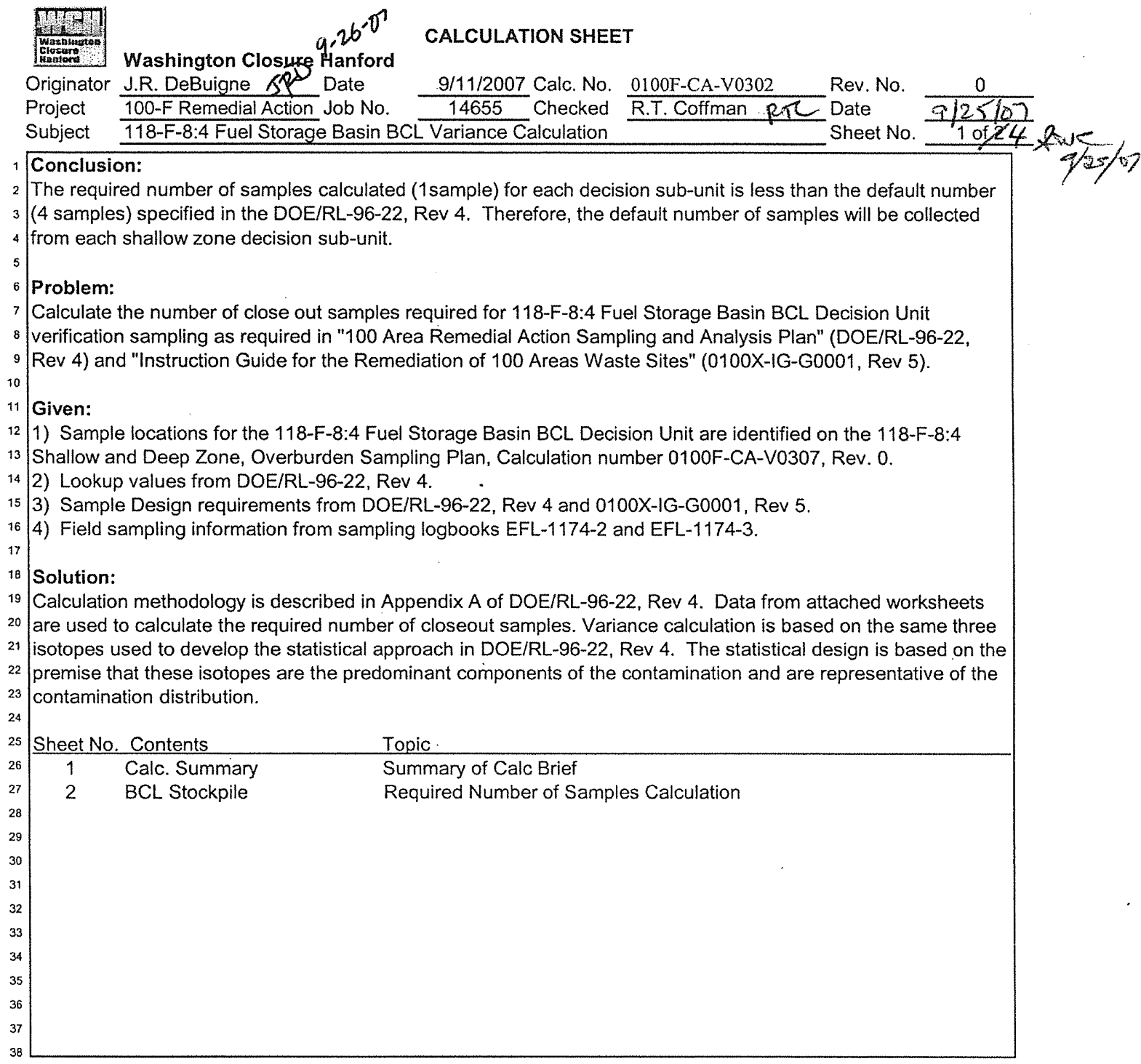


Dring

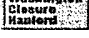

Originator

Project

Subject
Washington Closure Hanford

JR. DeBuigne TRD 9.26-57 Date 9/11/2007 100-F Remedial Action Job No

118-F-8:4 Fuel Storage Basin BCL Variance Calculation

1 Statistical Evaluation of Analytical Data

3 The required number of samples resulting from the calculation is highlighted at the bottom of the page.

4 Each value is reflective of the specific analyte evaluated.

5 The highest value of the three evaluations is used to determine the required number of samples as compared

6 against the default of four.

7 Sample locations are from Calculation 0100F-CA-V0307.

8 Mean, Standard Deviation, $t$, and Number of Samples formulas are from DOE/RL-96-22, Appendix A.

11 Decision Unit: 118-F-8:4 Fuel Storage Basin BCL Variance Calculation

12 Samples values from GEA analysis

13 Sample Areas , A1 thru D6

${ }_{14}$ Sample \# Sample Date Location Cobalt-60 Q $Q^{\mathrm{Cs}-137}$

\begin{tabular}{|c|c|c|c|c|c|c|c|c|c|}
\hline \multirow{2}{*}{\multicolumn{4}{|c|}{ 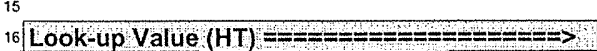 }} & \multirow{2}{*}{$\frac{p \mathrm{Ci} / \mathrm{g}}{1.4 \mathrm{~g}}$} & \multicolumn{3}{|c|}{$\mathrm{pCi} / \mathrm{g}$} & \\
\hline & & & & & & 6.2 & & 3.3 & \\
\hline & $\mathrm{J} 15592$ & Tा7/2007 & O-A1-2 & 0.076 & U & 0.08 & U & 0.193 & U \\
\hline & $J 15593$ & $6 / 7 / 2007$ & O-A1-3 & 0.091 & U & 0.088 & U & 0.181 & U \\
\hline & $\mathbf{J 1 5 5 9 4}$ & $6 / 7 / 2007$ & O-A1-4 & 0.081 & $\mathrm{U}$ & 0.074 & U & 0.184 & U \\
\hline & $J 15595$ & $6 / 7 / 2007$ & O-A1-10 & 0.052 & U & 0.103 & U & 0.151 & $U$ \\
\hline & $J 15596$ & $6 / 7 / 2007$ & O-A1-13 & 0.066 & U & 0.085 & & 0.196 & U \\
\hline & J15597 & $6 / 7 / 2007$ & $0-A 1-16$ & 0.07 & $\mathrm{U}$ & 0.18 & & 0.194 & U \\
\hline & $\mathrm{J15598}$ & $6 / 7 / 2007$ & $\mathrm{O}-\mathrm{A} 2 \mathrm{2}-3$ & 0.096 & $\mathrm{U}$ & 0.084 & Ut & 0.205 & U \\
\hline & J15599 & $6 / 7 / 2007$ & $0-A 2-6$ & 0.072 & U & 0.075 & U & 0.178 & U \\
\hline & J155B0 & $6 / 7 / 2007$ & O-A2-7 & 0.061 & U & 0.088 & & 0.195 & U \\
\hline & J155B1 & $6 / 7 / 2007$ & $0-A 2-10$ & 0.068 & U & 0.136 & & 0.172 & $U$ \\
\hline & $\mathrm{J155B2}$ & $6 / 7 / 2007$ & $\mathrm{O}-\mathrm{A} 2-14$ & 0.054 & U & 0.067 & U & 0.166 & U \\
\hline & J155B3 & $6 / 7 / 2007$ & $0-A 2-15$ & 0.091 & U & 0.199 & & 0.522 & 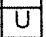 \\
\hline & J155B4 & $6 / 7 / 2007$ & O-A3-1 & 0.089 & U & 0.464 & & 1.14 & \\
\hline & J155B5 & $6 / 7 / 2007$ & O-A3-2 & 0.077 & U & 0.42 & & 1.6 & \\
\hline & J155B6 & $6 / 7 / 2007$ & $\mathrm{O}-\mathrm{A} 3-4$ & 0.065 & U & 0.365 & & 0.332 & \\
\hline & J155B 7 & $6 / 7 / 2007$ & O-A3-5 & 0.064 & U & 0.532 & & 0.379 & \\
\hline & J155B8 & $6 / 7 / 2007$ & O-A3-9 & 0.087 & U & 0.094 & & 0.184 & U \\
\hline & J155B9 & $6 / 7 / 2007$ & O-A3-11 & 0.067 & U & 1.12 & & 1.81 & \\
\hline & J155C0 & $6 / 7 / 2007$ & $\mathrm{O}-\mathrm{A} 4-3$ & 0.062 & U & 0.136 & & 0.163 & U \\
\hline & J155C1 & $6 / 7 / 2007$ & O-A4-4 & 0.05 & U & 0.273 & & 0.199 & $\mathrm{U}$ \\
\hline & J155C2 & $6 / 7 / 2007$ & O-A4-7 & 0.063 & U & 0.12 & & 0.169 & $\mathrm{U}$ \\
\hline & $\mathrm{J155C3}$ & $6 / 7 / 2007$ & $O-A 4-9$ & 0.092 & U & 0.203 & & 0.195 & U \\
\hline & J155C4 & $6 / 7 / 2007$ & O-A4-12 & 0.066 & 0 & 0.515 & & 0.37 & \\
\hline & J155C5 & $6 / 7 / 2007$ & O-A4-13 & 0.067 & U & 0.4 & & 0.34 & \\
\hline & $\mathrm{J155C6}$ & $6 / 7 / 2007$ & O-B5-1 & 0.075 & U & 0.431 & & 0.303 & \\
\hline & J155C7 & $6 / 7 / 2007$ & O-B5-2 & 0.074 & U & 0.066 & U & 0.175 & U \\
\hline & J155C8 & $6 / 7 / 2007$ & O-B5-5 & 0.074 & U & 0.07 & 0 & 0.176 & $U$ \\
\hline & J155C9 & $6 / 7 / 2007$ & O-B5-7 & 0.061 & U & 0.198 & & 0.172 & U \\
\hline & J155D0 & $6 / 7 / 2007$ & O-B5-13 & 0.076 & U & 0.374 & & 0.215 & U \\
\hline & J155D1 & $6 / 7 / 2007$ & O-B5-15 & 0.049 & U & 0.097 & & 0.133 & $U$ \\
\hline & $\mathrm{J} 155 \mathrm{D2}$ & $6 / 11 / 2007$ & $0-B 6-1$ & 0.048 & 0 & 0.078 & & 0.102 & 0 \\
\hline & J155D3 & $6 / 11 / 2007$ & $0-B 6-10$ & 0.066 & U & 1.26 & & 0.855 & \\
\hline & J155D4 & $6 / 111 / 2007$ & O-B6-12 & 0.05 & 0 & 0.054 & 0 & 0.14 & 0 \\
\hline 89 & J155D5 & $6 / 11 / 2007$ & O-B6-13 & 0.055 & U & 0.214 & & 0.139 & 0 \\
\hline & J155D6 & $6 / 11 / 2007$ & $0-B 6-15$ & 0.05 & U & 0.061 & U & 0.114 & 0 \\
\hline 2 & J155D7 & $6 / 11 / 2007$ & O-B6-16 & 0.051 & U & 0.055 & 0 & 0.135 & $U$ \\
\hline
\end{tabular}

118-F-8_4 FSB BCL 


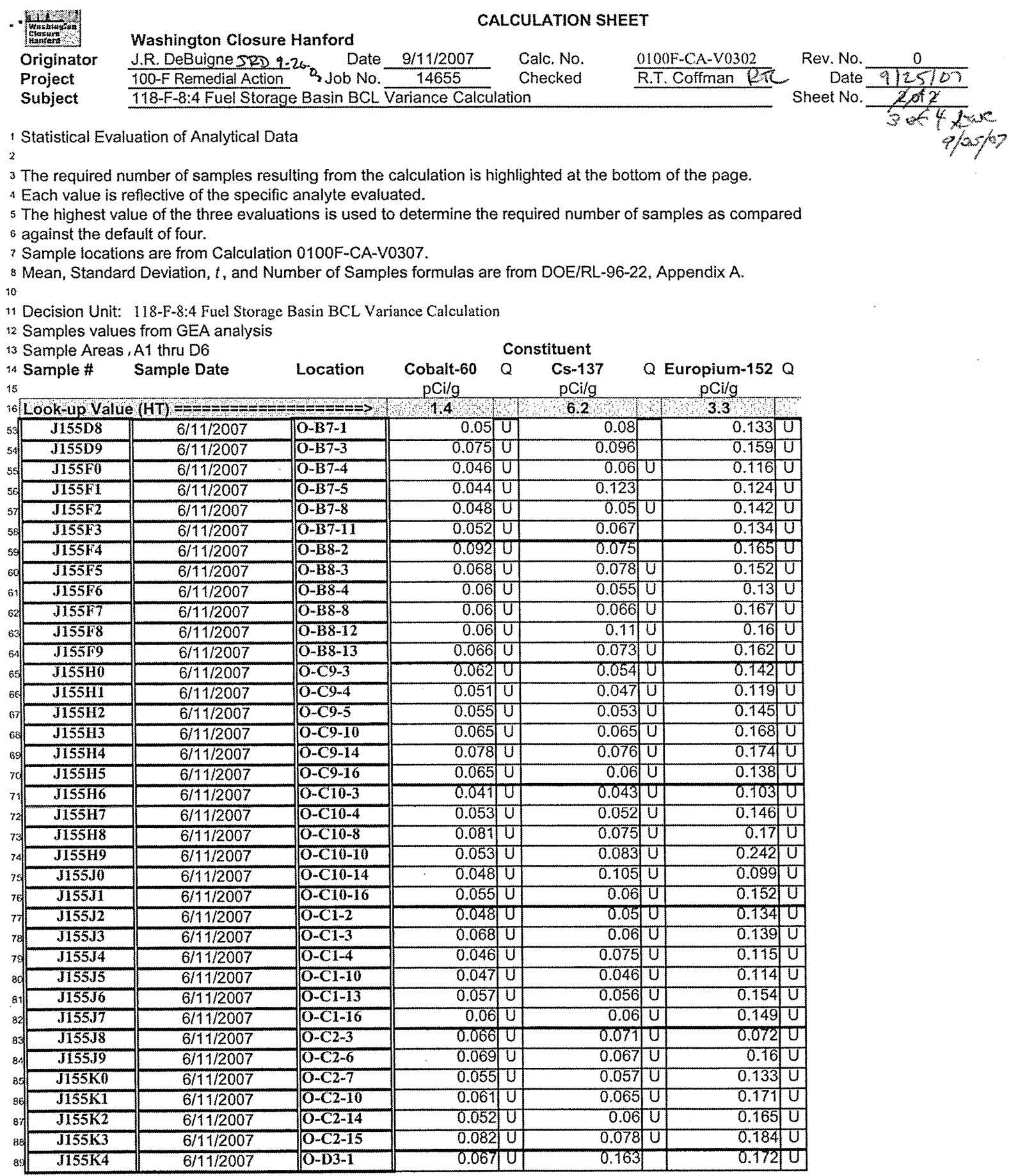




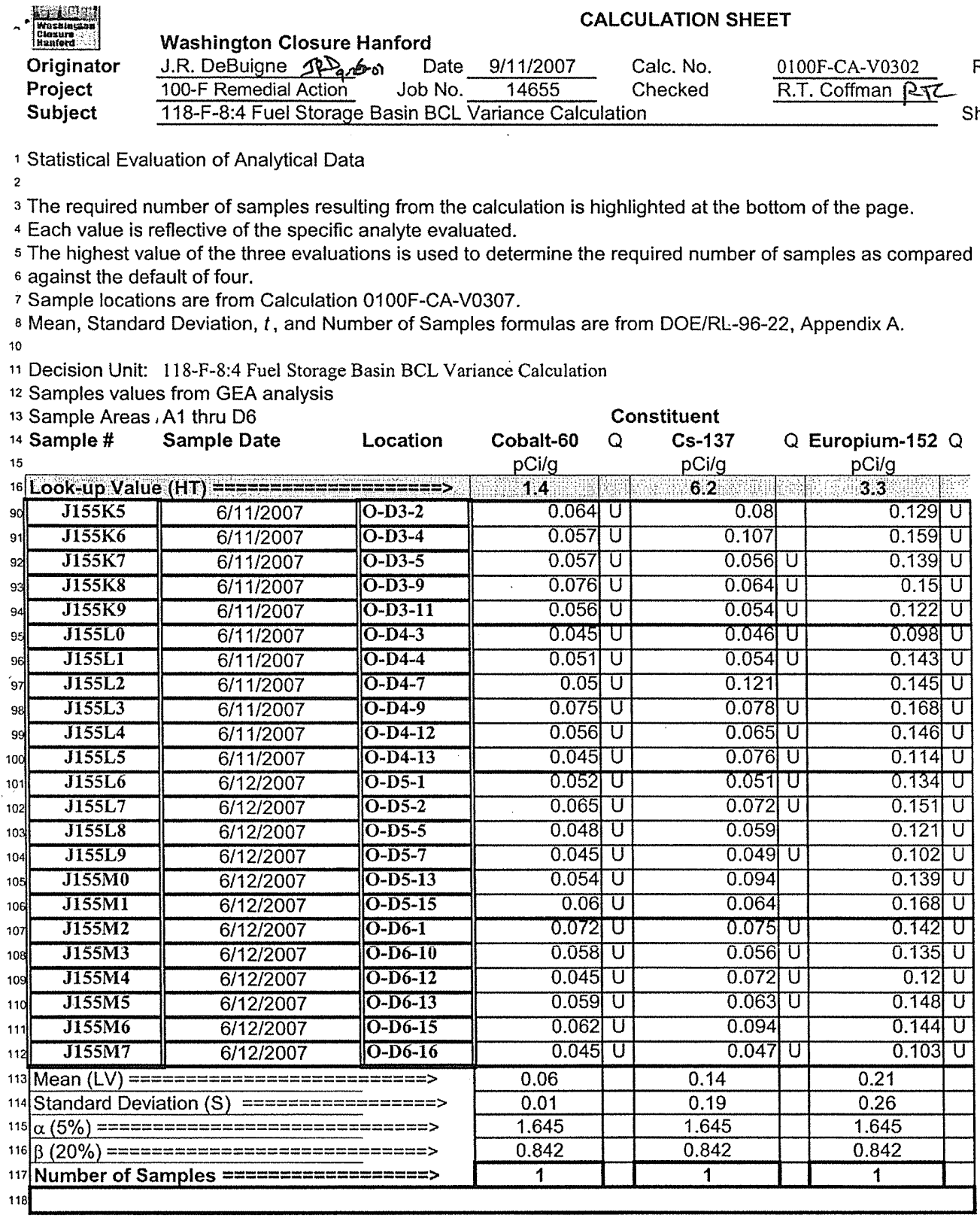

118-F-8_4 FSB BCL 


\section{CALCULATION COVER SHEET}

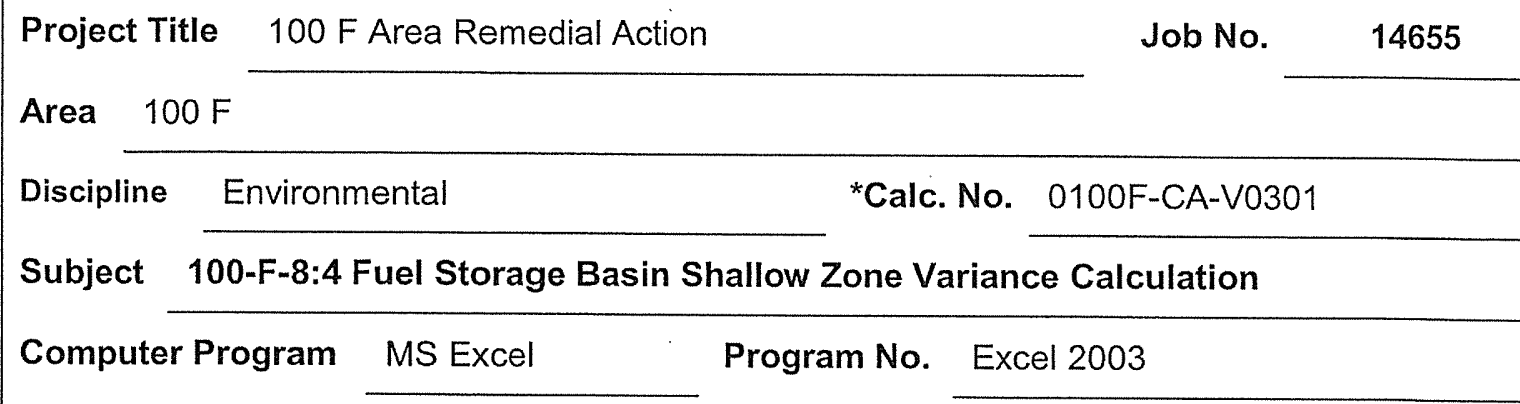

The attached calculations have been generated to document compliance with established cleanup levels. These calculations should be used in conjunction with other relevant documents in the administrative record.

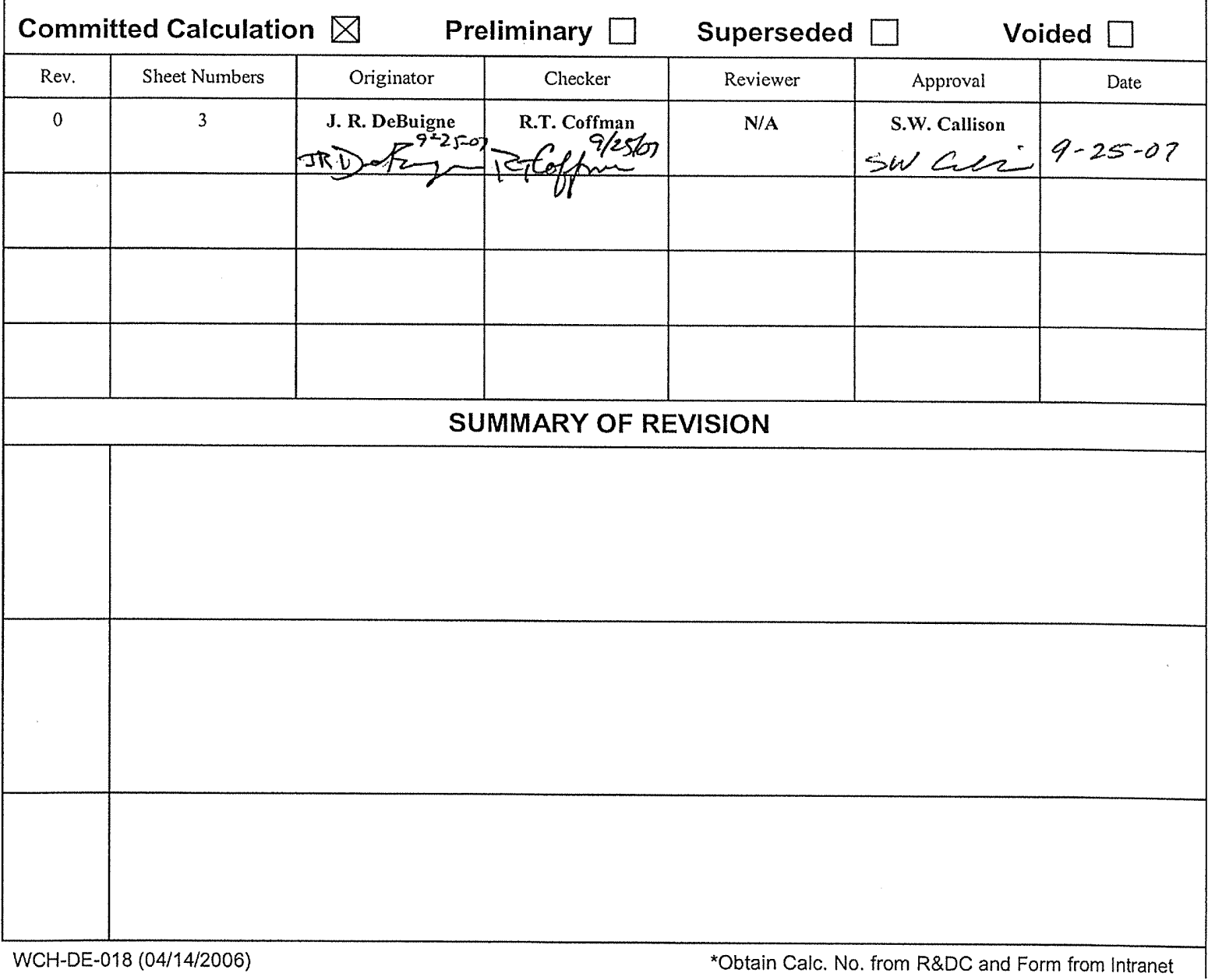


Conclusion:

2 The required number of samples calculated (1sample) for each decision sub-unit is less than the default number 3 (4 samples) specified in the DOE/RL-96-22, Rev 4. Therefore, the default number of samples will be collected 4 from each shallow zone decision sub-unit.

6 Problem:

7 Calculate the number of close out samples required for 118-F-8:4 Fuel Storage Basin Shallow Zone Decision Unit 8 verification sampling as required in "100 Area Remedial Action Sampling and Analysis Plan" (DOE/RL-96-22, 9 Rev 4) and "Instruction Guide for the Remediation of 100 Areas Waste Sites" (0100X-IG-G0001, Rev 5).

11 Given:

12 1) Sample locations for the 118-F-8:4 Fuel Storage Basin Shallow Zone Decision Unit are identified on the 118-F. 13 8:4 Shallow and Deep Zone, Overburden Sampling Plan, Calculation number 0100F-CA-V0307, Rev. 0.

14 2) Lookup values from DOE/RL-96-22, Rev 4.

15 3) Sample Design requirements from DOE/RL-96-22, Rev 4 and 0100X-1G-G0001, Rev 5.

16 4) Field sampling information from sampling logbooks EFL-1174-2 and EFL-1174-3.

18 Solution:

19 Calculation methodology is described in Appendix A of DOE/RL-96-22, Rev 4. Data from attached worksheets 20 are used to calculate the required number of closeout samples. Variance calculation is based on the same three 21 isotopes used to develop the statistical approach in DOE/RL-96-22, Rev 4. The statistical design is based on the 22 premise that these isotopes are the predominant components of the contamination and are representative of the 3 contamination distribution.

2 Shallow Zone Required Number of Samples Calculation

Calc. Summary 


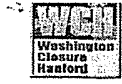

Originator

Project

Subject
Washington Closure Hanford

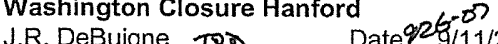
100-F Remedial Actic Job No.

CALCULATION SHEET

1 Statistical Evaluation of Analytical Data

3 The required number of samples resulting from the calculation is highlighted at the bottom of the page.

4 Each value is reflective of the specific analyte evaluated.

5 The highest value of the three evaluations is used to determine the required number of samples as compared

6 against the default of four.

7 Sample locations are from Calculation 0100F-CA-V0307.

8 Mean, Standard Deviation, $t$, and Number of Samples formulas are from DOE/RL-96-22, Appendix A.

10

11 Decision Unit: 118-F-8:4 Fuel Storage Basin Shallow Zone Variance Calculation

12 Samples values from GEA analysis

13 Sample Areas ، A1 thru A4

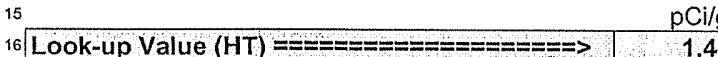

Constituent $\mathrm{pCi} / \mathrm{g} \quad \mathrm{pCi} / \mathrm{g}$

Q Europium-152 Q

$\mathrm{pCi} / \mathrm{g} \quad \mathrm{pCi} / \mathrm{g}$

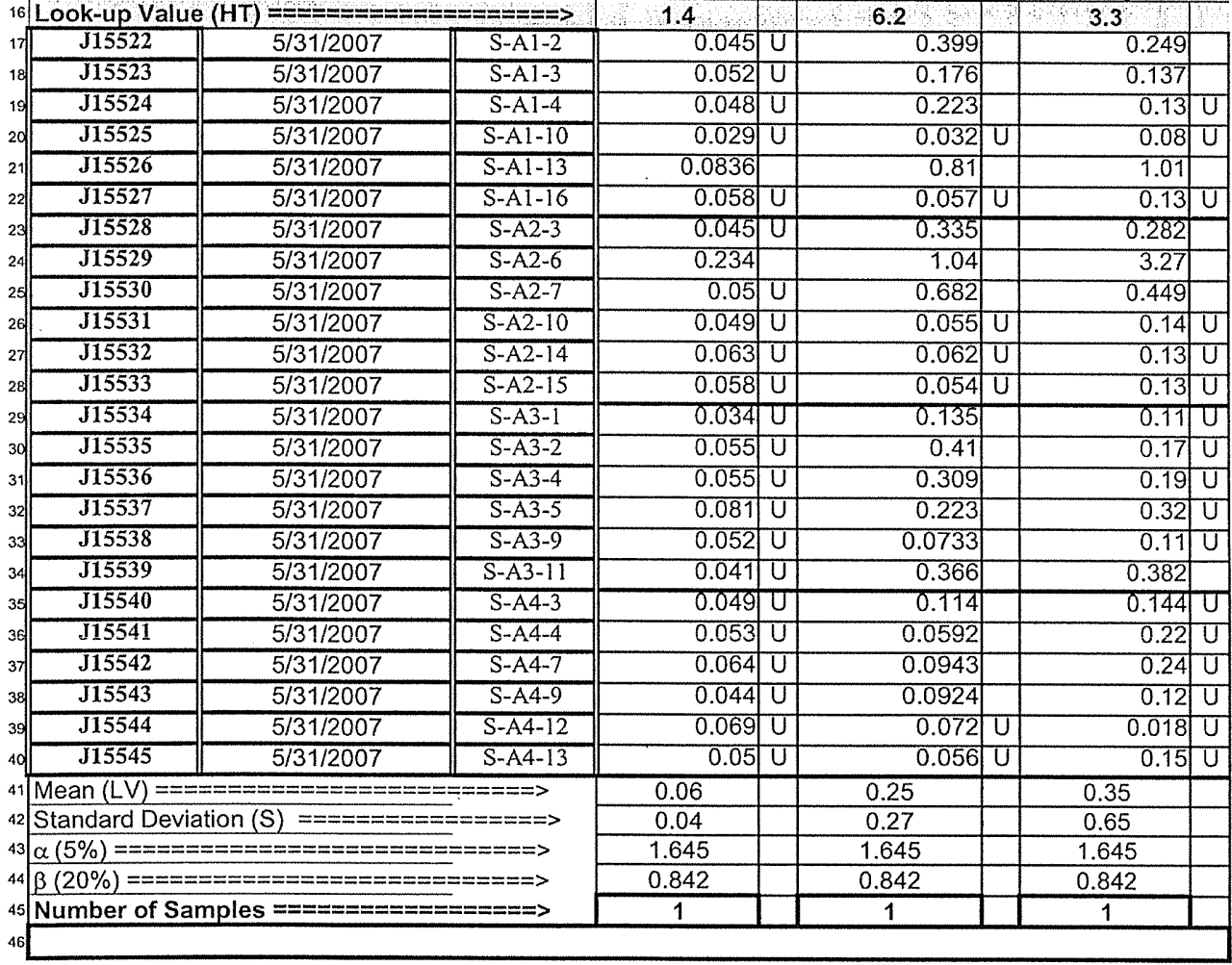

118-F-8_4 FSB Shallow Zone 


\section{APPENDIX D}

\section{DATA QUALITY ASSESSMENT}

D-i 
CVP-2007-00004

Rev. 0

D-ii 


\section{VERIFICATION SAMPLING DATA QUALITY ASSESSMENT}

A data quality assessment (DQA) was performed to compare the verification sampling approach and resulting analytical data with the sampling and data requirements specified in the site-specific sample design (DOE-RL 2005). A review of the sample design (DOE-RL 2005), the field logbooks (WCH 2007a, 2007b), and applicable analytical data packages has been performed as part of this DQA. This DQA was performed in accordance with site-specific data quality objectives found in the 100 Area Remedial Action Sampling and Analysis Plan (SAP) (DOE-RL 2001).

To ensure quality data, the SAP data assurance requirements and the data validation procedures for chemical and radiochemical analysis (BHI 2000a, 2000b) are used as appropriate. This review involves evaluation of the data to determine if they are of the right type, quality, and quantity to support the intended use (i.e., cleanup verification decisions). The DQA completes the data life cycle (i.e., planning, implementation, and assessment) that was initiated by the data quality objectives process (EPA 2000).

The cleanup verification sampling approach for the 118-F-8:4 Fuel Storage Basin included a sample design with multiple subunit areas. All samples were collected per the sample design. Verification sample data collected at the 118-F-8:4 waste site(s) were provided by the laboratories in eight sample delivery groups (SDGs). For the deep zone excavation, verification sample data were provided in two SDGs: SDG K0816 and SDG J00113. SDG K0816 was submitted for third-party validation. For the shallow zone excavation, verification sample data were provided in two SDGs: SDG K0829 and SDG J00115. For the below contaminant level (BCL) stockpiles, verification sample data was provided in five SDGs: SDG K0827, SDG K0829, SDG K0830, SDG J00116, and SDG K0844. No major deficiencies were identified in the analytical data set. Minor deficiencies are discussed below.

\section{SDG K0816}

This SDG comprises five field samples (J15515-J15519) collected from the 118-F-8:4 deep zone excavation. One field duplicate pair is included in this SDG (J15517/ J15518). These samples were analyzed for inductively coupled plasma (ICP) metals, mercury, hexavalent chromium, and polychlorinated biphenyls (PCBs) by gamma spectroscopy and alpha spectroscopy; total strontium by beta counting; and tritium, carbon-14, and nickel-63 by liquid scintillation counting (LSC). SDG K0816 was submitted for third-party validation. No major deficiencies were found in SDG K0816. Minor deficiencies are as follows:

No matrix spike (MS) analysis was performed for tritium or for carbon-14. Third-party validation qualified all tritium and carbon-14 results as estimated and flagged "J." The data are useable for decision-making purposes. 
No laboratory control sample (LCS) analysis was performed for curium-242. Third-party validation qualified all curium-242 results as estimated and flagged "J". The data are useable for decision-making purposes.

Reported analytical detection levels are compared against the required quantitation limits to ensure that laboratory detection levels meet the required criteria. In the radiochemical analysis, one result exceeded the required quantitation limit. Under the Washington Closure Hanford statement of work, no qualification is required.

In the ICP metals analysis, the LCS recovery for silicon is below the acceptance criteria at $70.4 \%$. The silicon data for SDG K0816 were qualified as estimated with a "J" flag by third-party validation. Estimated data are useable for decision-making purposes.

The MS recoveries for four ICP metals (aluminum, iron, antimony, and silicon) do not meet project acceptance criteria. For most of these analytes, the spiking concentration was insignificant compared to the native concentration in the sample from which the MS was prepared. The deficiency in the MS is a reflection of the analytical variability of the native concentration rather than a measure of the recovery from the sample. To confirm quantitation, post-digestion spikes and serial dilutions were prepared for each analyte with results ranging between $102.0 \%$ and $122.2 \%$. Antimony did not have mismatched spike and native concentrations in the original MS. The original MS recovery for antimony was $68.2 \%$. The antimony data for SDG K0816 were qualified as estimated with a "J" flag by third-party validation. Estimated data are useable for decision-making purposes.

\section{SDG J00113}

This SDG comprises one field sample (J15521), a split of sample J15517 from SDG K0816. This sample was analyzed at Severn Trent Laboratories, Inc. The analyses included ICP metals, mercury, hexavalent chromium, and PCBs by gamma spectroscopy and alpha spectroscopy; total strontium by gas proportional counting; and tritium, carbon-14, and nickel-63 by LSC. No major deficiencies were found in SDG J00113. Minor deficiencies are as follows:

For the alpha spectroscopy, the original analysis had no tracer yield for americium-241 and curium-243/244. The sample was reanalyzed, and the results from the reanalysis are reported. Also, curium-243/244 was reported in the method blank (MB) at a concentration of a similar magnitude to the concentration reported in the field sample. The curium-243/244 result may be considered estimated. The data are acceptable for decision-making purposes.

For the gamma spectroscopy, insufficient sample material was available to prepare a laboratory duplicate of sample J15521. The duplicate result was obtained by recounting sample J15521 using a different detector. 
In the ICP metals analysis, the LCS recovery for silicon is below the acceptance criteria at $63.0 \%$. The silicon result for the field sample may be considered estimated. Estimated data are considered acceptable for the intended use of the data.

In addition, the MS and/or matrix spike duplicate (MSD) recoveries for seven ICP metals (aluminum, chromium, iron, manganese, molybdenum, antimony, and silicon) do not meet project acceptance criteria. For aluminum, iron, and manganese, the spiking concentration was insignificant compared to the native concentration in the sample from which the MS was prepared. The deficiency in the MS and/or MSD is a reflection of the analytical variability of the native concentration rather than a measure of the recovery from the sample. Antimony, chromium, molybdenum, and silicon did not have mismatched spike and native concentrations in the original MS. The original MS and MSD recoveries for antimony were $69 \%$ and $71 \%$, respectively. The original MS recovery for chromium was $73 \%$. The original MS and MSD recoveries for molybdenum were $52 \%$, and $53 \%$, respectively. The original MS recovery for silicon was $38 \%$, and the MSD was $29 \%$. The data for these analytes in SDG J00113 may be considered estimated. Estimated data are useable for decision-making purposes.

\section{SDG K0829}

This SDG comprises five field samples (J15510 - J15514) collected from the 118-F-8:4 shallow zone excavation, and six field samples (J15578 - J15583) collected from the below cleanup level (BCL) stockpiles. One field duplicate pair is included in this SDG (J15510/ J15511). These samples were analyzed for ICP metals, mercury, hexavalent chromium, and PCBs by gamma spectroscopy and alpha spectroscopy; total strontium by beta counting; and tritium, carbon-14, and nickel-63 by LSC. No major deficiencies were found in SDG K0829. Minor deficiencies are as follows:

In the ICP metals analysis, the LCS recovery for silicon is below the acceptance criteria at $61.9 \%$. The silicon sample results may be considered estimated. Estimated data are considered acceptable for the intended use of the data.

Also, sodium was reported in the MB at a concentration below the contract-required quantitation limit (CRQL) but not less than one-fifth of the concentration reported in sample J15579 (i.e., the field sample concentration is low enough that the MB concentration is of similar magnitude). The sodium result for sample J15579 may be considered estimated. Estimated data are acceptable for decision-making purposes.

In addition, the MS recoveries for five ICP metals (aluminum, iron, manganese, antimony, and silicon) do not meet project acceptance criteria. For most of these analytes, the spiking concentration was insignificant compared to the native concentration in the sample from which the MS was prepared. The deficiency in the MS is a reflection of the analytical variability of the native concentration rather than a measure of the recovery from the sample. To confirm quantitation, post-digestion spikes and serial dilutions were prepared for each analyte with results ranging between $100.1 \%$ and $106.4 \%$. Antimony did not have mismatched spike and native 
concentrations in the original MS. The original MS recovery for antimony was $62.2 \%$. The antimony data for SDG K0829 may be considered estimated. Estimated data are useable for decision-making purposes.

\section{SDG J00115}

This SDG comprises one field sample (J15520), a split of sample J15510 from SDG K0829. This sample was analyzed at Severn Trent Laboratories, Inc. The analyses included ICP metals, mercury, hexavalent chromium, and PCBs by gamma spectroscopy and alpha spectroscopy; total strontium by gas proportional counting; and tritium, carbon-14, and nickel-63 by LSC. No major deficiencies were found in SDG J00115. Minor deficiencies are as follows:

For the alpha spectroscopy, the original analysis had no tracer recovery in the sample. The sample was reanalyzed, and the tracer recovery was acceptable for all analytes except the duplicate for uranium-234, uranium-235, and uranium-238. The tracer yield for the duplicate on the uranium isotopes was $19 \%$. The results from the reanalysis are reported. The data are acceptable for decision-making purposes.

For the gamma spectroscopy, insufficient sample material was available to prepare a laboratory duplicate of sample J15520. The duplicate result was obtained by recounting sample $\mathrm{J} 15520$ on a different detector.

The nickel-63 LCS recovery was below the acceptance criteria, at $74 \%$. The nickel-63 sample results may be considered estimated. Estimated data are useable for decisionmaking purposes.

In the ICP metals analysis, the LCS recovery for silicon is below the acceptance criteria at $59 \%$. The silicon sample results may be considered estimated. Estimated data are considered acceptable for the intended use of the data.

In addition, the MS recoveries for eight ICP metals (aluminum, barium, chromium, iron, manganese, antimony, silicon, and zinc) do not meet acceptance criteria. For aluminum, iron, and manganese, the spiking concentration was insignificant compared to the native concentration in the sample from which the MS was prepared. For these analytes, the deficiency in the MS is a reflection of the analytical variability of the native concentration rather than a measure of the recovery from the sample. Antimony, barium, chromium, silicon, and zinc did not have mismatched spike and native concentrations in the original MS. The original MS and MSD recoveries for antimony were $49 \%$ and $48 \%$, respectively. The original MS and MSD recoveries for silicon were $16 \%$ and $54 \%$, respectively. The MS recoveries for barium and chromium are both $72 \%$, and for zinc the recovery is $71 \%$. The antimony, barium, chromium, silicon, and zinc data for sample $\mathbf{J 1 5 5 2 0}$ may be considered estimated. The data are useable for decision-making purposes. 


\section{SDG K0827}

This SDG comprises four verification samples (J15574-J15577) from the BCL stockpiles. These samples were analyzed for ICP metals, mercury, hexavalent chromium, and PCBs by gamma spectroscopy and alpha spectroscopy; total strontium by beta counting; and tritium, carbon-14, and nickel-63 by LSC. No major deficiencies were found in SDG K0827. Minor deficiencies are as follows:

The laboratory duplicate relative percent difference (RPD) for hexavalent chromium is $79.4 \%$, exceeding the acceptance criteria $(30 \%)$. The primary result was from the duplicate pair below the detection limit, and the duplicate result was just above the detection limit. When the duplicate pair is near the detection limit, analysis of RPDs is not considered to be useful in the precision determination. The data are useable for decision-making purposes.

In the ICP metals analysis, the MS recoveries for four analytes (aluminum, iron, manganese, and antimony) do not meet acceptance criteria. For aluminum, iron, and manganese, the spiking concentration was insignificant compared to the native concentration in the sample from which the MS was prepared. For these analytes, the deficiency in the MS is a reflection of the analytical variability of the native concentration rather than a measure of the recovery from the sample. To confirm quantitation, postdigestion spikes and serial dilutions were prepared for all four analytes with results in the range of $93.5 \%$ to $109.4 \%$. Antimony did not have mismatched spike and native concentrations in the original MS. The original MS recovery for antimony is $65.8 \%$. The antimony data for SDG K0827 may be considered estimated. Estimated data are useable for decision-making purposes.

The RPD calculated for nickel (37.1\%) in the laboratory duplicate pair (sample J15577 and $\mathrm{J} 15577$ duplicate) are above the acceptance criteria of $30 \%$. Elevated RPDs in environmental soil samples are generally attributed to heterogeneities in the sample matrix and not to deficiencies in the laboratory procedures. The data are useable for decision-making purposes.

\section{SDG K0830}

This SDG comprises seven verification samples (J15584-J15590) from the BCL stockpiles. One field duplicate pair is included in this SDG (J15589/ J15590). These samples were analyzed for ICP metals, mercury, hexavalent chromium, and PCBs by gamma spectroscopy and alpha spectroscopy; total strontium by beta counting; and tritium, carbon-14, and nickel-63 by LSC. No major deficiencies were found in SDG K0830. Minor deficiencies are as follows:

In the ICP metals analysis, sodium was reported in the MB at a concentration below the CRQL but not less than one-fifth of the concentration reported in all samples for SDG K0830 (i.e., the field sample concentration is low enough that the MB concentration is of 
similar magnitude). The sodium result for SDG K0830 may be considered estimated. Estimated data are acceptable for decision-making purposes.

In addition, the MS recoveries for five ICP metals (aluminum, iron, manganese, antimony, and silicon) do not meet acceptance criteria. For aluminum, iron, manganese, and silicon, the spiking concentration was insignificant compared to the native concentration in the sample from which the MS was prepared. For these analytes, the deficiency in the MS is a reflection of the analytical variability of the native concentration rather than a measure of the recovery from the sample. Antimony did not have mismatched spike and native concentrations in the original MS. The original MS recovery for antimony is $52.7 \%$. The antimony data for SDG K0830 may be considered estimated. The data are useable for decision-making purposes.

The laboratory duplicate RPD for hexavalent chromium is $143 \%$, exceeding the acceptance criteria (30\%). The primary result was from the duplicate pair below the detection limit, and the duplicate result was just above the detection limit. When the duplicate pair is near the detection limit, analysis of RPDs is not considered to be useful in the precision determination. The data are useable for decision-making purposes.

The RPD calculated for plutonium-239/240 in the laboratory duplicate pair is above the acceptance criteria at $200 \%$. Elevated RPDs in environmental soil samples are generally attributed to heterogeneities in the sample matrix and not to deficiencies in the laboratory procedures. The data are useable for decision-making purposes.

\section{SDG J00116}

This SDG comprises one field sample (J15591), a split of sample J15589 from SDG K0829. This sample was analyzed at Severn Trent Laboratories, Inc. The analyses included ICP metals, mercury, hexavalent chromium, and PCBs by gamma spectroscopy and alpha spectroscopy; total strontium by gas proportional counting; and tritium, carbon-14, and nickel-63 by LSC. No major deficiencies were found in SDG J00116. Minor deficiencies are as follows:

For the gamma spectroscopy, insufficient sample material was available to prepare a laboratory duplicate of sample J15591. The duplicate result was obtained by recounting sample J15591 using a different detector.

For the nickel-63 analysis, the laboratory reported that the results for the duplicates from the original analysis did not agree. The sample was reanalyzed, and the results from the reanalysis are reported. The data are acceptable for decision-making purposes.

Elevated RPDs were reported for many radionuclide laboratory duplicates for sample J15591. For the isotopes with RPDs exceeding the acceptance criteria $(30 \%)$, the results were all below the detection limits or near the detection limits. When the 
duplicate pair is near the detection limit, analysis of RPDs is not considered to be useful in the precision determination. The data are useable for decision-making purposes.

The MB for curium-243/244 is greater than the practical quantitation limit, suggesting a high bias in the data. However, the data are listed as nondetected, and a high bias has no affect on nondetected analytical data. The data are useable for decision-making purposes.

The MS recovery for hexavalent chromium is out of acceptance criteria, with a result of $63 \%$. The LCS and blank spike results for hexavalent chromium were within acceptance criteria. The hexavalent chromium data for sample J15591 may be considered estimated. The data are useable for decision-making purposes.

Sample J15591 required an instrument dilution for aluminum, boron, iron, potassium, sodium, and silicon in the ICP metals analysis due to the sample matrix. The reporting limits were adjusted to reflect the necessary dilution for these analytes.

In the ICP metals analysis, potassium was reported in the MB at a concentration below the CRQL but not less than one-fifth of the concentration reported in sample J15591 (i.e., the field sample concentration is low enough that the MB concentration is of similar magnitude). The potassium result for sample J15591 may be considered estimated. Estimated data are acceptable for decision-making purposes.

In addition, the MS recoveries for nine ICP metals (aluminum, cadmium, iron, magnesium, manganese, antimony, selenium, silicon, and zinc) do not meet acceptance criteria. For aluminum, iron, and manganese, the spiking concentration was insignificant compared to the native concentration in the sample from which the MS was prepared. For these analytes, the deficiency in the MS is a reflection of the analytical variability of the native concentration rather than a measure of the recovery from the sample. Antimony, cadmium, magnesium, selenium, silicon, and zinc did not have mismatched spike and native concentrations in the original MS. The original MS and MSD recoveries for antimony were $43 \%$ and $47 \%$, respectively. The original MS and MSD recoveries for silicon were $16 \%$ and $31 \%$, respectively. The MS recoveries for cadmium, magnesium, selenium, and zinc are $71 \%, 66 \%, 73 \%$, and $72 \%$, respectively. The antimony, cadmium, magnesium, selenium, silicon, and zinc data for sample $\mathrm{J} 15591$ may be considered estimated. The data are useable for decision-making purposes.

\section{SDG K0844}

This SDG comprises one focus sample (J15690) from the 118-F-8:4 BCL stockpiles. This sample was analyzed for tritium and carbon-14 by LSC. The J15574 sample result reported in SDG K0827 indicated that the stockpile soil in area A1 of the overburden/BCL decision unit had detections of carbon-14 in excess of the remedial action goal. The contaminated soil was removed, and sample J15690 was taken to 
replace the $\mathrm{J} 15574$ carbon-14 result in statistical calculations for verification sampling of the 118-F-8:4 site. No major or minor deficiencies were found in SDG K0884.

\section{FIELD QUALITY ASSURANCE/QUALITY CONTROL}

RPD evaluations of main sample(s) versus the laboratory duplicate(s) are routinely performed and reported by the laboratory. Any deficiencies in those calculations are reported by SDG in the previous sections.

Field quality assurance (QA)/quality control (QC) measures are used to assess potential sources of error and cross contamination of samples that could bias results. Field QA/QC samples, listed in the field logbooks (WCH 2007a, 2007b), are summarized in Table D-1. The main and QA/QC sample results are presented in Appendix C.

Table D-1. Field Quality Assurance/Quality Control Samples.

\begin{tabular}{|l|c|c|c|}
\hline \multicolumn{1}{|c|}{ Sample Area } & Main Sample & $\begin{array}{c}\text { Duplicate } \\
\text { Sample }\end{array}$ & $\begin{array}{c}\text { Split } \\
\text { Sample }\end{array}$ \\
\hline Deep zone excavation & $\mathrm{J} 15517$ & $\mathrm{~J} 15518$ & $\mathrm{~J} 15521$ \\
\hline Shallow zone excavation & $\mathrm{J} 15510$ & $\mathrm{~J} 15511$ & $\mathrm{~J} 15520$ \\
\hline BCL Stockpile & $\mathrm{J} 15589$ & $\mathrm{~J} 15590$ & $\mathrm{~J} 15591$ \\
\hline
\end{tabular}

Field duplicate samples are collected to provide a relative measure of the degree of local heterogeneity in the sampling medium, unlike laboratory duplicates that are used to evaluate precision in the analytical process. The field duplicates are evaluated by computing the RPD of the duplicate samples for each contaminant of concern. Only analytes with values above five times the detection limits for both the main and duplicate samples are compared. The $95 \%$ upper confidence limit calculation brief in Appendix $\mathrm{C}$ provides details on duplicate pair evaluation and RPD calculation. The data are suitable for the intended purpose of cleanup verification.

Radionuclides. The RPD calculated for potassium-40 in the excavation deep zone duplicate (J15518) sample is above the acceptance criteria (30\%) at $73 \%$. An elevated RPD such as this, in the analysis of environmental soil samples, is largely attributed to heterogeneities in the soil matrix and only in small part attributed to precision and accuracy issues at the laboratory. The data are useable for decision-making purposes.

Nonradionuclides. The RPDs calculated for aluminum in the excavation shallow zone duplicate (J15511) and split (J15520), and the BCL split (J15591), samples are above the acceptance criteria (30\%) at $39 \%, 62 \%$, and $45 \%$, respectively. The RPDs calculated for barium in the excavation shallow zone duplicate (J15511) and split (J15520) samples are above the acceptance criteria (30\%) at $32 \%$ and $47 \%$, respectively. The RPD calculated for calcium in the excavation shallow zone split 
(J15520) sample is above the acceptance criteria (30\%) at 31\%. The RPDs calculated for chromium in the excavation shallow zone duplicate (J155511) and split (J15520) samples and the excavation deep zone split (J15521) sample are above the acceptance criteria, at $45 \%, 76 \%$, and $39 \%$, respectively. The RPDs calculated for iron in the excavation shallow zone duplicate (J155511) and split (J15520) samples and the BCL split (J15591) sample are above the acceptance criteria, at 36\%, 60\%, and $53 \%$, respectively. The RPDs calculated for magnesium in the excavation shallow zone duplicate (J155511) and split (J15520) samples and the excavation deep zone split (J15521) sample are above the acceptance criteria, at $32 \%, 57 \%$, and $31 \%$, respectively. The RPD calculated for manganese in the excavation shallow zone split (J15520) sample is above the acceptance criteria (30\%) at 44\%.The RPDs calculated for silicon in the BCL (J15591) and excavation deep zone (J15521) split samples are above the acceptance criteria (30\%) at $74 \%$ and $63 \%$, respectively. The RPDs calculated for vanadium in the excavation shallow zone duplicate (J155511) and split (J15520) samples and the BCL split (J15591) sample are above the acceptance criteria, at $31 \%, 61 \%$, and $48 \%$, respectively. Elevated RPDs such as these in the analysis of environmental soil samples are largely attributed to heterogeneities in the soil matrix and only in small part attributed to precision and accuracy issues at the laboratory. The data are useable for decision-making purposes.

RPDs for the remaining radionuclides and nonradionuclide analytes are not calculated because an evaluation of the data shows the analytes are not detected in both the main and duplicate sample at more than five times the target detection limit (TDL). RPDs of analytes detected at low concentrations (less than five times the detection limit) are not considered to be indicative of the analytical system performance. The data are useable for decision-making purposes.

A secondary check of the data variability is used when one or both of the samples being evaluated (main and duplicate) is less than five times the TDL, including undetected analytes. In these cases, a control limit of \pm 2 times the TDL is used (Appendix C) to indicate that a visual check of the data is required by the reviewer. For the excavation deep zone split samples, the cesium-137 result required this check. For the excavation shallow zone split sample, cobalt required this check. For the overburden/BCL stockpile split samples, the boron results required this check. These results are attributed to heterogeneities in the sample matrix from which the samples were collected. A visual inspection of all of the data is also performed. No additional major or minor deficiencies are noted. The data are useable for decision-making purposes.

\section{SUMMARY}

Limited, random, or sample matrix-specific influenced batch QC issues such as those discussed above are a potential for any analysis. The number and types seen in these data sets are within expectations for the matrix types and analyses performed. The DQA review of the 118-F-8:4 verification sampling data found that the analytical results are accurate within the standard errors associated with the analytical methods, 
sampling, and sample handling. The DQA review for 118-F-8:4 waste site concludes that the reviewed data are of the right type, quality, and quantity to support the intended use. Detection limits' precision, accuracy, and sampling data group completeness were assessed to determine if any analytical results should be rejected as a result of QA and QC deficiencies. The analytical data were found acceptable for decision-making purposes. The verification sample analytical data are stored in the Environmental Restoration project-specific database prior to being submitted for inclusion in the Hanford Environmental Information System database. The verification sample analytical data are also summarized in Appendix C.

\section{REFERENCES}

BHI, 2000a, Data Validation Procedure for Chemical Analysis, BHI-01435, Rev. 0, Bechtel Hanford, Inc., Richland, Washington.

BHI, 2000b, Data Validation Procedure for Radiochemical Analysis, BHI-01433, Rev. 0, Bechtel Hanford, Inc., Richland, Washington.

DOE-RL, 2005, 100 Area Remedial Action Sampling and Analysis Plan, DOE/RL-96-22, Rev. 4, U.S. Department of Energy, Richland Operations Office, Richland, Washington.

EPA, 2000, Guidance for Data Quality Assessment, EPA QA/G-9, QA00 Update, U.S. Environmental Protection Agency, Office of Environmental Information, Washington, D.C.

WCH, 2007a, 100F Remedial Sampling, Logbook EFL-1174-2, pp. 95, Washington Closure Hanford, Richland, Washington.

WCH, 2007b, 100F Remedial Sampling, Logbook EFL-1174-3, pp. 3-10 and 16, Washington Closure Hanford, Richland, Washington. 


\section{DISTRIBUTION}

U.S. Department of Energy

Richland Operations Office

D. C. Smith (5)

DOE-RL Public Reading Room

A3-04

$\mathrm{H} 2-53$

Fluor Hanford, Inc.

J. P. Shearer (WIDS) E6-35

Washington Closure Hanford

S. W. Clark

$\mathrm{H} 4-23$

J. M. Capron

$\mathrm{H} 4-23$

L. A. Dietz

$\mathrm{H} 4-22$

L. D. Habel

$\mathrm{H} 4-23$

J. D. Fancher

X9-07

M. T. Hughes

$\mathrm{H} 4-23$

K. A. Maki

X9-07

Document Control

H4-11

Hanford Technical Library

P8-55 
CVP-2007-00004

Rev. 0

Distr-2 